\title{
Music Similarity Models applied to Cover Song Identification and Classification
}

\author{
Roberto Piassi Passos Bodo \\ THESIS PRESENTED \\ TO \\ Institute OF MATHEMATICS AND StATistiCs \\ OF \\ UNIVERSITY OF SÃO PAULO \\ FOR \\ OBTAINING THE TITLE \\ $\mathrm{OF}$ \\ DoCTOR OF SCIENCE
}

Program: Graduate School in Computer Science

Advisor: Prof. Dr. Marcelo Gomes de Queiroz

This study was financed in part by the Coordenação de Aperfeiçoamento de Pessoal de Nível Superior - Brasil (CAPES) - Finance Code 001

São Paulo, August 2021 


\section{Music Similarity Models applied to Cover Song Identification and Classification}

This version of the thesis includes the corrections and modifications suggested by the Examining Committee during the defense of the original version of the work, which took place on July 28th, 2021. A copy of the original version is available at the Institute of Mathematics and Statistics of the University of São Paulo.

Examining Committee:

- Prof. Dr. Marcelo Queiroz - USP

- Prof. Dr. Emmanouil Benetos - QMUL

- Prof. Dr. Rodrigo Schramm - UFRGS

- Prof. Dr. Regis Faria - USP

- Prof. Dr. Tiago Tavares - UNICAMP 


\section{Acknowledgment}

This project would not have been possible without the support of Ana Clara Duarte Gavião,

Beatriz Maria Ferraz Noronha, Emmanouil Benetos, Marcelo Queiroz, my parents Luciana Piassi Passos Bodo and Roberto Bodo, and all my dear friends. 


\section{Abstract}

BODO, R. P. P. Music Similarity Models applied to Cover Song Identification and Classification. 2021. 165 p. Thesis (Doctorate) - Institute of Mathematics and Statistics, University of São Paulo, São Paulo, 2021.

The spread of digital music allowed the appearance of datasets with millions of music files. The processing of this huge number of audio files is carried out with techniques of Music Information Retrieval (MIR) that work directly with the audio content. The MIR task of most interest in this project is the modelling of Music Similarity. Our proposed approach follows this pipeline: extract audio features, aggregate local features into global features, and compute the similarities of every pair of songs from the dataset being processed. According to this approach, a triple $\left\{\right.$ extractor $_{i}$, aggregator $_{j}$, distance $\left._{k}\right\}$ defines a music similarity model, and our main goal is to investigate the ability of similarity models to distinguish audio files from different classes. The music similarity models are also used to address specific problems such as Cover Song Identification (CSI), which is an MIR application related to Music Similarity, and the closely-related Cover Song Classification (CSC) problem. MIR-related techniques, such as Dataset Modifications and Matrix Fusion, are explored in the context of improving the results of music similarity models. This work presents several contributions, among which a comprehensive benchmark of music similarity models; the definition of new similarity matrices within CSC as a solution approach to the CSI problem; the exploration of different types of dataset modifications and an investigation of their effect on music similarity metrics; and the fusion of similarity matrices computed from secondary datasets obtained via source separation. The experiments presented produced encouraging results, indicating that the methods proposed in this thesis point towards novel approaches to Music Similarity that are worth further investigation and development.

Keywords: computer music, music information retrieval, music similarity, cover song identification, cover song classification. 


\section{Resumo}

BODO, R. P. P. Modelos de Similaridade Musical aplicados à Identificação e Classificação de Covers. 2021. 165 f. Tese (Doutorado) - Instituto de Matemática e Estatística, Universidade de São Paulo, São Paulo, 2021.

A difusão da música digital permitiu o surgimento de conjuntos de dados com milhões de arquivos de áudio. O processamento deste enorme número de arquivos é realizado com técnicas de Recuperação de Informação Musical que analisam diretamente o conteúdo do áudio. A tarefa de maior interesse neste projeto é a modelagem de Similaridade Musical. Nossa proposta segue os seguintes passos: extrair descritores de áudio, agregar descritores locais em descritores globais e calcular as distâncias entre todos os pares de músicas dos conjuntos de dados sendo processados. De acordo com essa abordagem, uma tripla $\left\{\right.$ extrator $\left._{i}{\text {, } \text { agregador }_{j} \text {, distância }}_{k}\right\}$ define um modelo de similaridade musical, e nosso principal objetivo é investigar a capacidade dos modelos de similaridade de distinguir arquivos de áudio de classes diferentes. Os modelos de similaridade musical também são usados para resolver problemas específicos, como Identificação de Covers, que é uma aplicação de Similaridade Musical, e Classificação de Covers, que é um problema relacionado com o de Identificação. Outras técnicas de Recuperação de Informação Musical, como Modificação de Conjuntos de Dados e Fusão de Matrizes, são exploradas no contexto de melhorar os resultados de modelos de similaridade musical. Este trabalho apresenta várias contribuições, entre as quais o benchmark de modelos de similaridade musical; a definição de novas matrizes de similaridade no contexto de Classificação de Covers como abordagem de solução para a Identificação de Covers; a exploração de diferentes tipos de modificação de conjuntos de dados e uma investigação de seus efeitos nas métricas de similaridade musical; e a fusão de matrizes de similaridade calculadas a partir de conjuntos de dados secundários obtidos por meio de separação de fontes. Os experimentos apresentados produziram resultados encorajadores, indicando que os métodos propostos nesta tese apontam na direção de abordagens inovadoras que merecem investigação e desenvolvimento adicionais.

Palavras-chave: computação musical, recuperação de informação musical, similaridade musical, identificação de covers, classificação de covers. 


\section{Contents}

List of Figures $\quad$ xiii

List of Tables $\quad$ xvii

1 Introduction $\quad 1$

1.1 Context and Motivation . . . . . . . . . . . . . . . . . . 1

1.2 Problems addressed and Goals . . . . . . . . . . . . . . . . . . . . 2

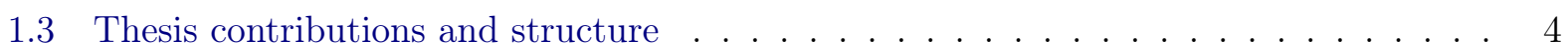

2 Music Similarity $\quad 5$

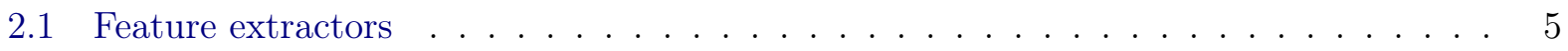

2.2 Feature aggregators . . . . . . . . . . . . . . . . . . . . . 22

2.3 Distance measures . . . . . . . . . . . . . . . . . . 27

2.4 Music similarity quality measures . . . . . . . . . . . . . . . . . . . . 32

3 Cover Song Identification $\quad 35$

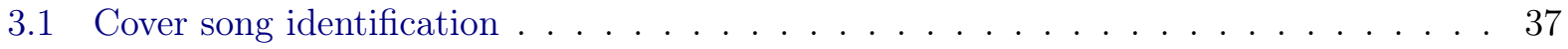

3.1 .1 CSI methods that use tonal features . . . . . . . . . . . . . . . . 37

3.1.2 CSI methods that use symbolic sequences . . . . . . . . . . . . . . . 39

3.1.3 CSI methods that use fusion methods . . . . . . . . . . . . . . . . . 41

3.1.4 Cover song identification quality measures . . . . . . . . . . . . . . . 44

3.2 Cover song classification . . . . . . . . . . . . . . . . . . . . 45

3.2 .1 Cover song classification quality measures . . . . . . . . . . . . . 47

4 Dataset Modifications $\quad 49$

4.1 Segment selection . . . . . . . . . . . . . . . . . . 49

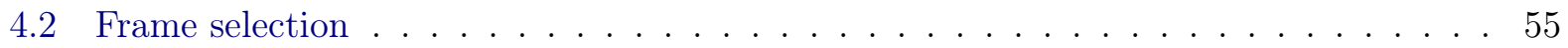

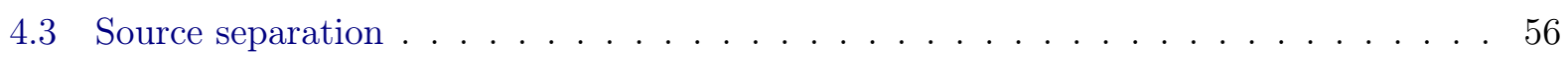

5 Methodology $\quad 59$

5.1 A Framework for Music Similarity and Cover Song Identification . . . . . . . . . . 59

5.2 Cover Song Classification . . . . . . . . . . . . . . . . 63

5.3 Cover Song Identification and Classification . . . . . . . . . . . . . . . . . . 67

5.4 Dataset Modifications . . . . . . . . . . . . . . . . . . 69

5.4 .1 Segment selection . . . . . . . . . . . . . . . . . . . . 69

5.4 Frame selection . . . . . . . . . . . . . . . . 71 
5.4 .3 Source separation . . . . . . . . . . . . . . . . . . . . . . 72

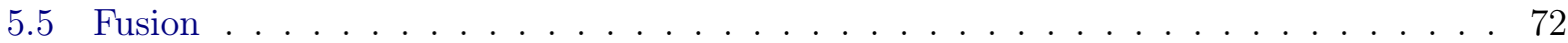

6 Experiments and Results $\quad 75$

6.1 Technical choices . . . . . . . . . . . . . . . . . . . . 75

6.2 Datasets . . . . . . . . . . . . . . . . . . . . 76

6.3 Data rearrangement and cleaning . . . . . . . . . . . . . . . 86

6.4 Experiment \#1: Music Similarity . . . . . . . . . . . . . . . . . . . 87

6.4 .1 Experiment design . . . . . . . . . . . . . . . . . . 87

6.4 .2 Results . . . . . . . . . . . . . . . . . . . . 87

6.4 .3 Discussion . . . . . . . . . . . . . . . . . . . . . . 89

6.5 Experiment \# 2: Cover Song Identification . . . . . . . . . . . . . . . . . . 93

6.5.1 Experiment design . . . . . . . . . . . . . . . . . . . . . 93

6.5 .2 Results . . . . . . . . . . . . . . . . . . . 93

6.5 .3 Discussion . . . . . . . . . . . . . . . . . . . . 96

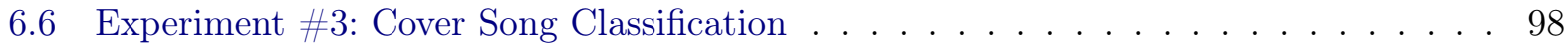

6.6 .1 Experiment design . . . . . . . . . . . . . . . . . . . . . . . . 98

6.6 .2 Results: experiment \#3a . . . . . . . . . . . . . . . . . . . . . . . . . 99

6.6.3 Discussion: experiment \#3a . . . . . . . . . . . . . . . . . . . . . . . . 101

6.6 .4 Results: experiment \#3b . . . . . . . . . . . . . . . . . . . . . 102

6.6.5 Discussion: experiment \#3b . . . . . . . . . . . . . . . . . . . . . . . 107

6.7 Experiment \#4: Cover Song Identification and Classification . . . . . . . . . . . . 108

6.7 .1 Experiment design . . . . . . . . . . . . . . . . . . . . . 108

6.7 .2 Results: experiment \#4a . . . . . . . . . . . . . . . . . . . . . 110

6.7 .3 Discussion: experiment \#4a . . . . . . . . . . . . . . . . . . . . . . . . . . . . 112

6.7 .4 Results: experiment \#4b . . . . . . . . . . . . . . . . . . . . . . . . 112

6.7.5 Discussion: experiment \#4b . . . . . . . . . . . . . . . . . . . . . . . 116

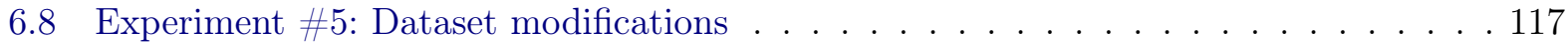

6.8.1 Experiment design . . . . . . . . . . . . . . . . . . . . 117

6.8 .2 Results . . . . . . . . . . . . . . . . . . . . . 118

6.8.3 Discussion . . . . . . . . . . . . . . . . . . . . . 120

6.9 Experiment $\# 6$ : Fusions $\ldots \ldots \ldots \ldots \ldots$

6.9 .1 Experiment design . . . . . . . . . . . . . . . . . . . . 123

6.9 .2 Results . . . . . . . . . . . . . . . . . . . . 124

6.9 .3 Discussion . . . . . . . . . . . . . . . . . . . . . . 126

6.10 Experiment \#7: Theoretical performance bounds $\ldots \ldots \ldots$. . . . . . . 127

7 Conclusions $\quad 133$

7.1 Analysing the Results and Recalling the Goals . . . . . . . . . . . . . . . . . . . 134

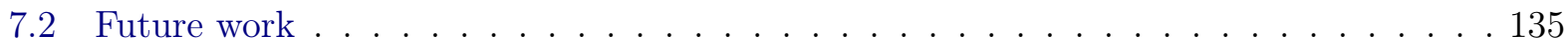

$\begin{array}{ll}\text { A Music Similarity: best models per class } & 137\end{array}$

B Cover Song Identification: top-50 results per dataset 143 
C Cover Song Identification: filtered results per dataset 149

D Cover Song Identification: unique top-50 results 153

E Cover Song Identification: unique filtered results $\quad 155$

$\begin{array}{lr}\text { Bibliography } & 157\end{array}$ 


\section{Acronyms}

CENS Chroma Energy Normalized Statistics. 8, 61, 90, 91, 107

CQT Constant-Q Transform. 6, 7, 61

CSC Cover Song Classification. 3, 4, 45-47, 63, 64, 67, 68, 73, 98, 101, 107-109, 117, 134

CSD Cover Song Detection. 37

CSI Cover Song Identification. 3, 4, 37, 38, 40-42, 44, 45, 51, 54, 56, 62, 63, 65, 67, 68, 72, 73, 83, 84, 93, 96-99, 101, 102, 108, 109, 116, 117, 121, 127, 133-136

CSM Cross-Similarity Matrix. 41, 42

CSR Cover Song Retrieval. 37

DCT Discrete Cosine Transform. 9

DPLA Dynamic Programming Local Alignment. 56

DTW Dynamic Time Warping. 38, 40, 54, 56, 135

EDM Electronic Dance Music. 79

EMD Earth Mover's Distance. 31

GMM Gaussian Mixture Model. 24, 39, 40, 61

HMM Hidden Markov Model. 70

HPCP Harmonic Pitch Class Profile. 37, 38, 41, 42, 46, 51, 56

IICSR Intra-Inter Class Similarity Ratio. 87, 89-92

KL Kullback-Leibler. 30

KNN K-Nearest Neighbors. 41

LCS Longest Common Subsequence. 29, 61, 92, 97

MAP Mean Average Precision. 45, 62, 67, 93, 120, 134

MDR Median Rank. 45, 62, 67, 93 
MFCC Mel-Frequency Cepstral Coefficients. 3, 9, 41, 42, 50, 51, 59, 61, 70, 75, 90, 91

MIDI Musical Instrument Digital Interface. 17, 26

MIR Music Information Retrieval. 1, 3, 5, 69, 82

MLP Multi-Layer Perceptron. 46

MR Mean Rank. 44, 45, 62, 65, 67, 93, 112, 116, 117, 120, 126, 134, 135

MRR Mean Reciprocal Rank. 44, 45, 62, 67, 93

NMF Non-negative Matrix Factorization. 70

OTI Optimal Transposition Index. 41, 62, 91, 97, 98, 134

PCA Principal Component Analysis. 70

PLCA Probabilistic Latent Component Analysis. 70

RMS Root Mean Square. 3, 15, 50, 59, 61, 71, 134

SHS Sub-Harmonic Summation. 40

SI-PLCA Shift-Invariant Probabilistic Latent Component Analysis. 70

SNF Similarity Network Fusion. 41-43, 73, 123, 126

SOM Self-Organizing Map. 135

SSM Self-Similarity Matrix. 40-42, 70

STFT Short-Time Fourier Transform. 5, 6, 8, 61

SVM Support Vector Machine. 46

THPCP Transposed Harmonic Pitch Class Profile. 38

ZCR Zero Crossing Rate. 16, 50, 51, 59, 61 


\section{List of Figures}

2.1 STFT spectrogram from a C-major scale sample performed by a piano. . . . . . . . 6

2.2 Filter bank that maps STFT-bins to pitch classes. . . . . . . . . . . . . . . 6

2.3 STFT-chromagram from a C-major scale sample performed by a piano. . . . . . . . 6

2.4 CQT-spectrogram from a C-major scale sample performed by a piano. . . . . . . . . 7

2.5 Filter bank that maps CQT-bins to pitch classes. . . . . . . . . . . . . 7

2.6 CQT-chromagram from a C-major scale sample performed by a piano. . . . . . . . . 8

2.7 CENS-chromagram from a C-major scale sample performed by a piano. . . . . . . 8

2.8 Filter bank that maps STFT-bins to Mel-frequency bins. . . . . . . . . . . . . . . 9

2.9 Mel-spectrogram from a C-major scale sample performed by a piano. . . . . . . . . . 9

2.10 MFCC extracted from a C-major scale sample performed by a piano. . . . . . . . . . 10

2.11 Spectral centroid values extracted from the same guitar sample before and after applying distortion. . . . . . . . . . . . . . . . . 10

2.12 Spectral bandwidth values extracted from the same guitar sample before and after applying distortion. . . . . . . . . . . . . . . . . 11

2.13 Spectral roll-off values extracted from the same guitar sample before and after ap-

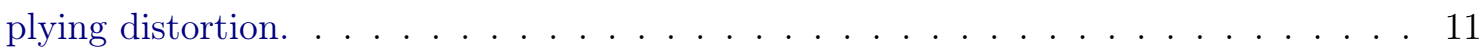

2.14 Spectral flatness values extracted from the same guitar sample before and after applying distortion. . . . . . . . . . . . . . . . . . . . . . . 12

2.15 Spectral contrast values extracted from the same guitar sample before and after applying distortion. . . . . . . . . . . . . . . . . 13

2.16 The tonnetz infinite planar representation of pitch relations. . . . . . . . . . . 14

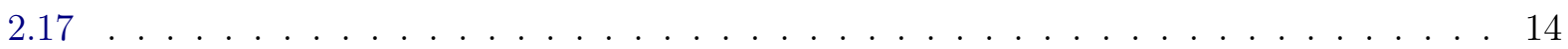

2.18 The three circles of tone relations and the tonal centroid for the A-major chord (reproduced from $[\mathrm{HSG} 06]) \ldots \ldots \ldots \ldots \ldots \ldots$

2.19 The tonnetz feature extracted from a C-major piano sample. . . . . . . . . . . . . 15

2.20 RMS values extracted from the tracks "Broken, Beat \& Scarred" (Metallica) and "Für Elise" (Lang Lang). . . . . . . . . . . . . . . . . . . . . 16

2.21 Zero crossing rate extracted from three basic signals. . . . . . . . . . . . . . . 16

2.22 Fundamental frequencies extracted from the predominant melody of a song using the Melodia software . . . . . . . . . . . . . . . . . . 17

2.23 Segmentation of a fundamental frequency sequence, converted to MIDI note numbers. 17

2.24 Processing pipeline to extract rhythm pattern and related features (reproduced from

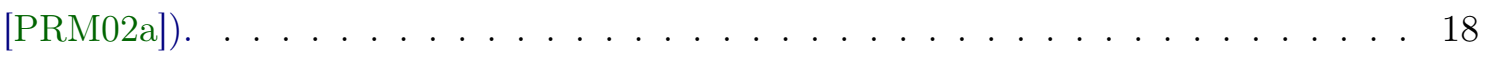

2.25 Rhythm pattern feature extracted for a 120 BPM drum sample. . . . . . . . . . . 19 
2.26 Rhythm histogram feature extracted for a 120 BPM drum sample. . . . . . . . . . . 19

2.27 Temporal rhythm histogram feature extracted for a 120 BPM drum sample. . . . . . 20

2.28 Statistical spectrum descriptor feature extracted for a 120 BPM drum sample. . . . . 20

2.29 Temporal statistical spectrum descriptor feature extracted for a 120 BPM drum sample. 21

2.30 Modulation variance descriptor feature extracted for a 120 BPM drum sample. . . . 22

2.31 Histogram of samples and the Gaussian curve obtained after estimating the param-

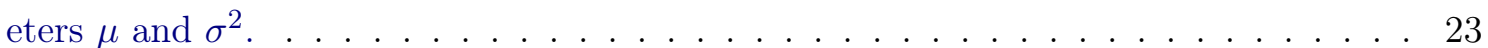

2.32 The discrete difference of the chroma_stft displayed in Figure 2.3. . . . . . . . . . . 23

2.33 Histogram of samples and the estimated two-component GMM. . . . . . . . . . . . . 24

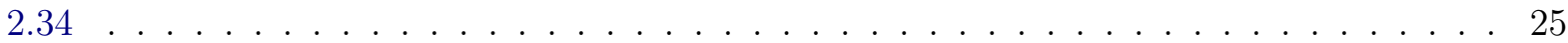

2.35 Markov chain built from a simple melody. . . . . . . . . . . . . . . . 26

2.36 MIDI note number sequence obtained discarding the duration values from the Pitch Contour Segmentation feature. . . . . . . . . . . . . . . . . 26

2.37 Octave abstraction to a melodic line . . . . . . . . . . . . . . . . 26

2.38 Interval abstraction to a melodic line. . . . . . . . . . . . . . . . . . 27

2.39 3-level pitch contour abstraction to a melodic line. . . . . . . . . . . . . . 27

2.405 -level pitch contour abstraction to a melodic line. . . . . . . . . . . . . . . 27

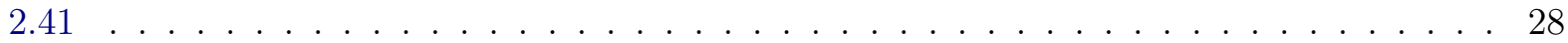

2.42 An example of the longest common subsequence computation. . . . . . . . . . . . . 29

2.43 An example of the edit distance computation. . . . . . . . . . . . . . . . . 30

2.44 Kullback-Leibler distance calculated for pairs of Gaussian distributions. . . . . . . . . 31

2.45 EMD calculated for pairs of Gaussian distributions. . . . . . . . . . . . . . . . 31

2.46 Monte carlo distance calculated for pairs of Gaussian distributions. . . . . . . . . . 32

2.47 Similarity matrix computed for a toy dataset with 20 tracks divided in 5 classes (4 tracks per class). . . . . . . . . . . . . . . . . . . . 33

3.1 Three distinct releases of the album Let It Be by The Beatles. . . . . . . . . . . . . . 35

3.2 Four distinct releases containing songs from the album The Wall by Pink Floyd. . . . 36

3.3 Labels from two singles of All Along the Watchtower originally recorded by Bob Dylan (a), and covered by Jimi Hendrix (b) . . . . . . . . . . . . . . 36

3.4 Labels from two singles of With a Little Help from My Friends originally recorded by The Beatles (a), and covered by Joe Cocker (b) . . . . . . . . . . . 36

3.5 Feature extraction pipeline and segments comparison scheme by Goméz et al. [GHO06]. 38

3.6 Filter bank applied by Jensen et al.[JCEJ08] in order to increase timing invariance. . 39

3.7 The complete processing diagram of the system proposed by Tralie [Tra17]. . . . . . 42

3.8 The parent Self-Similarity Matrix built by Tralie [Tra17] as input to the Similarity Network Fusion algorithm. . . . . . . . . . . . . . . . . . . . . . . . 42

3.9 The considered alignment constraints used by (a) $Q_{\max }$ and (b) $D_{\max }$ (reproduced from $[$ CLX18]) . . . . . . . . . . . . . . . . . . . . . . . . 43

3.10 The complete system diagram as proposed by Chen et al.[CLX18]. . . . . . . . . . . 43

3.11 An illustration of one column from the similarity matrix before and after being ordered, and how to determine the rank of the first retrieved cover of the column's correspondent song. . . . . . . . . . . . . . . . . . . . . . . . 
3.12 The diagram of the Cover Song Classification system developed by Ravuri and Ellis $[\mathrm{RE} 10] \ldots \ldots \ldots \ldots \ldots$

3.13 The complete diagram of the processing steps presented by Salamon et al. [SSG12]. . 47

4.1 An example time series (reproduced from $[\mathrm{SMGA12}]$ ) . . . . . . . . . . . 51

4.2 Time series computed considering past samples (reproduced from [SMGA12]) . . . . 52

4.3 Matrix that stores the comparisons between all samples (reproduced from [SMGA12]) 52

4.4 The time-lag matrix $L$ (reproduced from [SMGA12]) . . . . . . . . . . . . . 52

4.5 The estimated density $P$ (reproduced from $[$ SMGA12] $\ldots \ldots \ldots \ldots$

4.6 The novelty curve $c$ (reproduced from $[\mathrm{SMGA12}]) \ldots \ldots \ldots \ldots$

4.7 Results obtained by the use of frame selection (reproduced from [Sey10a]) . . . . . 55

4.8 System's pipeline proposed by Foucard et al. [FDLR10] . . . . . . . . . . . . . 57

5.1 Processing diagram for the implementation of our music similarity framework. . . . . 62

5.2 The proposed processing pipeline for the Cover Song Classification problem. . . . . . 63

5.3 An example of a tensor built from several similarity matrices, and the sample vector

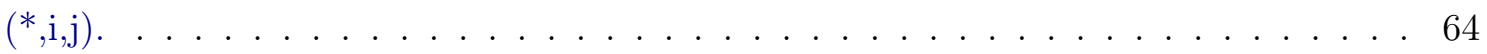

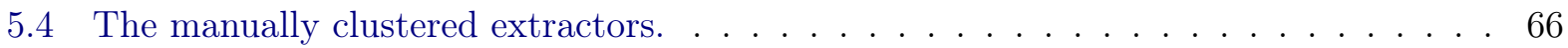

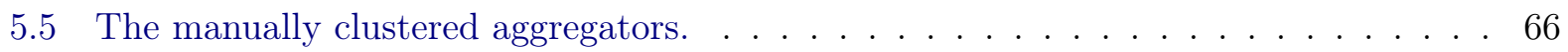

5.6 The manually clustered distances. . . . . . . . . . . . . . . . . . . 67

5.7 A naive solution to obtain the most of chance values for the test samples of a single

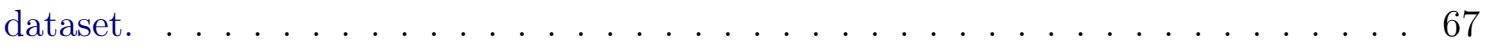

5.8 A proper solution to obtain chance values for all samples of one dataset using a second larger dataset for training. . . . . . . . . . . . . . . . . . 68

5.9 The full processing pipeline connecting cover song identification and cover song clas-

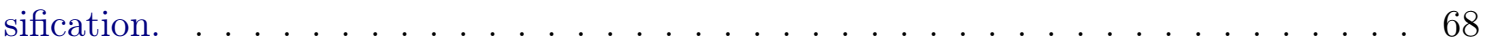

5.10 Frame selection keeping $50 \%$ of the frames with the highest RMS values. . . . . . . 71

5.11 Frame selection from the vocals-only (top) 50\% highest RMS values, and applying them to the $\operatorname{mix}($ bottom $) \ldots \ldots \ldots \ldots \ldots$. . . . . . . . . . . . . . . . . . .

6.1 Histogram of files per class from Ballroom dataset. . . . . . . . . . . . . 77

6.2 Histogram of files per class from IOACAS-QBH dataset. . . . . . . . . . . 78

6.3 Histogram of files per class from MAST dataset. . . . . . . . . . . . . . 80

6.4 Histogram of files per class from 1517-Artists dataset. . . . . . . . . . . . . 81

6.5 Histogram of files per class from MIR-QBSH dataset. . . . . . . . . . . . . . . . 82

6.6 Histogram of files per class from Covers1000 dataset. . . . . . . . . . . . . . . . 84

6.7 Histogram of files per class from Mazurkas dataset. . . . . . . . . . . . . . . . 85

6.8 Histogram of files per class from SHS9K dataset. . . . . . . . . . . . . . 86

6.9 Final tree structure after the rearrangement process. . . . . . . . . . . . . 86

6.10 Example recording with presence of silence in the end of the file. . . . . . . . . . 87

6.11 Example recording with vertical shift of the time domain audio signal. . . . . . . . 87

6.12 Summarized results to experiment \#3a by dimension (a) and by classifier (b) . . . . 102

6.13 Results for experiment \#3b, summarized by dimension (a) and by classifier (b) . . . 108

6.14 Summarized results to experiment \#4a by dimension (a), and by classifier (b). . . . 112 
6.15 Summarized results to experiment \#4a by dimension (a), and by classifier (b) . . . . 117

6.16 Comparison of best results obtained with source separation secondary datasets. . . . 121

6.17 Comparison of best results obtained with segment selection secondary datasets. . . . 122

6.18 Comparison of best results obtained with frame selection secondary datasets. . . . . 123

6.19 Number of tracks and theoretical mean rank as functions of the number of models for the Covers 80 dataset. . . . . . . . . . . . . . . . . . . . 130

6.20 Number of tracks and theoretical mean rank as functions of the number of models for the YouTubeCovers dataset. . . . . . . . . . . . . . . . . . . . 131

6.21 Number of tracks and theoretical mean rank as functions of the number of models for the Covers1000 dataset. . . . . . . . . . . . . . . . . . . . 131

6.22 Number of tracks and theoretical mean rank as functions of the number of models for the Mazurkas dataset. . . . . . . . . . . . . . . . . . . 132

6.23 Number of tracks and theoretical mean rank as functions of the number of models for the SHS9k dataset. . . . . . . . . . . . . . . . . . . . . . . 132 


\section{List of Tables}

4.1 Results obtained by the use of source separation (reproduced from [FDLR10]) . . . 57

5.1 Reviewed and explored list of extractors. . . . . . . . . . . . . . . . . 59

5.2 Reviewed and explored list of aggregators. . . . . . . . . . . . . . . . 60

5.3 Reviewed and explored list of distances. . . . . . . . . . . . . . . . . 60

5.4 An example segmentation computed using the SI-PLCA algorithm. . . . . . . . . . . 69

6.1 Extra information about Ballroom dataset. . . . . . . . . . . . . . 76

6.2 Extra information about GTZAN dataset. . . . . . . . . . . . . . . . 77

6.3 Extra information about IOACAS-QBH dataset. . . . . . . . . . . . . 78

6.4 Extra information about Panteli's melody dataset. . . . . . . . . . . . . . . 79

6.5 Extra information about Panteli's rhythm dataset. . . . . . . . . . . . . . . . 79

6.6 Extra information about MAST dataset. . . . . . . . . . . . . . . . . . . 80

6.7 Extra information about 1517-Artists dataset. . . . . . . . . . . . . . . 81

6.8 Extra information about MIR-QBSH dataset. . . . . . . . . . . . . . . . . . 82

6.9 Extra information about FMA-Small dataset. . . . . . . . . . . . . . . . . . . 82

6.10 Extra information about Covers80 dataset. . . . . . . . . . . . . . . . 83

6.11 Extra information about YouTubeCovers dataset. . . . . . . . . . . . . . . 83

6.12 Extra information about Covers1000 dataset. . . . . . . . . . . . . . . . . . 84

6.13 Extra information about Mazurkas dataset. . . . . . . . . . . . . . . . . 84

6.14 Extra information about SHS9K dataset. . . . . . . . . . . . . . . . . 85

6.15 The top-10 results for Ballroom dataset. . . . . . . . . . . . . . . . . . 87

6.16 The top-10 results for GTZAN dataset. . . . . . . . . . . . . . . . 88

6.17 The top-10 results for IOACAS-QBH dataset. . . . . . . . . . . . . . . . 88

6.18 The top-10 results for Maria Panteli's melody dataset. . . . . . . . . . . . . . 88

6.19 The top-10 results for Maria Panteli's rhythm dataset. . . . . . . . . . . . . . . 88

6.20 The top-10 results for MAST dataset. . . . . . . . . . . . . . . 88

6.21 The top-10 results for 1517 -Artists dataset. . . . . . . . . . . . . . . . . 89

6.22 The top-10 results for MIR-QBSH dataset. . . . . . . . . . . . . . . . . 89

6.23 The top-10 results for FMA-Small dataset. . . . . . . . . . . . . . . . . . . 89

6.24 Average results for models that do or do not use beat-synchronous features. . . . . . 91

6.25 Average results for models that do or do not use OTI . . . . . . . . . . . . . 91

6.26 Average results for models using minimum, mean or maximum to compute LCS-based distances. . . . . . . . . . . . . . . . . . . . . . . . . . . 92 
6.27 Average results for models that use minimum, mean or maximum to compute Levenshtein distances.

6.28 Average results for models that use the four variations of single Gaussian as aggregation model. . . . . . . . . . . . . . . . . . . . . . . . 92

6.29 Average results for vector quantization models using default or tailored number of

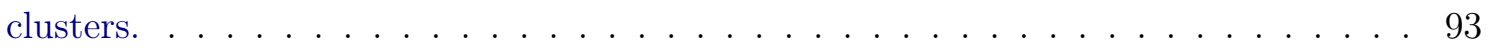

6.30 Average results for models with or without temporal summarization. . . . . . . . . 93

6.31 The top-10 results for Covers80 dataset. . . . . . . . . . . . . . . . . . . . 94

6.32 The top-10 results for YouTubeCovers dataset. . . . . . . . . . . . . . . . . 94

6.33 The top-10 results for Covers1000 dataset. . . . . . . . . . . . . . . . . . . . . 95

6.34 The top-10 results for Mazurkas dataset. . . . . . . . . . . . . . . . . . 95

6.35 The top-10 results for SHS9K dataset. . . . . . . . . . . . . . . . . 96

6.36 The best results for Experiment $\# 2 \ldots \ldots \ldots$. . . . . . . . . . . 96

6.37 Average results for models that do or do not use beat-synchronous features. . . . . . 97

6.38 Average results for models that do or do not use OTI. . . . . . . . . . . . . . . . 97

6.39 Average results for models using minimum, mean or maximum to normalise LCSbased distances. . . . . . . . . . . . . . . . . . . . . . . . . . . 97

6.40 Average results for models that use minimum, mean or maximum to normalise Levenshtein distances. . . . . . . . . . . . . . . . . . . . . 97

6.41 Average results for models that use the four variations of single Gaussian as aggregation model. . . . . . . . . . . . . . . . . . . . . . . . 97

6.42 Average results for vector quantization models using default or tailored number of

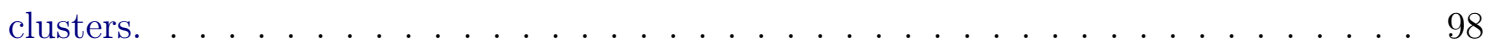

6.43 Average results for models with or without temporal summarization. . . . . . . . . 98

6.44 Comparative results for Music Similarity versus Cover Song Identification datasets. . 98

6.45 Top-10 results for Covers80 in Experiment \#3a of CSC. . . . . . . . . . . . . . . . . 99

6.46 Top-10 results for YouTubeCovers in Experiment \#3a of CSC. . . . . . . . . . . . . 99

6.47 Top-10 results for Covers1000 in Experiment \#3a of CSC . . . . . . . . . . . . . . . 100

6.48 Top-10 results for Mazurkas in Experiment \#3a of CSC . . . . . . . . . . . . . . . . 100

6.49 Top-10 results for SHS9K in Experiment \#3a of CSC. . . . . . . . . . . . . . . 101

6.50 Top-10 results for Covers80 in Experiment \#3b of the CSC problem. . . . . . . . . . 103

6.51 Top-10 results for YouTubeCovers in Experiment \#3b of the CSC problem. . . . . . 104

6.52 Top-10 results for Covers1000 in Experiment \#3b of the CSC problem. . . . . . . . . 105

6.53 Top-10 results for Mazurkas in Experiment \#3b of the CSC problem. . . . . . . . . 106

6.54 Top-10 results for SHS9K in Experiment \#3b of the CSC problem. . . . . . . . . . . 107

6.55 Best mean f1-score for all datasets in experiments \#3a and \#3b. . . . . . . . . . . . 108

6.56 List of all considered train and test datasets for Experiment \#4 . . . . . . . . . . . 109

6.57 Top-10 results for Covers80 to Experiment \#4a. . . . . . . . . . . . . . . . . . . 110

6.58 Top-10 results for YouTubeCovers to Experiment \#4a. . . . . . . . . . . . . . . . . . 110

6.59 Top-10 results for Covers1000 to Experiment \#4a. . . . . . . . . . . . . . . . . . . 111

6.60 Top-10 results for Mazurkas to Experiment \#4a. . . . . . . . . . . . . . . . . . 111

6.61 Top-10 results for Covers80 to Experiment \#4b. . . . . . . . . . . . . . . . . . . . . 113

6.62 Top-10 results for YouTubeCovers to Experiment \#4b. . . . . . . . . . . . . . . . . 114 
6.63 Top-10 results for Covers1000 to Experiment \#4b. . . . . . . . . . . . . . . . . 115

6.64 Top-10 results for Mazurkas to Experiment \#4b. . . . . . . . . . . . . . . . 116

6.65 Best results from Experiments \#4 compared with Experiment \#2. . . . . . . . . . 117

6.66 The best results for each modified dataset for Covers 80 . . . . . . . . . . . . . . 118

6.67 The best results for each modified dataset for YouTubeCovers. . . . . . . . . . . . . . 118

6.68 The best results for each modified dataset for Covers1000 . . . . . . . . . . . . . . . 119

6.69 The best results for each modified dataset for Mazurkas. . . . . . . . . . . . . . . . 119

6.70 The best results for each modified dataset for SHS9K. . . . . . . . . . . . . . 120

6.71 Top-5 results for Covers 80 fusing models using the minimum function. . . . . . . . . . 124

6.72 Top-5 results for Covers 80 fusing models using the maximum function. . . . . . . . . 124

6.73 Top-5 results for Covers 80 fusing models using the mean function. . . . . . . . . . . . . 124

6.74 Top-5 results for Covers80 fusing models using SNF. . . . . . . . . . . . . . . . . . 124

6.75 Top-5 results for YouTubeCovers fusing models using minimum function. . . . . . . . 124

6.76 Top-5 results for YouTubeCovers fusing models using maximum function. . . . . . . 124

6.77 Top-5 results for YouTubeCovers fusing models using mean function. . . . . . . . . . . 125

6.78 Top-5 results for YouTubeCovers fusing models using SNF. . . . . . . . . . . . . 125

6.79 Top-5 results for Covers1000 fusing models using minimum function. . . . . . . . . . . 125

6.80 Top-5 results for Covers1000 fusing models using maximum function. . . . . . . . . . 125

6.81 Top-5 results for Covers1000 fusing models using mean function. . . . . . . . . . . . . 125

6.82 Top-5 results for Covers1000 fusing models using SNF. . . . . . . . . . . . . . . . 125

6.83 Top-5 results for SHS9K fusing models using minimum function. . . . . . . . . . . 125

6.84 Top-5 results for SHS9K fusing models using maximum function. . . . . . . . . . . 126

6.85 Top-5 results for SHS9K fusing models using mean function. . . . . . . . . . . . . . 126

6.86 Top-5 results for SHS9K fusing models using SNF. . . . . . . . . . . . . . 126

6.87 Results obtained so far with 5 important CSI algorithms whose implementations are found in GitHub. . . . . . . . . . . . . . . . . . . . . 127

6.88 Rank distribution for the Covers80 dataset. . . . . . . . . . . . . . . . . . . 128

6.89 Rank distribution for the YouTubeCovers dataset. . . . . . . . . . . . . . . . 128

6.90 Rank distribution for the Covers1000 dataset. . . . . . . . . . . . . . . . . . . . . . 129

6.91 Rank distribution for the Mazurkas dataset. . . . . . . . . . . . . . . . . . . . . 129

6.92 Rank distribution for the SHS9k dataset. . . . . . . . . . . . . . . . . . . . . . 130

6.93 Comparison of metrics obtained in Experiment \#2 with the theoretical metrics. . . . 130

7.1 Best results for Covers80 through all CSI experiments. . . . . . . . . . . . . . 133

7.2 Best results for YouTubeCovers through all CSI experiments. . . . . . . . . . . . . 133

7.3 Best results for Covers1000 through all CSI experiments. . . . . . . . . . . . . . 133

7.4 Best results for Mazurkas through all CSI experiments. . . . . . . . . . . . . . . . 133

7.5 Best results for SHS9K through all CSI experiments. . . . . . . . . . . . . . . 134

7.6 The best results obtained for each CSI dataset. . . . . . . . . . . . . . . . . 135 


\section{Chapter 1}

\section{Introduction}

\subsection{Context and Motivation}

The dissemination of digital music has enabled the grouping of thousands of audio files (in personal collections) or millions of files (in databases of stream web-sites or online music stores). For instance, the company Spotify ${ }^{1}$ provides 70 million tracks to its users ${ }^{2}$.

Traditionally, searches on these datasets are done by objective terms (such as artist, album, track, year, record label, etc) or subjective (such as style, genre, etc). Searching with metadata can be interesting, but it has some inherent problems. For instance, the cold start problem: how to start the tagging process for a newly created dataset or for recently added audio files to a pre-existing database.

Some solutions used in the past are assignments by experts (as Pandora ${ }^{3}$ did) or by collective intelligence (as Last.fm ${ }^{4}$ did). However, both solutions have problems. When working with a team of specialists there is a high maintenance cost and, in addition, an intrinsic reduced speed of manual classification.

Regarding the classification by users, there is the bias created by communities with their own language and unique point of view when labeling the presented songs. In addition, there is the fairness problem: the most popular artists are classified before and in greater quantity, which makes them more prominent than unknown artists (who could be the most suitable answer to a search).

A second problem of searching (or browsing) with metadata is the limitation of the terms used. There is no consensus on which tag should be assigned to each song (or album, or artist), and also that it must be unique. Such attribution complexity generates a multitude of problems, such as the inability to fully capture the cultural context and the musical content of the tracks. Moreover, it is somewhat difficult for the lay public to assimilate the meaning of each tag, and it takes a while for the user to be able to search effectively.

For these and other reasons it has been an increasingly common practice to use Music Information Retrieval (MIR) techniques to deal with huge databases. The act of working directly with the audio and the musical contents has several advantages and this is the global goal of that research area.

MIR techniques can provide to the user the ability to hum to retrieve a melody, or to clap to fetch a rhythm, or even to use an audio file as the query object and receive similar tracks in response. The goal is to make content more accessible and in a more intuitive way.

The MIR task of most interest in this project is the Music Similarity one. Giving an annotated dataset, it is possible to calculate how similar are the songs within it (or to an external song) with the use of music similarity models. Thus, a solid foundation for navigation, organization, recommendation, and search in this database is created.

\footnotetext{
${ }^{1}$ https://www.spotify.com/

${ }^{2}$ https://newsroom.spotify.com/company-info/

${ }^{3}$ http://pandora.com/

${ }^{4}$ http://last.fm/
} 
Since there is no universally agreed-upon formalized concept of general musical similarity, our approach to this problem is to deal with its individual aspects. Some important perceptual properties of sound are pitch, duration, intensity and timbre. So similarities related to these properties are addressed: melodic, harmonic, rhythmic, dynamic, timbre, etc. It is important to explicitly state that the idea of music similarity completely depends on the context, which usually is established by the dataset and the application. We present a few examples below:

- query-by-humming

In this application the user has a melody in mind and makes a search for similar melodies humming it. Such a task benefits from melodic similarity models tolerant to changes in pitch, duration, and also the addition, deletion, and substitution of notes from the sought melody.

- automatic DJing

This application is directly linked to the automatic generation of playlists. However, the songs are appended in such a way that their tempi are equal. The onsets need to be synchronized, and the transitions between tracks should be as smooth as possible (no silences or breaks are allowed). Automatic DJing definitely benefits from rhythmic similarity models selecting tracks that can better mixed one after the other.

- recognition of musical instruments

The user may want to find all the songs on which there is a flute being played, or a trombone, or a harmonica. Maybe he wants to find a specific ensemble such as a string quartet (with two violins, a viola and a cello) or a jazz trio (with piano, bass and drums). Searches with these characteristics can be addressed by timbre similarity models.

The literature on audio-based music similarity presents several approaches, including the use of traditional information retrieval methods (such as extracting features from audio recordings, and computing their distances in a vector space) [Foo97, LO04, SSPK10, YZL ${ }^{+} 10$, KS13, Tra17]; modeling the extracted features distributions and comparing the models [LS01, $\mathrm{AP}^{+}$02, PA04, BLEW04, TWV05, SFW12]; feature learning [ $\mathrm{PBD}^{+}$16, YXCY20]; metric learning [SWW08, HKKL17]; deep neural networks [MSC19, FR21]; among others.

\subsection{Problems addressed and Goals}

The research presented here focuses on representations of musical content, and more specifically human-readable representations. This allows for a kind of audit of what happens at each stage of the modelling of music similarity, and how these models are used in specific tasks. We are mainly interested in offering tools that allows one to explain and understand exactly how music similarity models produce their scores, independently of how these models perform in those tasks. This focus on fully-explainable human-readable models and a desire to approach a multitude of alternative similarity models takes precedence over a quest for the highest achievable performance metrics.

\section{Music Similarity}

The main problem we address is the modelling of music similarity and the assessment of its quality. Our proposed approach, which generalizes the first two approaches above, is based on the following pipeline [KS16]: extract audio features, aggregate local features into global features, and compute the similarities of every pair of songs from the dataset being processed. A triple $\left\{\right.$ extractor $_{i}$, aggregator $_{j}$, distance ag $_{k}$ defines a music similarity model, and our main goal is to investigate the capacity of similarity models to distinguish tracks from different classes in selected datasets.

Regarding the feature extractors, we work with tonal features, such as chromagrams [JCEJ08, SGHS08, YZL ${ }^{+}$10, RE10], tonnetz [Tym09], and symbolic melodic sequences [GLCS95, TYW ${ }^{+} 05$, 
FM05, Mar06, RH06a, SSG13a]. Also with timbre features (Mel-Frequency Cepstral Coefficients (MFCC) [Foo97, LS01, $\mathrm{AP}^{+}$02, BLEW04, PA04, SFW12]), spectral features [LO04] (spectral centroid, bandwidth, contrast, flatness, etc), rhythmic features (Rhythm Pattern [PRM02a, $\mathrm{PFW}^{+} 05$, SSPK10]), dynamic features (Root Mean Square (RMS) [PT05, MF05]), and others.

Some of the aggregation techniques we employ are single Gaussian [SFW12], Gaussian Mixture Model [ $\mathrm{AP}^{+}$02, PA04, BLEW04], Vector Quantization [LS01], Markov Chain [HRG01], Octave Abstraction [SSG13a], Interval Abstraction [SSG13a], Pitch Contour (3-levels) [GLCS95], Pitch Contour (5-levels) [RH06a].

Lastly, the list of distances we use includes Manhattan distance [SSPK10, KS13], Euclidean distance [Foo97, LO04, YZL ${ }^{+}$10], Cosine distance [Foo97, Tra17], LCS-based distance [UZ99, Kel12], Levenshtein's distance [LU00, Kel12], Kullback-Leibler distance [BLEW04, SFW12], Earth Mover's distance [LS01, TWV05], Monte Carlo distance [AP+ 02, PA04].

\section{Cover Song Identification}

Alternatively, instead of looking for the music similarity problem as a "pure" problem, we also address one of its application: Cover Song Identification (CSI). This application involves identifying songs which are versions of each other, and it assumes that the covers of a given song must have some common music trait, which would be captured by a music similarity model.

Several papers from the CSI literature state that the best results can be achieved with tonal similarity, since the tonal aspects are the least likely to vary between version songs [SGH10, SSG12, CLX17]. Several projects use tonal features [GHO06, JCEJ08] or symbolic sequences [TYW ${ }^{+} 05$, Mar06]. Also it can be found that state-of-the-art CSI methods uses at least one type of (early or late) fusion [Tra17, CLX18].

The main goal of our approach to the CSI problem is quite similar to the one of the music similarity problem: we want to investigate the models that are best suited to distinguish groups of songs that are versions from groups of songs that are not. Regarding fusion methods, we use them in a distinct way than the one found in the literature, as will be fully explained in Section 5.5.

\section{Cover Song Classification}

The Cover Song Classification (CSC) problem, which is a sibling to the CSI problem, is also investigated. CSI produces ranked lists of version candidates with values between 0 and 1 determining the similarity scores between the query song and the songs in the database; CSC assigns 0 or 1 for a pair of songs (binary classification) saying if they are versions of each other. The literature for CSC is not as vast as the one for CSI, but a couple of papers could be identified [RE10, SSG12].

Our approach to the CSC problem basically consists in using the music similarity pipeline cited above to compute several similarity values (using several distinct models) between all pair of songs from a dataset, and use them to compose samples to the binary classification process. This is designed in order to exploit different points of view about that pair of songs. Our main goal here is to find out the best set of models that can achieve the best classification metrics.

We also explore the very promising bridge between CSI and CSC. Every trained classifier can be asked to return the chance of a pair of songs to be versions, instead of the usual binary classification value 0 or 1 . Our approach to link the CSC problem with CSI one is to obtain all these chance values for all pair of songs from a dataset, and build a new similarity matrix (as if we had a new music similarity model). Our main goal here is to check how this brand new similarity matrix can help to identify songs that are versions of each other from songs that are not.

\section{Dataset Modifications}

Finally we explore one last MIR-related method - dataset modification - applied to the CSI problem. Several remarks can be found in the literature about audio files modifications being used in 
order to obtain better results, or even to decrease processing time (when the modification technique returns a smallest representations of the audio files).

The main goal is to generate brand new secondary datasets using this modification methods, and check if it is possible to obtain better results for the CSI problem, compared to the ones obtained with the original datasets. As a bonus, we can also obtain better processing times with the derivative datasets (with a few specific cases). This is a very important aspect since the process of comparing every pair of songs from a dataset is already an expensive task (if $N$ is the number of tracks, the complexity is $O\left(N^{2}\right)$ ) and everything we can do to mitigate the time bottleneck is very promising.

Three main types of dataset modifications we experiment with are: frame selection [Sey10a], segment selection [LS06, BPVT08, SdSB15], and source separation [FDLR10]. Roughly, the first one discards a few frames from the audio files based on the injure they can potentially cause to the similarity computations; the second type replaces the full-length audio files to a fixed-length segment (for instance, 30 seconds); the later uses isolated sources (such as vocals, bass, drums, etc) in place of the whole mixtures.

\subsection{Thesis contributions and structure}

The main contributions of this thesis are:

- a comprehensive benchmark of music similarity models;

- a comparative discussion on related similarity models;

- an open-source music similarity framework;

- novel alternative ways to build samples for CSC;

- a new heuristic for train/test split;

- an experiment connecting the CSI and CSC problems;

- a comprehensive exploration of distinct dataset modifications and the proposal of novel dataset modification techniques;

- the use of source separation for dataset modifications and fusion of music similarity models;

- the study of theoretical performance bounds for the family of similarity models considered.

The remaining of this thesis is structured as follows: Chapter 2 presents the extractors, aggregators and distances to compose the considered music similarity models; Chapter 3 introduces all the works related to our approaches to Cover Song Identification and Classification; Chapter 4 gives all details about projects that use dataset modifications; Chapter 5 presents our individual approaches to every problem considered in this thesis; Chapter 6 displays the design and results of each one of our experiments; Chapter 7 shows our final conclusions and future works. 


\section{Chapter 2}

\section{Music Similarity}

\section{$2.1 \quad$ Feature extractors}

Despite the recent emergence of waveform-based music processing with deep learning [DS14, $\mathrm{ODZ}^{+} 16, \mathrm{PNP}^{+}$17], it is not common to use audio signals directly as samples of a Music Information Retrieval (MIR) task. Usually, alternative representations, called audio features or descriptors, are used. One of the main reasons is to avoid high dimensionality problems [Bel57], because time-domain audio vectors are very large (30 seconds sampled at $44100 \mathrm{~Hz}$ has over 1 million samples). Another reason is to highlight some kind of information contained in the audio signal, but not automatically (or trivially) deduced by the machine.

Choosing which features are extracted and used is therefore essential to solve a problem. Depending on the nature of the problem, we want to work with different music information, and audio features correlated with this information help the algorithms to get better results. In this section we present all the audio features used for the music similarity computations in our project.

\section{Chromagram STFT-based}

Pitch is made of two components: chroma and tone height [Mül15]. The chroma is a value in the set $\{\mathrm{C}, \mathrm{C} \#, \mathrm{D}, \mathrm{D} \#, \mathrm{E}, \mathrm{F}, \mathrm{F} \#, \mathrm{G}, \mathrm{G} \#, \mathrm{~A}, \mathrm{~A} \#, \mathrm{~B}\}$, and the tone height is a numeric value relative to the octave number. The pitches that have the same chroma form a group called pitch class, and a chromagram is a feature that aggregates the energy of all pitches of the same pitch class.

The chromagram is created from the spectrogram accumulating the amplitude of the bins associated to the same pitch class. The final result is a matrix with 12 rows (relative to all pitch classes) and the number of columns equal to the number of processed time frames. The chromagram referred to as chroma_stft in this text is specifically build using the Short-Time Fourier Transform (STFT) spectrogram [Ell07a]. Figure 2.1 shows an example spectrogram, Figure 2.2 shows the filter bank that maps the bins to pitch classes, and Figure 2.3 shows the resulting STFT-based chromagram obtained by the dot product of the spectrogram by the filter bank (followed by a normalization step). 


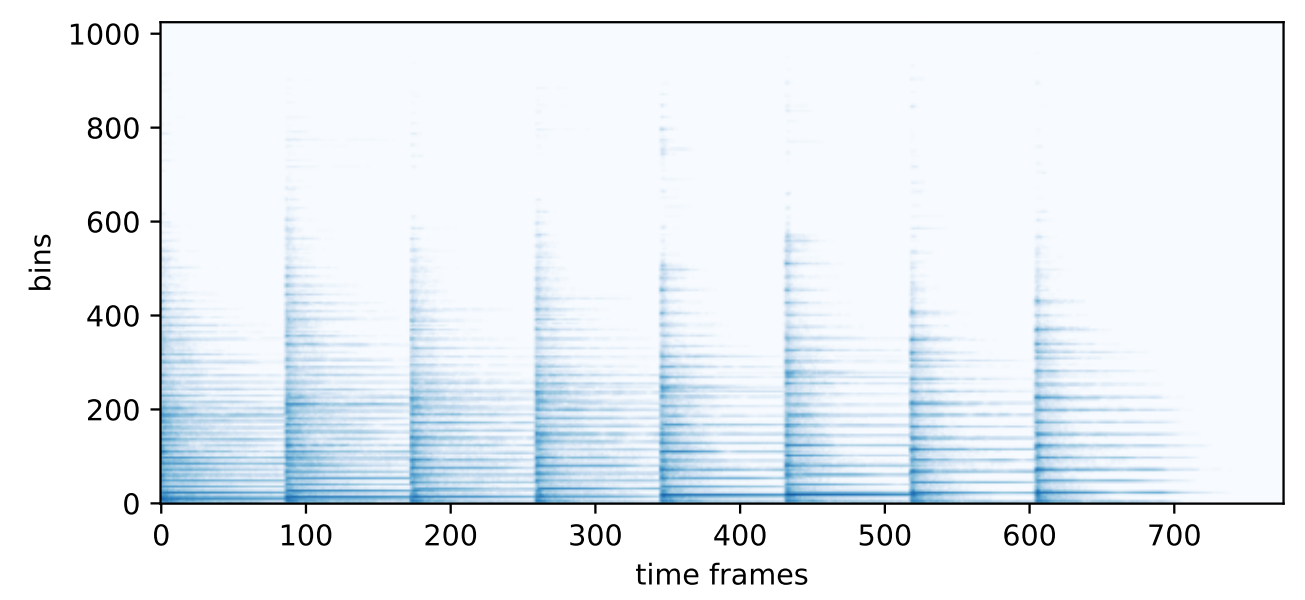

Figure 2.1: STFT spectrogram from a $C$-major scale sample performed by a piano.

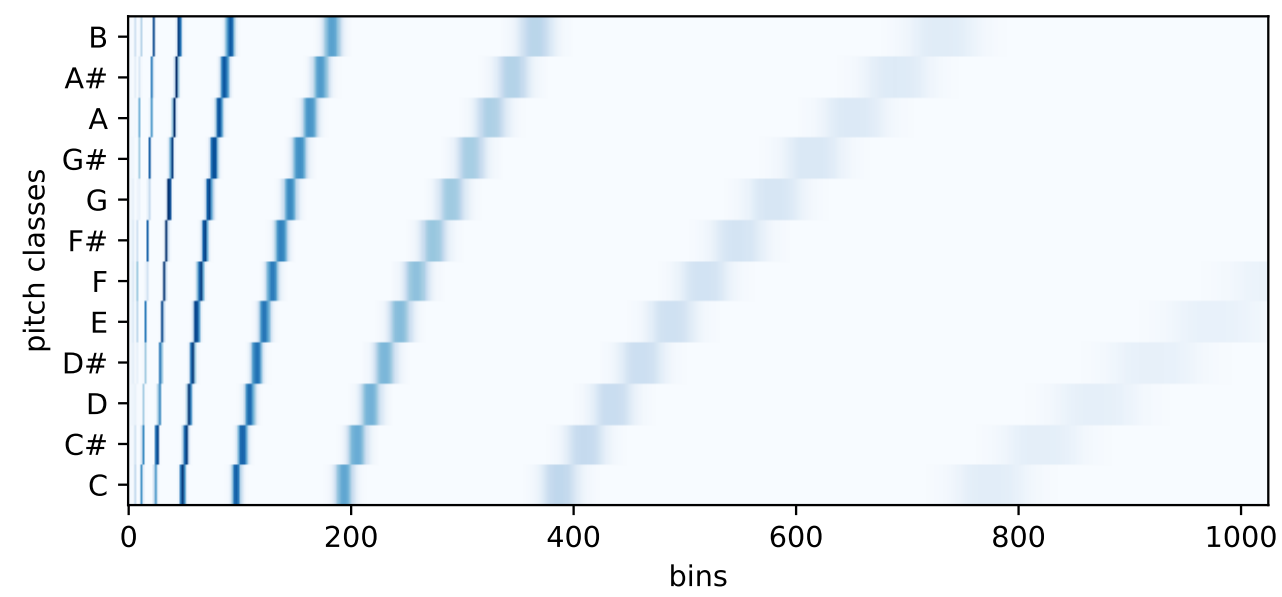

Figure 2.2: Filter bank that maps STFT-bins to pitch classes.

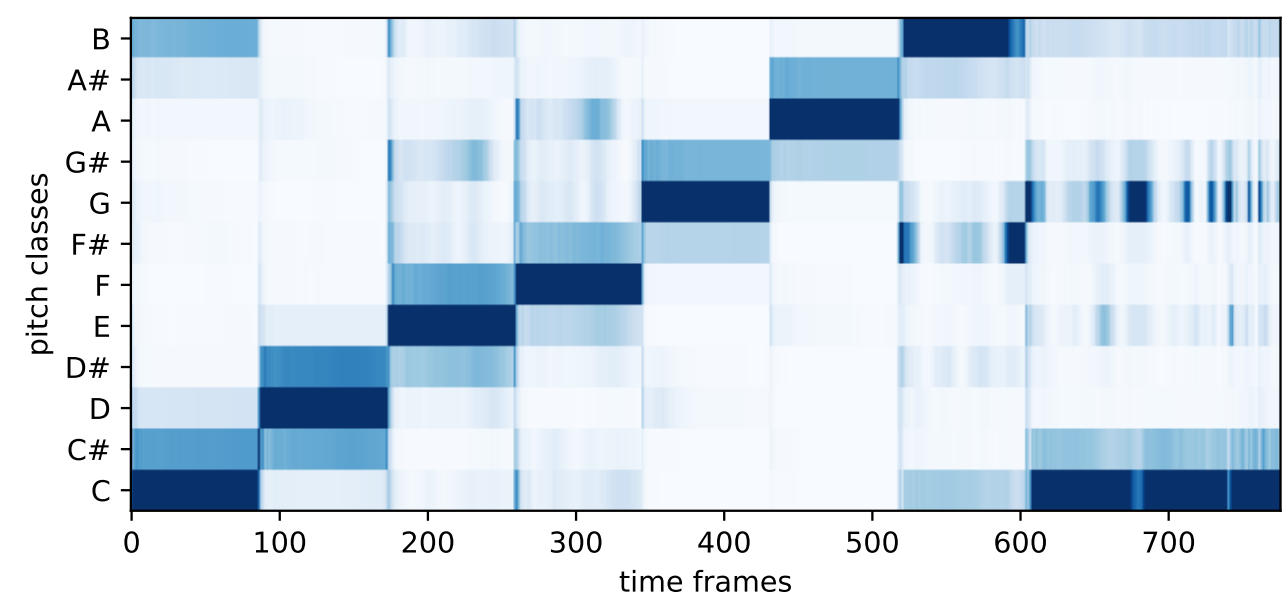

Figure 2.3: STFT-chromagram from a C-major scale sample performed by a piano.

\section{Chromagram CQT-based}

The feature referred to as chroma_cqt is another type of chromagram, but created using the Constant-Q Transform (CQT) instead of the STFT. The CQT have geometrically spaced frequency 
bins and the ratios between the center frequencies to the bandwidths are the same [SK10]. Therefore the CQT provides a high frequency resolution for low frequencies and high time resolution for high frequencies.

Figure 2.4 shows a CQT-spectrogram, Figure 2.5 shows the mapping filter, and Figure 2.6 shows the resulting CQT-based chromagram obtained by the dot product of the first and the latter (followed by thresholding and normalization steps).

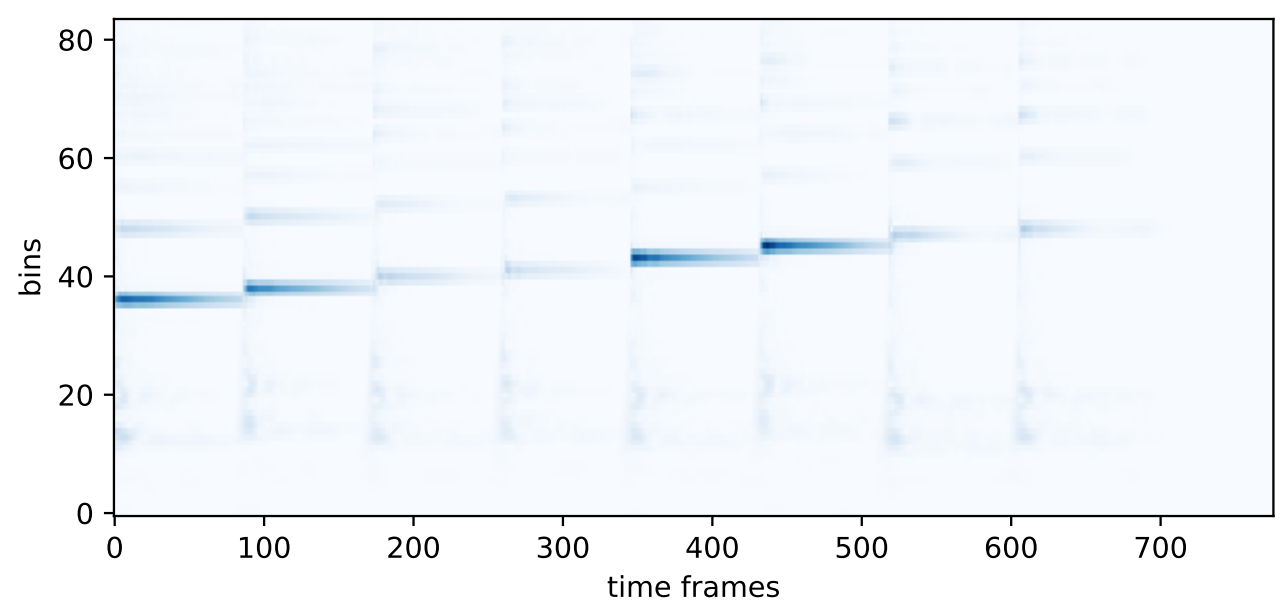

Figure 2.4: $C Q T$-spectrogram from a C-major scale sample performed by a piano.

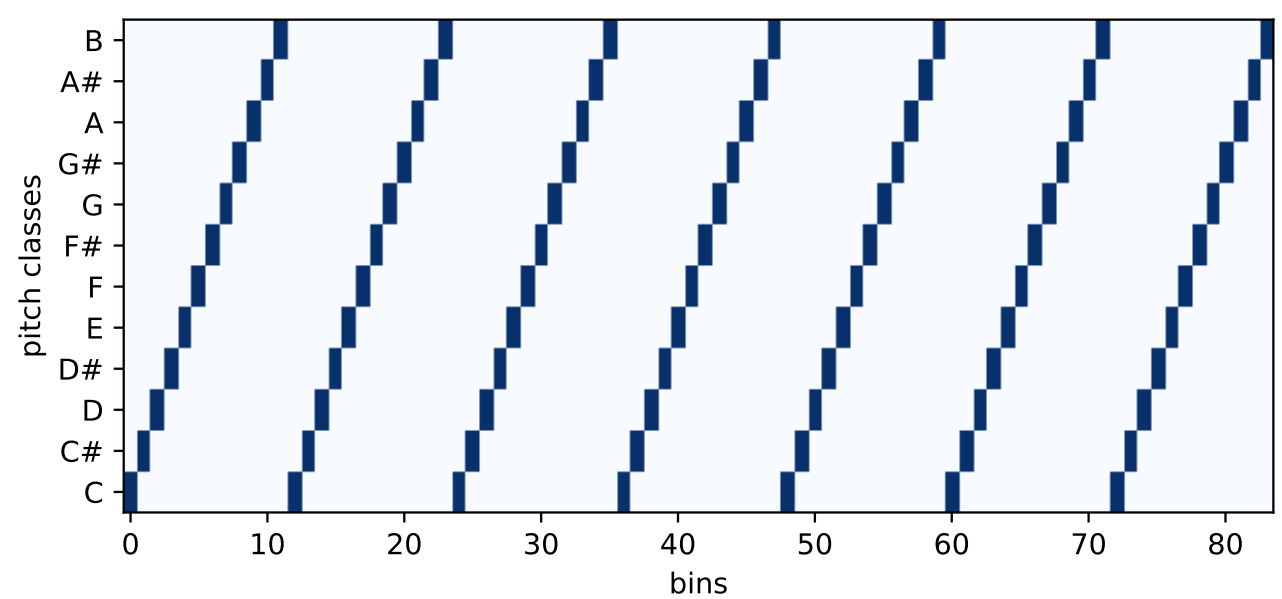

Figure 2.5: Filter bank that maps CQT-bins to pitch classes. 


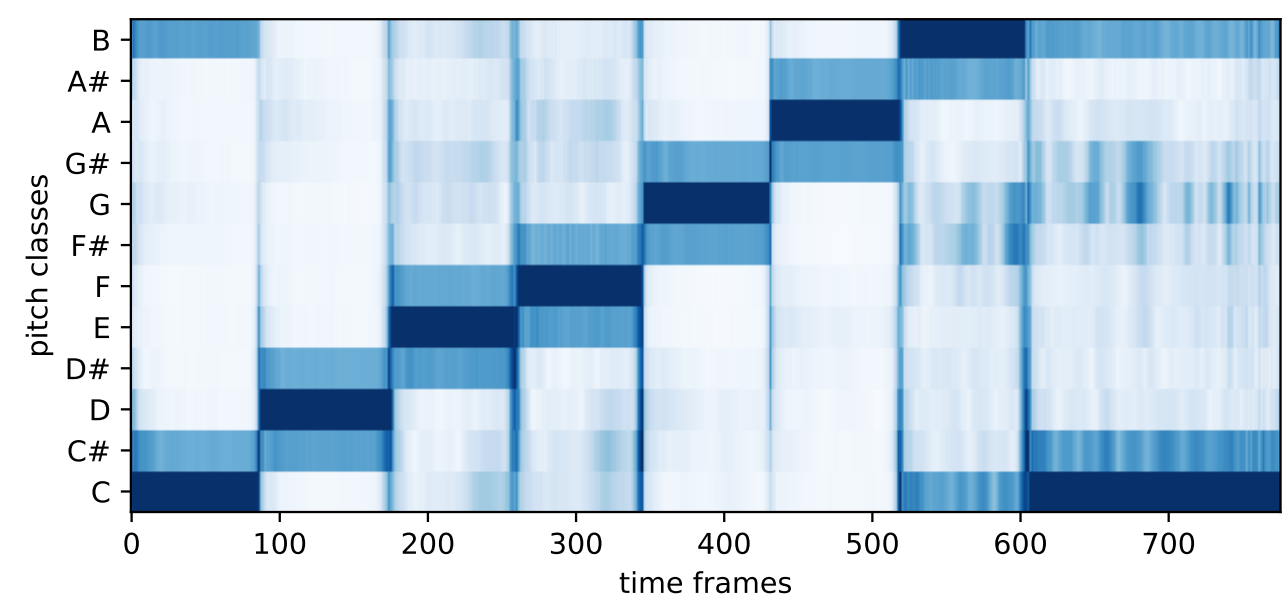

Figure 2.6: CQT-chromagram from a C-major scale sample performed by a piano.

\section{Chroma Energy Normalized Statistics}

The Chroma Energy Normalized Statistics (CENS) [ME11], referred to as chroma_cens, is built on top of the previous one. After the chroma_cqt is computed the following 5 steps are applied:

1. $L^{1}$-normalization

2. amplitude quantization (using logarithmic compression)

3. smoothing

4. downsampling

5. $L^{2}$-normalization

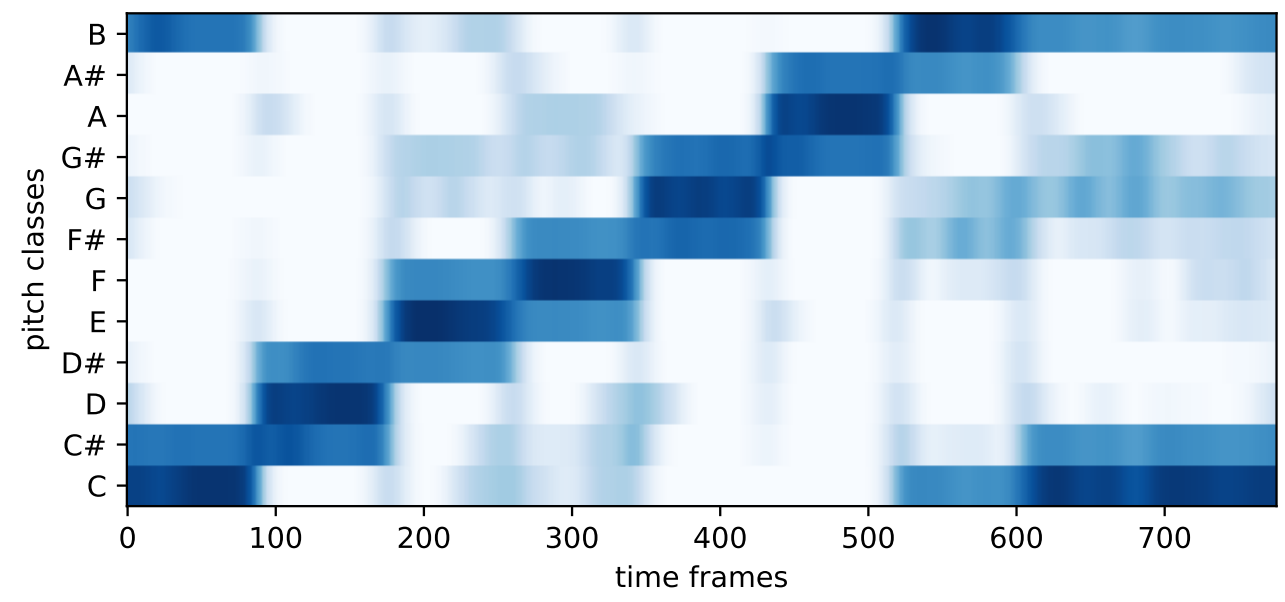

Figure 2.7: CENS-chromagram from a C-major scale sample performed by a piano.

\section{Mel-spectrogram}

This feature is a frequency-domain audio representation, but instead of using linear scale as seen in the STFT-spectrogram above, it uses a nonlinear one called mel-scale, which was created with the goal to reflect the human perception of pitch intervals, and the exact mapping was achieved in listening experiments [SVN37].

Figure 2.8 shows the filter-bank matrix that map frequencies to the mel-scale, and Figure 2.9 shows the resulting melspectrogram. 


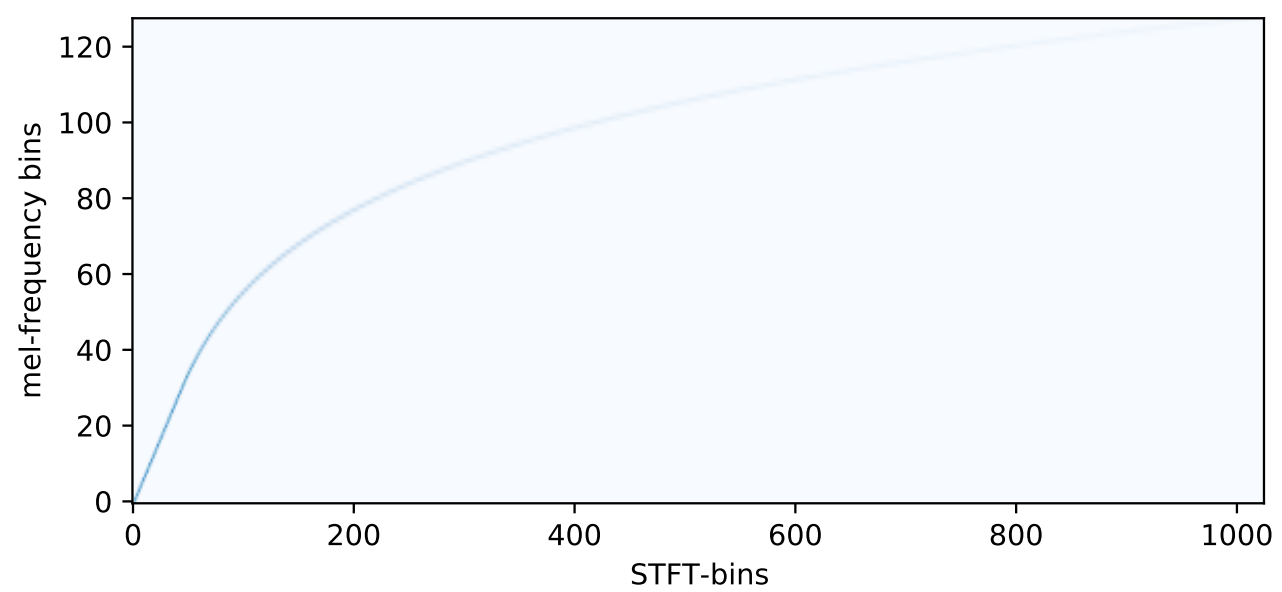

Figure 2.8: Filter bank that maps STFT-bins to Mel-frequency bins.

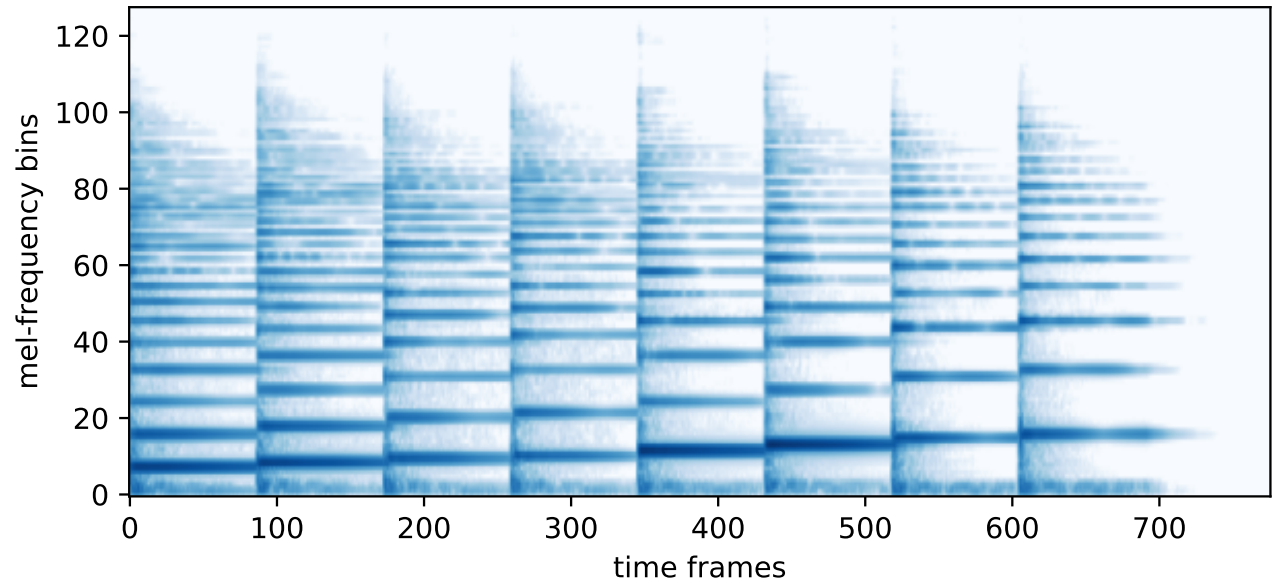

Figure 2.9: Mel-spectrogram from a C-major scale sample performed by a piano.

\section{Mel-Frequency Cepstral Coefficients}

The audio descriptor called Mel-Frequency Cepstral Coefficients (MFCC), widely used in the speech recognition problem [HCCP06, ISY12], is usually associated with timbre aspects of the songs [Ell07b, MEK09]. Its definition uses important aspects of the human auditory system [Pam06]. There are alternative implementations for the MFCC feature [GFK05], but the main steps are as follows [Pam06]:

1. compute the power-spectrum

2. apply a scale change to Mel-frequencies

3. apply another scale change to decibels

4. compute the Discrete Cosine Transform (DCT)

Figure 2.10 displays the coefficients for the $\mathrm{C}$ major scale performed by a piano whose spectrogram was displayed in Figure 2.1. 


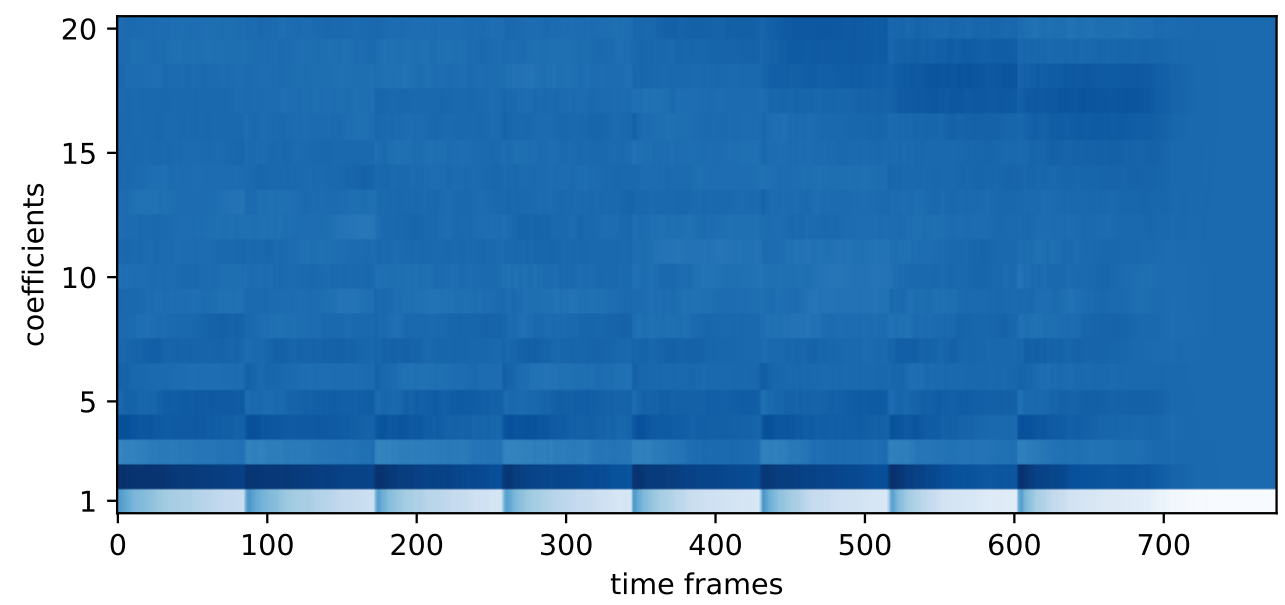

Figure 2.10: $M F C C$ extracted from a $C$-major scale sample performed by a piano.

\section{Spectral Centroid}

The spectral centroid is computed in the frequency-domain, it can be interpreted as the mean of the power-spectrum, and is defined as follows [Ler12]:

$$
\operatorname{spectral} \_ \text {centroid }(n)=\frac{\sum_{k=0}^{K / 2-1} k\left|X_{n}(k)\right|^{2}}{\sum_{k=0}^{K / 2-1}\left|X_{n}(k)\right|^{2}},
$$

where $n$ is the time frame index.

This feature is usually associated with brightness [TEC01] since more amplitude on high frequencies makes the value of spectral centroid higher. Figure 2.11 shows a clean guitar (blue line) versus a distorted guitar (orange line), and we can observe that the distorted one has larger values for almost all frames. This is expected since the distortion adds lots of harmonic partials leading to higher spectral centroids.

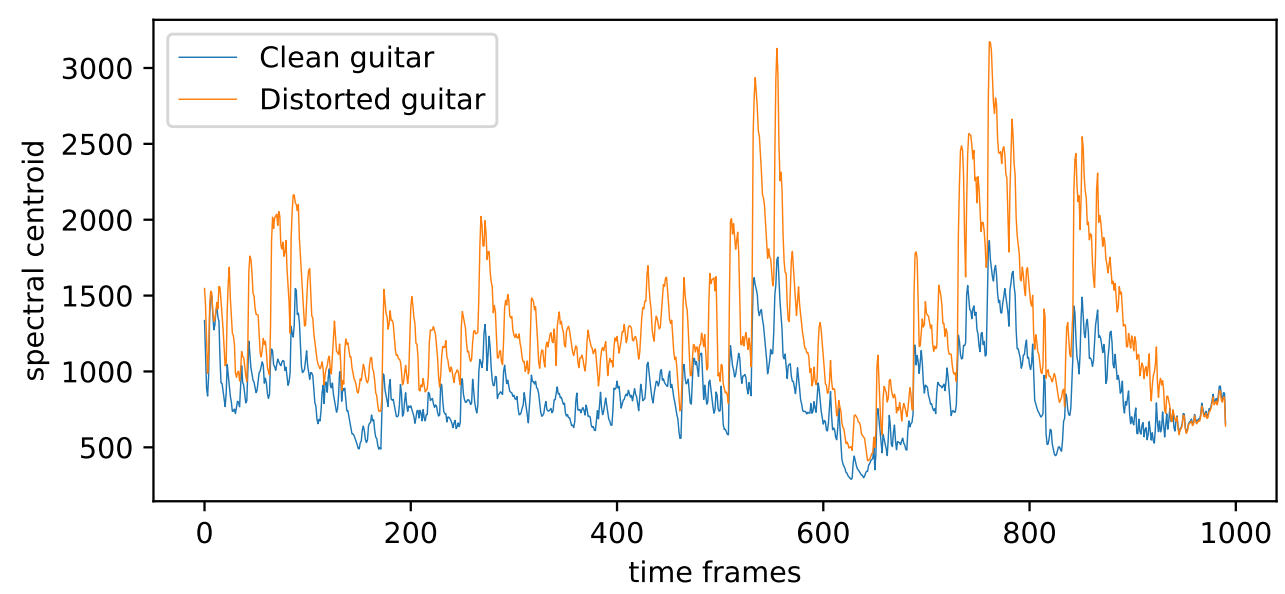

Figure 2.11: Spectral centroid values extracted from the same guitar sample before and after applying distortion.

\section{Spectral Bandwidth}

The spectral bandwidth is computed in the frequency-domain also, it can be interpreted as the standard deviation of the power-spectrum, and is defined as follows [Ler12]: 
spectral_bandwidth $(n)=\sqrt{\frac{\sum_{k=0}^{K / 2-1}(k-\text { spectral_centroid }(n))^{2}\left|X_{n}(k)\right|^{2}}{\sum_{k=0}^{K / 2-1}\left|X_{n}(k)\right|^{2}}}$,

where $n$ is the time frame index.

This feature is related to timbre characteristics of the audio signal, and can be used as a measure of spectrum spread. It can show if the spectrum has energy concentrated in a narrow band or if it has energy across a larger frequency interval. Figure 2.12 shows the spectral bandwidth for the same two guitar samples we saw before. The addition of distortion (i.e. the addition of harmonic partials) leads to higher spectral bandwidth values.

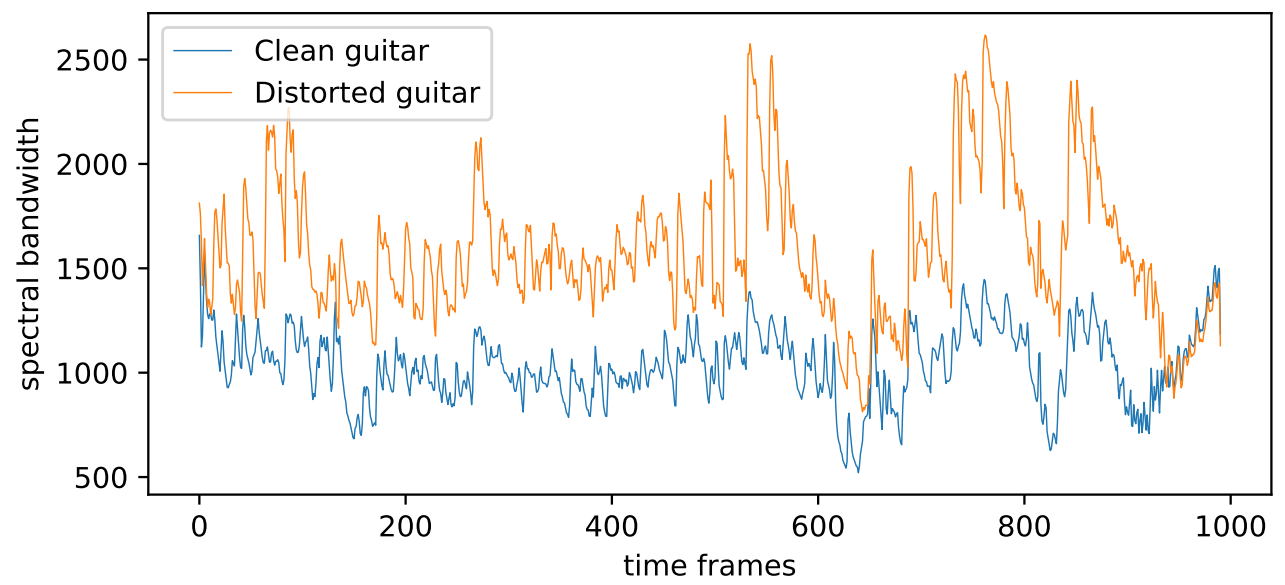

Figure 2.12: Spectral bandwidth values extracted from the same guitar sample before and after applying distortion.

\section{Spectral Roll-off}

The spectral roll-off is defined as the frequency value up to which the accumulated spectrum energy reaches a given percentage. Let this percentage be $85 \%$. If the spectral roll-off is $2000 \mathrm{~Hz}$ then it means that $85 \%$ of the spectrum energy is in the frequencies up to $2 \mathrm{kHz}$. This feature is correlated to the cutting frequency that separates harmonic content from noise [Pee04].

The two guitar samples used before illustrate this feature: the increased energy of the distorted guitar in higher frequencies produces a higher cutting point (i.e., higher roll-off), as seen in Figure 2.13 .

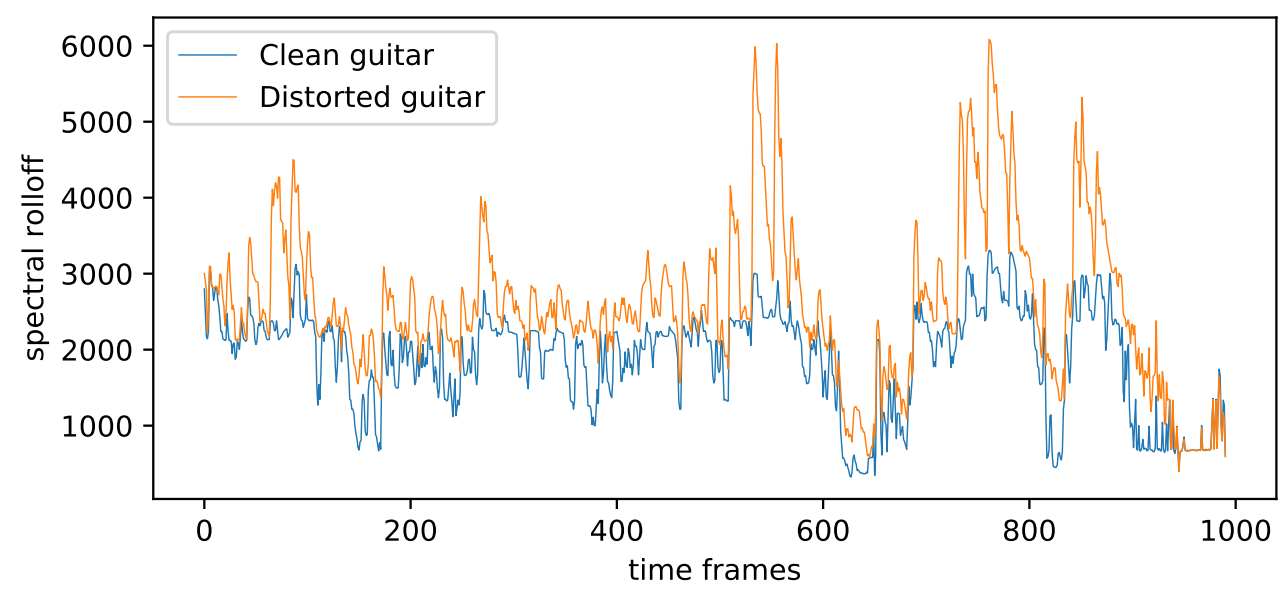

Figure 2.13: Spectral roll-off values extracted from the same guitar sample before and after applying distortion. 


\section{Spectral Flatness}

The spectral flatness, also called tonality coefficient, is another feature computed in the frequencydomain. Dubnov [Dub04] states that it can be used as a measure of randomness present in a signal. So it can be used as a measure of noisiness in the spectrum. It is defined as the ratio of the geometric mean to the arithmetic mean of the energy spectrum value [Pee04]. Figure 2.14 shows the two guitar samples, and we can observe the distorted guitar has highest values, which is consistent to the fact that it has more noise than the clean one.

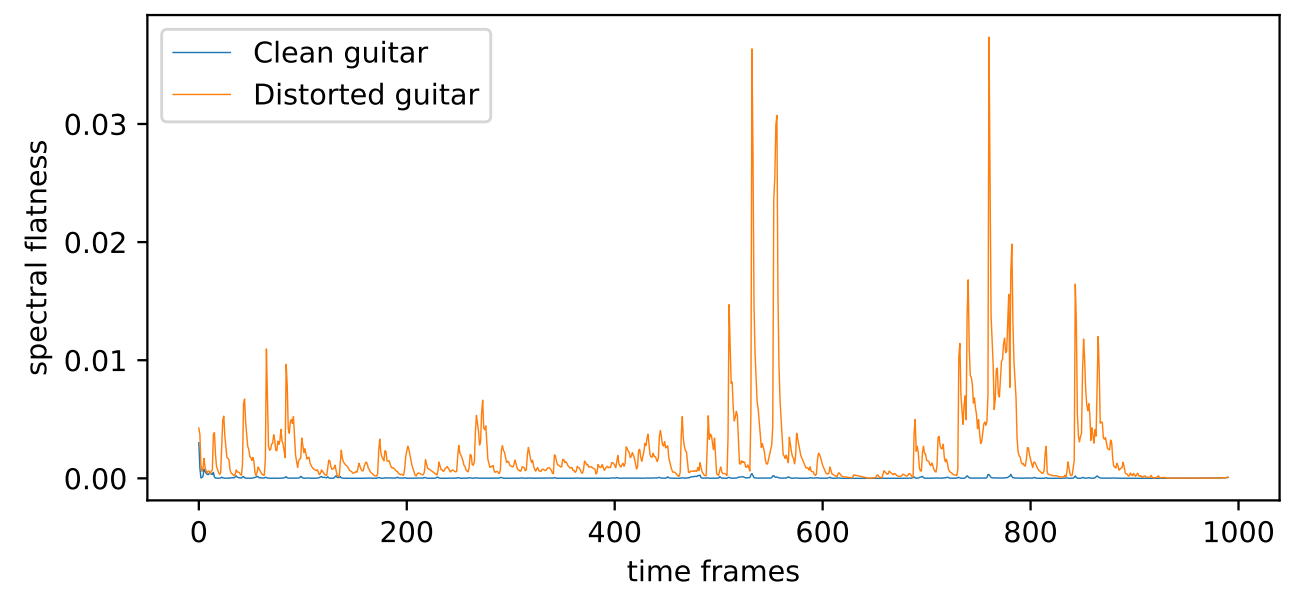

Figure 2.14: Spectral flatness values extracted from the same guitar sample before and after applying distortion.

\section{Spectral Contrast}

This feature divides the spectrum in sub-bands and computes the energy ratio between the spectral peaks and the spectral valleys for every sub-band. This ratio is called spectral contrast, and Jiang et al. [JLZ $\left.{ }^{+} 02\right]$ state that this feature loosely indicates the harmonic/non-harmonic relative distribution, since the peaks are related to harmonic components, and the valleys with non-harmonic components.

Figure 2.15 displays the spectral contrast values for the two guitar samples discussed above, computed over seven sub-bands. 


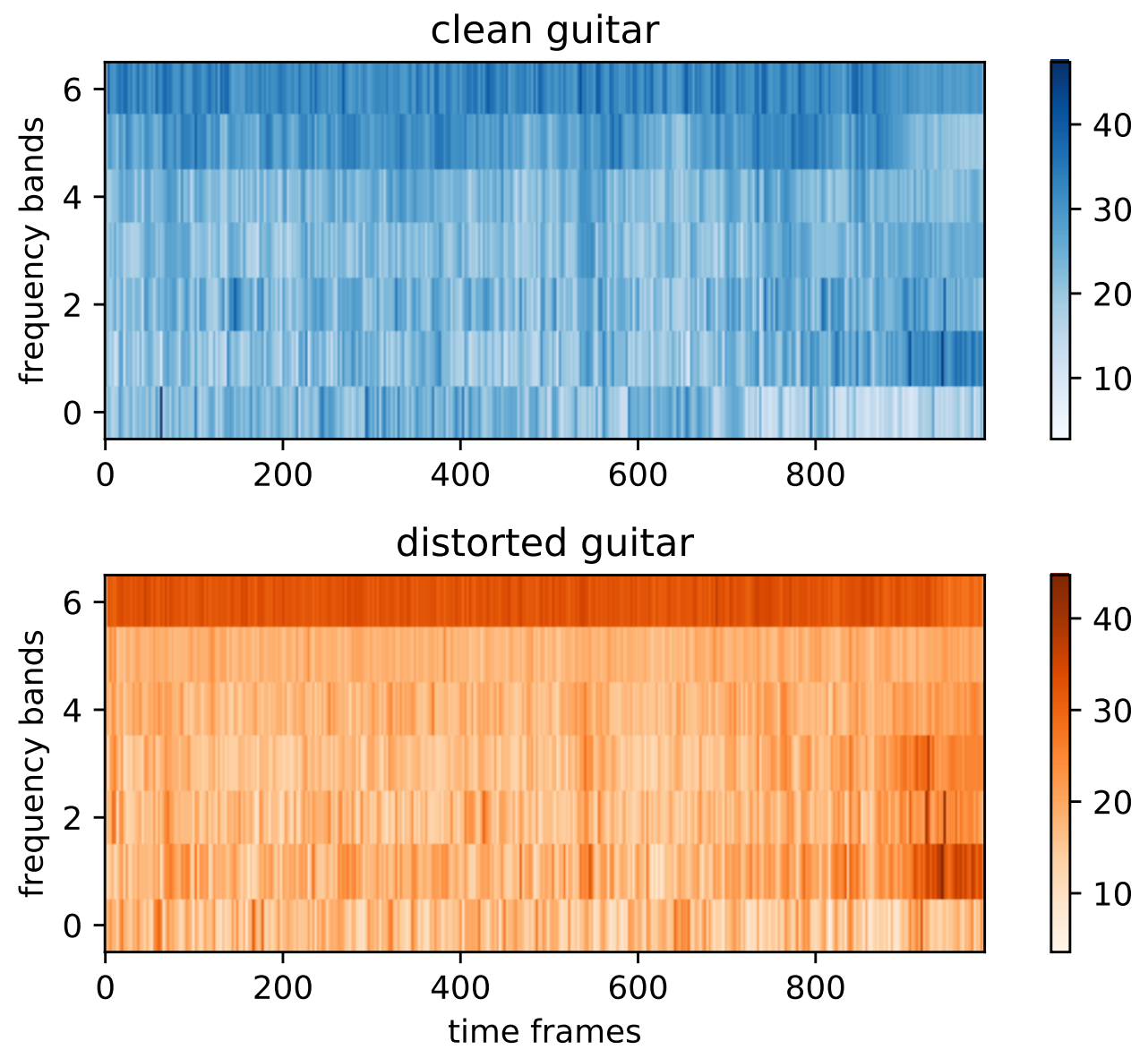

Figure 2.15: Spectral contrast values extracted from the same guitar sample before and after applying distortion.

\section{Tonnetz}

The tonnetz, also called harmonic network, is an infinite planar representation that displays the relations of the pitches from the twelve-tone equal temperament. Figure 2.16 shows part of it, and we can observe the basic three types of pitch relations:

1. horizontal lines: perfect fifths

2. upwards diagonals (from bottom-left to top-right nodes): minor thirds

3. downwards diagonals (from top-left to bottom-right nodes): major thirds

This infinite representation can be wrapped around to form an infinite tube in which the line of the fifths becomes a helix on its surface. This helix is known to be part of the spiral array model proposed by Elaine Chew [Che00] and can be seen in Figure 2.17a. For instance, a chord can be represented as a 3-dimensional point inside the tube, as the projection of the pitches in its surface.

A second transformation can be made if we assume enharmonic and octave equivalence. Part of the infinite tube can have both ends joined to compose a hypertorus. Here the circle of fifths goes around its surface three times, as we can see in Figure 2.17b. For instance, a chord can be represented as a 6 -dimensional point inside the hypertorus. The 6-tuple can be interpreted as $\left(x_{1}, y_{1}, x_{2}, y_{3}, x_{3}, y_{3}\right)$ where:

- $\left(x_{1}, y_{1}\right)$ is point in the circle of fifths

- $\left(x_{2}, y_{2}\right)$ is point in the circle of minor thirds 
- $\left(x_{3}, y_{3}\right)$ is point in the circle of major thirds

Figure 2.18 shows these three circles and the tonal centroid that represents the A-major chord.

Finally, every pitch class can be mapped to tonal centroids in this new hypertorus representation, converting 12-bin chromas into a 6 -dimensional vector. This defines the tonnetz feature that is accordingly associated with melodic and harmonic aspects. Figures 2.19 shows the tonnetz extracted from a C-major scale sample.

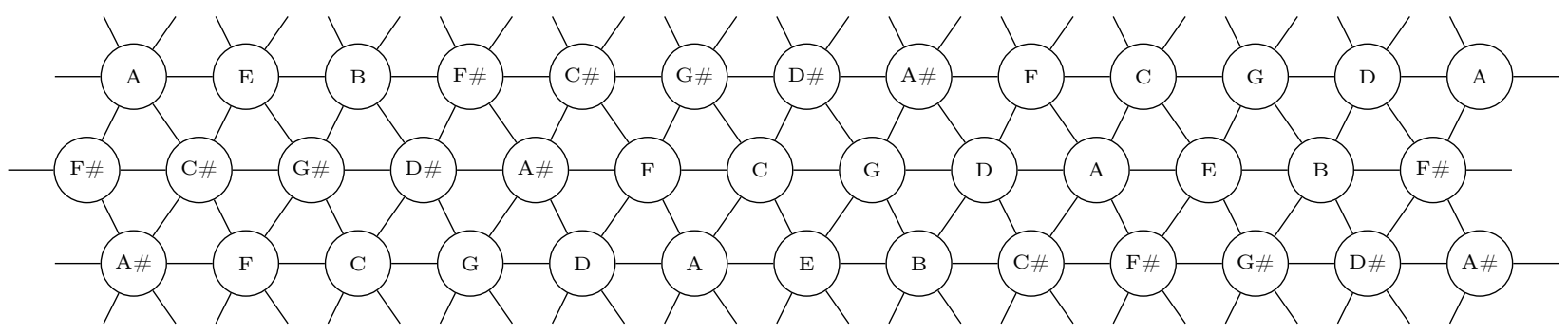

Figure 2.16: The tonnetz infinite planar representation of pitch relations.

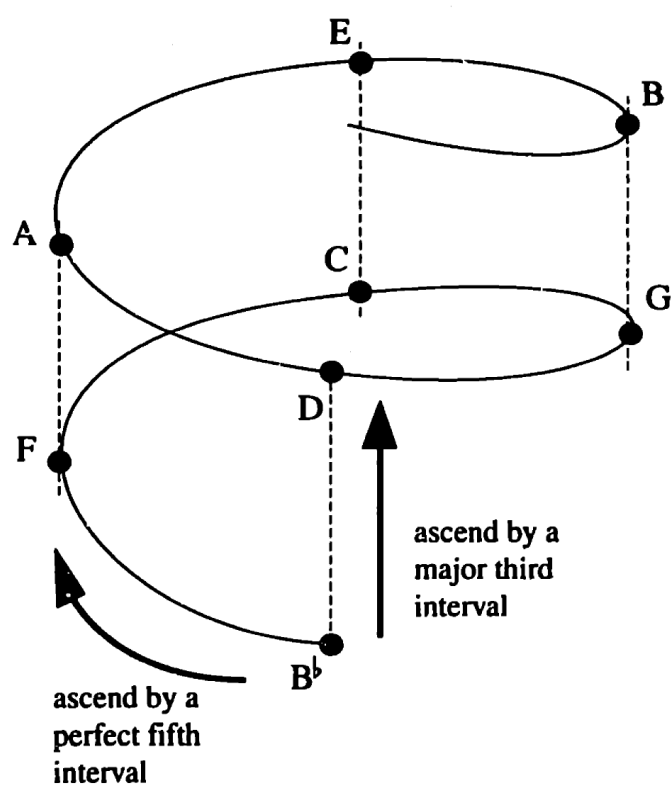

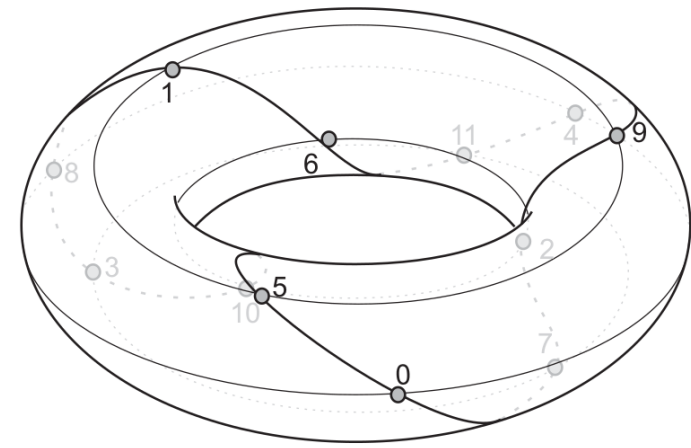

(b) The hypertorus representation of pitch relations with the helix of fifths going around it (reproduced from [HSG06]).

(a) The helix of fifths that compose the Chew's spiral array (reproduced from [Che00]).

Figure 2.17 

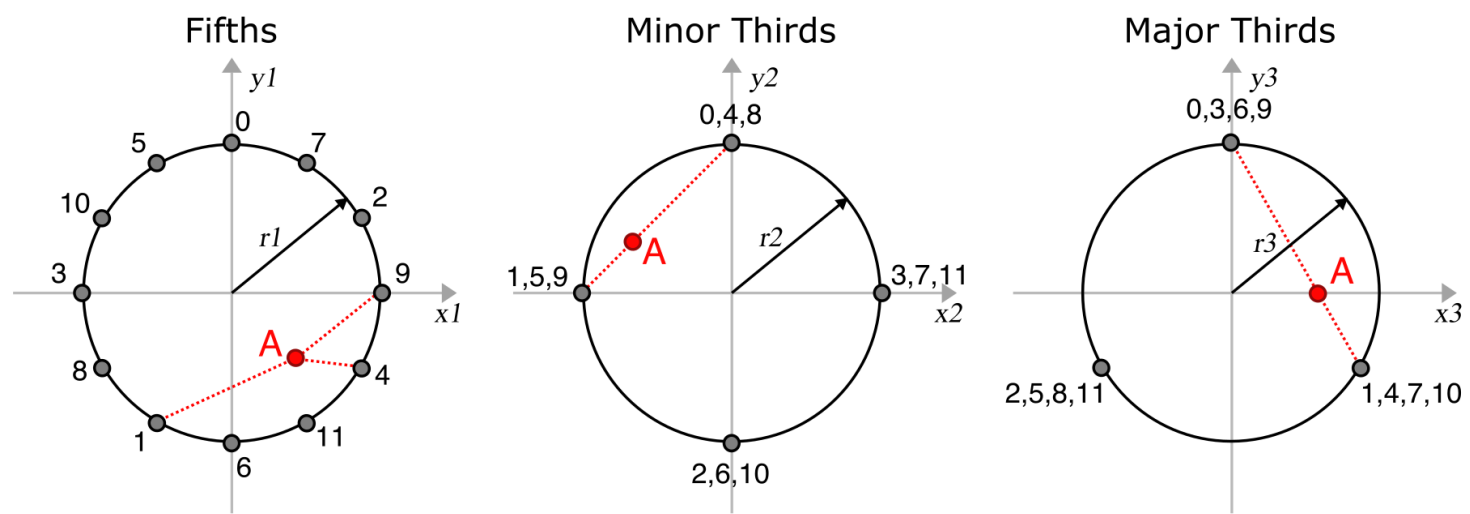

Figure 2.18: The three circles of tone relations and the tonal centroid for the A-major chord (reproduced from [HSG06]).

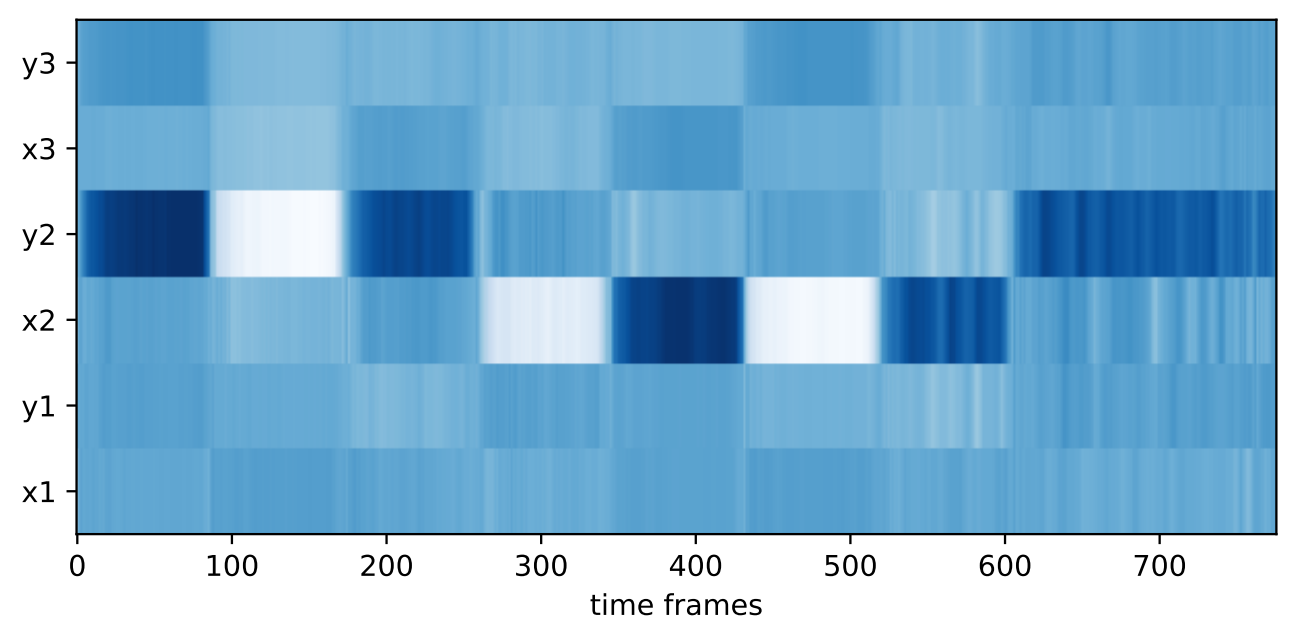

Figure 2.19: The tonnetz feature extracted from a C-major piano sample.

\section{Root Mean Square}

This feature is called Root Mean Square (RMS) and it is computed in the time-domain audio representation. It is defined as follows:

$$
r m s(x)=\sqrt{\frac{1}{N} \times \sum_{i=1}^{N} x_{i}^{2}}
$$

RMS is usually associated with dynamics, since it is a display of the signal levels over time. Figure 2.20 shows the RMS values of two songs with clear different dynamics. The first song (blue line) is the track "Broken, Beat \& Scarred" by the band Metallica, and the second song (orange line) is "Für Elise" performed by the pianist Lang Lang. 


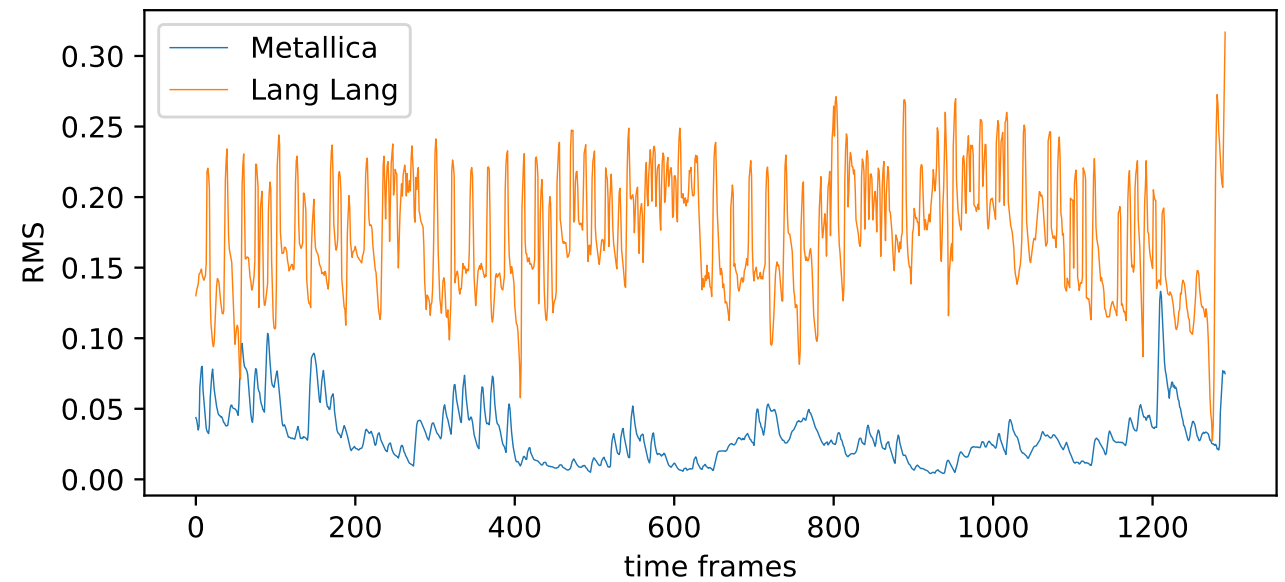

Figure 2.20: RMS values extracted from the tracks "Broken, Beat \& Scarred" (Metallica) and "Für Elise" (Lang Lang).

\section{Zero Crossing Rate}

This feature is computed in the time-domain and is a measure of how many times the audio signal crosses the x-axis. It can be used for several purposes. For instance, if the signal is very simple, such as a sinusoidal wave, the Zero Crossing Rate (ZCR) can be a useful measure to help us find the fundamental frequency. Figure 2.21 shows two sinusoids that have $110 \mathrm{~Hz}$ and $220 \mathrm{~Hz}$. Also shows that these signals have $Z C R$ values respectively $\approx 0.00488$ and $\approx 0.00976$ (notice that the ratio of $1: 2$ is respected).

ZCR can also be used to distinguish periodic and noisy sounds [Pee04]. Figure 2.21 also shows a white noise with its relative ZCR values, and we can see that they are considerably higher than the harmonic ones in the same Figure. ZCR can be used to classify percussive sounds $\left[\mathrm{GPD}^{+} 00\right]$ and categorize voiced/unvoiced signals [BKAB10].
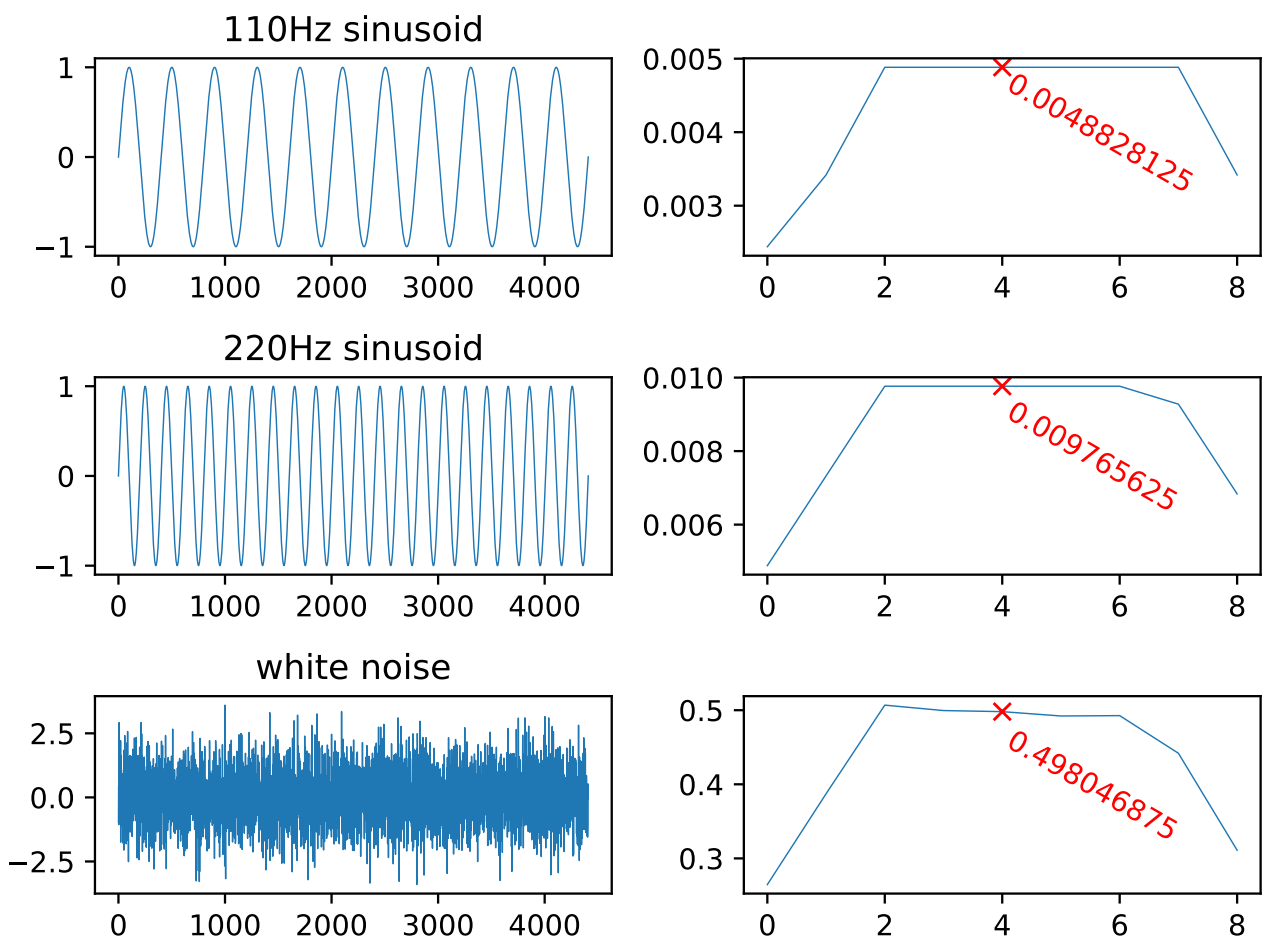

Figure 2.21: Zero crossing rate extracted from three basic signals. 


\section{Pitch Melodia}

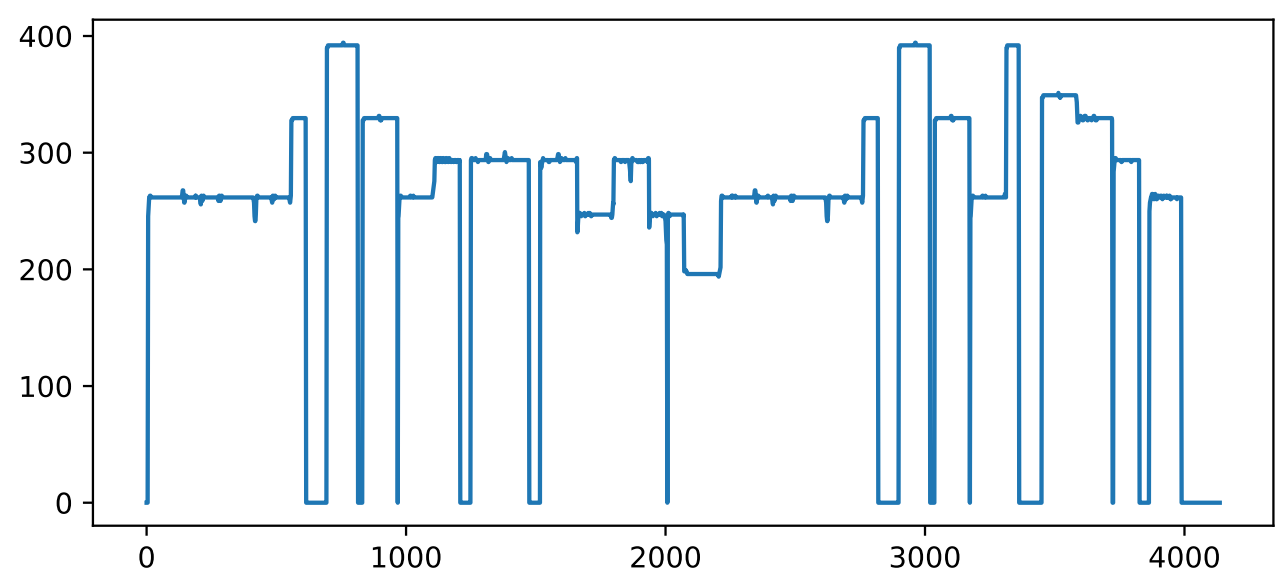

Figure 2.22: Fundamental frequencies extracted from the predominant melody of a song using the Melodia software.

The audio feature referred to as pitch_melodia is composed of the sequence of fundamental frequencies corresponding to the predominant melody in a piece of music. As we can see in Figure 2.22 in some moments the values may be zero. This occurs when no melodic component was detected in that moment (possibly in a silent or noisy part, or in a transition between two notes) [SG12a].

\section{Pitch Contour Segmentation}

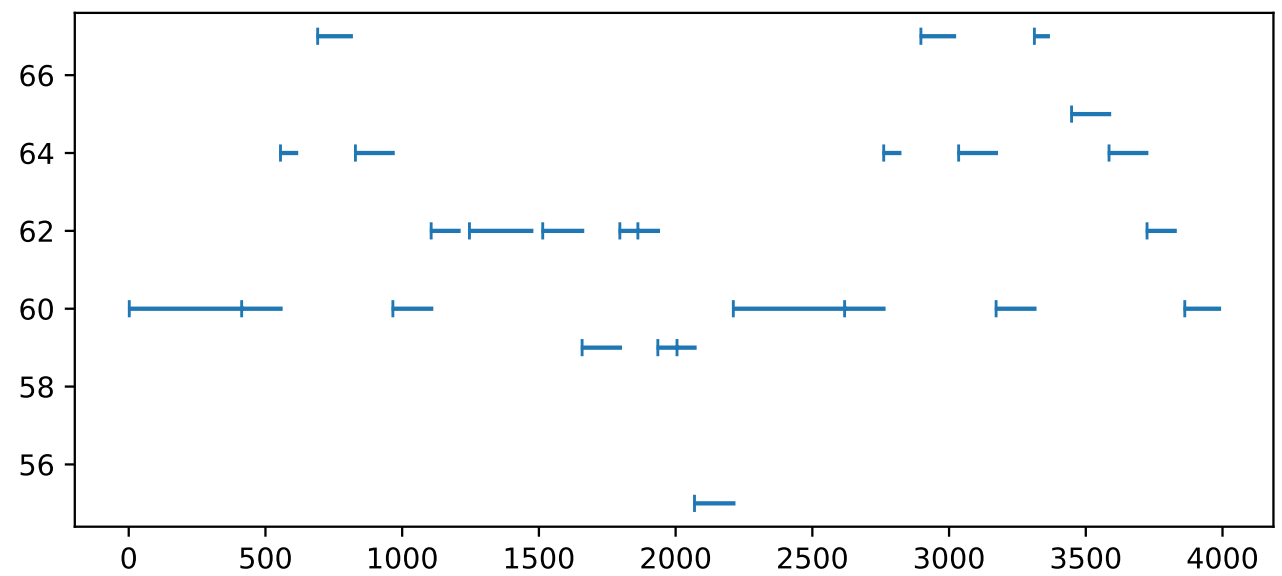

Figure 2.23: Segmentation of a fundamental frequency sequence, converted to MIDI note numbers.

The feature referred to as pitch_contour_segmentation is computed from the feature pitch_melodia. The sequence of fundamental frequencies in time is segmented to form a list of notes with their respective pitches and duration values. In addition, pitches are converted from Hertz to Musical Instrument Digital Interface (MIDI) note numbers. As we can see in Figure 2.23 the vertical axis domain is different from the one in Figure 2.22, and the same melody was used to generate both. 


\section{Rhythm Pattern}

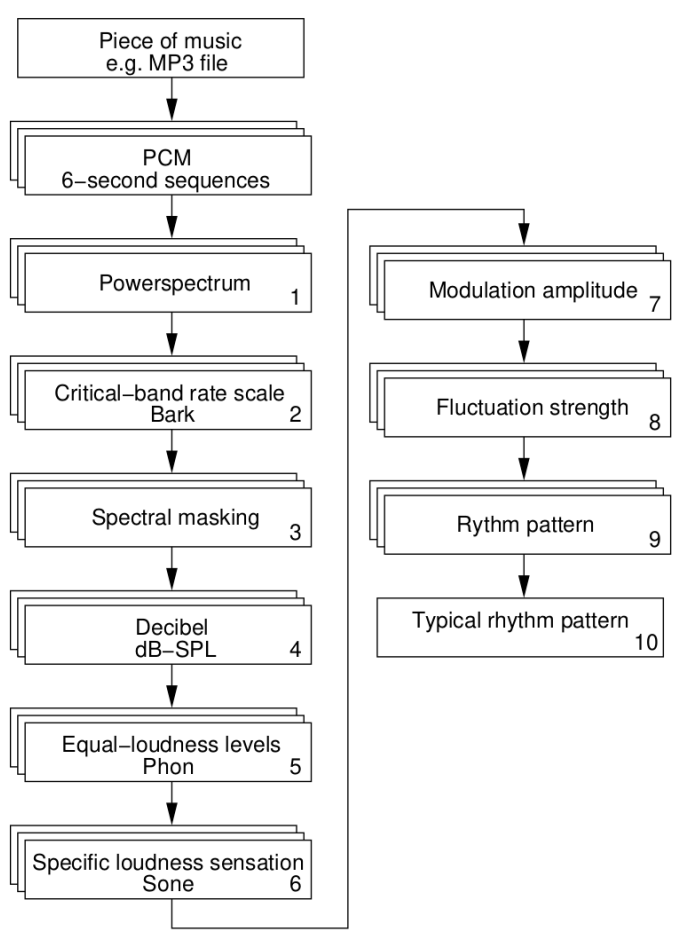

Figure 2.24: Processing pipeline to extract rhythm pattern and related features (reproduced from [PRM02a]).

Pampalk et al. [PRM02b] define the rhythm pattern audio feature computation through the processing steps below (illustrated in Figure 2.24):

1. (for every frame) compute the power-spectrum using Fourier transform;

2. convert frequencies to Bark-scale [ZF13];

3. calculate spectral masking effects [SAH79];

4. transform amplitudes to dB-SPL;

5. determine same amplitude levels with the Phon unit [ZF13];

6. recalculate the amplitudes using the Sone unit [BL81];

7. compute amplitude modulation for each critical band;

8. weight the amplitude modulation coefficients using the fluctuation strength psycho-acoustic model [Fas82];

9. apply gradient and Gaussian filters;

10. calculate the median of every frame result.

This audio feature determines fluctuation patterns for different frequency bands. The result of the steps above can be seen in Figure 2.25 for a simple 4/4 drum sample playing in 120 BPM. We can notice that near $2 \mathrm{~Hz}$ we have a column with noticeable amplitude, which is consistent with the track's tempo. Also there is observable amplitude in columns related to multiples of $2 \mathrm{~Hz}(1 \mathrm{~Hz}$ and $4 \mathrm{~Hz}$, for instance), which is expected regarding the tempo class of 120 BPM. 


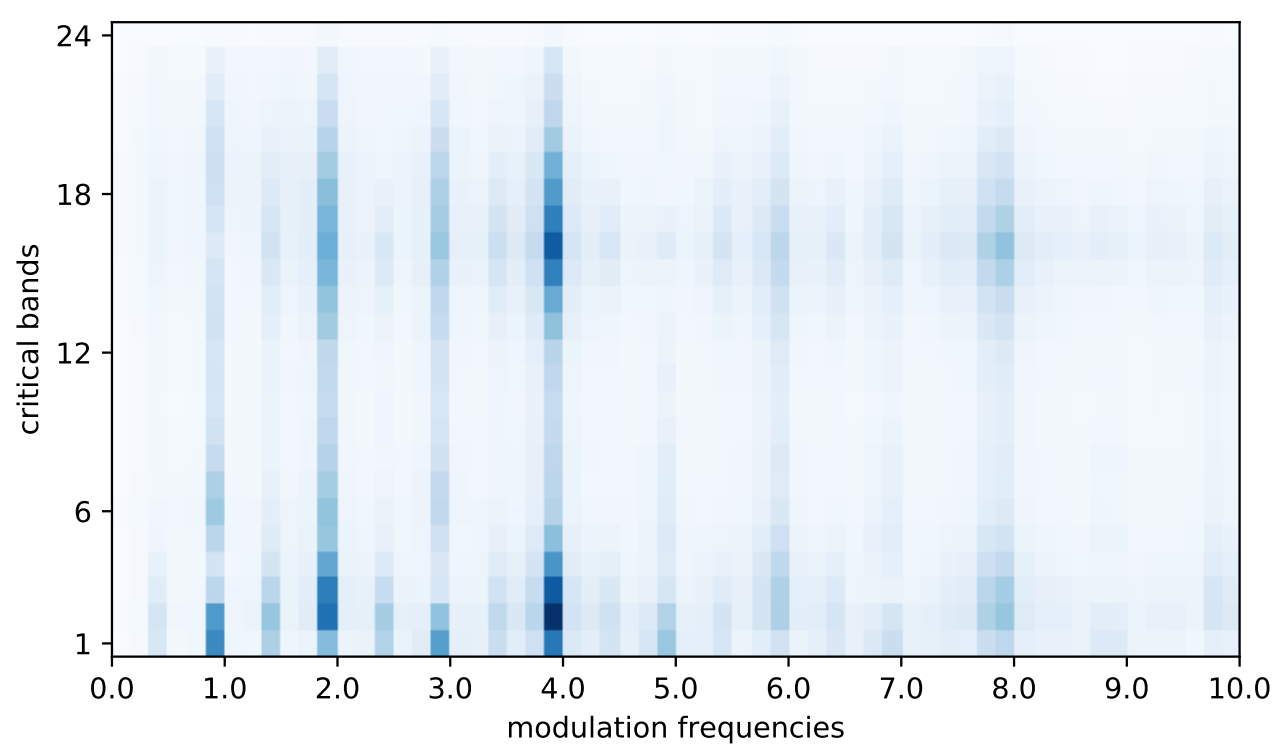

Figure 2.25: Rhythm pattern feature extracted for a 120 BPM drum sample.

\section{Rhythm Histogram}

In the processing steps of the previous feature, if we stopped before the fluctuation strength step (see Figure 2.24), we obtain the amplitude modulations that can be aggregated for each critical band resulting in a histogram of modulations. One histogram is computed for each frame of the song, and then the median of them is computed at the end. This is exactly the definition of the current feature called rhythm histogram [LR05], which is illustrated in Figure 2.26 with the same drum sample of the subsection above (and same distinguishable modulation frequencies).

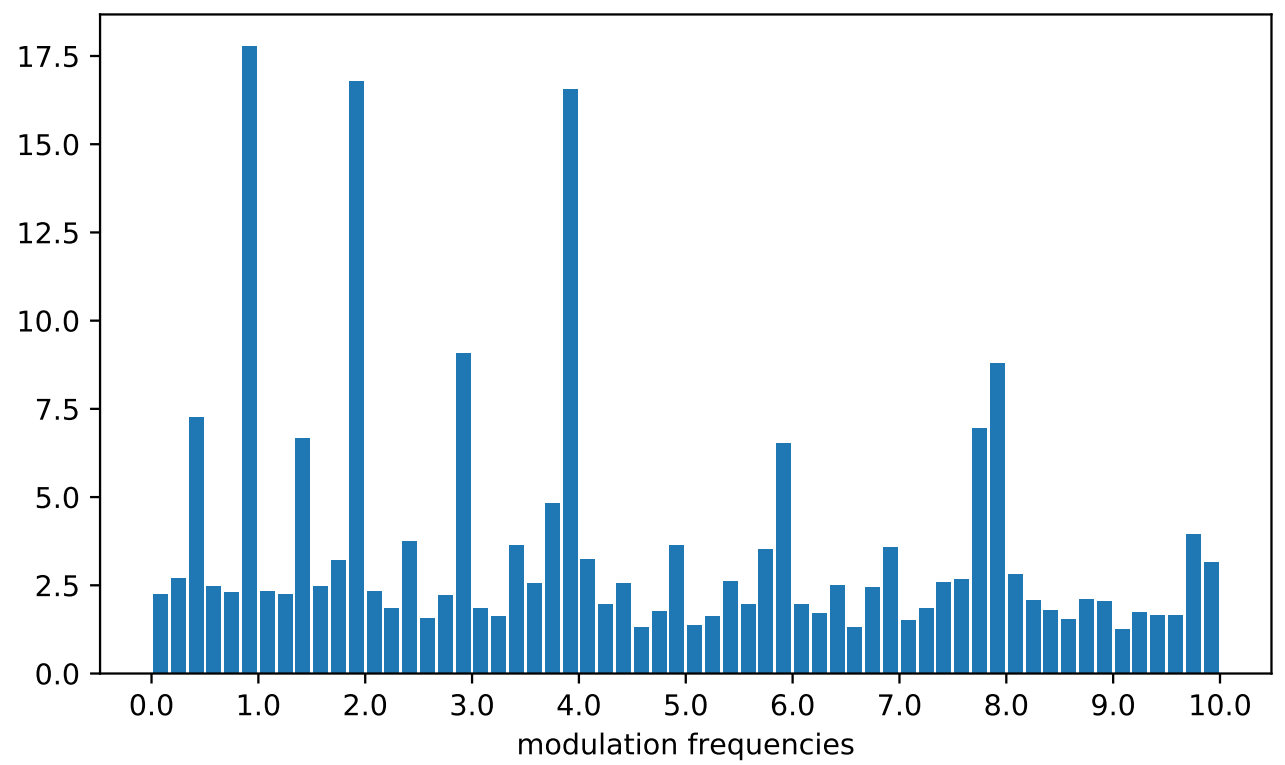

Figure 2.26: Rhythm histogram feature extracted for a 120 BPM drum sample.

\section{Temporal Rhythm Histogram}

This feature is a statistical summarization of the rhythm histograms computed in time. Rhythm histograms are computed for all frames of a song, and then a set of statistical measures (mean, variance, skewness, kurtosis, median, minimum, maximum) are calculated. The temporal rhythm 
histogram is supposed to capture change and variation of rhythmic aspects [Lid07]. Figure 2.27 shows these values for the drum sample whose rhythm histogram is displayed in Figure 2.26.

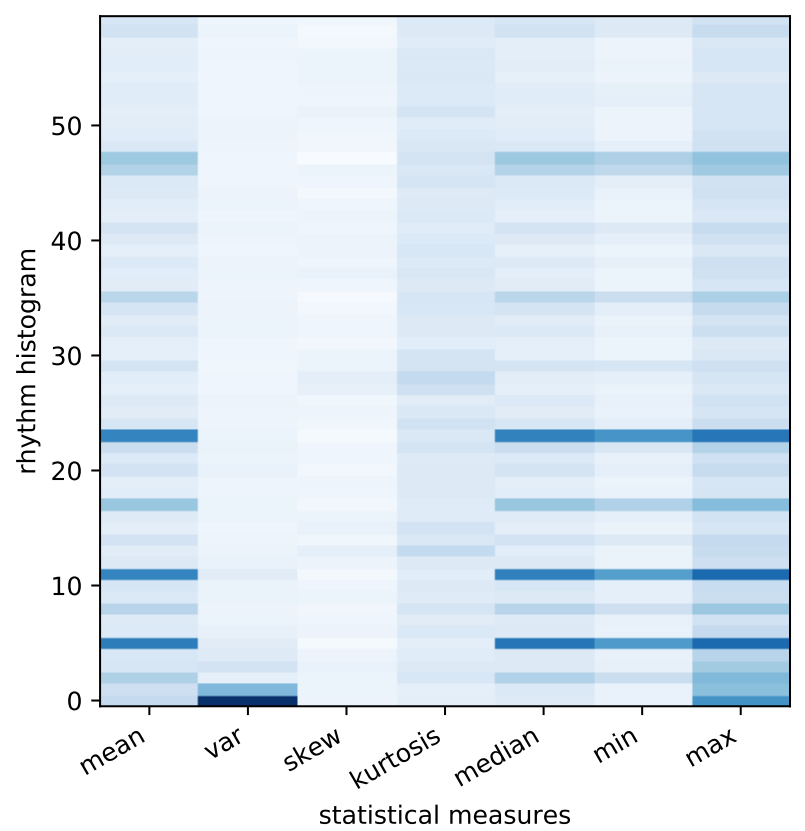

Figure 2.27: Temporal rhythm histogram feature extracted for a 120 BPM drum sample.

\section{Statistical Spectrum Descriptor}

The first six steps of the rhythm pattern processing can be used to define a feature usually called sonogram. For every frame of the song, the algorithm computes the sonogram, and then it calculates 7 statistical measures (mean, variance, skewness, kurtosis, median, minimum, maximum), for each critical band of the sonogram [Lid07]. The final statistical spectrum descriptor is the mean of these statistical vectors over time. An example of this feature is shown in Figure 2.28 extracted from the same drum sample that we have analyzed before.

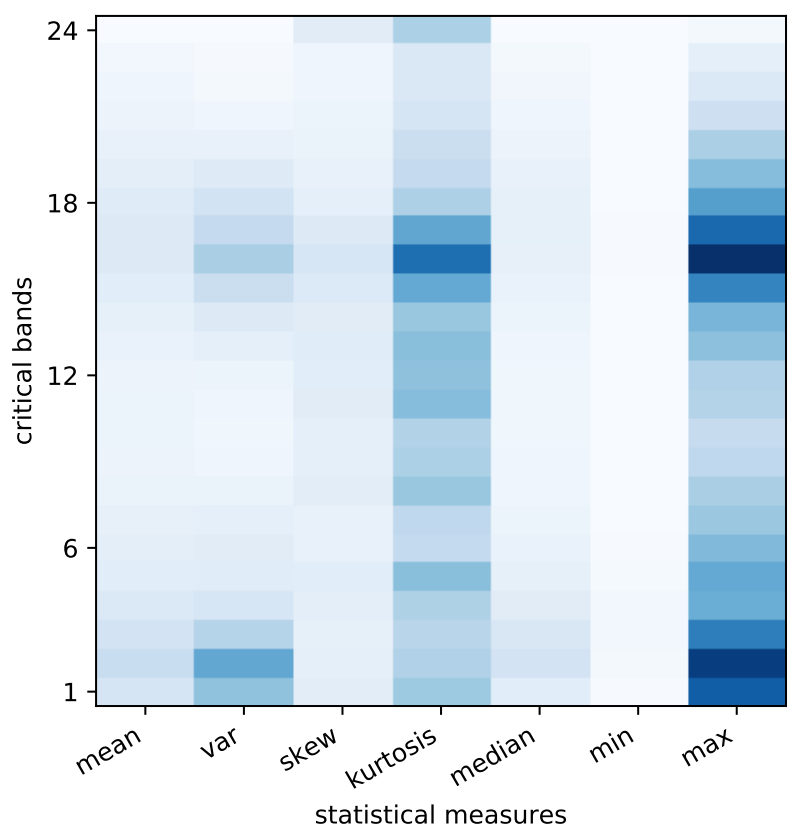

Figure 2.28: Statistical spectrum descriptor feature extracted for a 120 BPM drum sample. 


\section{Temporal Statistical Spectrum Descriptor}

This feature is derived from the statistical spectrum descriptor in a similar way as temporal rhythm histogram is derived from the rhythm histogram. The algorithm follows the same steps explained above, however at the end instead of computing the mean of the statistical vectors, it computes the same 7 statistical measures mentioned above to get the final feature. Lidy [Lid07] claims that this feature captures variations on timbre, besides the rhythmic ones. Following our analysis, the temporal statistical spectrum descriptor for the same drum sample is shown in Figure 2.29 .

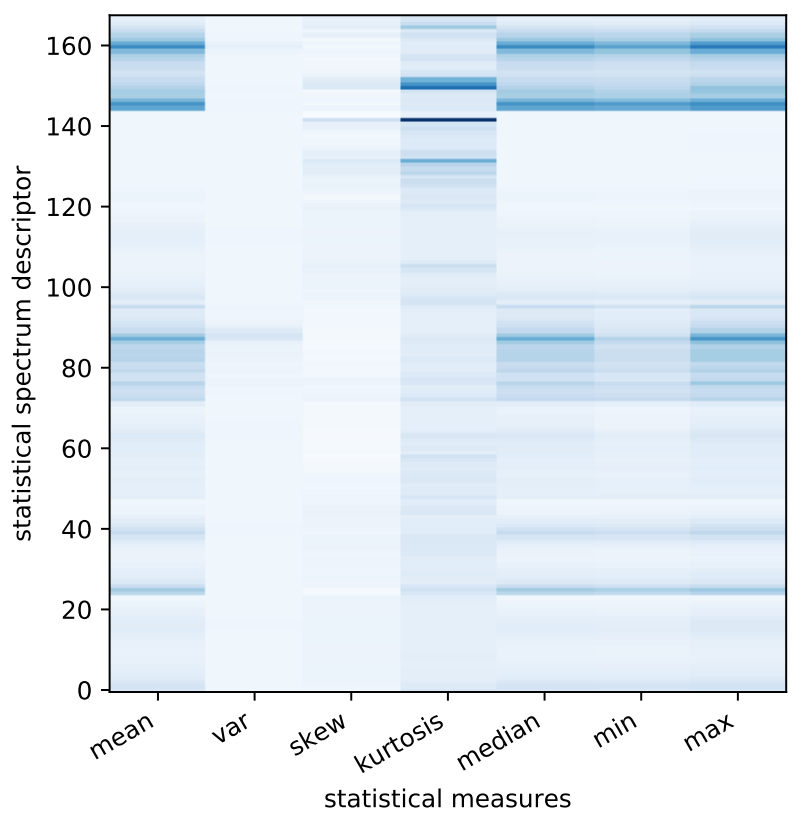

Figure 2.29: Temporal statistical spectrum descriptor feature extracted for a 120 BPM drum sample.

\section{Modulation Variance Descriptor}

For every frame of a song, the seven first processing steps of Figure 2.24 are applied. Then the 7 statistical measures are computed for the result vectors. For this feature the critical bands are aggregated, and these measures are intended to resume the modulation frequencies over time. As a final step, the mean of all statistical vectors is taken to conclude the modulation variance descriptor feature. Figure 2.30 shows an example of modulation variance descriptor for the same audio sample as above. 


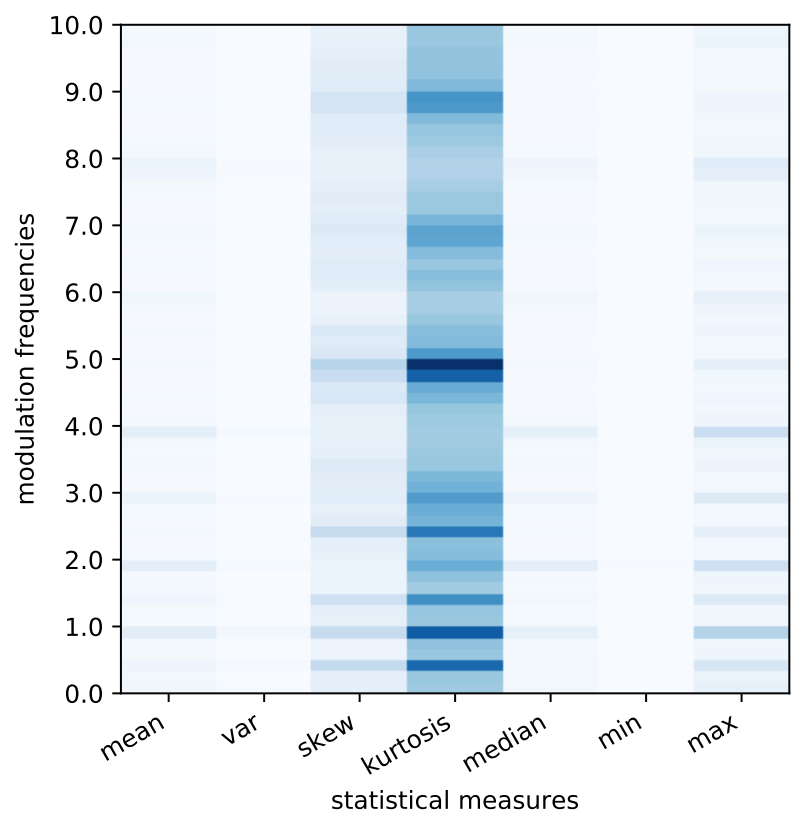

Figure 2.30: Modulation variance descriptor feature extracted for a 120 BPM drum sample.

\section{$2.2 \quad$ Feature aggregators}

Many features presented above are extracted frame by frame (i.e., they are local features) and, although this potentially reduces data dimension considerably (in comparison to the original audio), their dimension is still very high since it depends on the total length of the song. For example, even a one-dimensional local feature computed using 1024-sample frames without overlapping produces over a thousand values for 30 seconds of audio sampled at $44100 \mathrm{~Hz}$. This figure is in general multiplied by the dimension of the local feature.

In several cases, we want to do a second processing step to decrease the data sizes again. This step consists in aggregating the values of the local features to obtain a descriptor to represent the entire song (global feature). As a bonus, every global feature have the same dimension, which is not true for local features, since different audio files can have different duration values.

In this section we present some methods of local features aggregation used to generate global audio features. 


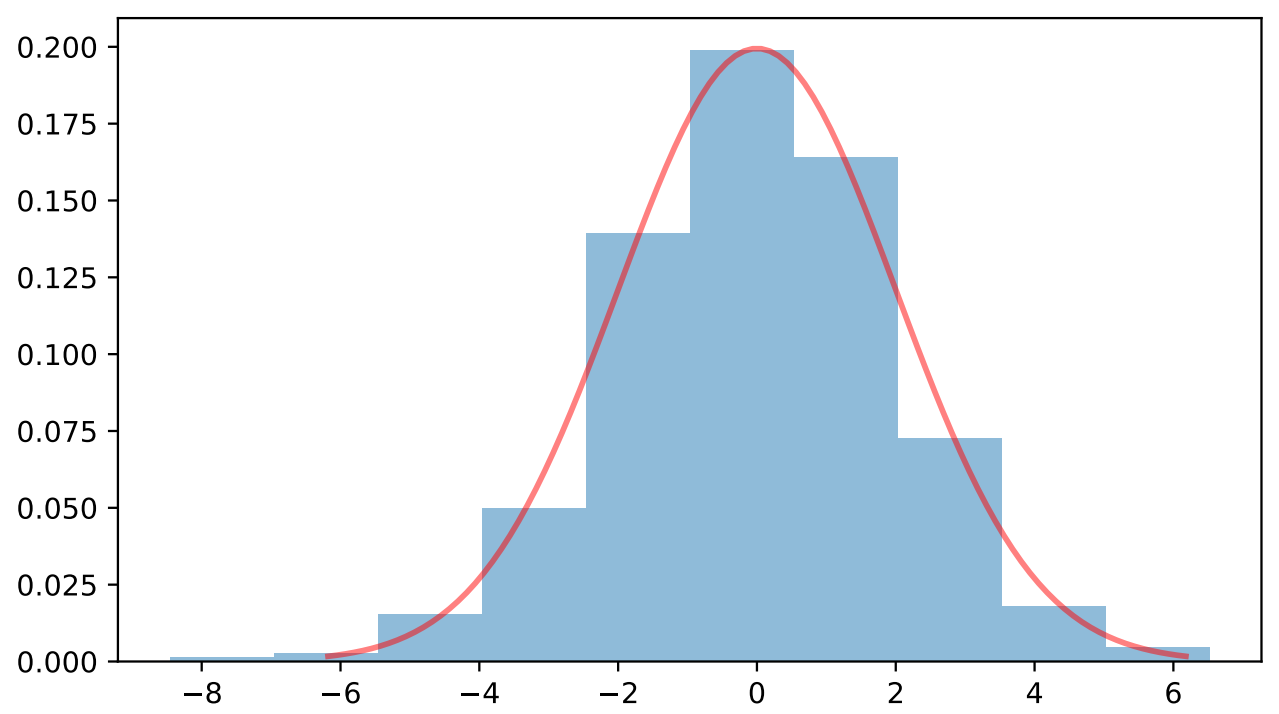

Figure 2.31: Histogram of samples and the Gaussian curve obtained after estimating the parameters $\mu$ and $\sigma^{2}$.

\section{Basic Statistics}

The aggregation method we call basic statistics consists in using a normal distribution (see Figure 2.31) to summarize the features frame by frame, that is, the evolution of local features over time. The parameters of a normal distribution are the mean $\mu$ and the variance $\sigma^{2}$.

Let $N$ be the dimension of the local feature for each frame. The global descriptor is a vector composed of the means $\left[\mu_{1}, \mu_{2}, \ldots, \mu_{N}\right]$ concatenated with the standard deviations $\left[\sigma_{1}, \sigma_{2}, \ldots, \sigma_{N}\right]$. This version is referred in Tables and Figures as basic_stats_1.

We also consider a second aggregation method where we append to the means and standard deviations the minimum and maximum values from the local features. Thus, the global feature vector becomes $\left[\mu_{1}, \ldots, \mu_{N}, \sigma_{1}, \ldots, \sigma_{N}, \min _{1}, \ldots, \min _{N}, \max _{1}, \ldots, \max _{N}\right]$, and is referred to as basic_stats_2.

\section{Difference Statistics}

This method of aggregation is quite similar to the previous one, but adds to the global feature information about the local feature's discrete difference, defined as $\operatorname{diff}[i]=$ feature $[i+1]-$ feature $[i]$, and illustrated in Figure 2.32.

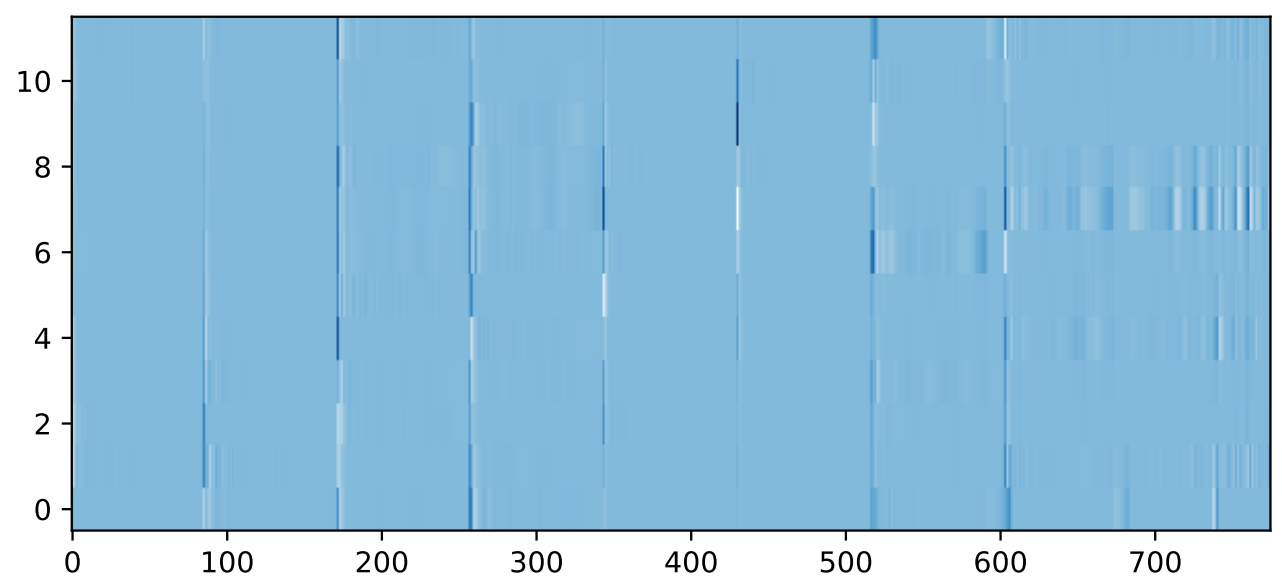

Figure 2.32: The discrete difference of the chroma_stft displayed in Figure 2.3. 
Here we define also two alternative aggregation methods. The first one, referred to as diff_stats_1, is defined as $\left[\mu_{1}, \ldots, \mu_{N}, \sigma_{1}, \ldots, \sigma_{N}\right]$ concatenated with $\left[\mu_{\text {diff }}, \ldots, \mu_{\text {dif } f_{N}}, \sigma_{\text {diff }}, \ldots, \sigma_{\text {diff }}\right]$ (the means and standard deviations of the feature's difference). The second one, referred to as diff_stats_2, is the diff_stats_1 vector concatenated with $\left[\min _{1}, \ldots, \min _{N}, \max _{1}, \ldots, \max _{N}\right]$ and $\left[\min _{\operatorname{diff} f_{1}}, \ldots, \min _{\text {diff }}\right.$, $\max _{\text {dif } f_{1}}, \ldots$, max $\left._{\text {dif } f_{N}}\right]$ (the minimum and maximum values of the feature's difference). Therefore, the difference statistics vectors have double the dimension of the basic statistics ones.

\section{Statistical Summarization}

This is the third aggregation method that uses a single Gaussian as the statistical model. The global feature is composed of $N$ means, $N$ variances and $\frac{N(N-1)}{2}$ covariances, having the dimension $2 N+\frac{N(N-1)}{2}$. The number of covariances is $\frac{N(N-1)}{2}$, because the covariance matrix is symmetric, and we can only use values below the main diagonal. This feature is referred in this text as statistical_summarization.

\section{Gaussian Mixture Model}

When the samples are organized in sub-populations, it is not enough to represent them with only one normal distribution. In this case, we can use the Gaussian Mixture Model (GMM) that considers $\mathrm{K}$ components to represent the data (see Figure 2.33).

Consider that the local features have the format $(M, N)$, where $M$ is the number of frames and $N$ is the dimension of each local feature. After the adjustment process by the model, the global feature is represented by an array of means in the $(K, N)$ format, a covariance tensor in the format $(K, N, N)$ and a vector of $K$ size weights (all flattened to form a vector).

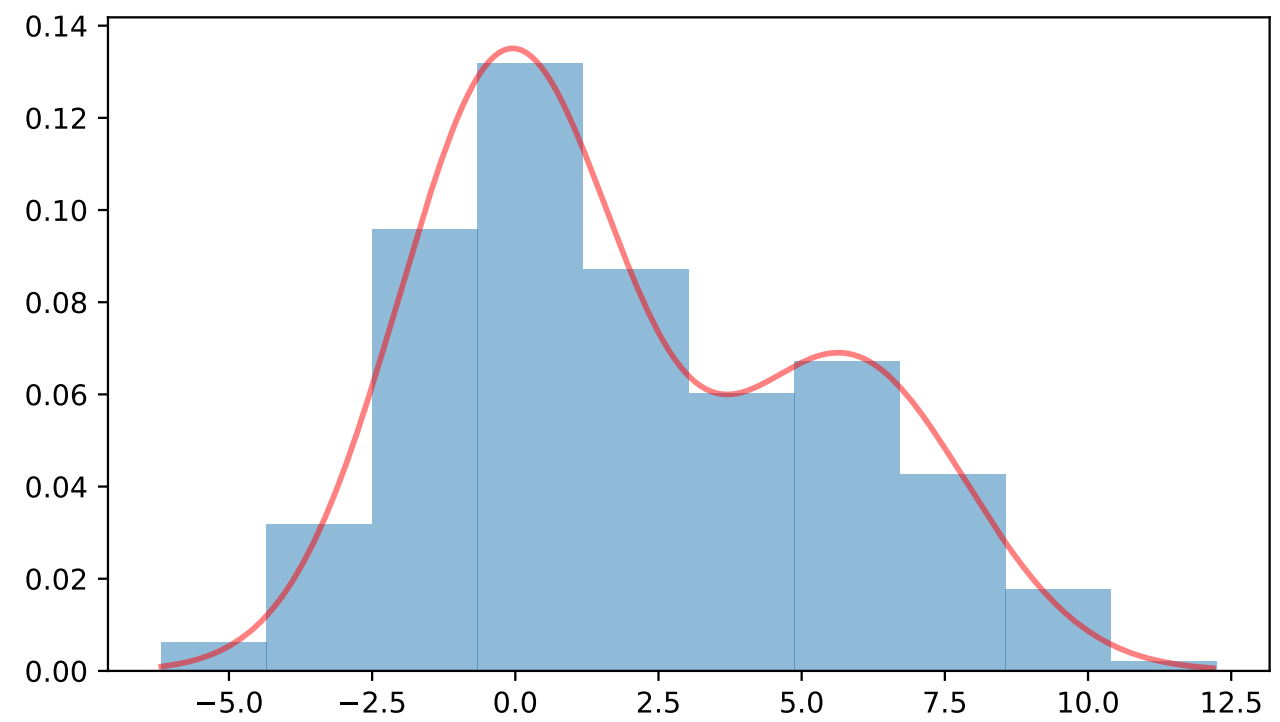

Figure 2.33: Histogram of samples and the estimated two-component GMM.

\section{Vector Quantization}

In this aggregation technique we start gathering all local features from every song in the dataset. Then, we use a clustering algorithm (in our project we use k-means) to partition these samples into separate $K$ groups. Each group is represented by their centroid. The set of all centroids is called dictionary. Then we scan each song and count for every local feature which is the closest centroid. The global feature that represents the whole song is the histogram of centroid occurrences (i.e. the histogram of the dictionary items). 
Papers that present this technique use small dictionary sizes (for instance, 8) and, although the processing takes more time and has a higher demand for memory (all songs are processed together), the global descriptor has a very small dimension compared to the local one.

Figure 2.34a shows 10 fictitious songs each one with 50 frames (so we have 500 sample points in the graph) and a fictitious feature whose dimension is 2 . The samples of one song are highlighted in red, and we can observe that a subset of these points is closer to the first centroid, and the rest is closer to the second centroid. Figure 2.34b displays the final histogram for this song: the local features from 13 frames were closer to centroid 1, and 37 to centroid 2. The global descriptor is the vector $[13,37]$.

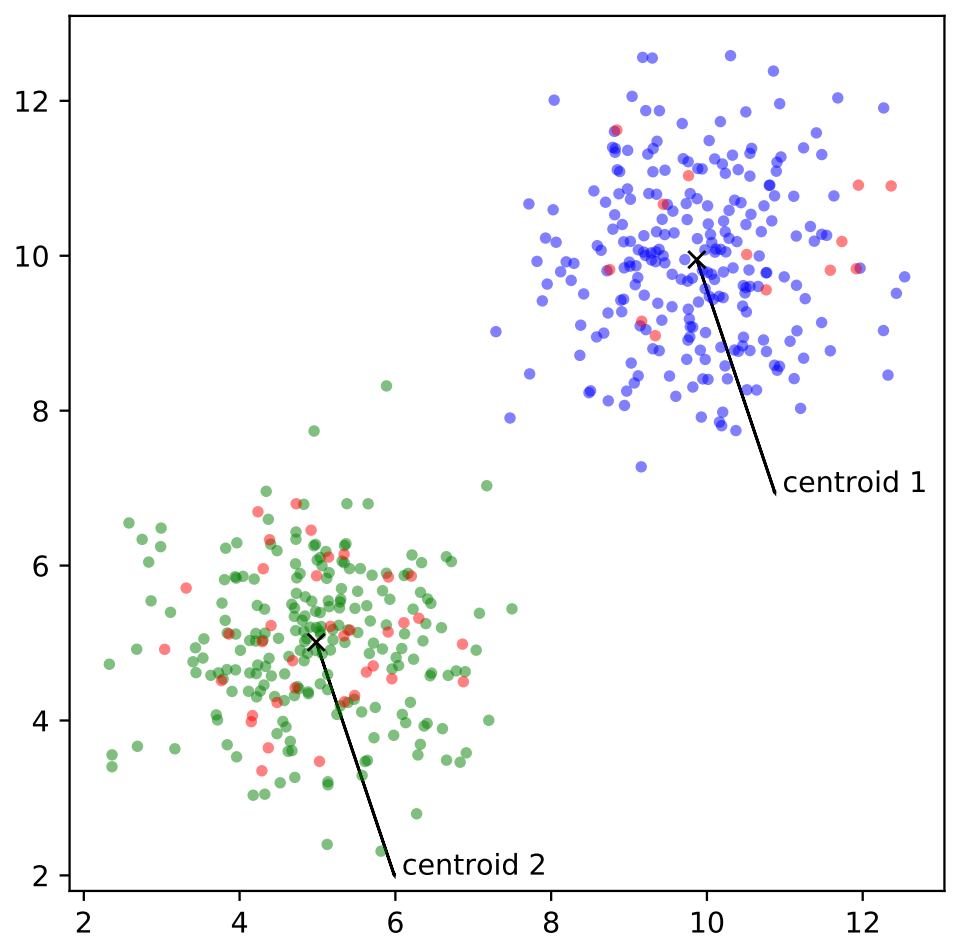

(a) Example with two sample populations and the respective centroids computed by the k-means algorithm, and the frames from one song highlighted in red color.

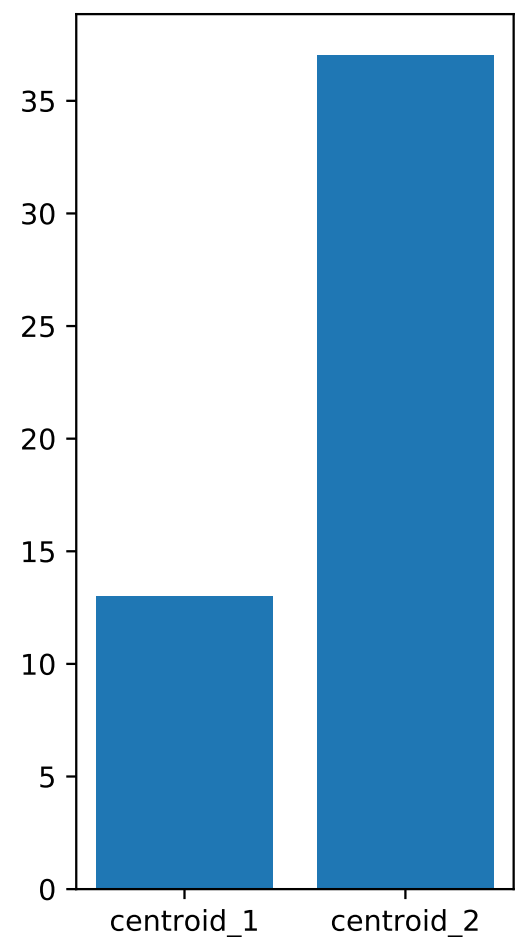

(b) An example histogram of counting the closest centroids to the samples marked as red.

Figure 2.34

\section{Markov Chains}

Audio signals are time series, that is, the occurrence of a sample is not independent from previous samples. This remains true after extracting the local descriptors, so it is desirable to use some aggregation method that considers the order of the descriptors.

Note that in the previous techniques, any global feature is the same if we perform a random permutation of the respective local features. For instance, the means and variances of a data set remain the same regardless of the order in which they are processed; the histogram of dictionary items remains the same regardless of the order in which the samples are counted.

With the purpose of not ignoring the order in which local features temporally occur, an alternative is to use Markov chains. They have states that represent values and edges that represent transitions between states, each edge associated with a probability of transition between states (as we can see in Figure 2.35). 


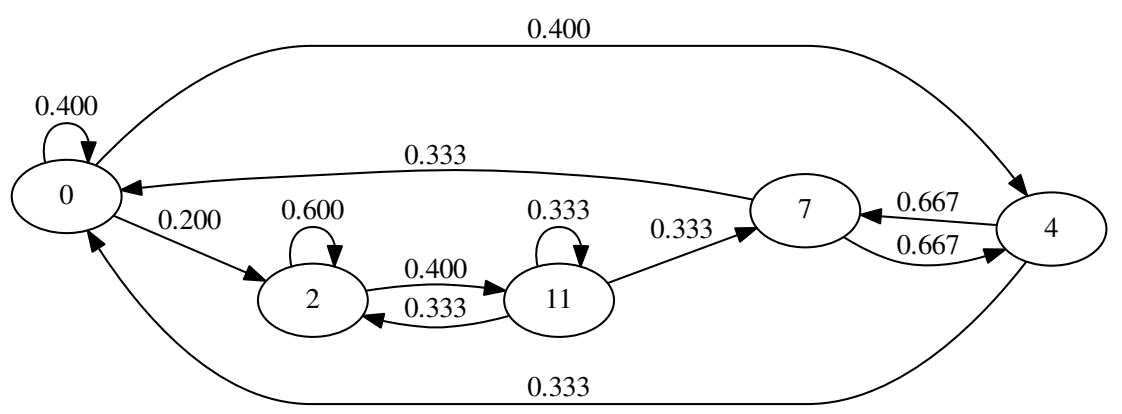

Figure 2.35: Markov chain built from a simple melody.

\section{Octave Abstraction}

This method is designed for pitch vectors that can be obtained by discarding the duration values from the Pitch Contour Segmentation feature (as displayed in Figure 2.36). We can apply some abstractions to this list. The first, which defines the current technique, is to take only the chromas instead of the pitches [SSG13b].

The result can be seen in Figure 2.37, and the formula that defines it from the original list of pitches is:

octave_abstraction $(i)=\operatorname{pitch}(i) \% 12$

$[60,60,64,67,64,60,62,62,62,59,62,62,59,59,55,60,60,64,67,64]$

Figure 2.36: MIDI note number sequence obtained discarding the duration values from the Pitch Contour Segmentation feature.

$[0,0,4,7,4,0,2,2,2,11,2,2,11,11,7,0,0,4,7,4]$

Figure 2.37: Octave abstraction to a melodic line.

\section{Interval Abstraction}

This method is also designed for the sequence of MIDI note numbers. It is called interval abstraction, considers the finite difference of the melodic line, and is defined according to the following formula [SSG13b]:

interval_abstraction $(i)=\operatorname{pitch}(i+1)-\operatorname{pitch}(i)$

The outcome for the same melodic sequence presented in Figure 2.36 above can be seen in Figure 2.38. 
$[0,4,3,-3,-4,2,0,0,-3,3,0,-3,0,-4,5,0,4,3,-3]$

Figure 2.38: Interval abstraction to a melodic line.

\section{Pitch Contour: 3 levels}

This technique is also constructed from a sequence of pitches. In this case, we have another interval abstraction, but instead of computing the difference, we only check if the values go up (' $u$ '), down (' $\mathrm{d}$ '), or repeat (' $\mathrm{r}$ ') [GLSC95]. The formula that defines it is:

$$
p_{3 \_ \text {levels }}(i)=\left\{\begin{array}{l}
\text { ' } u \text { ', } \operatorname{pitch}(i)>\operatorname{pitch}(i-1) \\
\text { ' } d^{\prime}, \operatorname{pitch}(i)<\operatorname{pitch}(i-1) \\
\text { ' } r \text { ', otherwise. }
\end{array}\right.
$$

The result for the melodic sequence from the previous examples can be seen in Figure 2.39.

$$
\text { ['r', 'u', 'u', 'd', 'd', 'u', 'r', 'r', 'd', 'u', 'r', 'd', 'r', 'd', 'u', 'r', 'u', 'u', 'd'] }
$$

Figure 2.39: 3-level pitch contour abstraction to a melodic line.

\section{Pitch Contour: 5 levels}

This method is very similar to the previous one, but considers 5 levels: up (' $u$ '), up a lot (' $U$ '), down ('d'), down a lot ('D'), and repeat ('r') [RH06b]. Its definition is as follows:

$$
p_{5 \_ \text {levels }}(i)=\left\{\begin{array}{l}
\text { ' } U^{\prime}, \operatorname{pitch}(i)>\operatorname{pitch}(i-2) \\
\text { ' } D^{\prime}, \operatorname{pitch}(i)<\operatorname{pitch}(i-2) \\
\left\{\begin{array}{l}
\text { ' } u \text { ', } \operatorname{pitch}(i)>\operatorname{pitch}(i-1) \\
\text { ' } d^{\prime}, \operatorname{pitch}(i)<\operatorname{pitch}(i-1) \\
\text { ' } r^{\prime}, \operatorname{pitch}(i)=\operatorname{pitch}(i-1)
\end{array} \quad, \operatorname{pitch}(i)=\operatorname{pitch}(i-2)\right.
\end{array}\right.
$$

The values obtained from the melody in Figure 2.36 are shown in Figure 2.40.

$$
\text { ['U', 'U', 'd', 'D', 'D', 'U', 'r', 'D', 'u', 'U', 'D', 'D', 'D', 'U', 'U', 'U', 'U', 'd'] }
$$

Figure 2.40: 5-level pitch contour abstraction to a melodic line.

\subsection{Distance measures}

The next step, after selecting an audio descriptor and a feature aggregator, is to decide how to compare global features of two songs. The computation of the similarity between two songs is based on the distance between them. In this section we present all the distance measurements we use. 

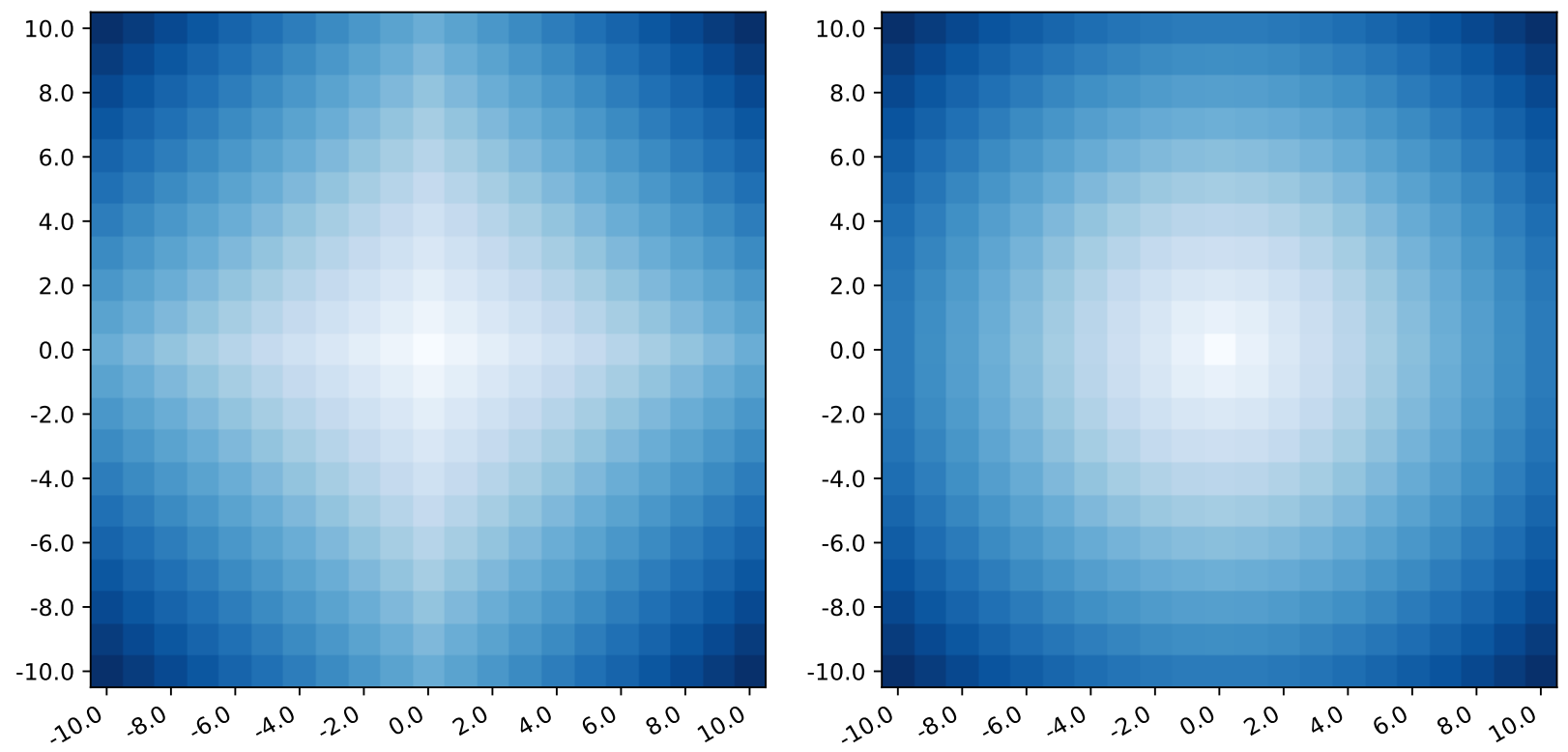

(a) Manhattan distances to $(0,0)$ computed for values in a (b) Euclidean distances to $(0,0)$ computed for values in a discrete rectangle in $\mathbb{R}^{2}$ for illustration.

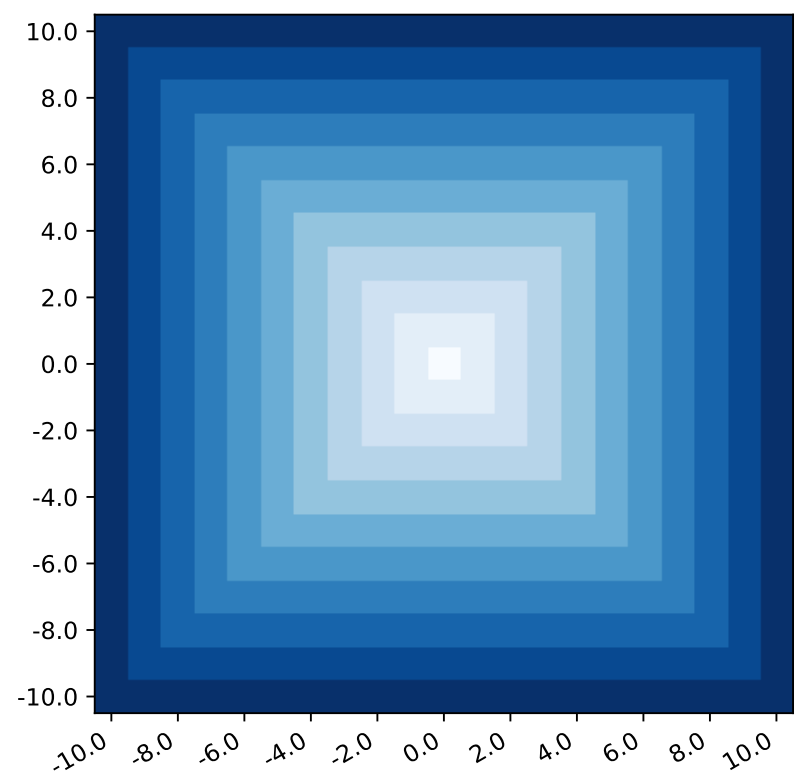
discrete rectangle in $\mathbb{R}^{2}$ for illustration.

(c) Chebyshev distances to $(0,0)$ computed for values in a (d) Cosine distances to $(1,1)$ computed for values in a discrete rectangle in $\mathbb{R}^{2}$ for illustration.

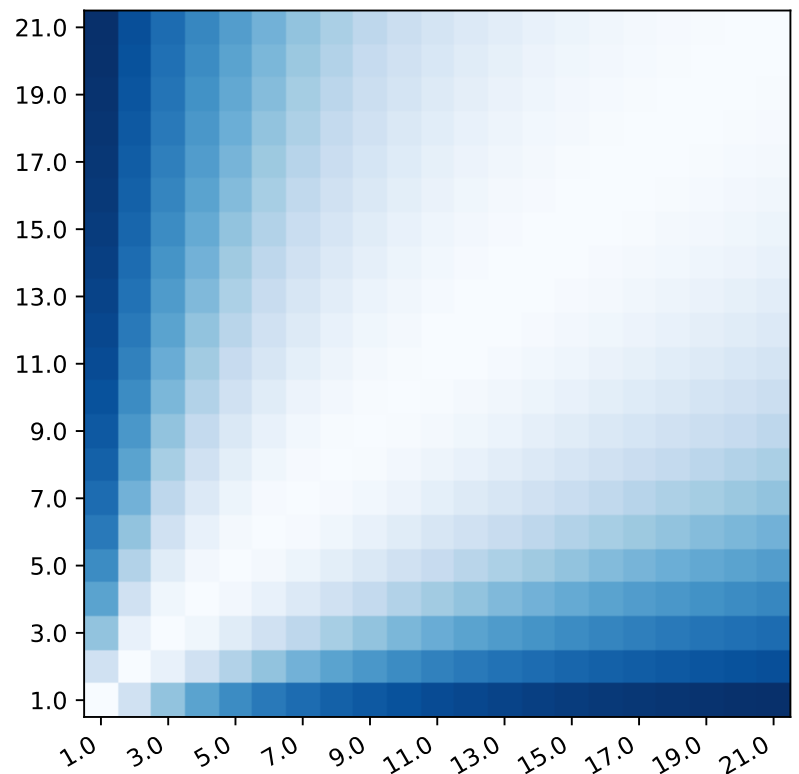
discrete rectangle in $\mathbb{R}^{2}$ for illustration.

Figure 2.41

\section{Manhattan distance}

The first distance function is the Manhattan that computes the distance between two elements of a vector space ( $\mathbb{R}^{n}$ in our case) according to the following formula:

manhattan_distance $(u, v)=\sum_{i=1}^{n}\left|u_{i}-v_{i}\right|$

\section{Euclidean distance}

The Euclidean distance is also computed in the $\mathbb{R}^{n}$ vector space and follows the formula below: 
euclidean_distance $(u, v)=\sqrt{\sum_{i=1}^{n}\left(u_{i}-v_{i}\right)^{2}}$

\section{Chebyshev distance}

The Chebyshev distance is computed between two vectors in $\mathbb{R}^{n}$ according to the following formula:

$\operatorname{chebyshev\_ distance}(u, v)=\max \left(\left|u_{i}-v_{i}\right|\right)$

\section{Cosine distance}

The cosine distance is defined for non-zero vectors in $\mathbb{R}^{n}$ space as the formula below:

$$
\text { cosine_distance }(u, v)=1-\frac{u \cdot v}{\|u\|\|v\|}
$$

\section{LCS-based distance}

The algorithm that computes the Longest Common Subsequence (LCS) can be used to obtain a distance between two sequences of symbols. This dynamic programming algorithm handles additions, deletions and substitutions, and returns the size of the largest common subsequence. This is actually a value of similarity between the two sequences. To obtain a distance measure one possibility is to use the ratio between the LCS-length and the length of the longest sequence, as defined by the following formula:

$$
l c s \_\operatorname{distance}(u, v)=1-\frac{\text { lcs_length }(u, v)}{\max (|u|,|v|)}
$$

Alternatively, this ratio can be computed using the length of the shortest subsequence being compared, and the mean length of both sequences. Figure 2.42 shows the comparison between two sequences of length 9 . The length of the longest common subsequence is 7 . So the distance is $1-7 / 9 \approx 0.222$.

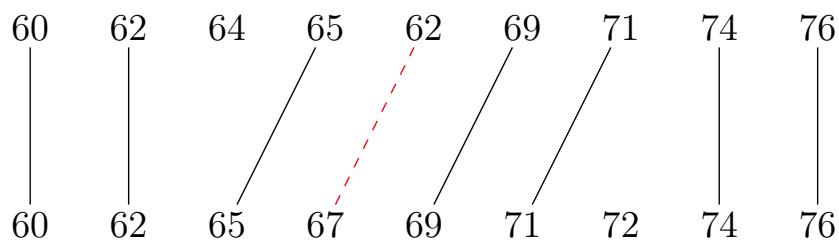

Figure 2.42: An example of the longest common subsequence computation.

\section{Levenshtein's distance}

Levenshtein's distance, also known as edit distance, is defined between two sequences of symbols and provides us with the number of insertion, deletion or substitution operations necessary to transform one sequence into another. It is defined according to the formula below:

$$
\text { levenshtein_distance }_{u, v}(i, j)=\left\{\begin{array}{l}
\max (i, j), i f \min (i, j)=0 \\
\min \left\{\begin{array}{l}
\text { levenshtein_distance } u, v(i-1, j)+1 \\
\text { levenshtein_distance }{ }_{u, v}(i, j-1)+1 \\
\text { levenshtein_distance }{ }_{u, v}(i-1, j-1)+I\left(u_{i}, v_{j}\right)
\end{array},\right. \text { otherwise. }
\end{array}\right.
$$


where $I(x, y)=0$ if $x=y$ and $I(x, y)=1$ if $x \neq y$.

Here the ratio between the Levenshtein distance and the minimum, maximum, and mean of the sequences' lengths is also computed. Figure 2.43 gives an example of two sequences of size 9 . The edit distance is 3 , and the final distance value is $3 / 9 \approx 0.333$.

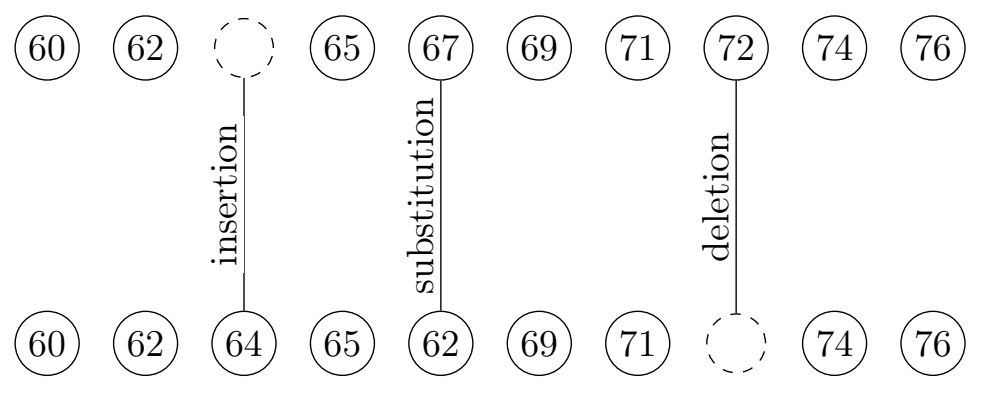

Figure 2.43: An example of the edit distance computation.

\section{Kullback-Leibler distance}

The Kullback-Leibler (KL) divergence [KL51], also known as relative entropy, gives us a measure of how much two probability distributions differ from each other. If $\mathrm{P}$ and $\mathrm{Q}$ are two continuous distributions, the KL-divergence is defined as:

$$
k l \_\operatorname{divergence}(P, Q)=\int_{-\infty}^{\infty} p(x) \log \left(\frac{p(x)}{q(x)}\right) d x
$$

If $\mathrm{P}$ and $\mathrm{Q}$ are two discrete distributions, the KL-divergence is defined as:

$$
k l \_\operatorname{divergence}(P, Q)=\sum_{i} P(i) \log \left(\frac{P(i)}{Q(i)}\right)
$$

However, one of our premises is that the distance functions should be symmetrical. Since this is not true for a divergence, a distance measure can be defined according to the formula below [KS16]:

$$
k l \_\operatorname{distance}(P, Q)=\frac{1}{2}\left(k l \_\operatorname{divergence}(P, Q)+k l \_ \text {divergence }(Q, P)\right)
$$

Figure 2.44 shows how this distance behaves by comparing several distributions with a base distribution (with mean equals 0 and standard deviation equals 1 ). The parameters of these distributions are shown on the axes, the horizontal axis represents the means and the vertical one the standard deviations.

Note that the distance increases as the mean increases, and also when the standard deviation increases. However there is a slight inequality in this growth. The distance is more sensitive to the variation of the standard deviation than to the mean. 


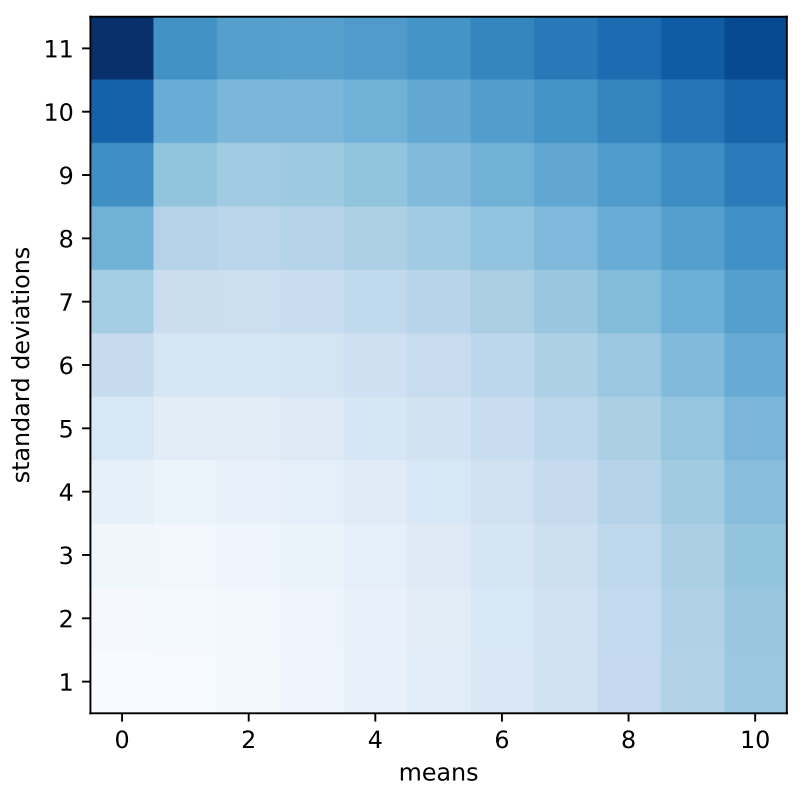

Figure 2.44: Kullback-Leibler distance calculated for pairs of Gaussian distributions.

\section{Earth Mover's distance}

The Earth Mover's Distance (EMD), also called Wasserstein distance, is defined between two distributions of probability. It has this name as an analogy where each distribution is a mound of land and the metric is related to the minimum cost of transforming one mound into another, considering the distance and also the amount of earth to be transported between them.

To illustrate how this distance behaves, we computed the EMD for several Gaussian distributions against one fixed distribution (with mean equals 0 and standard deviation equals 1 ). The results are shown in Figure 2.45.

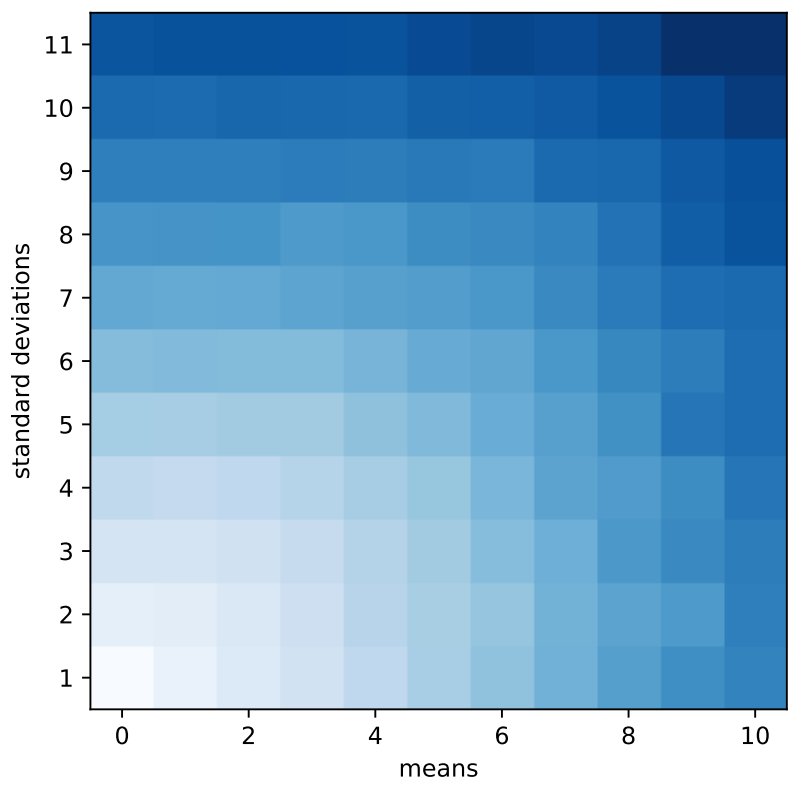

Figure 2.45: EMD calculated for pairs of Gaussian distributions.

\section{Monte Carlo distance}

When comparing two distributions we can determine how distant they are by taking samples from the first one and computing the probability that these samples were generated by the second. 
This method known as Monte Carlo sampling can be used to create a measure of distance, according to the following formula [AP04]:

$$
\begin{aligned}
\text { monte_carlo_distance }(P, Q) & =\sum_{i=1}^{s} \log p\left(S_{i}^{P} \mid P\right)+\sum_{i=1}^{s} \log p\left(S_{i}^{Q} \mid Q\right) \\
& -\sum_{i=1}^{s} \log p\left(S_{i}^{P} \mid Q\right)-\sum_{i=1}^{s} \log p\left(S_{i}^{Q} \mid P\right),
\end{aligned}
$$

where $P, Q$ are the probability distributions, $S_{i}^{A}$ is the i-th sample of $A$, and $p\left(S_{i}^{A} \mid B\right)$ is the probability that the i-th sample of $A$ is in $B$.

Figure 2.46 shows the values of Monte Carlo distances calculated for the same pairs of normal distributions we considered in the two previous distance measures.

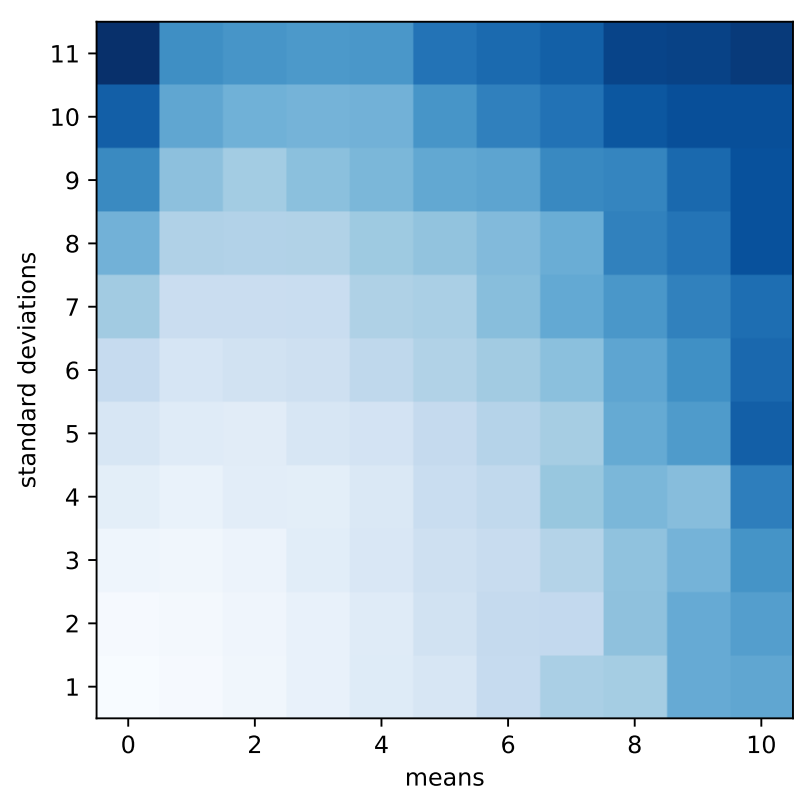

Figure 2.46: Monte carlo distance calculated for pairs of Gaussian distributions.

\subsection{Music similarity quality measures}

After choosing an audio feature, an aggregation technique and a distance measure (that is, a triplet \{extractor, aggregator, distance\} which defines a music similarity model), we would like to know the quality of this model on estimating similarity values for a dataset. In this section we will look at two representations commonly seen in the literature, and propose a new one.

\section{Similarity matrix}

The most popular way found in the literature to obtain a representation of the music similarity model quality is the similarity matrix. Its creation consists in computing the similarity of all possible music pairs in the dataset. The $i, j$ position of this matrix contains the similarity between the i-th and the $\mathrm{j}$-th songs. It can be defined from a distance measure according to the following formula (where dist is normalized between 0 and 1 ):

$$
\operatorname{sim}\left(t_{i}, t_{j}\right)=1-\hat{d i s t}\left(t_{i}, t_{j}\right)
$$

As we can see in Figure 2.47 the matrix main diagonal has the highest values (because the songs have maximum similarity with themselves) and we can also observe some darker and lighter regions. The darker regions show a subset of the data with high intra-similarity. In Figure 2.47, for example, 
we were able to identify darker square regions $(4 \times 4)$ related to songs of a class with high internal similarity. The lighter colored regions represent a lower inter-similarity. Again, in Figure 2.47 we can identify a cross-shaped region showing a low external similarity of the second class.

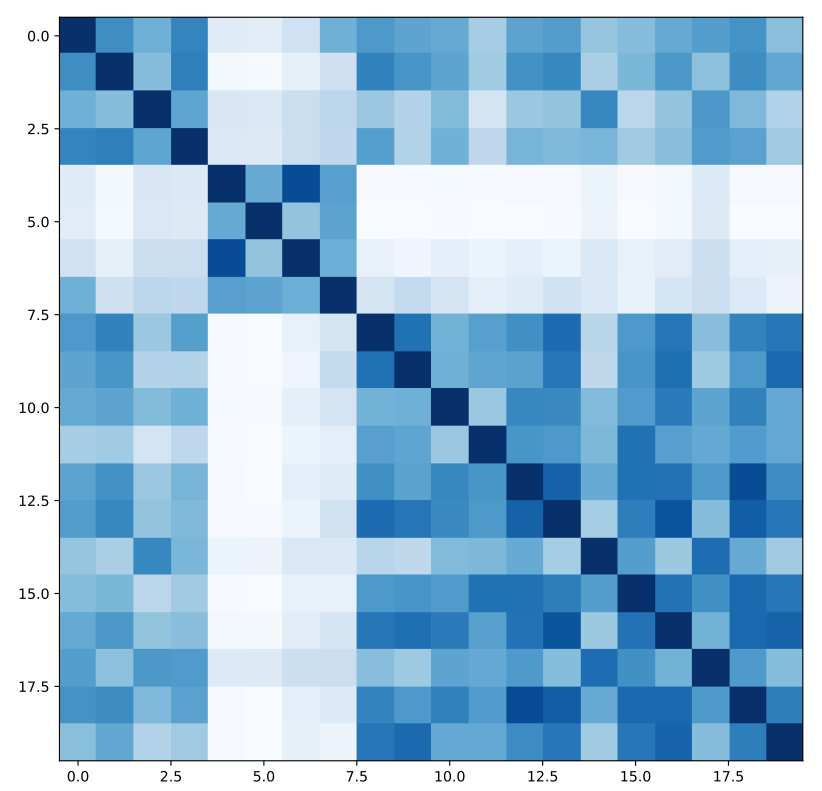

Figure 2.47: Similarity matrix computed for a toy dataset with 20 tracks divided in 5 classes (4 tracks per class).

\section{Intra-inter-class similarity ratio}

We can also compute more succinct values to measure a music similarity model's quality. The authors of the book entitled "Music Similarity and Retrieval" [KS16] define them in terms of tracks' genres in order to compare the intra-genre similarity with the inter-genre similarity (for all genres). We are changing their terminology from "genre" to "class" to address more generic cases. For every class annotated in the dataset we compute the intra-inter-class similarity ratio from the similarity matrix according to the following formula:

$$
i i c s r(c)=\frac{\frac{\sum_{t_{1} \in T_{c}} \sum_{t_{2} \in T_{c}, t_{1} \neq t_{2}} \operatorname{sim}\left(t_{1}, t_{2}\right)}{\left(\left|T_{c}\right|^{2}+\left|T_{c}\right|\right)}}{\frac{\sum_{t_{1} \in T_{c}} \sum_{t_{2} \in T_{C} \backslash \backslash c} \operatorname{sim}\left(t_{1}, t_{2}\right)}{\left(\left|T_{C \backslash \backslash c}\right|\left|T_{C}\right|\right)}},
$$

where $T_{c}$ is the set of all tracks in the class $c$ and $T_{C \backslash \backslash c}$ is the set of all tracks not in the class $c$.

This measure quantifies the similarity between tracks in the same class (weighted by the number of tracks in the class) over the similarity between tracks in that class and tracks in other classes (weighted by the number of tracks in the other classes). If this ratio is greater than 1 , then the intra-class similarity is greater than the inter-class, and this means that the model is able to better separate tracks from that specific class.

\section{Weighted mean intra-inter-class similarity ratio}

Since the intra-inter-class similarity ratio is computed for every class we still have a group of values, which makes the task of comparing two similarity models still not straightforward (this was our goal when decreasing the number of values to be compared from $n_{\text {tracks }}^{2}$ of the similarity matrix to $\left.n_{\text {classes }}\right)$. We can define an even leaner quality measure which is the mean of all intra-inter-class similarity ratios weighted by the size of the classes:

$$
i i c s r_{\text {weighted_mean }}=\frac{1}{\sum_{c \in C}\left|T_{c}\right|} \sum_{c \in C} i i c s r(c) \times\left|T_{c}\right|
$$




\section{Chapter 3}

\section{Cover Song Identification}

A cover song consists in an alternative recording of another song that is usually referred as the original one. The term version can also be used in a similar way. The versions of a song can be different from the original one in several musical aspects. Serrà et al. [SGH10] make an extensive list of these variant characteristics.

Considering the perceptual properties of the sound as pitch, duration, intensity and timbre, every single one of the related musical attributes (e.g., melody, harmony, rhythm, tempo, dynamics, timbre) can change from one version to the other.

For instance, timbre can vary between versions. From countless scenarios, timbre can change due to differences in mixing or mastering, and also due to different instrumentations on recordings of the same songs. Figure 3.1 illustrates three releases of the album Let It Be by The Beatles. Figure 3.1a illustrates the original album cover as released on 1970, Figure 3.1b illustrates the 2012 stereo remastered version, and Figure 3.1c illustrates the cover of the alternative mixing released on 2003 in which several orchestral and other types of embellishments introduced by the producer Phil Spector were removed, among other differences.

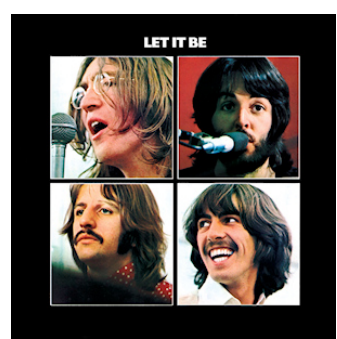

(a)

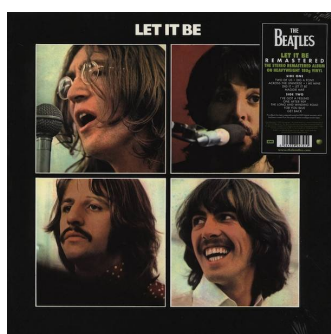

(b)

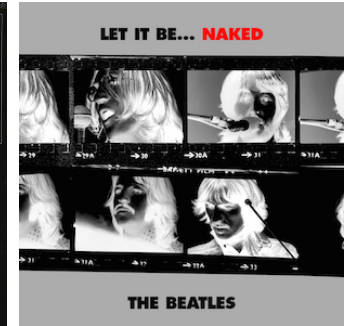

(c)

Figure 3.1: Three distinct releases of the album Let It Be by The Beatles.

Distinct timbre can be observed in live recordings, even if performed by the same band, and even if the same instruments were used, as a result of the recording environment. Figure 3.2 illustrates four versions of the album The Wall: Figure 3.2a contains the original studio one by Pink Floyd, Figure 3.2b displays a live version recorded on 1980-81 also by Pink Floyd, and Figures 3.2c and 3.2d illustrate two live recordings performed by Pink Floyd's bassist Roger Waters, recorded on 1990 and 2012 respectively, with different personnel and equipment. 


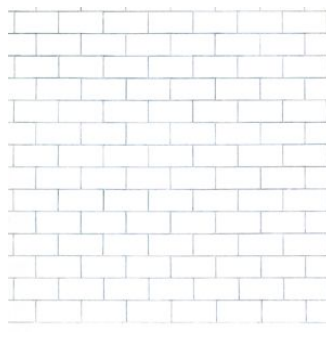

(a)

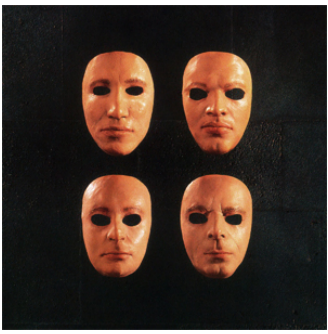

(b)

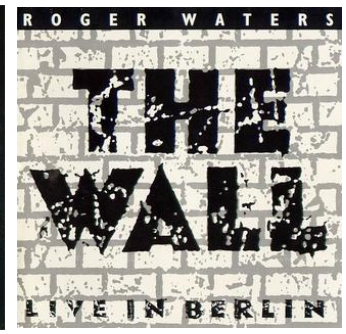

(c)

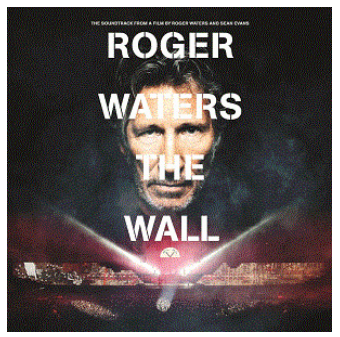

(d)

Figure 3.2: Four distinct releases containing songs from the album The Wall by Pink Floyd.

Versions can have distinct tempi also. This can happen for several reasons, but usually the tempo is changed for expressiveness and contextual purposes [SGH10]. The song All Along the Watchtower, performed by Jimi Hendrix (as illustrated in Figure 3.3b), has the tempo of 116BPM (according to the website Note Discover ${ }^{1}$ ), while the original version, recorded by Bob Dylan (as illustrated in Figure 3.3a), has the tempo of 130BPM (also according to Note Discover ${ }^{2}$ ).

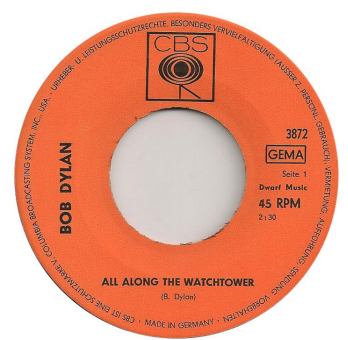

(a)

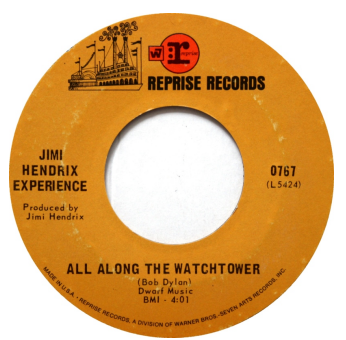

(b)

Figure 3.3: Labels from two singles of All Along the Watchtower originally recorded by Bob Dylan (a), and covered by Jimi Hendrix (b).

Rhythm is composed of several aspects besides tempo, and all of them can change between versions. For instance, distinct versions can have completely different time signatures. The song With a Little Help from My Friends was originally recorded by The Beatles (as displayed in Figure 3.4a) in a ${ }_{4}^{4}$ beat (according to the sheet music available on Music Notes ${ }^{3}$ ), while the version of it performed by Joe Cocker (as illustrated in Figure 3.4b) was recorded in a ${ }_{8}^{12}$ beat (according to the Music Notes' sheet music $\left.{ }^{4}\right)$.

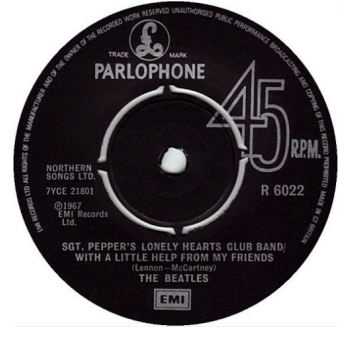

(a)

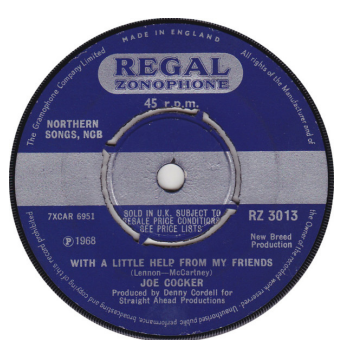

(b)

Figure 3.4: Labels from two singles of With a Little Help from My Friends originally recorded by The Beatles (a), and covered by Joe Cocker (b).

As we can observe in the literature (and illustrate in the following quotes), tonal aspects tend to be the least variant factor between version songs.

\footnotetext{
${ }^{1}$ https://www.notediscover.com/song/jimi-hendrix-all-along-the-watchtower

${ }^{2}$ https://www.notediscover.com/song/bob-dylan-all-along-the-watchtower

${ }^{3}$ https://www.musicnotes.com/sheetmusic/mtd.asp?ppn=MN0053730

${ }^{4}$ https://www.musicnotes.com/sheetmusic/mtd.asp?ppn=MN0054613
} 
"In general, we can assume that different versions of the same piece mostly preserve the main melodic line and/or the harmonic progression, regardless of its main key. For this reason, tonal or harmonic content is a mid-level characteristic that should be considered to robustly identify covers."

Joan Serrà et al. [SGH10]

"Systems for the automatic detection of versions exploit musical facets which remain mostly unchanged across different renditions, primarily the harmonic (or tonal) progression."

Justin Salamon et al. [SSG12]

"What remains almost invariable among the various cover versions are harmonic progressions and melody evolution, which form the basis of the most existing CSI descriptor extraction algorithms."

Ning Chen et al. [CLX17]

However, we still need to face a few challenges regarding tonal information. For instance, the key can be different. It can change to adjust to the singer vocal range or for aesthetics reasons [SGH10]. The versions of All Along the Watchtower, previously referenced, were recorded in different keys (the original was in A Minor, and the version was in C\# Minor).

Harmony can also vary between versions. The chord progression can be altered in several ways: chords can be added, removed or altered (adding and removing intervals). For instance, George Benson recorded an album called The Other Side of Abbey Road where he recorded several Beatles' songs. His version of Here Comes the Sun is completely re-harmonized.

Still regarding tonal changes, even though it is very rare, melody is subject to change as well. It can occur with the use of vocal ornaments, improvisation, among other reasons. Frank Zappa's live cover of Sunshine of Your Love (originally by The Cream) is a radically different rendition of it, where one can identify the main theme of the song, but the overall main melody is extinguished.

Other aspects can change such as dynamics (e.g., the overly compressed metal recording done by Children of Bodom of the song Oops I Did It Again! by Britney Spears), lyrics (e.g., the Brazilian singer Zé Ramalho who recorded a Portuguese translated version from Knockin' on Heaven's Door by Bob Dylan, called Bate-Bate-Bate na Porta do Céu), noise level (e.g., the early Beatles live recordings with quite loud audience talking and screaming), and also the structure, which is particularly challenging since bands can decide to add a non-pre-existing introduction, or to repeat the chorus, or even completely change the order of the original sections.

\subsection{Cover song identification}

The Cover Song Identification (CSI) problem, that can also be called Cover Song Detection (CSD) [EC07, RE10, LS10] or Cover Song Retrieval (CSR) [LH06, Bel07, SZA09], is basically the task of identifying songs that are versions of each other in a group of songs. Ravuri and Ellis [RE10] define it as retrieving $n$ cover songs of a query track from a set of $m$ songs.

Serrà et al. [SGH10] state that the standard approach is to use music similarity techniques since the cover songs share some music elements between them, and this should be explored. Furthermore, the use of tonal similarity is considered very robust to identify covers [SGH10, SSG12, CLX17], and it is the most common approach in the literature.

\subsubsection{CSI methods that use tonal features}

Gómez et al. [GHO06] present a technique for the CSI problem that uses tonal descriptors. More specifically, they use the Harmonic Pitch Class Profile (HPCP) feature, which is very similar 
to chromagrams (as seen in Section 2.1). Among the differences between them we have: HPCP is usually computed with higher interval resolution (e.g., 36-bins [SGHS08] or 120-bins [GHO06]) and for a narrower frequency band (e.g., $50-5000 \mathrm{~Hz}$ [SSG12] or 100-5000Hz [Góm06]).

They compute HPCPs for every frame of a song, and then they perform two distinct processing pipelines. The first one estimates the song's key and converts the HPCP vectors into Transposed Harmonic Pitch Class Profile (THPCP) vectors, which is a transposition-invariant feature [GHO06]. The second pipeline performs a structural analysis using an improved version of the algorithm introduced by Goto [Got03].

This analysis returns segmentation profiles of the songs, and then two segments are selected for each song as their music summaries using the following two criteria: the segment should repeat at least once in the song, and the segment's group should retain the majority of the song's duration.

At the end of these two pipelines, that are illustrated in Figure 3.5a, they obtain a THPCP for the whole song, and two HPCP vectors (one for each selected segment). These feature vectors are used in two different versions: the instantaneous evolution and their global average.

When comparing two songs, the similarity values are computed for both features extracted from the entire songs, and from the two segments of each song. The global averages are compared using Pearson's correlation coefficients, and the instantaneous evolutions are compared using a Dynamic Time Warping (DTW) algorithm. In the segment's comparison case, they obtain four values (as shown in Figure 3.5b) and the final similarity is the highest one.
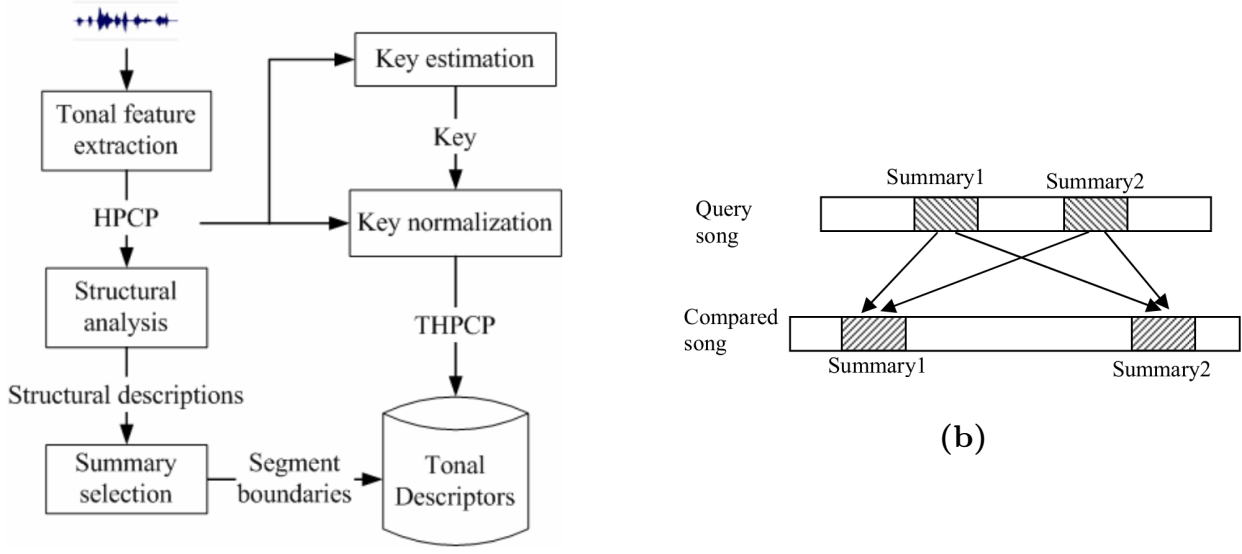

(b)

(a)

Figure 3.5: Feature extraction pipeline and segments comparison scheme by Goméz et al. [GHO06].

Jensen et al. [JCEJ08] propose a system for the CSI problem that is invariant to different instrumentation, timing, tempo and transposition. They start the feature extraction process computing 12-bin chromagrams for every song, following the implementation introduced by Ellis and Poliner [EP07], which is invariant to instrumentation.

$$
C=\left[\begin{array}{ccc}
c_{1}(1) & \ldots & c_{1}(N) \\
\vdots & & \vdots \\
c_{12}(1) & \ldots & c_{12}(N)
\end{array}\right]
$$

where $c_{i}$ is the $\mathrm{i}$-th row of the chromagram related to the i-th pitch class.

Next, they apply an element-wise logarithm scaling on the chromagram matrices based on the argument that this will increase the quality of the results, because the human perception of loudness can be roughly comparable to log-scale. 


$$
C_{\log }=\left[\begin{array}{ccc}
\log \left(1+\frac{c_{1}(1)}{\delta}\right) & \ldots & \log \left(1+\frac{c_{1}(N)}{\delta}\right) \\
\vdots & & \vdots \\
\log \left(1+\frac{c_{12}(1)}{\delta}\right) & \ldots & \log \left(1+\frac{c_{12}(N)}{\delta}\right)
\end{array}\right]
$$

where $\delta$ is a small constant

Then, they compute the power spectrum of each line of the chromagram to prevent time alignment problems allowing invariance to time shifts or tempo changes.

$$
C_{p w r}=\left[\begin{array}{c}
\left|\mathcal{F}\left(c_{1}^{\log }\right)\right|^{2} \\
\vdots \\
\left|\mathcal{F}\left(c_{12}^{\log }\right)\right|^{2}
\end{array}\right]
$$

where $\mathcal{F}$ is the Fourier transform.

After that, they sample the rows of $C_{p w r}$ using a bank with 25 logarithmically spaced filters with $50 \%$ overlapping, as displayed in Figure 3.6. This is done to further increase tolerance to timing variations.

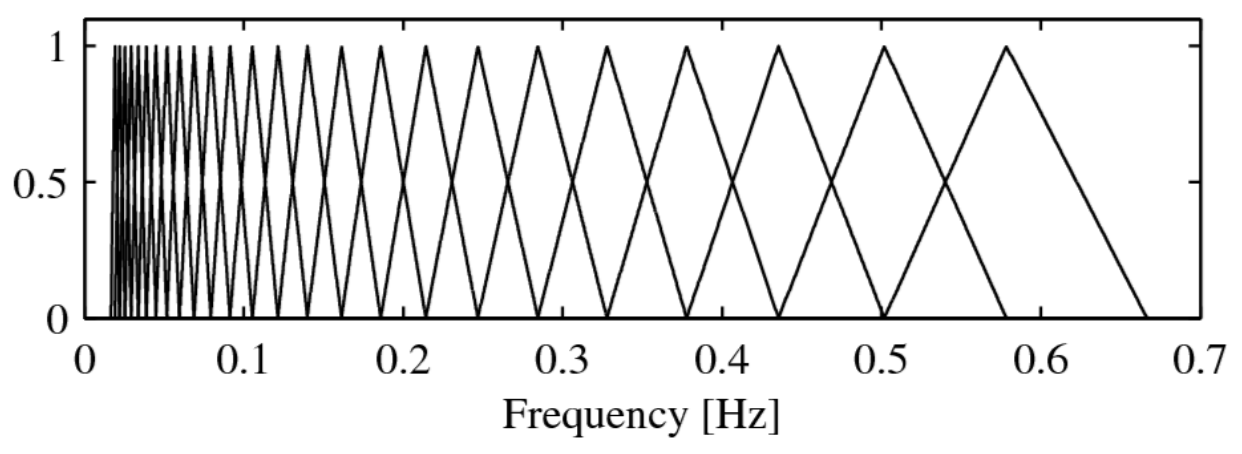

Figure 3.6: Filter bank applied by Jensen et al.[JCEJ08] in order to increase timing invariance.

Finally, the similarity between two songs' features is computed using the Frobenius distance. First they normalize the feature matrices, then they select the minimum Frobenius distance when comparing one matrix to all 12 possible transpositions of the other one, in order to achieve transposition invariance. Later, they also shifted the matrices up to 2 columns to increase the tolerance to time deviations. The shift that led to the smallest Frobenius distance is the final distance measure between the two songs.

\subsubsection{CSI methods that use symbolic sequences}

Some other approaches try to extract the main melody from the audio files, and then compare sequences in the symbolic-domain. Tsai et al. [TYW $\left.{ }^{+} 05\right]$ present a technique to extract the main melody of non-vocal segments of the songs. This is done under the argument that accompaniments can vary substantially between versions, and if non-vocal segments are passed to the melody extraction algorithm, it can lead to spurious melody sequences that are not related to singing voice.

In order to remove the non-vocal segments cepstral-based feature vectors are extracted from the segments, and two different Gaussian Mixture Model (GMM)s are used: one to model the vocal segments; the other to model the non-vocal segments. The training is performed with manually 
annotated samples. Then, when the query song is processed, features are extracted from it and likelihoods are computed from both GMMs in order to classify each segment as vocal or non-vocal.

The process of extracting the main melody follows several processing steps that can be consulted in the original paper $\left[\mathrm{TYW}^{+} 05\right]$. The signal is fragmented, windowed, then for every frame the spectrum is computed and spectral intensity analysis is performed. Since the fundamental frequency can not be located at the spectral maxima, Sub-Harmonic Summation (SHS) [PG79] is applied.

Another issue that has to be faced is when the detected note number is related to the accompaniment. So, under the assumption that the accompaniment is often repeated in neighboring segments, a method to identify which notes are more likely to be produced by instruments is applied, in order to mitigate the impact of partials that belong to the accompaniment.

As post-processing steps short- and long-term constraints are applied. First a median filter is used to remove jitters, then the octaves of outlier notes are corrected, based on the premise that the range of singing voices is somewhat narrow.

Finally, the similarity computation between note sequences is done using a DTW algorithm to find the temporal alignment between them. This algorithm computes a matrix $D$ that contains the distances between the note sequences. The similarity of two note sequences is computed as follows:

$$
S(q, u)=\left\{\begin{array}{l}
\max _{\frac{T}{2} \leq l \leq \min (2 T, L)}\left(\frac{1}{D(T, l)}\right), \text { if } L \geq \frac{T}{2} \\
\infty, \text { if } L<\frac{T}{2}
\end{array}\right.
$$

where $T=|q|, L=|u|$, and D is the matrix computed by the DTW algorithm.

Finally, in order to handle different transpositions the previous formula is computed to transposed versions of one of the sequences as follows:

$$
S_{\text {final }}(q, u)=\max _{-K \leq k \leq+K} S\left(q^{(k)}, u\right)
$$

where $\mathrm{K}$ is the maximum allowed transposition value in semitones, $q^{(i)}$ is the $\mathrm{i}$-th transposition of the sequence $q$.

Marolt [Mar06] presents a melody-based approach to the CSI problem that handles variations on rhythm and structure. It starts using a melody extraction algorithm proposed by Marolt in a past work [Mar05]. This algorithm returns a set of melodic fragments, each one with start and end times, loudness and pitch variations in that time interval. The melodic representations have their frequency mapped to 24 values per octave thus alleviating the effects of pitch variations (such as in vibratos). Octave abstraction is also applied, mapping every frequency values to one octave.

In order to be a rhythmic invariant technique, a beat tracker is used and an average filter is applied to the melody representation (6 frames for every beat). Beyond that, to be invariant to structure variations, a Self-Similarity Matrix (SSM) of the beat-synchronous melody representations is computed using cosine distance, followed by several post-processing steps.

The SSMs helps the author to look for melodic patterns, which are melody fragments that appear more than once in the song. These patterns are selected using a greedy algorithm that locates the most repeated fragment and its duplicates, removes them all from the SSM, and then redo these two steps until $90 \%$ of the SSM is gone.

When comparing two songs, the algorithm computes correlations of the first song to circularshifted versions of the second one to be able to find the best transposition. Then all patterns from both songs are compared using cosine similarity. When comparing two patterns, since they can be of different sizes (also not temporally aligned), the similarities of the largest one versus shifted versions of the smallest one are all computed. Then, the final similarity of a pair of patterns is the highest computed one. The final similarity value between the two songs is the mean of the best $n$ matching patterns similarity values ( $n$ empirically set to 2 ). 


\subsubsection{CSI methods that use fusion methods}

One important technique that is applied in the CSI problem is the Similarity Network Fusion (SNF) proposed by Wang et al. [WMD $\left.{ }^{+} 14\right]$. Let us consider one dataset with $\mathrm{n}$ samples and $\mathrm{m}$ different similarity measures. We can represent a similarity network as a graph $G=(V, E)$, where $V$ is the set of the samples $x_{1}, x_{2}, \ldots, x_{n}$, and $E$ is the set of the edges which are weighted by how similar the samples are.

For one fixed distance measure represented by the function $\rho$, the authors define the exponential kernel $W$ as follows:

$$
W(i, j)=\exp \left(-\frac{\rho^{2}\left(x_{i}, x_{j}\right)}{\mu \epsilon_{i, j}}\right)
$$

where $\left\{\begin{array}{l}\epsilon_{i, j}=\frac{\operatorname{mean}\left(\rho\left(x_{i}, N_{i}\right)\right), \text { mean }\left(\rho\left(x_{j}, N_{j}\right)\right), \rho\left(x_{i}, x_{j}\right)}{3} \\ \text { mean }\left(\rho\left(x_{i}, N_{i}\right)\right) \text { is the average value of the distances between } x_{i} \text { and its neighbors } \\ \left.N_{i} \text { represents the set of the neighbors of } x_{i} \text { (including } x_{i}\right) \\ \mu \text { is a hyperparameter that can be empirically set }(\mu \in[0.3,0.8])\end{array}\right.$

Then they define the transition matrix $P$ as we can see below:

$$
P(i, j)=\left\{\begin{array}{l}
\frac{1}{2} \frac{W(i, j)}{\sum_{k \neq i} W(i, k)}, \text { if } j \neq i \\
\frac{1}{2}, \text { otherwise }
\end{array}\right.
$$

Next, they define a K-Nearest Neighbors $(\mathrm{KNN})$ version of $P$ with this formula:

$$
S(i, j)=\left\{\begin{array}{l}
\frac{W(i, j)}{\sum_{k \in N_{i}} W(i, k)}, \text { if } j \in N_{i} \\
0, \text { otherwise }
\end{array}\right.
$$

We should note that $P$ stores the similarities of each sample to all others, while $S$ only stores the $\mathrm{K}$ highest similarity values of each sample. Lastly, the final fused network using $m$ different measures is defined as:

$$
P^{(v)}=S^{(v)} \times\left(\frac{\sum_{k \neq v} P^{(k)}}{m-1}\right) \times\left(S^{(v)}\right)^{T}
$$

for $v=1,2, \ldots, m$

Tralie [Tra17] proposes a CSI system that uses the SNF method in order to combine the strengths of each considered feature. It starts with the extraction of three different features. The first one is HPCP which is followed by the computation of a stacked delay embedding using delay coordinates (as done by Joan Serrà et al. [SSA09]). Also Optimal Transposition Index (OTI) is applied to achieve transposition invariance.

Then Mel-Frequency Cepstral Coefficients (MFCC) features are computed for every beat-synchronous block (20 beats per block). These features are then exponentially scaled, interpolated (400 MFCCs per block), and normalized using the technique by Tralie and Bendich [TB15]. As the third feature, a list of SSMs is computed between every block of the song being analysed (instead of a unique SSM for the whole song). Euclidean distances are calculated for all windows inside each block, taking window length of 0.5 second and hop size of 512 samples.

After these three features are extracted, three Cross-Similarity Matrices (CSMs) are computed using the feature's sequences from the pair of songs. The CSM of the HPCP is built using cosine distance; the CSMs of MFCC and MFCC SSMs are built using Euclidean distance. Then all CSM are converted into binary matrices, and the Smith Waterman algorithm is applied to them in order to find the best local alignments.

Additionally to using the isolated features, the application of SNF, the method proposed by Wang et al. [WMD $\left.{ }^{+} 14\right]$ that was presented before, is utilized. Both early and late fusions are 
applied, as can be seen in Figure 3.7.

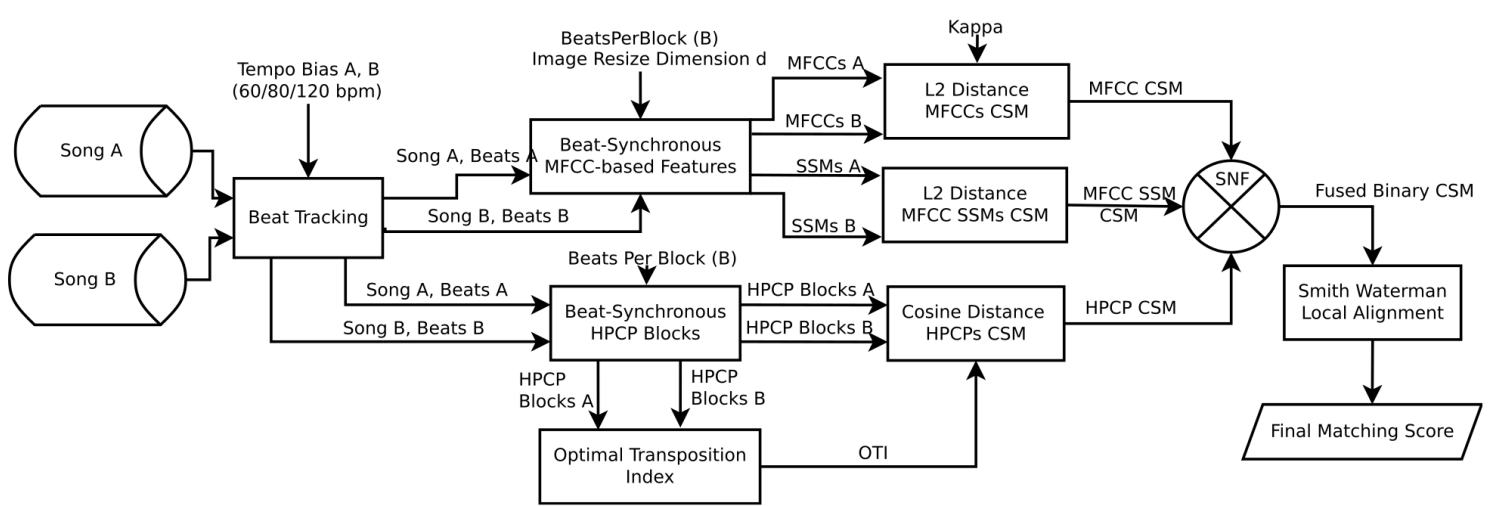

Figure 3.7: The complete processing diagram of the system proposed by Tralie [Tra17].

The early fusion is performed at feature level before calling the Smith Waterman algorithm. For every type of feature - HPCP, MFCC, MFCC SSMs - the algorithm builds a matrix called "parent SSM". As displayed in Figure 3.8 we have four distinct regions: the SSM of the song A (top-left), the SSM of the song B (bottom-right), the CSM of the songs A and B (top-right), and the CSM of the songs B and A (bottom-left). Since the values on the SSM regions are much higher than the ones on the CSM regions, a normalization step that made the values to be scaled properly is performed. Then these "parent SSMs" are fused using the SNF, and the output of it is passed as an argument to the Smith Waterman algorithm.

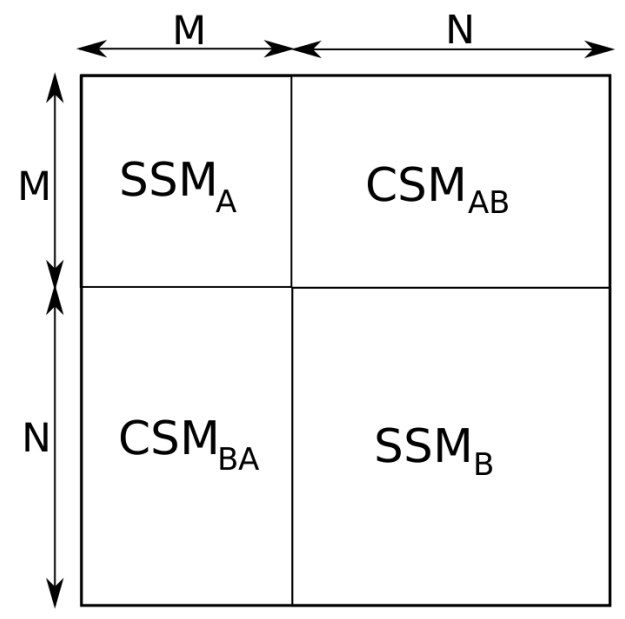

Figure 3.8: The parent Self-Similarity Matrix built by Tralie [Tra17] as input to the Similarity Network Fusion algorithm.

The late fusion step is much simpler. The SNF technique is applied to fuse score matrices between all pairs of songs for the feature set. Since the CSMs are already computed for each feature, elementwise inverse mapping is applied, and then the fused transition matrix is obtained from the SNF, which is used to compute the final score.

Chen et al. [CLX18] also use SNF to the CSI problem. They use only one type of feature 12-bin HPCP - extracted for every $464 \mathrm{~ms}$ frame, in the $5 \mathrm{~Hz}-5000 \mathrm{~Hz}$ frequency range, with loudness normalization, tuning estimation, and spectral whitening.

For the similarity computations they use two functions. The first one is $Q_{\max }$, created by Serrà et al. [SSA09] and the second one, called $D_{\max }$, is a modification of $Q_{\max }$. The only difference between them is the considered alignment constraints, as can be seen in Figure 3.9. 


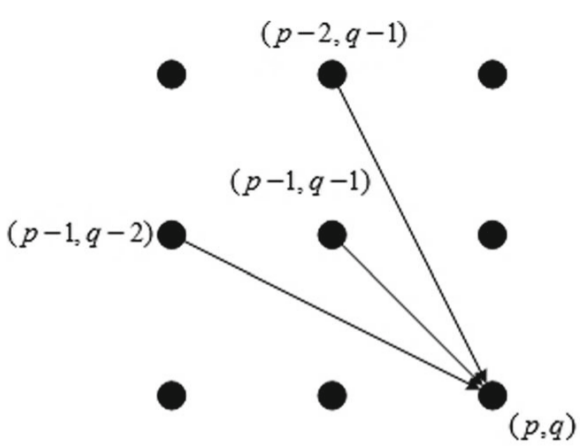

(a)

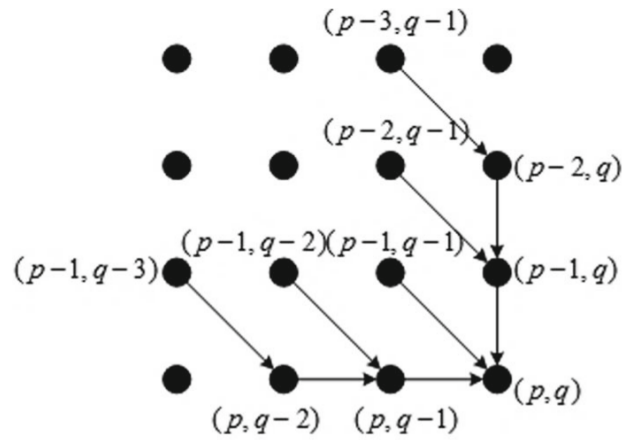

(b)

Figure 3.9: The considered alignment constraints used by (a) $Q_{\max }$ and (b) $D_{\max }$ (reproduced from [CLX18]).

After they obtain two similarity matrices (one for each similarity function above), they use SNF to fuse them, as we saw earlier on this section. The complete process is illustrated in Figure 3.10.

(a)

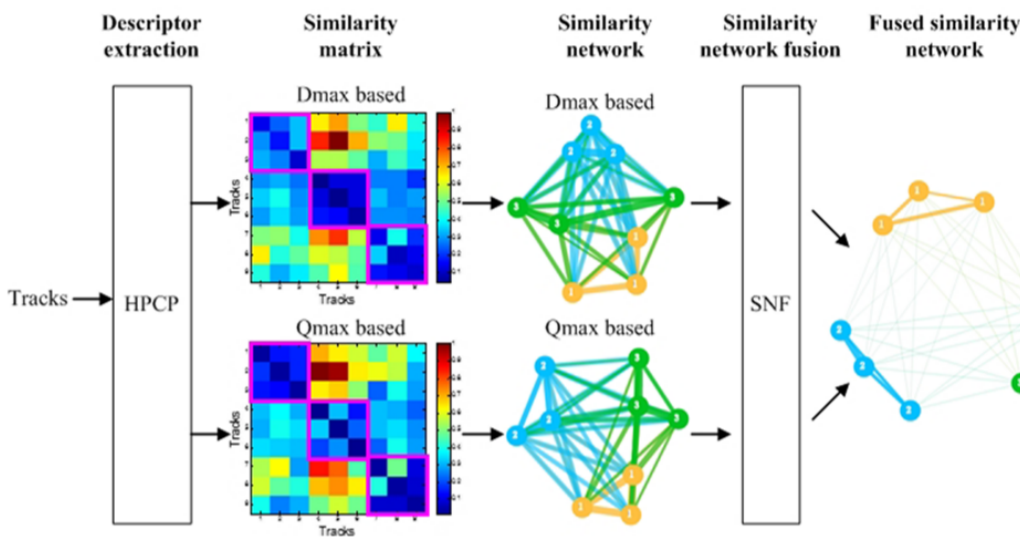

(f)

Fused similarity matrix (g)

Performances evaluation

Figure 3.10: The complete system diagram as proposed by Chen et al.[CLX18]. 


\subsubsection{Cover song identification quality measures}

i-th column

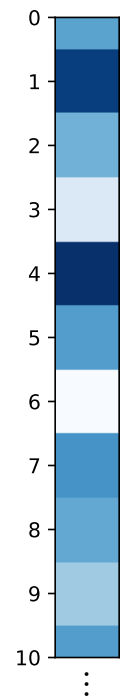

sorted i-th column

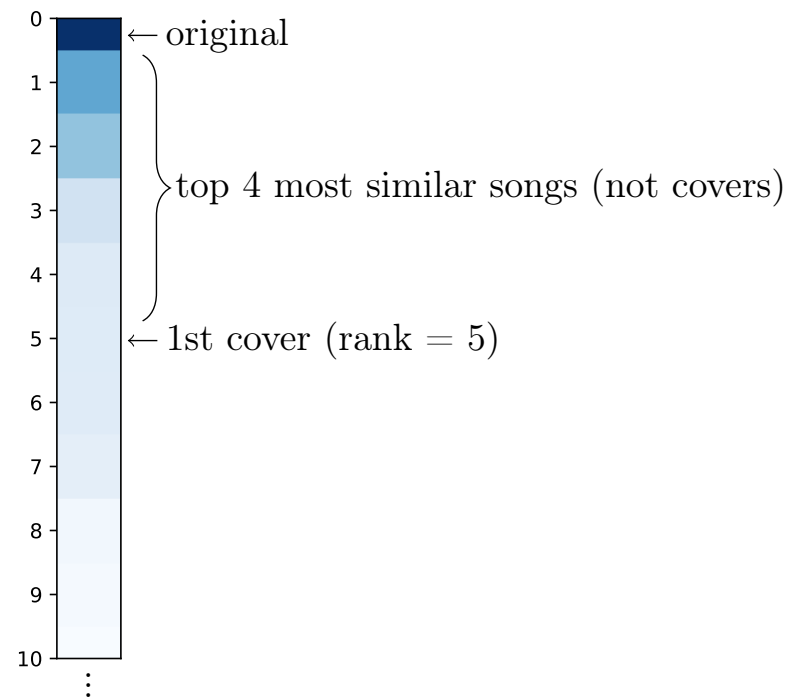

Figure 3.11: An illustration of one column from the similarity matrix before and after being ordered, and how to determine the rank of the first retrieved cover of the column's correspondent song.

\section{Mean Rank (MR)}

The Mean Rank (MR) can be used as a measure of the quality of a CSI system given a dataset. We can interpret it as the expected position where the first cover will appear in the result list when querying a song. It is broadly used in the literature [Tra17, SHD19] and it is defined from the similarity matrix $S \in \mathbb{R}^{N \times N}$ as follows:

1. Sort in descending order every column (song) $j=1,2, \ldots, N$ of the similarity matrix $S$, producing a matrix $K$ where $K_{i, j}$ is the index of the $i$-th most similar song to $j$. In other words, the sorted values of the $j$-th column of $S$ are

$$
S_{K_{1, j}, j} \geq S_{K_{2, j}, j} \geq \cdots S_{K_{N, j}, j}
$$

in particular, $K_{1, j}=j$, since song $j$ is most similar to itself;

2. For each column (song) compute the rank of song $j$ as $R_{j}=\min \left\{l>1 \mid K_{l, j}\right.$ is a cover song of $\left.j\right\}$, i.e., $R_{j}$ is the rank (position) of the most similar cover song of $j$;

3. Compute $M R=\frac{1}{N} \sum_{j} R_{j}$.

This algorithm is illustrated in Figure 3.11.

\section{Mean Reciprocal Rank (MRR)}

The reciprocal rank is defined as the inverse of a rank [DBEJ08]. In the CSI problem we are accounting ranks to the first version song that appears on the list ordered by similarity values of retrieved songs. We compute the ranks of the first retrieved version for every song on the database, then we take the inverse of these ranks. Finally, we compute the mean of these reciprocal ranks, obtaining the Mean Reciprocal Rank (MRR). This quality measure is computed from the similarity matrix $S \in \mathbb{R}^{N \times N}$ as follows: 
1. Compute $R_{j}, j=1, \ldots, N$ as in Mean Rank;

2. Compute $M R R=\frac{1}{N} \sum_{j} \frac{1}{R_{j}}$.

\section{Median Rank (MDR)}

The Median Rank (MDR) is another metric based on statistics of the first retrieved songs' ranks. The ranks are computed equally to MR and MRR, and after that the median is computed.

\section{Mean Average Precision (MAP)}

Kim Falk [Fal19] defines Mean Average Precision (MAP) in the context of recommender systems, in which users perform queries, and each query returns a list of ranked items.

- Precision at $K(\mathrm{P} @ \mathrm{k})$ :

is the number of relevant items found on the first $k$ items

- Average Precision (AP):

$A P(u)=\frac{1}{m} \sum_{k=1}^{m} P @ k(u)$

where $m$ is the length of the ranked list, and $u$ is the user performing the query

- Mean Average Precision (MAP):

$M A P=\frac{1}{|U|} \sum_{u \in U} A P(u)$

where $U$ is the set of all users performing queries

\section{Top at $\mathrm{N}$}

The group of metrics called Top at N (Top@N) is simply the number of relevant items that are present in the top $N$ items. The parameter $N$ usually is set to $1,10,100$, and 1000 .

\subsection{Cover song classification}

There is a related problem to the CSI one that it is called Cover Song Classification (CSC). Instead of receiving one query song as input, a CSC system receives a pair of songs. The goal of this system is to classify this pair as versions of each other or not. We clearly have here a binary classification problem. From this point on, we will refer to an original/cover pair as "a pair labeled as 1", and an original/non-cover pair as "a pair labeled as 0". We also will refer to a input pair of songs as "reference/test" or "query/target" when it is not clear if it is a pair labeled as 1 or 0 .

Ravuri and Ellis [RE10] are the first authors to implement a system that handles the CSC problem. The system receives a pair of songs as input, then computes beat-synchronous chromagrams for both. The beat tracker is executed 3 times, each time trying to favor the detected song's basic beat to be as close as possible to $240 \mathrm{BPM}, 120 \mathrm{BPM}$, and $60 \mathrm{BPM}$, respectively. The authors propose 3 ways to compare the features from the pair of songs, which leads to 9 score values per pair, since each pair are represented by 3 distinct beat-synchronous chromagrams.

The first method to compare chromagrams starts with a square-root compression (as a postprocessing step to the feature extraction process). Then a cross-correlation of the reference chromagram and the test chromagram is performed in order to highlight fragments that match. In this process all 12 circular shifts of the test chromagram are considered. Next, high-pass filtering is performed to emphasize sharp local maxima, where the authors consider to be the genuine matches. Lastly, the score is calculated as the value of the maximum peak. The second method to compare chromagrams has its score equally defined, only with an extra normalization of each chroma before computing the cross-correlation, so that the energy in each beat has unit sum. 
The third method is built on top of a binary similarity matrix, computed from all chromagram pairs using the method defined by Serrà and Gómez [SG07]. Then the Smith Waterman algorithm is used to find the best local alignment in this matrix, using the technique published by Serrà et al. [SGHS08]. Finally, the score is the highest value related to the maximum similarity between the two chromagrams.

After computing the 9 scores (3 scores for each of the 3 types of features), the values are postprocessed and placed in a vector. This 9D vector is used as the sample to represent the pair of reference/test songs in the classification step. For that they used two different classifiers - Support Vector Machine (SVM) and Multi-Layer Perceptron (MLP) - which are trained with manually annotated samples, and at last they are able to classify pairs of songs as being 1's or 0's. Figure 3.12 shows the block diagram of the system.

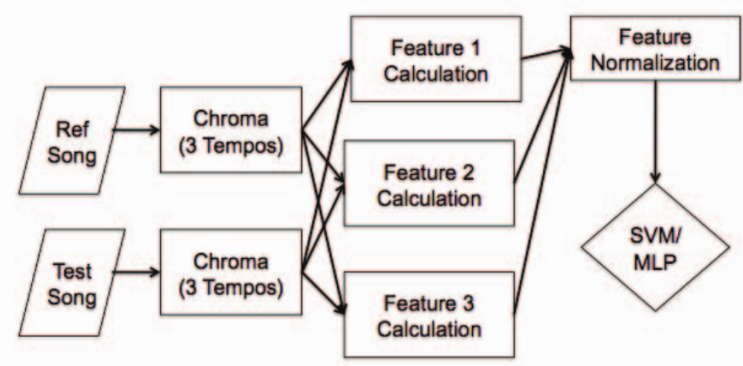

Figure 3.12: The diagram of the Cover Song Classification system developed by Ravuri and Ellis [RE10].

Salamon et al. [SSG12] when approaching the CSC problem consider three different representations: harmony, melody and bass line. The harmonic feature is a 12-bin HPCP computed in the range of $50 \mathrm{~Hz}$ to $5000 \mathrm{~Hz}$, and through the use of normalization, tuning estimation and spectral whitening.

The melody representation is obtained using a melody extraction algorithm developed by Salamon and Gómez [SG12b]. This same algorithm is adapted to extract the bass line. A low-pass filter is applied at $261.6 \mathrm{~Hz}$, the window size is increased to $185 \mathrm{~ms}$ (for better frequency resolution at low frequencies), only 2 octaves are considered (within the range of $27.5 \mathrm{~Hz}$ to $110 \mathrm{~Hz}$ ), among other details [SSG12].

After that, the melody and bass line sequences are post-processed in order to handle variations between versions. All frequency values are converted to cents, and two types of abstraction are applied: semitone abstraction (to filter local variations) and octave abstraction (to remove octave displacements).

The similarity between two songs is computed using the $Q_{\max }$ algorithm, which is proposed by Serrà et al. [SSA09] as a dynamic programming method for computing the best local alignment from two time series. The distances from the 3 representations (HPCP, melody, bass line) are gathered to be the sample to represent the pair of songs.

The classification process is as follows: 5 disjoint balanced sets of query/target pairs of songs are selected; the corresponding samples are used to train 5 different classifiers (random forest, SVM, logistic regression, k-star, and Bayesian network); the classifiers are used to determine if two songs are versions or not. 


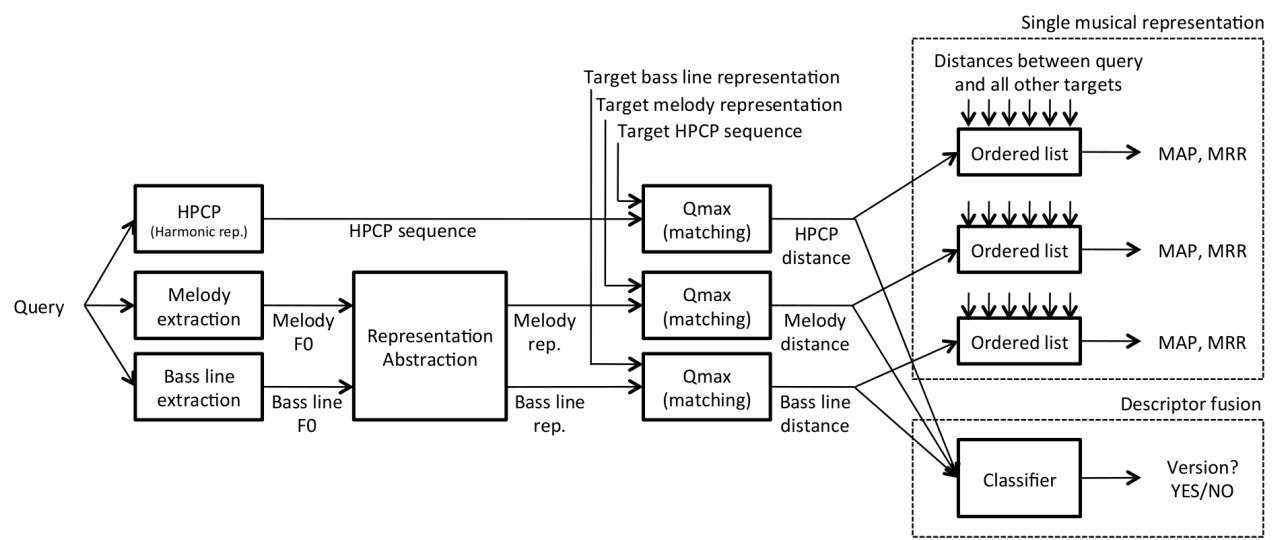

Figure 3.13: The complete diagram of the processing steps presented by Salamon et al. [SSG12].

\subsubsection{Cover song classification quality measures}

\section{Accuracy}

Our first quality measure for the CSC problem is the accuracy. Let $y_{\text {test }}$ be the annotations of the tested samples, and $y_{\text {predicted }}$ be the predicted classes from a classifier, the accuracy value is:

$$
\operatorname{accuracy}\left(y_{\text {test }}, y_{\text {predicted }}\right)=\frac{1}{N} \sum_{i=1}^{N} \text { equals }\left(y_{i_{\text {test }}}, y_{i_{\text {predicted }}}\right)
$$

where equals $(a, b)=\left\{\begin{array}{l}1, \text { if } a=b \\ 0, \text { if } a \neq b\end{array}\right.$

\section{Precision, recall and f1-score}

In a binary classification problem such as the CSC we can use other quality measures such as precision, recall and f1-score. The table below shows how samples can be called according to how they are classified.

\begin{tabular}{cc|cc} 
& & \multicolumn{2}{|c}{ Annotated label } \\
& & 1 & 0 \\
\hline \multirow{2}{*}{ Predicted label } & 1 & true positive & false positive \\
& 0 & false negative & true negative
\end{tabular}

Using this taxonomy we can define the three quality measures with the following formulas:

$$
\begin{aligned}
& \text { precision }=\frac{\text { true positive }}{\text { true positive }+ \text { false positive }} \\
& \text { recall }=\frac{\text { true positive }}{\text { true positive }+ \text { false negative }} \\
& \text { f1-score }=\frac{2 \times \text { precision } \times \text { recall }}{\text { precision }+ \text { recall }}
\end{aligned}
$$


Precision is the number of correct classifications of covers over the number of all (correct or wrong) classifications of covers, recall is the number of correct classifications of covers over the number of samples labeled as covers (independent if they are classified correctly), and f1-score is the harmonic mean of precision and recall. 


\section{Chapter 4}

\section{Dataset Modifications}

In order either to achieve better performance or to achieve better results several works on the literature perform modifications on selected datasets by replacing the full-length recordings to alternative versions of them.

It is important to clarify that the dataset modifications considered here are fundamentally different from dataset augmentation. Dataset modifications aim at replacing the entire dataset with new data based on a single type of transformation applied to the original data. In contrast, dataset augmentation aims at enlarging the dataset, by creating new modified data using several types of transformation.

For instance, consider a basic binary classification problem aiming at identifying cats and dogs. One plausible dataset modification might introduce noise to all images, replacing the original noisefree data, in order to improve the tolerance of the classifier to noise. A typical data augmentation process would perform several transformations to the image files (rotation, scaling, cropping, etc), adding the transformed files to the same dataset, alongside the original images. The main goal would be to obtain a considerably larger training set, which would indirectly improve the robustness of the classifier.

The main difference therefore between the two techniques is related to their goals: dataset modifications focus on specific transformations of the data, whereas dataset augmentation focuses on producing more training data.

This research explores dataset modifications in the context of music similarity, based on transformations that are presented below.

\subsection{Segment selection}

Levy and Sandler [LS06] present a system to search for songs that uses a content-based music similarity technique. This technique relies on segment models in order to achieve better time and memory performances. The algorithm that performs the segmentation of the songs, and selects thumbnails to replace the full-length songs, works as follows:

- extract beat-synchronous constant-Q spectrogram

- train a HMM on these features where each hidden state represents a distinct type of timbre found over the song

- use Viterbi algorithm to decode features into sequence of types of timbre

- label each beat with its type of timbre

- compute normalized histograms of these types of timbre for every 7-beat window

- cluster the histogram in order to define the segment boundaries (details about this clustering step are presented by Levy et al. [LSC06]) 
The algorithm that receives the segmentation as input and decides which thumbnail should be selected works as follows:

- count the segments

- find the most frequent types of timbre

- (if necessary) untie the first place by choosing the type of timbre whose segments have the highest mean energy (under the hypothesis that some sections such as choruses - the desired ones - are stronger than other ones such as verses)

- select the second segment of the most frequent type of timbre (under the assumption that sections towards the middle of a song are more representative of it, comparing with sections at the boundaries)

In a search task, the thumbnail for the query song is computed, and then the first 20 MelFrequency Cepstral Coefficients (MFCC) are extracted from it. Then a single Gaussian is fitted to these features, and a vector composed of the means and covariances concatenated is selected as global feature. Finally, two parameter vectors are compared using a symmetric version of the Kullback-Leibler divergence. The most similar (less distant) songs from the collection are presented to the user in an ordered list.

Bozzon et al. [BPVT08] develop a method for content-based music recommendation that relies on the similarity of semantic segments. They argue that the exploitation of semantic segmentation can lead to more meaningful results to the users. The semantic segmentation algorithm is proposed by Ong and Herrera [OH05] and can be divided in two main phases:

1. Phase 1: "detecting boundaries"

- segment the audio file using window length of 4096 samples, and hop size of 512 samples

- compute MFCC and sub-bands energy

- calculate all inter-frame cosine distances, and store the values in one similarity matrix for each feature

- enhance each similarity matrix using morphological filtering [BWFW04]

- compute novelty measures correlating the matrices with a $2 \times 2$ kernel [Foo00]

- detect the 40 local maxima on the novelty curves, and use them as candidates for segment boundaries

2. Phase 2: "refining detected boundaries"

- perform a second segmentation of the audio file, but now using the detected boundaries

- compute 8 new features - Zero Crossing Rate (ZCR), spectral centroid, flatness, rolloff, flux, Root Mean Square (RMS), low bass energy, high-medium energy - for every segment

- calculate inter-segment Euclidean distances from the average values of these features

- repeat the same steps of phase 1 in order to compute new novelty curves

- divide the peaks in two groups: $P$ and $E$, where $P$ contains peaks above a predefined threshold, and $E$ below this threshold

- order all peaks in $P$ according to their indexes, order all peaks in $E$ according to their values, and apply the following routine:

- starting with the highest peak on $E$

- if it is temporally distant 4 seconds (at most) from any peak on $P$, then insert it in $P$ 
- otherwise, delete it

- start over until there are no peaks left in $E$

- return $P$

After song semantic segmentation, the method extracts a 25-dimensional feature vector $(1 \mathrm{ZCR}$ +1 spectral centroid +1 spectral roll-off +1 spectral flux +1 spectral flatness +20 MFCC) from each segment windowed every $100 \mathrm{~ms}$ with $40 \mathrm{~ms}$ overlapping. The feature vectors for all segments are summarized using a single Gaussian, and the global feature is the concatenation of the means and covariances. Finally, the inter-segment similarity is computed using Kullbak-Leibler divergence, and the inter-song similarity is computed according to the process described below.

For a query song $q$ and the collection of songs $B$, they define the sets of segments $S_{q}$ and $S_{b}, \forall b \in B$. Both sets are filtered (segments shorter than 10 s are deleted) and then a graph is constructed with nodes that represent all elements from the sets, and edges that connect all nodes from $S_{q}$ to all nodes from $S_{b}, \forall b \in B$. The edges are weighted by the distances between the segments. Finally, a graph reduction algorithm is applied, discarding edges with low similarity values, but maintaining full connectivity between the segments from both songs. The remaining edges are used to compute the overall similarity between the two songs as shown in this formula:

$$
\operatorname{similarity}(q, b)=\frac{1}{|A(q, b)|} \sum_{u=1}^{|A(q, b)|} w_{u}
$$

where $A(q, b)$ is the set of edges that guarantees full coverage on the sets $S_{q}$ and $S_{b}$, and $w_{u}$ is the weight associated with the edge $e_{u} \in A(q, b)$.

Balen et al. [VBBWV14] perform a few experiments on Cover Song Identification (CSI) using cognitive audio descriptors. In the first experiment they use full-length songs from the chosen dataset; in the second experiment they use segment candidates returned by the algorithm proposed by Serrá et al. [SMGA12] that works as follows:

- start with a time series $X=\left[x_{1}, \ldots, x_{N^{\prime}}\right]$ with length $N^{\prime}$, where $x_{i}$ can be a column vector

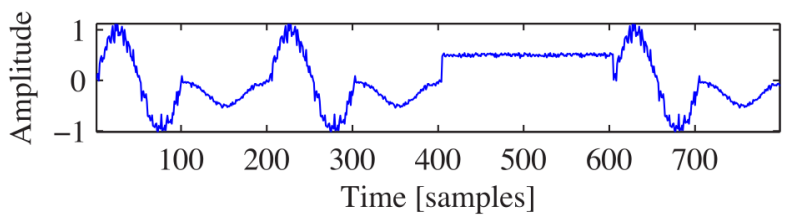

Figure 4.1: An example time series (reproduced from [SMGA12])

The time series presented in Figure 4.1 is just for illustration, since the actual time series used as input for the segmentation task is Harmonic Pitch Class Profile (HPCP) [Góm06].

- compute $\hat{X}=\left[\hat{x}_{1}, \ldots, \hat{x}_{N}\right]$ with length $N$, where $\hat{x}_{i}=\left[x_{i}^{T} x_{i-\tau}^{T} \ldots x_{i-(m-1) \tau}^{T}\right]$, for $i=w+1, \ldots, N^{\prime}$, $w=(m-1) \tau, m$ is the parameter that determines how much of the past is considered (chosen value by the authors is between 0 and 5 secs), $\tau$ is the parameter called time delay (chosen value is 1 ). 


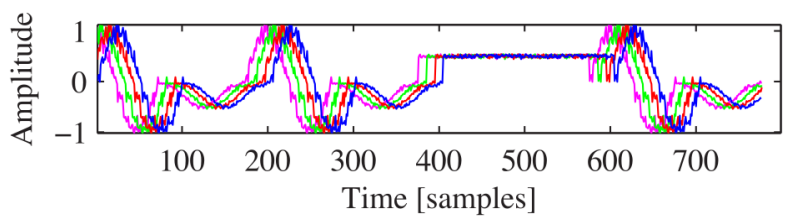

Figure 4.2: Time series computed considering past samples (reproduced from [SMGA12])

This step tries to mimic the human short-term memory by aggregating in the sample $\hat{x}_{i}$ information contained in the recent past. Figure 4.2 illustrates the new time series computed.

- calculate a square matrix $R$, where $R_{i, j}=\Theta\left(\epsilon_{i, j}-\left\|\hat{x}_{i}-\hat{x}_{j}\right\|\right), \Theta(z)=\left\{\begin{array}{l}1, \text { if } z>0 \\ 0, \text { otherwise }\end{array}\right.$

\|\| is the Euclidean norm, and $\epsilon_{i, j}$ represents a different threshold for each position $(i, j)$ of the matrix $R$ (details on how to compute $\epsilon_{i, j}$ can be found in the original paper [VBBWV14]).

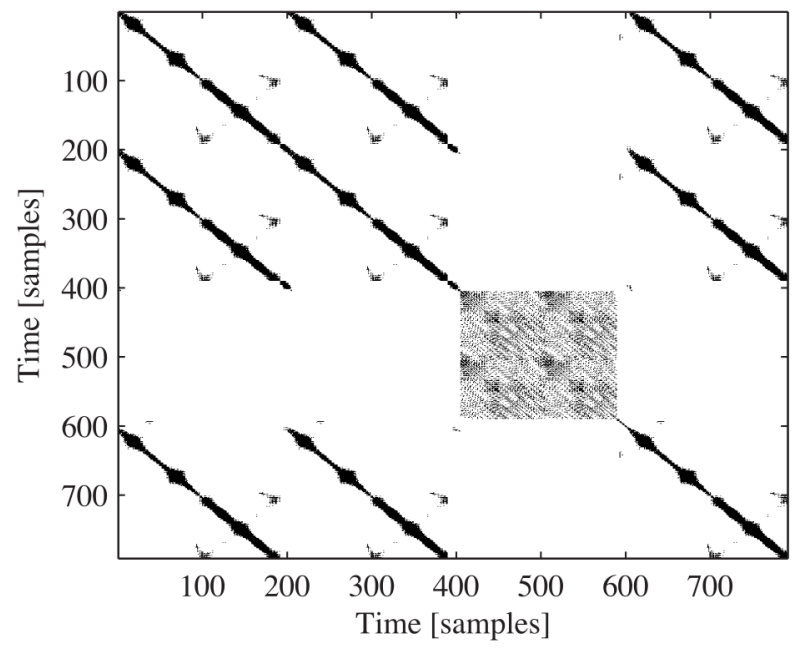

Figure 4.3: Matrix that stores the comparisons between all samples (reproduced from [SMGA12])

This third step is performed to look for repeated patterns, comparing all possible pairs of samples, and an example can be visualized on Figure 4.3.

- create a time-lag matrix $L$, where $L_{i, j}=R_{i, k+1}$ for $i, j$ in $1, \ldots, N, k=(i+j-2) \% N$.

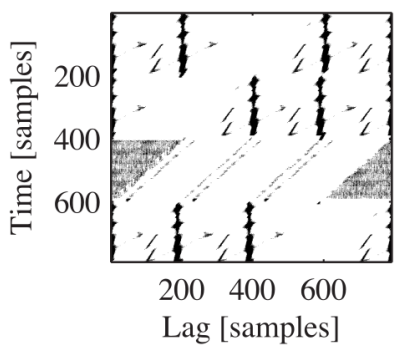

Figure 4.4: The time-lag matrix L (reproduced from [SMGA12])

This step (along with the next one) computes the so-called "structure features", starting with the application of temporal lags, as illustrated in Figure 4.4. 
- compute $P=L * G$, where $G=g_{t} g_{l}^{T}, g_{t}$ and $g_{l}$ being two Gaussian windows (with variances chosen to be $\left.\sigma^{2}=0.16\right)$ of sizes $s_{t}$ and $s_{l}\left(s_{t}\right.$ set to around $30 \mathrm{sec} ; s_{l}$ set to $0.3 \mathrm{sec}$ ).

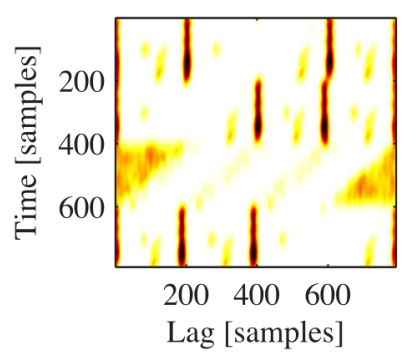

Figure 4.5: The estimated density $P$ (reproduced from [SMGA12])

This is the second step of computing the "structure features", and it consists in estimating a "bivariate probability density with Gaussian kernels" (the result can be seen in Figure 4.5).

- calculate $c=\left[c_{1}, \ldots, c_{N-1}\right]$, a uni-dimensional novelty curve, where each $c_{i}=\left\|p_{i+1}-p_{i}\right\|^{2},\|\|$ is the Euclidean norm, and $c$ is linearly normalized between 0 and 1 .

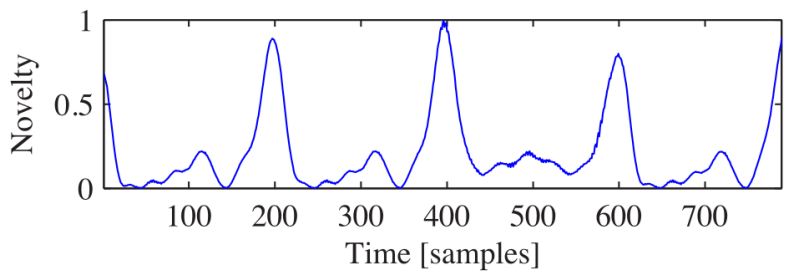

Figure 4.6: The novelty curve $c$ (reproduced from [SMGA12])

This last step computes a novelty curve, and the peaks are considered as candidates for segment boundaries (as shown in Figure 4.6).

Finally, every $c_{i}$ is considered as a peak if is higher than a predefined threshold $\delta$ (set to 0.05), and also if it is a global maximum of a window centered in $c_{i}$ and with length $\lambda$ (set to $12 \mathrm{sec}$ ). The last step before declaring the segment boundaries is to add up $\frac{w}{2}$ to the peak indexes, to make up for the displacement included in the delay coordinates.

Returning to the experiments of Balen et al. [VBBWV14], they extract three features - Pitch Bihistogram, Chroma Correlation Coefficients, and Harmonisation Feature - from all full-length songs in the first experiment. Then the features are whitened, transposed, weighted, and concatenated. The final representations are called global fingerprints which are compared to each other using cosine distance. When the program receives a query song, 12 global fingerprints are computed from it (regarding every transposition), and then compared with all stored fingerprints pre-computed from the entire collection. Then, a ranked list is returned for evaluation.

The second experiment uses the segmentation method presented above, and two non-overlapping thumbnails are selected from the returned segments list as follows:

- perform a simplification on the sequence of segment labels by merging repeated patterns:

$$
a b a b X a b X X \Longrightarrow Y Y X Y X X
$$

- compute the duration of segments for all labels 
- find the 2 labels whose segments cover the music most

- return the boundaries of the first instances of the 2 selected labels

Finally the method computes 24 new fingerprints, in the same manner as in the previous experiment, regarding 12 transpositions of each thumbnail.

Silva et al. [SdSB15] propose a technique for CSI which is based on the similarity of music shapelets. The concept of shapelets comes from time series classification, and the authors of this paper are inspired in the work done by Ye and Keogh [YK09]. In the context of time series classification, a shapelet is a subsequence of one time series which is most representative of its class. For every class on the classification problem they select a shapelet from one of its samples to be used to train a decision tree. The steps of the training phase can be summarized as follows:

- select candidates for shapelets computing all subsequences whose lengths are between $\min _{\text {len }}$ and $\max _{l e n}$ (user-defined parameters) from all training time series of the dataset $D$

- compute the distance values of each candidate to all original time series (since the length $l$ of a subsequence $s$ is smaller than the length of a time series $T$, the minimum of the Euclidean distances of $s$ and every subsequence $s^{\prime}$ of $T$ whose length is also $l$ is computed)

- (for every candidate) order the distances and finds the optimal splitting point

- find a distance threshold that, from it, $D$ is split into two subsets $D_{1}$ and $D_{2}$, such that $\forall T_{1} \in D_{1}, \operatorname{dist}\left(T_{1}, S\right)<d_{t h}$ and $\forall T_{2} \in D_{2}, \operatorname{dist}\left(T_{2}, S\right) \geq d_{t h}$ ( $S$ is the shapelet candidate)

- this distance threshold $d_{t h}$ is considered optimal when $\operatorname{Gain}\left(S, d_{t h}\right) \geq \operatorname{Gain}\left(S, d_{t h}^{\prime}\right)$ for any other possible distance threshold $d_{t h}^{\prime}$,

- Gain is defined as Gain(splitting_point $)=I(D)-\hat{I}(D)$

- $I(D)$ is the entropy of $\mathrm{D}$ before the split; $I(\hat{D})$ is the entropy of $\mathrm{D}$ after the split

$-I(D)=-p(A) \log (p(A))-p(B) \log (p(B))$, where $p$ defines the proportion of elements in each class

- I( $\hat{D})=f\left(D_{1}\right) I\left(D_{1}\right)+f\left(D_{2}\right) I\left(D_{2}\right)$, where $f$ defines the fraction of elements in each subset

- finally, after computing all optimal split points for all shapelet candidates (and their respective gain of information values), the chosen shapelet is the one that has the highest gain.

This method of shapelets is adapted by Silva et al. [SdSB15] in 5 aspects in order to be used in the CSI problem.

1. instead of using candidates with several different sizes (which can be very cumbersome) the authors reduce the set of possible subsequence lengths, and also use a hop size higher than 1

2. they replace the Euclidean distance by the Dynamic Time Warping (DTW) to compare the distance between subsequences and time series, since DTW is invariant with respect to time warpings

3. rather than performing a classification task based on information gain, they propose an alternative to assess a shapelet candidate's quality, according to this formula:

$$
\operatorname{DistDiff}(s)=\min _{i=1, \ldots, n}(\operatorname{dist}(s, \operatorname{OtherClass}(i)))-\frac{1}{m} \sum_{i=1}^{m} \operatorname{dist}(s, \operatorname{SameClass}(i))
$$


4. they select one shapelet for each song from the training set (as opposed to one shapelet per class)

5. the authors use three shapelets for each song (since the use of only one can be not very descriptive) which are selected by dividing the feature vector in three parts and using the method by Ye and Keogh [YK09] to select one shapelet for each third of the song

Finally, the process of querying is made by computing the distances from the input song to the triplets of every training song, averaging these three distances, and returning a ranked list.

\subsection{Frame selection}

The full-length songs can also be modified by selecting subsequences of them but now with non-contiguous segments, i.e., with a subset of frames that do not compose a contiguous segment. Seyerlehner [Sey10a] presents a tool to analyze which frames contributed the most to a similaritybased task when comparing two songs.

This tool shows that frames with low energy interfere substantially to the similarity value, because low energy frames have small Euclidean distance between them. Therefore, songs with several low frames are considered very similar by the computer, which contradicts what the author expects to be useful to a human listener (two songs are not going to reported as similar simply because they share silent parts).

In order to obtain better similarity values, he proposes a method that perform the following steps:

- compute the spectrum for each frame

- map them to mel-scale

- sum the energy across all mel-bands for every frame

- sort the frames by their duration

- drop a percentile (for instance, $50 \%$ ) of the low energy frames

- compute the similarity values using this new representations

Seyerlehner [Sey10a] also evaluates this frame selection method using two datasets, and removing low-energy frames corresponding to percentiles of $10 \%, 20 \%, \ldots, 90 \%$. Besides comparing to the original dataset $(0 \%)$ he also creates secondary datasets by removing random frames (also with percentiles from $10 \%$ to $90 \%$ ) to verify if the selection of frames by energy actually makes sense.

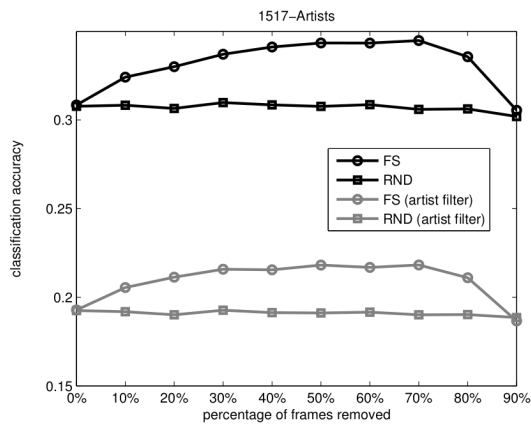

(a)

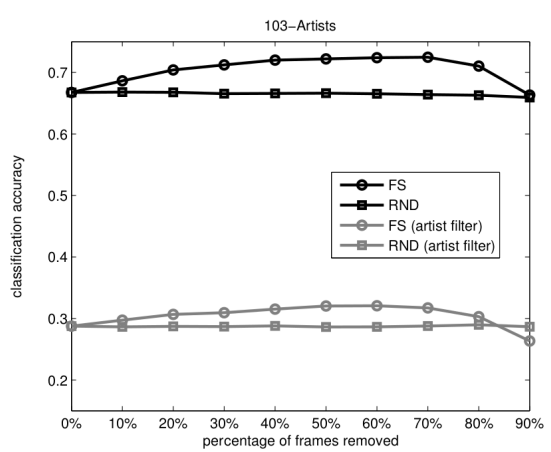

(b)

Figure 4.7: Results obtained by the use of frame selection (reproduced from [Sey10a]) 
Figures $4.7 \mathrm{a}$ and $4.7 \mathrm{~b}$ show the obtained results. We can observe that the datasets present best results with $70 \%$ and $80 \%$ respectively, comparing to the original dataset $(0 \%)$ and to randomly removing frames (an artist filter is also applied, and details about it can be seen in the original paper [Sey10a]).

\subsection{Source separation}

As we mentioned before, the CSI literature states that the least common variable musical aspects between versions are the tonal ones [SGH10, SSG12, CLX17]. In Section 3.2 we cite a work done by Salamon et al. [SSG12] where they explore the isolated similarities from melody, bass line, and harmony representations. Foucard et al. [FDLR10] takes this approach to a next step, and uses a source separation algorithm (by Durrieu et al. [DRD09]) to isolate the main melody from the accompaniment.

The method by Foucard et al. [FDLR10] uses three representations for each song: the original mixture, the main melody, and the accompaniment. From each representation they extract a 36-bin HPCP averaged over all frames to compose the global features. When comparing two songs $A$ and $B$ they compute a matrix $S$ according to the following formula:

$$
S_{n, m}=\left\{\begin{array}{l}
+1, \text { if } O T I\left(h_{A, n}, h_{B, m}^{T r}\right) \in\{0,1,35\} \\
-0.9, \text { otherwise }
\end{array}\right.
$$

where $O T I\left(h_{A}, h_{B}\right)=\operatorname{argmax}_{0 \leq i \leq 35}\left\{h_{A}\right.$.circular_shift $\left.\left(h_{B}, i\right)\right\}, h_{A, n}$ is the $\mathrm{n}$-th frame of song $A$, and $h_{B, m}^{T r}$ is the (already transposed) $\mathrm{m}$-th frame of song $B$.

From this matrix $S$ they compute an alignment matrix $H$ using an algorithm called Dynamic Programming Local Alignment (DPLA) (which is similar to DTW). This process is done for the three representations, so they obtain three matrices $-H_{m i x}, H_{m e l}$, and $H_{a c c}$ - which are combined to produce the final similarity value.

They select the maximum of each matrix as candidate for the overall similarity value, and consider the following sets: $\left\{M_{m i x}, M_{m e l}\right\},\left\{M_{m i x}, M_{a c c}\right\},\left\{M_{m e l}, M_{a c c}\right\}$, and $\left\{M_{m i x}, M_{m e l}, M_{a c c}\right\}$, where $M_{m i x}$ is the maximum of $H_{m i x}, M_{m e l}$ is the maximum of $H_{m e l}$, and $M_{a c c}$ is the maximum of $H_{a c c}$. For each set - which represents a different fusion scheme - they select the maximum again as final similarity value to be reported.

They also propose an early fusion process performed before calling DPLA. Using the three matrices $S_{m i x}, S_{m e l}$, and $S_{a c c}$ they produce a new matrix $S$, before calling DPLA, as follows:

$$
S_{n, m}=\max \left(S_{n, m}^{m i x}, S_{n, m}^{m e l}, S_{n, m}^{a c c}\right)
$$

All these alternatives are computed and illustrated in Figure 4.8. Table 4.1 shows the results they obtain, from which we consider the use of source separation encouraging as a pre-processing step for similarity-based tasks. 


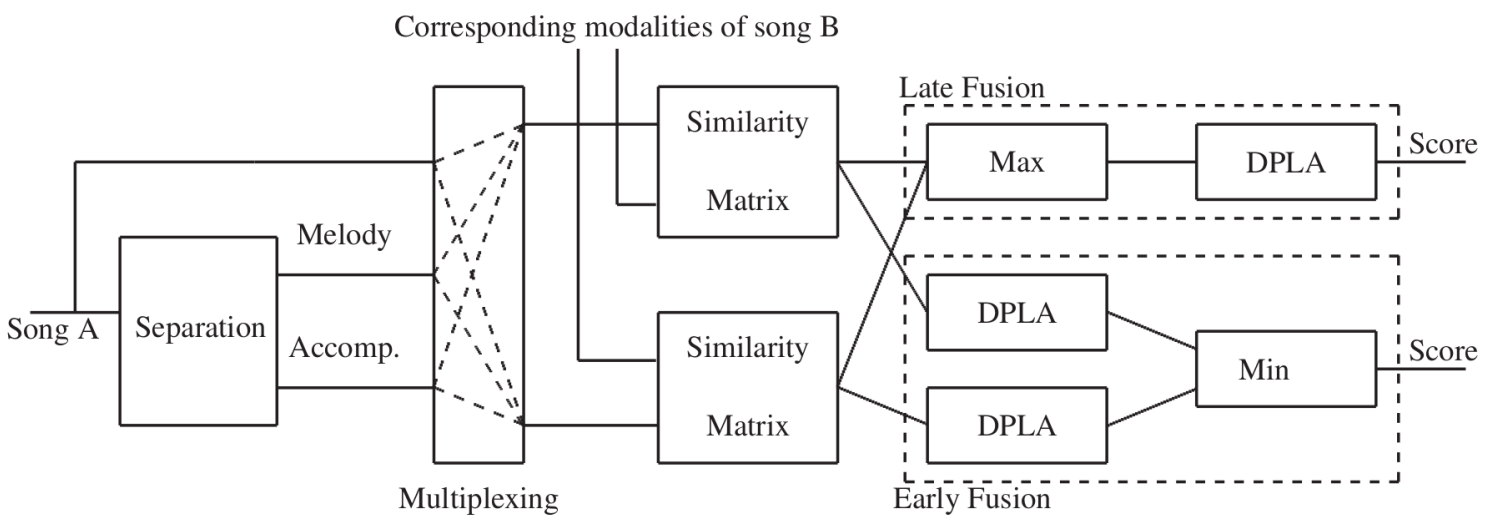

Figure 4.8: System's pipeline proposed by Foucard et al. [FDLR10].

\begin{tabular}{|l|c|c|c|}
\hline & Precision & Mean rank $_{1}$ & Median rank $_{1}$ \\
\hline Mix. & $\mathbf{0 . 3 5}$ & $\mathbf{3 2 . 0 2}$ & $\mathbf{7}$ \\
\hline Acc. & 0.34 & 33.18 & $\mathbf{7}$ \\
\hline Mel. & 0.21 & 43.98 & 21 \\
\hline \hline Mix., Acc. & $\mathbf{0 . 3 7}$ & 31.41 & $\mathbf{5}$ \\
\hline Acc., Mel. & 0.34 & 32.28 & 6.5 \\
\hline Mix., Mel. & 0.35 & $\mathbf{3 1 . 1 8}$ & 7 \\
\hline Mix., Acc., Mel. & $\mathbf{0 . 3 7}$ & 31.24 & $\mathbf{5}$ \\
\hline \hline Early fusion & 0.34 & $\mathbf{3 0 . 4 9}$ & 5.5 \\
\hline
\end{tabular}

Table 4.1: Results obtained by the use of source separation (reproduced from [FDLR10]) 


\section{Chapter 5}

\section{Methodology}

In this chapter we concentrate all the original contributions of this research, which include implementations and experiments related to music similarity, cover song identification and classification, and dataset modifications.

\subsection{A Framework for Music Similarity and Cover Song Identifica- tion}

The first step in our exploration of music similarity corresponded to reviewing and exploring, through implementations and small experiments, extractors, aggregators, and distance functions appearing in the literature. These are summarized in Tables 5.1, 5.2 and 5.3 below, and form the building blocks for a proposed music similarity framework.

\begin{tabular}{|c|c|}
\hline Chromagrams & $\begin{array}{l}\text { Jensen et al. [JCEJ08]; Serrá et al. [SGHS08]; } \\
\left.\text { Yu et al. [YZL }{ }^{+} 10\right] \text {; Ravuri and Ellis [RE10]; }\end{array}$ \\
\hline MFCC & $\begin{array}{l}\text { Foote [Foo97]; Logan and Salomon [LS01]; Au- } \\
\left.\text { couturier and Pachet [AP }{ }^{+} 02\right] \text {; Berenzweig et } \\
\text { al. [BLEW04]; Pachet and Aucouturier [PA04]; } \\
\text { Schnitzer et al. [SFW12]; }\end{array}$ \\
\hline Spectral features (centroid, bandwidth, etc) & Li and Ogihara [LO04]; \\
\hline Tonnetz & Tymoczko [Tym09]; \\
\hline RMS & $\begin{array}{l}\text { Panagiotakis and Tziritas [PT05]; McKay and } \\
\text { Fujinaga [MF05]; }\end{array}$ \\
\hline ZCR & Gouyon et al. [GPD $\left.{ }^{+} 00\right]$; Li and Ogihara [LO04]; \\
\hline symbolic melodic sequences & $\begin{array}{l}\left.\text { Ghias et al. [GLCS95]; Tsai et al. } \text { [TYW }^{+} 05\right] \text {; } \\
\text { Frieler and Müllensiefen [FM05]; } \\
\text { Marolt [Mar06]; Rho and Hwang [RH06a]; } \\
\text { Salamon et al. [SSG13a]; }\end{array}$ \\
\hline Rhythm Pattern & $\begin{array}{l}\text { Pampalk et al. [PRM02a]; Pampalk et } \\
\text { al. }\left[\mathrm{PFW}^{+} \text {05];Seyerlehner et al. [SSPK10]; }\right.\end{array}$ \\
\hline
\end{tabular}

Table 5.1: Reviewed and explored list of extractors. 


\begin{tabular}{|l|l|}
\hline single Gaussian & Schnitzer et al. [SFW12]; \\
\hline Gaussian Mixture Model & $\begin{array}{l}\text { Aucouturier and Pachet [AP }{ }^{+} \text {02]; Pachet and Aucouturier [PA04]; } \\
\text { Berenzweig et al. [BLEW04]; }\end{array}$ \\
\hline Vector Quantization & Logan and Salomon [LS01]; \\
\hline Markov Chain & Hoos et al. [HRG01]; \\
\hline Octave Abstraction & Salamon et al. [SSG13a]; \\
\hline Interval Abstraction & Salamon et al. [SSG13a]; \\
\hline Pitch Contour (3-levels) & Ghias et al. [GLCS95]; \\
\hline Pitch Contour (5-levels) & Rho and Hwang [RH06a]; \\
\hline
\end{tabular}

Table 5.2: Reviewed and explored list of aggregators.

\begin{tabular}{|l|l|}
\hline Manhattan distance & Seyerlehner et al. [SSPK10]; Knees and Schedl [KS13]; \\
\hline Euclidean distance & Foote [Foo97]; Li and Ogihara [LO04]; Yu et al. [YZL $\left.{ }^{+} 10\right] ;$ \\
\hline Cosine distance & Foote [Foo97]; Tralie [Tra17]; \\
\hline LCS-based distance & Uitdenbogerd and Zobel [UZ99]; Kelly [Kel12]; \\
\hline Levenshtein's distance & Lemström and Ukkonen [LU00]; Kelly [Kel12]; \\
\hline Kullback-Leibler distance & Berenzweig et al. [BLEW04]; Schnitzer et al. [SFW12]; \\
\hline Earth Mover's distance & Logan and Salomon [LS01]; Typke et al. [TWV05]; \\
\hline Monte Carlo distance & Aucouturier and Pachet [AP+ $\left.{ }^{+} 02\right] ;$ Pachet and Aucouturier [PA04]; \\
\hline
\end{tabular}

Table 5.3: Reviewed and explored list of distances.

Notice that the previous lists do not contemplate all the techniques presented in Chapter 2. We actually started with a huge list of techniques that was altered in many ways (both discarding and collecting techniques along the research process).

An important criterion for this revision consisted in removing the techniques that do not have open source implementations. The rationale for this is to avoid producing implementations that might differ from the original techniques due to ambiguous or insufficient details in their textual descriptions. The survey of open source libraries (more details on Section 6.1) also led us to include techniques not found in the music similarity literature. For instance, the library that computes Rhythm Patterns ${ }^{1}$ also implements Rhythm Histograms, Statistical Spectrum Descriptors, and Modulation Variance Descriptors, which have also been considered in our work.

This same criterion was applied to aggregator and distance techniques, but in a softer way, since they are simpler to implement. For instance, it only took one implementation of one clustering algorithm - k-means - to keep the vector quantization technique in the list (we implemented the remaining processing steps after the clustering of local features).

\section{Music similarity models}

The detailed analysis of the techniques in the literature produces one major observation: several papers do not explicitly argue as for why a particular extractor (or aggregator, or distance) is selected to solve a particular problem. Even less frequent are arguments about why a specific set of techniques are used in combination (instead of many other plausible alternatives). This prompted us to try to explore (the hundreds of) combinations that are not considered in the literature, instead of trying to reproduce (very closely) only a few papers. It was thus natural to look at this problem as a benchmark, exhaustively experimenting with lots of combinations of extractors, aggregators, distances, here referred to as music similarity models.

All the combinations of features, aggregators, and distances considered result in a total of 690 music similarity models; the complete list is available at https://rppbodo.github.io/phd/music-similarity-models html. The number of models is not a basic multiplication of $n_{\text {features }} \times n_{\text {aggregators }} \times n_{\text {distances }}$ due

\footnotetext{
${ }^{1}$ https://github.com/tuwien-musicir/rp extract
} 
to compatibility issues: not all aggregators can be used with all features, and not all distances can be used to compare all global features. Below we summarize how the music similarity models are built.

Every feature that corresponds to a numerical value (1-D feature), or a numerical vector (2D feature), associated with each audio frame allows the use of statistical aggregation methods. Thus the melspectrogram, all the chromagrams (Short-Time Fourier Transform (STFT), ConstantQ Transform (CQT), Chroma Energy Normalized Statistics (CENS)), tonnetz, MFCC, all spectral features (centroid, bandwidth, roll-off, flatness, contrast), RMS, and ZCR can be aggregated through single or multivariate Gaussians (please refer to Section 2.2 to check all the Gaussian variations considered). Alternatively, Gaussian Mixture Model and Vector Quantization can also be used as aggregation methods for these features.

The symbolic domain feature called Pitch Contour Segmentation is aggregated with 5 methods: octave abstraction, interval abstraction, pitch contour with 3 levels, pitch contour with 5 levels, and Markov chain.

The remaining features - Rhythm Pattern, Rhythm Histogram, Temporal Rhythm Histogram, Statistical Spectrum Descriptor, Temporal Statistical Spectrum Descriptor, and Modulation Variance Descriptor - are already aggregations of local features by definition, and thus we do not aggregate them further (this is identified by the aggregation method bypass).

Every global feature that corresponds to a numerical vector (for instance, concatenated parameters from statistical models) can be seen as a point in the vector space $\mathbb{R}^{n}$, and can be compared to one another using spatial distances such as Euclidean, Manhattan, Chebyshev, and cosine. The aggregators that are compatible with these 4 distances are basic statistics, difference statistics, statistical summarization, Gaussian Mixture Model (GMM), vector quantization, and Markov chain.

Statistical models can also be viewed as computational models, allowing us to draw random samples from them and access their probability density functions. Thereby we can compare them using Kullback Leibler, Earth Mover's and Monte Carlo distances. Finally, all symbolic global features can be compared with Longest Common Subsequence (LCS)-based and Levenshtein distances.

\section{Implementation details}

In our work, all these models and methods were incorporated into a music similarity framework, implemented according to the architecture illustrated in Figure 5.1. 


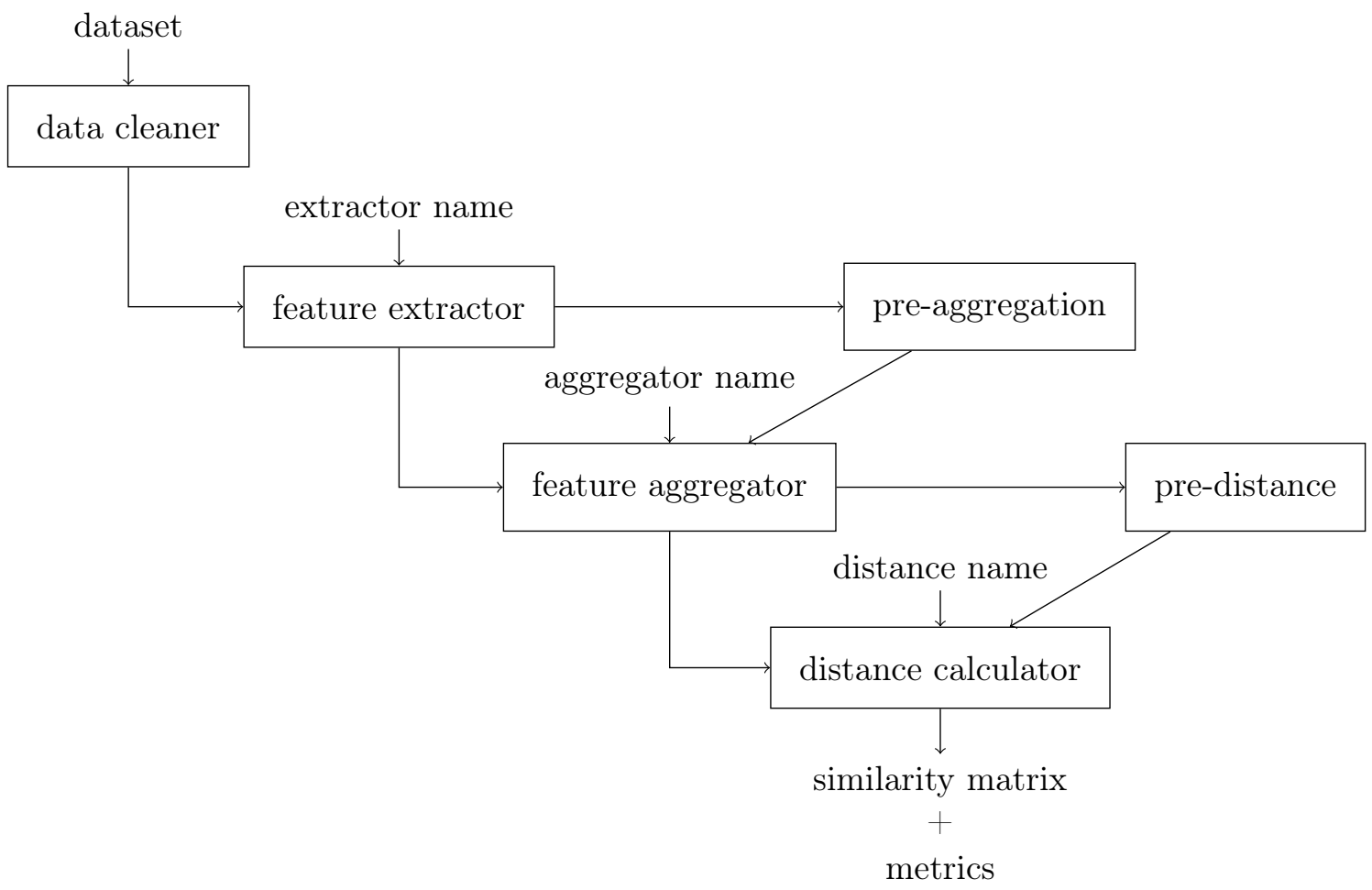

Figure 5.1: Processing diagram for the implementation of our music similarity framework.

The data cleaner module processes an input dataset and performs data cleaning tasks, as presented in Section 6.3; the feature extractor module extracts the named feature for all audio files of the dataset; the pre-aggregation module computes information that is required by some aggregation functions (for instance, if the selected aggregation method is vector quantization, this module computes the codebook); the feature aggregator module computes global features from local ones; the pre-distance module computes information required by some distance functions (for instance, computes the Optimal Transposition Index (OTI) from every pair of songs before measuring the distance between them); the distance calculator module computes the distances between global features for all possible track pairs, and returns the similarity matrix (as described in Section 2.4) and a set of chosen metrics. If the "pure" music similarity problem is selected, it returns intra-inter-class similarity ratios; if the Cover Song Identification (CSI) problem is selected, it returns Mean Rank (MR), Mean Reciprocal Rank (MRR), Median Rank (MDR), Mean Average Precision (MAP), etc. The source code of our music similarity framework is available at https://github.com/rppbodo/music-similarity-framework.

Every module may be executed as a single thread or in multi-thread mode. They also save all computed data to disk for future executions, which can lead to a considerable time gain. For illustration, it takes $14 \mathrm{~m} 54.096 \mathrm{~s}$ to extract melspectrograms for a dataset of 160 tracks with a single thread of a Intel(R) Core(TM) i5-5200U CPU @ 2.20GHz processor, while it only takes 0m7.861s to load the same pre-computed data from the hard disk.

\section{Relevant metrics for task-specific music similarity models}

Even though the inter-intra-class similarity ratio is an useful metric to assess a music similarity model's overall quality, specific tasks often require specific metrics that best capture the specificities of the application domain. For instance, we use two datasets that were designed for the query by singing/humming task, and MIREX $2020^{2}$ defined top-10 hit rate as the evaluation metric for the Query by Singing/Humming competition. As already pointed out in Chapter 1, the very notion of

\footnotetext{
${ }^{2}$ https://www.music-ir.org/mirex/wiki/2020:Query_by_Singing/Humming
} 
music similarity always exist within some specified context, which influences how music similarity expresses itself and how music similarity models should be evaluated.

In order to evaluate the use of our music similarity framework within the context of one specific application of music similarity, and its related datasets, we consider the Cover Song Identification problem, introduced in Section 3.1. This is a well-suited problem for the evaluation of the framework for two main reasons: on the one hand, a cover song always has musical elements which are similar to those of the original song; on the other hand, the specific musical elements (melody, rhythm, etc), as well as the specific way in which they are similar (between cover song and original) can be very subtle and diverse, suggesting that different models may be better suited to identify cover songs written in different styles or using different music strategies.

\subsection{Cover Song Classification}

Besides considering CSI, we also investigate the Cover Song Classification (CSC) problem, in order to address the differences between the search for covers within a dataset and the binary classification of a pair of songs (whether they are versions of each other or not). Inspired by fusion CSI methods (see Section 3.1.3) we investigated the use of combined information from several music similarity models, in order to attempt to produce better similarity models in a constructive (or collaborative) way. The act of gathering information from different models can be categorized as an early fusion scheme, since we are blending data before the classification step, according to Ning Chen et al. [CLX17]. Figure 5.2 presents the proposed processing pipeline for the CSC problem.
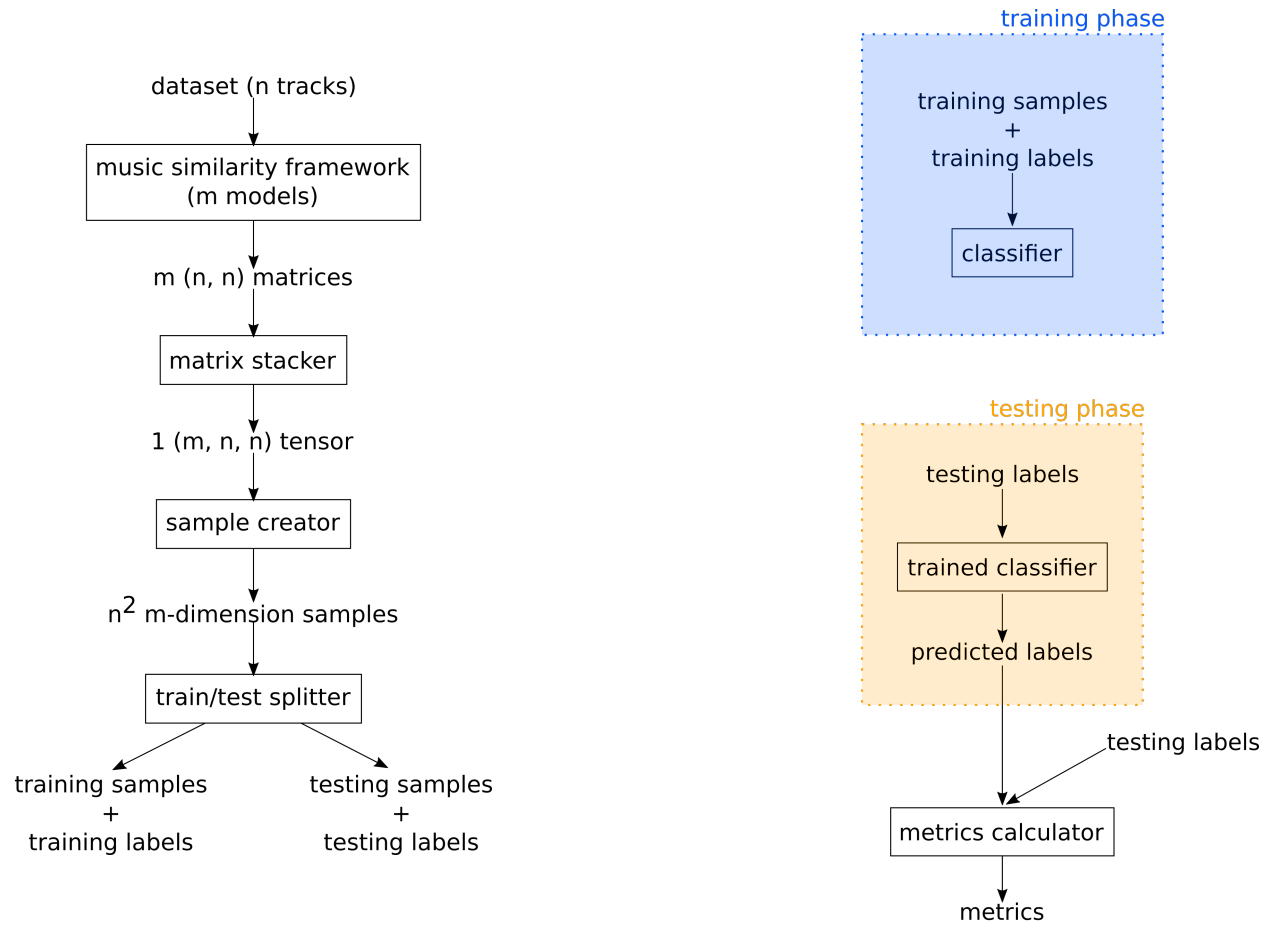

Figure 5.2: The proposed processing pipeline for the Cover Song Classification problem.

The first element in the pipeline of Figure 5.2 is the music similarity framework presented above. A group of $m$ similarity models is selected, and the corresponding similarity matrices are gathered to be fused, incorporating information from distinct models. In order not to interrupt the presentation of the pipeline, we postpone the discussion of model selection strategies to the end of this section. The $m$ similarity matrices returned by the music similarity framework are stacked to form a tensor with dimension $(m, n, n)$, where $n$ is the number of tracks in the processed dataset.

In the sample creator module the tensor is traversed (as seen in Figure 5.3) to produce the samples for classification, which in this case correspond to pairs of songs. The sample that represents 
the $\left(\operatorname{song}_{i}\right.$, song $\left._{j}\right)$ pair is the vector obtained by traversing the tensor through the positions $(*, i, j)$. Semantically, the samples may be viewed as sets of different opinions on how similar song $i$ and song $_{j}$ are from different perspectives (i.e., lists of scores from distinct music similarity models).

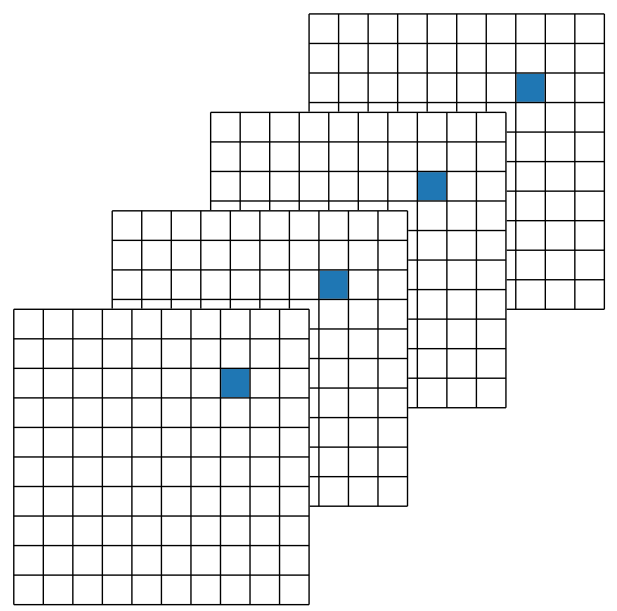

Figure 5.3: An example of a tensor built from several similarity matrices, and the sample vector $\left({ }^{*}, i, j\right)$.

Experiments with classification require disjoint training and testing sets. This makes sense since it is not fair to test the classifier with a sample that has been already seen in the training phase. In the CSC problem, a sample holds information about 2 songs, so if we select a sample $(*, i, j)$ to be in the training set, every sample $(*, k, l)$ in the test set must satisfy $k \neq i, j$ and $l \neq i, j$. This shows that in order to create such a train test split it is necessary to place original songs along with their versions together in the same split.

Considering that a train test split has to partition the samples, which represent song pairs, according to a given ratio, and at the same time satisfy the above constraint, some care must be taken as we assign groups of songs (originals along with their versions) to the train or test set. It should be clear that in order to produce for instance a 70\%-30\% train test split, i.e., having $70 \%$ of the pairs in the training set and 30\% of the pairs in the test set, one cannot simply assign $70 \%$ and $30 \%$ of the original songs respectively to the train and test sets, because the number of versions for each original song may be wildly different.

We propose the following heuristic approach to solve this problem. We start with empty train and test sets, and sort the original songs by their corresponding number of versions, in descending order. Beginning with the first original song (i.e. the one that has the largest number of versions and thus can have the largest impact in the split percentages), we assign it to the split that is farthest from its target percentage (this would typically be the train split). We then proceed to the next original song, and compute the difference between the current split percentages and the desired split. If the train set corresponds to the largest difference, the current original song (along with its versions) is added to the train set. Analogously, the current song with its versions are added to the test set if this split is farthest from its target percentage. This heuristic is formalized in the following pseudo-code. 


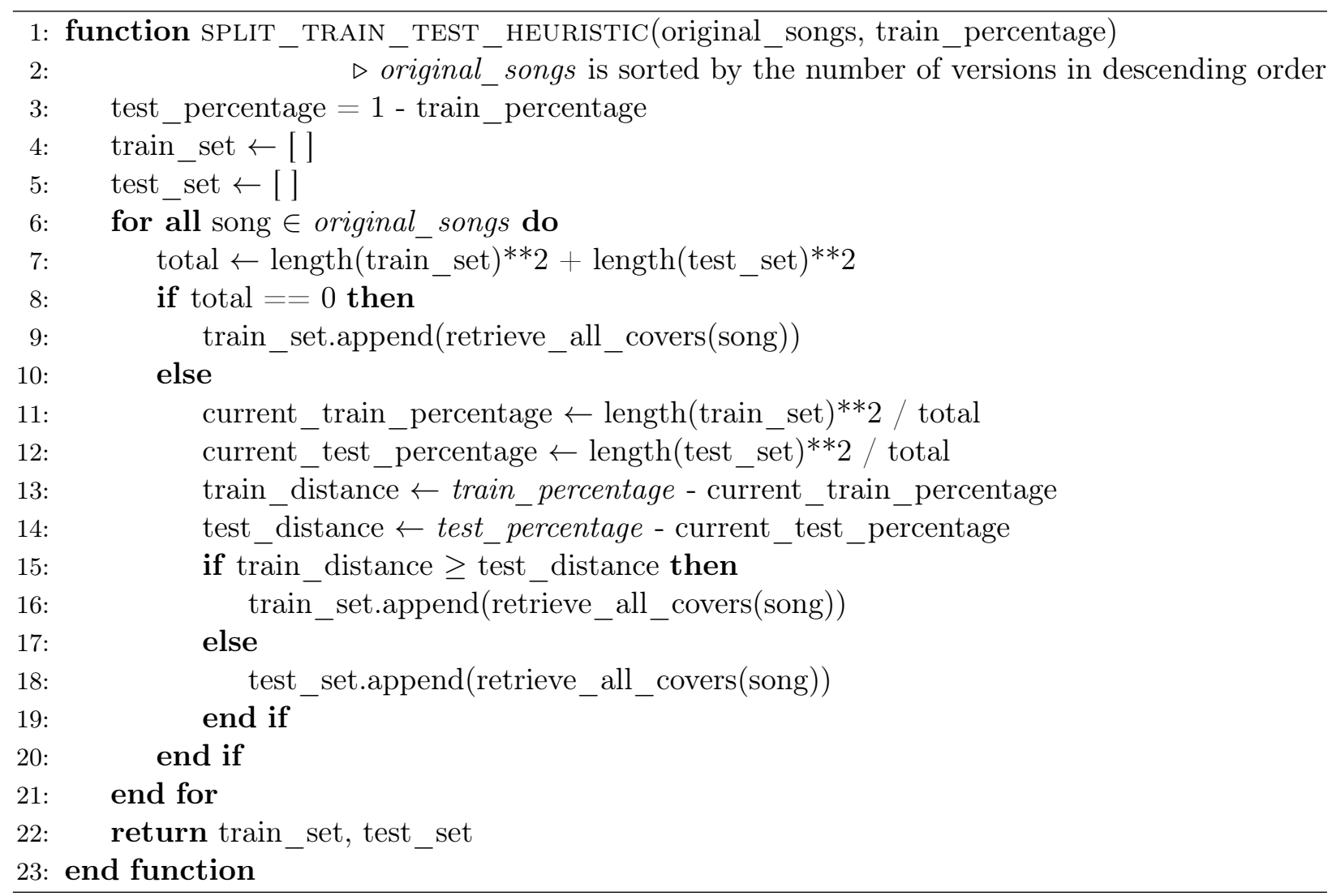

Regarding the actual classification step, just as in any machine learning problem, we start with the learning step, where the classification model is fitted to the training samples. After that, we ask the classifier to predict the classes for all testing samples. In the implemented pipeline we worked with the following classification models:

- naive Bayes

- logistic regression

- decision tree

- random forest

- ada boost

The last module, called Metrics Calculator, receives the predicted and ground-truth labels, and computes all the metrics discussed in Section 3.1.4.

We now discuss how to select the music similarity models to create the tensors from which we extract the samples for the classification task. We start by ordering the models according to their quality in the CSI problem using MR values, which proved to be a quite reliable measure of the models' quality.

A somewhat natural idea is to select the Top- $\mathrm{N}$ models, since they are supposed to provide the most reliable similarity measures for the classification task. However, the best models are usually very similar to each other: they are usually tonal features, with statistical aggregation methods, and vector space distances. Thus, we aimed to explore alternative model selection strategies that would avoid to include very similar techniques (e.g., chroma_stft and beat_synchronous_chroma_stft) that appear close in the Top- $\mathrm{N}$ list, and prefer more complementary ideas (e.g., a rhythmic descriptor) that might appear just a few positions ahead in the same list.

The solution we propose is to cluster similar techniques (feature, aggregator or distance) into equivalence classes, and, when traversing the sorted list of models, we only select models with at 
least one technique that has not been selected before. The manually clustered techniques are shown in Figures 5.4, 5.5, and 5.6.

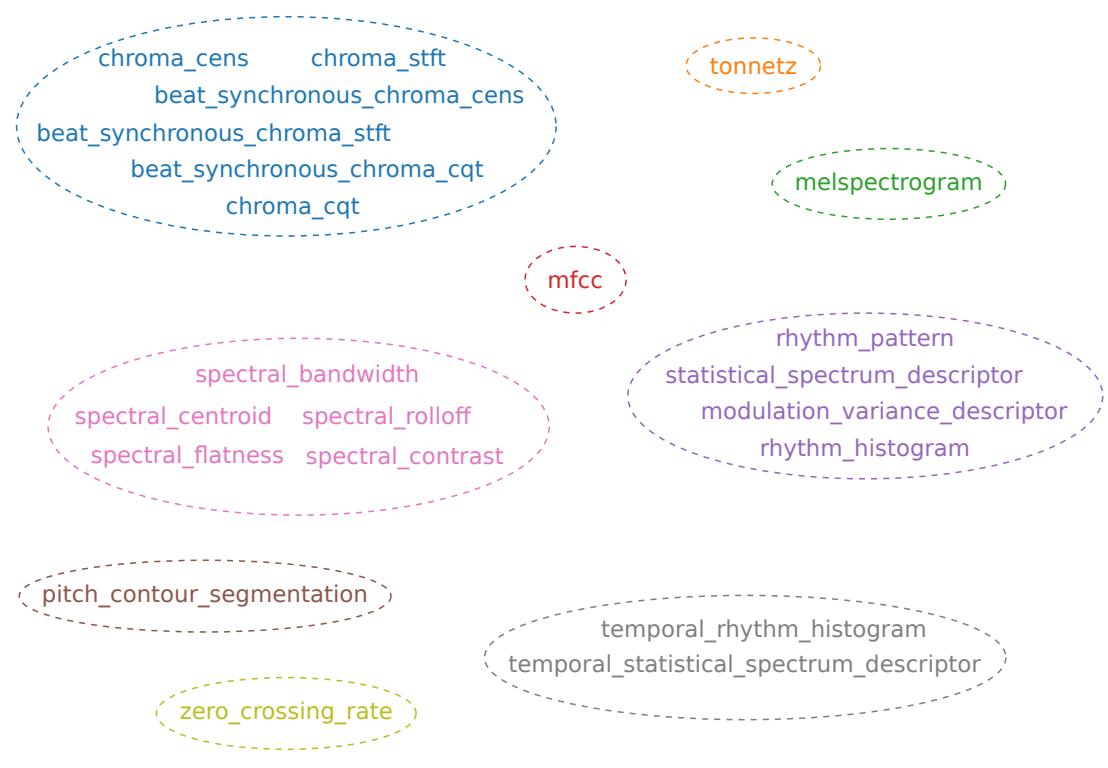

Figure 5.4: The manually clustered extractors.

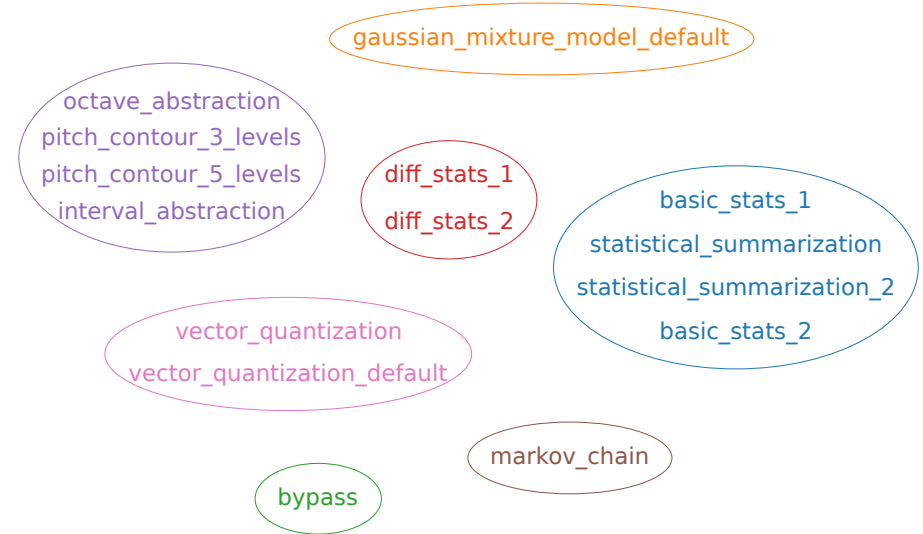

Figure 5.5: The manually clustered aggregators. 


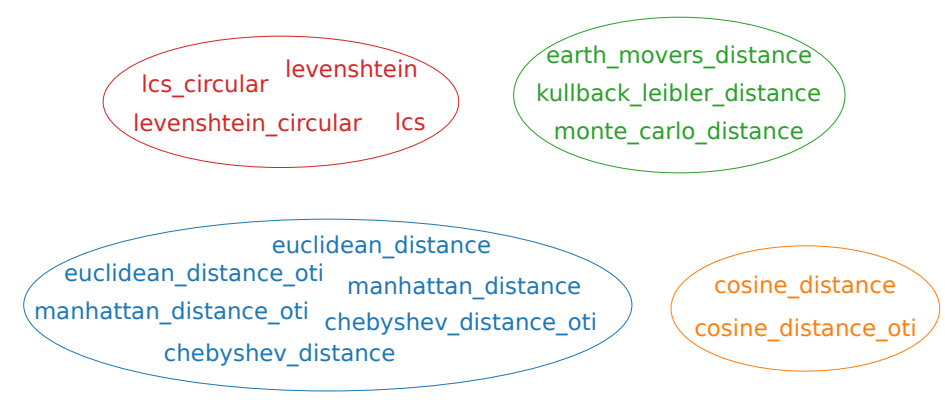

Figure 5.6: The manually clustered distances.

\subsection{Cover Song Identification and Classification}

For every classifier in the previous section it is possible to obtain a likelihood estimation for samples (pairs of songs), prior to the binary classification. For our specific problem, this means that for every trained classifier we may obtain a chance for a pair of songs to be versions of the same work, according to the trained model. This secondary information obtained from the CSC experiments can be used in the CSI context as scores, and by aggregating these scores for every pair of songs from a dataset, we may define a new similarity matrix. This similarity matrix can thus be used to solve the CSI problem, and be evaluated within that context using the relevant metrics (MR, MRR, MDR, MAP, etc).

There is one issue with this idea: in the CSC problem, described in the preceding section, samples are divided into two disjoint sets (training and test). According to good machine learning practices, the classifier should only be used to obtain these chance values of the test samples (because it knows the ground truth values for the training samples). Even if we did try to train two separate classifiers by swapping the train/test sets as illustrated in Figure 5.7, we would still be far from solving the issue: on the one hand, it would be unusual and ineffective to train a classifier on a set smaller than the test set; on the other hand, this would still leave out many samples (the white cells in Figure 5.7). This issue does not appear to have a simple solution if both training and test samples are obtained from the same dataset.
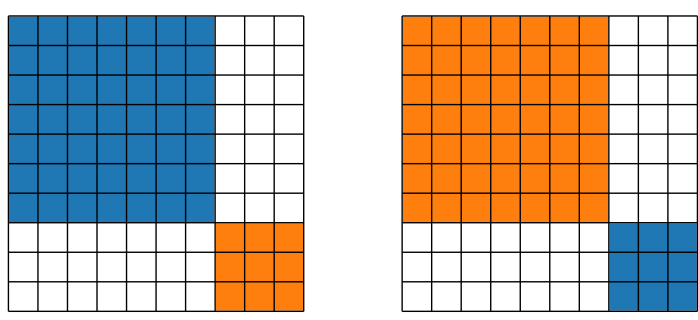

Figure 5.7: A naive solution to obtain the most of chance values for the test samples of a single dataset.

An alternative (and better) method is to perform a cross-dataset classification, using a larger dataset $A$ to train the classifier, and a smaller dataset $B$ to obtain the estimated chance values for all pairs of songs in $B$, as exhibited in Figure 5.8. This method allows us to produce a similarity matrix for dataset $B$ that embodies the knowledge of the classifier trained on dataset $A$. Thus, a 
solution to the CSC problem can be used in the CSI problem, as long as the classifier computes likelihood estimates prior to the binary classification.
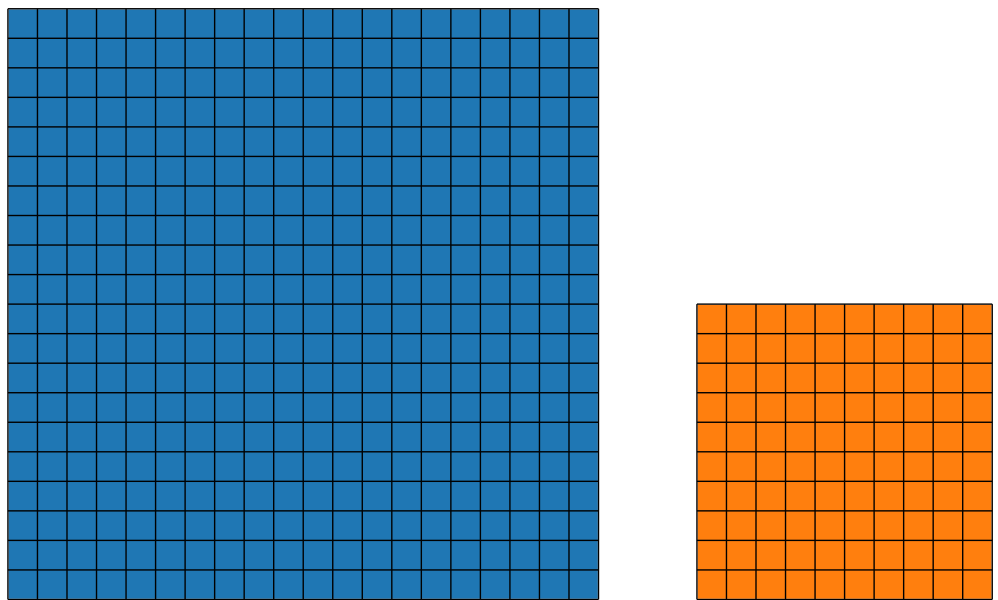

Figure 5.8: A proper solution to obtain chance values for all samples of one dataset using a second larger dataset for training.

The complete process of using a CSC classifier for CSI is quite similar to the CSC pipeline, so we skip a step-by-step explanation, but refer to Figure 5.9, where two parallel pipelines represent the training phase (with dataset $A$ ) and predicting phase (with dataset $B$ ). We observe that, due to the different datasets involved, these parallel lines could be executed concurrently. The classifier is trained with $k^{2}$ samples from dataset $A$, and only after that, it receives the $l^{2}$ pairs of songs from dataset $B$ to produce values between 0 and 1 , interpreted as chances of the samples to be from pairs of versions.
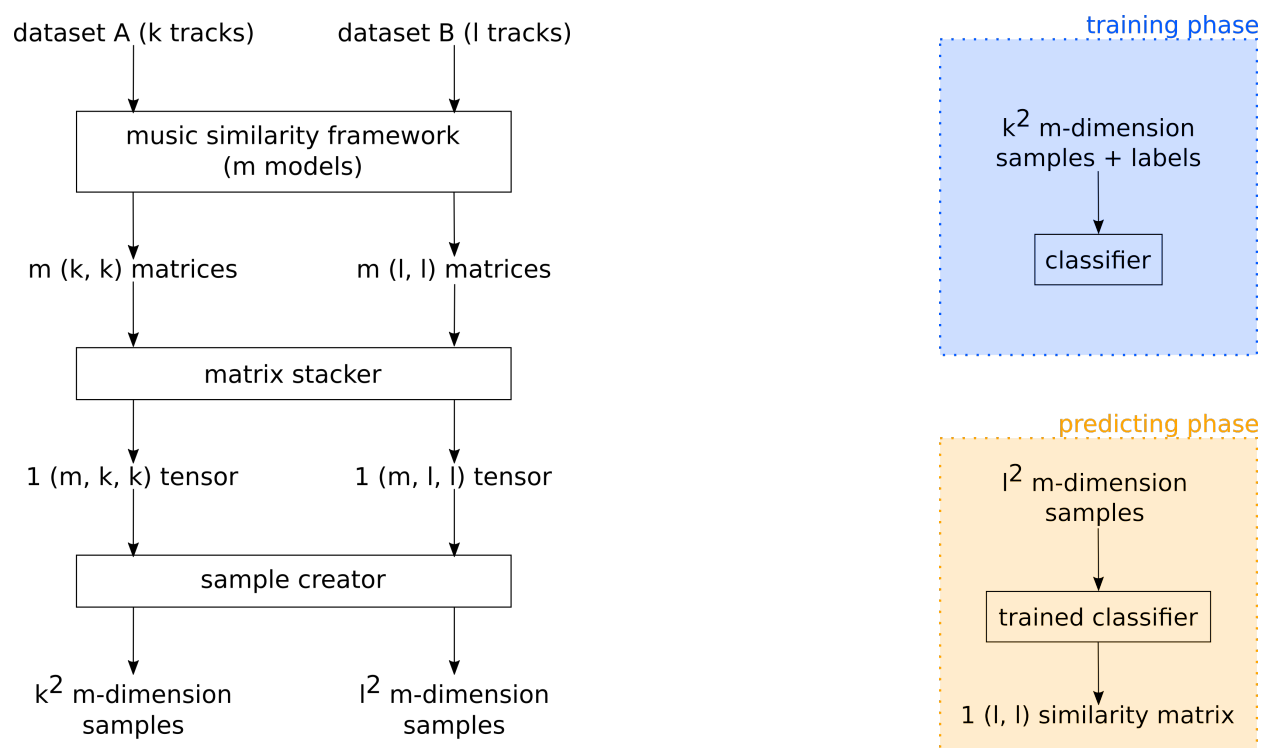

Figure 5.9: The full processing pipeline connecting cover song identification and cover song classification.

Finally, a small adjustment is required in the choice of the music similarity models that are combined, because we have now two datasets (train and test). Each dataset produces a different list for the Top-N best similarity models, with potentially conflicting quality values for the similarity models that are common to both lists. Additionally, there is the issue of filtering out models which are considered equivalent (for combining similar feature/aggregation/distance techniques).

The solution we propose builds a centralized list of music similarity models ordered by their quality values (mean ranks), and works as follows. Consider that we have $N$ datasets, along with 
the mean rank values for all music similarity models considered (in our research, this corresponds to 690 models). Switching perspectives, we can see that for each similarity model we have $N$ mean ranks (one for each dataset). We want to represent each model using a single value, so we can order the models (chain ordering is not useful here, because there is no a priori preference for any particular dataset).

Considering that the same similarity model may perform very differently on different datasets, we would like to get a sense of the central tendency of the values corresponding to each particular model, and also of their dispersion (which could be used to break ties). The most obvious statistics that come to mind are mean and standard deviation; however, these are quite sensitive to outliers. For this reason we propose using robust statistics for representing central tendency and statistical dispersion, namely median and median absolute deviation, respectively.

\subsection{Dataset Modifications}

As we have seen on Chapter 4, data modifications have been used in order to improve results for Music Information Retrieval (MIR) tasks, with a decrease in computational effort when the modified audio files tend to be considerably smaller than the original ones.

\subsubsection{Segment selection}

Segment selection is the first dataset modification considered here. It is motivated by the potential computational impact of substituting full-length songs for much smaller songs snippets, and also by the fact that online datasets are subject to copyright restrictions and usually cannot provide complete songs.

The basic idea of segment selection is to create a modified dataset where each song is replaced by a representative segment, such as a chorus or refrain. Several strategies for selecting representative song segments are detailed in the sequel.

\section{Segment selection via music segmentation}

Music segmentation algorithms are natural candidates for segment selection, since they look for repetitive patterns in a song by analyzing music structure. These algorithms produce segmentation profiles such as the one displayed in Table 5.4, with labels denoting repeating segments.

\begin{tabular}{lll} 
start time & end time & segment label \\
\hline 0.0 & 2.9000 & $\mathrm{G}$ \\
2.9000 & 17.8200 & $\mathrm{~F}$ \\
17.8200 & 30.9880 & $\mathrm{D}$ \\
30.9880 & 86.6440 & $\mathrm{~A}$ \\
86.6440 & 99.2800 & $\mathrm{~F}$ \\
99.2800 & 108.3440 & $\mathrm{~A}$ \\
108.3440 & 121.7800 & $\mathrm{D}$ \\
121.7800 & 158.3240 & $\mathrm{~A}$ \\
158.3240 & 181.6040 & $\mathrm{~F}$ \\
181.6040 & 204.9400 & $\mathrm{~A}$ \\
204.9400 & 224.9280 & $\mathrm{~F}$ \\
224.9280 & 265.4800 & $\mathrm{E}$ \\
265.4800 & 265.5200 & $\mathrm{G}$
\end{tabular}

Table 5.4: An example segmentation computed using the SI-PLCA algorithm.

Given a segmentation of a song, a representative segment could be chosen for instance as the most frequent segment with duration above 30 seconds. This allows us to generate a new modified 
dataset, by substituting a song for its representative segment. Three different music segmentation algorithms are included in our evaluation and detailed below.

Levy and Sandler [LS08] propose an algorithm for audio segmentation that consists in extracting spectral features, post-processing them, and reducing their dimensionality using Principal Component Analysis (PCA). The resulting 20-dimensional vector with the principal components of each frame is then appended with the corresponding spectrum norm, resulting in the final feature vector. An Hidden Markov Model (HMM) is then trained with all the feature vectors, and Viterbi algorithm is used to decode each song into a sequence of labels, which represents a low-level labeling for every frame of the song. Histograms are produced to represent the distribution of these low-level labels and their neighboring labels. Frame sequences are finally grouped into labeled segments by clustering these histograms, using an algorithm that considers temporal continuity constraints via a Markov random field. Such labeled segments correspond to the structural segmentations for each track.

Mauch et al. [MND09] present a segmentation algorithm in the context of automatic chord transcription. It starts by extracting chroma vectors for every beat of each song. Then it builds a selfsimilarity matrix comparing all pairs of chromas using Pearson correlation coefficients. This matrix is smoothed via low-pass filtering, and diagonals are traversed to locate repetitions. Repeating segments are then clustered using heuristics to discard sub-segments and choose among overlapping segments, and favoring those segments with largest coverage of the song (coverage is measured by a segment's length multiplied by its number of repetitions).

Weiss and Bello [Bel10] propose a technique called Shift-Invariant Probabilistic Latent Component Analysis (SI-PLCA) which is used to decompose beat-synchronous chromagrams into repetitive patterns and their temporal occurrences, which encodes the temporal structure within the songs. SI-PLCA is built incrementally on top of the Non-negative Matrix Factorization (NMF) technique, which decomposes a non-negative matrix $V$, representing an audio signal, into two matrices $V \approx W H$ - where the columns of $W$ are templates occurring in $V$, and the rows of $H$ are the activations of these templates in $V$. They first define the Probabilistic Latent Component Analysis (PLCA), which is a probabilistic variant of NMF, where both columns of $W$ and rows of $H$ are interpreted as multinomial probability distributions, and columns of $W$ are interpreted as basis functions. A new distribution is added as weights for each basis function, resulting in a new factorization $V \approx W Z H$, where $Z$ is a diagonal matrix with the weight distributions. The technique SI-PLCA is derived from PLCA by adding the property of shift invariance. This is done by modifying the basis functions to include $L$ templates instead of a single template as in PLCA. So the basis functions become matrices instead of vectors, and $W$ becomes a tensor.

\section{Segment selection via thumbnailing}

Thumbnailing algorithms can also be used to produce candidate segments meant to replace full-length recordings in modified datasets. Two such algorithms are included in our evaluation.

Cooper and Foote [CF02] propose a method to select thumbnails from songs that uses parameterized versions of audio signals through MFCC analysis, producing compact representations for every frame. From the MFCC it builds a Self-Similarity Matrix (SSM) $S$ using cosine distance between frames, and then for every segment starting at index $i$ and ending at $i+L$ the method defines its average similarity with respect to the whole song as $\hat{S}(i, i+L)=\frac{1}{N L} \sum_{m=i}^{i+L} \sum_{n=1}^{N} S(m, n)$. The thumbnail is selected by looking for the segment with maximum similarity to the entire song, that is, by finding $i$ and $L$ that maximizes $\hat{S}(i, i+L)$, subject to constraints on starting time and duration.

Bartsch and Wakefield [BW05] also work with a self-similarity matrix, but this is computed using the correlation of 12-bin chroma vectors extracted from beat-synchronous frames. A step of correlation filtering produces a modified self-similarity matrix, from which the thumbnail is obtained by selecting the maximum value of the new matrix, also subject to constraints on starting time and duration. 


\section{Other strategies for segment selection}

Besides segmentation and thumbnailing algorithms, we also considered the selection of the beginning, middle and end portions of the songs, as is frequently done in the context of music information retrieval [TC02a]. We used the initial, mid-point and final slices of 30s for each song as representative segments, thus generating three modified datasets.

\subsubsection{Frame selection}

As in Seyerlehner's work [Sey10a], discussed in Section 4.2, we also included frame selection in our experimental evaluation.

RMS values are computed for each song using 1024-sample frames. These values are sorted, and then a corresponding percentile (we use 10\%,20\%, .., 90\%) of the lowest valued frames is discarded, resulting in a shortened version of the song with the remaining highest valued frames (in their original order). This produces nine secondary datasets (one modified dataset for each percentile). Figure 5.10 shows a 30 second audio clip (from the song Creep by Radiohead) and the selected $50 \%$ highest RMS valued frames highlighted in red.

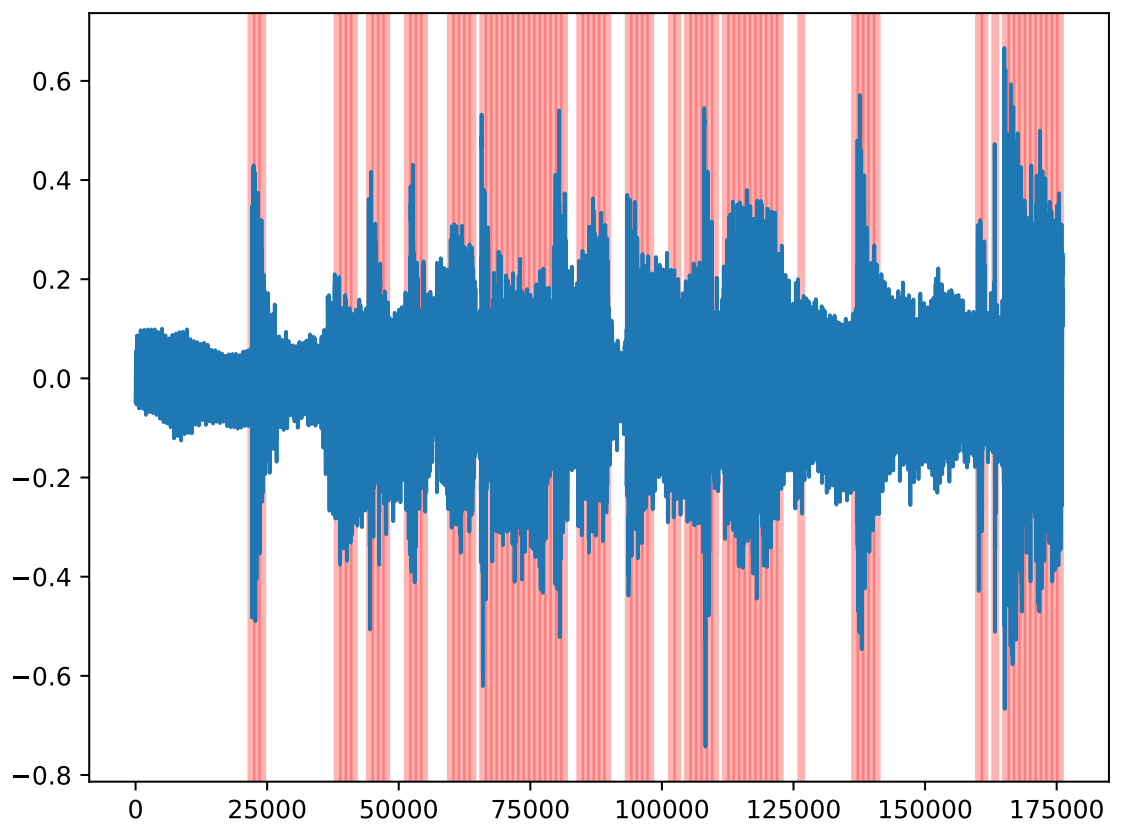

Figure 5.10: Frame selection keeping $50 \%$ of the frames with the highest RMS values.

In addition to the above mentioned method, we aim to verify whether frames containing vocals would be more relevant to similarity measures than instrumental frames For this purpose, we propose a novel frame selection method, using the RMS values from the vocal track ${ }^{3}$ as the sorting criterion and then applying a given percentile (again 10\%, 20\%, ...,90\%), similarly to the above mentioned frame selection method. Figure 5.11 (top audio clip) shows the isolated vocals from the same song as in Figure 5.10, and the 50\% selected frames with higher (vocal) RMS values. Then the selected frame indexes are applied to the original track (bottom audio clip). This method produces nine modified datasets, one for each percentile.

\footnotetext{
${ }^{3}$ Vocal tracks are obtained using the Open-Unmix [SULM19] source separation algorithm.
} 

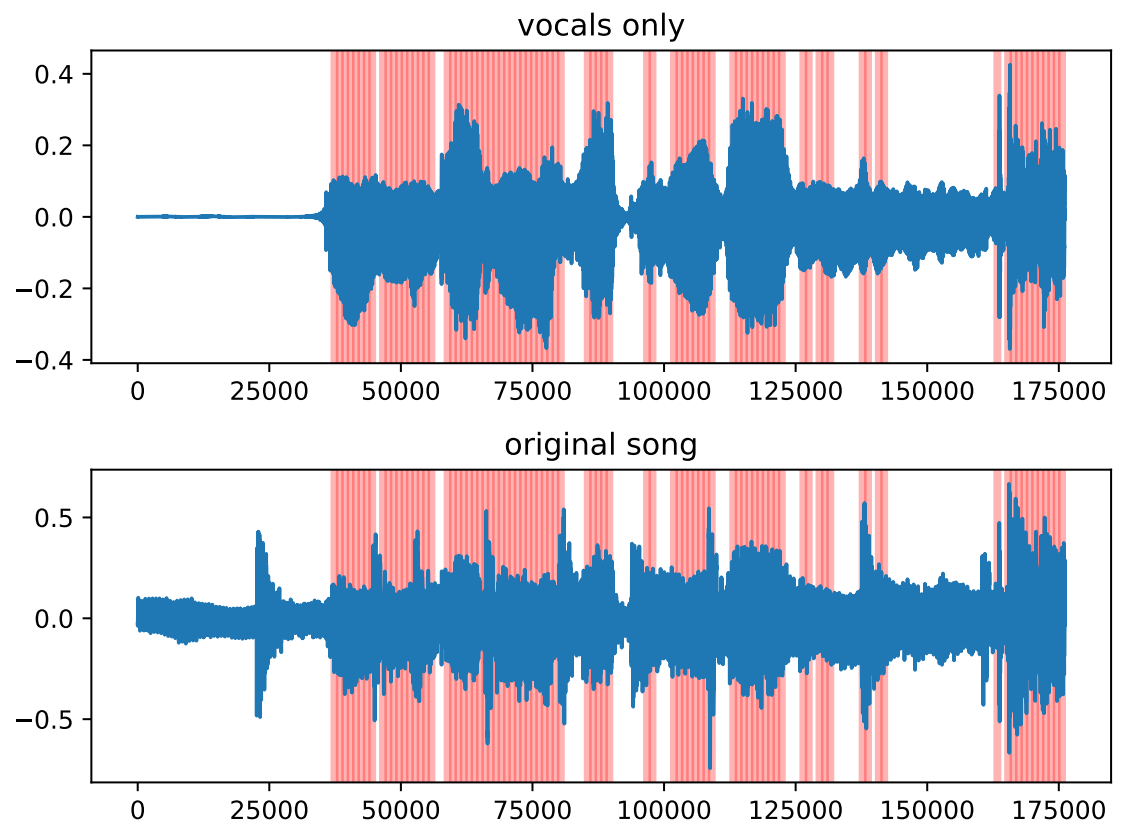

Figure 5.11: Frame selection from the vocals-only (top) 50\% highest RMS values, and applying them to the mix (bottom).

\subsubsection{Source separation}

Foucard et al.[FDLR10] consider that songs consist in a leading voice mixed with an accompaniment, and use separated sources in the context of music similarity and cover song detection. They compare not only the original songs but also the extracted melodies and the accompaniments, and fuse the comparison results using different strategies.

Based on their work, we consider the use of separated sources to measure different aspects of music similarity. For instance, we can use the separated vocal tracks of two songs to produce a measure of melodic similarity between them, and the isolated drum tracks for rhythmic similarity. We expect that, with the combined use of similarities obtained from different musical perspectives, we should be able to improve CSI results.

For the task of separating audio sources within a song recording, we use the Open-Unmix model [SULM19], which is currently considered the state-of-the-art in audio source separation. This algorithm returns four audio sources for each input, labeled "vocals", "bass", "drums", and "other" (a generic track containing the remaining sources, after the separation of the previous three). For each given dataset, we use these separate tracks to build four new datasets, one corresponding to each individual source.

\subsection{Fusion}

As seen on Section 3.1.3, fusion techniques have been successfully applied in the context of CSI research. In the previous two sections we discussed dataset modification methods and source separation, where the idea is to explore different music similarity models applied to isolated sources. We propose here the use of fusion techniques to similarity matrices obtained from datasets containing distinct individual sources.

We start by combining the four self-similarity matrices, corresponding to the four sources produced by Open-Unmix [SULM19], by using the mean, minimum and maximum of the corresponding similarity values for each pair of songs. Specifically, if $B, D, V, O$ are similarity matrices respectively obtained from the separate "bass", "drums", "vocals" and "other" modified datasets, we define the three following matrices: 
- $\operatorname{Mean}_{i, j}=\operatorname{mean}\left(\left[V_{i, j}, B_{i, j}, D_{i, j}, O_{i, j}\right]\right)$;

- $\operatorname{Min}_{i, j}=\min \left(\left[V_{i, j}, B_{i, j}, D_{i, j}, O_{i, j}\right]\right)$;

- $\operatorname{Max}_{i, j}=\max \left(\left[V_{i, j}, B_{i, j}, D_{i, j}, O_{i, j}\right]\right)$.

We also include the Similarity Network Fusion (SNF) technique presented in Section 3.1.3 as a fourth fusion method, which corresponds to a more sophisticated approach to fusion in comparison to the basic statistics above.

In order to decide which models are included in the source separation fusion process, we explore the use of filtered lists of similarity models, as discussed in the context of the CSC problem. Basically, the Top-N list of the best similarity models (ranked according to their mean rank in the CSI problem) is filtered, considering the equivalence classes of similar techniques (feature, aggregator or distance), as illustrated in Figures 5.4, 5.5, and 5.6. This produces $M<N$ similarity models, which in turn are applied to the separate "bass", "drums", "vocals" and "other" modified datasets, producing 4 sets of $M$ similarity matrices, one for each source. Finally, each possible 4-tuple of similarity matrices (i.e., "bass", "drums", "vocals" and "other") is fused, producing $M^{4}$ fused similarity matrices.

Because it considers all such combinations of models and sources, the above method provides yet another way of combining multiple musical perspectives, by fusing for instance a melodic similarity model applied to vocals and bass, a rhythmic similarity model to the drums and a timbre-related similarity model to other sources, among many other possibilities. 


\section{Chapter 6}

\section{Experiments and Results}

\subsection{Technical choices}

The programming language chosen for the implementation is Python, a generic language, with a large and active community, widely used in areas such as data analysis, machine learning, artificial intelligence, etc. In addition, there are several modules for Python directly linked to the tasks involved in our project.

\section{- $\operatorname{LibROSA}^{1}$}

LibROSA is a module for audio analysis implemented and maintained by LabROSA ${ }^{2}$ from Columbia University. It has several feature extractors, including melspectrogram, chromagrams, tonnetz, etc.

- Essentia ${ }^{3}$

Essentia is a library implemented in $\mathrm{C}++$ with an encapsulation for Python, developed by the $\mathrm{MTG}^{4}$ at Pompeu Fabra University. It focus on musical analysis, and has a huge range of extractors: PitchMelodia, PitchContourSegmentation, Mel-Frequency Cepstral Coefficients (MFCC), etc.

- RP_extract ${ }^{5}$

This is the Python library that implements the Rhythm Pattern feature extractor along with its sibling features: Rhythm Histogram, Statistical Spectrum Descriptors, Modulation Variance Descriptors, etc.

- $\mathrm{NumPy}^{6}$

NumPy is a scientific computing module that has an N-dimensional array object (ndarray) widely used in our project to store and manipulate data (local features, global features, similarity matrices, etc). Any operation performed on tensors (such as ordering, searching, extracting basic statistics, adding, multiplying, among others) thus becomes trivial to implement with NumPy. Under the hood, these operations are also heavily optimized for speed.

- $\mathrm{SciPy}^{7}$

SciPy is a scientific computing library that contains most of the distance functions and statistical models used in our project.

\footnotetext{
${ }^{1}$ http://librosa.github.io/

${ }^{2}$ https://labrosa.ee.columbia.edu/

${ }^{3}$ http://essentia.upf.edu/

${ }^{4}$ https://www.upf.edu/web/mtg

${ }^{5}$ https://github.com/tuwien-musicir/rp_extract

${ }^{6}$ http://www.numpy.org/

${ }^{7}$ https://www.scipy.org/
} 
- matplotlib ${ }^{8}$

Matplotlib is a visual library for plotting graphics, and is used to produce virtually all figures presented in this thesis. It was also used to produce several graphics during the course of implementation, which served as guides for the process.

- scikit-learn $^{9}$

This is arguably the most widely used tool for data analysis and machine learning in Python. Built on top of NumPy, SciPy, and matplotlib, it is extremely easy to use, with fast initial learning curve. It implements all the classifiers used in our experiments.

- multiprocessing

This module was used to implement all parallel computations, which are essential to reduce the processing time of the experiments, due to the large amount of audio files used.

- gzip + joblib

Two other modules of great importance for reducing the processing times of the experiments are gzip and joblib, which are used for storing data on disk, such as features, aggregations, distances and similarity matrices, so as not to perform those costly operations multiple times.

\subsection{Datasets}

\section{Ballroom}

The Ballroom ${ }^{10}$ dataset is composed of excerpts from several dance styles typically used in ballroom dancing. The audio files are provided by Ballroom Dancers website ${ }^{11}$, and several annotations can also be found online. We use this dataset mainly for rhythmic similarity. All excerpts are classified according to the names of the dance styles. More details about the Ballroom dataset can be seen in Table 6.1.

number of classes 10

number of files 698

files per class distribution heterogeneous (see Figure 6.1)

file format wav

duration (mean) 31.28103

duration (std) 0.75731

duration $(\min ) \quad 29.35$

duration $(\max ) \quad 34.06$

Table 6.1: Extra information about Ballroom dataset.

\footnotetext{
${ }^{8}$ https://matplotlib.org/

${ }^{9}$ https://scikit-learn.org/stable/

${ }^{10} \mathrm{http}: / / \mathrm{mtg}$. upf.edu/ismir2004/contest/tempoContest/node5.html

${ }^{11}$ https://www.ballroomdancers.com/
} 


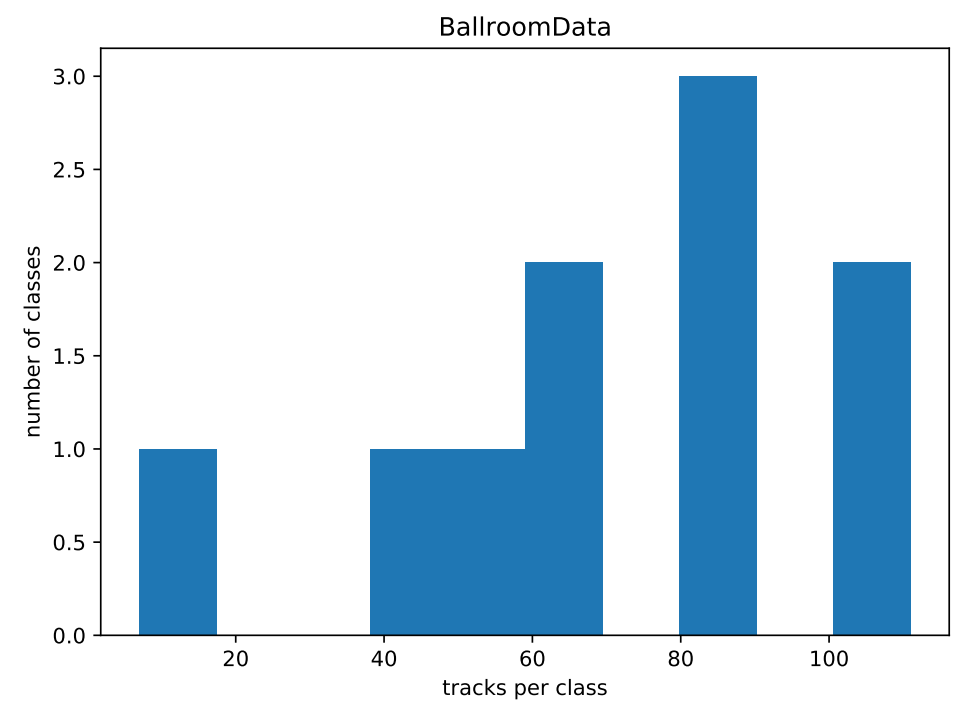

Figure 6.1: Histogram of files per class from Ballroom dataset.

\section{GTZAN}

Tzanetakis and Cook [TC02b] created a dataset called GTZAN ${ }^{12}$ for the task of music genre classification. The authors claim that an effort was made to ensure that the audio files were representative of the corresponding musical genres. Furthermore, the files were obtained from several different sources (records, radios, ambient recordings with microphones, etc.) in order to represent a variety of recording conditions.

GTZAN is annotated with the musical genres of each audio file, and we use it mainly for timbre similarity, based on papers that seek to establish a correlation between genre and timbre, on the assumption that different genres employ different instruments. It is important to note that this is a simplifying hypothesis for which countless counter-examples can be easily produced. Meta-data for this dataset can be found in Table 6.2.

$$
\begin{aligned}
\text { number of classes } & 10 \\
\text { number of files } & 1000
\end{aligned}
$$

files per class distribution homogeneous (100 files per class)

file format .au

$\begin{aligned} \text { duration (mean) } & 30.02187 \\ \text { duration (std) } & 0.08117 \\ \text { duration (min) } & 29.93 \\ \text { duration (max) } & 30.65\end{aligned}$

Table 6.2: Extra information about GTZAN dataset.

\section{IOACAS-QBH}

IOACAS ${ }^{13}$ is a dataset compiled by the Institute of Acoustics (IOA) of the Chinese Academy of Sciences (CAS) for the query by humming task. It is composed of 298 ground-truth melodies (MIDI files), and 759 query melodies (WAVE files of singers humming the melodies). The MIDI files are converted to WAVE files using the FluidSynth ${ }^{14}$ command line tool. This dataset is used for melodic similarity, and the classes correspond to the individual melodies (each class contains

\footnotetext{
${ }^{12}$ http://marsyas.info/downloads/datasets.html

${ }^{13} \mathrm{http}: / /$ mirlab.org/dataSet/public/IOACAS_QBH.rar

${ }^{14}$ https://www.fluidsynth.org/
} 
a synthesized ground-truth melody and all of corresponding queries). More information about this dataset can be seen in Table 6.3.

$\begin{aligned} \text { number of classes } & 298 \\ \text { number of files } & 1057\end{aligned}$

files per class distribution heterogeneous (see Figure 6.2)

file format .wav

duration (mean) 65.58255

duration (std) 94.87213

duration $(\min ) \quad 7.22$

duration $(\max ) \quad 721.25$

Table 6.3: Extra information about IOACAS-QBH dataset.

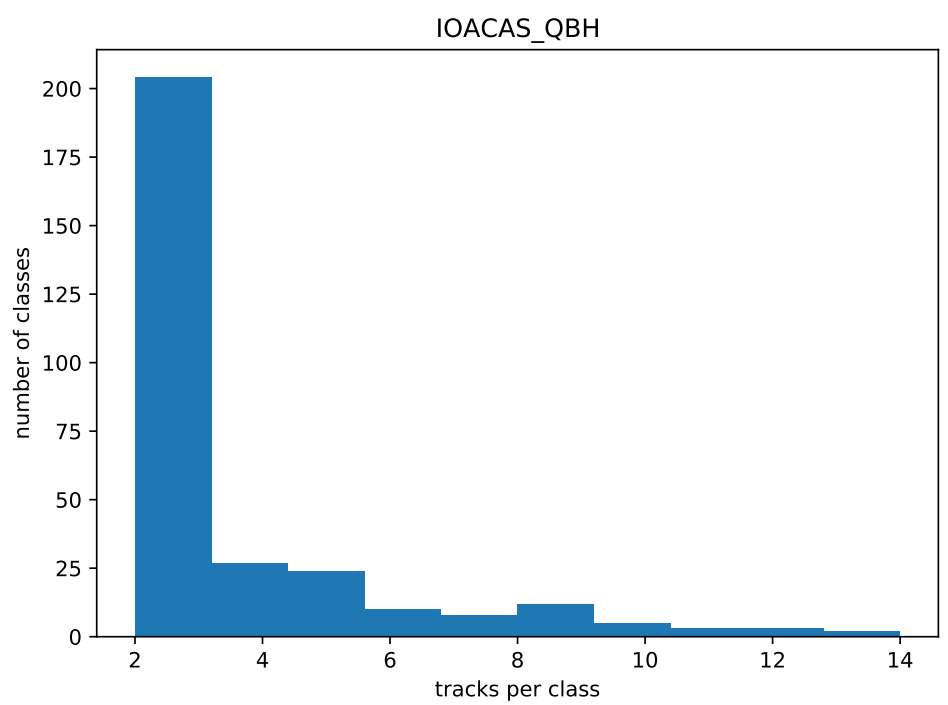

Figure 6.2: Histogram of files per class from IOACAS-QBH dataset.

\section{Maria Panteli's melody dataset}

Panteli and Dixon $\left[\mathrm{PD}^{+} 16\right]$ perform an evaluation of audio features in the context of melodic and rhythmic similarities. They create two datasets (one for each task) composed of synthesized audio, with controlled transformations applied to timbre, recording quality, global tempo, key transposition, and local tempo. These transformations are meant to enforce invariance properties upon the features used.

The melody dataset ${ }^{15}$ is composed of 30 main melodies divided in six musical styles, as follows:

- monophonic melodies:

- 5 Dutch Folk

- 5 Classical

- 5 Byzantine

-5 Pop

- polyphonic melodies:

- 5 Classical

\footnotetext{
${ }^{15}$ https://archive.org/details/panteli_maria_melody_dataset
} 


$$
-5 \text { Pop }
$$

Each main melody selected by the authors is transformed in several ways, and each class is composed of the original melody and its transformations. More details about this dataset can be found in Table 6.4 .

$$
\begin{aligned}
\text { number of classes } & 30 \\
\text { number of files } & 3000
\end{aligned}
$$

files per class distribution homogeneous (100 files per class)

file format .flac

$$
\begin{aligned}
\text { duration }(\text { mean }) & 25.84829 \\
\text { duration }(\mathrm{std}) & 6.51136 \\
\text { duration }(\min ) & 9.36 \\
\text { duration }(\max ) & 37.45
\end{aligned}
$$

Table 6.4: Extra information about Panteli's melody dataset.

\section{Maria Panteli's rhythm dataset}

The rhythm dataset ${ }^{16}$ created by Panteli and Dixon $\left[\mathrm{PD}^{+} 16\right]$ is composed of 30 main rhythms divided in six musical styles, as follows:

- monophonic rhythms:

- 5 Afro-American

-5 North-Indian

-5 African

-5 Classical

- polyphonic rhythms:

- 5 Electronic Dance Music (EDM)

- 5 Latin-Brazilian

Similarly to the previous dataset, each original rhythm are subject to various transformations, and each class is formed by the original rhythm and its transformations. Extra information about this dataset is located in Table 6.5.

$$
\begin{aligned}
\text { number of classes } & 30 \\
\text { number of files } & 3000
\end{aligned}
$$

files per class distribution homogeneous (100 files per class)

file format .flac

$\begin{aligned} \text { duration }(\text { mean }) & 8.0103 \\ \text { duration }(\mathrm{std}) & 0.51466 \\ \text { duration }(\min ) & 6.56 \\ \text { duration }(\max ) & 10.0\end{aligned}$

Table 6.5: Extra information about Panteli's rhythm dataset.

\footnotetext{
${ }^{16}$ https://archive.org/details/panteli_maria_rhythm_dataset
} 


\section{MAST}

The MAST ${ }^{17}$ dataset is composed of 3721 audio files recorded at several examinations of reproductions of rhythmic patterns performed at a conservatory in Turkey in the years 2005/2006. Besides the identification of the rhythm pattern, each audio file is annotated with one of the 3 labels "reference", "pass", or "fail". The first is the original recording, which was played for the musicians as reference; the second is a recording of an examination in which the musician passed; the third one in which the musician failed.

Since the failed recordings have no use to us, they are dropped. The resulting dataset is classified according to the identification of the rhythm patterns (each class contains the reference recording along with the corresponding recordings of the musicians that passed the exam). Refer to Table 6.6 for further information.

$$
\begin{aligned}
\text { number of classes } & 40 \\
\text { number of files } & 3104
\end{aligned}
$$

files per class distribution heterogeneous (see Figure 6.3)

$\begin{aligned} \text { file format } & . \mathrm{m} 4 \mathrm{a} \\ \text { duration (mean) } & 4.64725 \\ \text { duration (std) } & 0.58415 \\ \text { duration (min) } & 2.41 \\ \text { duration (max) } & 7.2\end{aligned}$

Table 6.6: Extra information about MAST dataset.

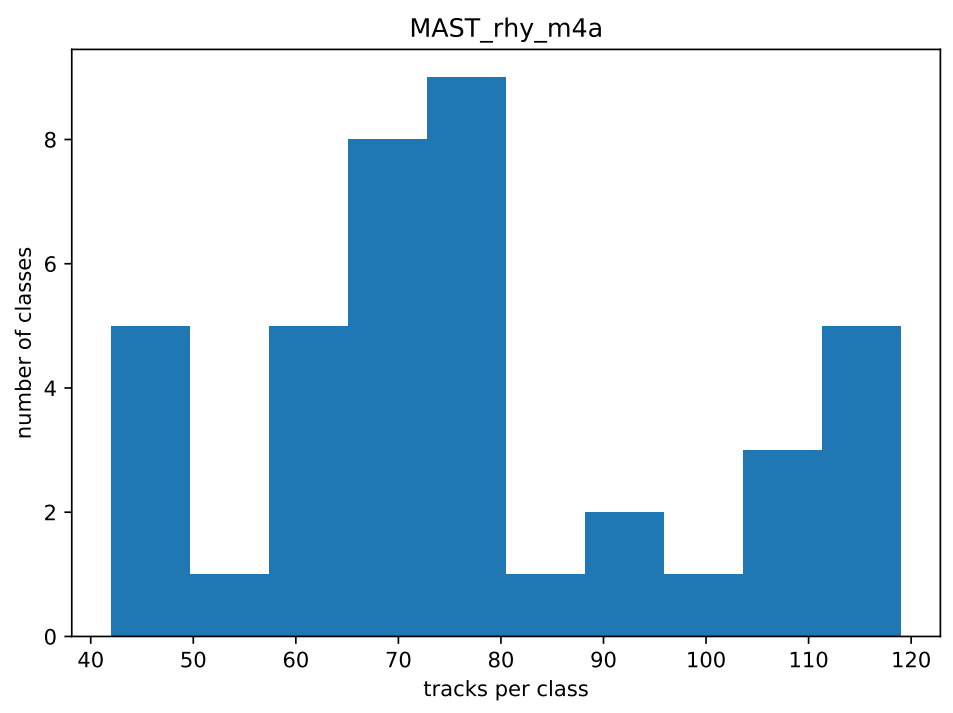

Figure 6.3: Histogram of files per class from MAST dataset.

\section{7-Artists}

The 1517-Artists ${ }^{18}$ dataset is presented in the $\mathrm{PhD}$ thesis by Klaus Seyerlehner [Sey10b]. It is a dataset for musical similarity with 3180 tracks performed by 1517 artists.

In his thesis, Seyerlehner states that a musical recommendation system based on audio similarity can suffer from an artist (or album) effect, in which only the songs by the same artist (or album) of the query song are recommended. In order to avoid this, the 1517-Artists dataset is constructed with just a few tracks by each artist, and is supposed to be used in such a way that no track from the same artist appears in both training and testing sets simultaneously.

\footnotetext{
${ }^{17}$ https://zenodo.org/record/2620357

${ }^{18}$ http://www.seyerlehner.info/index.php? $\mathrm{p}=1$ _ 3 _Download
} 
Even though we do not use this dataset for classification tasks, the 1517-Artists is annotated with musical genres, and so can also be used for timbre similarity. More information can be seen in Table 6.7.

$\begin{aligned} \text { number of classes } & 19 \\ \text { number of files } & 3180\end{aligned}$

files per class distribution heterogeneous (see Figure 6.4)

file format .mp3

duration (mean) 233.08766

duration (std) 85.27873

duration (min) 87.75

duration $(\max ) \quad 698.7$

Table 6.7: Extra information about 1517-Artists dataset.

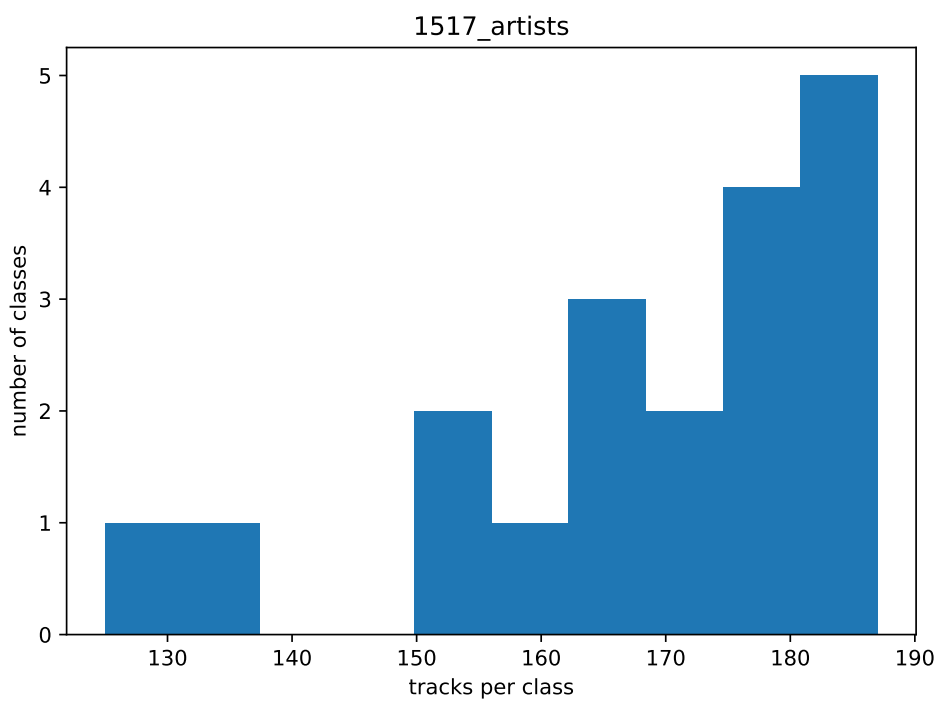

Figure 6.4: Histogram of files per class from 1517-Artists dataset.

\section{MIR-QBSH}

The dataset MIR-QBSH ${ }^{19}$ was created by the Multimedia Information Retrieval Lab from the National University of Tsing Hua (Taiwan) for the task of query by singing/humming. It consists of 48 MIDI files with the reference melodies and 4431 audio files of these melodies hummed by 195 people.

The dataset went through a process where each MIDI file was synthesized using the FluidSynth ${ }^{20}$ command line tool. Each reference melody forms a class of files along with their respective hummed versions. More detailed information about this dataset can be seen in Table 6.8.

\footnotetext{
${ }^{19}$ http://mirlab.org/dataSet/public/MIR-QBSH-corpus.rar

${ }^{20}$ http://www.fluidsynth.org/
} 
number of classes 48

number of files 4479

files per class distribution heterogeneous (see Figure 6.5)

file format wav

duration (mean) 8.34834

duration (std) 6.46538

duration $(\mathrm{min}) \quad 8.0$

duration $(\max ) \quad 278.07$

Table 6.8: Extra information about MIR-QBSH dataset.

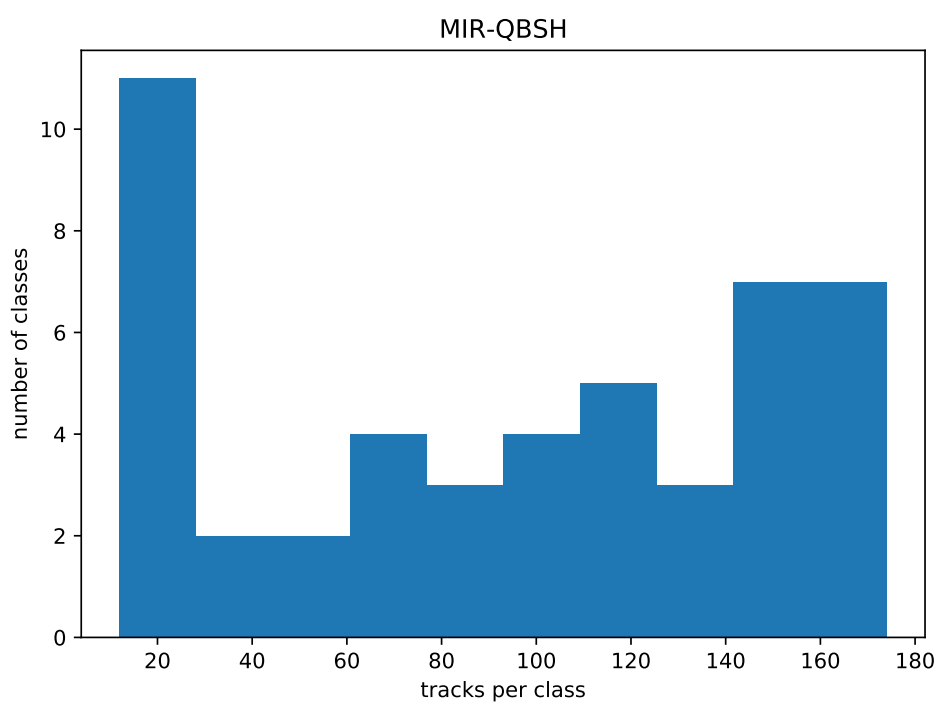

Figure 6.5: Histogram of files per class from MIR-QBSH dataset.

\section{FMA-Small}

The Free Music Archive dataset [DBVB17] was published in 2017 with the purpose of evaluating various distinct tasks in Music Information Retrieval (MIR). Its full version has $917 \mathrm{~Gb}$ comprising 106,574 tracks, all under a Creative-Commons ${ }^{21}$ license.

The version we are using is called FMA-Small ${ }^{22}$ and is a subset with 8000 files equally divided in 8 musical genres. Detailed information for this dataset is shown in Table 6.9.

$$
\begin{aligned}
\text { number of classes } & 8 \\
\text { number of files } & 8000
\end{aligned}
$$

files per class distribution homogeneous (1000 files per class)

file format .mp3

duration (mean) 29.95643

duration (std) 0.51339

duration $(\min ) \quad 6.0$

duration $(\max ) \quad 33.24$

Table 6.9: Extra information about FMA-Small dataset.

\footnotetext{
${ }^{21}$ https://creativecommons.org/

${ }^{22}$ https://github.com/mdeff/fma/
} 


\section{Covers80}

The Covers 80 dataset $^{23}$ is the first of 5 datasets we use for experiments with the Cover Song Identification (CSI) problem, which is a music similarity application selected to further explore the proposed similarity models. It was published in 2007 and is formed by 80 pairs of song/cover, from artists such as Aerosmith, Beatles, Cream, Deep Purple, etc. More details about this dataset can be found in Table 6.10 .

$$
\begin{aligned}
\text { number of classes } & 80 \\
\text { number of files } & 160
\end{aligned}
$$

files per class distribution homogeneous (2 files per class)

file format .mp3

$\begin{aligned} \text { duration (mean) } & 250.30606 \\ \text { duration (std) } & 79.4877 \\ \text { duration (min) } & 117.83 \\ \text { duration (max) } & 595.37\end{aligned}$

Table 6.10: Extra information about Covers80 dataset.

\section{YouTubeCovers}

The YouTubeCovers ${ }^{24}$ dataset was released in 2015 and is formed by 350 tracks divided in 50 groups of 7 versions of each original track. Artists appearing in this dataset include Amy Winehouse, Bee Gees, Cake, Daft Punk, among others. Meta-information about the YouTubeCovers dataset can be found in Table 6.11.

$$
\begin{aligned}
\text { number of classes } & 50 \\
\text { number of files } & 350
\end{aligned}
$$

files per class distribution homogeneous ( 7 files per class)

file format .mp3

$$
\begin{aligned}
\text { duration (mean) } & 263.11166 \\
\text { duration }(\mathrm{std}) & 100.26204 \\
\text { duration }(\min ) & 91.9 \\
\text { duration }(\max ) & 826.93
\end{aligned}
$$

Table 6.11: Extra information about YouTubeCovers dataset.

\section{Covers1000}

The Covers 1000 dataset $^{25}$ was created in 2017 by Christopher Tralie, and is constituted by 395 classes with 2, 3 or 4 cover songs, adding up to 1000 tracks. The author states that the songs were randomly selected from the Second Hand Songs ${ }^{26}$ website, and later manually curated and checked. From the available meta-data we recognize the presence of similar artists from the two previous datasets. More details can be found in Table 6.6.

\footnotetext{
${ }^{23}$ https://labrosa.ee.columbia.edu/projects/coversongs/covers $80 /$

${ }^{24}$ https://sites.google.com/site/ismir2015shapelets/data

${ }^{25}$ http://www.covers1000.net/

${ }^{26}$ https://secondhandsongs.com/
} 
number of classes 395

number of files 1000

files per class distribution heterogeneous (see Figure 6.6)

file format $659 . \mathrm{ebm}(65.9 \%) / 322 . \mathrm{m} 4 \mathrm{a}(32.2 \%) / 19 . \mathrm{mp} 3(1.9 \%)$

duration (mean) 222.32492

duration (std) 87.54064

duration (min) 71.96

duration $(\max ) \quad 927.92$

Table 6.12: Extra information about Covers1000 dataset.

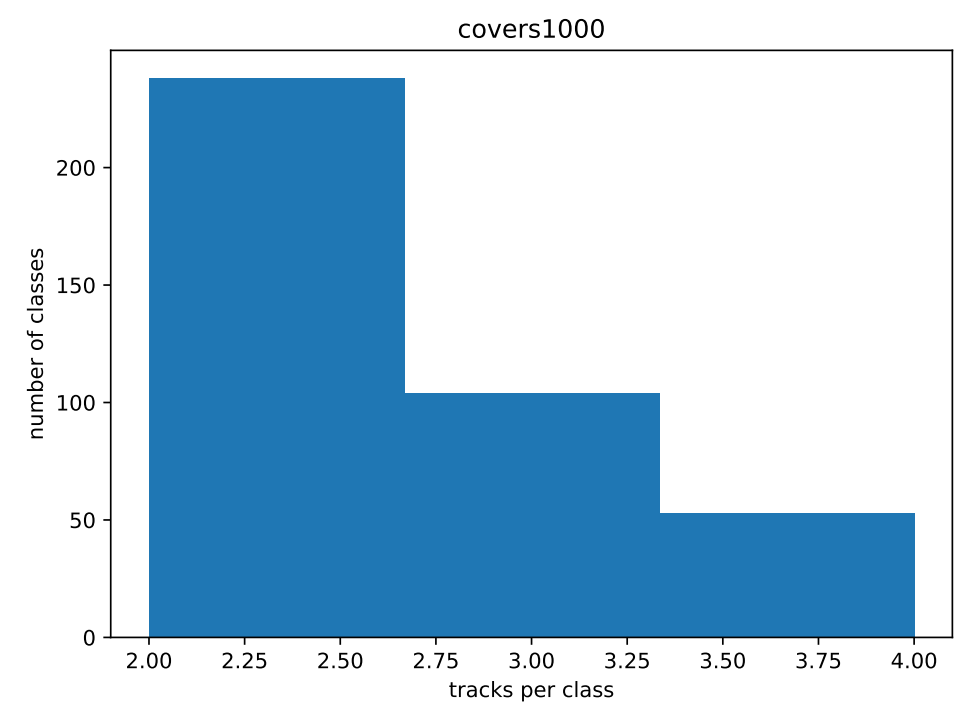

Figure 6.6: Histogram of files per class from Covers1000 dataset.

\section{Mazurkas}

The Mazurkas dataset ${ }^{27}$ is unique among those selected for the CSI experiments. It is composed of several performances of 49 selected Chopin Mazurkas. This dataset is widely known for its continued use in the MIREX Audio Cover Song Identification task ${ }^{28}$. Further information is shown in Table 6.13.

$$
\begin{aligned}
\text { number of classes } & 49 \\
\text { number of files } & 2741
\end{aligned}
$$

files per class distribution heterogeneous (see Figure 6.7)

$$
\begin{aligned}
\text { file format } & . \mathrm{mp} 4 \\
\text { duration (mean) } & 163.75769 \\
\text { duration (std) } & 74.36453 \\
\text { duration (min) } & 27.25 \\
\text { duration (max) } & 487.31
\end{aligned}
$$

Table 6.13: Extra information about Mazurkas dataset.

\footnotetext{
${ }^{27}$ http://www.mazurka.org.uk/

${ }^{28}$ https://www.music-ir.org/mirex/wiki/2020:Audio_Cover_Song_Identification
} 


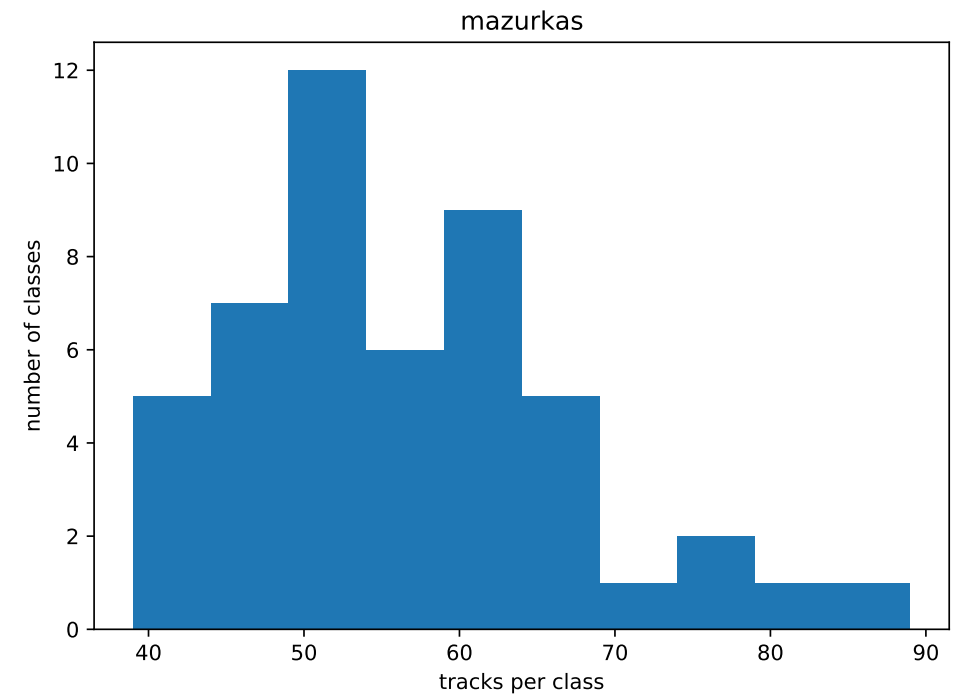

Figure 6.7: Histogram of files per class from Mazurkas dataset.

\section{SHS9K}

The dataset we call SHS9K ${ }^{29}$ is also a selection from the Second Hand Songs massive database. More specifically, it is a sub-set of the SHS100K dataset ${ }^{30}$ crafted by us by selecting the original songs that have from 50 to 100 covers. This resulted in a new dataset with slightly more than 9000 tracks, hence its name. Detailed information can be found in Table 6.14.

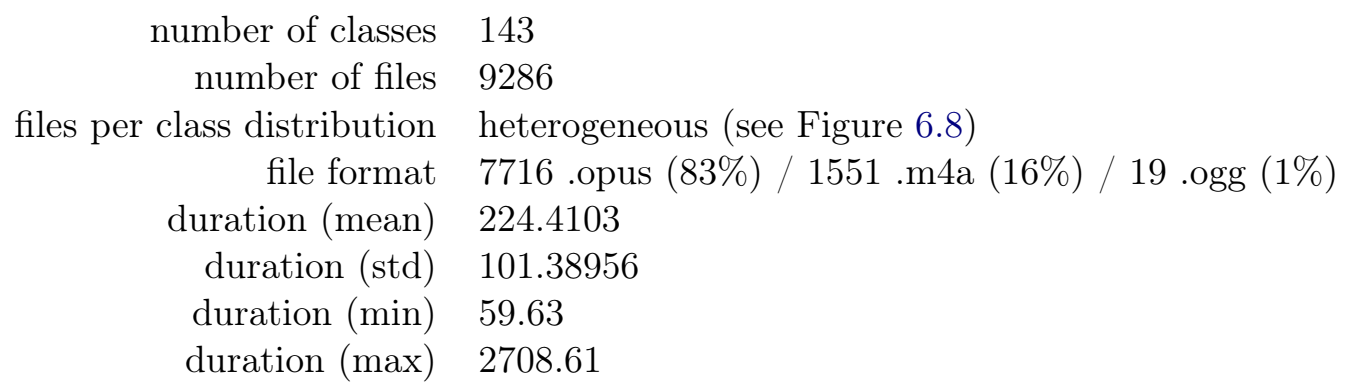

Table 6.14: Extra information about SHS9K dataset.

\footnotetext{
${ }^{29}$ https://rppbodo.github.io/phd/shs9k.html

${ }^{30}$ https://github.com/NovaFrost/SHS100K
} 


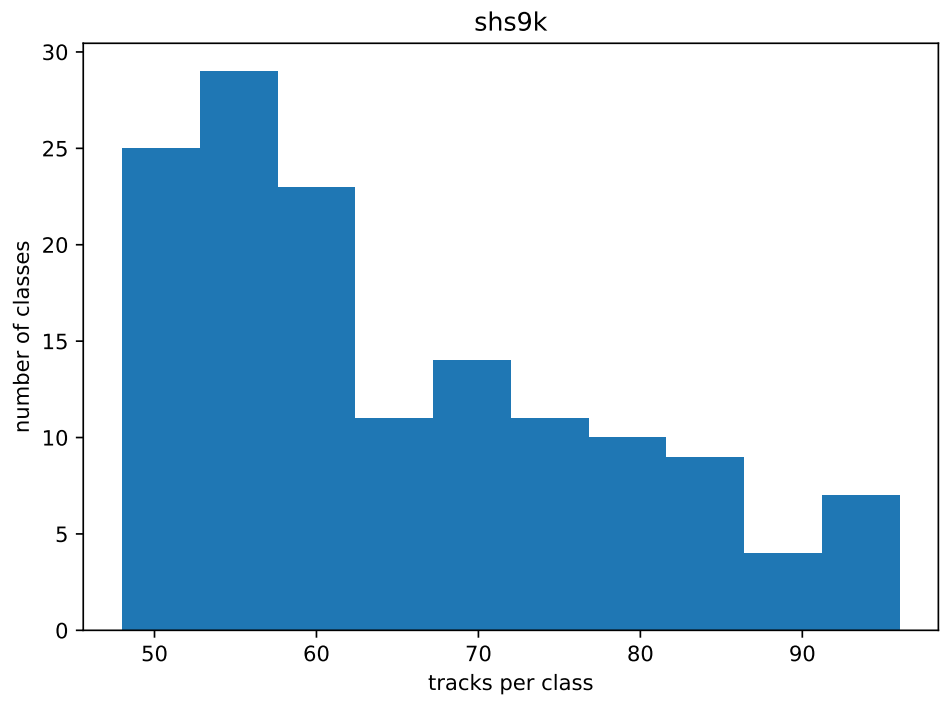

Figure 6.8: Histogram of files per class from SHS9K dataset.

\subsection{Data rearrangement and cleaning}

Every dataset we use in our experiments presents issues either with the directories/files hierarchy tree, or with the audio recordings content. Regarding the hierarchy tree, all datasets have been standardized in order to be manipulated by the implemented framework. The final tree structure can be seen in Figure 6.9.

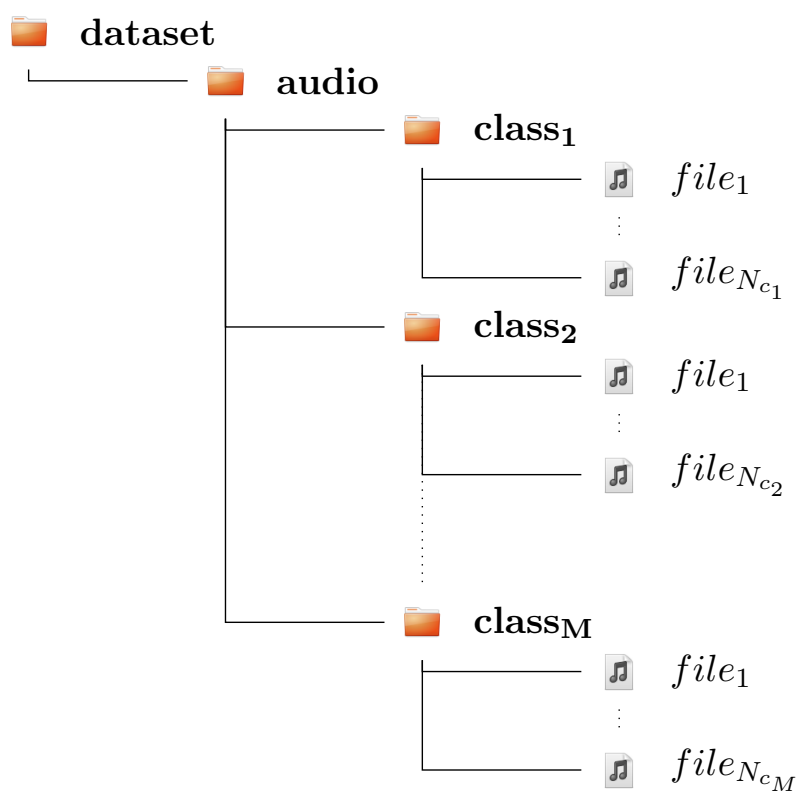

Figure 6.9: Final tree structure after the rearrangement process.

Regarding data cleaning, we had to deal with two main issues. The most common one is the presence of silence in the beginning or end of the file (or both) that takes up a significant fraction of the file duration, as we can see in the example in Figure 6.10. The solution adopted here is to perform a trim on both ends of all tracks. 


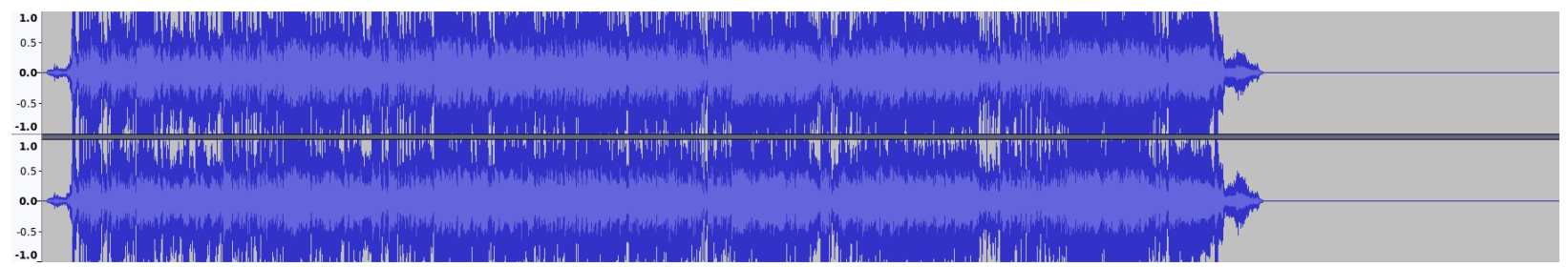

Figure 6.10: Example recording with presence of silence in the end of the file.

The second issue relates to DC components, which although less frequent, can be more dangerous than the previous issue. Basically, it consists of a vertical shift of the time domain audio signal, sometimes making the amplitude values all above (or below) zero, as shown in Figure 6.11. The solution for this problem is to filter out the DC component by subtracting the mean from all samples.

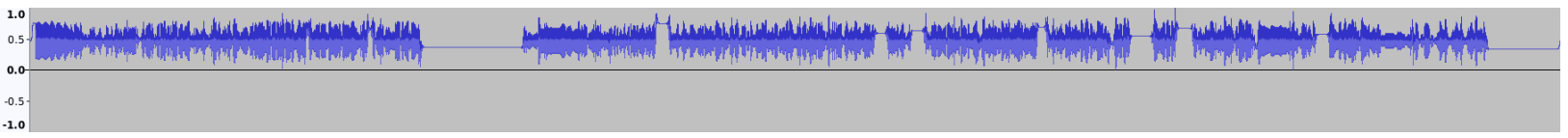

Figure 6.11: Example recording with vertical shift of the time domain audio signal.

\subsection{Experiment \#1: Music Similarity}

\subsubsection{Experiment design}

The goal of our first experiment is to check which music similarity models lead to best results for the annotated datasets we selected. In order to accomplish this we run each one of the 9 datasets considered through our music similarity framework, compute the Intra-Inter Class Similarity Ratio (IICSR) for every class, and finally compute the weighted mean IICSR for each one of the 690 music similarity models considered.

\subsubsection{Results}

The results are provided for the reader as follows:

- the top-10 weighted mean IICSR values for each dataset are presented in this section (Tables 6.15-6.23);

- the best IICSR values for each class and dataset are listed in Appendix A;

- the entire list of IICSR values computed in this experiment is published in https://rppbodo. github.io/phd/experiment_1.html

\section{Ballroom}

\begin{tabular}{llll} 
weighted mean iicsr & extractor & aggregator & distance \\
\hline 1.3540499634178205 & spectral_bandwidth & vector_quantization & cosine_distance \\
1.3534165441182575 & spectral_bandwidth & vector_quantization_default & cosine_distance \\
1.34745124592136 & spectral_rolloff & vector_quantization_ & cosine_distance \\
1.3218322081020337 & spectral_bandwidth & vector_quantization_default & manhattan_distance \\
1.3181242579060137 & spectral_flatness & vector_quantization_default & manhattan_distance \\
1.3176688901939193 & spectral_rolloff & vector_quantization_default & cosine_distance \\
1.3162289921171548 & spectral_bandwidth & vector_quantization & manhattan_distance \\
1.3123177903304417 & spectral_rolloff & vector_quantization & manhattan_distance \\
1.30713484050078 & spectral_flatness & vector_quantization & manhattan_distance \\
1.3040807412653603 & spectral_flatness & vector_quantization & chebyshev_distance
\end{tabular}

Table 6.15: The top-10 results for Ballroom dataset. 
GTZAN

\begin{tabular}{llll} 
weighted mean iicsr & extractor & aggregator & distance \\
\hline 1.7494572740656718 & spectral_contrast & vector_quantization & cosine_distance \\
1.7131831605622414 & spectral_contrast & vector_quantization_default & cosine_distance \\
1.6788328390171978 & spectral_contrast & vector_quantization_ & manhattan_distance \\
1.6759432689949039 & spectral_contrast & vector_quantization_default & manhattan_distance \\
1.418845639658447 & spectral_bandwidth & vector_quantization_default & cosine_distance \\
1.4103487415673173 & spectral_bandwidth & vector_quantization_ & cosine_distance \\
1.3836662833613098 & spectral_bandwidth & vector_quantization & manhattan_distance \\
1.3812174678017382 & spectral_bandwidth & vector_quantization_default & manhattan_distance \\
1.3581029443038866 & spectral_flatness & vector_quantization_ & manhattan_distance \\
1.351530977309869 & spectral_rolloff & vector_quantization_default & cosine_distance
\end{tabular}

Table 6.16: The top-10 results for GTZAN dataset.

IOACAS-QBH

\begin{tabular}{l} 
weighted mean iicsr \\
\hline 1.092476072741732 \\
1.087884691051669 \\
1.073704506284947 \\
1.061096398839759 \\
1.0351712811724265 \\
1.023542856425961 \\
1.0221451612555899 \\
1.021471201783239 \\
1.020460038429503 \\
1.018800012510516
\end{tabular}

extractor
pitch_contour_segmentation
pitch_contour_segmentation
pitch_contour_segmentation
pitch_contour_segmentation
tonnetz
chroma_stft
chroma_stft
chroma_stft
beat_synchronous_chroma_stft
beat_synchronous_chroma_stft

aggregator octave_abstraction interval_abstraction pitch_contour_5_levels pitch_contour_3_levels statistical_summarization vector_quantization_default vector_quantization_default vector_quantization_default vector quantization default statistical summarization

distance
lcs_circular_min
lcs_min
lcs_min
lcs_min
cosine_distance
manhattan_distance
euclidean_distance
cosine_distance
manhattan_distance
chebyshev_distance

Table 6.17: The top-10 results for IOACAS-QBH dataset.

\section{Maria Panteli's melody dataset}

\begin{tabular}{llll} 
weighted mean iicsr & extractor & aggregator & distance \\
\hline 3.121672460498691 & pitch_contour_segmentation & interval_abstraction & levenshtein_max \\
2.8784933361226916 & pitch_contour_segmentation & interval_abstraction & lcs_max \\
2.7668967351583764 & melspectrogram & statistical_summarization & cosine_distance \\
2.7037201080229716 & melspectrogram & gaussian_mixture_model_default & cosine_distance \\
2.506759190743482 & pitch_contour_segmentation & octave_abstraction & levenshtein_circular_max \\
2.4480779120261142 & spectral_contrast & vector_quantization & cosine_distance \\
2.4177974266180406 & pitch_contour_segmentation & interval_abstraction & lcs_mean \\
2.2584899152688735 & mfcc & vector_quantization & manhattan_distance \\
2.2526835343526903 & spectral_contrast & vector_quantization & manhattan_distance \\
2.2435619283602906 & pitch_contour_segmentation & octave_abstraction & lcs_circular_max
\end{tabular}

Table 6.18: The top-10 results for Maria Panteli's melody dataset.

\section{Maria Panteli's rhythm dataset}

\begin{tabular}{llll} 
weighted mean iicsr & extractor & aggregator & distance \\
\hline 2.8293656707245263 & chroma_cens & vector_quantization & manhattan_distance \\
2.7623598781359173 & chroma_cens & vector_quantization & cosine_distance \\
2.48723963483041 & beat_synchronous_chroma_stft & vector_quantization & manhattan_distance \\
2.393660849090048 & beat_-synchronous_chroma_cens & vector_quantization & manhattan_distance \\
2.361307847543314 & beat_synchronous_chroma_-stft & vector_quantization & cosine_distance \\
2.345796969404894 & beat_synchronous_chroma_cens & vector_quantization & cosine_distance \\
2.187603115508135 & mfcc & vector_quantization & manhattan_distance \\
2.1371463415826595 & beat_synchronous_chroma_cqt & vector_quantization & manhattan_distance \\
2.1105097583986066 & beat_-synchronous_chroma_cqt & vector_quantization & cosine_distance \\
2.0369103515947824 & mfcc & vector_quantization & cosine_distance
\end{tabular}

Table 6.19: The top-10 results for Maria Panteli's rhythm dataset.

MAST

\begin{tabular}{llll} 
weighted mean iicsr & extractor & aggregator & distance \\
\hline 1.4396680845011323 & mfcc & vector_quantization & cosine_distance \\
1.3125029718125802 & mfcc & vector_quantization & manhattan_distance \\
1.2465251362753116 & melspectrogram & vector_quantization & cosine_distance \\
1.1924435178522057 & chroma_cens & vector_quantization & cosine_distance \\
1.1840461600719778 & melspectrogram & vector_quantization & manhattan_distance \\
1.1729248978438367 & beat_synchronous_chroma_cqt & vector_quantization & cosine_distance \\
1.166218396960286 & beat_synchronous_chroma_cens & vector_quantization & cosine_distance \\
1.1645781941317102 & beat_synchronous_chroma_stft & vector_quantization & cosine_distance \\
1.157322340846282 & beat_synchronous_chroma_cqt & vector_quantization & manhattan_distance \\
1.155902837959492 & chroma_cens & vector_quantization & manhattan_distance
\end{tabular}

Table 6.20: The top-10 results for MAST dataset. 


\section{7-Artists}

\begin{tabular}{|c|c|c|c|}
\hline weighted mean iicsr & extractor & aggregator & distance \\
\hline 1.2055620886915397 & mfcc & vector quantization & cosine distance \\
\hline 1.176134102621637 & $\mathrm{mfcc}$ & vector_quantization_default & cosine_distance \\
\hline 1.1568750811844912 & spectral_flatness & vector_quantization $^{-}$ & manhātan_distance \\
\hline 1.1553044988931647 & $\operatorname{mfcc}-$ & vector-quantization & manhattan_distance \\
\hline 1.1539750706463232 & spectral bandwidth & vector-quantization & cosine_distance \\
\hline 1.1509643592853198 & spectral-rolloff & vector-quantization & cosine $e^{-}$distance \\
\hline 1.1500943736026472 & temporal_statistical_spectrum_descriptor & bypass ${ }^{-1}$ & cosine $e^{-}$distance \\
\hline 1.1493571493864683 & spectral_bandwidth ${ }^{-}$ & vector_quantization_default & cosine_distance \\
\hline 1.1486351584539187 & spectral_rolloff & vector_quantization_default & cosine $e^{-}$distance \\
\hline 1.1464940564416854 & $\operatorname{mfcc}-$ & vector-quantization ${ }^{-}$default & manhātan_distance \\
\hline
\end{tabular}

Table 6.21: The top-10 results for 1517-Artists dataset.

\section{MIR-QBSH}

\begin{tabular}{llll} 
weighted mean iicsr & extractor & aggregator & distance \\
\hline 1.2949131442350692 & pitch_contour_segmentation & octave_abstraction & levenshtein_circular_max \\
1.2402936615590263 & pitch_contour_segmentation & interval_abstraction & levenshtein_max \\
1.233022209189783 & pitch_contour_segmentation & octave_abstraction & lcs_circular_min \\
1.2061107813026728 & pitch_contour_segmentation & octave_abstraction & lcs_circular_max \\
1.2047184610592656 & pitch_contour_segmentation & pitch_contour_5_levels & levenshtein_max \\
1.1796156638383086 & pitch_contour_segmentation & octave_abstraction & lcs_circular_mean \\
1.177118878994491 & pitch_contour_segmentation & interval_abstraction & lcs_max \\
1.1764569956217827 & pitch_contour_segmentation & pitch_contour_3_levels & levenshtein_max \\
1.1623291012208554 & pitch_contour_segmentation & pitch_contour_5_levels & lcs_max \\
1.1582764726064485 & pitch_contour_segmentation & markov_chain- & circular_cosine
\end{tabular}

Table 6.22: The top-10 results for MIR-QBSH dataset.

\section{FMA-Small}

\begin{tabular}{llll} 
weighted mean iicsr & extractor & aggregator & distance \\
\hline 1.2112879995990873 & $\mathrm{mfcc}$ & vector_quantization_default & cosine_distance \\
1.2074322324746771 & $\mathrm{mfcc}$ & vector_quantization & cosine_distance \\
1.1839280987042582 & $\mathrm{mfcc}$ & vector_quantization & manhattan_distance \\
1.1824114904970324 & mfcc & vector_quantization_default & manhattan_distance \\
1.1775203487886956 & melspectrogram & statistical_summarization & cosine_distance \\
1.1552211718086771 & spectral_bandwidth & vector_quantization_default & cosine_distance \\
1.1520489565884702 & spectral_rolloff & vector_quantization & cosine_distance \\
1.1484443004872782 & spectral_bandwidth & vector_quantization & cosine_distance \\
1.1480826442713625 & spectral_rolloff & vector_quantization_default & cosine_distance \\
1.147194473827082 & spectral_rolloff & vector_quantization & manhattan_distance
\end{tabular}

Table 6.23: The top-10 results for FMA-Small dataset.

\subsubsection{Discussion}

\section{Melodic, Rhythmic and Genre datasets}

Three of the datasets considered are aimed specifically to test melodic similarity models: MIQQBSH and IOACAS-QBH are designed for the query-by-humming task (classes are composed of a reference melody and a set of recordings of people trying to hum it), and Panteli's melody dataset uses synthesis to test similarity models against several melodic transformations. The models that achieved the best weighted mean IICSR are:

- $\{$ pitch_contour_segmentation, octave_abstraction, levenshtein_circular_max $\}$ for MIQ-QBSH;

- $\{$ pitch_contour_segmentation, octave_abstraction, lcs_circular_min\} for IOACAS-QBH;

- \{pitch_contour_segmentation, interval_abstraction, levenshtein_max\} for Panteli's melody dataset.

All of the models above have Pitch Contour Segmentation as their feature, which matches the hypothesis that melodic features lead to better results for melodic datasets. Regarding the aggregators we have two models using octave abstraction and one using interval abstraction. This is somehow expected, since the alternatives - 3-level and 5-level pitch contours - are relatively weaker due to their simplistic representations of the original pitch sequences. 
Three other datasets - Ballroom, MAST and Panteli's rhythm dataset - are designed for tasks related to rhythm similarity. The first one is composed of recordings from distinct dance styles; the second one has recordings of students successfully reproducing rhythmic patterns; the later is composed of different rhythms under several transformations. The best models for each dataset are:

- \{spectral_bandwidth, vector_quantization, cosine_distance\} for Ballroom;

- $\{m f c c$, vector_quantization, cosine_distance $\}$ for MAST;

- $\{$ chroma_cens, vector_quantization, manhattan_distance $\}$ for Panteli's rhythm dataset.

The models that performed best for these datasets are relatively surprising, not only because none of the features are specifically designed for rhythm similarity tasks, but also because they are very different from each other: Spectral Bandwidth is related to the spectrum spread, MFCC is usually associated with timbre, and Chroma Energy Normalized Statistics (CENS) is a tonal feature.

The three remaining datasets - GTZAN, 1517-Artists, FMA-Small - are annotated with the tracks' genres. As discussed before, several papers in the literature claim that there is a relationship between genre and timbre, and under this assumption, these datasets could be used to test timbre similarity models. The highest weighted mean IICSR are obtained with the following models:

- \{spectral_contrast, vector_quantization, cosine_distance\} for GTZAN;

- $\{m f c c$, vector_quantization, cosine_distance $\}$ for 1517-Artists;

- $\{m f c c$, vector_quantization_default, cosine_distance $\}$ for FMA-Small.

Two out of the three best models mentioned above have MFCC as their feature, and the other one has Spectral Contrast. MFCC is a feature usually related to timbre, so this matches the initial hypothesis. According to Jiang et al. $\left[\mathrm{JLZ}^{+} 02\right]$, Spectral Contrast is reported to have a better discriminating power for different music types than MFCC, so it is noteworthy that this feature has also emerged here.

\section{Results per class}

Next we analyse the IICSR for each individual class, according to the results presented in Appendix A.

Starting with the melodic datasets, MIR-QBSH has 26 classes (melodies) out of $48(\approx 54 \%)$ whose best model is \{pitch_contour_segmentation, octave_abstraction, levenshtein_circular_max\}, followed by \{pitch_contour_segmentation, interval_abstraction, levenshtein_max $\}$ for 9 classes $(\approx 18 \%)$. The model that appear more frequently (28 classes out of $298-\approx 9 \%)$ for IOACAS$\mathrm{QBH}$ is \{pitch_contour_segmentation, interval_abstraction, lcs_min\}, technically tied with $\{m f c c$, vector_quantization, cosine_distance\} which is best for 27 classes $(\approx 9 \%)$. For Panteli's melody dataset the first model is \{pitch_contour_segmentation, interval_abstraction, levenshtein_max\}, which is best for 10 out of 30 classes $(\approx 33 \%)$, and the second best is \{melspectrogram, statistical_summarization, cosine_distance\} (for 6 out of 30 classes, or 20\%).

The observations here are quite similar to the ones referring to the weighted mean IICSR, where we are mainly concerned to check if the types of features appearing in the best models matches the tasks for which the datasets were designed. In this case, the datasets designed for melodic tasks produce best results (for most classes) using models with Pitch Contour Segmentation as feature, but noteworthy exceptions are MFCC with IOACAS-QBH and melspectrogram with Panteli's dataset.

For the rhythmic datasets, Ballroom has the model \{spectral_rolloff, vector_quantization, cosine_distance\}, with 2 classes (dance types) out of 10 (20\%), tied with \{spectral_flatness, diff_stats_2, chebyshev_distance\} (also 2 classes or 20\%). MAST has 32 classes (rhythmic patterns) out of 40 
$(80 \%)$ whose best model is \{mfcc, vector_quantization, cosine_distance $\}$, followed by \{melspectrogram, vector_quantization, cosine_distance\} which is best for 5 classes (12.5\%). Panteli's rhythm dataset has 11 out of 30 classes $(\approx 36 \%)$ with best model being \{chroma_cens, vector_quantization, cosine_distance $\}$, and the second best model \{chroma_cens, vector_quantization, manhattan_distance\}, for 7 classes of $30(\approx 23 \%)$.

The rhythmic datasets best models per class followed nearly the same pattern as when analysing the weighted mean IICSR values: the Ballroom dataset presents spectral features as the most frequent, $80 \%$ of the classes from MAST dataset have the best model using MFCC as feature, and Panteli's rhythm dataset has the CENS feature as the most frequent, considering the number of classes for which it appears in the best models.

The genre (or timbre) datasets have the following best models, when analysing the individual classes. GTZAN has in first place \{spectral_contrast, vector_quantization, cosine_distance\} for 4 classes out of $10(40 \%)$, and in second place $\{$ melspectrogram, gaussian_mixture_model_default, cosine_distance\} for 2 classes out of 10 (20\%). 1517-Artists has the best model \{melspectrogram, gaussian_mixture_model_default, cosine_distance\} for 6 out of 19 classes $(\approx 31 \%)$, followed by \{temporal_statistical_spectrum_descriptor, bypass, cosine_distance $\}$ for 4 out of 19 classes $(\approx 21 \%)$. The FMA-Small dataset has as best models \{mfcc, vector_quantization_default, cosine_distance\} for 2 classes out of $8(25 \%)$, tied with \{melspectrogram, gaussian_mixture_model_default, cosine_distance $\}$ also for 2 classes out of $8(25 \%)$.

Some remarkable results appear with the genre datasets in the above analysis. Besides the already expected models, which have timbre (or other spectral) features as their first element, some distinct models appeared here for the first time. For instance, Temporal Statistical Spectrum Descriptor shows up as the feature for the second most frequent model for 1517-Artists dataset. This is roughly a rhythmic feature, and this finding suggests an alternative approach to distinguish genres by music perspectives other than timbre.

We also would like to register that the model that leads to the best weighted mean IICSR for a dataset is not necessarily the most frequent model when counting the best models for individual classes. For instance, the best model in average for 1517-Artists has MFCC as its feature, while the most frequent models have melspectrogram and Temporal Statistical Spectrum Descriptor as their features, for $\approx 31 \%$ and $\approx 21 \%$ of the classes respectively.

\section{Comparative analysis}

Next we present a comparative analysis of the results, considering IICSR values obtained from very similar models (considering variations of specific details).

Several models include features which may or may not be synchronized to the beats. According to Table 6.24 , the synchronized models achieved a slightly higher average performance, i.e., the highest mean value of IICSR over all 9 datasets.

\begin{tabular}{lll} 
beat-synchronous? & weighted mean iicsr (mean) & weighted mean iicsr (std) \\
\hline yes & 1.0613942664419753 & 0.13502158454978952 \\
no & 1.054403957112558 & 0.11633719623232362
\end{tabular}

Table 6.24: Average results for models that do or do not use beat-synchronous features.

Table 6.25 compares models that apply (or not) the Optimal Transposition Index (OTI). Results are marginally better when OTI is applied.

\begin{tabular}{lll} 
OTI applied? & weighted mean iicsr (mean) & weighted mean iicsr (std) \\
\hline yes & 1.0453967661190973 & 0.06992686333055692 \\
no & 1.0446341713815084 & 0.06949308225223967
\end{tabular}

Table 6.25: Average results for models that do or do not use OTI. 
As seen in Section 2.3, LCS-based distances are computed by dividing the length of the Longest Common Subsequence (LCS) by three alternatives values: the minimum, mean and maximum of the lengths of the two sequences being compared. Table 6.26 shows that the best average result is obtained by dividing the LCS-length to the maximum, although the spread of the IICSR values is larger; the smaller spread occurs using the minimum as denominator, but this also produces the smaller weighted mean IICSR.

\begin{tabular}{lll} 
function used & weighted mean iicsr (mean) & weighted mean iicsr (std) \\
\hline min & 1.062289167637259 & 0.17594215851354272 \\
mean & 1.1209214132881897 & 0.28139660828286006 \\
$\max$ & 1.1724594908899157 & 0.3824835767516491
\end{tabular}

Table 6.26: Average results for models using minimum, mean or maximum to compute LCS-based distances.

The same comparison can be made for the models that use the Levenshtein distance to compare two symbolic melodic sequences. As Table 6.27 shows, the highest average result across all datasets is obtained using the maximum of the sequences lengths, but again the spread is much worse in this case.

\begin{tabular}{lll} 
function used & weighted mean iicsr (mean) & weighted mean iicsr (std) \\
\hline min & 1.0022681075684954 & 0.006668255790810766 \\
mean & 1.071226607689819 & 0.15633168399303013 \\
max & 1.1824659151025125 & 0.43507425892616736
\end{tabular}

Table 6.27: Average results for models that use minimum, mean or maximum to compute Levenshtein distances.

When aggregating local features into global ones using single Gaussian models we used a few alternative versions. As seen in Section 2.2, the method called basic_stats_ 1 is composed of the mean and standard deviation values of each dimension of the local feature; basic_stats_2 is basic_stats_1 with added minimum and maximum values for each dimension; diff_stats_ 1 is basic_stats_ 1 concatenated with mean and standard deviation values of the difference of the features in each dimension; and diff_stats_2 is obtained from diff_stats_ 1 by adding the minimum and maximum values from both the feature sequence and its difference.

\begin{tabular}{lll} 
aggregator & weighted mean iicsr (mean) & weighted mean iicsr (std) \\
\hline basic_stats_1 & 1.0442789316659908 & 0.07682125963980427 \\
basic_stats_2 & 1.0430074642691964 & 0.08077065712859963 \\
diff_stats_1 & 1.0467260309527056 & 0.07955852655272544 \\
diff_stats_2 & 1.0428426784620992 & 0.08511169059676862
\end{tabular}

Table 6.28: Average results for models that use the four variations of single Gaussian as aggregation model.

From Table 6.28 we verify that basic_stats_2 has worst mean result than basic_stats_ 1 and diff_stats_2 has worse mean result than diff_stats_1, so we can conclude that, for the models and datasets experimented, adding minimum and maximum values does not help. Comparing $b a-$ sic_stats_1 with diff_stats_ 1 we verify that the use of extra information regarding the difference of the features leads to slightly improved results.

All model using vector quantization as aggregation method was used in the experiment with the default number of clusters, and also with one cluster per class (as discussed in Section 2.2). Table 6.29 shows that using the default values leads to worst results, so the proposed idea of matching the number of clusters with the number of classes appears to be worth it (although the spread becomes larger). 


\begin{tabular}{lll} 
number of clusters & weighted mean iicsr (mean) & weighted mean iicsr (std) \\
\hline number of classes & 1.1562207356318348 & 0.27738676416067043 \\
function default (8) & 1.1198686700737968 & 0.17850814034079127
\end{tabular}

Table 6.29: Average results for vector quantization models using default or tailored number of clusters.

The last comparative analysis is related to features implemented by the $R P_{-}$extract module. Some of the features are provided with statistical summarization over time, besides the regular version. We can verify in Table 6.30 that the regular versions yield marginally better results for the datasets considered in the experiment.

\begin{tabular}{lll} 
temporal features? & weighted mean iicsr (mean) & weighted mean iicsr (std) \\
\hline yes & 1.0597115107027533 & 0.1332907243029386 \\
no & 1.0612171968855186 & 0.08395955789035918
\end{tabular}

Table 6.30: Average results for models with or without temporal summarization.

\subsection{Experiment \#2: Cover Song Identification}

\subsubsection{Experiment design}

This experiment has a similar goal to the previous one, namely to check which music similarity models lead to best results, but now with CSI datasets. We compute similarity matrices using all 690 models for all 5 CSI datasets, and then calculate metrics which are specific to this problem: Mean Rank (MR), Mean Reciprocal Rank (MRR), Median Rank (MDR), Mean Average Precision (MAP), etc.

\subsubsection{Results}

The results are displayed in the following manner:

- the top-10 models for each dataset are presented in Tables 6.31 to 6.35 , which are broken in two parts because they do not fit horizontally on the pages (please use line id as guidance);

- the top-50 models for each dataset are listed in Appendix B;

- the entire list of metrics computed in this experiment is published in https://rppbodo.github. io/phd/experiment_2.html 


\section{Covers 80}

\begin{tabular}{l|llllllll} 
line id & MR & MRR & MDR & MAP & Top 1 & Top 10 & Top 100 & Top 1000 \\
\hline 1 & 41.575 & 0.19359 & 31.0 & 0.19359 & 21.0 & 46.0 & 142.0 & 160.0 \\
2 & 41.99375 & 0.19206 & 32.0 & 0.19206 & 20.0 & 44.0 & 142.0 & 160.0 \\
3 & 42.3625 & 0.20598 & 27.5 & 0.20598 & 20.0 & 56.0 & 138.0 & 160.0 \\
4 & 42.44375 & 0.1901 & 24.0 & 0.1901 & 18.0 & 57.0 & 141.0 & 160.0 \\
5 & 42.4625 & 0.18129 & 32.0 & 0.18129 & 19.0 & 47.0 & 143.0 & 160.0 \\
6 & 42.675 & 0.19459 & 24.0 & 0.19459 & 19.0 & 57.0 & 136.0 & 160.0 \\
7 & 42.71875 & 0.17317 & 31.0 & 0.17317 & 18.0 & 46.0 & 142.0 & 160.0 \\
8 & 43.01875 & 0.18078 & 34.0 & 0.18078 & 17.0 & 48.0 & 136.0 & 160.0 \\
9 & 43.08125 & 0.20681 & 25.0 & 0.20681 & 23.0 & 48.0 & 137.0 & 160.0 \\
10 & 43.35625 & 0.16701 & 29.5 & 0.16701 & 16.0 & 46.0 & 137.0 & 160.0
\end{tabular}

(a)

\begin{tabular}{l|lll} 
line id & extractor & aggregator & distance \\
\hline 1 & chroma_stft & diff_stats_1 & cosine_distance_oti \\
2 & chroma_stft & basic_stats_1 & cosine_distance_oti \\
3 & beat_synchronous_chroma_cens & basic_stats_2 & manhattan_distance_oti \\
4 & beat_synchronous_chroma_cens & basic_stats_2 & euclidean_distance_oti \\
5 & chroma_stft & diff_stats_1 & manhattan_distance_oti \\
6 & beat_synchronous_chroma_cens & basic_stats_2 & cosine_distance_oti \\
7 & chroma_stft & statistical_summarization & manhattan_distance_oti \\
8 & chroma_stft & diff_stats_1 & euclidean_distance_oti \\
9 & chroma_stft & statistical_summarization & cosine_distance_oti \\
10 & chroma_stft & basic_stats_2 & chebyshev_distance_oti
\end{tabular}

(b)

Table 6.31: The top-10 results for Covers 80 dataset.

\section{YouTubeCovers}

\begin{tabular}{l|llllllll} 
line id & MR & MRR & MDR & MAP & Top 1 & Top 10 & Top 100 & Top 1000 \\
\hline 1 & 7.97143 & 0.6942 & 1.0 & 0.36114 & 219.0 & 296.0 & 345.0 & 350.0 \\
2 & 9.41143 & 0.63231 & 1.0 & 0.2977 & 189.0 & 279.0 & 345.0 & 350.0 \\
3 & 14.1 & 0.4344 & 3.5 & 0.19221 & 110.0 & 231.0 & 343.0 & 350.0 \\
4 & 14.13714 & 0.40846 & 5.0 & 0.1805 & 103.0 & 233.0 & 344.0 & 350.0 \\
5 & 14.71143 & 0.38021 & 5.0 & 0.15415 & 85.0 & 227.0 & 344.0 & 350.0 \\
6 & 15.89429 & 0.37811 & 5.0 & 0.15807 & 93.0 & 216.0 & 346.0 & 350.0 \\
7 & 15.89429 & 0.37811 & 5.0 & 0.15807 & 93.0 & 216.0 & 346.0 & 350.0 \\
8 & 16.46286 & 0.37605 & 5.5 & 0.15219 & 86.0 & 223.0 & 343.0 & 350.0 \\
9 & 16.85429 & 0.5271 & 2.0 & 0.21466 & 153.0 & 247.0 & 335.0 & 350.0 \\
10 & 17.06 & 0.35579 & 6.0 & 0.14139 & 78.0 & 225.0 & 342.0 & 350.0
\end{tabular}

(a)

\begin{tabular}{l|lll} 
line id & extractor & aggregator & distance \\
\hline 1 & pitch_contour_segmentation & octave_abstraction & lcs_circular_mean \\
2 & pitch_contour_segmentation & octave_abstraction & levenshtein_circular_max \\
3 & pitch_contour_segmentation & markov_chain & circular_manhattan \\
4 & pitch_contour_segmentation & markov_chain & circular_cosine \\
5 & chroma_stft & statistical_summarization & cosine_distance_oti \\
6 & pitch_contour_segmentation & markov_chain & circular_euclidean \\
7 & pitch_contour_segmentation & markov_chain & circular_chebyshev \\
8 & chroma_stft_ & diff_stats_1 & cosine_distance_oti \\
9 & pitch_contour_segmentation & octave_abstraction & lcs_circular_max \\
10 & chroma_stft & basic_stats_1 & cosine_distance_oti
\end{tabular}

(b)

Table 6.32: The top-10 results for YouTubeCovers dataset. 


\section{Covers1000}

\begin{tabular}{l|llllllll} 
line id & MR & MRR & MDR & MAP & Top 1 & Top 10 & Top 100 & Top 1000 \\
\hline 1 & 144.041 & 0.25731 & 35.0 & 0.19159 & 201.0 & 366.0 & 636.0 & 1000.0 \\
2 & 175.546 & 0.09845 & 74.5 & 0.06977 & 52.0 & 188.0 & 548.0 & 1000.0 \\
3 & 180.808 & 0.13566 & 75.0 & 0.09752 & 77.0 & 249.0 & 551.0 & 1000.0 \\
4 & 181.48 & 0.15934 & 72.0 & 0.11552 & 103.0 & 261.0 & 558.0 & 1000.0 \\
5 & 183.802 & 0.0861 & 95.0 & 0.06054 & 48.0 & 155.0 & 515.0 & 1000.0 \\
6 & 184.645 & 0.12143 & 81.0 & 0.08497 & 70.0 & 213.0 & 533.0 & 1000.0 \\
7 & 185.462 & 0.13359 & 79.0 & 0.09661 & 80.0 & 236.0 & 540.0 & 1000.0 \\
8 & 185.521 & 0.14401 & 84.5 & 0.10473 & 93.0 & 228.0 & 525.0 & 1000.0 \\
9 & 185.781 & 0.09549 & 97.0 & 0.06776 & 49.0 & 183.0 & 512.0 & 1000.0 \\
10 & 185.884 & 0.14356 & 86.0 & 0.10421 & 93.0 & 229.0 & 526.0 & 1000.0
\end{tabular}

(a)

\begin{tabular}{|c|c|c|c|}
\hline line id & extractor & aggregator & distance \\
\hline 1 & pitch_contour_segmentation & octave_abstraction & lcs_circular_mean \\
\hline 2 & beat synchronous chroma stft & diff stats 1 & cosine distance oti \\
\hline 3 & beat ${ }^{-}$synchronous ${ }^{-}$chroma ${ }^{-}$cqt & $\operatorname{diff}^{-}$stats $^{-} 1$ & manhattan distance oti \\
\hline 4 & rhythm histogram & bypass $-{ }^{-}$ & cosine distance \\
\hline 5 & beat synchronous chroma stft & basic stats 1 & cosine $^{-}$distance oti \\
\hline 6 & chroma cens & basic ${ }^{-}$stats $^{-}{ }_{2}$ & manhattan distance oti \\
\hline 7 & beat synchronous chroma cqt & diff stats $\overline{1}$ & euclidean distance oti \\
\hline 8 & beat ${ }^{-}$synchronous ${ }^{-}$chroma ${ }^{-}$cens & $\operatorname{diff}^{-}$stats $^{-} 1$ & cosine distance ot $\overline{\mathrm{i}}$ \\
\hline 9 & beat ${ }^{-}$synchronous ${ }^{-}$chroma ${ }^{-}$stft & $\operatorname{diff}^{-}$stats $^{-} 1$ & manhattan distance oti \\
\hline 10 & beat ${ }^{-}$synchronous ${ }^{-}$chroma ${ }^{-}$cens & $\operatorname{diff}^{-}$stats $^{-} 1$ & euclidean distance oti \\
\hline
\end{tabular}

(b)

Table 6.33: The top-10 results for Covers1000 dataset.

\section{Mazurkas}

\begin{tabular}{l|llllllll} 
line id & MR & MRR & MDR & MAP & Top 1 & Top 10 & Top 100 & Top 1000 \\
\hline 1 & 4.15724 & 0.95774 & 1.0 & 0.82286 & 2561.0 & 2714.0 & 2728.0 & 2739.0 \\
2 & 4.46479 & 0.87347 & 1.0 & 0.33623 & 2251.0 & 2638.0 & 2722.0 & 2740.0 \\
3 & 4.6297 & 0.83039 & 1.0 & 0.28564 & 2076.0 & 2620.0 & 2721.0 & 2740.0 \\
4 & 4.65268 & 0.85999 & 1.0 & 0.32391 & 2197.0 & 2622.0 & 2717.0 & 2741.0 \\
5 & 4.86173 & 0.8785 & 1.0 & 0.3994 & 2241.0 & 2671.0 & 2718.0 & 2741.0 \\
6 & 4.86647 & 0.83115 & 1.0 & 0.30325 & 2067.0 & 2615.0 & 2723.0 & 2739.0 \\
7 & 4.95659 & 0.81238 & 1.0 & 0.24961 & 2018.0 & 2583.0 & 2722.0 & 2740.0 \\
8 & 4.9865 & 0.80264 & 1.0 & 0.2673 & 1965.0 & 2593.0 & 2726.0 & 2741.0 \\
9 & 5.08063 & 0.95648 & 1.0 & 0.86734 & 2551.0 & 2713.0 & 2728.0 & 2737.0 \\
10 & 5.14447 & 0.79237 & 1.0 & 0.30303 & 1921.0 & 2602.0 & 2722.0 & 2741.0
\end{tabular}

(a)

\begin{tabular}{l|lll} 
line id & extractor & aggregator & distance \\
\hline 1 & pitch_contour_segmentation & octave_abstraction & levenshtein_circular_max \\
2 & beat_synchronous_chroma_cqt & statistical_summarization & manhattan_distance_oti \\
3 & chroma_cqt & statistical_summarization & manhattan_distance_oti \\
4 & beat_synchronous_chroma_cens & statistical_summarization & manhattan_distance_oti \\
5 & beat_synchronous_chroma_stft & statistical_summarization & manhattan_distance_oti \\
6 & beat_synchronous_chroma_cens & diff_stats_-2 & manhattan_distance_oti \\
7 & chroma_stft & statistical_summarization & manhattan_distance_oti \\
8 & chroma_stft & statistical_summarization & cosine_distance_oti \\
9 & pitch_contour_segmentation & octave_abstraction & lcs_circular_mean \\
10 & chroma_stft & diff_stats_1 & cosine_distance_oti
\end{tabular}

(b)

Table 6.34: The top-10 results for Mazurkas dataset. 
SHS9K

\begin{tabular}{l|llllllll} 
line id & MR & MRR & MDR & MAP & Top 1 & Top 10 & Top 100 & Top 1000 \\
\hline 1 & 47.57883 & 0.40387 & 6.0 & 0.05102 & 3008.0 & 5188.0 & 8067.0 & 9265.0 \\
2 & 56.67866 & 0.33549 & 11.0 & 0.03429 & 2385.0 & 4527.0 & 7741.0 & 9273.0 \\
3 & 59.06128 & 0.31115 & 13.0 & 0.02701 & 2182.0 & 4334.0 & 7658.0 & 9272.0 \\
4 & 62.9398 & 0.23255 & 15.0 & 0.02777 & 1275.0 & 3990.0 & 7836.0 & 9240.0 \\
5 & 63.73713 & 0.22736 & 16.0 & 0.02813 & 1234.0 & 3914.0 & 7809.0 & 9238.0 \\
6 & 64.75038 & 0.22813 & 16.0 & 0.02696 & 1265.0 & 3888.0 & 7787.0 & 9237.0 \\
7 & 65.22755 & 0.22587 & 16.0 & 0.02669 & 1222.0 & 3899.0 & 7766.0 & 9236.0 \\
8 & 65.88273 & 0.18724 & 20.0 & 0.02269 & 927.0 & 3407.0 & 7716.0 & 9243.0 \\
9 & 66.06203 & 0.29483 & 16.0 & 0.02617 & 2038.0 & 4100.0 & 7485.0 & 9270.0 \\
10 & 66.90114 & 0.18625 & 20.0 & 0.02065 & 903.0 & 3429.0 & 7646.0 & 9252.0
\end{tabular}

(a)

\begin{tabular}{l|lll} 
line id & extractor & aggregator & distance \\
\hline 1 & pitch_contour_segmentation & octave_abstraction & lcs_circular_mean \\
2 & pitch_contour_segmentation & octave_abstraction & levenshtein_circular_max \\
3 & pitch_contour_segmentation & octave_abstraction & lcs_circular_max \\
4 & beat_synchronous_chroma_cqt & diff_stats_1 & manhattan_distance_oti \\
5 & beat_synchronous_chroma_cqt & diff_stats_1 & cosine_distance_oti_ \\
6 & beat_synchronous_chroma_cqt & statistical_summarization & manhattan_distance_oti \\
7 & beat_synchronous_chroma_cqt & diff_stats_1 & euclidean_distance_oti \\
8 & beat_synchronous_chroma_cqt & diff_stats_2 & manhattan_distance_oti \\
9 & pitch_contour_segmentation & octave_abstraction & levenshtein_circular_mean \\
10 & beat_synchronous_chroma_stft & diff_stats_1 & manhattan_distance_oti
\end{tabular}

(b)

Table 6.35: The top-10 results for SHS9K dataset.

\subsubsection{Discussion}

\section{Best results}

The models that result in the best mean rank values for the CSI datasets are shown in Table 6.36. Four models out of five share the same feature (pitch_contour_segmentation) and the same aggregator (octave_abstraction), which is a very good indication of the relevance of these methods for CSI, while the remaining model uses a chromagram as feature. All features appearing in the best models encode tonal information, which corroborates the observation in the literature that tonal differences are less frequent between versions of the same song [SGH10, SSG12, CLX17].

\begin{tabular}{llll} 
dataset & extractor & aggregator & distance \\
\hline Covers80 & chroma_stft & diff_stats_1 & cosine_distance_oti \\
YouTubeCovers & pitch_contour_segmentation & octave_abstraction & lcs_circular_mean \\
Covers1000 & pitch_contour_segmentation & octave_abstraction & lcs_circular_mean \\
Mazurkas & pitch_contour_segmentation & octave_abstraction & levenshtein_circular_max \\
SHS9K & pitch_contour_segmentation & octave_abstraction & lcs_circular_mean
\end{tabular}

Table 6.36: The best results for Experiment \#2.

Moreover, if we analyse the Top-50 list of best features for each dataset (see Appendix B) we may verify that 249 models out of 250 (99.6\%) use tonal features: 231 models use Chromagrams, 17 models use Pitch Contour Segmentation, and 1 model uses Tonnetz. This is a stronger evidence that tonal similarity has indeed the highest relevance for the CSI problem. As a side note, the only model that doesn't include a tonal feature is \{rhythm_histogram, bypass, cosine_distance $\}$, the fourth best model for Covers1000, which uses a rhythmic feature.

\section{Comparative results}

As seen in the comparative analysis of Experiment \#1, some techniques may differ by only a single setting, for instance, models that share the same feature, aggregator, and distance, but where the feature is beat-synchronized in one model and not in the other. Similarly to what was done in the preceding section, we analyse which settings are best, but now for CSI datasets.

Comparing models that may (or not) use beat-synchronous features, we can verify (see Table 6.37) that in average it is better not to beat-sync features for the CSI datasets and the models we included in the experiment. 


\begin{tabular}{lll} 
beat-synchronous? & mean rank (mean) & mean rank $($ std $)$ \\
\hline yes & 89.3480537370109 & 172.35261733957287 \\
no & 88.3282453970704 & 90.53508052977543
\end{tabular}

Table 6.37: Average results for models that do or do not use beat-synchronous features.

According to Table 6.38 we conclude that applying OTI leads to better results for the models and datasets compared.

\begin{tabular}{lll} 
OTI applied? & mean rank (mean) & mean rank $($ std $)$ \\
\hline yes & 82.69112416120925 & 192.75918501547838 \\
no & 85.20164733110768 & 84.94848924394955
\end{tabular}

Table 6.38: Average results for models that do or do not use OTI.

When computing the LCS-based distances, the best results are obtained using the mean length for normalisation, as shown in Table 6.39.

\begin{tabular}{lll} 
function used & mean rank (mean) & mean rank (std) \\
\hline min & 321.7782208039525 & 314.83518453601465 \\
mean & 105.87476892655063 & 110.58916821207373 \\
max & 108.87226174873874 & 105.8208158479763
\end{tabular}

Table 6.39: Average results for models using minimum, mean or maximum to normalise LCS-based distances.

Table 6.40 shows that better results can be achieved by using the mean length for normalisation when computing Levenshtein distances.

\begin{tabular}{lll} 
function used & mean rank (mean) & mean rank (std) \\
\hline min & 104.611049187205 & 92.176838784475 \\
mean & 100.96905325877044 & 91.34877090282131 \\
max & 109.92441834426197 & 105.13459286919078
\end{tabular}

Table 6.40: Average results for models that use minimum, mean or maximum to normalise Levenshtein distances.

As explained in Section 2.2, we compare several distinct aggregators based on the single Gaussian model. Comparing the average mean ranks in Table 6.41 we may verify that, in average, basic_stats_1 is better than basic_stats_2, and diff_stats_1 is better than both diff_stats_2 and basic_stats_1. Thereby we can conclude that adding the minimum and maximum values to the global features is not worth it, and that it is beneficial to add information about the feature differences to the global features.

\begin{tabular}{lll} 
aggregator & mean rank (mean) & mean rank (std) \\
\hline basic_stats_1 & 95.26268075728171 & 92.57033667079618 \\
basic_stats_2 & 99.63181852081064 & 98.3795194071255 \\
diff_stats_1 & 93.58111901686559 & 91.45156497941215 \\
diff_stats_2 & 118.82704802637727 & 232.48864683342376
\end{tabular}

Table 6.41: Average results for models that use the four variations of single Gaussian as aggregation model.

As we can see in Table 6.42, for the CSI datasets, the models that use vector quantization as aggregation method lead to better results with the default number of clusters ${ }^{31}$.

\footnotetext{
${ }^{31}$ https://scikit-learn.org/stable/modules/generated/sklearn.cluster.KMeans.html
} 


\begin{tabular}{lll} 
number of clusters & mean rank (mean) & mean rank (std) \\
\hline number of classes & 118.19316996341703 & 260.85976735055647 \\
function default (8) & 106.07161721064126 & 97.17924297824821
\end{tabular}

Table 6.42: Average results for vector quantization models using default or tailored number of clusters.

Finally, when comparing features from the $R P_{-}$extract library that have temporal summarization as an option, it is better not to use it according to Table 6.43.

\begin{tabular}{lll} 
temporal features? & mean rank (mean) & mean rank $($ std) \\
\hline yes & 113.86163326823744 & 102.01929218250392 \\
no & 104.53442949216674 & 89.31943704335092
\end{tabular}

Table 6.43: Average results for models with or without temporal summarization.

Analysing these comparative results from the CSI datasets with those of Experiment \#1, we can verify what both experiments have in common: we should apply OTI to chromagrams, not add minimum and maximum values to single Gaussian aggregators, consider the feature differences for single Gaussian aggregators, and avoid temporal features when using the $R P_{-}$extract library.

\begin{tabular}{lll} 
comparative analysis & music similarity & cover song identification \\
\hline beat-synchronous? & yes & no \\
OTI applied? & yes & yes \\
LCS function & max & mean \\
Levenshtein function & max & mean \\
add min and max to Gaussian aggregator? & no & no \\
add diff to Gaussian aggregator? & yes & yes \\
number of clusters (vector quantization) & number of classes & function default \\
temporal features? & no & no
\end{tabular}

Table 6.44: Comparative results for Music Similarity versus Cover Song Identification datasets.

\subsection{Experiment \#3: Cover Song Classification}

\subsubsection{Experiment design}

The main goal of Experiment \#3 is to explore different sample definitions, i.e. different ways to combine similarity models, for the Cover Song Classification (CSC) problem and verify which model combinations lead to the best results. As explained in Section 5.2, we consider two alternative approaches: fusing models using the Top- $\mathrm{N}$ best models ( $\mathrm{N}$ equals to 10, 20, 30, 40, and 50), and using the filtered list of models (skipping so-called "equivalent" ones). We are going to refer to these alternatives as Experiments \#3a and \#3b.

In both experiments we use samples from the same dataset to train and test classifiers, setting split percentages to 70/30. Since our samples share information of pairs of songs, and a song that appears in the train set cannot appear again in the test set, we apply a heuristic (as described in Section 5.2) to separate classes of tracks.

We use five classifiers (Naive Bayes, Logistic Regression, Decision Tree, Random Forest, and Ada Boost), run each configuration 10 times to make good use of the random splits, and compute mean and standard deviation of every classification metric (f-score, precision, recall, accuracy, 0's accuracy, and 1's accuracy).

The best results by dataset are presented below and, for consultation, we present the Top-50 models per dataset in Appendix B and the filtered models per dataset in Appendix C. Also, all classification results are published in https://rppbodo.github.io/phd/experiment_3.html. 


\subsubsection{Results: experiment \#3a}

In Experiment \#3a we build tensors using the Top- $\mathrm{N}$ best models, so the dimension of the samples is defined by the number of models we use. Since we have 5 classifiers, 5 different $N$ values, and 10 different splits for each setting, we have 250 classification runs in total. Tables 6.45-6.49 display the best 10 results for each 5 CSI datasets.

\section{Covers80}

\begin{tabular}{|c|c|c|c|c|c|c|c|c|}
\hline line id & dimension & classifier & f1-score (mean) & f1-score (std) & precision (mean) & precision (std) & recall (mean) & recall $(\mathrm{std})$ \\
\hline 1 & 30 & random forest & 0.70751 & 0.01326 & 0.9702 & 0.03995 & 0.55781 & 0.02102 \\
\hline 2 & 50 & random ${ }^{-}$forest & 0.70083 & 0.01211 & 0.98122 & 0.02956 & 0.54531 & 0.01094 \\
\hline 3 & 10 & random ${ }^{-}$forest & 0.69955 & 0.01926 & 0.96295 & 0.029 & 0.55 & 0.024 \\
\hline 4 & 40 & random ${ }^{-}$forest & 0.69906 & 0.01882 & 0.96491 & 0.02663 & 0.54844 & 0.02031 \\
\hline 5 & 30 & ada boost & 0.69254 & 0.02162 & 0.9151 & 0.06552 & 0.55937 & 0.02688 \\
\hline 6 & 20 & random forest & 0.69068 & 0.0211 & 0.97847 & 0.0327 & 0.53438 & 0.024 \\
\hline 7 & 50 & ada boost & 0.6875 & 0.02586 & 0.92149 & 0.03513 & 0.54844 & 0.02259 \\
\hline 8 & 40 & $\mathrm{ada}^{-}$boost & 0.68512 & 0.03228 & 0.91654 & 0.07159 & 0.54844 & 0.02747 \\
\hline 9 & 20 & $\mathrm{ada}^{-}$boost & 0.68185 & 0.02352 & 0.94547 & 0.05262 & 0.53438 & 0.02596 \\
\hline 10 & 10 & $\mathrm{ada}^{-}$boost & 0.68101 & 0.03351 & 0.9071 & 0.08111 & 0.54688 & 0.0221 \\
\hline
\end{tabular}

(a)

\begin{tabular}{l|llllll} 
line id & accuracy (mean) & accuracy (std) & 0's acc. (mean) & 0's acc. (std) & 1's acc. (mean) & 1's acc. (std) \\
\hline 1 & 0.9856 & 0.00063 & 0.9994 & 0.00084 & 0.55781 & 0.02102 \\
2 & 0.98545 & 0.00065 & 0.99965 & 0.00055 & 0.54531 & 0.01094 \\
3 & 0.98525 & 0.00078 & 0.99929 & 0.00056 & 0.55 & 0.024 \\
4 & 0.98525 & 0.00084 & 0.99934 & 0.00051 & 0.54844 & 0.02031 \\
5 & 0.98447 & 0.00134 & 0.99819 & 0.00155 & 0.55937 & 0.02688 \\
6 & 0.98506 & 0.00091 & 0.9996 & 0.00063 & 0.53438 & 0.024 \\
7 & 0.98442 & 0.00126 & 0.99849 & 0.00068 & 0.54844 & 0.02259 \\
8 & 0.98423 & 0.00181 & 0.99829 & 0.0015 & 0.54844 & 0.02747 \\
9 & 0.98442 & 0.00119 & 0.99894 & 0.00104 & 0.53438 & 0.02596 \\
10 & 0.98394 & 0.00216 & 0.99803 & 0.00196 & 0.54688 & 0.0221
\end{tabular}

(b)

Table 6.45: Top-10 results for Covers80 in Experiment \#3a of CSC.

\section{YouTubeCovers}

\begin{tabular}{|c|c|c|c|c|c|c|c|c|}
\hline line id & dimension & classifier & f1-score (mean) & f1-score (std) & precision (mean) & precision (std) & recall (mean) & recall $(\mathrm{std})$ \\
\hline 1 & 40 & ada_boost & 0.48029 & 0.02518 & 0.82439 & 0.05955 & 0.3402 & 0.02604 \\
\hline 2 & 40 & random_forest & 0.47871 & 0.02079 & 0.88495 & 0.07285 & 0.32959 & 0.02162 \\
\hline 3 & 20 & random ${ }^{-}$forest & 0.47015 & 0.03995 & 0.89132 & 0.04495 & 0.32122 & 0.0398 \\
\hline 4 & 10 & random_forest & 0.46446 & 0.03359 & 0.82369 & 0.056 & 0.32571 & 0.03643 \\
\hline 5 & 20 & ada_boōst & 0.46408 & 0.03529 & 0.84501 & 0.02923 & 0.32122 & 0.03496 \\
\hline 6 & 30 & random_forest & 0.45923 & 0.0365 & 0.8269 & 0.10298 & 0.32571 & 0.05414 \\
\hline 7 & 50 & ada_boōst & 0.4587 & 0.02132 & 0.81802 & 0.07667 & 0.32 & 0.01843 \\
\hline 8 & 50 & random_forest & 0.45395 & 0.0256 & 0.90094 & 0.04924 & 0.30449 & 0.02484 \\
\hline 9 & 10 & ada boost & 0.45043 & 0.03082 & 0.82771 & 0.077 & 0.31163 & 0.03201 \\
\hline 10 & 30 & ada_boost $^{-}$ & 0.44774 & 0.03767 & 0.75775 & 0.09581 & 0.32531 & 0.05271 \\
\hline
\end{tabular}

(a)

\begin{tabular}{l|llllll} 
line id & accuracy (mean) & accuracy (std) & 0's acc. (mean) & 0's acc. (std) & 1's acc. (mean) & 1's acc. (std) \\
\hline 1 & 0.96326 & 0.00152 & 0.99605 & 0.00161 & 0.3402 & 0.02604 \\
2 & 0.96414 & 0.00167 & 0.99754 & 0.00203 & 0.32959 & 0.02162 \\
3 & 0.96401 & 0.00157 & 0.99784 & 0.0011 & 0.32122 & 0.0398 \\
4 & 0.96263 & 0.00132 & 0.99615 & 0.00163 & 0.32571 & 0.03643 \\
5 & 0.96307 & 0.00137 & 0.99685 & 0.00085 & 0.32122 & 0.03496 \\
6 & 0.96207 & 0.00188 & 0.99556 & 0.00425 & 0.32571 & 0.05414 \\
7 & 0.96223 & 0.00204 & 0.99604 & 0.0022 & 0.32 & 0.01843 \\
8 & 0.96346 & 0.00114 & 0.99814 & 0.00121 & 0.30449 & 0.02484 \\
9 & 0.96211 & 0.00165 & 0.99635 & 0.00194 & 0.31163 & 0.03201 \\
10 & 0.96029 & 0.00228 & 0.99371 & 0.00434 & 0.32531 & 0.05271
\end{tabular}

(b)

Table 6.46: Top-10 results for YouTubeCovers in Experiment \#3a of CSC. 


\section{Covers1000}

\begin{tabular}{|c|c|c|c|c|c|c|c|c|}
\hline line id & dimension & classifier & f1-score (mean) & f1-score (std) & precision (mean) & precision (std) & recall (mean) & recall (std) \\
\hline 1 & 10 & random_forest & 0.57966 & 0.00478 & 0.96275 & 0.01583 & 0.4148 & 0.0074 \\
\hline 2 & 10 & ada_boōst & 0.5781 & 0.01072 & 0.96817 & 0.01531 & 0.41221 & 0.0111 \\
\hline 3 & 20 & ada boost & 0.57484 & 0.00896 & 0.95694 & 0.02049 & 0.41092 & 0.0082 \\
\hline 4 & 20 & random_forest & 0.5726 & 0.00714 & 0.97836 & 0.01466 & 0.40481 & 0.00712 \\
\hline 5 & 50 & ada_boōst & 0.5724 & 0.00794 & 0.95081 & 0.01816 & 0.40962 & 0.00945 \\
\hline 6 & 30 & $\mathrm{ada}^{-}$boost & 0.57194 & 0.01057 & 0.96565 & 0.02579 & 0.40648 & 0.01023 \\
\hline 7 & 40 & random_forest & 0.57154 & 0.00576 & 0.97678 & 0.01609 & 0.40407 & 0.00728 \\
\hline 8 & 40 & ada boost & 0.5713 & 0.01368 & 0.9643 & 0.02279 & 0.40629 & 0.01622 \\
\hline 9 & 50 & random_forest & 0.56818 & 0.00629 & 0.97666 & 0.01953 & 0.40074 & 0.0069 \\
\hline 10 & 30 & random ${ }^{-}$forest & 0.56739 & 0.0063 & 0.98546 & 0.01738 & 0.39852 & 0.00783 \\
\hline
\end{tabular}

(a)

\begin{tabular}{l|llllll} 
line id & accuracy (mean) & accuracy (std) & 0's acc. (mean) & 0's acc. (std) & 1's acc. (mean) & 1's acc. (std) \\
\hline 1 & 0.99583 & $2 \mathrm{e}-05$ & 0.99989 & $5 \mathrm{e}-05$ & 0.4148 & 0.0074 \\
2 & 0.99583 & $8 \mathrm{e}-05$ & 0.9999 & $5 \mathrm{e}-05$ & 0.41221 & 0.0111 \\
3 & 0.99579 & $9 \mathrm{e}-05$ & 0.99987 & $6 \mathrm{e}-05$ & 0.41092 & 0.0082 \\
4 & 0.99581 & $6 \mathrm{e}-05$ & 0.99994 & $4 \mathrm{e}-05$ & 0.40481 & 0.00712 \\
5 & 0.99576 & $6 \mathrm{e}-05$ & 0.99985 & $6 \mathrm{e}-05$ & 0.40962 & 0.00945 \\
6 & 0.99579 & 0.0001 & 0.9999 & $8 \mathrm{e}-05$ & 0.40648 & 0.01023 \\
7 & 0.9958 & $4 \mathrm{e}-05$ & 0.99993 & $5 \mathrm{e}-05$ & 0.40407 & 0.00728 \\
8 & 0.99578 & $8 \mathrm{e}-05$ & 0.99989 & $8 \mathrm{e}-05$ & 0.40629 & 0.01622 \\
9 & 0.99578 & $6 \mathrm{e}-05$ & 0.99993 & $6 \mathrm{e}-05$ & 0.40074 & 0.0069 \\
10 & 0.99579 & $4 \mathrm{e}-05$ & 0.99996 & $5 \mathrm{e}-05$ & 0.39852 & 0.00783
\end{tabular}

(b)

Table 6.47: Top-10 results for Covers1000 in Experiment \#3a of CSC.

\section{Mazurkas}

\begin{tabular}{|c|c|c|c|c|c|c|c|c|}
\hline line id & dimension & classifier & f1-score (mean) & f1-score (std) & precision (mean) & precision (std) & recall (mean) & recall (std) \\
\hline 1 & 50 & random_forest & 0.91457 & 0.0134 & 0.97536 & 0.01013 & 0.86116 & 0.02086 \\
\hline 2 & 30 & random ${ }^{-}$forest & 0.90372 & 0.01394 & 0.96467 & 0.01556 & 0.85021 & 0.0178 \\
\hline 3 & 40 & random_forest & 0.90307 & 0.01612 & 0.95885 & 0.02565 & 0.85384 & 0.01811 \\
\hline 4 & 50 & logistic regression & 0.90007 & 0.01664 & 0.98039 & 0.00913 & 0.83225 & 0.02584 \\
\hline 5 & 20 & random_forest & 0.89885 & 0.00701 & 0.95285 & 0.0121 & 0.85084 & 0.01191 \\
\hline 6 & 10 & random ${ }^{-}$forest & 0.89372 & 0.01176 & 0.95761 & 0.03267 & 0.83897 & 0.02123 \\
\hline 7 & 40 & logistic_regression & 0.88701 & 0.01165 & 0.97486 & 0.02065 & 0.8142 & 0.01902 \\
\hline 8 & 30 & logistic regression & 0.88592 & 0.01386 & 0.97366 & 0.01337 & 0.81297 & 0.02066 \\
\hline 9 & 20 & logistic regression & 0.88552 & 0.00725 & 0.96497 & 0.01052 & 0.81837 & 0.01374 \\
\hline 10 & 20 & ada boost & 0.87581 & 0.00976 & 0.94003 & 0.01046 & 0.81997 & 0.01491 \\
\hline
\end{tabular}

(a)

\begin{tabular}{l|llllll} 
line id & accuracy (mean) & accuracy (std) & 0 's acc. (mean) & 0 's acc. (std) & 1's acc. (mean) & 1's acc. (std) \\
\hline 1 & 0.99123 & 0.0013 & 0.99874 & 0.00052 & 0.86116 & 0.02086 \\
2 & 0.99012 & 0.0014 & 0.9982 & 0.00082 & 0.85021 & 0.0178 \\
3 & 0.98998 & 0.00171 & 0.99785 & 0.00139 & 0.85384 & 0.01811 \\
4 & 0.98993 & 0.00156 & 0.99904 & 0.00045 & 0.83225 & 0.02584 \\
5 & 0.98957 & 0.0007 & 0.99757 & 0.00066 & 0.85084 & 0.01191 \\
6 & 0.98911 & 0.00132 & 0.99777 & 0.00185 & 0.83897 & 0.02123 \\
7 & 0.98867 & 0.00114 & 0.99876 & 0.00106 & 0.8142 & 0.01902 \\
8 & 0.98858 & 0.00131 & 0.99872 & 0.00066 & 0.81297 & 0.02066 \\
9 & 0.98848 & 0.00068 & 0.99828 & 0.00054 & 0.81837 & 0.01374 \\
10 & 0.98734 & 0.00094 & 0.99698 & 0.00056 & 0.81997 & 0.01491
\end{tabular}

(b)

Table 6.48: Top-10 results for Mazurkas in Experiment \#3a of CSC. 


\section{SHS9K}

\begin{tabular}{|c|c|c|c|c|c|c|c|c|}
\hline line id & dimension & classifier & f1-score (mean) & f1-score (std) & precision (mean) & precision (std) & recall (mean) & recall (std) \\
\hline 1 & 20 & naive_bayes & 0.08792 & 0.00458 & 0.0552 & 0.00446 & 0.22045 & 0.01992 \\
\hline 2 & 40 & decision_tree & 0.06982 & 0.00294 & 0.07181 & 0.00344 & 0.06796 & 0.0028 \\
\hline 3 & 50 & decision_tree & 0.06968 & 0.00206 & 0.07229 & 0.00157 & 0.06732 & 0.00317 \\
\hline 4 & 30 & naive_bāyes & 0.06761 & 0.00601 & 0.03701 & 0.00396 & 0.40306 & 0.03381 \\
\hline 5 & 30 & decision_tree & 0.06549 & 0.00131 & 0.06812 & 0.00184 & 0.06308 & 0.00157 \\
\hline 6 & 20 & decision_tree & 0.06516 & 0.00164 & 0.0691 & 0.00192 & 0.06169 & 0.00212 \\
\hline 7 & 10 & decision_tree & 0.06183 & 0.0018 & 0.06799 & 0.00133 & 0.05673 & 0.00234 \\
\hline 8 & 40 & naive_bāyes & 0.06097 & 0.00397 & 0.03252 & 0.00236 & 0.496 & 0.02807 \\
\hline 9 & 50 & naive_bayes & 0.05754 & 0.00376 & 0.03046 & 0.00221 & 0.52758 & 0.0276 \\
\hline 10 & 10 & naive_bayes & 0.05038 & 0.00253 & 0.05069 & 0.00389 & 0.05028 & 0.00267 \\
\hline
\end{tabular}

(a)

\begin{tabular}{l|llllll} 
line id & accuracy (mean) & accuracy (std) & 0 's acc. (mean) & 0 's acc. (std) & 1's acc. (mean) & 1 's acc. (std) \\
\hline 1 & 0.91604 & 0.01183 & 0.92896 & 0.0124 & 0.22045 & 0.01992 \\
2 & 0.96698 & 0.00051 & 0.98368 & 0.00052 & 0.06796 & 0.0028 \\
3 & 0.96724 & 0.00065 & 0.98395 & 0.00071 & 0.06732 & 0.00317 \\
4 & 0.79433 & 0.03371 & 0.80159 & 0.03496 & 0.40306 & 0.03381 \\
5 & 0.96719 & 0.00052 & 0.98397 & 0.00055 & 0.06308 & 0.00157 \\
6 & 0.96774 & 0.00056 & 0.98456 & 0.0006 & 0.06169 & 0.00212 \\
7 & 0.96863 & 0.0004 & 0.98556 & 0.00045 & 0.05673 & 0.00234 \\
8 & 0.71927 & 0.03408 & 0.72341 & 0.03521 & 0.496 & 0.02807 \\
9 & 0.68257 & 0.0359 & 0.68545 & 0.03707 & 0.52758 & 0.0276 \\
10 & 0.96542 & 0.00161 & 0.98241 & 0.00166 & 0.05028 & 0.00267
\end{tabular}

(b)

Table 6.49: Top-10 results for SHS9K in Experiment \#3a of CSC.

\subsubsection{Discussion: experiment \#3a}

\section{Best results}

The best results for Covers 80 are obtained using random forest classifier with Top-30 models; for YouTubeCovers these are obtained using ada boost with Top-40 models; for Covers1000 these are obtained using random forest with Top-10 models; for Mazurkas these are obtained using random forest with Top-50 models; for SHS9K these are obtained using naive Bayes with Top-20 models.

Comparing the mean f1-score of these results, Mazurkas achieves the highest score for the CSC problem (as for the CSI problem) with $\mathrm{f} 1=0.91457$. This dataset is mainly composed of piano recordings which have more potential to generate cleaner tonal features (such as chromagrams) and better automatic transcriptions (as with Pitch Contour Segmentation). Following Mazurkas, the datasets along with the best mean $\mathrm{f1}$-scores achieved are Covers80 ( $\mathrm{f} 1=0.70751$ ), Covers1000 ( $\mathrm{f} 1=0.57966)$, YouTubeCovers $(\mathrm{f} 1=0.48029)$, and SHS9K $(\mathrm{f} 1=0.08792)$ which is by far the most difficult dataset in the context of this experiment.

Recalling the CSI results, we had the following Mean Ranks: Mazurkas (with MR=4.15724), YouTubeCovers $(\mathrm{MR}=7.97143)$, Covers80 $(\mathrm{MR}=41.575)$, SHS9K $(\mathrm{MR}=47.57883)$, and Covers1000 $(\mathrm{MR}=144.041)$. We should notice two datasets in particular: Covers1000 achieved bad results in the CSI problem, but considerably good results in the CSC, while for SHS9K we observed the opposite performance of our models in the CSI and CSC problems. 


\section{Comparative results}

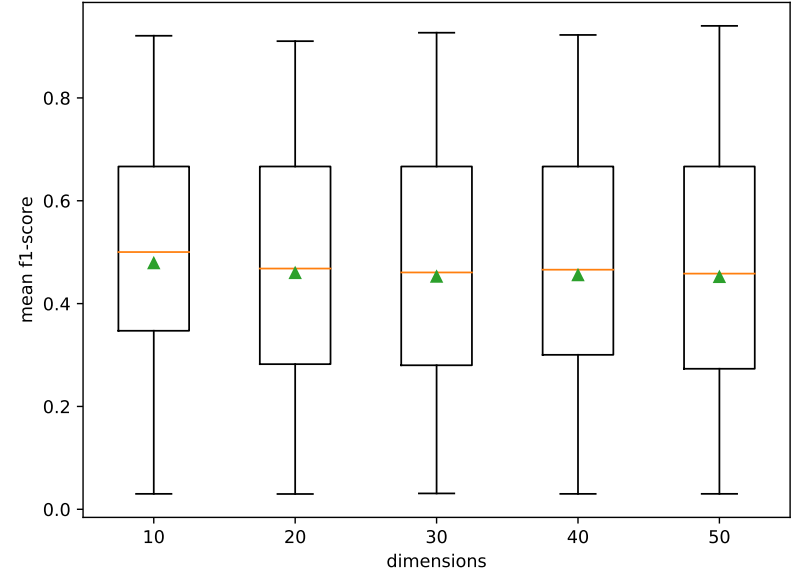

(a)

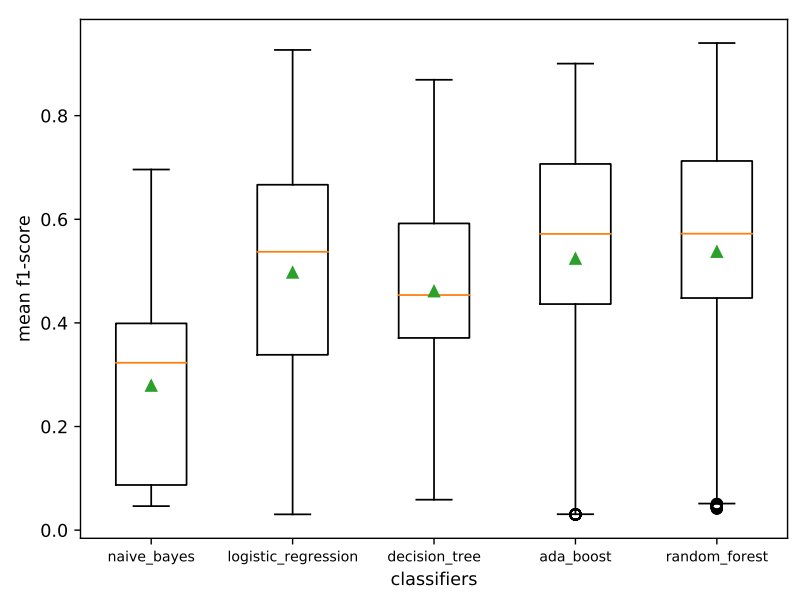

(b)

Figure 6.12: Summarized results to experiment \#3a by dimension (a) and by classifier (b).

Analyzing Figure 6.12a, which resumes all the classification runs according to the dimension of the Top- $\mathrm{N}$ models, we can see a slightly higher mean f1-score for the Top-10 models. Moreover, it does not present any evidence that using more models yields better results. One plausible reason for this is the fact that, even though the larger Top-N lists do include more information about the pair of songs, the models added are very similar to the previous ones, so they do not actually help the classification process. As discussed before, from the Top-50 models of the 5 CSI datasets, there are 231 models with chromagrams as features, 17 models with Pitch Contour Segmentation, and 1 model with tonnetz, so most models can be seen as variations of the topmost models.

Figure $6.12 \mathrm{~b}$ shows all the results according to the classifier used. Random forest and ada boost have the best results (random forest is the classifier with the best results for Covers80, Covers1000, and Mazurkas; ada boost is the classifier with the best result for YouTubeCovers). These classifiers are followed by logistic regression, decision tree, and naive Bayes, in this order.

\subsubsection{Results: experiment \#3b}

In Experiment \#3b we build tensors using the filtered models (see Section 5.2), exploring the individual models as well as all combinations of models in pairs and in groups of three. This is done in order to exploit distinct information obtained by fusing different models. The sample dimension is defined by the number of models (1-3) in each combination, and the number of settings is determined by the number of combinations. The model filtering process leads to lists of 16 models, so adding up 16, 120 (number of combinations of size 2), and 560 (number of combinations of size 3) we obtain 696 in total. Multiplying it by 5 classifiers and 10 different splits, we have in total 34,800 classification runs. Tables 6.50-6.54 show the best 10 results for all datasets. 


\section{Covers80}

\begin{tabular}{|c|c|c|c|}
\hline line id & length & models & classifier \\
\hline 1 & 3 & $\begin{array}{l}\text { \{chroma_stft, diff_stats_1, cosine_distance_oti }\} \\
\text { \{chroma_stft, basic_stats_1, cosine_distance_oti }\} \\
\text { \{beat_synchronous_chroma_cens, basic_stats_2, manhattan_distance_oti }\end{array}$ & naive_bayes \\
\hline 2 & 3 & $\begin{array}{l}\left.\text { \{beat_synchronous_chroma_cens, basic }{ }^{-}{ }^{-}{ }^{-} \text {stats }{ }^{-} 2 \text {, manhattan }{ }^{-} \text {distance }{ }^{-} \text {oti }\right\} \\
\text { \{beat_synchronous_chroma_cens, statistical_summarization_- } \\
\text { \{pitch_contour segmentation, octave_abstraction, lcs_circular_mean }\}\end{array}$ & random_forest \\
\hline 3 & 2 & $\begin{array}{l}\{\text { beat_synchronous_chroma_cens, statistical_summarization_2, monte_carlo_distance }\} \\
\{\text { pitch_contour_segmentation, octave_abstraction, lcs_circular_mean }\}\end{array}$ & random_forest \\
\hline 4 & 3 & $\begin{array}{l}\text { \{chroma_stft, basic_stats_1, cosine_distance_oti }\} \\
\text { \{beat_synchronous_chroma_cens, basic_stats_2, manhattan_distance_oti }\} \\
\text { \{beat_synchronous_chroma_cens, statistical_summarization_2, monte_carlo_distance }\}\end{array}$ & random_forest \\
\hline 5 & 3 & 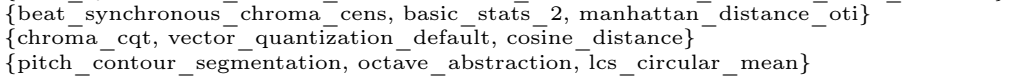 & random_forest \\
\hline 6 & 3 & $\begin{array}{l}\text { \{chroma_stft, diff_stats_1, cosine_distance_oti }\} \\
\text { \{beat_synchronous_chroma_cens, basic_stats_2, manhattan_distance_oti }\} \\
\text { \{chroma_cqt, vector_quantization_default, cosine_distance }\}\end{array}$ & naive_bayes \\
\hline 7 & 3 & $\begin{array}{l}\{\text { beat_synchronous_chroma_cens, } \bar{b} \text { asic_stats_2, } \text { manhattan_distance_oti }\} \\
\text { \{pitch_contour_segmentation, octave_abstraction, lcs_circular_mean }\} \\
\{\text { mfcc, statistical_summarization_2, monte_carlo_distance }\end{array}$ & random_forest \\
\hline 8 & 3 & $\begin{array}{l}\{\text { beat_synchronoüs_chroma_cens, statistical_summarization_2, monte_carlo_distance }\} \\
\text { \{pitch_contour_segmentation, octave_abstraction, lcs_circular_mean }\} \\
\{\text { pitch_contour_segmentation, markov_chain, circular_manhattan }\}\end{array}$ & random_forest \\
\hline 9 & 3 & $\begin{array}{l}\{\text { beat_synchronous_chroma_cens, basic stats_2, manhattan_distance_oti }\} \\
\text { \{pitch_contour_segmentation, octave abstraction, lcs_circular mean }\} \\
\text { \{chroma_stft, gaussian mixture model default, manhattan distance }\end{array}$ & random_forest \\
\hline 10 & 3 & $\begin{array}{l}\text { \{chroma_stft, basic_stäts_1, cosine_distance_oti }\} \\
\{\text { beat_synchronous_chroma_cens, basic_stats_2, manhattan } \\
\text { \{chroma_cqt, vector_quantization_default, cosine_distance }\}\end{array}$ & naive_bayes \\
\hline
\end{tabular}

(a)

\begin{tabular}{l|llllll} 
line id & f1-score (mean) & f1-score (std) & precision (mean) & precision $(\mathrm{std})$ & recall (mean) & recall (std) \\
\hline 1 & 0.70709 & 0.01742 & 0.97635 & 0.03197 & 0.55469 & 0.01881 \\
2 & 0.70106 & 0.0267 & 0.95889 & 0.03469 & 0.55312 & 0.02856 \\
3 & 0.7003 & 0.01416 & 0.94322 & 0.04184 & 0.55781 & 0.01888 \\
4 & 0.69998 & 0.01896 & 0.97792 & 0.02491 & 0.54531 & 0.01907 \\
5 & 0.69993 & 0.01439 & 0.97907 & 0.02973 & 0.54531 & 0.02031 \\
6 & 0.69949 & 0.01787 & 0.99744 & 0.00769 & 0.53906 & 0.02344 \\
7 & 0.69888 & 0.02203 & 0.96329 & 0.03483 & 0.54922 & 0.02685 \\
8 & 0.69735 & 0.0173 & 0.97277 & 0.02978 & 0.54375 & 0.01646 \\
9 & 0.69716 & 0.01778 & 0.96221 & 0.02964 & 0.54688 & 0.01712 \\
10 & 0.69635 & 0.01549 & 1.0 & 0.0 & 0.53438 & 0.01822
\end{tabular}

(b)

\begin{tabular}{l|llllll} 
line id & accuracy (mean) & accuracy $($ std) & 0 's acc. (mean) & 0's acc. (std) & 1 's acc. (mean) & 1 's acc. (std) \\
\hline 1 & 0.98564 & 0.00082 & 0.99955 & 0.00062 & 0.55469 & 0.01881 \\
2 & 0.98528 & 0.00119 & 0.99922 & 0.00067 & 0.55312 & 0.02856 \\
3 & 0.98508 & 0.00075 & 0.99887 & 0.00086 & 0.55781 & 0.01888 \\
4 & 0.9854 & 0.00085 & 0.9996 & 0.00045 & 0.54531 & 0.01907 \\
5 & 0.9854 & 0.0006 & 0.9996 & 0.00059 & 0.54531 & 0.02031 \\
6 & 0.98555 & 0.00063 & 0.99995 & 0.00015 & 0.53906 & 0.02344 \\
7 & 0.98523 & 0.00097 & 0.99929 & 0.0007 & 0.54922 & 0.02685 \\
8 & 0.98525 & 0.00083 & 0.9995 & 0.00056 & 0.54375 & 0.01646 \\
9 & 0.98516 & 0.00085 & 0.99929 & 0.00056 & 0.54688 & 0.01712 \\
10 & 0.98545 & 0.00057 & 1.0 & 0.0 & 0.53438 & 0.01822
\end{tabular}

(c)

Table 6.50: Top-10 results for Covers80 in Experiment \#3b of the CSC problem. 


\section{YouTubeCovers}

\begin{tabular}{|c|c|c|c|}
\hline line id & length & models & classifier \\
\hline 1 & 3 & $\begin{array}{l}\text { \{pitch_contour_segmentation, octave abstraction, lcs_circular_mean }\} \\
\text { \{rhythm_histogram, bypass, cosine_distance } \\
\text { chroma }\end{array}$ & random_forest \\
\hline 2 & 3 & $\begin{array}{l}\text { \{pitch_contour_segmentation, octave abstraction, } \overline{\text { lcs }} \text { _circular_mean } \\
\{\text { chroma_stft, statistical_summarization, cosine_distance_oti }\} \\
\text { rhythm_histogram, bypass, cosine_distance }\end{array}$ & random_forest \\
\hline 3 & 3 & $\begin{array}{l}\text { \{pitch_contour_segmentation, octave_abstraction, lcs_circular_mean }\} \\
\{\text { chroma_stft, statistical_summarization, cosine_distance_oti }\} \\
\{\text { chroma-cens, statistical summarization } 2, \text { monte carlo distance }\}\end{array}$ & random_forest \\
\hline 4 & 3 & $\begin{array}{l}\text { \{pitch_contour_segmentation, octave_abstraction, } \overline{\mathrm{l}} \mathrm{cs} \text { _circular_mean } \\
\{\text { pitch_contour_segmentation, markov_chain, circular_manhattan } \\
\{\text { chroma_cens, statistical_summarization_2, monte_carlo_distance }\end{array}$ & random_forest \\
\hline 5 & 3 & $\begin{array}{l}\text { \{pitch_contour_segmentation, octave_abstraction, } \overline{\mathrm{lcs}} \text { _circular_mean }\} \\
\text { \{rhythm_histogram, bypass, cosine_distance } \\
\text { \{chroma cens, statistical summarization } 2, \text { monte carlo distance }\end{array}$ & ada_boost \\
\hline 6 & 3 & $\begin{array}{l}\text { \{itch_contour_segmentation, octave_abstraction, } \overline{\text { lcs_circular_mean }} \\
\text { \{pitch_contour_segmentation, markov_chain, circular_cosine } \\
\text { \{chroma_stft, statistical_summarization, cosine_distance_oti }\end{array}$ & random_forest \\
\hline 7 & 3 & $\begin{array}{l}\text { \{pitch_contour_segmentätion, octave_abstraction, lcs_circular_mean }\} \\
\text { \{chroma_stft, statistical_summarization, cosine_distance_oti\} } \\
\text { \{rhythm_histogram, bypass, cosine_distance }\end{array}$ & ada_boost \\
\hline 8 & 3 & $\begin{array}{l}\text { \{pitch_contour_segmentation, octave abstraction, lcs_circular_mean } \\
\text { \{chroma_stft, diff_stats_1, cosine_distance_oti } \\
\text { \{chroma_cens, statistical_summarization 2, monte carlo_distance }\end{array}$ & random_forest \\
\hline 9 & 3 & $\begin{array}{l}\text { \{pitch_contour_segmentation, octave_abstraction, } \overline{\mathrm{l}} \mathrm{cs} \text { _circular_mean } \\
\text { \{chroma_stft, diff_stats_1, cosine_distance_oti\} } \\
\text { \{chroma-cens, statistical summarization 2, monte carlo distance }\end{array}$ & ada_boost \\
\hline 10 & 3 & 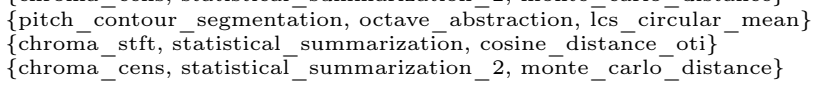 & ada_boost \\
\hline
\end{tabular}

(a)

\begin{tabular}{l|llllll} 
line id & f1-score (mean) & f1-score $(\mathrm{std})$ & precision $($ mean $)$ & precision $(\mathrm{std})$ & recall $(\mathrm{mean})$ & recall $(\mathrm{std})$ \\
\hline 1 & 0.48576 & 0.02425 & 0.82668 & 0.07015 & 0.34643 & 0.02989 \\
2 & 0.47995 & 0.02784 & 0.83543 & 0.05175 & 0.33796 & 0.02815 \\
3 & 0.47957 & 0.02361 & 0.82868 & 0.04793 & 0.33827 & 0.02356 \\
4 & 0.47918 & 0.02456 & 0.79661 & 0.11895 & 0.34806 & 0.02965 \\
5 & 0.47338 & 0.02846 & 0.86892 & 0.05007 & 0.32694 & 0.03034 \\
6 & 0.47238 & 0.0278 & 0.81299 & 0.06603 & 0.33449 & 0.02685 \\
7 & 0.46963 & 0.03598 & 0.87965 & 0.05308 & 0.32184 & 0.03395 \\
8 & 0.46948 & 0.03245 & 0.83914 & 0.05695 & 0.32694 & 0.02979 \\
9 & 0.46847 & 0.03373 & 0.86959 & 0.04984 & 0.32153 & 0.03039 \\
10 & 0.46778 & 0.03173 & 0.86596 & 0.06568 & 0.32194 & 0.03107
\end{tabular}

(b)

\begin{tabular}{l|llllll} 
line id & accuracy (mean) & accuracy $(\mathrm{std})$ & 0 's acc. (mean) & 0 's acc. (std) & 1's acc. (mean) & 1's acc. (std) \\
\hline 1 & 0.96342 & 0.00161 & 0.9959 & 0.00237 & 0.34643 & 0.02989 \\
2 & 0.96347 & 0.00147 & 0.99639 & 0.0014 & 0.33796 & 0.02815 \\
3 & 0.96334 & 0.00144 & 0.99624 & 0.0013 & 0.33827 & 0.02356 \\
4 & 0.96214 & 0.00368 & 0.99446 & 0.00475 & 0.34806 & 0.02965 \\
5 & 0.96376 & 0.00116 & 0.99727 & 0.00138 & 0.32694 & 0.03034 \\
6 & 0.96269 & 0.00206 & 0.99576 & 0.00212 & 0.33449 & 0.02685 \\
7 & 0.9638 & 0.00168 & 0.99758 & 0.00132 & 0.32184 & 0.03395 \\
8 & 0.96314 & 0.00181 & 0.99663 & 0.0013 & 0.32694 & 0.02979 \\
9 & 0.96362 & 0.00175 & 0.99741 & 0.00107 & 0.32153 & 0.03039 \\
10 & 0.96347 & 0.00187 & 0.99723 & 0.00165 & 0.32194 & 0.03107
\end{tabular}

(c)

Table 6.51: Top-10 results for YouTubeCovers in Experiment \#3b of the CSC problem. 


\section{Covers1000}

\begin{tabular}{|c|c|c|c|}
\hline line id & length & models & classifier \\
\hline 1 & 3 & $\begin{array}{l}\text { \{pitch_contour_segmentation, octave_abstraction, lcs_circular_mean }\} \\
\text { \{rhythm_histogram, bypass, cosine_distance } \\
\text { \{tonnetz, diff stats 1, manhattan distance }\end{array}$ & random_forest \\
\hline 2 & 3 & $\begin{array}{l}\text { \{pitch_contoūr_segmentation, octave abstraction, lcs_circular_mean } \\
\text { \{rhythm_histogram, bypass, cosine_distance } \\
\text { \{beat synchronous chroma cens statistical }\end{array}$ & random_forest \\
\hline 3 & 3 & $\begin{array}{l}\text { \{pitch_contour_segmentation, octave_abstraction, lcs_circular_mean\} } \\
\text { \{rhythm_histogram, bypass, cosine_distance\} } \\
\text { \{beat synchronous chroma stft, vector quantization, manhattan distance }\end{array}$ & random_forest \\
\hline 4 & 3 & $\begin{array}{l}\text { \{pitch_contour_segmentation, octave_abstraction, lcs_circular_mean } \\
\text { \{beat_synchronous_chroma_cqt, diff-stats_1, manhattan_distance_oti } \\
\text { \{rhythm histogram, bypass, cosine distance\} }\end{array}$ & random_forest \\
\hline 5 & 3 & $\begin{array}{l}\text { \{pitch_contour_segmentation, octave_abstraction, lcs_circular_mean }\} \\
\text { \{beat_synchronous_chroma_stft, vector_quantization, manhattan_distance } \\
\text { \{beat }- \text { synchronous chroma-cens, statistical summarization 2, monte carlo distance }\end{array}$ & random_forest \\
\hline 6 & 3 & $\begin{array}{l}\text { \{pitch_contour_segmentation, octave_abstraction, lcs_circulär_mean }\}^{-} \\
\text {\{rhythm_histogram, bypass, cosine distance\} } \\
\text { \{pitch_contour_segmentation, markov_chain, circular_manhattan }\end{array}$ & random_forest \\
\hline 7 & 3 & $\begin{array}{l}\text { \{pitch_contour_segmentation, octave_abstraction, lcs_circular_mean } \\
\text { \{beat_synchronous_chroma_stft, diff_-stats_1, cosine_distance_oti } \\
\text { \{beat_synchronous_chroma_cens, statistical_summarization_2, } \\
\text { [bonte_carlo_distance }\}\end{array}$ & random_forest \\
\hline 8 & 3 & $\begin{array}{l}\{\text { pitch_contour_segmentation, octave_abstraction, lcs_circular_mean }\}^{-} \\
\{\text {beat_synchronous_chroma_cens, statistical_summarization_2, monte_carlo_distance }\} \\
\text { temporal rhythm }- \text { histogram, bypass, cosine_distance }\}\end{array}$ & random_forest \\
\hline 9 & 3 & $\begin{array}{l}\text { \{pitch_contour_segmentation, octave_abstraction, lcs_circular_mean }\} \\
\{\text { beat_synchronous_chroma_cens, statistical_summarization_2, monte_carlo_distance }\} \\
\text { pitch contour segmentation, markov chain, circular manhattan }\}\end{array}$ & random_forest \\
\hline 10 & 3 & $\begin{array}{l}\text { \{pitch }{ }^{-}{ }^{-} \text {contour } \\
\text { \{tonnetz, diff_stats_1, manhattan_distance } \\
\text { \{beat_synchronous_chroma_cens, statistical_summarization_2, monte_carlo_distance }\end{array}$ & random_forest \\
\hline
\end{tabular}

(a)

\begin{tabular}{l|llllll} 
line id & f1-score (mean) & f1-score (std) & precision (mean) & precision (std) & recall (mean) & recall (std) \\
\hline 1 & 0.58666 & 0.00989 & 0.95689 & 0.01797 & 0.42313 & 0.00992 \\
2 & 0.58364 & 0.00708 & 0.95884 & 0.01848 & 0.41961 & 0.00768 \\
3 & 0.57913 & 0.00747 & 0.95357 & 0.01372 & 0.41591 & 0.00749 \\
4 & 0.57452 & 0.00856 & 0.94528 & 0.01711 & 0.41277 & 0.00845 \\
5 & 0.57434 & 0.00804 & 0.94644 & 0.01512 & 0.4123 & 0.00682 \\
6 & 0.57396 & 0.00833 & 0.9579 & 0.0173 & 0.40981 & 0.00745 \\
7 & 0.57353 & 0.00933 & 0.95497 & 0.02364 & 0.4099 & 0.00685 \\
8 & 0.57296 & 0.00671 & 0.95172 & 0.022 & 0.40999 & 0.00714 \\
9 & 0.57287 & 0.00614 & 0.96514 & 0.01853 & 0.4074 & 0.00594 \\
10 & 0.57223 & 0.00807 & 0.93664 & 0.02649 & 0.41221 & 0.00975
\end{tabular}

(b)

\begin{tabular}{l|llllll} 
line id & accuracy (mean) & accuracy (std) & 0 's acc. (mean) & 0's acc. (std) & 1's acc. (mean) & 1 's acc. (std) \\
\hline 1 & 0.99587 & $8 \mathrm{e}-05$ & 0.99987 & $6 \mathrm{e}-05$ & 0.42313 & 0.00992 \\
2 & 0.99585 & $6 \mathrm{e}-05$ & 0.99987 & $6 \mathrm{e}-05$ & 0.41961 & 0.00768 \\
3 & 0.99581 & $6 \mathrm{e}-05$ & 0.99986 & $4 \mathrm{e}-05$ & 0.41591 & 0.00749 \\
4 & 0.99576 & $7 \mathrm{e}-05$ & 0.99983 & $6 \mathrm{e}-05$ & 0.41277 & 0.00845 \\
5 & 0.99577 & $8 \mathrm{e}-05$ & 0.99984 & $5 \mathrm{e}-05$ & 0.4123 & 0.00682 \\
6 & 0.99579 & $8 \mathrm{e}-05$ & 0.99987 & $5 \mathrm{e}-05$ & 0.40981 & 0.00745 \\
7 & 0.99578 & 0.0001 & 0.99986 & $7 \mathrm{e}-05$ & 0.4099 & 0.00685 \\
8 & 0.99577 & $7 \mathrm{e}-05$ & 0.99985 & $7 \mathrm{e}-05$ & 0.40999 & 0.00714 \\
9 & 0.99579 & $6 \mathrm{e}-05$ & 0.9999 & $6 \mathrm{e}-05$ & 0.4074 & 0.00594 \\
10 & 0.99573 & $8 \mathrm{e}-05$ & 0.9998 & $9 \mathrm{e}-05$ & 0.41221 & 0.00975
\end{tabular}

(c)

Table 6.52: Top-10 results for Covers1000 in Experiment \#3b of the CSC problem. 


\section{Mazurkas}

\begin{tabular}{|c|c|c|c|}
\hline line id & length & models & classifier \\
\hline 1 & 3 & $\begin{array}{l}\text { \{pitch_contour_segmentation, octave_abstraction, levenshtein_circular_max }\} \\
\text { pitch_contour_segmentation, markov_chain, circular_manhattan }\} \\
\{\text { chroma cens, statistical summarization } 2, \text { monte carlo distance }\}\end{array}$ & random_forest \\
\hline 2 & 3 & $\begin{array}{l}\text { \{pitch_contour_segmentation, octave_abstraction, } \overline{\text { levenshtein_circular_max }} \\
\{\text { chroma_cens, statistical_summarization_2, monte_carlo_distance } \\
\text { \{melspectrogram, vector quantization, manhattan distance }\end{array}$ & random_forest \\
\hline 3 & 3 & $\begin{array}{l}\text { \{pitch_contour_segmentation, octave_abstraction, levenshtein_circular_max } \\
\text { \{chroma_cens, statistical_summarization_2, monte_carlo_distance } \\
\text { \{statistical spectrum descriptor, bypass, manhattan distance\} }\end{array}$ & random_forest \\
\hline 4 & 3 & $\begin{array}{l}\text { \{pitch_contour_segmentation, octave_abstraction, levenshtein_circular_max } \\
\text { \{chroma_cens, statistical_summarization_2, monte_carlo_distance } \\
\text { \{temporal rhythm histogram, bypass, manhattan_distance }\end{array}$ & random_forest \\
\hline 5 & 3 & $\begin{array}{l}\text { \{pitch_contour_segmentation, octave abstraction, levenshtein_circular_max }\} \\
\{\text { chroma_cens, gaussian mixture_model_default, manhattan_distance\} } \\
\text { \{chroma-cens, statistical summarization } 2 \text {, monte carlo distance }\end{array}$ & random_forest \\
\hline 6 & 3 & $\begin{array}{l}\text { \{pitch_contour_segmentation, octave_abstraction, levenshtein_circular_max } \\
\{\text { chroma_cens, statistical_summarization_2, monte_carlo_distance } \\
\text { \{mfcc, statistical_summarization, manhattan_distance }\end{array}$ & random_forest \\
\hline 7 & 3 & $\begin{array}{l}\text { \{pitch_contour_segmentation, octave_abstraction, levenshtein circular_max } \\
\{\text { chroma_cens, } \\
\text { zero_crostistical_summarization_2, monte_carlo_distance }\end{array}$ & random_forest \\
\hline 8 & 3 & $\begin{array}{l}\text { \{pitch_contour_segmentation, octave abstraction, levenshtein_circular_max } \\
\{\text { chroma_stft, statistical_summarization, cosine_distance_oti }\} \\
\text { \{chroma_cens, statistical_summarization } 2, \text { monte carlo_distance }\}\end{array}$ & random_forest \\
\hline 9 & 3 & $\begin{array}{l}\text { \{pitch_contour_segmentation, octave_abstraction, levenshtein_circular_max }\} \\
\{\text { beat_synchronous_chroma_cqt, vector_quantization, chebyshev_distance } \\
\text { \{chroma cens, statistical summarization } 2, \text { monte carlo distance }\end{array}$ & random_forest \\
\hline 10 & 3 & $\begin{array}{l}\text { \{pitch_contour_segmentation, octave_abstraction, } \overline{\text { levenshtein_circular_max }} \\
\text { \{beat_synchronous_chroma_cqt, statistical_summarization, manhattan_distance_oti }\} \\
\text { \{chroma_cens, statistical_summarization_2, monte_carlo_distance }\end{array}$ & random_forest \\
\hline
\end{tabular}

(a)

\begin{tabular}{l|llllll} 
line id & f1-score (mean) & f1-score $($ std) & precision (mean) & precision (std) & recall (mean) & recall (std) \\
\hline 1 & 0.89848 & 0.00728 & 0.9499 & 0.0107 & 0.85271 & 0.01768 \\
2 & 0.8952 & 0.00805 & 0.96593 & 0.01266 & 0.83441 & 0.01543 \\
3 & 0.89085 & 0.01256 & 0.95649 & 0.01063 & 0.83421 & 0.02521 \\
4 & 0.89037 & 0.01264 & 0.94522 & 0.0259 & 0.84251 & 0.02438 \\
5 & 0.89035 & 0.01204 & 0.96719 & 0.01589 & 0.82545 & 0.02453 \\
6 & 0.88954 & 0.01124 & 0.94116 & 0.03692 & 0.84437 & 0.01174 \\
7 & 0.88855 & 0.00354 & 0.96728 & 0.02177 & 0.82233 & 0.01641 \\
8 & 0.88799 & 0.01225 & 0.96704 & 0.01977 & 0.82169 & 0.02575 \\
9 & 0.88669 & 0.01444 & 0.97294 & 0.01948 & 0.81595 & 0.036 \\
10 & 0.88258 & 0.01713 & 0.96247 & 0.03001 & 0.81592 & 0.0263
\end{tabular}

(b)

\begin{tabular}{l|llllll} 
line id & accuracy (mean) & accuracy $(\mathrm{std})$ & 0 's acc. (mean) & 0 's acc. (std) & 1's acc. (mean) & 1's acc. (std) \\
\hline 1 & 0.98953 & 0.00066 & 0.9974 & 0.00061 & 0.85271 & 0.01768 \\
2 & 0.98936 & 0.00075 & 0.99829 & 0.00065 & 0.83441 & 0.01543 \\
3 & 0.98888 & 0.00111 & 0.9978 & 0.0006 & 0.83421 & 0.02521 \\
4 & 0.98871 & 0.00127 & 0.99713 & 0.00144 & 0.84251 & 0.02438 \\
5 & 0.9889 & 0.00112 & 0.99836 & 0.00084 & 0.82545 & 0.02453 \\
6 & 0.98857 & 0.00143 & 0.99686 & 0.00208 & 0.84437 & 0.01174 \\
7 & 0.98873 & 0.0004 & 0.99835 & 0.00115 & 0.82233 & 0.01641 \\
8 & 0.9887 & 0.00113 & 0.99835 & 0.00108 & 0.82169 & 0.02575 \\
9 & 0.98866 & 0.00117 & 0.99864 & 0.00104 & 0.81595 & 0.036 \\
10 & 0.98815 & 0.00172 & 0.9981 & 0.00162 & 0.81592 & 0.0263
\end{tabular}

(c)

Table 6.53: Top-10 results for Mazurkas in Experiment \#3b of the CSC problem.

\section{SHS9K}

Experiments for the dataset SHS9K are in progress. Table 6.54 shows the best results obtained with $\approx 20 \%$ of the classification runs obtained so far. 


\begin{tabular}{|c|c|c|c|}
\hline line id & length & models & classifier \\
\hline 1 & 2 & $\begin{array}{l}\text { \{pitch_contour_segmentation, octave abstraction, lcs_circular_mean }\} \\
\text { \{beat_synchronous chroma cqt, statistical summarization 2, monte carlo distance }\}\end{array}$ & naive_bayes \\
\hline 2 & 2 & $\begin{array}{l}\text { \{pitch_contour_segmentation, octave abstrāction, lcs_circular mean } \overline{\}} \\
\text { \{beat_synchronous chroma cens, vector quantization, manhattan distance }\end{array}$ & naive_bayes \\
\hline 3 & 2 & $\begin{array}{l}\text { \{pitch_contour_segmentation, octave abstraction, lcs_circular_meān } \\
\text { \{tonnetz, vector quantization, manhattan distance }\end{array}$ & naive_bayes \\
\hline 4 & 2 & $\begin{array}{l}\text { \{pitch_contour_segmentation, octave abstraction, lcs_circular_mean } \\
\text { \{beat_synchronous chroma cqt, diff stats 1, manhattan distance oti }\end{array}$ & naive_bayes \\
\hline 5 & 2 & $\begin{array}{l}\left\{\text { pitch_contour_segmentation, octave }{ }^{-} \text {abstraction, lcs_circular_mean }\right\} \\
\text { \{rhythm_histogram, bypass, cosine_distance }\end{array}$ & naive_bayes \\
\hline 6 & 2 & $\begin{array}{l}\text { \{pitch_contour_segmentation, octave_abstraction, lcs_circular_mean } \\
\{\text { beat_synchronous_chroma_cqt, diff_stats_1, cosine_distance_oti\} }\end{array}$ & naive_bayes \\
\hline 7 & 2 & $\begin{array}{l}\text { \{pitch_contour_segmentation, octave abstraction, lcs_circular }{ }^{-} \text {mean } \\
\text { \{beat_synchronous_chroma_cqt, statistical_summarization, manhattan_distance_oti\} }\end{array}$ & naive_bayes \\
\hline 8 & 2 & $\begin{array}{l}\text { \{pitch_contour_segmentation, octave_abstrāction, lcs_circular_mean }{ }^{-} \\
\text {\{mfcc, diff_stats_2, manhattan_distance\} }\end{array}$ & naive_bayes \\
\hline 9 & 2 & $\begin{array}{l}\text { \{pitch_contour_segmentation, octave_abstraction, lcs_circular_mean } \\
\text { \{pitch_contour_segmentation, markov_chain, circular_cosine }\end{array}$ & naive_bayes \\
\hline 10 & 2 & $\begin{array}{l}\text { \{pitch_contour_segmentation, octave_abstraction, lcs_circular_mean }\} \\
\text { \{temporal_rhythm_histogram, bypass, manhattan_distance }\end{array}$ & naive_bayes \\
\hline
\end{tabular}

\begin{tabular}{l|llllll}
\multicolumn{1}{l}{ line id } & f1-score (mean) & f1-score $($ std) & precision (mean) & $\begin{array}{c}(\mathbf{a}) \\
\text { precision (std) }\end{array}$ & recall (mean) & recall (std) \\
\hline 1 & 0.03317 & 0.00072 & 0.04586 & 0.00341 & 0.02612 & 0.00142 \\
2 & 0.03303 & 0.00086 & 0.05709 & 0.00898 & 0.02356 & 0.00167 \\
3 & 0.03247 & 0.00074 & 0.05777 & 0.00834 & 0.02285 & 0.00161 \\
4 & 0.03236 & 0.00087 & 0.05597 & 0.00989 & 0.02325 & 0.00232 \\
5 & 0.03233 & 0.00063 & 0.05248 & 0.00527 & 0.02351 & 0.00112 \\
6 & 0.03212 & 0.00056 & 0.0532 & 0.00374 & 0.02309 & 0.00102 \\
7 & 0.03209 & 0.00035 & 0.0535 & 0.00954 & 0.02334 & 0.00173 \\
8 & 0.03175 & 0.00053 & 0.05119 & 0.00642 & 0.02326 & 0.00148 \\
9 & 0.03158 & 0.00052 & 0.05863 & 0.00926 & 0.02187 & 0.00126 \\
10 & 0.03147 & 0.00064 & 0.05412 & 0.00733 & 0.02246 & 0.00176
\end{tabular}

(b)

\begin{tabular}{l|llllll} 
line id & accuracy (mean) & accuracy (std) & 0's acc. (mean) & 0's acc. (std) & 1's acc. (mean) & 1's acc. (std) \\
\hline 1 & 0.97224 & 0.00127 & 0.98981 & 0.00132 & 0.02612 & 0.00142 \\
2 & 0.97486 & 0.00154 & 0.99253 & 0.0016 & 0.02356 & 0.00167 \\
3 & 0.97519 & 0.00137 & 0.99288 & 0.00142 & 0.02285 & 0.00161 \\
4 & 0.97468 & 0.00204 & 0.99235 & 0.00212 & 0.02325 & 0.00232 \\
5 & 0.97435 & 0.00106 & 0.99201 & 0.0011 & 0.02351 & 0.00112 \\
6 & 0.97464 & 0.00079 & 0.99231 & 0.00083 & 0.02309 & 0.00102 \\
7 & 0.97435 & 0.00179 & 0.992 & 0.00186 & 0.02334 & 0.00173 \\
8 & 0.97413 & 0.00161 & 0.99179 & 0.00167 & 0.02326 & 0.00148 \\
9 & 0.97554 & 0.00144 & 0.99325 & 0.0015 & 0.02187 & 0.00126 \\
10 & 0.97481 & 0.0016 & 0.99249 & 0.00166 & 0.02246 & 0.00176
\end{tabular}

(c)

Table 6.54: Top-10 results for SHS9K in Experiment \#3b of the CSC problem.

\subsubsection{Discussion: experiment \#3b}

\section{Best results}

The best results obtained in experiment $\# 3 \mathrm{~b}$ are as follows: mean f1-score $=0.89848$ for the Mazurkas dataset using random forest; $\mathrm{f} 1=0.70709$ for Covers 80 using naive Bayes; $\mathrm{f} 1=0.58666$ for Covers1000 using random forest; $\mathrm{f} 1=0.48576$ for YouTubeCovers using random forest, all obtained with 3 fused models. Inspecting the models that are fused, we find two models for Mazurkas using Pitch Contour Segmentation and the third with CENS; all three models for Covers 80 use chromagrams; models for Covers1000 use Pitch Contour Segmentation, Rhythm Histogram and Tonnetz; models for YouTubeCovers use Pitch Contour Segmentation, Rhythm Histogram and CENS.

The models for Covers1000 and YouTubeCovers are very unique since they blend tonal features of two different types with a rhythmic feature. Consulting Appendix $\mathrm{C}$ we can further observe that the model that uses Rhythm Histogram is the fourth in the list of best models for Covers1000, while the next model using Rhythm Histogram appears as the 53rd in the list for YouTubeCovers. Two conclusions may be drawn from these observations: features that are not tonal as well as features that are not necessarily high ranked may produce good results for the CSC problem. This is a point in favor of using the proposed filtered models for CSC. 


\section{Comparative results}

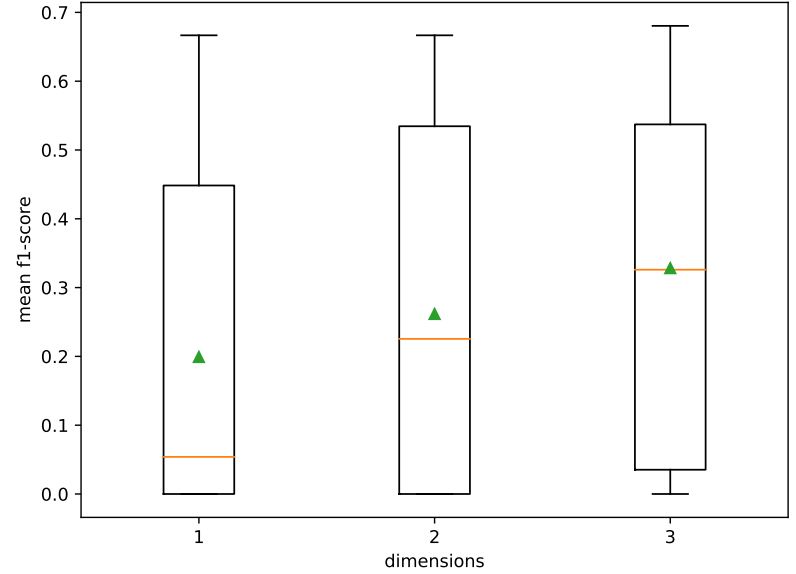

(a)

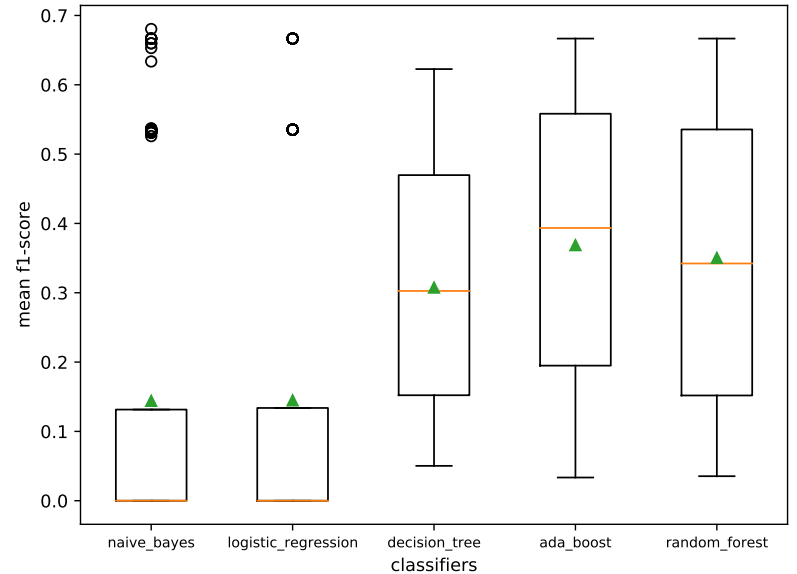

(b)

Figure 6.13: Results for experiment \#3b, summarized by dimension (a) and by classifier (b).

Figure 6.13a shows us a summarization of all classification results by dimension, i.e., the number of models fused. It is clear that the mean (or median) f1-score increases for larger dimensions, which underlines the relevance of the proposed filtered models. The results summarized by classifier are shown in Figure 6.13b, and again both random forest and ada boost appear as the best classifiers. Comparing it with Experiment $\# 3 \mathrm{a}$, the most prominent difference is observed with logistic regression, which was the third best in the previous experiment, but now dropped to the last position alongside naive bayes.

\begin{tabular}{lll} 
dataset & best mean f1-score (experiment \#3a) & best mean f1-score (experiment \#3b) \\
\hline Covers80 & 0.70751 & 0.70709 \\
YouTubeCovers & 0.48029 & 0.48576 \\
Covers1000 & 0.57966 & 0.58666 \\
Mazurkas & 0.91457 & 0.89848 \\
SHS9K & 0.08792 & 0.03317
\end{tabular}

Table 6.55: Best mean f1-score for all datasets in experiments \#3a and \#3b.

Finally, we also compare experiments \#3a and \#3b in terms of their best results. Table 6.55 highlights the best mean f1-score for all datasets in both experiments (considering the partial results for SHS9K). These results are quite similar, indicating that the two approaches to fuse models (using plain Top-N lists or filtering out equivalent models) are equally promising. However, fused models in experiment \#3b use samples with much smaller dimensions, another finding that favors the use of filtered models. Considering that we stop the combination of models at length 3 , and larger dimension values $(4,5$, etc) can still be tested, we strongly recommend skipping equivalent models, instead of using the original Top- $\mathrm{N}$ lists, in the process of fusing similarity models within the CSC problem.

\subsection{Experiment \#4: Cover Song Identification and Classification}

\subsubsection{Experiment design}

This experiment exploits the CSC problem in order to return to the CSI problem. Every trained classifier can return values between 0 and 1 (corresponding to a likelihood of a pair of songs being versions) instead of values equal to 0 or 1 (that say if the pair of songs are versions of each other 
or not). By gathering this information for all possible pairs of songs from a dataset it is possible to build a brand new similarity matrix, and compute novel CSI metrics. Considering that, the main goal of Experiment \#4 is to check if we can get better CSI results using the matrices manufactured by CSC runs.

The classification settings are very similar to the ones in experiment $\# 3$, with the following important differences: cross-dataset classification is performed, an unique list of music similarity models is created, and the train sets are balanced to have the same number of 0 's and 1's samples (all details are explained in Section 5.2). Moreover, since it is a good practice in machine learning to have the train set larger than the test set, we only consider pairs of datasets for experiment \#4 when the train dataset has more tracks than the test dataset, as listed below:

\begin{tabular}{ll} 
test dataset & train datasets \\
\hline Covers80 & YouTubeCovers; Covers1000; Mazurkas; SHS9K \\
YouTubeCovers & Covers1000; Mazurkas; SHS9K \\
Covers1000 & Mazurkas; SHS9K \\
Mazurkas & SHS9K
\end{tabular}

Table 6.56: List of all considered train and test datasets for Experiment \#4.

Experiment \#4 is also divided in two: \#4a uses the Top- $N$ best models ( $N$ equals to $10,20,30$, 40, and 50), and \#4b uses the filtered models. From Table 6.56 it is possible to count 10 pairs of classification datasets. This makes experiment \#4 ten times larger than experiment \#3. Experiment \#4a has 10 pairs of datasets, 5 classifiers, 5 dimension values, 10 sample batches randomly varying the 0's samples, resulting in 2,500 classifications runs. Experiment \#4b has 10 pairs of datasets, 5 classifiers, 696 possible combinations of filtered models to produce the samples, 10 random batches of samples, resulting in 348,000 classifications runs.

This Section shows the Top-10 results for each dataset, Appendix D shows the unique Top-50 list, and Appendix E the unique filtered list. The entire list of classification results is published in https://rppbodo.github.io/phd/experiment_4.html. 


\subsubsection{Results: experiment \#4a}

\section{Covers 80}

\begin{tabular}{l|lllllll} 
line id & train dataset & dimension & classifier & MR (mean) & MR (std) & MRR (mean) & MRR (std) \\
\hline 1 & covers1000 & 50 & naive_bayes & 33.97812 & 0.06698 & 0.28545 & 0.00179 \\
2 & covers1000 & 40 & naive_bayes & 34.64375 & 0.03412 & 0.27822 & 0.00269 \\
3 & youtubecovers & 40 & naive_bayes & 34.93875 & 0.16623 & 0.27856 & 0.00232 \\
4 & youtubecovers & 50 & naive_bayes & 34.98062 & 0.46308 & 0.27935 & 0.00601 \\
5 & youtubecovers & 10 & naive_bayes & 35.1275 & 0.03793 & 0.24055 & 0.00157 \\
6 & covers1000 & 50 & logistic_regression & 35.18938 & 0.199 & 0.28261 & 0.00346 \\
7 & covers1000 & 30 & naive_bayes & 35.34 & 0.03625 & 0.25136 & 0.00262 \\
8 & youtubecovers & 30 & naive_bayes & 35.57625 & 0.32109 & 0.2622 & 0.00379 \\
9 & shs9k & 50 & naive_bayes & 35.7075 & 0.02179 & 0.24941 & 0.00033 \\
10 & shs9k & 40 & naive_bayes & 35.77813 & 0.02742 & 0.24354 & 0.00202
\end{tabular}

(a)

\begin{tabular}{l|llllll} 
line id & MDR (mean) & MDR (std) & MAP (mean) & MAP (std) & Top-1 (mean) & Top-1 (std) \\
\hline 1 & 16.25 & 0.51235 & 0.28545 & 0.00179 & 34.8 & 0.6 \\
2 & 16.15 & 0.22913 & 0.27822 & 0.00269 & 33.8 & 0.87178 \\
3 & 16.1 & 0.3 & 0.27856 & 0.00232 & 33.5 & 0.67082 \\
4 & 17.15 & 0.50249 & 0.27935 & 0.00601 & 31.9 & 1.13578 \\
5 & 18.1 & 0.4899 & 0.24055 & 0.00157 & 26.6 & 0.4899 \\
6 & 14.25 & 1.00623 & 0.28261 & 0.00346 & 34.1 & 0.83066 \\
7 & 17.0 & 0.0 & 0.25136 & 0.00262 & 29.2 & 0.9798 \\
8 & 15.55 & 0.41533 & 0.2622 & 0.00379 & 30.8 & 0.0 \\
9 & 18.3 & 0.24495 & 0.24941 & 0.00033 & 29.0 & 0.45826 \\
10 & 18.1 & 0.43589 & 0.24354 & 0.00202 & 28.3 &
\end{tabular}

(b)

\begin{tabular}{l|llllll} 
line id & Top-10 (mean) & Top-10 (std) & Top-100 (mean) & Top-100 (std) & Top-1000 (mean) & Top-1000 (std) \\
\hline 1 & 62.8 & 0.4 & 145.0 & 0.0 & 160.0 & 0.0 \\
2 & 59.0 & 0.0 & 145.0 & 0.0 & 160.0 & 0.0 \\
3 & 62.7 & 0.64031 & 143.7 & 1.00499 & 160.0 & 0.0 \\
4 & 63.0 & 1.61245 & 144.0 & 1.09545 & 160.0 & 0.0 \\
5 & 63.0 & 0.89443 & 141.7 & 0.9 & 160.0 & 0.0 \\
6 & 72.5 & 1.62788 & 141.1 & 1.04403 & 160.0 & 0.0 \\
7 & 61.0 & 0.44721 & 144.7 & 0.45826 & 160.0 & 0.0 \\
8 & 61.0 & 0.63246 & 141.7 & 0.64031 & 160.0 & 0.0 \\
9 & 59.1 & 0.3 & 145.0 & 0.0 & 160.0 & 0.0 \\
10 & 56.7 & 0.78102 & 145.0 & 0.0 & 160.0 & 0.0
\end{tabular}

(c)

Table 6.57: Top-10 results for Covers80 to Experiment \#4a.

\section{YouTubeCovers}

\begin{tabular}{l|lllllll} 
line id & train dataset & dimension & classifier & MR (mean) & MR (std) & MRR (mean) & MRR (std) \\
\hline 1 & covers1000 & 40 & naive_bayes & 13.42257 & 0.04173 & 0.50354 & 0.00152 \\
2 & covers1000 & 50 & naive_bayes & 13.79743 & 0.03304 & 0.48866 & 0.00165 \\
3 & mazurkas & 20 & ada_boost & 13.85914 & 0.35326 & 0.44791 & 0.01374 \\
4 & covers1000 & 30 & naive_bayes & 14.01743 & 0.02389 & 0.49034 & 0.00194 \\
5 & mazurkas & 20 & random_forest & 14.04343 & 0.33195 & 0.44897 & 0.01022 \\
6 & covers1000 & 40 & logistic_regression & 14.11686 & 0.19979 & 0.49318 & 0.00408 \\
7 & shs9k & 40 & naive_bayes & 14.16543 & 0.01763 & 0.48278 & 0.00059 \\
8 & shs9k & 50 & naive_bayes & 14.20629 & 0.02359 & 0.47954 & 0.00077 \\
9 & covers1000 & 40 & random_forest & 14.20857 & 0.80384 & 0.48012 & 0.01119 \\
10 & shs9k & 30 & naive_bayes & 14.46914 & 0.02324 & 0.47304 & 0.00011
\end{tabular}

(a)

\begin{tabular}{l|llllll} 
line id & MDR (mean) & MDR (std) & MAP (mean) & MAP (std) & Top-1 (mean) & Top-1 (std) \\
\hline 1 & 3.0 & 0.0 & 0.21716 & 0.00041 & 138.4 & 1.11355 \\
2 & 3.0 & 0.0 & 0.21384 & 0.00047 & 132.2 & 1.32665 \\
3 & 3.7 & 0.45826 & 0.19596 & 0.00404 & 115.5 & 7.76209 \\
4 & 3.0 & 0.0 & 0.20772 & 0.00058 & 134.9 & 0.9434 \\
5 & 3.7 & 0.45826 & 0.1939 & 0.00261 & 115.6 & 4.84149 \\
6 & 3.0 & 0.0 & 0.2188 & 0.00245 & 136.5 & 2.15639 \\
7 & 3.4 & 0.2 & 0.20693 & 0.00017 & 133.1 & 0.3 \\
8 & 3.0 & 0.0 & 0.20695 & 0.00016 & 130.0 & 0.0 \\
9 & 3.15 & 0.32016 & 0.202 & 0.00441 & 131.4 & 5.55338 \\
10 & 4.0 & 0.0 & 0.19993 & 0.0001 & 130.0 & 0.0
\end{tabular}

(b)

\begin{tabular}{l|llllll} 
line id & Top-10 (mean) & Top-10 (std) & Top-100 (mean) & Top-100 (std) & Top-1000 (mean) & Top-1000 (std) \\
\hline 1 & 258.9 & 0.53852 & 345.0 & 0.0 & 350.0 & 0.0 \\
2 & 254.3 & 1.9 & 343.9 & 0.3 & 350.0 & 0.0 \\
3 & 242.4 & 3.72022 & 343.9 & 1.22066 & 350.0 & 0.0 \\
4 & 252.6 & 1.35647 & 343.7 & 0.64031 & 350.0 & 0.0 \\
5 & 239.0 & 5.76194 & 344.4 & 1.35647 & 350.0 & 0.0 \\
6 & 245.4 & 2.2 & 344.3 & 0.78102 & 350.0 & 0.0 \\
7 & 249.0 & 0.0 & 344.0 & 0.44721 & 350.0 & 0.0 \\
8 & 249.8 & 0.4 & 344.0 & 0.0 & 350.0 & 0.0 \\
9 & 243.6 & 6.43739 & 342.2 & 1.66132 & 350.0 & 0.0 \\
10 & 244.1 & 0.3 & 342.0 & 0.0 & 350.0 & 0.0
\end{tabular}

(c)

Table 6.58: Top-10 results for YouTubeCovers to Experiment \#4a. 


\section{Covers1000}

\begin{tabular}{l|lllllll} 
line id & train dataset & dimension & classifier & MR (mean) & MR (std) & MRR (mean) & MRR (std) \\
\hline 1 & shs9k & 50 & naive_bayes & 146.5084 & 0.06913 & 0.17431 & 0.00068 \\
2 & shs9k & 40 & naive_bayes & 148.0769 & 0.07151 & 0.18237 & 0.00046 \\
3 & mazurkas & 20 & logistic_regression & 151.2592 & 0.24362 & 0.14936 & 0.00092 \\
4 & shs9k & 30 & naive_bayes & 152.7983 & 0.05249 & 0.17726 & 0.00013 \\
5 & mazurkas & 20 & ada_boost & 155.75 & 1.88268 & 0.13797 & 0.00617 \\
6 & mazurkas & 20 & random_forest & 158.4418 & 2.60728 & 0.11805 & 0.00392 \\
7 & shs9k & 20 & naive_bayes & 158.8768 & 0.09422 & 0.15025 & 0.00019 \\
8 & mazurkas & 10 & ada_boost & 162.3155 & 2.08897 & 0.13362 & 0.00717 \\
9 & shs9k & 10 & naive_bayes & 164.3159 & 0.04239 & 0.1561 & 0.00027 \\
10 & mazurkas & 10 & logistic_regression & 164.5202 & 0.08982 & 0.14963 & 0.00048
\end{tabular}

(a)

\begin{tabular}{l|llllll} 
line id & MDR (mean) & MDR (std) & MAP (mean) & MAP (std) & Top-1 (mean) & Top-1 (std) \\
\hline 1 & 42.35 & 0.32016 & 0.12918 & 0.00042 & 103.9 & 1.51327 \\
2 & 46.1 & 0.2 & 0.13363 & 0.00031 & 120.2 & 0.6 \\
3 & 59.85 & 0.22913 & 0.10694 & 0.00074 & 85.7 & 1.18743 \\
4 & 55.55 & 0.15 & 0.12952 & $6 \mathrm{e}-05$ & 121.0 & 0.0 \\
5 & 60.35 & 3.42819 & 0.09908 & 0.00631 & 76.6 & 8.45222 \\
6 & 65.55 & 2.8058 & 0.08357 & 0.00413 & 60.4 & 4.75815 \\
7 & 64.1 & 0.43589 & 0.10734 & 0.0001 & 96.9 & 0.3 \\
8 & 69.15 & 3.3989 & 0.09524 & 0.00476 & 73.5 & 6.97496 \\
9 & 59.4 & 0.3 & 0.11227 & 0.00025 & 100.8 & 0.4 \\
10 & 68.0 & 0.54772 & 0.11009 & 0.0004 & 95.1 & 0.7
\end{tabular}

(b)

\begin{tabular}{l|llllll} 
line id & Top-10 (mean) & Top-10 (std) & Top-100 (mean) & Top-100 (std) & Top-1000 (mean) & Top-1000 (std) \\
\hline 1 & 311.0 & 0.44721 & 615.2 & 0.6 & 1000.0 & 0.0 \\
2 & 295.9 & 1.22066 & 619.8 & 1.249 & 1000.0 & 0.0 \\
3 & 270.3 & 0.78102 & 599.5 & 1.28452 & 1000.0 & 0.0 \\
4 & 288.9 & 0.3 & 592.3 & 0.45826 & 1000.0 & 0.0 \\
5 & 261.8 & 8.61162 & 597.6 & 6.46838 & 1000.0 & 0.0 \\
6 & 223.8 & 5.86174 & 584.6 & 8.89044 & 1000.0 & 0.0 \\
7 & 267.5 & 0.5 & 591.6 & 0.8 & 1000.0 & 0.0 \\
8 & 244.9 & 8.45518 & 578.6 & 6.28013 & 1000.0 & 0.0 \\
9 & 258.2 & 0.6 & 595.4 & 0.66332 & 1000.0 & 0.0 \\
10 & 250.6 & 1.49666 & 571.2 & 1.46969 & 1000.0 & 0.0
\end{tabular}

(c)

Table 6.59: Top-10 results for Covers 1000 to Experiment \#4a.

\section{Mazurkas}

\begin{tabular}{l|lllllll} 
line id & train dataset & dimension & classifier & MR (mean) & MR (std) & MRR (mean) & MRR (std) \\
\hline 1 & shs9k & 40 & naive_bayes & 3.99186 & 0.01127 & 0.92073 & 0.00125 \\
2 & shs9k & 50 & naive_bayes & 4.06895 & 0.01931 & 0.91092 & 0.00295 \\
3 & shs9k & 30 & naive_bayes & 4.06899 & 0.00264 & 0.91694 & 0.00033 \\
4 & shs9k & 20 & naive_bayes & 4.34174 & 0.00247 & 0.88371 & 0.00031 \\
5 & shs9k & 10 & naive_bayes & 4.3483 & 0.00235 & 0.87817 & 0.00021 \\
6 & shs9k & 10 & ada_boost & 16.28179 & 2.61392 & 0.7186 & 0.01662 \\
7 & shs9k & 50 & random_forest & 27.99208 & 6.54633 & 0.23056 & 0.02339 \\
8 & shs9k & 40 & random_forest & 32.92525 & 6.68131 & 0.20585 & 0.02657 \\
9 & shs9k & 10 & logistic_regression & 37.11022 & 0.9395 & 0.26813 & 0.00619 \\
10 & shs9k & 10 & random_forest & 38.40423 & 13.84079 & 0.17541 & 0.03637
\end{tabular}

(a)

\begin{tabular}{l|llllll} 
line id & MDR (mean) & MDR (std) & MAP (mean) & MAP (std) & Top-1 (mean) & Top-1 (std) \\
\hline 1 & 1.0 & 0.0 & 0.44795 & 0.00135 & 2419.5 & 5.55428 \\
2 & 1.0 & 0.0 & 0.46185 & 0.00172 & 2380.7 & 12.9387 \\
3 & 1.0 & 0.0 & 0.42365 & 0.0004 & 2405.8 & 1.66132 \\
4 & 1.0 & 0.0 & 0.37273 & 0.00028 & 2277.8 & 1.53623 \\
5 & 1.0 & 0.0 & 0.36039 & 0.0007 & 2254.8 & 1.249 \\
6 & 1.0 & 0.0 & 0.15394 & 0.00788 & 1807.6 & 43.26708 \\
7 & 14.3 & 3.68917 & 0.04349 & 0.00431 & 371.2 & 38.98153 \\
8 & 17.1 & 3.93573 & 0.04268 & 0.00495 & 323.7 & 50.68343 \\
9 & 10.6 & 0.4899 & 0.08062 & 0.00195 & 424.2 & 11.94822 \\
10 & 22.3 & 9.74731 & 0.04108 & 0.00572 & 253.3 & 61.62637
\end{tabular}

(b)

\begin{tabular}{l|llllll} 
line id & Top-10 (mean) & Top-10 (std) & Top-100 (mean) & Top-100 (std) & Top-1000 (mean) & Top-1000 (std) \\
\hline 1 & 2685.7 & 0.9 & 2726.0 & 0.0 & 2740.0 & 0.0 \\
2 & 2683.2 & 1.4 & 2725.3 & 0.45826 & 2740.0 & 0.0 \\
3 & 2679.0 & 0.0 & 2725.0 & 0.0 & 2740.0 & 0.0 \\
4 & 2662.9 & 0.7 & 2724.6 & 0.4899 & 2740.0 & 0.0 \\
5 & 2657.0 & 0.0 & 2726.0 & 0.0 & 2740.0 & 0.0 \\
6 & 2234.7 & 52.84515 & 2616.2 & 26.06837 & 2740.0 & 0.44721 \\
7 & 1191.5 & 141.44981 & 2592.0 & 78.01538 & 2741.0 & 0.0 \\
8 & 1069.0 & 147.85331 & 2536.7 & 76.17749 & 2741.0 & 0.0 \\
9 & 1361.2 & 24.95115 & 2472.1 & 8.6539 & 2737.3 & 0.45826 \\
10 & 943.1 & 207.5902 & 2486.7 & 178.39566 & 2741.0 & 0.0
\end{tabular}

(c)

Table 6.60: Top-10 results for Mazurkas to Experiment \#4a. 


\subsubsection{Discussion: experiment \#4a}

\section{Best results}

Covers80 gets the best mean MR from the classification done by naive Bayes being trained with Covers1000 and using the Top-50 models. YouTubeCovers has the best result by the classification with naive Bayes also, train dataset as Covers1000, and the samples being produced with the Top40 models. The best mean MR for Covers1000 is obtained with naive Bayes classification trained with SHS9K and using Top-50 models. Lastly, Mazurkas produce the best result with a naive Bayes model trained with SHS9K and with 40-dimensional samples. All best results are obtained using naive Bayes, so this suggests that this classifier has good potential for the task of this experiment.

Regarding the actual values of mean MR from the best results we have: 3.99186 for Mazurkas, 13.42257 for YouTubeCovers, 33.97812 for Covers80, and 146.5084 for Covers1000. The order is the same we had in Experiment $\# 2$, reflecting the intrinsic difficulty of retrieving versions from each dataset.

\section{Comparative results}

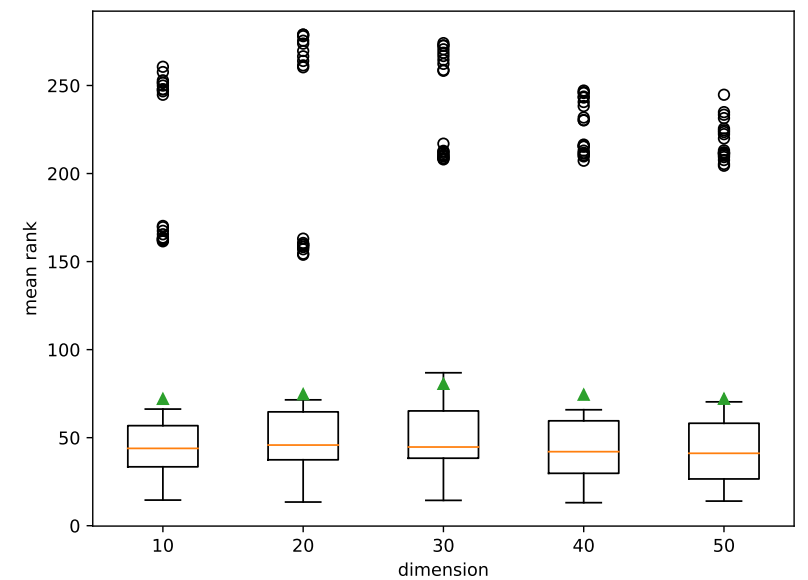

(a)

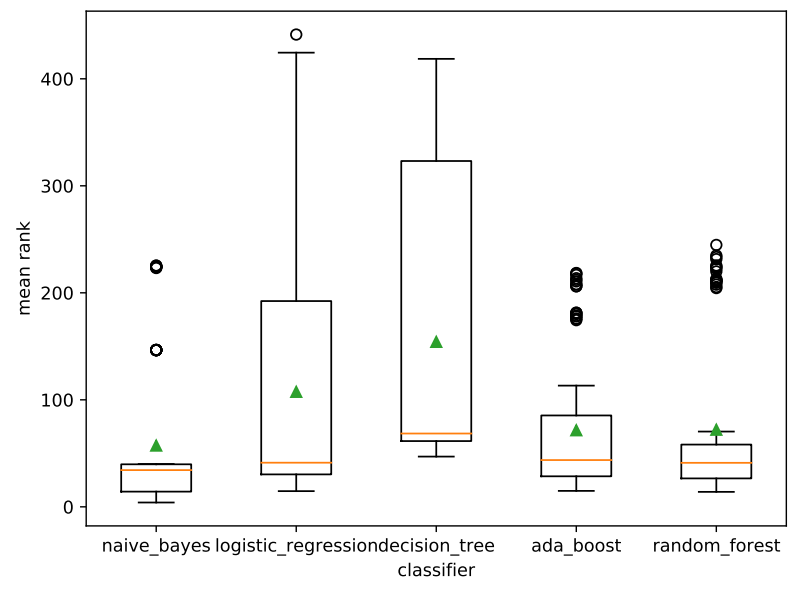

(b)

Figure 6.14: Summarized results to experiment \#4a by dimension (a), and by classifier (b).

Analysing results beyond the Top-10, we can see in Figure $6.14 \mathrm{a}$ that the quality of the experiments do not grow up as the dimension gets higher. This is also observed in Experiment \#3a, so our hypothesis that fusing similar information does not actually help in the context of these experiments gets confirmed.

Figure $6.14 \mathrm{~b}$ shows us that naive Bayes is indeed the best performing classifier for this specific experiment. Random forest also has good average performance, and this was also observed in experiment $\# 3 \mathrm{a}$, so it is safe to consider it a robust classifier in the context of our datasets and our experiments.

\subsubsection{Results: experiment \#4b}

While analysing the following results, be aware that the classification runs done with SHS9K as training dataset are still running to this date $(\approx 35 \%$ is finished). So Tables 6.61 to 6.64 display the current partial results. 


\section{Covers 80}

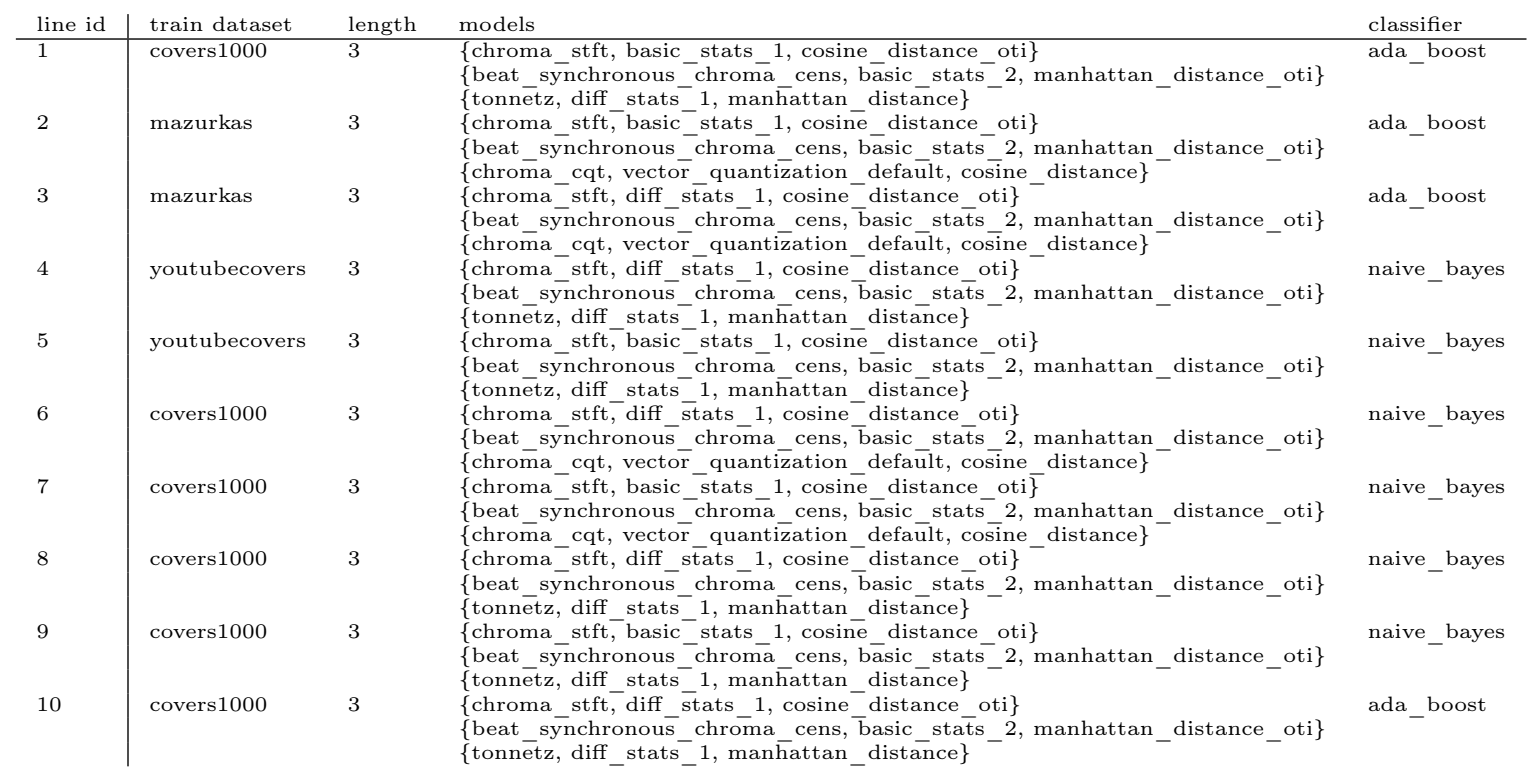

(a)

\begin{tabular}{|c|c|c|c|c|c|c|c|c|}
\hline \\
\hline line id & MR (mean) & MR (std) & MRR (mean) & MRR (std) & MDR (mean) & MDR (std) & MAP (mean) & MAP (std) \\
\hline 1 & 33.33313 & 1.28237 & 0.2385 & 0.01361 & 19.4 & 1.57797 & 0.2385 & 0.01361 \\
\hline 2 & 33.37063 & 0.53799 & 0.27669 & 0.00578 & 19.0 & 1.18322 & 0.27669 & 0.00578 \\
\hline 3 & 33.37438 & 0.64621 & 0.27402 & 0.00556 & 17.7 & 1.07703 & 0.27402 & 0.00556 \\
\hline 4 & 33.48 & 0.17184 & 0.26881 & 0.0041 & 13.75 & 0.40311 & 0.26881 & 0.0041 \\
\hline 5 & 33.53375 & 0.12432 & 0.26831 & 0.00399 & 14.7 & 0.5099 & 0.26831 & 0.00399 \\
\hline 6 & 33.655 & 0.11812 & 0.26308 & 0.00168 & 17.25 & 0.25 & 0.26308 & 0.00168 \\
\hline 7 & 33.83375 & 0.0738 & 0.26111 & 0.00115 & 19.0 & 0.22361 & 0.26111 & 0.00115 \\
\hline 8 & 33.97812 & 0.02728 & 0.27669 & 0.00206 & 15.0 & 0.3873 & 0.27669 & 0.00206 \\
\hline 9 & 34.0225 & 0.09451 & 0.27478 & 0.00113 & 15.35 & 0.39051 & 0.27478 & 0.00113 \\
\hline 10 & 34.11688 & 1.58241 & 0.22903 & 0.02695 & 18.6 & 2.36432 & 0.22903 & 0.02695 \\
\hline
\end{tabular}

(b)

\begin{tabular}{|c|c|c|c|c|c|c|c|c|}
\hline line id & Top-1 (mean) & Top-1 (std) & Top-10 (mean) & Top-10 (std) & Top-100 (mean) & Top-100 (std) & Top-1000 (mean) & Top-1000 (std) \\
\hline 1 & 24.1 & 2.62488 & 64.6 & 1.90788 & 147.8 & 2.4 & 160.0 & 0.0 \\
\hline 2 & 31.9 & 0.7 & 65.7 & 1.9 & 147.2 & 0.9798 & 160.0 & 0.0 \\
\hline 3 & 31.8 & 1.07703 & 65.3 & 1.34536 & 147.0 & 1.18322 & 160.0 & 0.0 \\
\hline 4 & 31.3 & 0.9 & 66.3 & 1.48661 & 142.3 & 0.64031 & 160.0 & 0.0 \\
\hline 5 & 31.0 & 0.89443 & 68.2 & 1.07703 & 142.6 & 0.66332 & 160.0 & 0.0 \\
\hline 6 & 31.2 & 0.4 & 63.0 & 0.63246 & 148.1 & 0.3 & 160.0 & 0.0 \\
\hline 7 & 31.2 & 0.4 & 62.5 & 0.92195 & 147.0 & 0.0 & 160.0 & 0.0 \\
\hline 8 & 32.9 & 0.53852 & 68.6 & 0.4899 & 146.9 & 1.04403 & 160.0 & 0.0 \\
\hline 9 & 32.3 & 0.45826 & 67.0 & 0.63246 & 145.8 & 0.6 & 160.0 & 0.0 \\
\hline 10 & 22.2 & 5.34416 & 64.5 & 4.34166 & 145.5 & 2.33452 & 160.0 & 0.0 \\
\hline
\end{tabular}

(c)

Table 6.61: Top-10 results for Covers80 to Experiment \#4b. 


\section{YouTubeCovers}

\begin{tabular}{|c|c|c|c|c|}
\hline line id & train dataset & length & models & classifier \\
\hline 1 & covers 1000 & 3 & $\begin{array}{l}\text { \{pitch_contour_segmentation, octave abstraction, lcs_circular_mean }\} \\
\text { \{beat_synchronous_chroma_cens, statistical_summarization_2, monte_carlo_distance }\} \\
\text { \{pitch contour segmentation, markov chain, circular manhattan }\}\end{array}$ & logistic_regression \\
\hline 2 & mazurkas & 3 & 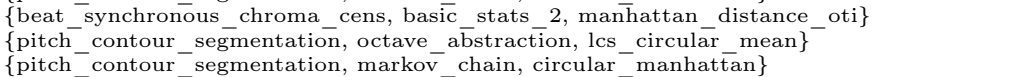 & logistic_regression \\
\hline 3 & covers 1000 & 3 & $\begin{array}{l}\text { \{pitch_contour_segmentation, octave_abstraction, lcs_circular_mean } \\
\text { \{beat_synchronous_chroma_cens, statistical_summarization_2, monte_carlo_distance }\} \\
\text { \{rms, diff stats 1, cosine distance }\end{array}$ & logistic_regression \\
\hline 4 & covers 1000 & 3 & $\begin{array}{l}\text { pitch_contour_segmentation, octave abstraction, lcs_circular_mean } \\
\text { \{beat_synchronous_chroma_cens, statistical_summarization_2, monte_carlo_distance } \\
\text { \{mfcc, statistical_summarization_2, monte_carlo_distance }\end{array}$ & logistic_regression \\
\hline 5 & mazurkas & 3 & $\begin{array}{l}\text { \{chroma_cqt, vector_quantization_default, cosine_distance }\} \\
\text { \{pitch_contour_segmentation, octave_abstraction, lcs_circular_mean } \\
\text { \{pitch }\end{array}$ & logistic_regression \\
\hline 6 & covers 1000 & 2 & $\begin{array}{l}\{\text { pitch_contour_segmentation, octave_abstraction, lcs_circular_mean } \\
\text { \{beat_synchronous_chroma_cens, statistical_summarization_2, monte_carlo_distance }\}\end{array}$ & logistic_regression \\
\hline 7 & mazurkas & 3 & $\begin{array}{l}\text { \{pitch_contour_segmentation, octave_abstraction, lcs_circular_mean }\}^{-} \\
\text {\{chroma_stft, gaussian_mixture_model_default, manhattan_distance\} } \\
\text { \{pitch_contour_segmentation, markov_chain, circular_manhattan\} }\end{array}$ & logistic_regression \\
\hline 8 & covers 1000 & 3 & $\begin{array}{l}\{\text { beat_synchronous_chroma_cens, basic_stats_2, manhattan_distance_oti }\} \\
\text { \{pitch_contour_segmentation, octave_abstraction, lcs_circular_mean } \\
\text { \{pitch_contour_segmentation, markov_chain, circular_manhattan }\}\end{array}$ & logistic_regression \\
\hline 9 & covers 1000 & 3 & $\begin{array}{l}\left\{\text { pitch_contour_segmentation, octave_abstraction, lcs }{ }^{-} \text {circular_mean }\right. \\
\text { \{beat_synchronous_chroma_cens, statistical_summarization_2, monte_carlo_distance } \\
\text { \{temporal_rhythm_histogram, bypass, cosine_distance }\end{array}$ & logistic_regression \\
\hline 10 & mazurkas & 3 & $\begin{array}{l}\text { \{chroma_stft, basic_stats_1, cosine_distance_oti } \\
\text { \{pitch_contour_segmentation, octave_abstraction, lcs_circular_mean } \\
\text { \{pitch_contour_segmentation, markov_chain, circular_manhattan }\end{array}$ & logistic_regression \\
\hline
\end{tabular}

(a)

\begin{tabular}{|c|c|c|c|c|c|c|c|c|}
\hline \multirow[b]{2}{*}{ line id } & \\
\hline & MR (mean) & MR (std) & MRR (mean) & MRR (std) & MDR (mean) & MDR (std) & MAP (mean) & MAP (std) \\
\hline 1 & 5.63714 & 0.05424 & 0.70923 & 0.00208 & 1.0 & 0.0 & 0.39507 & 0.00157 \\
\hline 2 & 6.37714 & 0.01054 & 0.73284 & 0.00068 & 1.0 & 0.0 & 0.40336 & 0.00026 \\
\hline 3 & 6.43486 & 0.06823 & 0.69203 & 0.00196 & 1.0 & 0.0 & 0.35837 & 0.00077 \\
\hline 4 & 6.44857 & 0.0814 & 0.69852 & 0.00121 & 1.0 & 0.0 & 0.36465 & 0.00077 \\
\hline 5 & 6.47743 & 0.00564 & 0.6997 & 0.00017 & 1.0 & 0.0 & 0.37871 & 0.00023 \\
\hline 6 & 6.48914 & 0.06202 & 0.69808 & 0.00101 & 1.0 & 0.0 & 0.36424 & 0.00059 \\
\hline 7 & 6.63886 & 0.01071 & 0.72393 & 0.00021 & 1.0 & 0.0 & 0.39434 & 0.00014 \\
\hline 8 & 6.63914 & 0.06962 & 0.68407 & 0.00193 & 1.0 & 0.0 & 0.36068 & 0.00217 \\
\hline 9 & 6.64743 & 0.01311 & 0.70585 & 0.00251 & 1.0 & 0.0 & 0.36096 & 0.0022 \\
\hline 10 & 6.67971 & 0.0088 & 0.72049 & 0.00115 & 1.0 & 0.0 & 0.39148 & 0.00018 \\
\hline
\end{tabular}

(b)

\begin{tabular}{|c|c|c|c|c|c|c|c|c|}
\hline line id & Top-1 (mean) & Top-1 (std) & Top-10 (mean) & Top-10 (std) & Top-100 (mean) & Top-100 (std) & Top-1000 (mean) & Top-1000 (std) \\
\hline 1 & 221.9 & 1.04403 & 306.2 & 1.16619 & 348.9 & 0.3 & 350.0 & 0.0 \\
\hline 2 & 232.3 & 0.45826 & 307.0 & 0.0 & 348.0 & 0.0 & 350.0 & 0.0 \\
\hline 3 & 210.9 & 1.22066 & 298.4 & 1.0198 & 349.0 & 0.0 & 350.0 & 0.0 \\
\hline 4 & 217.6 & 0.4899 & 299.8 & 0.74833 & 348.9 & 0.3 & 350.0 & 0.0 \\
\hline 5 & 211.0 & 0.0 & 304.2 & 0.4 & 349.0 & 0.0 & 350.0 & 0.0 \\
\hline 6 & 217.6 & 0.4899 & 299.5 & 0.67082 & 348.7 & 0.45826 & 350.0 & 0.0 \\
\hline 7 & 230.0 & 0.0 & 305.0 & 0.0 & 348.0 & 0.0 & 350.0 & 0.0 \\
\hline 8 & 209.4 & 1.2 & 296.9 & 0.83066 & 348.0 & 0.0 & 350.0 & 0.0 \\
\hline 9 & 218.0 & 1.48324 & 303.9 & 0.3 & 346.6 & 0.4899 & 350.0 & 0.0 \\
\hline 10 & 227.4 & 0.66332 & 300.0 & 0.0 & 349.0 & 0.0 & 350.0 & 0.0 \\
\hline
\end{tabular}

(c)

Table 6.62: Top-10 results for YouTubeCovers to Experiment \#4b. 


\section{Covers1000}

\begin{tabular}{|c|c|c|c|c|}
\hline line id & train dataset & length & models & classifier \\
\hline 1 & mazurkas & 3 & $\begin{array}{l}\text { \{pitch_contour_segmentation, octave_abstraction, lcs_circular_mean }\} \\
\{\text { tonnetz, diff_stats_1, manhattan_distance } \\
\text { \{rhythm histogram, bypass, cosine distance }\end{array}$ & logistic_regression \\
\hline 2 & mazurkas & 3 & $\begin{array}{l}\text { \{chroma_stft, basic_stats_1, cosine_distance_oti\} } \\
\text { \{pitch_contour_segmentation, octave abstraction, lcs_circular_mean } \\
\text { \{tonnetz, diff stats } 1 \text {, manhattan distance }\end{array}$ & logistic_regression \\
\hline 3 & mazurkas & 3 & $\begin{array}{l}\text { \{chroma_stft, diff_stats_1, cosine_distance_oti }\} \\
\text { \{pitch_contour_segmentation, octave abstraction, lcs_circular_mean }\} \\
\text { \{tonnetz, diff stats } 1 \text {, manhattan distance }\end{array}$ & logistic_regression \\
\hline 4 & mazurkas & 3 & $\begin{array}{l}\text { \{pitch_contour_segmentation, octave_abstraction, lcs_circular_mean }\} \\
\text { \{tonnetz, diff_stats_1, manhattan_distance } \\
\text { \{beat synchronous chroma cens, statistical summarization } 2, \text { monte carlo distance }\end{array}$ & logistic_regression \\
\hline 5 & mazurkas & 3 & $\begin{array}{l}\text { \{pitch_contour_segmentation, octave abstraction, lcs_circular_mean\} } \\
\text { \{tonnetz, diff_stats_1, manhattan_distance\} } \\
\text { \{pitch contour segmentation, markov chain, circular manhattan\} }\end{array}$ & logistic_regression \\
\hline 6 & mazurkas & 3 & $\begin{array}{l}\{\text { chroma_cqt, vector_quantization_default, cosine_distance }\} \\
\text { \{pitch_contour_segmentation, octave_abstraction, lcs_circular_mean } \\
\text { \{tonnetz, diff_stats_1, manhattan_distance }\end{array}$ & logistic_regression \\
\hline 7 & mazurkas & 3 & $\begin{array}{l}\text { \{chroma_cqt, vector_quantization_default, cosine_distance }\} \\
\left.\text { \{pitch_contour_segmentation, octave_abstraction, }{ }_{\text {lcs_circular_mean }}\right\} \\
\text { \{rhythm histogram, bypass, cosine distance }\end{array}$ & logistic_regression \\
\hline 8 & mazurkas & 3 & $\begin{array}{l}\text { \{beat_synchronous_chroma_cens, } \bar{b} \text { asic_stats_2, manhattan_distance_oti }\} \\
\text { \{pitch_contour_segmentation, octave_abstraction, lcs_circular_mean }{ }^{-}{ }^{o} \\
\text { \{tonnetz, diff_stats_1, manhattan_distance }\end{array}$ & logistic_regression \\
\hline 9 & mazurkas & 3 & $\begin{array}{l}\text { \{beat_synchronous_chroma_cens, } \overline{b a s i c} \text { stats_2, manhattan_distance_oti }\} \\
\text { \{pitch_contour_segmentation, octave_abstraction, lcs_circular_mean }{ }^{-} \\
\text {\{rhythm histogram, bypass, cosine distance\} }\end{array}$ & logistic_regression \\
\hline 10 & mazurkas & 3 & $\begin{array}{l}\{\text { pitch_contour_segmentation, octave_abstraction, lcs_circular_mean }\} \\
\text { tonnetz, diff_stats_1, manhattan_distance } \\
\text { \{melspectrogram, basic_stats_1, cosine_distance }\end{array}$ & logistic_regression \\
\hline
\end{tabular}

(a)

\begin{tabular}{|c|c|c|c|c|c|c|c|c|}
\hline \multirow[b]{2}{*}{ line id } & \\
\hline & MR (mean) & MR (std) & MRR (mean) & MRR (std) & MDR (mean) & MDR (std) & MAP (mean) & MAP (std) \\
\hline 1 & 109.0794 & 0.23776 & 0.30175 & 0.00065 & 16.5 & 0.3873 & 0.23293 & 0.00034 \\
\hline 2 & 110.2433 & 0.05417 & 0.29784 & 0.00022 & 17.0 & 0.0 & 0.23014 & 0.00019 \\
\hline 3 & 110.2546 & 0.05547 & 0.29754 & 0.00048 & 17.0 & 0.0 & 0.22995 & 0.00031 \\
\hline 4 & 110.4845 & 0.02306 & 0.30212 & 0.00015 & 17.0 & 0.0 & 0.23172 & 0.00022 \\
\hline 5 & 111.1542 & 0.0444 & 0.3025 & 0.0002 & 18.1 & 0.2 & 0.23237 & 0.00023 \\
\hline 6 & 111.9633 & 0.03316 & 0.29852 & 0.00021 & 18.0 & 0.0 & 0.23083 & 0.0002 \\
\hline 7 & 112.0256 & 0.108 & 0.30206 & 0.00028 & 17.0 & 0.0 & 0.22708 & 0.00019 \\
\hline 8 & 112.1167 & 0.13261 & 0.2939 & 0.00042 & 18.0 & 0.0 & 0.22709 & 0.00044 \\
\hline 9 & 112.8789 & 0.19713 & 0.31051 & 0.0005 & 20.0 & 0.0 & 0.23483 & 0.00037 \\
\hline 10 & 114.492 & 0.05107 & 0.29445 & 0.00057 & 19.75 & 0.25 & 0.22728 & 0.00034 \\
\hline
\end{tabular}

(b)

\begin{tabular}{|c|c|c|c|c|c|c|c|c|}
\hline line id & Top-1 (mean) & Top-1 (std) & Top-10 (mean) & Top-10 (std) & Top-100 (mean) & Top-100 (std) & Top-1000 (mean) & Top-1000 (std) \\
\hline 1 & 228.7 & 0.9 & 440.0 & 0.89443 & 707.0 & 0.0 & 1000.0 & 0.0 \\
\hline 2 & 225.0 & 0.0 & 432.9 & 0.53852 & 700.9 & 0.3 & 1000.0 & 0.0 \\
\hline 3 & 224.4 & 0.4899 & 433.4 & 0.4899 & 701.1 & 0.3 & 1000.0 & 0.0 \\
\hline 4 & 233.8 & 0.4 & 442.9 & 1.57797 & 700.8 & 0.6 & 1000.0 & 0.0 \\
\hline 5 & 232.6 & 0.4899 & 436.5 & 0.80623 & 708.8 & 1.46969 & 1000.0 & 0.0 \\
\hline 6 & 223.9 & 0.3 & 436.5 & 0.80623 & 692.8 & 0.74833 & 1000.0 & 0.0 \\
\hline 7 & 236.0 & 0.44721 & 437.1 & 0.3 & 697.0 & 0.0 & 1000.0 & 0.0 \\
\hline 8 & 222.5 & 0.80623 & 425.8 & 0.6 & 698.4 & 0.66332 & 1000.0 & 0.0 \\
\hline 9 & 253.4 & 0.66332 & 413.0 & 1.18322 & 715.2 & 0.74833 & 1000.0 & 0.0 \\
\hline 10 & 226.7 & 1.00499 & 423.1 & 0.83066 & 698.4 & 0.66332 & 1000.0 & 0.0 \\
\hline
\end{tabular}

(c)

Table 6.63: Top-10 results for Covers1000 to Experiment \#4b. 


\section{Mazurkas}

\begin{tabular}{|c|c|c|c|c|}
\hline line id & train dataset & length & models & classifier \\
\hline 1 & shs9k & 2 & $\begin{array}{l}\text { \{pitch_contour_segmentation, octave abstraction, lcs_circular_mean }\} \\
\{\text { zero_crossing rate, basic stats } 1 \text {, chebyshev distance }\end{array}$ & random_forest \\
\hline 2 & shs $9 \mathrm{k}$ & 2 & $\{$ chroma_cqt, vector_quantization_default, cosine_distance $\}$ & random_forest \\
\hline 3 & shs $9 \mathrm{k}$ & 2 & $\begin{array}{l}\left\{\text { pitch_contour }{ }_{-}^{-} \text {segmentation, octave }{ }_{-}^{-} \text {abstraction, } \text { lcs }_{-}^{-}{ }^{-} \text {circular }\right. \\
\text { \{melspectrogram, basic stats } 1, \text { cosine distance }\end{array}$ & random_forest \\
\hline 4 & shs $9 k$ & 2 & $\begin{array}{l}\{\text { beat_synchronous_chroma_cens, basic_stats_2, manhattan_distance_oti }\} \\
\{\text { pitch_contour_segmentation, octave_abstraction, lcs_circular_mean }\}\end{array}$ & random_forest \\
\hline 5 & shs9k & 2 & $\begin{array}{l}\left\{\text { pitch_contour }^{-} \text {segmentation, octave_abstraction, lcs_circular_mean }\right\} \\
\{\text { temporal_rhythm histogram, bypass, cosine_distance }\}\end{array}$ & random_forest \\
\hline 6 & shs9k & 2 & $\begin{array}{l}\{\text { pitch contour_segmentation, octave_abstraction, lcs_circular_mean }\} \\
\{\text { rms, diff stats } 1, \text { cosine distance }\}\end{array}$ & random_forest \\
\hline 7 & shs $9 \mathrm{k}$ & 2 & $\begin{array}{l}\text { \{pitch_contour_-segmentation, octave abstraction, lcs circular_mean }\} \\
\{\text { chroma_stft, gaussian_mixture_model_default, manhattan_distance }\end{array}$ & random_forest \\
\hline 8 & shs $9 \mathrm{k}$ & 2 & $\begin{array}{l}\text { \{pitch_contour_segmentation, octave_abstraction, lcs_circular_mean }\} \\
\{\text { spectral_contrast, statistical_summarization_2, earth_movers_distance }\}\end{array}$ & random_forest \\
\hline 9 & shs $9 \mathrm{k}$ & 2 & $\begin{array}{l}\text { \{pitch_contour_segmentation, octave abstraction, lcs_circular_mean }\} \\
\{\text { tonnetz, diff stats } 1 \text {, manhattan distance }\end{array}$ & random_forest \\
\hline 10 & shs $9 \mathrm{k}$ & 2 & $\begin{array}{l}\text { \{pitch_contoūr_segmentation, octave_abstraction, lcs_circular_mean }\} \\
\{\text { beat_synchronous_chroma_cens, statistical_summarization_2, monte_carlo_distance }\}\end{array}$ & random_forest \\
\hline
\end{tabular}

\begin{tabular}{|c|c|c|c|c|c|c|c|c|}
\hline \multirow[b]{2}{*}{ line id } & \multicolumn{8}{|c|}{ (a) } \\
\hline & MR (mean) & MR (std) & MRR (mean) & MRR (std) & MDR (mean) & MDR (std) & MAP (mean) & MAP (std) \\
\hline 1 & 1.97322 & 0.05831 & 0.93372 & 0.00406 & 1.0 & 0.0 & 0.62158 & 0.01322 \\
\hline 2 & 1.9873 & 0.08763 & 0.94935 & 0.00224 & 1.0 & 0.0 & 0.7264 & 0.0059 \\
\hline 3 & 2.00795 & 0.05173 & 0.94463 & 0.00199 & 1.0 & 0.0 & 0.62359 & 0.00578 \\
\hline 4 & 2.03068 & 0.05444 & 0.94547 & 0.00261 & 1.0 & 0.0 & 0.62956 & 0.00884 \\
\hline 5 & 2.05932 & 0.10032 & 0.93815 & 0.00291 & 1.0 & 0.0 & 0.57592 & 0.0083 \\
\hline 6 & 2.0633 & 0.07103 & 0.93469 & 0.00446 & 1.0 & 0.0 & 0.5783 & 0.01642 \\
\hline 7 & 2.0903 & 0.0641 & 0.93903 & 0.00249 & 1.0 & 0.0 & 0.58748 & 0.00799 \\
\hline 8 & 2.09861 & 0.06603 & 0.93325 & 0.00291 & 1.0 & 0.0 & 0.66314 & 0.00584 \\
\hline 9 & 2.12331 & 0.11285 & 0.95074 & 0.0023 & 1.0 & 0.0 & 0.73001 & 0.00662 \\
\hline 10 & 2.13769 & 0.09337 & 0.93815 & 0.00565 & 1.0 & 0.0 & 0.59706 & 0.01346 \\
\hline
\end{tabular}

(b)

\begin{tabular}{|c|c|c|c|c|c|c|c|c|}
\hline line id & Top-1 (mean) & Top-1 (std) & Top-10 (mean) & Top-10 (std) & Top-100 (mean) & Top-100 (std) & Top-1000 (mean) & Top-1000 (std) \\
\hline 1 & 2457.3 & 21.89087 & 2697.8 & 3.73631 & 2734.5 & 1.62788 & 2741.0 & 0.0 \\
\hline 2 & 2526.4 & 9.40425 & 2700.7 & 4.42832 & 2732.2 & 1.32665 & 2741.0 & 0.0 \\
\hline 3 & 2507.0 & 10.30534 & 2699.5 & 2.06155 & 2734.5 & 1.36015 & 2741.0 & 0.0 \\
\hline 4 & 2515.5 & 11.47388 & 2696.5 & 4.00625 & 2733.3 & 1.79165 & 2741.0 & 0.0 \\
\hline 5 & 2474.9 & 12.87206 & 2700.3 & 3.13209 & 2732.8 & 1.46969 & 2741.0 & 0.0 \\
\hline 6 & 2458.6 & 21.22828 & 2701.6 & 3.90384 & 2732.9 & 1.22066 & 2741.0 & 0.0 \\
\hline 7 & 2483.1 & 9.67936 & 2698.2 & 3.84187 & 2732.7 & 1.48661 & 2741.0 & 0.0 \\
\hline 8 & 2451.2 & 11.83047 & 2699.3 & 2.05183 & 2732.9 & 1.81384 & 2741.0 & 0.0 \\
\hline 9 & 2537.5 & 11.1018 & 2697.7 & 3.0348 & 2731.2 & 2.44131 & 2741.0 & 0.0 \\
\hline 10 & 2482.0 & 26.95923 & 2697.4 & 4.22374 & 2732.8 & 1.46969 & 2741.0 & 0.0 \\
\hline
\end{tabular}

(c)

Table 6.64: Top-10 results for Mazurkas to Experiment \#4b.

\subsubsection{Discussion: experiment \#4b}

\section{Best results}

The best results for experiment \#4b are: Covers 80 by ada boost trained with Covers 1000 and 3-dimensional samples; YouTubeCovers by logistic regression with Covers1000 as train dataset and using 3 models to obtain the samples; Covers1000 by logistic regression, trained with Mazurkas and 3-D samples; Mazurkas by random forest trained with SHS9K and 2-dimensional samples.

Both Covers 80 and YouTubeCovers have the three models that lead to the best CSI results with tonal features, while Covers1000 have Pitch Contour Segmentation, Tonnetz, and Rhythm Histogram which is a rhythmic feature. In Experiment \#3b, Covers1000 also needed a rhythmic feature to achieve the best results, and this can be an indication that versions in Covers1000 can be better retrieved with the aid of rhythmic similarity models. The order of the datasets is the same: Mazurkas (with 1.97322 mean MR), YouTubeCovers (5.63714), Covers80 (33.33313), and Covers1000 (109.0794). 


\section{Comparative results}

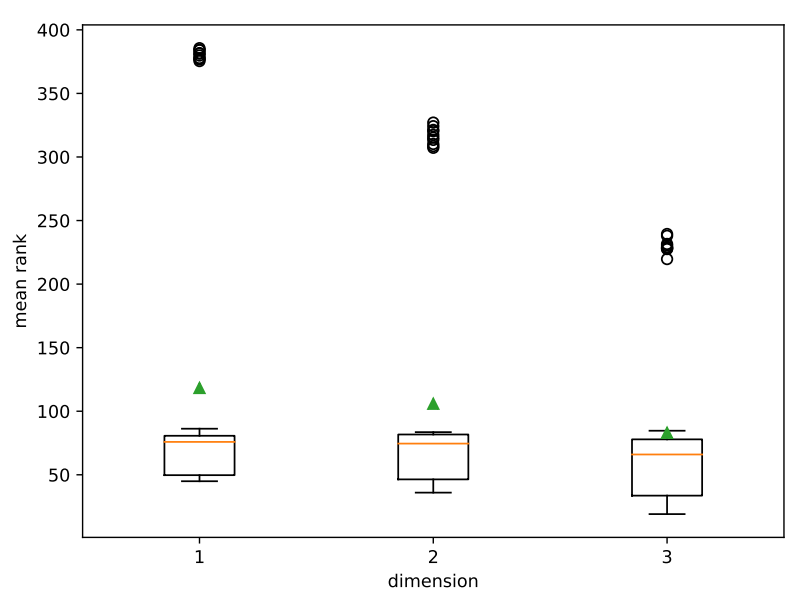

(a)

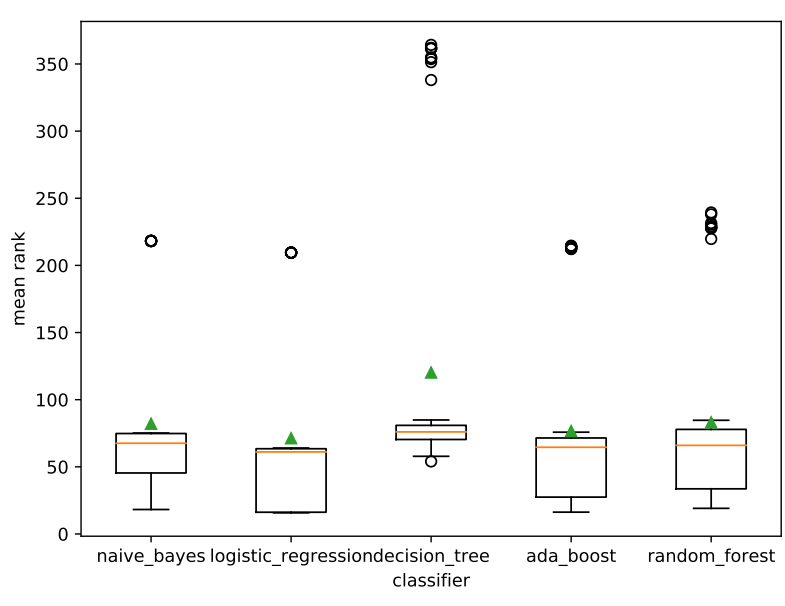

(b)

Figure 6.15: Summarized results to experiment \#4a by dimension (a), and by classifier (b).

Investigating the overall results, Figure 6.15a summarizes the MR values by the dimension of the samples. As observed in experiment \#3b, the filtered models lead to better results as the dimension grows. Figure $6.15 \mathrm{~b}$ displays the values of MR by classifier. All classifiers, excluding decision tree, have their average MR quite close to each other, with a slightly better mean for logistic regression, which shows up in all Top-10 results for YouTubeCovers and Covers1000.

\begin{tabular}{l|lll} 
dataset & best MR (experiment \#2) & best mean MR (experiment \#4a) & best mean MR (experiment \#4b) \\
\hline Covers80 & 41.575 & 33.97812 & 33.33313 \\
YouTubeCovers & 7.97143 & 13.42257 & 5.63714 \\
Covers1000 & 144.041 & 146.5084 & 109.0794 \\
Mazurkas & 4.15724 & 3.99186 & 1.97322
\end{tabular}

Table 6.65: Best results from Experiments \#4 compared with Experiment \#2.

Finally, we can compare results from both experiments presented in this Section with the results from the main CSI experiment. Table 6.65 shows that, in experiment \#4a, Covers 80 and Mazurkas already have better results compared to experiment \#2. Experiment \#4b leads to all datasets having better results than the original CSI experiment. This is a strong result in favor of the use of CSC methods for the CSI problem.

\subsection{Experiment \#5: Dataset modifications}

\subsubsection{Experiment design}

In this experiment we apply all dataset modifications presented in Section 5.4, and then we compute all CSI metrics presented in Section 3.1.4. The main goal of Experiment $\# 5$ is to verify if any modification leads to better CSI results when compared to the metrics obtained with the original datasets.

In the following Section the best results are displayed for every one of the 5 CSI datasets. All results are published in https://rppbodo.github.io/phd/experiment_5.html. 


\subsubsection{Results}

\section{Covers 80}

\begin{tabular}{|c|c|c|c|c|c|c|c|c|}
\hline dataset & MR & MRR & MDR & MAP & Top 1 & Top 10 & Top 100 & Top 1000 \\
\hline covers 80 & 41.575 & 0.19359 & 31.0 & 0.19359 & 21.0 & 46.0 & 142.0 & 160.0 \\
\hline covers80_30_beg & 52.00625 & 0.13789 & 37.5 & 0.13789 & 13.0 & 42.0 & 128.0 & 160.0 \\
\hline covers80-30-end & 53.9 & 0.13827 & 45.0 & 0.13827 & 14.0 & 35.0 & 131.0 & 160.0 \\
\hline covers $80^{-} 30^{-}$mid & 46.19375 & 0.17388 & 28.0 & 0.17388 & 18.0 & 50.0 & 134.0 & 160.0 \\
\hline covers $80^{-}$bartsch_wakefield & 52.4875 & 0.11902 & 44.0 & 0.11902 & 9.0 & 35.0 & 134.0 & 160.0 \\
\hline covers $80_{-}^{-}$bass ${ }^{-}$ & 42.125 & 0.20927 & 24.0 & 0.20927 & 22.0 & 57.0 & 135.0 & 160.0 \\
\hline covers80_cooper_foote & 51.025 & 0.14076 & 40.0 & 0.14076 & 11.0 & 42.0 & 136.0 & 160.0 \\
\hline covers $80^{-}$drums ${ }^{-}$ & 54.3375 & 0.07624 & 43.0 & 0.07624 & 3.0 & 30.0 & 129.0 & 160.0 \\
\hline covers $80^{-}$other & 46.33125 & 0.15671 & 34.5 & 0.15671 & 12.0 & 54.0 & 134.0 & 160.0 \\
\hline covers $80^{-}$qm-segmenter & 51.55 & 0.1138 & 36.5 & 0.1138 & 6.0 & 43.0 & 126.0 & 160.0 \\
\hline covers $80^{-} \mathrm{rms} \quad 10$ & 61.7875 & 0.07634 & 49.5 & 0.07634 & 5.0 & 24.0 & 120.0 & 160.0 \\
\hline covers $80^{-} \mathrm{rms}^{-} 20$ & 52.4 & 0.08832 & 40.0 & 0.08832 & 6.0 & 24.0 & 130.0 & 160.0 \\
\hline covers80_rms_30 & 50.0625 & 0.13294 & 39.0 & 0.13294 & 14.0 & 33.0 & 136.0 & 160.0 \\
\hline covers80_rms_40 & 48.6125 & 0.14707 & 37.5 & 0.14707 & 15.0 & 41.0 & 135.0 & 160.0 \\
\hline covers $80^{-} \mathrm{rms}^{-} 50$ & 47.11875 & 0.15756 & 36.0 & 0.15756 & 15.0 & 43.0 & 137.0 & 160.0 \\
\hline covers $80^{-}$rms $^{-} 60$ & 43.5125 & 0.18049 & 28.0 & 0.18049 & 17.0 & 51.0 & 134.0 & 160.0 \\
\hline covers $80^{-} \mathrm{rms}^{-} 70$ & 43.95625 & 0.17219 & 29.0 & 0.17219 & 15.0 & 52.0 & 137.0 & 160.0 \\
\hline covers $80^{-} \mathrm{rms}^{-} 80$ & 42.975 & 0.18578 & 28.0 & 0.18578 & 19.0 & 52.0 & 142.0 & 160.0 \\
\hline covers80_rms_-90 & 42.06875 & 0.20586 & 26.5 & 0.20586 & 20.0 & 56.0 & 141.0 & 160.0 \\
\hline covers $80^{-}$-segmentino & 58.0625 & 0.08312 & 49.5 & 0.08312 & 5.0 & 28.0 & 123.0 & 160.0 \\
\hline covers $80^{-}$_siplca & 53.46875 & 0.10518 & 45.5 & 0.10518 & 8.0 & 31.0 & 131.0 & 160.0 \\
\hline covers $80^{-}$vocals & 51.19375 & 0.21095 & 34.5 & 0.21095 & 23.0 & 52.0 & 127.0 & 160.0 \\
\hline covers $80^{-}$vocals rms 10 & 49.34375 & 0.18072 & 37.0 & 0.18072 & 19.0 & 46.0 & 134.0 & 160.0 \\
\hline covers $80^{-}$vocals ${ }^{-} \mathrm{rms}^{-} 20$ & 47.65 & 0.14985 & 33.5 & 0.14985 & 12.0 & 48.0 & 134.0 & 160.0 \\
\hline covers $80^{-}$vocals ${ }^{-} \mathrm{rms}^{-}-30$ & 44.35625 & 0.19798 & 35.5 & 0.19798 & 21.0 & 51.0 & 141.0 & 160.0 \\
\hline covers $80^{-}$vocals ${ }^{-} \mathrm{rms}^{-} 40$ & 42.64375 & 0.16433 & 31.0 & 0.16433 & 15.0 & 48.0 & 140.0 & 160.0 \\
\hline covers $80^{-}$vocals ${ }^{-} \mathrm{rms}^{-} 50$ & 42.38125 & 0.16205 & 32.0 & 0.16205 & 16.0 & 43.0 & 141.0 & 160.0 \\
\hline covers $80^{-}$vocals ${ }^{-} \mathrm{rms}^{-} 60$ & 42.3375 & 0.16502 & 28.5 & 0.16502 & 16.0 & 48.0 & 140.0 & 160.0 \\
\hline covers $80^{-}$vocals ${ }^{-} \mathrm{rms}^{-} 70$ & 41.3 & 0.20834 & 22.5 & 0.20834 & 20.0 & 61.0 & 134.0 & 160.0 \\
\hline covers $80^{-}$vocals ${ }^{-} \mathrm{rms}^{-} 80$ & 41.975 & 0.17761 & 28.5 & 0.17761 & 18.0 & 45.0 & 144.0 & 160.0 \\
\hline covers $80^{-}$vocals ${ }^{-} \mathrm{rms}^{-} 90$ & 41.75 & 0.18389 & 31.0 & 0.18389 & 20.0 & 47.0 & 141.0 & 160.0 \\
\hline
\end{tabular}

Table 6.66: The best results for each modified dataset for Covers80.

\section{YouTubeCovers}

\begin{tabular}{|c|c|c|c|c|c|c|c|c|}
\hline dataset & MR & MRR & MDR & MAP & Top 1 & Top 10 & Top 100 & Top 1000 \\
\hline youtubecovers & 7.97143 & 0.6942 & 1.0 & 0.36114 & 219.0 & 296.0 & 345.0 & 350.0 \\
\hline youtubecovers_30_beg & 20.96571 & 0.34045 & 9.0 & 0.1322 & 88.0 & 186.0 & 341.0 & 350.0 \\
\hline youtubecovers ${ }^{-} 30^{-}$end & 27.49143 & 0.24078 & 13.0 & 0.0861 & 48.0 & 152.0 & 328.0 & 350.0 \\
\hline youtubecovers ${ }^{-} 30^{-} \mathrm{mid}$ & 22.80286 & 0.29309 & 9.0 & 0.11305 & 61.0 & 193.0 & 335.0 & 350.0 \\
\hline youtubecovers ${ }^{-}$bartsch wakefield & 19.92571 & 0.38321 & 5.0 & 0.13723 & 95.0 & 213.0 & 337.0 & 350.0 \\
\hline youtubecovers ${ }^{-}$bass & 16.37143 & 0.5491 & 2.0 & 0.27495 & 166.0 & 241.0 & 340.0 & 350.0 \\
\hline youtubecovers_cooper_foote & 28.00857 & 0.22246 & 14.0 & 0.08266 & 41.0 & 152.0 & 332.0 & 350.0 \\
\hline youtubecovers ${ }^{-}$drums & 37.15429 & 0.14012 & 23.0 & 0.05417 & 21.0 & 114.0 & 320.0 & 350.0 \\
\hline youtubecovers_other & 14.06 & 0.47627 & 3.0 & 0.23085 & 131.0 & 240.0 & 343.0 & 350.0 \\
\hline youtubecovers_qm-segmenter & 27.48 & 0.22917 & 13.0 & 0.08615 & 44.0 & 160.0 & 331.0 & 350.0 \\
\hline youtubecovers rms 10 & 37.76286 & 0.13124 & 22.0 & 0.05137 & 20.0 & 105.0 & 322.0 & 350.0 \\
\hline youtubecovers $\mathrm{rms}^{-} 20$ & 27.07143 & 0.25139 & 12.5 & 0.09752 & 53.0 & 165.0 & 334.0 & 350.0 \\
\hline youtubecovers $\mathrm{rms}^{-} 30$ & 16.70857 & 0.39305 & 5.0 & 0.16382 & 97.0 & 229.0 & 340.0 & 350.0 \\
\hline youtubecovers ${ }^{-} \mathrm{rms}^{-}-40$ & 14.53429 & 0.48738 & 3.0 & 0.21466 & 137.0 & 243.0 & 342.0 & 350.0 \\
\hline youtubecovers ${ }^{-} \mathrm{rms}^{-} 50$ & 11.89714 & 0.54654 & 2.0 & 0.25512 & 158.0 & 266.0 & 342.0 & 350.0 \\
\hline youtubecovers ${ }^{-} \mathrm{rms}^{-} 60$ & 11.44286 & 0.59266 & 2.0 & 0.28625 & 174.0 & 275.0 & 338.0 & 350.0 \\
\hline youtubecovers ${ }^{-} \mathrm{rms}^{-}-70$ & 9.13429 & 0.62006 & 1.0 & 0.32162 & 184.0 & 286.0 & 344.0 & 350.0 \\
\hline youtubecovers ${ }^{-} \mathrm{rms}^{-} 80$ & 8.18857 & 0.66438 & 1.0 & 0.34196 & 202.0 & 300.0 & 346.0 & 350.0 \\
\hline youtubecovers_rms_-90 & 8.77143 & 0.67878 & 1.0 & 0.35952 & 211.0 & 296.0 & 346.0 & 350.0 \\
\hline youtubecovers_segmentino & 28.6 & 0.23981 & 16.5 & 0.08817 & 52.0 & 145.0 & 329.0 & 350.0 \\
\hline youtubecovers_siplca & 22.59429 & 0.32043 & 8.0 & 0.12486 & 73.0 & 192.0 & 335.0 & 350.0 \\
\hline youtubecovers_-vocals & 12.77143 & 0.6162 & 1.0 & 0.35571 & 192.0 & 261.0 & 339.0 & 350.0 \\
\hline youtubecovers_vocals_rms 10 & 18.93714 & 0.32488 & 7.0 & 0.127 & 70.0 & 204.0 & 341.0 & 350.0 \\
\hline youtubecovers ${ }^{-}$vocals ${ }^{-} \mathrm{rms}^{-} 20$ & 10.34 & 0.55453 & 2.0 & 0.25723 & 157.0 & 264.0 & 347.0 & 350.0 \\
\hline youtubecovers ${ }^{-}$vocals ${ }^{-} \mathrm{rms}^{-} 30$ & 10.81429 & 0.64884 & 1.0 & 0.3301 & 198.0 & 287.0 & 342.0 & 350.0 \\
\hline youtubecovers ${ }^{-}$vocals ${ }^{-} \mathrm{rms}^{-} 40$ & 8.42571 & 0.68883 & 1.0 & 0.36878 & 216.0 & 287.0 & 347.0 & 350.0 \\
\hline youtubecovers ${ }^{-}$vocals ${ }^{-} \mathrm{rms}^{-} 50$ & 9.80286 & 0.67666 & 1.0 & 0.37006 & 207.0 & 292.0 & 343.0 & 350.0 \\
\hline youtubecovers ${ }^{-}$vocals ${ }^{-} \mathrm{rms}^{-} 60$ & 9.19143 & 0.67039 & 1.0 & 0.37298 & 205.0 & 291.0 & 343.0 & 350.0 \\
\hline youtubecovers ${ }^{-}$vocals ${ }^{-} \mathrm{rms}^{-} 70$ & 8.42857 & 0.67708 & 1.0 & 0.37043 & 212.0 & 293.0 & 344.0 & 350.0 \\
\hline youtubecovers ${ }^{-}$vocals ${ }^{-} \mathrm{rms}^{-} 80$ & 9.01714 & 0.68146 & 1.0 & 0.36879 & 213.0 & 293.0 & 343.0 & 350.0 \\
\hline youtubecovers ${ }^{-}$vocals ${ }^{-} \mathrm{rms}^{-} 90$ & 9.17143 & 0.66096 & 1.0 & 0.35772 & 201.0 & 289.0 & 342.0 & 350.0 \\
\hline
\end{tabular}

Table 6.67: The best results for each modified dataset for YouTubeCovers. 


\section{Covers1000}

\begin{tabular}{|c|c|c|c|c|c|c|c|c|}
\hline dataset & MR & MRR & MDR & MAP & Top 1 & Top 10 & Top 100 & Top 1000 \\
\hline covers1000 & 144.041 & 0.25731 & 35.0 & 0.19159 & 201.0 & 366.0 & 636.0 & 1000.0 \\
\hline covers1000_30_beg & 213.562 & 0.12522 & 106.0 & 0.08702 & 81.0 & 200.0 & 490.0 & 1000.0 \\
\hline covers $1000^{-} 30^{-}$end & 205.748 & 0.08836 & 117.5 & 0.06245 & 45.0 & 172.0 & 469.0 & 1000.0 \\
\hline covers1000-30- mid & 206.592 & 0.08778 & 109.5 & 0.0622 & 42.0 & 161.0 & 485.0 & 1000.0 \\
\hline covers1000_bartsch_wakefield & 225.575 & 0.09385 & 115.5 & 0.06538 & 50.0 & 165.0 & 480.0 & 1000.0 \\
\hline covers $1000^{-}$bass & 150.027 & 0.32957 & 27.0 & 0.26457 & 280.0 & 421.0 & 649.0 & 1000.0 \\
\hline covers1000_cooper_foote & 212.222 & 0.07075 & 126.0 & 0.05355 & 35.0 & 125.0 & 457.0 & 1000.0 \\
\hline covers1000_drums ${ }^{-}$ & 246.203 & 0.1013 & 119.0 & 0.07178 & 54.0 & 178.0 & 468.0 & 1000.0 \\
\hline covers1000_other & 164.52 & 0.139 & 67.0 & 0.1007 & 86.0 & 237.0 & 582.0 & 1000.0 \\
\hline covers1000_qm-segmenter & 214.766 & 0.08124 & 118.5 & 0.05836 & 43.0 & 141.0 & 459.0 & 1000.0 \\
\hline covers $1000^{-}$rms 10 & 264.19 & 0.02966 & 184.5 & 0.02228 & 7.0 & 60.0 & 343.0 & 1000.0 \\
\hline covers1000_rms_20 & 242.019 & 0.04186 & 149.5 & 0.02992 & 14.0 & 79.0 & 392.0 & 1000.0 \\
\hline covers $1000_{-}^{-} \mathrm{rms}_{-}^{-} 30$ & 226.976 & 0.06397 & 135.0 & 0.04325 & 30.0 & 125.0 & 428.0 & 1000.0 \\
\hline covers $1000_{-}^{-} \mathrm{rms}_{-}^{-} 40$ & 212.291 & 0.14401 & 99.5 & 0.10373 & 102.0 & 214.0 & 503.0 & 1000.0 \\
\hline covers $1000_{-}^{-} \mathrm{rms}_{-}^{-} 50$ & 192.557 & 0.18258 & 78.0 & 0.135 & 139.0 & 263.0 & 527.0 & 1000.0 \\
\hline covers $1000^{-} \mathrm{rms}^{-} 60$ & 163.671 & 0.20892 & 61.0 & 0.15403 & 160.0 & 301.0 & 592.0 & 1000.0 \\
\hline covers $1000^{-} \mathrm{rms}^{-} 70$ & 156.124 & 0.22716 & 52.5 & 0.16557 & 176.0 & 331.0 & 612.0 & 1000.0 \\
\hline covers $1000^{-} \mathrm{rms}^{-} 80$ & 149.235 & 0.24245 & 39.5 & 0.17682 & 186.0 & 352.0 & 629.0 & 1000.0 \\
\hline covers1000_rms_-90 & 139.806 & 0.27382 & 30.0 & 0.20739 & 218.0 & 381.0 & 662.0 & 1000.0 \\
\hline covers1000_segmentino & 218.62 & 0.07973 & 126.0 & 0.05763 & 39.0 & 152.0 & 458.0 & 1000.0 \\
\hline covers1000_siplca & 186.598 & 0.07564 & 99.0 & 0.05182 & 33.0 & 152.0 & 503.0 & 1000.0 \\
\hline covers $1000^{-}$vocals & 156.711 & 0.31149 & 32.0 & 0.24498 & 264.0 & 404.0 & 638.0 & 1000.0 \\
\hline covers $1000_{-}^{-}$vocals_rms_10 & 227.618 & 0.06649 & 141.5 & 0.0465 & 35.0 & 115.0 & 424.0 & 1000.0 \\
\hline covers $1000^{-}$vocals ${ }^{-} \mathrm{rms}^{-} 20$ & 174.339 & 0.15585 & 72.0 & 0.1097 & 107.0 & 239.0 & 558.0 & 1000.0 \\
\hline covers $1000^{-}$vocals ${ }^{-} \mathrm{rms}^{-} 30$ & 155.591 & 0.2271 & 46.0 & 0.16291 & 169.0 & 336.0 & 616.0 & 1000.0 \\
\hline covers1000_vocals_rms_- 40 & 141.942 & 0.25919 & 35.0 & 0.18761 & 200.0 & 369.0 & 648.0 & 1000.0 \\
\hline covers1000_vocals_rms_-50 & 154.75 & 0.25097 & 37.0 & 0.18189 & 190.0 & 369.0 & 635.0 & 1000.0 \\
\hline covers1000_vocals_rms_-60 & 152.472 & 0.26559 & 37.0 & 0.19624 & 213.0 & 381.0 & 633.0 & 1000.0 \\
\hline covers $1000^{-}$vocals ${ }^{-} \mathrm{rms}^{-} 70$ & 150.976 & 0.2475 & 38.0 & 0.18228 & 192.0 & 358.0 & 635.0 & 1000.0 \\
\hline covers $1000^{-}$vocals $^{-} \mathrm{rms}^{-} 80$ & 151.975 & 0.2476 & 39.5 & 0.18165 & 195.0 & 336.0 & 639.0 & 1000.0 \\
\hline covers $1000^{-}$vocals ${ }^{-} \mathrm{rms}^{-}-90$ & 142.65 & 0.26677 & 40.0 & 0.19668 & 212.0 & 366.0 & 654.0 & 1000.0 \\
\hline
\end{tabular}

Table 6.68: The best results for each modified dataset for Covers1000.

\section{Mazurkas}

Mazurkas is a dataset whose tracks are mainly piano recordings, so it is not possible to explore some dataset modification techniques: the 4 source separation datasets can not be created; the 9 secondary datasets build using frame selection by vocals' RMS also can not be created. Thus we have only 17 modified datasets to experiment.

\begin{tabular}{lllllllll} 
dataset & MR & MRR & MDR & MAP & Top 1 & Top 10 & Top 100 & Top 1000 \\
\hline mazurkas & 4.15724 & 0.95774 & 1.0 & 0.82286 & 2561.0 & 2714.0 & 2728.0 & 2739.0 \\
\hline mazurkas_30_beg & $\mathbf{2 . 8 1 2 8 4}$ & 0.92509 & 1.0 & 0.55828 & 2434.0 & 2690.0 & 2727.0 & $\mathbf{2 7 4 0 . 0}$ \\
mazurkas_30_end & 4.33382 & 0.87207 & 1.0 & 0.36153 & 2239.0 & 2633.0 & 2722.0 & $\mathbf{2 7 4 1 . 0}$ \\
mazurkas_30_mid & $\mathbf{3 . 1 2 7 3 3}$ & 0.88732 & 1.0 & 0.41965 & 2294.0 & 2645.0 & 2728.0 & $\mathbf{2 7 4 1 . 0}$ \\
mazurkas_bartsch_wakefield & $\mathbf{3 . 8 0 4 0 9}$ & 0.88995 & 1.0 & 0.38092 & 2308.0 & 2648.0 & 2720.0 & $\mathbf{2 7 4 1 . 0}$ \\
mazurkas_cooper_foote & 4.72273 & 0.82289 & 1.0 & 0.31283 & 2062.0 & 2614.0 & 2720.0 & $\mathbf{2 7 4 1 . 0}$ \\
mazurkas_qm-segmenter & 5.94856 & 0.76276 & 1.0 & 0.22742 & 1832.0 & 2545.0 & 2713.0 & $\mathbf{2 7 4 0 . 0}$ \\
mazurkas_rms_10 & 6.76323 & 0.8015 & 1.0 & 0.36347 & 1978.0 & 2581.0 & 2718.0 & 2738.0 \\
mazurkas_rms_20 & 5.72492 & 0.73234 & 1.0 & 0.22176 & 1719.0 & 2538.0 & 2719.0 & $\mathbf{2 7 4 0 . 0}$ \\
mazurkas_rms_30 & 4.62532 & 0.85361 & 1.0 & 0.38133 & 2182.0 & 2629.0 & 2727.0 & 2739.0 \\
mazurkas_rms_40 & $\mathbf{3 . 9 4 8 9 2}$ & 0.91145 & 1.0 & 0.52903 & 2385.0 & 2687.0 & 2727.0 & 2739.0 \\
mazurkas_rms_50 & $\mathbf{3 . 8 9 8 2 1}$ & 0.92668 & 1.0 & 0.52952 & 2448.0 & 2691.0 & 2725.0 & 2739.0 \\
mazurkas_rms_60 & $\mathbf{3 . 5 5 3 0 8}$ & 0.94471 & 1.0 & 0.63254 & 2512.0 & 2701.0 & $\mathbf{2 7 2 9 . 0}$ & 2739.0 \\
mazurkas_rms_70 & $\mathbf{3 . 5 2 4 2 6}$ & 0.95366 & 1.0 & 0.71281 & 2543.0 & 2711.0 & $\mathbf{2 7 3 0 . 0}$ & 2739.0 \\
mazurkas_rms_80 & $\mathbf{3 . 9 9 9 6 4}$ & 0.90595 & 1.0 & 0.46992 & 2363.0 & 2671.0 & 2725.0 & $\mathbf{2 7 4 0 . 0}$ \\
mazurkas_rms_90 & $\mathbf{3 . 9 5 8 4 1}$ & 0.92943 & 1.0 & 0.60055 & 2467.0 & 2676.0 & 2722.0 & $\mathbf{2 7 4 1 . 0}$ \\
mazurkas_segmentino & 4.77599 & 0.83857 & 1.0 & 0.23812 & 2136.0 & 2584.0 & 2721.0 & $\mathbf{2 7 4 1 . 0}$ \\
mazurkas_siplca & 5.97337 & 0.81504 & 1.0 & 0.25614 & 2042.0 & 2548.0 & 2712.0 & $\mathbf{2 7 4 1 . 0}$
\end{tabular}

Table 6.69: The best results for each modified dataset for Mazurkas.

\section{SHS9K}

The experiments with SHS9K modified datasets are still running to the date of this publication, so the following table shows the partial best results. 


\begin{tabular}{|c|c|c|c|c|c|c|c|c|}
\hline dataset & MR & MRR & MDR & MAP & Top 1 & Top 10 & Top 100 & Top 1000 \\
\hline shs9k & 47.57883 & 0.40387 & 6.0 & 0.05102 & 3008.0 & 5188.0 & 8067.0 & 9265.0 \\
\hline $\begin{array}{lll}\text { shs9k } 30 & \text { beg }\end{array}$ & 83.35225 & 0.17503 & 34.0 & 0.01713 & 1013.0 & 2767.0 & 6859.0 & 9275.0 \\
\hline shs $9 \mathrm{k}^{-} 30^{-}$mid & 45.83082 & 0.2384 & 13.0 & 0.03149 & 1282.0 & 4161.0 & 8272.0 & 9267.0 \\
\hline shs $9 \mathrm{k}^{-} 30^{-}$end & 87.48568 & 0.14304 & 33.0 & 0.01848 & 661.0 & 2619.0 & 7028.0 & 9235.0 \\
\hline shs $9 \mathrm{k}$ bartsch wakefield & 81.08249 & 0.16252 & 29.0 & 0.01845 & 819.0 & 2868.0 & 7210.0 & 9257.0 \\
\hline shs $9 \mathrm{k}^{-}$bass & 59.93216 & 0.36286 & 10.0 & 0.0621 & 2729.0 & 4644.0 & 7685.0 & 9253.0 \\
\hline shs9k_cooper foote & 90.88768 & 0.13307 & 36.0 & 0.01722 & 620.0 & 2413.0 & 6968.0 & 9233.0 \\
\hline shs $9 \mathrm{k}^{-}$drums ${ }^{-}$ & 127.94648 & 0.0541 & 77.0 & 0.00954 & 163.0 & 979.0 & 5441.0 & 9253.0 \\
\hline shs $9 \mathrm{k}^{-}$other & 63.8459 & 0.22859 & 16.0 & 0.02854 & 1268.0 & 3892.0 & 7779.0 & 9242.0 \\
\hline shs $9 k^{-}$qm-segmenter & 81.95057 & 0.16444 & 28.0 & 0.02021 & 807.0 & 2910.0 & 7196.0 & 9242.0 \\
\hline shs $9 \mathrm{k}$ rms 10 & 109.81047 & 0.08204 & 57.0 & 0.0112 & 324.0 & 1475.0 & 6119.0 & 9249.0 \\
\hline shs $9 k^{-}{ }^{-}{ }^{-}{ }^{-} 20$ & 91.6583 & 0.14435 & 40.0 & 0.01622 & 757.0 & 2440.0 & 6643.0 & 9273.0 \\
\hline shs $9 \mathrm{k}^{-} \mathrm{rms}^{-} 30$ & 74.96059 & 0.22647 & 24.0 & 0.02432 & 1425.0 & 3404.0 & 7236.0 & 9266.0 \\
\hline shs $9 \mathrm{k}^{-} \mathrm{rms}^{-} 40$ & 62.03565 & 0.29329 & 15.0 & 0.03327 & 2004.0 & 4102.0 & 7545.0 & 9274.0 \\
\hline shs $9 \mathrm{k}^{-} \mathrm{rms}-50$ & 55.80713 & 0.34819 & 11.0 & 0.04142 & 2504.0 & 4640.0 & 7768.0 & 9270.0 \\
\hline shs $9 \mathrm{k}^{-} \mathrm{rms}-60$ & 50.94012 & 0.37985 & 8.0 & 0.04729 & 2775.0 & 4980.0 & 7929.0 & 9268.0 \\
\hline shs $9 k-\mathrm{kms}^{-} 70$ & 48.25942 & 0.40286 & 7.0 & 0.05088 & 3037.0 & 5144.0 & 8000.0 & 9274.0 \\
\hline shs $9 \mathrm{k}^{-} \mathrm{rms}^{-} 80$ & 46.27256 & 0.41185 & 6.0 & 0.05264 & 3096.0 & 5274.0 & 8050.0 & 9275.0 \\
\hline $\mathrm{shs} 9 \mathrm{k}^{-} \mathrm{rms}^{-}-90$ & 46.07915 & 0.41066 & 6.0 & 0.05257 & 3080.0 & 5267.0 & 8076.0 & 9271.0 \\
\hline shs $9 \mathrm{k}-$ segmentino & 77.40965 & 0.18467 & 25.0 & 0.02181 & 957.0 & 3201.0 & 7355.0 & 9236.0 \\
\hline shs9k- siplca & 81.09412 & 0.14743 & 31.0 & 0.01787 & 689.0 & 2724.0 & 7154.0 & 9255.0 \\
\hline shs $9 \mathrm{k}$ vocals & 49.71732 & 0.48504 & 3.0 & 0.09081 & 3956.0 & 5498.0 & 7940.0 & 9273.0 \\
\hline shs $9 k^{-}$vocals_rms 10 & 90.95994 & 0.12264 & 36.0 & 0.01608 & 505.0 & 2340.0 & 6888.0 & 9250.0 \\
\hline shs9k ${ }^{-}$vocals ${ }^{-}{ }^{-} 20$ & 68.31585 & 0.25591 & 18.0 & 0.03019 & 1629.0 & 3834.0 & 7409.0 & 9266.0 \\
\hline shs $9 k^{-}$vocals ${ }^{-} \mathrm{rms}^{-} 30$ & 56.14236 & 0.37507 & 8.0 & 0.05122 & 2754.0 & 4861.0 & 7809.0 & 9266.0 \\
\hline shs $9 k^{-}$vocals ${ }^{-} \mathrm{rms}^{-} 40$ & 49.30336 & 0.43569 & 5.0 & 0.06648 & 3341.0 & 5409.0 & 7999.0 & 9275.0 \\
\hline shs $9 k^{-}$vocals ${ }^{-} \mathrm{rms}^{-} 50$ & 45.41536 & 0.4578 & 4.0 & 0.07188 & 3571.0 & 5551.0 & 8085.0 & 9270.0 \\
\hline shs9k-vocals_rms 60 & 43.63246 & 0.4593 & 4.0 & 0.0693 & 3575.0 & 5602.0 & 8148.0 & 9276.0 \\
\hline shs $9 k^{-}$vocals ${ }^{-} \mathrm{rms}^{-} 70$ & 43.42462 & 0.44726 & 4.0 & 0.06443 & 3420.0 & 5550.0 & 8126.0 & 9270.0 \\
\hline shs $9 k^{-}$vocals ${ }^{-} \mathrm{rms}^{-} 80$ & 43.30401 & 0.43289 & 5.0 & 0.05862 & 3289.0 & 5433.0 & 8182.0 & 9278.0 \\
\hline shs $9 k^{-}$vocals ${ }^{-} \mathrm{rms}^{-} 90$ & 44.47351 & 0.41457 & 6.0 & 0.0536 & 3089.0 & 5354.0 & 8107.0 & 9275.0 \\
\hline
\end{tabular}

Table 6.70: The best results for each modified dataset for SHS9K.

\subsubsection{Discussion}

\section{Best results}

The analysis of the best results corresponds to looking for modified datasets that have better mean ranks than the original ones. Covers 80 has one modified dataset (vocals rms 70) with better mean rank; YouTubeCovers has none; Covers1000 has three (rms_90, vocals_rms_90, vocals_rms_40); Mazurkas has nine (rms_40, rms_50, rms_60, rms_70, rms_80, rms_90, 30_beg, 30_mid, bartsch_wakefield); and SHS9K has eight (30_mid, rms_80, rms_90, vocals_rms_50, vocals_rms_60, vocals_rms_70, vocals_rms_80,vocals_rms_90).

Clearly, datasets modified by frame selection appear more often (17 of the 21 that lead to better results), than the other types of modification techniques, followed by 4 datasets modified by segment selection. Analysing only the MR, none of the source separation modified datasets appeared.

While MR takes account of the ranks of the first versions in the retrieved lists, MAP uses the ranks from all versions (as explained in Section 3.1.4), so we also look for better results obtained by MAP. Covers 80 has five datasets (bass, rms_90, vocals, vocals_rms_30, vocals_rms_70) with better mean rank; YouTubeCovers has also five (vocals_rms_40, vocals_rms_50, vocals_rms_60, vocals_rms_70, vocals_rms_80); Covers1000 has five likewise (bass, rms_90, vocals, vocals_rms_60, vocals_rms_90); Mazurkas has none; and SHS9K has eleven (bass, rms_80, rms_90, vocals, vocals_rms_30, vocals_rms_40, vocals_rms_50,vocals_rms_60, vocals_rms_70, vocals_rms_80, vocals_rms_90).

Again, the datasets modified by frame selection emerge more frequently (20 out of 26 techniques that produce higher MAP values), than the other types of dataset modifications. The other two types of modifications - segment selection and source separation - now switch places. All the remaining 6 modifications use source separation, and none better result is obtained using segment selection, when analysing the MAP metric. 


\section{Comparative results}

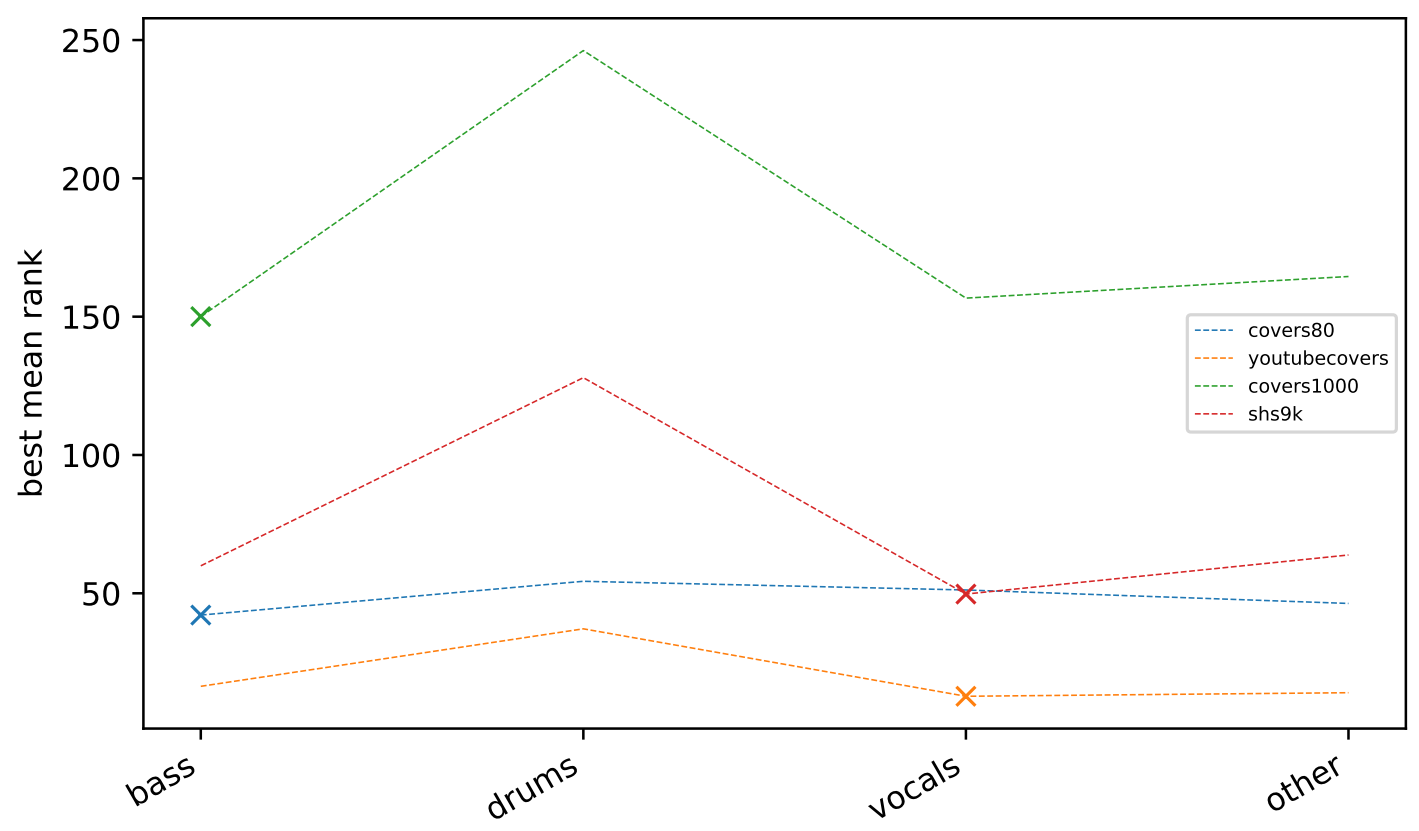

Figure 6.16: Comparison of best results obtained with source separation secondary datasets.

Figure 6.16 shows us the best mean ranks obtained for 4 cover song datasets (please remember that it is not possible to extract sources from Mazurkas dataset). In this figure, the best mean rank for each dataset is highlighted with an ' $x$ '. Two datasets - Covers 80 and Covers 1000 - have their best results via source separation by the bass secondary datasets. The other two datasets YouTubeCovers and SHS9K - have their best results with the isolated vocals datasets. Both sources hold mainly tonal information, which confirm the statements in the literature about this being the best music characteristic for CSI problem [SGH10, SSG12, CLX17]. 


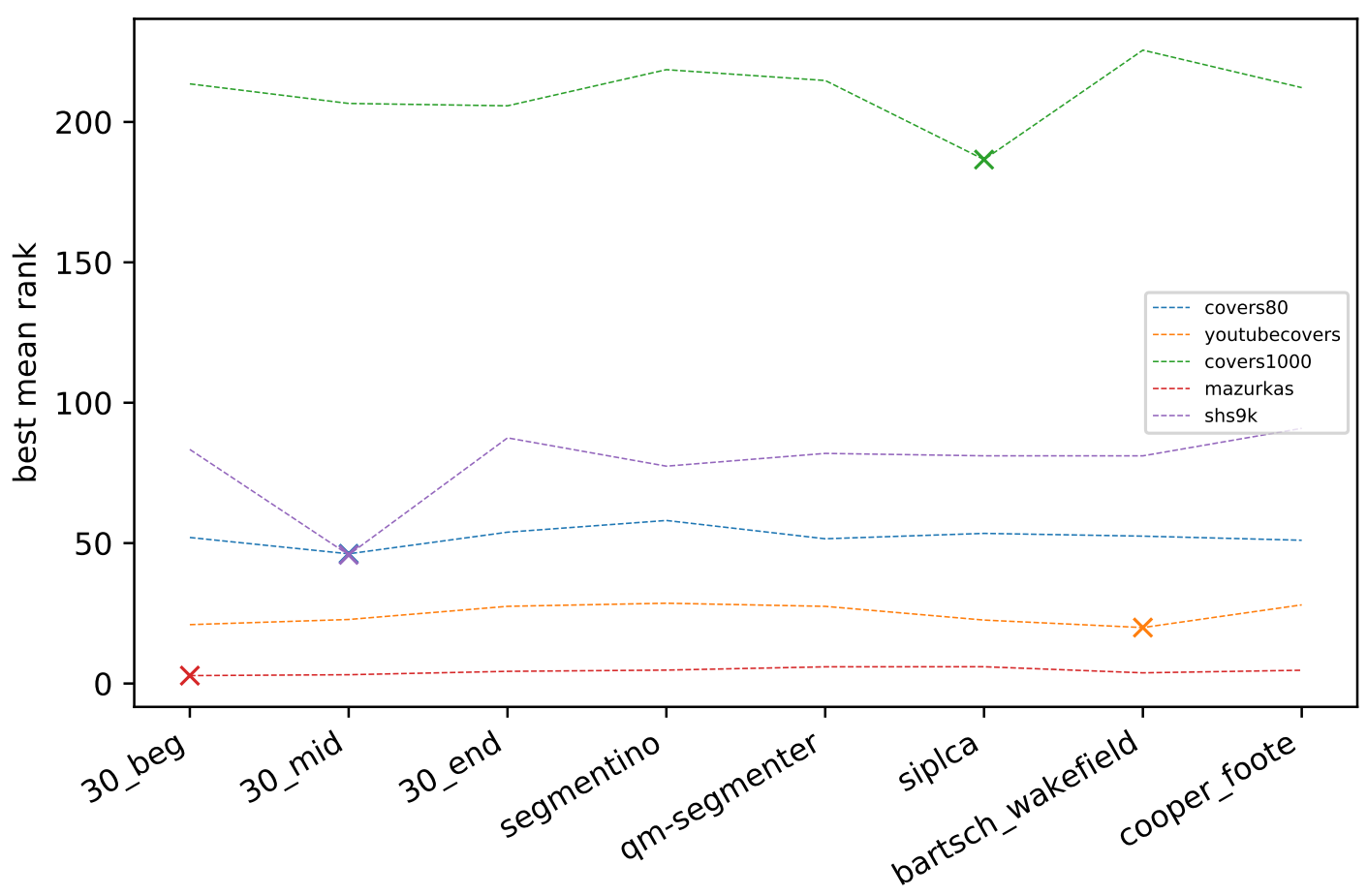

Figure 6.17: Comparison of best results obtained with segment selection secondary datasets.

Comparing the best results obtained with segment selection, Figure 6.17 shows that YouTubeCovers has the best result with a thumbnailing technique (Bartsch and Wakefield), Covers1000 has the best result with a segmentation technique (SIPLCA), and three remaining datasets have their best results with the selection of arbitrary segments (Covers80 and SHS9K with the middle 30-second segments, and Mazurkas with the initial 30-second segments). 


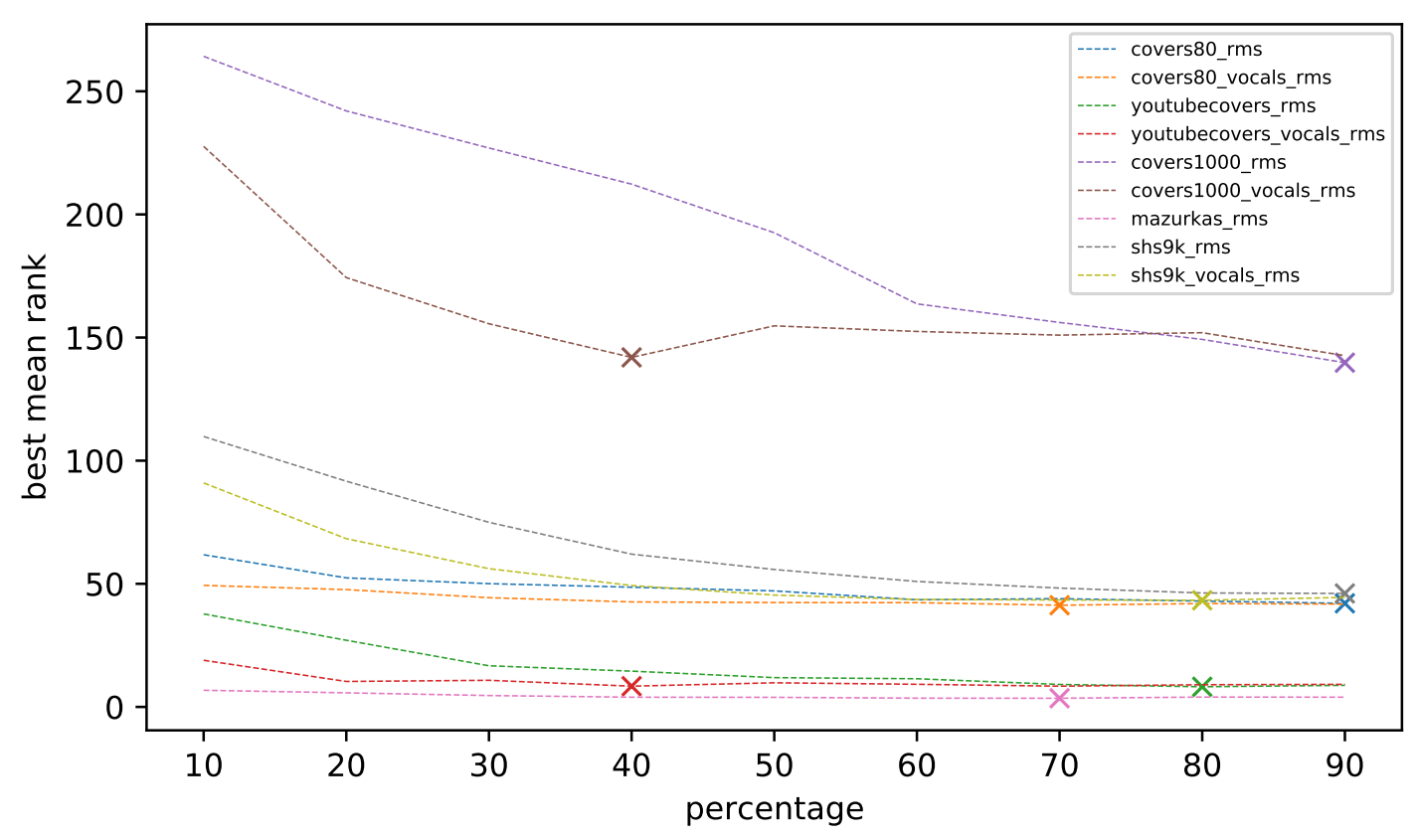

Figure 6.18: Comparison of best results obtained with frame selection secondary datasets.

Finally, Figure 6.18 shows us the best results for datasets obtained with frame selection methods. Firstly, we can check that the best mean rank values do not monotonically decrease with the increase of the percentage of retained frames. Secondly, the best results are not always obtained with the maximum percentage. There are two best results with $80 \%$ (shs $9 k_{\text {_v vocals_rms }}$ and youtubecovers_rms), two with $70 \%$ (mazurkas_rms and covers80_vocals_rms), and also two with $40 \%$ (youtubecovers_vocals_rms and covers1000_vocals_rms).

Lastly, it is possible to compare the frame selection process made with the songs' RMS values and with the vocals' RMS values (except with Mazurkas whose tracks do not have vocals). From 36 results (4 datasets $\times 9$ percentage values) comparing relative pairs (same dataset with same percentage value), 32 values $(\approx 88 \%)$ are better with the vocals' RMS. This is a good discovery in favor of the frame selection technique we propose.

\subsection{Experiment \#6: Fusions}

\subsubsection{Experiment design}

The main goal of this experiment is to try different fusion functions with similarity matrices obtained from the source separation modified datasets. From experiments $\# 3$ and $\# 4$ it is doubly verified that fusing filtered models leads to better results compared to Top- $\mathrm{N}$ models. So we fuse only the filtered models from the derivative dataset of each individual source.

We experiment with 4 alternative matrix fusion functions: 3 simple ones - minimum, maximum, and mean - and a more complex one, as presented in Section 5.5, Similarity Network Fusion (SNF). Therefore we have here 4 modified datasets, 16 models, and 4 functions, leading to $262,144\left(16^{4} \times 4\right)$ fusion schemes. It should be remembered that Mazurkas recordings do not have the sources identified and separated by Open-Unmix [SULM19], so this dataset is not used in this experiment.

Tables 6.71 to 6.86 show the Top- 5 results for each individual source dataset, each fusion function, and which models are fused to obtain the results. Please, refer to Appendix C to identify 
which models are listed below, since they are displayed by their line identifiers. Also, all results are published in https://rppbodo.github.io/phd/experiment_6.html.

\subsubsection{Results}

\section{Covers80}

\begin{tabular}{|c|c|c|c|c|c|c|c|c|c|c|c|}
\hline bass & $\begin{array}{l}\text { model } \\
\text { drums }\end{array}$ & $\begin{array}{l}\text { line id } \\
\text { vocals }\end{array}$ & other & MR & MRR & MDR & MAP & Top-1 & Top-10 & Top-100 & Top-1000 \\
\hline 6 & 2 & 6 & 13 & 27.675 & 0.36057 & 9.5 & 0.36057 & 47.0 & 81.0 & 149.0 & 160.0 \\
\hline 6 & 9 & 6 & 10 & 27.675 & 0.36057 & 9.5 & 0.36057 & 47.0 & 81.0 & 149.0 & 160.0 \\
\hline 6 & 5 & 6 & 5 & 27.675 & 0.36057 & 9.5 & 0.36057 & 47.0 & 81.0 & 149.0 & 160.0 \\
\hline 6 & 13 & 6 & 2 & 27.675 & 0.36057 & 9.5 & 0.36057 & 47.0 & 81.0 & 149.0 & 160.0 \\
\hline 6 & 10 & 6 & 9 & 27.675 & 0.36057 & 9.5 & 0.36057 & 47.0 & 81.0 & 149.0 & 160.0 \\
\hline
\end{tabular}

Table 6.71: Top-5 results for Covers80 fusing models using the minimum function.

\begin{tabular}{|c|c|c|c|c|c|c|c|c|c|c|c|}
\hline bass & $\begin{array}{c}\text { model' } \\
\text { drums }\end{array}$ & $\begin{array}{l}\text { line id } \\
\text { vocals }\end{array}$ & other & MR & MRR & MDR & MAP & Top-1 & Top-10 & Top-100 & Top- 1000 \\
\hline 6 & 12 & 14 & 12 & 28.0 & 0.32869 & 13.5 & 0.32869 & 41.0 & 77.0 & 150.0 & 160.0 \\
\hline 6 & 12 & 14 & 4 & 28.0125 & 0.32711 & 13.5 & 0.32711 & 41.0 & 77.0 & 150.0 & 160.0 \\
\hline 6 & 12 & 14 & 8 & 28.01875 & 0.32711 & 13.5 & 0.32711 & 41.0 & 77.0 & 150.0 & 160.0 \\
\hline 6 & 12 & 14 & 0 & 28.01875 & 0.32711 & 13.5 & 0.32711 & 41.0 & 77.0 & 150.0 & 160.0 \\
\hline 6 & 12 & 14 & 11 & 28.01875 & 0.32711 & 13.5 & 0.32711 & 41.0 & 77.0 & 150.0 & 160.0 \\
\hline
\end{tabular}

Table 6.72: Top-5 results for Covers80 fusing models using the maximum function.

\begin{tabular}{|c|c|c|c|c|c|c|c|c|c|c|c|}
\hline bass & $\begin{array}{c}\text { model } \\
\text { drums }\end{array}$ & $\begin{array}{l}\text { line id } \\
\text { vocals }\end{array}$ & other & MR & MRR & MDR & MAP & Top-1 & Top-10 & Top-100 & Top- 1000 \\
\hline 6 & 0 & 6 & 11 & 23.73125 & 0.41531 & 10.0 & 0.41531 & 57.0 & 83.0 & 152.0 & 160.0 \\
\hline 6 & 1 & 6 & 11 & 23.84375 & 0.41438 & 9.5 & 0.41438 & 57.0 & 83.0 & 152.0 & 160.0 \\
\hline 6 & 0 & 6 & 0 & 24.5125 & 0.4273 & 9.0 & 0.4273 & 59.0 & 83.0 & 150.0 & 160.0 \\
\hline 6 & 0 & 6 & 1 & 24.54375 & 0.42729 & 9.0 & 0.42729 & 59.0 & 84.0 & 150.0 & 160.0 \\
\hline 6 & 1 & 6 & 0 & 24.5625 & 0.42689 & 9.0 & 0.42689 & 59.0 & 83.0 & 150.0 & 160.0 \\
\hline
\end{tabular}

Table 6.73: Top-5 results for Covers80 fusing models using the mean function.

\begin{tabular}{|c|c|c|c|c|c|c|c|c|c|c|c|}
\hline bass & $\begin{array}{l}\text { model } \\
\text { drums }\end{array}$ & $\begin{array}{l}\text { line id } \\
\text { vocals }\end{array}$ & other & MR & MRR & MDR & MAP & Top-1 & Top-10 & Top-100 & Top- 1000 \\
\hline 3 & 8 & 11 & 5 & 44.08125 & 0.13177 & 29.5 & 0.13177 & 9.0 & 42.0 & 142.0 & \\
\hline 3 & 8 & 11 & 3 & 44.275 & 0.11449 & 29.0 & 0.11449 & 7.0 & 46.0 & 140.0 & \\
\hline 2 & 8 & 8 & 5 & 44.91875 & 0.1084 & 26.0 & 0.1084 & 7.0 & 41.0 & 137.0 & \\
\hline 3 & 8 & 4 & 5 & 45.025 & 0.14034 & 30.0 & 0.14034 & 11.0 & 44.0 & 141.0 & \\
\hline 3 & 8 & 8 & 5 & 45.2625 & 0.12966 & 31.0 & 0.12966 & 9.0 & 43.0 & 137.0 & \\
\hline
\end{tabular}

Table 6.74: Top-5 results for Covers80 fusing models using SNF.

\section{YouTubeCovers}

\begin{tabular}{|c|c|c|c|c|c|c|c|c|c|c|c|}
\hline bass & $\begin{array}{l}\text { model's } \\
\text { drums }\end{array}$ & $\begin{array}{l}\text { line id } \\
\text { vocals }\end{array}$ & other & MR & MRR & MDR & MAP & Top-1 & Top-10 & Top-100 & Top- 1000 \\
\hline 1 & 10 & 0 & 0 & 9.04857 & 0.56417 & 2.0 & 0.31359 & 163.0 & 272.0 & 347.0 & \\
\hline 8 & 8 & 0 & 0 & 9.04857 & 0.56417 & 2.0 & 0.31359 & 163.0 & 272.0 & 347.0 & \\
\hline 12 & 8 & 0 & 0 & 9.04857 & 0.56417 & 2.0 & 0.31359 & 163.0 & 272.0 & 347.0 & \\
\hline 1 & 8 & 0 & 0 & 9.04857 & 0.56417 & 2.0 & 0.31359 & 163.0 & 272.0 & 347.0 & \\
\hline 1 & 12 & 0 & 0 & 9.04857 & 0.56417 & 2.0 & 0.31359 & 163.0 & 272.0 & 347.0 & \\
\hline
\end{tabular}

Table 6.75: Top-5 results for YouTubeCovers fusing models using minimum function.

\begin{tabular}{|c|c|c|c|c|c|c|c|c|c|c|c|}
\hline bass & $\begin{array}{r}\text { mode } \\
\text { drums }\end{array}$ & $\begin{array}{l}\text { line id } \\
\text { vocals }\end{array}$ & other & MR & MRR & MDR & MAP & Top-1 & Top-10 & Top-100 & Top-1000 \\
\hline 2 & 6 & 0 & 6 & 11.82571 & 0.61532 & 1.0 & 0.32795 & 190.0 & 261.0 & 340.0 & \\
\hline 2 & 6 & 0 & 2 & 11.82571 & 0.61544 & 1.0 & 0.32507 & 190.0 & 260.0 & 340.0 & \\
\hline 2 & 6 & 0 & 9 & 11.86571 & 0.61523 & 1.0 & 0.32741 & 190.0 & 260.0 & 341.0 & \\
\hline 2 & 6 & 0 & 4 & 11.87143 & 0.61514 & 1.0 & 0.32739 & 190.0 & 260.0 & 340.0 & \\
\hline 2 & 6 & 0 & 5 & 11.87143 & 0.61514 & 1.0 & 0.32739 & 190.0 & 260.0 & 340.0 & \\
\hline
\end{tabular}

Table 6.76: Top-5 results for YouTubeCovers fusing models using maximum function. 


\begin{tabular}{|c|c|c|c|c|c|c|c|c|c|c|c|}
\hline bass & $\begin{array}{l}\text { model' } \\
\text { drums }\end{array}$ & $\begin{array}{l}\text { line id } \\
\text { vocals }\end{array}$ & other & MR & MRR & MDR & MAP & Top-1 & Top-10 & Top-100 & Top- 1000 \\
\hline 0 & 4 & 0 & 2 & 7.63429 & 0.64876 & 1.0 & 0.33159 & 196.0 & 286.0 & 346.0 & \\
\hline 0 & 3 & 0 & 2 & 7.74857 & 0.64938 & 1.0 & 0.33093 & 195.0 & 288.0 & 346.0 & \\
\hline 4 & 3 & 0 & 0 & 8.00286 & 0.64513 & 1.0 & 0.35413 & 194.0 & 297.0 & 347.0 & 350.0 \\
\hline 4 & 4 & 0 & 0 & 8.1 & 0.64107 & 1.0 & 0.35168 & 192.0 & 291.0 & 347.0 & 350.0 \\
\hline 0 & 4 & 0 & 0 & 8.11429 & 0.69677 & 1.0 & 0.38713 & 218.0 & 294.0 & 344.0 & 350.0 \\
\hline
\end{tabular}

Table 6.77: Top-5 results for YouTubeCovers fusing models using mean function.

\begin{tabular}{|c|c|c|c|c|c|c|c|c|c|c|c|}
\hline bass & $\begin{array}{l}\text { model } \\
\text { drums }\end{array}$ & $\begin{array}{l}\text { line id } \\
\text { vocals }\end{array}$ & other & MR & MRR & MDR & MAP & Top-1 & Top-10 & Top-100 & Top-1000 \\
\hline 7 & 0 & 8 & 9 & 22.30857 & 0.28747 & 8.0 & 0.12021 & 57.0 & 198.0 & 335.0 & \\
\hline 4 & 0 & 8 & 9 & 22.51429 & 0.29568 & 11.0 & 0.12344 & 66.0 & 174.0 & 336.0 & \\
\hline 9 & 5 & 5 & 0 & 22.60571 & 0.25172 & 9.0 & 0.11368 & 42.0 & 186.0 & 333.0 & \\
\hline 8 & 5 & 8 & 0 & 22.83714 & 0.30203 & 10.0 & 0.14037 & 67.0 & 177.0 & 337.0 & \\
\hline 9 & 5 & 8 & 0 & 22.85714 & 0.27738 & 10.0 & 0.12357 & 59.0 & 179.0 & 338.0 & \\
\hline
\end{tabular}

Table 6.78: Top-5 results for YouTubeCovers fusing models using SNF.

\section{Covers1000}

\begin{tabular}{|c|c|c|c|c|c|c|c|c|c|c|c|}
\hline bass & $\begin{array}{r}\text { model } \\
\text { drums }\end{array}$ & $\begin{array}{l}\text { line id } \\
\text { vocals }\end{array}$ & other & MR & MRR & MDR & MAP & Top-1 & Top-10 & Top-100 & Top- 1000 \\
\hline 0 & 11 & 0 & 0 & 97.947 & 0.34529 & 18.5 & 0.28203 & 293.0 & 439.0 & 739.0 & \\
\hline 0 & 11 & 0 & 7 & 103.246 & 0.38502 & 12.0 & 0.3162 & 332.0 & 483.0 & 738.0 & \\
\hline 0 & 8 & 0 & 5 & 103.246 & 0.38502 & 12.0 & 0.3162 & 332.0 & 483.0 & 738.0 & \\
\hline 0 & 8 & 0 & 14 & 103.246 & 0.38502 & 12.0 & 0.3162 & 332.0 & 483.0 & 738.0 & \\
\hline 0 & 8 & 0 & 8 & 103.246 & 0.38502 & 12.0 & 0.3162 & 332.0 & 483.0 & 738.0 & \\
\hline
\end{tabular}

Table 6.79: Top-5 results for Covers1000 fusing models using minimum function.

\begin{tabular}{|c|c|c|c|c|c|c|c|c|c|c|c|}
\hline bass & $\begin{array}{c}\text { model' } \\
\text { drums }\end{array}$ & $\begin{array}{l}\text { line id } \\
\text { vocals }\end{array}$ & other & MR & MRR & MDR & MAP & Top-1 & Top-10 & Top-100 & Top-1000 \\
\hline 0 & 1 & 15 & 6 & 149.799 & 0.33058 & 27.0 & 0.26457 & 281.0 & 425.0 & 649.0 & \\
\hline 0 & 4 & 1 & 6 & 149.799 & 0.33058 & 27.0 & 0.26457 & 281.0 & 425.0 & 649.0 & \\
\hline 0 & 1 & 3 & 6 & 149.799 & 0.33058 & 27.0 & 0.26457 & 281.0 & 425.0 & 649.0 & \\
\hline 0 & 4 & 6 & 6 & 149.831 & 0.33118 & 28.0 & 0.26525 & 282.0 & 424.0 & 649.0 & \\
\hline 0 & 1 & 6 & 6 & 149.831 & 0.33118 & 28.0 & 0.26525 & 282.0 & 424.0 & 649.0 & \\
\hline
\end{tabular}

Table 6.80: Top-5 results for Covers1000 fusing models using maximum function.

\begin{tabular}{|c|c|c|c|c|c|c|c|c|c|c|c|}
\hline bass & $\begin{array}{l}\text { model } \\
\text { drums }\end{array}$ & $\begin{array}{l}\text { line id } \\
\text { vocals }\end{array}$ & other & MR & MRR & MDR & MAP & Top-1 & Top-10 & Top-100 & Тор-1000 \\
\hline 0 & 1 & 0 & 3 & 93.464 & 0.43794 & 7.0 & 0.35771 & 385.0 & 537.0 & 779.0 & \\
\hline 0 & 1 & 0 & 1 & 94.771 & 0.43178 & 8.0 & 0.35226 & 375.0 & 525.0 & 772.0 & \\
\hline 0 & 0 & 0 & 0 & 98.65 & 0.36548 & 12.0 & 0.29382 & 301.0 & 483.0 & 750.0 & \\
\hline 0 & 0 & 0 & 1 & 102.164 & 0.38365 & 11.0 & 0.30681 & 320.0 & 499.0 & 742.0 & \\
\hline 0 & 15 & 0 & 0 & 102.758 & 0.38889 & 11.0 & 0.31825 & 331.0 & 496.0 & 760.0 & \\
\hline
\end{tabular}

Table 6.81: Top-5 results for Covers1000 fusing models using mean function.

\begin{tabular}{|c|c|c|c|c|c|c|c|c|c|c|c|}
\hline bass & $\begin{array}{l}\text { model' } \\
\text { drums }\end{array}$ & $\begin{array}{l}\text { line id } \\
\text { vocals }\end{array}$ & other & MR & MRR & MDR & MAP & Top-1 & Top-10 & Top-100 & Top-1000 \\
\hline 8 & 3 & 3 & 6 & 153.648 & 0.12984 & 65.0 & 0.09162 & 72.0 & 230.0 & 594.0 & \\
\hline 0 & 3 & 3 & 6 & 154.185 & 0.139 & 66.0 & 0.10154 & 82.0 & 239.0 & 588.0 & \\
\hline 0 & 3 & 3 & 5 & 161.74 & 0.11418 & 65.5 & 0.08688 & 57.0 & 220.0 & 583.0 & \\
\hline 3 & 3 & 3 & 6 & 162.273 & 0.13289 & 67.0 & 0.09446 & 78.0 & 227.0 & 579.0 & \\
\hline 0 & 11 & 3 & 6 & 163.958 & 0.11783 & 79.0 & 0.08224 & 67.0 & 205.0 & 551.0 & \\
\hline
\end{tabular}

Table 6.82: Top-5 results for Covers1000 fusing models using SNF.

\section{SHS9K}

\begin{tabular}{|c|c|c|c|c|c|c|c|c|c|c|c|}
\hline bass & $\begin{array}{l}\text { model } \\
\text { drums }\end{array}$ & $\begin{array}{l}\text { line id } \\
\text { vocals }\end{array}$ & other & MR & MRR & MDR & MAP & Top-1 & Top-10 & Top-100 & Top-1000 \\
\hline 0 & 1 & 0 & 6 & 41.97728 & 0.31233 & 10.0 & 0.06505 & 1992.0 & 4738.0 & 8197.0 & \\
\hline 0 & 13 & 0 & 2 & 67.60414 & 0.20474 & 19.0 & 0.02875 & 1066.0 & 3629.0 & 7705.0 & \\
\hline 4 & 5 & 13 & 2 & 67.60575 & 0.20463 & 19.0 & 0.02875 & 1064.0 & 3629.0 & 7704.0 & \\
\hline 5 & 3 & 3 & 5 & 69.6596 & 0.17229 & 27.0 & 0.01985 & 910.0 & 2964.0 & 7285.0 & \\
\hline 13 & 4 & 9 & 5 & 73.58421 & 0.22815 & 20.0 & 0.02439 & 1335.0 & 3670.0 & 7423.0 & \\
\hline
\end{tabular}

Table 6.83: Top-5 results for SHS9K fusing models using minimum function. 


\begin{tabular}{|c|c|c|c|c|c|c|c|c|c|c|c|}
\hline bass & $\begin{array}{l}\text { model } \\
\text { drums }\end{array}$ & $\begin{array}{l}\text { line id } \\
\text { vocals }\end{array}$ & other & MR & MRR & MDR & MAP & Top-1 & Top-10 & Top-100 & Top-1000 \\
\hline 0 & 0 & 0 & 8 & 51.90308 & 0.33782 & 10.0 & 0.03659 & 2353.0 & 4737.0 & 7920.0 & \\
\hline 0 & 0 & 0 & 7 & 52.00118 & 0.33741 & 10.0 & 0.03655 & 2350.0 & 4738.0 & 7916.0 & \\
\hline 15 & 11 & 0 & 1 & 67.25867 & 0.19913 & 19.0 & 0.02638 & 1003.0 & 3595.0 & 7675.0 & \\
\hline 5 & 14 & 11 & 1 & 71.31262 & 0.19336 & 22.0 & 0.0241 & 1018.0 & 3348.0 & 7561.0 & \\
\hline 0 & 1 & 2 & 3 & 73.36496 & 0.16625 & 25.0 & 0.01841 & 798.0 & 3041.0 & 7397.0 & \\
\hline
\end{tabular}

Table 6.84: Top-5 results for SHS9K fusing models using maximum function.

\begin{tabular}{llllllllllll}
\multicolumn{1}{c}{ model's line id } & \multicolumn{1}{l}{} & & & & & \\
bass & drums & vocals & other & MR & MRR & MDR & MAP & Top-1 & Top-10 & Top-100 & Top-1000 \\
\hline 0 & 0 & 0 & 8 & 38.61393 & 0.46388 & 4.0 & 0.06979 & 3581.0 & 5727.0 & 8291.0 & \\
0 & 0 & 0 & 7 & 38.86377 & 0.39672 & 6.0 & 0.05259 & 2811.0 & 5422.0 & 8319.0 & \\
5 & 14 & 11 & 1 & 60.45348 & 0.21987 & 18.0 & 0.02653 & 1203.0 & 3763.0 & 7796.0 & 7796.0 \\
15 & 11 & 0 & 1 & 60.92796 & 0.20144 & 18.0 & 0.02459 & 1037.0 & 3586.0 & 7796.0 \\
0 & 1 & 2 & 3 & 64.36528 & 0.18218 & 21.0 & 0.0207 & 886.0 & 3343.0 & 7656.0
\end{tabular}

Table 6.85: Top-5 results for SHS9K fusing models using mean function.

\begin{tabular}{|c|c|c|c|c|c|c|c|c|c|c|c|}
\hline bass & $\begin{array}{l}\text { model' } \\
\text { drums }\end{array}$ & $\begin{array}{l}\text { line id } \\
\text { vocals }\end{array}$ & other & MR & MRR & MDR & MAP & Top-1 & Top-10 & Top-100 & Top- 1000 \\
\hline 5 & 3 & 3 & 5 & 93.12061 & 0.10387 & 45.0 & 0.01248 & 412.0 & 1973.0 & 6639.0 & \\
\hline 8 & 0 & 2 & 5 & 93.17779 & 0.09779 & 45.0 & 0.01238 & 370.0 & 1876.0 & 6592.0 & \\
\hline 0 & 1 & 0 & 6 & 96.06612 & 0.12136 & 41.0 & 0.01424 & 526.0 & 2267.0 & 6683.0 & \\
\hline 0 & 13 & 0 & 2 & 96.87982 & 0.08756 & 41.0 & 0.0136 & 278.0 & 1822.0 & 6786.0 & \\
\hline 8 & 2 & 13 & 6 & 97.1106 & 0.08988 & 45.0 & 0.01304 & 287.0 & 1943.0 & 6578.0 & \\
\hline
\end{tabular}

Table 6.86: Top-5 results for SHS9K fusing models using SNF.

\subsubsection{Discussion}

The best results obtained with each dataset are: Covers80 best MR equals to 23.73125; YouTubeCovers best MR equals to 7.63428571; Covers1000 best MR equals to 93.464; SHS9K best MR equals to 38.61393 (all of them using the mean function). It was unexpected to see that SNF does not perform well in this experiment settings for these datasets, since it is such a complex fusion function. The models fused to obtain each one of these results are:

- Covers 80

- bass: \{pitch_contour_segmentation, octave_abstraction, lcs_circular_mean\}

- drums: \{chroma_stft, diff_stats_1, cosine_distance_oti\}

- vocals: \{pitch_contour_segmentation, octave_abstraction, lcs_circular_mean\}

- other: \{zero_crossing_rate, basic_stats_1, chebyshev_distance\}

- YouTubeCovers

- bass: \{pitch_contour_segmentation, octave_abstraction, lcs_circular_mean\}

- drums: \{chroma_stft, diff_stats_1, cosine_distance_oti\}

- vocals: \{pitch_contour_segmentation, octave_abstraction, lcs_circular_mean\}

- other: \{pitch_contour_segmentation, markov_chain, circular_cosine\}

- Covers1000

- bass: \{pitch_contour_segmentation, octave_abstraction, lcs_circular_mean\}

- drums: \{beat_synchronous_chroma_stft, diff_stats_1, cosine_distance_oti\}

- vocals: \{pitch_contour_segmentation, octave_abstraction, lcs_circular_mean\}

- other: \{rhythm_histogram, bypass, cosine_distance\}

- SHS9K

- bass: \{pitch_contour_segmentation, octave_abstraction, lcs_circular_mean\} 
- drums: \{pitch_contour_segmentation, octave_abstraction, lcs_circular_mean\}

- vocals: \{pitch_contour_segmentation, octave_abstraction, lcs_circular_mean\}

- other: \{rhythm_histogram, bypass, cosine_distance\}

Regarding the features from the models of "bass" and "vocals" best results, all of them have Pitch Contour Segmentation. Actually, if we look further, the models for the "bass" and "vocals" that lead to the best results are all the same: \{pitch_contour_segmentation, octave_abstraction, lcs_circular_mean\}. Tonal models are expected to appear in such positions, but the fact that they are all equal is quite surprising.

Analysing the remaining sources, the drums' models from Covers80 and YouTubeCovers are exactly the same. The drums' model from the Covers1000 is almost the same: the only difference is the feature being synchronized to beats. SHS9K, however, has a distinct model, the only resemblance is the tonal feature.

Finally, inspecting the models from the "other" source we have a vast group. However, this is expected since this source is the remaining of the separation process after excluding the bass, drums, and vocals from the full mix. So it can vary very much, according to the nature of the tracks of each dataset.

\subsection{Experiment \#7: Theoretical performance bounds}

At this point, we have seen how our music similarity models perform on 5 CSI datasets, and we wish to compare our results with other CSI algorithms. For instance, it is possible to find on GitHub $^{32}$ implementations of five important CSI algorithms: FTM2D [ET12], SiMPle [SYB $\left.{ }^{+} 16\right]$, Serra09 [SSA09], LateFusionChen [CLX17], and EarlyFusionTraile [Tra17]. Table 6.87 shows the results from the algorithms we managed to run so far with our 5 selected CSI datasets.

\begin{tabular}{llllllllll} 
algorithm & dataset & MR & MRR & MDR & MAP & Top 1 & Top 10 & Top 100 & Top 1000 \\
\hline FTM2D & Covers80 & 26.3 & 0.476 & 3 & 0.476 & 64 & 105 & 144 & 160 \\
SiMPle & Covers80 & 28.2 & 0.444 & 6.5 & 0.444 & 62 & 88 & 145 & 160 \\
Serra09 & Covers80 & 17.6 & 0.69 & 1 & 0.69 & 101 & 125 & 148 & 160 \\
LateFusionChen & Covers80 & 15 & 0.709 & 1 & 0.709 & 107 & 125 & 152 & 160 \\
EarlyFusionTraile & Covers80 & 9.19 & 0.759 & 1 & 0.759 & 116 & 132 & 157 & 160 \\
\hline FTM2D & YouTubeCovers & 8.74 & 0.774 & 1 & 0.498 & 257 & 303 & 342 & 350 \\
SiMPle & YouTubeCovers & 7.59 & 0.786 & 1 & 0.484 & 256 & 315 & 344 & 350 \\
Serra09 & YouTubeCovers & 5.47 & 0.919 & 1 & 0.751 & 317 & 331 & 347 & 350 \\
\hline FTM2D & Covers1000 & 71.4 & 0.439 & 5 & 0.368 & 372 & 569 & 826 & 1000 \\
SiMPle & Covers1000 & 91.5 & 0.458 & 5 & 0.391 & 404 & 564 & 767 & 1000 \\
Serra09 & Covers1000 & 61.6 & 0.749 & 1 & 0.677 & 720 & 800 & 874 & 1000 \\
\hline FTM2D & Mazurkas & 10.9 & 0.843 & 1 & 0.422 & 2190 & 2540 & 2680 & 2740 \\
Serra09 & Mazurkas & 7.79 & 0.96 & 1 & 0.91 & 2590 & 2700 & 2720 & 2730 \\
\hline FTM2D & SHS9K & 43.3 & 0.436 & 5 & 0.106 & 3220 & 5740 & 8370 & 9250
\end{tabular}

Table 6.87: Results obtained so far with 5 important CSI algorithms whose implementations are found in GitHub.

Comparing our results with the state-of-the-art, we verify that there is still room for improvement, especially with Covers1000, the dataset for which the applied models obtained the worst results. The low performance of our approach on this dataset led us to question the idea of attempting to solve the CSI problem for a whole dataset using a unique similarity model, which we termed "one-size-fits-all".

Our current hypothesis is that this problem is not due to the discriminating power of the individual similarity models, but to the fact that the type of similarity between a song and its versions changes according to the musical decisions involved in writing/arranging each version. For instance, one cover song could keep the melody and change the harmony or the rhythm with respect to the original song, whereas another version might keep the rhythm and change a singing voice for a spoken one. This led us to conjecture that a similarity model should be selected for each specific query song, or even for each query and potential version candidate.

\footnotetext{
${ }^{32}$ https://github.com/
} 
In order to further investigate this we hypothesize the existence of an oracle that is capable of choosing the best possible model for each CSI query. In our previous experiments, each query produces a ranked list which corresponds to the similarity values of the query with respect to each other song within a chosen model. The hypothesized oracle should be able to instantly select the model that would place the first version of the query in the lowest possible rank of the corresponding list. The idea is to determine some type of upper bound for the performance of models within the context of our framework, and based on this upper bound to decide whether it would be beneficial to explore model selection techniques that would decide, based on a given query, which similarity model is most likely to produce good results. This will guide further research on model selection strategies for similarity-based music retrieval tasks.

An extra experiment is proposed, using the same 690 music similarity models, in order to assess the best possible outcome of these models, given the above-mentioned oracle ${ }^{33}$. For each CSI dataset, we loop over all possible queries, and for each query we use best possible model provided by the oracle, thus producing a ranked list where the first retrieved cover appears at the earliest possible position. We then group all queries that produced the same rank, in order to understand the statistical distribution of the rank values and also to obtain a Mean Rank estimate for the theoretical algorithm proposed. Tables 6.88 to 6.92 display the rank distribution for all datasets considered, and Table 6.93 shows the corresponding Mean Ranks, alongside the MR values obtained in Experiment \#2 for comparison.

\begin{tabular}{lll} 
rank (1st cover retrieved) & number of tracks & percentage \\
\hline 1 & 142 & 88.75 \\
2 & 8 & 5.0 \\
3 & 2 & 1.25 \\
4 & 4 & 2.5 \\
5 & 1 & 0.625 \\
6 & 1 & 0.625 \\
14 & 1 & 0.625 \\
18 & 1 & 0.625
\end{tabular}

Table 6.88: Rank distribution for the Covers80 dataset.

\begin{tabular}{lll} 
rank (1st cover retrieved) & number of tracks & percentage \\
\hline 1 & 350 & 100.0
\end{tabular}

Table 6.89: Rank distribution for the YouTubeCovers dataset.

It is clear from these tables that, with the help of such an oracle, it is indeed possible to obtain very good results for the majority of queries. For instance, on YouTubeCovers the theoretical algorithm proposed achieves a perfect score where all queries produce a cover in the first rank of the retrieved list. Even the worst results attained by this algorithm, in the Covers1000 dataset, with the smallest percentage of queries producing a rank of 1 and a distribution with the longest tail, ends up with a Mean Rank of 1.709 , which is impressive.

Although unrealistic, these numbers offer scientific evidence that the family of similarity models considered include very good combinations of features, aggregators and distances that are capable, in principle, of identifying covers with excellent performance. The question that remains open is how to identify such models that are perfectly fit for a given query.

In an attempt to devise a more realistic approach to help future heuristics identifying fitting models for input queries, we investigated how many different models would be required by such a heuristic in order to keep the mean rank close to the oracle's ideal. The underlying assumption here is that this model selection problem could be cast as a classification problem, where a trained

\footnotetext{
${ }^{33}$ In this experiment, the oracle is implemented via a brute-force search over all available models.
} 


\begin{tabular}{lll} 
rank (1st cover retrieved) & number of tracks & percentage \\
\hline 1 & 811 & 81.1 \\
2 & 86 & 8.6 \\
3 & 30 & 3.0 \\
4 & 13 & 1.3 \\
5 & 12 & 1.2 \\
6 & 9 & 0.9 \\
7 & 7 & 0.7 \\
8 & 6 & 0.6 \\
9 & 3 & 0.3 \\
10 & 3 & 0.3 \\
11 & 7 & 0.7 \\
12 & 3 & 0.3 \\
13 & 3 & 0.3 \\
16 & 3 & 0.3 \\
19 & 1 & 0.1 \\
24 & 1 & 0.1 \\
31 & 1 & 0.1 \\
42 & 1 & 0.1
\end{tabular}

Table 6.90: Rank distribution for the Covers1000 dataset.

\begin{tabular}{lll} 
rank (1st cover retrieved) & number of tracks & percentage \\
\hline 1 & 2738 & 99.89055089383437 \\
2 & 3 & 0.10944910616563298
\end{tabular}

Table 6.91: Rank distribution for the Mazurkas dataset.

classifier would be able to predict the best model for each query within a small set of choices. We conducted a secondary theoretical experiment exploring the number of models required to produce the theoretical best results for a certain fraction of the dataset.

The complete list of models is sorted in descending order by the number of tracks for which they produce the best possible rank. We consider subsets of the $n$ first models in this list. For each $n$, we compute the theoretical mean rank with a restricted version of the oracle that selects the best among the available models, as well as the number of tracks in the dataset for which this selection is optimal. We then plot, for each dataset, the number of tracks optimally covered as well as the theoretical mean rank attained as functions of $n$. Figures 6.19-6.23 display these results.

In these Figures it is possible to observe that the mean rank values drop abruptly with the addition of very few similarity models. Even for the Covers1000 dataset, which attained the worst metrics with a single model, the mean rank obtained in Experiment \#2 drops from 144.041 to 72.0205 with the use of 4 models, and to 36.01025 with 8 models.

This theoretical experiment is meant as an abstract exercise to explore the limits of an approach based on the class of similarity models that use the pipeline described in Section 5.1. It allows us to conclude that such an approach is capable of producing very good results, if extended beyond the "one-size-fits-all" assumption that underlies this research. 


\begin{tabular}{lll} 
rank (1st cover retrieved) & number of tracks & percentage \\
\hline 1 & 9133 & 98.35235838897265 \\
2 & 127 & 1.3676502261468877 \\
3 & 19 & 0.20460908895110919 \\
4 & 6 & 0.0646133965108766 \\
6 & 1 & 0.010768899418479432
\end{tabular}

Table 6.92: Rank distribution for the SHS9k dataset.

\begin{tabular}{lll} 
dataset & best mean rank from experiment \#2 & oracle's theoretical mean rank \\
\hline Covers80 & 41.575 & 1.39375 \\
YouTubeCovers & 7.97143 & 1.0 \\
Covers1000 & 144.041 & 1.709 \\
Mazurkas & 4.15724 & 1.0010944910616564 \\
SHS9K & 47.57883 & 1.0202455309067413
\end{tabular}

Table 6.93: Comparison of metrics obtained in Experiment \#2 with the theoretical metrics.

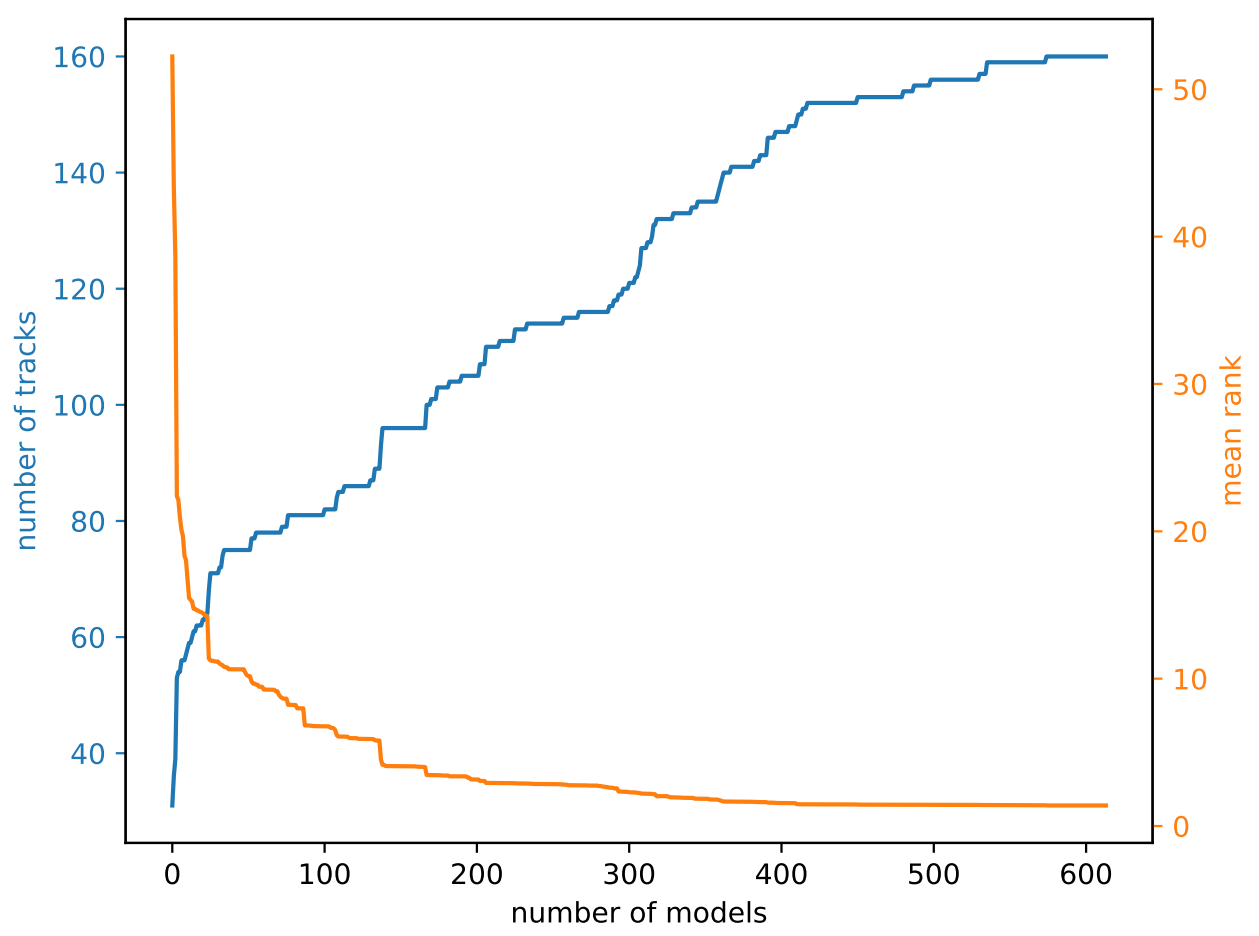

Figure 6.19: Number of tracks and theoretical mean rank as functions of the number of models for the Covers 80 dataset. 


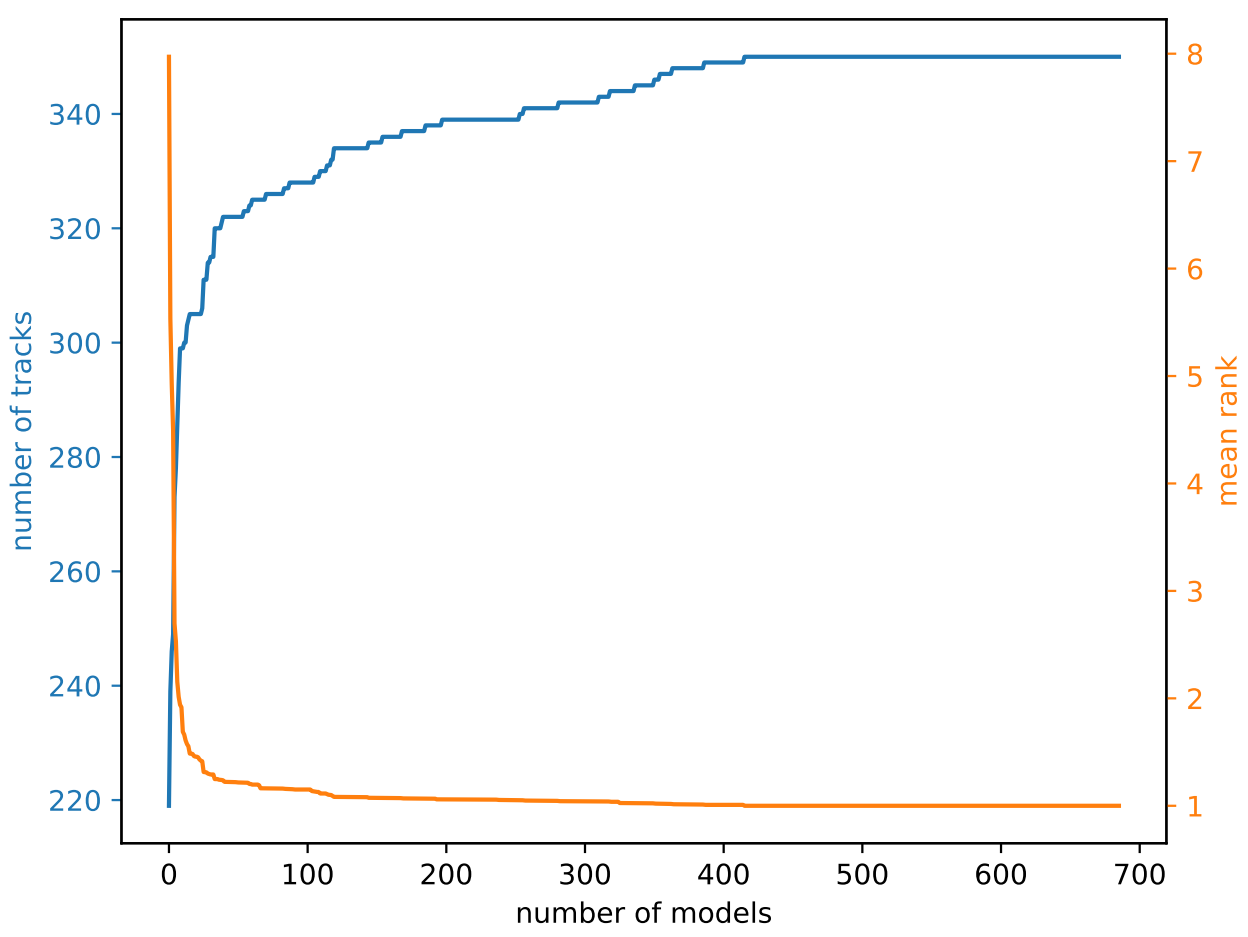

Figure 6.20: Number of tracks and theoretical mean rank as functions of the number of models for the YouTubeCovers dataset.

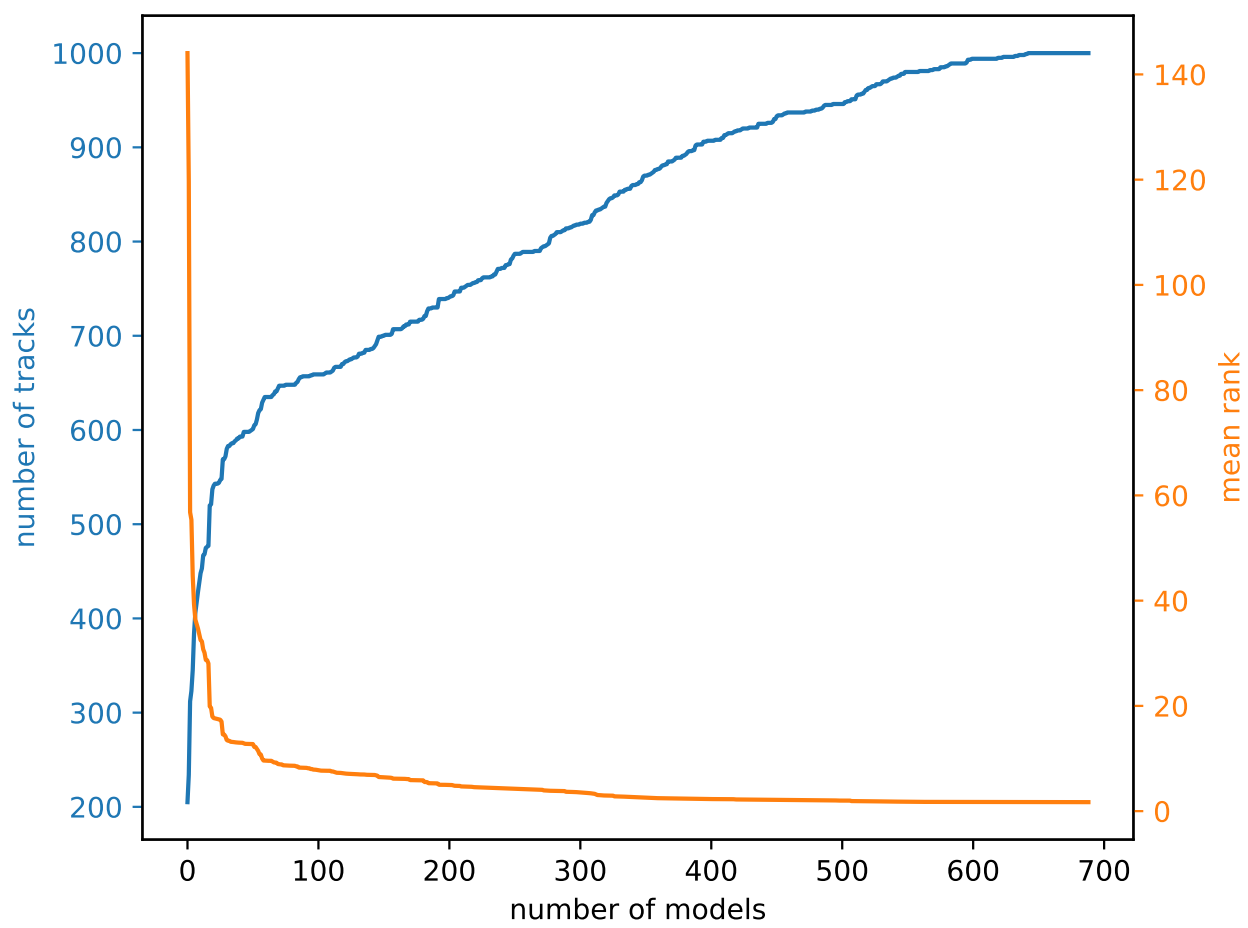

Figure 6.21: Number of tracks and theoretical mean rank as functions of the number of models for the Covers1000 dataset. 


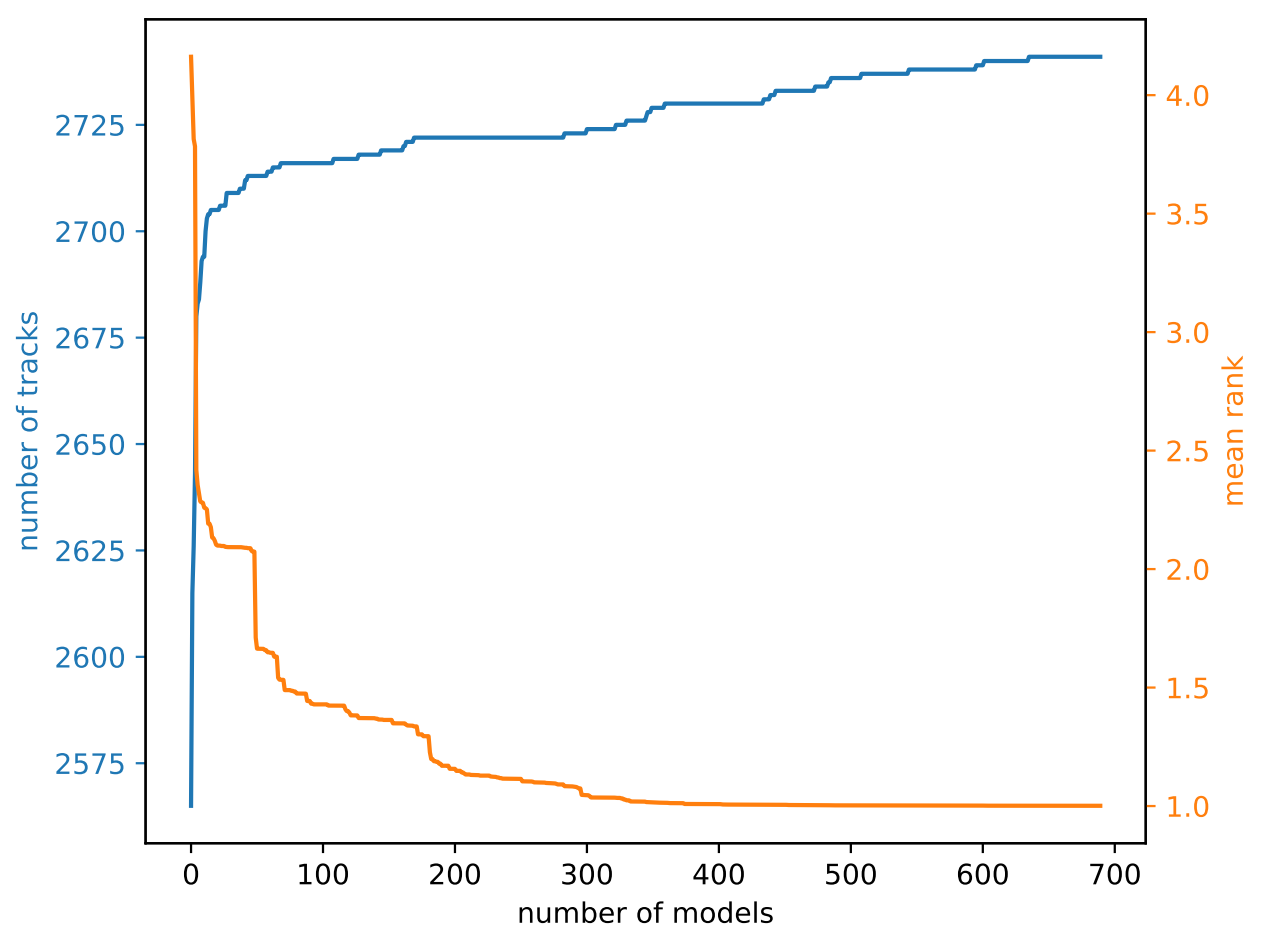

Figure 6.22: Number of tracks and theoretical mean rank as functions of the number of models for the Mazurkas dataset.

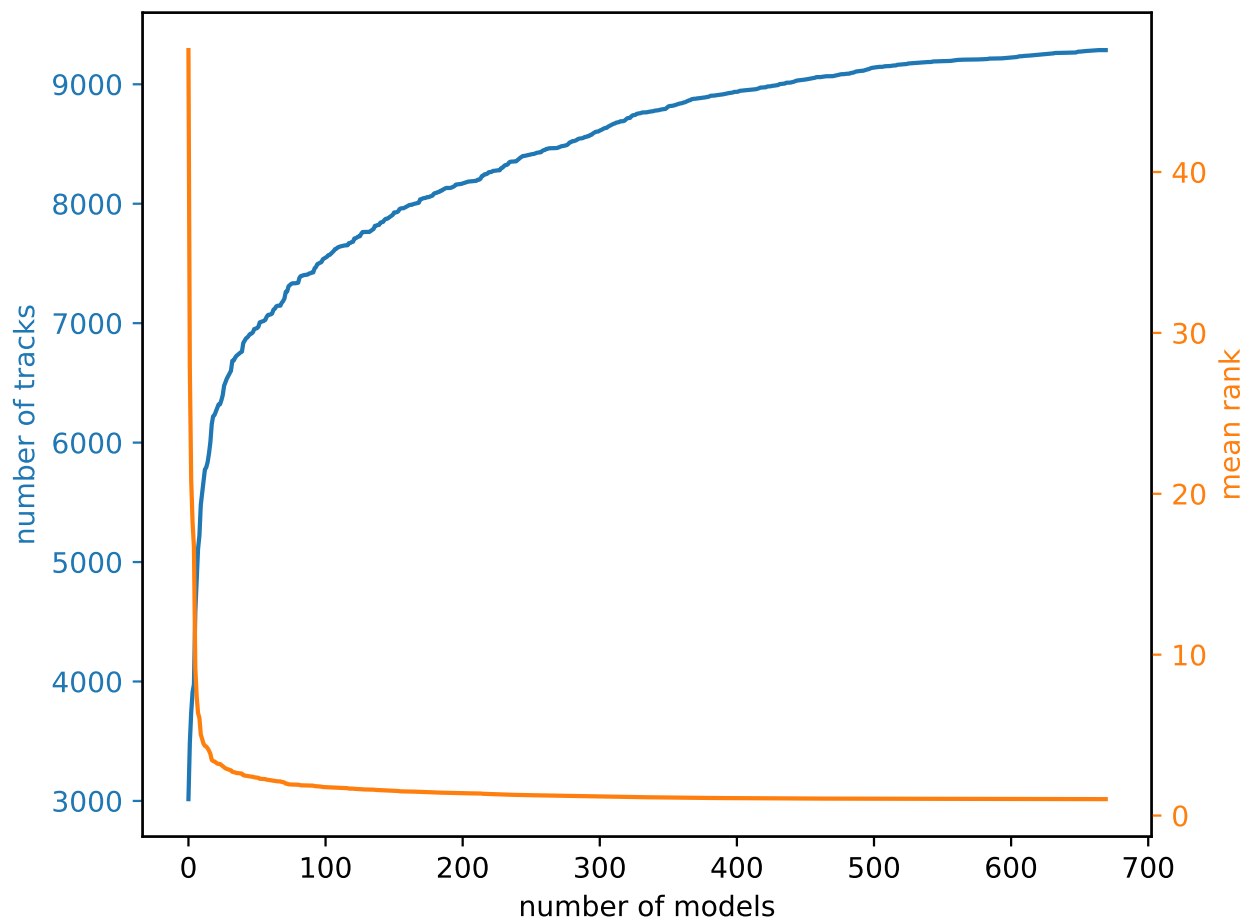

Figure 6.23: Number of tracks and theoretical mean rank as functions of the number of models for the SHS9k dataset. 


\section{Chapter 7}

\section{Conclusions}

In this final Chapter, the best results for every Cover Song Identification (CSI) experiment are recalled in Tables 7.1 to 7.5. Remembering that Mazurkas is not used in Experiment \#6 (since it is not possible to extract the four individual sources), and that SHS9K is only used as training dataset in Experiments \#4a and \#4b.

\section{Covers80}

\begin{tabular}{lllllllll} 
& MR & MRR & MDR & MAP & Top 1 & Top 10 & Top 100 & Top 1000 \\
\hline experiment \#2 & 41.575 & 0.19359 & 31.0 & 0.19359 & 21.0 & 46.0 & 142.0 & 160.0 \\
\hline experiment \#4a & 33.97812 & 0.28545 & 16.25 & 0.28545 & 34.8 & 62.8 & 145.0 & 160.0 \\
experiment \#4b & 33.33313 & 0.2385 & 19.4 & 0.2385 & 24.1 & 64.6 & 147.8 & 160.0 \\
experiment \#5 & 41.3 & 0.20834 & 22.5 & 0.20834 & 20.0 & 61.0 & 134.0 & 160.0 \\
experiment \#6 & 23.73125 & 0.41531193 & 10.0 & 0.41531193 & 57.0 & 83.0 & 152.0 & 160.0
\end{tabular}

Table 7.1: Best results for Covers80 through all CSI experiments.

\section{YouTubeCovers}

\begin{tabular}{lllllllll} 
& MR & MRR & MDR & MAP & Top 1 & Top 10 & Top 100 & Top 1000 \\
\hline experiment \#2 & 7.97143 & 0.6942 & 1.0 & 0.36114 & 219.0 & 296.0 & 345.0 & 350.0 \\
\hline experiment \#4a & 13.42257 & 0.50354 & 3.0 & 0.21716 & 138.4 & 258.9 & 345.0 & 350.0 \\
experiment \#4b & 5.63714 & 0.70923 & 1.0 & 0.39507 & 221.9 & 306.2 & 348.9 & 350.0 \\
experiment \#5 & 8.18857 & 0.66438 & 1.0 & 0.34196 & 202.0 & 300.0 & 346.0 & 350.0 \\
experiment \#6 & 7.63428571 & 0.648763101 & 1.0 & 0.331591159 & 196.0 & 286.0 & 346.0 & 350.0
\end{tabular}

Table 7.2: Best results for YouTubeCovers through all CSI experiments.

\section{Covers1000}

\begin{tabular}{lllllllll} 
& MR & MRR & MDR & MAP & Top 1 & Top 10 & Top 100 & Top 1000 \\
\hline experiment \#2 & 144.041 & 0.25731 & 35.0 & 0.19159 & 201.0 & 366.0 & 636.0 & 1000.0 \\
\hline experiment \#4a & 146.5084 & 0.17431 & 42.35 & 0.12918 & 103.9 & 311.0 & 615.2 & 1000.0 \\
experiment \#4b & 109.0794 & 0.30175 & 16.5 & 0.23293 & 228.7 & 440.0 & 707.0 & 1000.0 \\
experiment \#5 & 139.806 & 0.27382 & 30.0 & 0.20739 & 218.0 & 381.0 & 662.0 & 1000.0 \\
experiment \#6 & 93.464 & 0.437937535 & 7.0 & 0.357714675 & 385.0 & 537.0 & 779.0 & 1000.0
\end{tabular}

Table 7.3: Best results for Covers1000 through all CSI experiments.

\section{Mazurkas}

\begin{tabular}{lllllllll} 
& MR & MRR & MDR & MAP & Top 1 & Top 10 & Top 100 & Top 1000 \\
\hline experiment \#2 & 4.15724 & 0.95774 & 1.0 & 0.82286 & 2561.0 & 2714.0 & 2728.0 & 2739.0 \\
\hline experiment \#4a & 3.99186 & 0.92073 & 1.0 & 0.44795 & 2419.5 & 2685.7 & 2726.0 & 2740.0 \\
experiment \#4b & 1.97322 & 0.93372 & 1.0 & 0.62158 & 2457.3 & 2697.8 & 2734.5 & 2741.0 \\
experiment \#5 & 2.81284 & 0.92509 & 1.0 & 0.55828 & 2434.0 & 2690.0 & 2727.0 & 2740.0
\end{tabular}

Table 7.4: Best results for Mazurkas through all CSI experiments. 


\section{SHS9K}

\begin{tabular}{lllllllll} 
& MR & MRR & MDR & MAP & Top 1 & Top 10 & Top 100 & Top 1000 \\
\hline experiment \#2 & 47.57883 & 0.40387 & 6.0 & 0.05102 & 3008.0 & 5188.0 & 8067.0 & 9265.0 \\
\hline experiment \#5 & 43.30401 & 0.43289 & 5.0 & 0.05862 & 3289.0 & 5433.0 & 8182.0 & 9278.0 \\
experiment \#6 & 38.613935 & 0.463884786 & 4.0 & 0.0697890851 & 3581.0 & 5727.0 & 8291.0 & 9276.0
\end{tabular}

Table 7.5: Best results for SHS9K through all CSI experiments.

\subsection{Analysing the Results and Recalling the Goals}

From Tables 7.1 to 7.5 above it is possible to see that every dataset has improved results with at least one experiment, compared to experiment $\# 2$ (which is our base experiment). This is an encouraging outcome of our proposed approaches and methodology for the CSI problem.

\section{Music Similarity and Cover Song Identification}

Regarding each individual experiment, $\# 1$ and \#2 are, to the author's knowledge, the first attempt to benchmark music similarity models, where several models not considered in the literature are tested. Moreover, several comparative conclusions are obtained: we should apply Optimal Transposition Index (OTI) to chromagrams, not add minimum and maximum values to single Gaussian aggregators, consider the feature differences for single Gaussian aggregators, among others.

The main goal of experiments $\# 1$ and $\# 2$ is to check which music similarity models lead to best results for the annotated datasets, whether with generic datasets used in Experiment \#1 or CSI datasets in Experiment \#2, and this is completely accomplished.

\section{Cover Song Classification}

Experiment \#3 presents a new heuristic for train/test split for the Cover Song Classification (CSC) problem, a unique way of building samples (by the fusion of similarity matrices obtained by distinct models), and performs binary classification with 5 datasets and 5 classifiers (several combinations not published before). The goals include verifying which model combination scheme lead to the best results, and if our suggestion to use filtered models (skipping equivalent ones) achieves the best results (which is indeed observed).

Experiment \#4 tests our proposal to exploit trained classifiers to build new similarity matrices, and to return to the CSI problem. The main goal of this experiment is to check if it possible to improve CSI results using the matrices manufactured by CSC runs. Here again the use of filtered models to build samples lead to better results than using the Top- $\mathrm{N}$ models, and indeed better results are reached for every CSI dataset.

\section{Dataset Modifications}

Also to the author's knowledge, Experiment \#5 is the first attempt to explore different types of dataset modifications together in a comparative study to investigate how they affect music similarity metrics. The main goal of this experiment is to verify if any modification leads to better CSI results when compared to the metrics obtained with the original datasets. Whether comparing Mean Rank (MR) or Mean Average Precision (MAP), the most important metrics for the CSI problem, every dataset presented at least one modification that lead to a better result. The frame selection secondary datasets are the most prominent ones, and our proposal of using the Root Mean Square (RMS) values of the isolated vocals (instead of the RMS values of the original mixes) achieves better metrics.

\section{Fusion}

Experiment \#6 uses four distinct functions to fuse music similarity matrices obtained from the modified datasets by source separation. The goal of this experiment is to verify if it is possible to 
achieve better results when compared to the original dataset alone. This indeed is verified by the experiment that lead to better MR values for all datasets.

\section{Theoretical performance bounds}

After comparing the best results obtained for each dataset (as displayed in Table 7.6) with the state-of-the-art, we investigated and concluded that the problem lies on the attempt to solve the CSI problem for a whole dataset using a unique similarity model (the "one-size-fits-all" approach), rather than on the discriminating power of the individual similarity models. Experiment $\# 7$ provides scientific evidence that the family of similarity models considered in this project are capable of identifying covers with excellent performance.

\begin{tabular}{lll} 
dataset & best MR & experiment \\
\hline Covers80 & 23.73125 & $\# 6$ \\
YouTubeCovers & 5.63714 & $\# 4 \mathrm{~b}$ \\
Covers1000 & 93.464 & $\# 6$ \\
Mazurkas & 1.97322 & $\# 4 \mathrm{~b}$ \\
SHS9K & 38.613935 & $\# 6$
\end{tabular}

Table 7.6: The best results obtained for each CSI dataset.

\subsection{Future work}

Every one of these experiments still has space for improvements. In this Section we discuss our future work plans.

\section{Music Similarity and Cover Song Identification}

The list of improvements for Experiments $\# 1$ and $\# 2$ includes the expansion of the extractors (HPCP, crema-PCP, Onset Patterns, Scale Transform, Pitch Bihistogram, Intervalgram, etc), aggregators (Dynamic Time Warping (DTW), Self-Organizing Map (SOM), vector quantization using tree-based clustering, n-grams, etc), and distances (Mahalanobis, Jensen-Shannon, SmithWaterman, Mongeau-Sankoff, etc) for the music similarity framework.

Modules of the music similarity framework can also be expanded with approaches not currently contemplated. For instance, the extractor module can be extended with feature learning techniques $\left[\mathrm{PBD}^{+} 16, \mathrm{YXCY} 20\right]$, and the distance module can benefit with the use of metric learning techniques [SWW08, HKKL17].

\section{Cover Song Classification}

Experiments \#3 and \#4 can be further explored with different models to be fused, alternative ways to build samples, new classifiers (such as SVM, KNN-based, neural networks, etc) and more parameters for classifiers. We would like also to try in the future the python toolkit called autosklearn ${ }^{1}$ that can perform automated algorithm selection and hyperparameter tuning. Particularly in experiment \#4 we wish to train classifiers with a larger dataset in order to collect metrics for SHS9K.

\section{Dataset Modifications}

Regarding Experiment \#5 we have a vast list of possible future improvements, from variations on the settings of the current techniques (frames with more samples, segments with variable duration values, etc) to the adoption of new techniques (alternative segmentation, thumbnailing, or source

\footnotetext{
${ }^{1}$ https://automl.github.io/auto-sklearn/master/
} 
separation algorithms). The latter has a very good candidate, Spleeter [HKVM20] by Deezer ${ }^{2}$, that can separate the tracks into 5 stems: vocals, drums, bass, piano, and other.

Also, we desire to integrate at least one method that compares the sets of segments when comparing two songs $A$ and $B$. For instance, this can be done similarly to what Bozzon et al. [BPVT08] propose, using a graph that contains the distances from all segments of song $A$ to all the segments of song $B$, and explore this graph in order to reduce it to a unique distance value.

\section{Fusion}

The sixth experiment is also full of potential. The fusion can be done using matrices from other modified datasets of Experiment \#5 (besides only the source separation ones). It can be also done using matrices build in Experiment \#4. We can fusion virtually any similarity matrix obtained for a CSI dataset, even inter-experiments matrices, and matrices obtained by other algorithms than our own.

\section{Theoretical performance bounds}

Lastly, Experiment \#7 encourages us to investigate how to identify the music similarity models that are perfectly fit for a given query. As mentioned in Section 6.10 one idea is to cast the problem as a classification one, where a trained classifier would be able to predict the best model for each query. The design of classification strategies for selecting appropriate similarity models (music perspectives) for identifying versions of a given query is the subject of future work.

\footnotetext{
${ }^{2}$ https://www.deezer.com/
} 


\section{Appendix A}

\section{Music Similarity: best models per class}

\section{Ballroom}

\begin{tabular}{|c|c|c|c|c|}
\hline class & iicsr & extractor & aggregator & distance \\
\hline Jive & 1.440056195358931 & spectral bandwidth & vector quantization & cosine $e^{-}$distance \\
\hline Quickstep & 1.2568875004786253 & $\mathrm{mfcc}-$ & vector ${ }^{-}$quantization & cosine ${ }^{-}$distance \\
\hline Rumba-American & 1.9796567522114297 & spectral_flatness & diff_stäts_2 & chebyshev_distance \\
\hline Rumba-Misc & 1.4688978853570556 & spectral_flatness & $\operatorname{diff}^{-}$stats $^{-} 2$ & chebyshev $^{-}$distance ${ }^{-}$ \\
\hline Samba & 1.7050151994083254 & melspectrogram & gaussian_mixture_model default & cosine_distance \\
\hline Tango & 1.3269934151838436 & spectral rolloff & vector quantization & cosine $e^{-}$distance \\
\hline VienneseWaltz & 1.3527489891887357 & spectral rolloff & vector ${ }^{-}$quantization & cosine $e^{-}$distance \\
\hline
\end{tabular}

\section{GTZAN}

\begin{tabular}{lllll} 
class & iicsr & extractor & aggregator & distance \\
\hline blues & 2.0634199115352496 & spectral_contrast & vector_quantization & cosine_distance \\
classical & 2.644307967413323 & melspectrogram & vector_quantization & manhattan_distance \\
country & 1.2617036717897034 & spectral_contrast & vector_quantization_default & cosine_distance \\
disco & 1.4027194091593116 & melspectrogram & gaussian_mixture_model_default & cosine_distance \\
hiphop & 1.719408802575485 & melspectrogram & gaussian_mixture_model_default & cosine_distance \\
jazz & 1.3459618632721098 & spectral_contrast & vector_quantization & cosine_distance \\
metal & 3.187620155034533 & spectral_contrast & vector_quantization & cosine_distance \\
pop & 2.7107378150455474 & spectral_contrast & vector_quantization & cosine_distance \\
reggae & 1.3653166221767408 & melspectrogram & statistical_summarization & cosine_distance \\
rock & 1.1913570919251713 & spectral_bandwidth & vector_quantization & cosine_distance
\end{tabular}

\section{IOACAS-QBH}

\begin{tabular}{ll} 
class & iicsr \\
\hline 00024 & 1.931033773676616 \\
00025 & 1.901487447845127 \\
00065 & 1.6718779950388922 \\
00075 & 1.2647310006280135 \\
00080 & 2.0424897695993898 \\
00083 & 1.4697457241534404 \\
00089 & 1.3488308166318899 \\
00101 & 1.706905481098727 \\
00106 & 1.4425725406149095 \\
00108 & 1.7193037170909928 \\
00110 & 1.400753203469558 \\
00111 & 1.7994128763939803 \\
00114 & 1.6178691674810624 \\
00116 & 2.960345510079841 \\
00120 & 2.0047212679696647 \\
00128 & 1.436903729562698 \\
00129 & 1.5756637211240436 \\
00133 & 1.698328767142286 \\
00139 & 1.5321662211203018 \\
00140 & 1.5673438296221571 \\
00160 & 1.3946192661995784 \\
00162 & 1.7709617258242663 \\
00167 & 1.799982100675112 \\
00185 & 1.6031910027744627 \\
00192 & 1.6328450865612627 \\
00193 & 1.91270620701724 \\
00195 & 1.7381033411120395 \\
00197 & 1.598649434779093 \\
00210 & 1.4270926614362023 \\
00232 & 1.5986903612139411 \\
00233 & 1.543336613016316 \\
00245 & 1.6270473736295192 \\
00250 & 1.5896671948964811
\end{tabular}

extractor
mfcc
mfcc
chroma_cqt
pitch_contour_segmentation
tonnetz
spectral_flatness
pitch_contour_segmentation
temporal_statistical_spectrum_descriptor
mfcc
melspectrogram
beat_synchronous_chroma_stft
tonnetz
temporal_statistical_spectrum_descriptor
chroma_stft
mfcc
chroma_stft
chroma_cens
melspectrogram
melspectrogram
pitch_contour_segmentation
tonnetz
mfcc
pitch_contour_segmentation
melspectrogram
chroma_cqt
mfcc
pitch_contour_segmentation
melspectrogram
pitch_contour_segmentation
melspectrogram
pitch_contour_segmentation
temporal_statistical_spectrum_descriptor
beat_synchronous_chroma_cqt

aggregator
vector_quantization
vector_quantization
gaussian_mixture_model_default
markov_chain
gaussian_mixture_model_default
gaussian_mixture__model_default
octave_abstraction
bypass
vector_quantization
gaussian_mixture_model_default
gaussian_mixture_model_default
gaussian_mixture_model_default
bypass
gaussian_mixture_model_default
vector_quantization
gaussian_mixture_model_default
gaussian_mixture_model_default
statistical_summarization
vector_quantization
interval_abstraction
gaussian_mixture_model_default
vector_quantization
interval_abstraction
gaussian_mixture_model_default
gaussian_mixture_model_default
vector_quantization
interval_abstraction
gaussian_mixture_model_default
interval_abstraction
gaussian_mixture_model_default
interval_abstraction
bypass_
vector_quantization

distance cosine_distance cosine_distance euclidean_distance circular cosine chebyshev distance chebyshev_distance chebyshev_distance cosine_distance cosine_distance cosine_distance chebyshev_distance chebyshev_distance cosine distance chebyshev distance cosine distance chebyshev distance chebyshev_distance cosine_distance cosine distance lcs_min cosine distance cosine - distance lcs $\min$ cosine distance chebyshev_distance cosine_distance levensh̄tein_max cosine_distānce lcs_min cosine distance lcs min cosine distance cosine_distance 


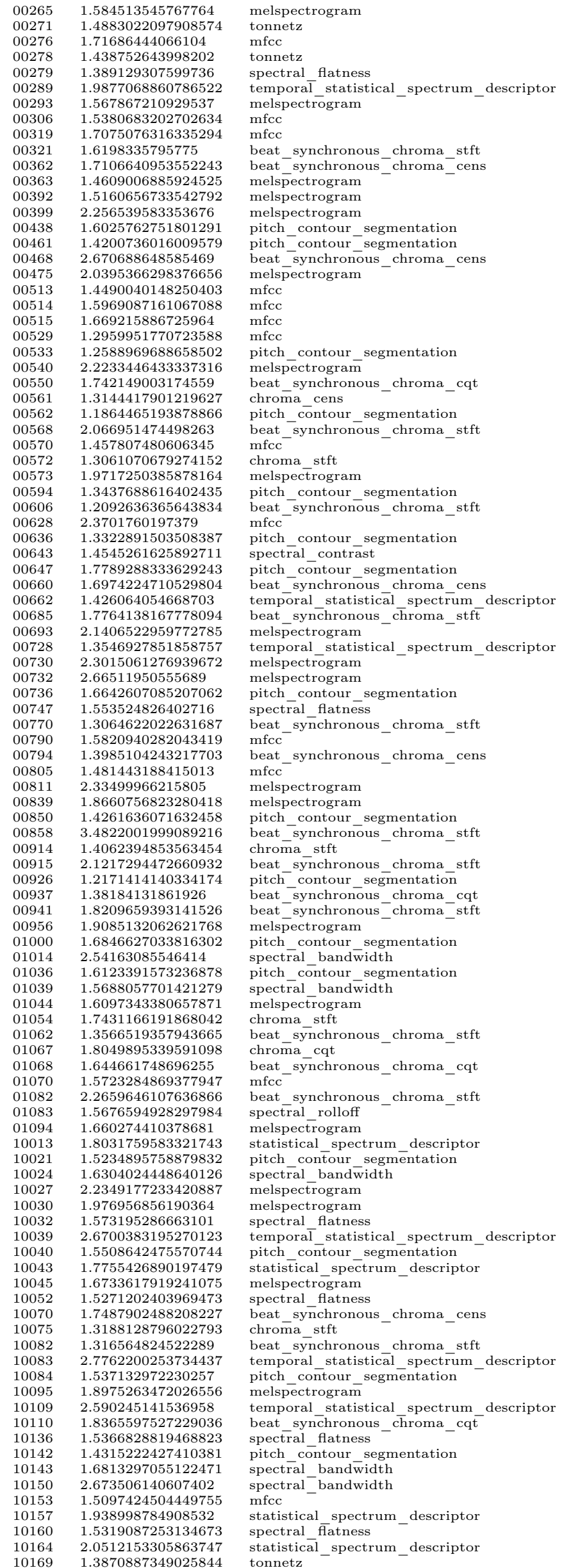

vector_quantization gaussian_mixture _ gaussian mixture model default gaussian_mixture-model_default gaussian_mixture_model_default gaussian_mixture_model_default vector_quantization vector_quantization vector_quantization vector quantization gaussian_mixture model default gaussian mixture model default gaussian_mixture_model_default octave_ābstraction

octave_abstraction

vector quantization

gaussian mixture model default vector_quantization vector quantization vector ${ }^{-}$quantization vector quantization nterval abstraction gaussian_mixture_model_default gaussian_mixture_model_default gaussian_mixture_model_default pitch_contour_5_levels

vector quantization

vector quantization

gaussian_mixture_model_default gaussian_mixture_model_default octave ābstraction

vector -quantization

vector-quantization

inter - quantization

vector quantization

vector quantization

vector

vector_quantization

gaussian_mixture_model_default

bypass

gaussian_mixture_model_default statistical_summarization

interval_abstraction

gaussian mixture model default gaussian mixture ${ }^{-}$model ${ }^{-}$default vector quantization

gaussian_mixture_model_default vector_quantization

vector-quantization

gaussian mixture model default interval abstraction

interval_abstraction

vector_quantization

vector_quantization

octave_abstraction

vector_quantization

vector quantization

gaussian_mixture model default interval_abstraction

gaussian_mixture model_default interval_abstraction

gaussian mixture model default gaussian ${ }^{-}$mixture ${ }^{-}$model $^{-}$default gaussian_mixture_model_default vector quantization

gaussian mixture model default gaussian_mixture- ${ }^{2}$ model - default vector quantization

vector_quantization

gaussian mixture model default gaussian_mixture_model_default gaussian

bypass

markov_chain

gaussian_mixture_model_default statistical summarization

gaussian mixture model default gaussian ${ }^{-}$mixture ${ }^{-}$model ${ }^{-}$default bypass

interval abstraction

bypass

vector quantization default

gaussian mixture - default gaussian_mixture_model_default gaus__ gaussian mixture $-{ }_{\text {model }}{ }_{\text {default }}$ gaussia

interval abstraction

statistical_summarization

bypass

vector_quantization

gaussian_mixture model_default interval abstraction

gaussian mixture model default gaussian ${ }^{-}$mixture ${ }^{-}$model $^{-}$default vector_quantization

bypass

gaussian_mixture_model_default bypass

gaussian mixture model default cosine distance

chebyshev distance

cosine distance

cosine ${ }^{-}$distance

chebyshev distance

cosine_distance

cosine_distance

cosine_distance

cosine_distance

cosine_distance

cosine_distance

cosine_distance

cosine-distance

cosine distance

lcs_circular_min

lcs ${ }^{-}$circular ${ }^{-}$min

cosine distance

cosine distance

cosin_distance

cosine - distance

cosine_distance

cosine - distance

cosine_distance

levenshtein_max

cosine distance

chebyshev_distance

lcs min

cosine distance

cosine distance

chebyshev distance

cosine distance

lcs circular min

cosine distance

cosine ${ }^{-}$distance

lcs min

cosine distance

cosine distance
levenshtein max

cosine distance

cosine-distance

manhattan_distance

cosine_distance

cosine_distance

cosine_distance

cosine_distance

lcs min

chebyshev distance chebyshev distance cosine_distance

chebysh̄ev_distance

manhattan distance

cosine distānce

cosine ${ }^{-}$distance

lcs min

cosine distance

euclidean_distance

cosine_distance

lcs_circular_min
manhattan_distance

cosine_distance

cosine_distance

lcs_min

cosine distance

lcs min

cosine distance

cosine distance

chebyshev_distance

cosine distance

chebyshev distance

chebyshev-distance

cosine distance

cosine ${ }^{-}$distance

cosine ${ }^{-}$distance

cosine ${ }^{-}$distance

cosine_distance

circular_cosine

cosine_distance

cosine_distance

cosine distance

chebyshev distance

cosine distance

lcs $\max$

chebyshev_distance

chebyshev distance

chebyshev ${ }^{-}$distance

chebyshev ${ }^{-}$distance

cosine distance

lcs $\mathrm{min}$

cosine_distance

cosine_distance

cosine distance

chebyshevedistance

levenshtein $\max$

cosine distance

cosine_distance

cosine distance

cosine distance

chebyshev distance

cosine distance

chebyshev distance 
10173

888162209837

$10183 \quad 1.6076388010800153$

$\begin{array}{ll}10186 & 2.7663688067746244 \\ 10196 & 1.745295866553635\end{array}$

$10204 \quad 2.511697571571461$

$10209 \quad 1.6695775951188168$

$10220 \quad 6.6664838256588785$

$10222 \quad 2.373718824769523$

$10223 \quad 1.3412999798625447$

$\begin{array}{ll}10227 & 1.5279188065497633 \\ 10230 & 1.719142224660351\end{array}$

$10231 \quad 1.3477355744393593$

$10237 \quad 1.9704596548284965$

$10251 \quad 2.769220761200123$

$10254 \quad 1.5323379659784537$

$10255 \quad 1.3533636113070917$

$10257 \quad 2.988035414397908$

$10258 \quad 1.3401408486384718$

$10263 \quad 1.6641893151902698$

$\begin{array}{ll}10266 & 1.8018945834085849 \\ 10272 & 2.756024488179296\end{array}$

$10274 \quad 1.5144212736951403$

$10285 \quad 1.6099899438392196$

$10291 \quad 1.478070134482988$

$10294 \quad 2.9038263543029452$

$10295 \quad 1.5581485419502679$

$10298 \quad 1.456357575354308$

$10300 \quad 1.5028123090526297$

$10302 \quad 1.7178068115878014$

$10303-1.643511082683424$

$10306 \quad 1.8911515811625417$

$10310 \quad 1.7274132114889453$

$10312 \quad 1.630677818567374$

$10314 \quad 1.4927312317267682$

$10314 \quad 1.4927312317267682$

$10325 \quad 1.806610736255341$

$\begin{array}{ll}10329 & 1.5766901845760417 \\ 10331 & 1.5816827111829836\end{array}$

$\begin{array}{ll}10331 & 1.5816827111829836 \\ 10332 & 2.6188891770030245\end{array}$

$10340 \quad 1.3901279920961627$

$10341 \quad 1.5273397406011338$

$10343 \quad 3.3538335226166898$

$10345 \quad 2.2300102100355916$

$10354 \quad 1.4448355992220743$

$10370 \quad 1.6506145936162089$

$10372 \quad 1.5902382411784608$

$10382-1.6337097456117633$

$10383 \quad 2.326723300626564$

$10399 \quad 1.6307156671730356$

$10404 \quad 1.6658829861244748$

$10412 \quad 1.633439172987491$

$10430 \quad 1.9303340435202792$

$\begin{array}{ll}10430 & 1.9303340435202792 \\ 10435 & 1.298987798068178\end{array}$

$\begin{array}{ll}10435 & 1.298987798068178 \\ 10437 & 1.6907215914040645\end{array}$

$\begin{array}{ll}10437 & 1.6907215914040645 \\ 10442 & 1.958619801094365\end{array}$

$10445-1.6670816361105545$

$10446 \quad 2.6071473651721258$

$10447 \quad 1.6503733090503572$

$10453 \quad 1.6307144432291583$

$10477 \quad 2.0465638148683643$

$10478 \quad 1.8298612382962005$

$10479 \quad 1.605006696430526$

$10491 \quad 1.8393406113163555$

$10494 \quad 1.2802261157408032$

$10503 \quad 1.525643690929738$

$10512 \quad 1.5023627702936215$

$\begin{array}{ll}10520 & 1.7497136888081002\end{array}$

$10521 \quad 1.2701680775141684$

$10529 \quad 1.5066769087050793$

$10541 \quad 1.240651266466125$

$10546 \quad 1.9340688364268341$

$10567 \quad 1.5769625001697865$

$10583 \quad 1.6621578166850806$

$10594 \quad 1.934582370662252$

$10606 \quad 2.028325575503405$

$10611 \quad 1.4900579714482276$

$10613 \quad 1.7565730125955301$

$10617 \quad 1.6579914360927908$

$10628-1.2952872501491235$

$10642 \quad 1.6341991551161794$

$10985 \quad 1.5601756667287836$

$10989 \quad 2.9787331838475213$

$11007 \quad 1.6418619342691785$

$11070 \quad 1.36617649261048$

$\begin{array}{ll}11209 & 2.8354945524090436\end{array}$

$\begin{array}{ll}11209 & 2.8354945524090436 \\ 11228 & 4.637017841072229\end{array}$

$\begin{array}{ll}11228 & 4.637017841072229 \\ 11299 & 1.5367149092967716\end{array}$

$\begin{array}{ll}11299 & 1.5367149092967716 \\ 11342 & 1.6218561646113163\end{array}$

$\begin{array}{ll}11342 & 1.621856164611316 \\ 11441 & 1.79236450430892\end{array}$

$11485 \quad 1.83798537624978$

$11488 \quad 1.833900137837313$

$\begin{array}{ll}11521 & 1.5169401341463162 \\ 11566 & 1.6347424316914292\end{array}$

$\begin{array}{ll}11566 & 1.6347424316914292 \\ 11591 & 1.7404129644795805\end{array}$

$\begin{array}{ll}11591 & 1.7404129644795805 \\ 11620 & 1.521483989143152\end{array}$

$11650 \quad 1.601884774605334$

$11657 \quad 2.144991384859208$

$\begin{array}{ll}11682 & 3.711098176521339\end{array}$

$11710 \quad 1.6597745170769258$

$11719 \quad 1.5253920120123463$

$11739 \quad 2.7151715276746735$ melspectrogram

emporal statistical spectrum descriptor

pitch contour segmentation

emporal statistical_spectrum_descriptor

pitch_contour_segmentation

melspectrogran

chroma_stft

pitch_contour_segmentation

spectral_flatness

spectral_centroid

chroma_cqt

chroma-stft

temporal statistical spectrum descriptor temporal_statistical_spectrum_descriptor

zero_crossing rate

temporal_statistical_spectrum_descriptor

beat_synchronous_chroma_cqt

beat_synchronous_chroma_cq

chroma stft

proma_stft

pectral_contrast

beat_synchronous_chroma_stft

chroma_stft

temporal_statistical_spectrum_descriptor

spectral_fflatness

onnetz

pectral flatness

melspectrogram

mfcc

spectral_bandwidth

pitch_contour_segmentation

melspectrogram

beat_synchronous_chroma_stft

chroma_cqt

$\mathrm{mfcc}$

pectral flatness

beat_synchronous chroma stft

temporal_statistical_spectrum_descriptor

chroma_stft

beat_synchronous_chroma_stft

$\mathrm{mfcc}^{-}$

spectral_bandwidth

chroma_cens

pitch_contour_segmentation

$\mathrm{mfcc}$

melspectrogram

pectral bandwidth

pitch contour segmentation

mfcc

beat_synchronous_chroma_cqt

spectral flatnes

beat_synchronous_chroma_stft

pitch_contour_segmentation

beat_synchronous_chroma_cens

pitch_contour_segmentation

beat_synchronous_chroma_cqt

temporal_statisticāl_spectrum_descriptor chroma_cens

tonnetz

beat_synchronous_chroma_cqt

tonnetz

spectral flatness

chroma stft

beat_synchronous_chroma_stft

spect

mfcc

hroma_cens

beat_synchronous_chroma_cens

chroma_cqt

mfcc

spectral_bandwidth

pitch_contour_segmentation

beat_synchronous_chroma_cqt

spectral_contrast

pitch contour segmentation

chroma_cqt

temporal_statistical_spectrum_descriptor

beat_synchronous_chroma_cens

pitch contour segmentation

temporal_statistical_spectrum_descriptor melspectrogram

spectral flatness

beat_synchronous_chroma_cens

pitch_contour_segmentation

beat_synchronous_chroma_stft

tonnetz

pitch_contour_segmentation

pitch_contour_segmentation

spectral_flatness

pitch_contour_segmentation

statistical_spectrum_descriptor

melspectrogram

beat_synchronous_chroma_cqt

temporal statistical_spectrum_descriptor

vector quantization

gaussian_mixture_model_default

bypass

interval abstraction

bypass

interval abstraction

gaussian_mixture_model_default

gaussian_mixture_model_defaul

interval_abstraction

gaussian_mixture model default

gaussian_mixture_model_default

gaussian_mixture model default

gaussian mixture ${ }^{-}$model ${ }^{-}$default

bypass

gaussian_mixture model_default

bypass

gaussian mixture model default

interval abstraction

gaussian mixture model default

bypass

vector_quantization

vector_quantization

gaussian_mixture_model_default

bypass

gaussian_mixture_model_default

statistical summarization

gaussian_mixture model_default

gaussian mixture ${ }^{-}$model $^{-}$default

vector_quantization

gaussian mixture model default

interval abstraction

gaussian_mixture_model_default

vector quantization

gaussian mixture model_default

vector quantization

gaussian mixture model default

gaussian_mixture ${ }^{-}$model_default

bypass

gaussian_mixture_model_default

gaussian_mixture_model_default

vector_quantization

gaussian_mixture_model_default

vector_quantization

interval abstraction

vector quantization

gaussian mixture model default

gaussian ${ }^{-}$mixture ${ }^{-}$model $^{-}$default

interval_abstraction

vector-quantization

gaussian_mixture

gaussian mixture model default

gaussian ${ }^{-}$mixture ${ }^{-}$model $^{-}$default

vector_quantization

interval_abstraction

vector quantization

interval_abstraction

gaussian_mixture_model_default

bypass

vector_quantization

vector quantization default

gaussian mixture_model default

statistical summarization

gaussian_mixture_model_default

gaussian mixture model default

gaussian mixture ${ }^{-}$model $^{-}$default

vector_quantization

vector quantization

vector quantization

vector quantization

gaussian mixture model default

gaussian mixture model default

vector quantization

gaussian_mixture_model_default

interval_abstraction

vector quantization

vector quantization

vector_quantization_default

interval abstraction

gaussian mixture model default

bypass

vector quantization default

octave-abstraction

bypass

gaussian mixture model default gaussian ${ }^{-}$mixture ${ }^{-}$model ${ }^{-}$default vector_quantization

gaussian_mixture_model_default

interval_abstraction

vector_quantization

statistical_summarization

interval_abstraction

interval-abstraction

gaussian_mixture_model_default

interval_abstraction

bypass

gaussian mixture model default 
melspectrogram

statistical spectrum descriptor

beat_synchronous_chroma_cens

chroma stft

beat_synchronous chroma_stft

pitch_contour_segmentation

melspectrogram

beat_synchronous_chroma_cqt

tonnetz

chroma_cqt

melspectrogram

melspectrogram

beat_synchronous chroma cqt

pitch ${ }^{-}$contour segmentation

spectral_flatness

melspectrogram

spectral_contrast

chroma_ stft

beat_synchronous_chroma_stft

beat_synchronou

spectral ${ }^{-}$flatness

chroma cens

spectral flatness

temporal_statistical_spectrum_descriptor chroma stft

pitch_contour_segmentation

statistical_spectrum_descriptor

melspectrogram

statistical_spectrum_descriptor

spectral flatness

pitch contour segmentation

melspectrogram

melspectrogram

tonnetz

beat_synchronous_chroma_stft

beat synchronous

zero_crossing_rate

melspectrogram

melspectrogram

melspectrogram

melspectrogram

temporal_statistical_spectrum_descripto

temporal_statistical_spectrum_descriptor

beat_synchronous_chroma_stft

melspectrogram

beat synchronous chroma stft

pitch_contour_segmentation

beat synchronous chroma stft

statistical spectrum descriptor

spectral bandwidth

melspectrogram

beat_synchronous_chroma_stft

beat_synchronous_chroma_-

pitch_contour

melspectrogram

(n)

statistical_spectrum_descriptor

statistical - spectrum_descriptor

beat_synchronous_chroma_cqt

spectral_centroid

melspectrogram

beat_synchronous_chroma_cens

beat_synchronous_chroma_stft

beat_synchronous_chroma_stft

temporal statisticāl spectrum descriptor statistical summarization

bypass

vector_quantization

gaussian mixture model default

gaussian ${ }^{-}$mixture ${ }^{-}$model $^{-}$default

interval abstraction

statistical summarization

gaussian_mixture model default

gaussian ${ }^{-}$mixture ${ }^{-}$model $^{-}$default

gaussian_mixture ${ }^{-}$model_default

statistical_summarization

vector quantization default

gaussian_mixture_model default

interval_abstraction

gaussian mixture model default

gaussian ${ }^{-}$mixture ${ }^{-}$model ${ }^{-}$default

vector quantization

gaussian mixture model_default

gaussian mixture _

vector_quantization

(aussian_mixture_model_default

gaussian_mixture ${ }^{\text {model }}$ - default

gaussian_mixture_model_default

gaussian_mixture_model_default

gaussian_mixture_model_default

interval_abstraction

bypass

gaussian_mixture_model_default bypass

gaussian_mixture_model_default interval abstraction

statisticāl_summarization

statistical summarization

gaussian_mixture_model_default

gaussian - mixture ${ }^{-}$model ${ }^{-}$default

gaussian mixture model ${ }^{-}$default

statistical summarization

statistical- summarization

statistical- summarization

vector_quantization_default

bypass

bypass

vector_quantization

vector_quantization_default

vector quantization

interval abstraction

vector quantization

bypass

gaussian_mixture_model_default

vector_quantization_defaült

vector quantization_default

interval abstraction

statistical summarization

vector quantization default

bypass

bypass

vector_quantization

gaussian mixture model default

vector_quantization_default

vector_quantization

vector quantization

gaussian mixture model default

bypass cosine distance

cosine_distance

cosine ${ }^{-}$distance

chebyshever distance

euclidean distance

lcs $\min$

cosine distance

euclidean_distance

cosine distance

chebyshev_distance

cosine_distance

cosine distance

chebyshev distance

lcs min

cosine distance

cosine ${ }^{-}$distance

cosine distance

chebyshev distance

cosine_distance

cosine ${ }^{-}$distance

chebyshev distance

chebyshev distance

cosine_distance

cosine distance

chebysh̄ev_distance

lcs_min

cosine_distance

cosine_distance

cosine ${ }^{-}$distance

chebyshev distance

lcs_min

chebyshev distance

cosine distance

cosine ${ }^{-}$distance

cosine ${ }^{-}$distance

cosine ${ }^{-}$distance

cosine ${ }^{-}$distance

cosine_distance

cosine distance

manhattan_distance

cosine_distance

cosine distance

lcs $\min$

cosine distance

cosine ${ }^{-}$distance

cosine ${ }^{-}$distance

cosine ${ }^{-}$distance

cosine ${ }^{-}$distance

lcs $\min$

cosine distance

cosine ${ }^{-}$distance

cosine ${ }^{-}$distance

cosine ${ }^{-}$distance

cosine distance

cosine - distance

cosine distance

manhattan distance

cosine distance

chebyshev distance

cosine distance

\section{Maria Panteli's melody dataset}

\begin{tabular}{lll} 
class & iicsr & extractor \\
\hline 1 & 4.572860360301707 & pitch_contour_segmentation \\
2 & 4.595488591478775 & pitch_contour_segmentation \\
3 & 4.667953017999352 & pitch_contour_segmentation \\
4 & 3.7801805173521292 & pitch_contour_segmentation \\
5 & 4.253788622841754 & pitch_contour_segmentation \\
6 & 3.0931585472915883 & pitch_contour_segmentation \\
7 & 4.136376432826418 & pitch_contour_segmentation \\
8 & 7.132299323200648 & pitch_contour_segmentation \\
9 & 2.7462094457222794 & pitch_contour_segmentation \\
10 & 3.301147886316078 & pitch_contour_segmentation \\
11 & 3.4250252510795147 & pitch_contour_segmentation \\
12 & 2.8428993629829065 & melspectrogram \\
13 & 3.7872063093146475 & pitch_contour_segmentation \\
14 & 2.9213992384749026 & melspectrogram \\
15 & 3.7533687103512112 & pitch_contour_segmentation \\
16 & 4.9527143368654345 & pitch_contour_segmentation \\
17 & 5.128621876014979 & pitch_contour_segmentation \\
18 & 2.7718426025520984 & pitch_contour_segmentation \\
19 & 2.30990245660279 & melspectrogram \\
20 & 2.9569345777365337 & melspectrogram \\
21 & 2.924364382427016 & mfcc \\
22 & 4.455118828580867 & spectral_contrast \\
23 & 3.43882193082738 & melspectrogram \\
24 & 2.656617917998237 & melspectrogram \\
25 & 2.9080653951108073 & pitch_contour_segmentation \\
26 & 3.9429393369828185 & melspectrogram \\
27 & 4.472476063911515 & spectral_contrast \\
28 & 4.241831353861312 & melspectrogram \\
29 & 4.742320714782647 & spectral_contrast \\
30 & 3.236193595007395 & melspectrogram \\
& &
\end{tabular}

aggregator
interval_abstraction
interval_abstraction
interval_abstraction
interval_abstraction
interval_abstraction
octave_abstraction
interval_abstraction
interval_abstraction
octave_abstraction
marko-_chain
interval_abstraction
statistical_summarization
interval_abstraction
statistical_summarization
interval_abstraction
interval_abstraction
interval_abstraction
octave_abstraction
statistical_summarization
statistical_summarization
vector_quantization
vector_quantization
gaussian_mixture_model_default
gaussian_mixture_model_default
interval_abstraction
gaussian_mixture_model_default
vector_quantization
statistical_summarization
vector_quantization
statistical_summarization

distance

levenshtein_max

levenshtein_max

levenshtein_max

levenshtein circular max

levenshtein_max

levenshtein_max

levenshtein_circular_max

circular_cosine

levenshtein_max

cosine_distance

cs max

cosine distance

lcs max

levenshtein_max

levenshtein circular max

cosine ${ }^{-}$distance

cosin- distance

cosine-distance

cosine ${ }^{-}$distance

cosine- distance

lcs max

cosine distance

manhattan_distance

cosine_distance

cosine_distance

tatistical summarization 


\section{Maria Panteli's rhythm dataset}

\begin{tabular}{|c|c|c|c|c|}
\hline class & iicsr & extractor & aggregator & distance \\
\hline 1 & 1.8670298087033916 & beat_synchronous_chroma_cens & vector_quantization & manhattan_distance \\
\hline 2 & 2.2926001988195237 & chroma_cens $\quad-$ & vector-quantization & manhattan ${ }^{-}$distance \\
\hline 3 & 1.6188468257146416 & chroma_cens & vector quantization & cosine_distance \\
\hline 4 & 1.9375919403849882 & chroma ${ }^{-}$cens & vector quantization & cosine $e^{-}$distance \\
\hline 5 & 2.0600823372158277 & chroma_cens & vector_quantization & cosine_distance \\
\hline 6 & 2.249490571880482 & chroma_cens & vector_quantization & cosine distance \\
\hline 7 & 2.4496064675664773 & chroma_cens & vector-quantization & cosine $e^{-}$distance \\
\hline 8 & 1.8294307561901648 & beat_synchronous_chroma_cens & vector-quantization & manhātan_distance \\
\hline 9 & 2.2143484693635 & chroma_cens $-{ }^{-}$ & vector-quantization & cosine_distance \\
\hline 10 & 3.2620774461342745 & beat_synchronous_chroma_stft & vector_quantization & cosine_distance \\
\hline 11 & 1.8691095336651231 & chroma cens & vector quantization default & manhātan distance \\
\hline 12 & 1.8418994918528444 & chroma_cens & vector_quantization_default & manhattan_distance \\
\hline 13 & 1.8522476160762056 & chroma_cens & vector $^{-}$quantization ${ }^{-}$ & cosine_distance \\
\hline 14 & 1.7902546240434094 & chroma_cens & vector-quantization_default & manhātan_distance \\
\hline 15 & 1.8187801111781927 & chroma_cens & vector ${ }^{-}$quantization ${ }^{-}$ & cosine_distance \\
\hline 16 & 1.9485394898754373 & chroma_cens & vector_quantization & cosine_distance \\
\hline 17 & 1.7026595161486144 & chroma_cens & vector_quantization & cosine_distance \\
\hline 18 & 2.314321102047266 & chroma_cens & vector_quantization & cosine $e^{-}$distance \\
\hline 19 & 1.9083899662609827 & beat_synchronous_chroma_stft & vector_quantization & manhattan_distance \\
\hline 20 & 1.7401751410973576 & beat_synchronous_chroma-stft & vector-quantization & manhattan ${ }^{-}$distance \\
\hline 21 & 3.4049155987800286 & chroma_cens $\quad-$ & vector_quantization & manhattan_distance \\
\hline 22 & 3.4885142295054643 & beat synchronous chroma stft & vector ${ }^{-}$quantization & manhattan ${ }^{-}$distance \\
\hline 23 & 3.6681551328269824 & beat_synchronous_chroma-stft & vector_quantization & manhattan_distance \\
\hline 24 & 4.164128240158876 & chroma_cens $\quad-$ & vector_quantization & manhattan_distance \\
\hline 25 & 4.463776112464625 & chroma_cens & vector_quantization & manhattan_distance \\
\hline 26 & 3.4231621827272445 & melspectrogram & vector-quantization & cosine_distance \\
\hline 27 & 4.647600957936456 & beat synchronous chroma cens & vector ${ }^{-}$quantization & cosine ${ }^{-}$distance \\
\hline 28 & 3.851326718857485 & chroma cens & vector ${ }^{-}$quantization & manhātan distance \\
\hline 29 & 5.390396490175905 & chroma_cens & vector_quantization & manhattan_distance \\
\hline 30 & 13.207691825597978 & chroma_cens & vector-quantization & manhattan ${ }^{-}$distance \\
\hline
\end{tabular}

\section{MAST}

\begin{tabular}{|c|c|c|c|c|}
\hline class & iicsr & extractor & aggregator & distance \\
\hline 51 rhy 1 & 1.2710345335966193 & $\mathrm{mfcc}$ & vector quantization & cosine distance \\
\hline $51^{-}$rhy 2 & 1.197985004287646 & $\mathrm{mfcc}$ & vector ${ }^{-1}$ quantization & cosine $^{-}$distance \\
\hline $52^{-}$rhy 1 & 1.2958063945996323 & beat synchronous chroma stft & vector quantization & cosine ${ }^{-}$distance \\
\hline $52^{-}$rhy 2 & 1.3303252480126277 & $\mathrm{mfcc}^{-}$ & vector quantization & cosine ${ }^{-}$distance \\
\hline $53^{-}$rhy 1 & 1.3105333145845612 & melspectrogram & vector ${ }^{-1}$ quantization & cosine $e^{-}$distance \\
\hline $53^{-}$rhy2 & 1.365515475052475 & melspectrogram & vector ${ }^{-1}$ quantization & cosine ${ }^{-}$distance \\
\hline $54^{-}$rhy 1 & 1.2473952719687482 & rhythm histogram & bypass & manhattan distance \\
\hline $54^{-}$rhy 2 & 1.2915682840332523 & mfcc - & vector quantization & cosine distance \\
\hline $55^{-}$rhy 1 & 1.324655702228605 & $\mathrm{mfcc}$ & vector quantization & cosine ${ }^{-}$distance \\
\hline $55^{-}$rhy 2 & 1.157098644355708 & $\mathrm{mfcc}$ & vector quantization & cosine ${ }^{-}$distance \\
\hline $56^{-}$rhy 1 & 1.4030468790783808 & melspectrogram & vector quantization & cosine ${ }^{-}$distance \\
\hline $56^{-}$rhy2 & 1.2637637104874466 & $\mathrm{mfcc}$ & vector ${ }^{-}$quantization & cosine $^{-}$distance \\
\hline $57^{-}$rhy 1 & 1.4301647322521653 & mfcc & vector ${ }^{-}$quantization & cosine ${ }^{-}$distance \\
\hline $57^{-}$rhy 2 & 1.331310654157978 & mfcc & vector ${ }^{-}$quantization & cosine ${ }^{-}$distance \\
\hline $58^{-}$rhy 1 & 1.442056154281866 & $\mathrm{mfcc}$ & vector ${ }^{-}$quantization & cosine ${ }^{-}$distance \\
\hline $58^{-}$rhy 2 & 1.329289417750898 & melspectrogram & vector quantization & cosine $e^{-}$distance \\
\hline $59^{-}$rhy 1 & 1.3757720348279594 & $\mathrm{mfcc}$ & vector ${ }^{-}$quantization & cosine ${ }^{-}$distance \\
\hline $59^{-}$rhy2 & 1.4163457314191061 & $\mathrm{mfcc}$ & vector quantization & cosine ${ }^{-}$distance \\
\hline $61^{-}$rhy 1 & 1.5787841569442553 & $\mathrm{mfcc}$ & vector quantization & cosine ${ }^{-}$distance \\
\hline $61^{-}$rhy 2 & 1.5478398738313663 & $\mathrm{mfcc}$ & vector quantization & cosine ${ }^{-}$distance \\
\hline $62^{-}$rhy 1 & 1.7420472205121904 & mfcc & vector quantization & cosine ${ }^{-}$distance \\
\hline $62^{-}$rhy 2 & 1.8102568891528337 & mfcc & vector quantization & cosine ${ }^{-}$distance \\
\hline $63^{-}$rhy 1 & 1.5051097622800966 & mfcc & vector quantization & cosine ${ }^{-}$distance \\
\hline $63^{-}$rhy 2 & 1.5319079904177115 & $\mathrm{mfcc}$ & vector quantization & cosine $e^{-}$distance \\
\hline $64^{-}$rhy 1 & 1.573149397178731 & $\mathrm{mfcc}$ & vector quantization & cosine ${ }^{-}$distance \\
\hline $64^{-}$rhy2 & 1.5771004756691684 & mfcc & vector quantization & cosine ${ }^{-}$distance \\
\hline $65^{-}$rhy 1 & 1.7214771508170008 & mfcc & vector quantization & cosine ${ }^{-}$distance \\
\hline $65^{-}$rhy 2 & 1.702037258445737 & mfcc & vector quantization & cosine ${ }^{-}$distance \\
\hline $66^{-}$rhy 1 & 1.5756120013491706 & $\mathrm{mfcc}$ & vector quantization & cosine $e^{-}$distance \\
\hline $66^{-}$rhy 2 & 1.7584688986199082 & $\mathrm{mfcc}$ & vector quantization & cosine ${ }^{-}$distance \\
\hline $67^{-}$rhy 1 & 1.5631061272516145 & $\mathrm{mfcc}$ & vector quantization & cosine ${ }^{-}$distance \\
\hline $67^{-}$rhy2 & 1.7222187702130034 & $\mathrm{mfcc}$ & vector quantization & cosine ${ }^{-}$distance \\
\hline $68^{-}$rhy 1 & 1.5207842589703118 & $\mathrm{mfcc}$ & vector quantization & cosine ${ }^{-}$distance \\
\hline $68^{-}$rhy 2 & 1.404187410154036 & $\mathrm{mfcc}$ & vector quantization & cosine ${ }^{-}$distance \\
\hline $69^{-}$rhy 1 & 1.7458435351239012 & $\mathrm{mfcc}$ & vector quantization & cosine ${ }^{-}$distance \\
\hline $69^{-}$rhy2 & 1.6958878785452038 & mfcc & vector - quantization & cosine ${ }^{-}$distance \\
\hline $51 \overline{0}$ rhy 1 & 1.3524313981519618 & melspectrogram & vector quantization & cosine $^{-}$distance \\
\hline $510^{-}$rhy2 & 1.2268756183928735 & chroma cens & vector quantization & cosine ${ }^{-}$distance \\
\hline $610^{-}$rhy 1 & 1.952292573383342 & $\operatorname{mfcc}-$ & vector quantization & cosine ${ }^{-}$distance \\
\hline $610^{-}$rhy2 & 1.8806557150616792 & $\mathrm{mfcc}$ & vector quantization & cosine ${ }^{-}$distance \\
\hline
\end{tabular}

\section{7-Artists}

\begin{tabular}{|c|c|c|c|c|}
\hline class & iicsr & extractor & aggregator & distance \\
\hline alternative_and_punk & 1.4999695490436216 & melspectrogram & gaussian_mixture_model_default & cosine_distance \\
\hline blues $\quad-1-\infty$ & 1.1098871819330052 & beat_synchronous_chroma_stft & vector_quantization & cosine_distance \\
\hline childrens & 1.09430 & temporal_statisticāl_sp & bypass ${ }^{-}$ & cosine_distance \\
\hline isical & 2.1164904537244684 & $\mathrm{mfcc}-{ }_{-}^{-}$ & vector_quantization & cosine_distance \\
\hline comedy_and_spoken_word & 1.1974831893872648 & melspectrogram & vector_quantization & manhattan_distance \\
\hline ountry $^{-}--{ }^{-}-$ & 1.2554695639407525 & $\mathrm{mfcc}$ & vector_quantization & cosine_distance \\
\hline easy_listening_and_vocals & 1.1939354506841648 & spectral_flatness & vector_quantization_default & manhattan_distance \\
\hline electronic_and_dan & 1.840397912731588 & melspectrogram & gaussian_mixture_model_default & cosine_distance \\
\hline folk & 1.2152099198606334 & temporal_statistical_spectrum_descriptor & bypass & cosine_distance \\
\hline
\end{tabular}




\author{
hip_hop \\ jazz \\ new ag \\ $\mathrm{rb}$ and soul \\ reggae \\ religious \\ rock_and_pop \\ soundtracks__and_more \\ world
}

1.822437782260004

1.2021325504515423

1.1231307443059342

1.251975624140489

1.476072914544827

1.542428608725201

1.0668655201385653

1.2498143324639641

1.1794783186030748
1.0778001669749437 melspectrogram

$\mathrm{mfcc}$

temporal_statistical_spectrum_descriptor emporal_statistical_spectrum_descriptor melspectrogram

beat_synchronous_chroma_stft

melspectrogram

rms

pitch_contour_segmentation gaussian mixture model default vector quantization

bypass

gaussian mixture model default gaussian_mixture_model_default gaussian mixture m
vector quantization

vector_quantization gaussian_mixture_model_defaul vector_quantization_defaūt interval_abstraction cosine distance cosine ${ }^{-}$distance cosine ${ }^{-}$distance cosine ${ }^{-}$distance cosine ${ }^{-}$distance cosine ${ }^{-}$distance manhattan_distance cosine_distance cosine ${ }_{-}^{-}$distance lcs $\min$

\section{MIR-QBSH}

\begin{tabular}{|c|c|c|c|c|}
\hline class & iicsr & extractor & aggregator & distance \\
\hline 00002 & 1.1854384487609289 & beat ${ }^{-}$synchronous ${ }^{-}$chroma ${ }^{-}$cqt & vector quantization & cosine $e^{-}$distance \\
\hline 00003 & 1.2089379804091687 & beat_synchronous _chroma-cqt & gaussian_mixture_model_default & chebyshev_distance \\
\hline 00004 & 1.5140063780774038 & pitch_contour_segmentation & octave_ābstraction & levenshtein__circular_max \\
\hline 00007 & 1.4810408546023004 & beat ${ }^{-}$synchronous ${ }^{-}$chroma ${ }^{-}$stft & vector quantization & cosine ${ }^{-}$distance \\
\hline 00008 & 1.7058914361938693 & beat_synchronous_chroma-cens & vector_quantization & cosine $e^{-}$distance \\
\hline 00009 & 1.2584274546232286 & pitch_contour_segmentation & octave abstraction & levensh̄tein circular $\max$ \\
\hline 00013 & 1.2408659176153771 & pitch $^{-}$contour ${ }^{-}$segmentation & octave abstraction & levenshtein ${ }^{-}$circular $\max$ \\
\hline 00014 & 1.3710733579205252 & pitch $^{-}$contour ${ }^{-}$segmentation & octave $^{-}$abstraction & levenshtein ${ }^{-}$circular ${ }^{-} \max$ \\
\hline 00015 & 2.8752141436215215 & pitch_contour_segmentation $^{-}$ & interval_abstraction & levenshtein_-max - \\
\hline 00016 & 1.2416637016174383 & beat_synchronous_chroma_stft & gaussian_mixture_model default & chebyshev_distance \\
\hline 00017 & 1.2330353215917977 & pitch_contour_segmentation & octave_abstraction & levenshtein_circular_max \\
\hline 00018 & 1.2735425266442444 & pitch ${ }^{-}$contour ${ }^{-}$segmentation & octave $^{-}$abstraction & levenshtein ${ }^{-}$circular ${ }^{-} \max$ \\
\hline 00019 & 1.3002635184876157 & pitch ${ }^{-}$contour ${ }^{-}$segmentation & interval abstraction & levenshtein ${ }^{-} \max -$ \\
\hline 00025 & 1.365174657637962 & pitch_contour_segmentation & octave_abstraction & levenshtein_circular_max \\
\hline 00026 & 1.2129547851601532 & pitch $^{-}$contour ${ }^{-}$segmentation & octave abstraction & levenshtein ${ }^{-}$ircular ${ }^{-} \max$ \\
\hline 00027 & 1.2870527989905776 & pitch_contour_segmentation & octave-abstraction & levenshtein_circular_max \\
\hline 00028 & 1.213312386482332 & pitch $^{-}$contour ${ }^{-}$segmentation & octave-abstraction & levenshtein ${ }^{-}$circular $^{-} \max$ \\
\hline 00029 & 1.2694733437999601 & pitch $^{-}$contour ${ }^{-}$segmentation & interval abstraction & levenshtein ${ }^{-} \max \quad-$ \\
\hline 00030 & 1.4310178987924125 & pitch_contour_segmentation & octave_ābstraction & levenshtein_circular_max \\
\hline 00031 & 1.2926576412946402 & pitch $^{-}$contour ${ }^{-}$segmentation & octave $^{-}$abstraction & levenshtein ${ }^{-}$circular $^{-} \max$ \\
\hline 00032 & 1.3987053026930807 & pitch_contour_segmentation & interval_abstraction & levenshtein_max \\
\hline 00033 & 1.240558326933691 & pitch_contour_segmentation & octave_àbstraction & levenshtein_circular_max \\
\hline 00034 & 1.4092555463527239 & pitch_contour_segmentation & octave_abstraction & levenshtein_circular_max \\
\hline 00035 & 1.3928981122158477 & pitch $^{-}$contour ${ }^{-}$segmentation & interval abstraction & levenshtein ${ }^{-} \max \quad-$ \\
\hline 00036 & 1.2186706262622518 & pitch ${ }^{-}$contour ${ }^{-}$segmentation & octave abstraction & levenshtein circular max \\
\hline 00037 & 1.2591839456105867 & pitch ${ }^{-}$contour ${ }^{-}$segmentation & octave $^{-}$abstraction & levenshtein ${ }^{-}$ircular ${ }^{-} \max$ \\
\hline 00038 & 1.4380810494107832 & pitch_contour_segmentation & pitch_contour_5_levels & levenshtein_max \\
\hline 00039 & 1.295451309499923 & pitch_contour_segmentation & pitch_contour ${ }^{-} 5^{-}$levels & levenshtein_-max \\
\hline 00040 & 1.2838996250824963 & pitch ${ }^{-}$contour ${ }^{-}$segmentation & octave abstraction & levenshtein_circular_max \\
\hline
\end{tabular}

\section{FMA-Small}

\begin{tabular}{lllll} 
class & iicsr & extractor & aggregator & distance \\
\hline electronic & 1.571253188813728 & melspectrogram & gaussian_mixture_model_default & cosine_distance \\
experimental & 1.079796885278995 & chroma_cqt & gaussian_mixture_model_default & chebyshev_distance \\
folk & 1.4259507088603285 & mfcc & vector_quantization_default & cosine_distance \\
hiphop & 1.7413277755764476 & melspectrogram & gaussian_mixture_model_default & cosine_distance \\
instrumental & 1.3366911248426074 & spectral_flatness & vector_quantization_default & manhattan_distance \\
international & 1.1580329180897504 & spectral_rolloff & vector_quantization_ & cosine_distance \\
pop & 1.1103872709568248 & spectral_rolloff & vector_quantization_default & manhattan_distance \\
rock & 1.2649471222355302 & mfcc & vector_quantization_ddefault & cosine_distance
\end{tabular}




\section{Appendix B}

\section{Cover Song Identification: top-50 results per dataset}

\section{Covers80}

\begin{tabular}{|c|c|c|c|c|c|c|c|c|}
\hline line id & MR & MRR & MDR & MAP & Top 1 & Top 10 & Top 100 & Top 1000 \\
\hline 1 & 41.575 & 0.19359 & 31.0 & 0.19359 & 21.0 & 46.0 & 142.0 & 160.0 \\
\hline 2 & 41.99375 & 0.19206 & 32.0 & 0.19206 & 20.0 & 44.0 & 142.0 & 160.0 \\
\hline 3 & 42.3625 & 0.20598 & 27.5 & 0.20598 & 20.0 & 56.0 & 138.0 & 160.0 \\
\hline 4 & 42.44375 & 0.1901 & 24.0 & 0.1901 & 18.0 & 57.0 & 141.0 & 160.0 \\
\hline 5 & 42.4625 & 0.18129 & 32.0 & 0.18129 & 19.0 & 47.0 & 143.0 & 160.0 \\
\hline 6 & 42.675 & 0.19459 & 24.0 & 0.19459 & 19.0 & 57.0 & 136.0 & 160.0 \\
\hline 7 & 42.71875 & 0.17317 & 31.0 & 0.17317 & 18.0 & 46.0 & 142.0 & 160.0 \\
\hline 8 & 43.01875 & 0.18078 & 34.0 & 0.18078 & 17.0 & 48.0 & 136.0 & 160.0 \\
\hline 9 & 43.08125 & 0.20681 & 25.0 & 0.20681 & 23.0 & 48.0 & 137.0 & 160.0 \\
\hline 10 & 43.35625 & 0.16701 & 29.5 & 0.16701 & 16.0 & 46.0 & 137.0 & 160.0 \\
\hline 11 & 43.35625 & 0.16701 & 29.5 & 0.16701 & 16.0 & 46.0 & 137.0 & 160.0 \\
\hline 12 & 43.35625 & 0.16701 & 29.5 & 0.16701 & 16.0 & 46.0 & 137.0 & 160.0 \\
\hline 13 & 43.45 & 0.1628 & 29.5 & 0.1628 & 15.0 & 44.0 & 137.0 & 160.0 \\
\hline 14 & 43.925 & 0.1763 & 36.0 & 0.1763 & 17.0 & 45.0 & 138.0 & 160.0 \\
\hline 15 & 44.5 & 0.17416 & 36.0 & 0.17416 & 17.0 & 45.0 & 137.0 & 160.0 \\
\hline 16 & 44.53125 & 0.17385 & 36.0 & 0.17385 & 17.0 & 45.0 & 137.0 & 160.0 \\
\hline 17 & 44.78125 & 0.22168 & 33.0 & 0.22168 & 26.0 & 51.0 & 136.0 & 160.0 \\
\hline 18 & 44.80625 & 0.20759 & 34.5 & 0.20759 & 23.0 & 53.0 & 139.0 & 160.0 \\
\hline 19 & 45.0 & 0.20318 & 29.0 & 0.20318 & 22.0 & 53.0 & 137.0 & 160.0 \\
\hline 20 & 45.0125 & 0.17628 & 35.0 & 0.17628 & 20.0 & 45.0 & 134.0 & 160.0 \\
\hline 21 & 45.0625 & 0.17673 & 32.5 & 0.17673 & 17.0 & 53.0 & 140.0 & 160.0 \\
\hline 22 & 45.0625 & 0.17687 & 31.5 & 0.17687 & 17.0 & 53.0 & 139.0 & 160.0 \\
\hline 23 & 45.15 & 0.16971 & 38.5 & 0.16971 & 17.0 & 45.0 & 136.0 & 160.0 \\
\hline 24 & 45.15 & 0.20324 & 31.5 & 0.20324 & 22.0 & 52.0 & 137.0 & 160.0 \\
\hline 25 & 45.15625 & 0.1726 & 37.0 & 0.1726 & 17.0 & 45.0 & 138.0 & 160.0 \\
\hline 26 & 45.2875 & 0.2088 & 27.0 & 0.2088 & 23.0 & 50.0 & 135.0 & 160.0 \\
\hline 27 & 45.36875 & 0.24177 & 26.5 & 0.24177 & 29.0 & 56.0 & 135.0 & 160.0 \\
\hline 28 & 45.38125 & 0.21505 & 27.0 & 0.21505 & 23.0 & 57.0 & 133.0 & 160.0 \\
\hline 29 & 45.4625 & 0.17864 & 33.0 & 0.17864 & 20.0 & 42.0 & 137.0 & 160.0 \\
\hline 30 & 45.61875 & 0.20712 & 25.5 & 0.20712 & 20.0 & 57.0 & 128.0 & 160.0 \\
\hline 31 & 45.83125 & 0.22481 & 30.0 & 0.22481 & 26.0 & 51.0 & 134.0 & 160.0 \\
\hline 32 & 46.04375 & 0.19432 & 29.5 & 0.19432 & 20.0 & 50.0 & 132.0 & 160.0 \\
\hline 33 & 46.06875 & 0.1945 & 29.5 & 0.1945 & 20.0 & 50.0 & 132.0 & 160.0 \\
\hline 34 & 46.09375 & 0.17987 & 31.0 & 0.17987 & 19.0 & 46.0 & 135.0 & 160.0 \\
\hline 35 & 46.16875 & 0.16105 & 31.5 & 0.16105 & 13.0 & 50.0 & 136.0 & 160.0 \\
\hline 36 & 46.19375 & 0.21467 & 29.5 & 0.21467 & 23.0 & 49.0 & 134.0 & 160.0 \\
\hline 37 & 46.2 & 0.21456 & 29.5 & 0.21456 & 23.0 & 49.0 & 134.0 & 160.0 \\
\hline 38 & 46.21875 & 0.21466 & 29.5 & 0.21466 & 23.0 & 49.0 & 134.0 & 160.0 \\
\hline 39 & 46.2375 & 0.21455 & 29.5 & 0.21455 & 23.0 & 49.0 & 134.0 & 160.0 \\
\hline 40 & 46.25625 & 0.2138 & 28.0 & 0.2138 & 24.0 & 53.0 & 134.0 & 160.0 \\
\hline 41 & 46.2625 & 0.20077 & 32.0 & 0.20077 & 20.0 & 51.0 & 136.0 & 160.0 \\
\hline 42 & 46.3375 & 0.21427 & 29.5 & 0.21427 & 24.0 & 53.0 & 133.0 & 160.0 \\
\hline 43 & 46.41875 & 0.20396 & 29.0 & 0.20396 & 21.0 & 49.0 & 140.0 & 160.0 \\
\hline 44 & 46.48125 & 0.1864 & 31.0 & 0.1864 & 20.0 & 47.0 & 134.0 & 160.0 \\
\hline 45 & 46.48125 & 0.21301 & 30.0 & 0.21301 & 22.0 & 53.0 & 136.0 & 160.0 \\
\hline 46 & 46.55 & 0.2003 & 29.0 & 0.2003 & 20.0 & 49.0 & 140.0 & 160.0 \\
\hline 47 & 46.6125 & 0.21394 & 33.0 & 0.21394 & 21.0 & 57.0 & 137.0 & 160.0 \\
\hline 48 & 46.7375 & 0.20593 & 26.5 & 0.20593 & 22.0 & 51.0 & 129.0 & 160.0 \\
\hline 49 & 46.76875 & 0.21671 & 29.5 & 0.21671 & 24.0 & 50.0 & 135.0 & 160.0 \\
\hline 50 & 46.8 & 0.18481 & 29.5 & 0.18481 & 18.0 & 46.0 & 130.0 & 160.0 \\
\hline
\end{tabular}

\begin{tabular}{l|lll} 
line id & extractor & aggregator & distance \\
\hline 1 & chroma_stft & diff_stats_1 & cosine_distance_oti \\
2 & chroma_stft & basic_stats_1 & cosine_distance_oti \\
3 & beat_synchronous_chroma_cens & basic_stats_- & manhattan_distance_oti \\
4 & beat_synchronous_chroma_cens & basic_stats_- & euclidean_distance_oti \\
5 & chroma_stft & diff_stats_1 & manhattan_distance_oti \\
6 & beat_synchronous_chroma_cens & basic_stats_2 & cosine_distance_oti \\
7 & chroma_stft & statistical_summarization & manhattan_distance_oti \\
8 & chroma_stft & diff_stats_1 & euclidean_distance_oti \\
9 & chroma_stft & statistical_summarization & cosine_distance_oti \\
10 & chroma_stft & basic_stats_2 & chebyshev_distance_oti \\
11 & chroma_stft & basic_stats_1 & chebyshev_distance_oti \\
12 & chroma_stft & diff_stats_1 & chebyshev_distance_oti
\end{tabular}




\begin{tabular}{l|l}
13 & chroma_stft \\
14 & chroma_stft \\
15 & chroma_stft \\
16 & chroma_stft \\
17 & beat_synchronous_chroma_cqt \\
18 & beat_synchronous_chroma_cqt \\
19 & beat_synchronous_chroma_cens \\
20 & chroma_stft \\
21 & beat_synchronous_chroma_cens \\
22 & beat_synchronous_chroma_cens \\
23 & chroma_stft \\
24 & beat_synchronous_chroma_cens \\
25 & chroma_stft \\
26 & beat_synchronous_chroma_cens \\
27 & beat_synchronous_chroma_cqt \\
28 & beat_synchronous_chroma_cqt \\
29 & chroma_stft \\
30 & beat_synchronous_chroma_cens \\
31 & beat_synchronous_chroma_cqt \\
32 & beat_synchronous_chroma_cens \\
33 & beat_synchronous_chroma_cens \\
34 & chroma_cqt \\
35 & beat_synchronous_chroma_cens \\
36 & chroma_cens \\
37 & chroma_cens \\
38 & chroma_cens \\
39 & chroma_cens \\
40 & beat_synchronous_chroma_cens \\
41 & beat_synchronous_chroma_cens \\
42 & beat_synchronous_chroma_cens \\
43 & chroma_cens \\
44 & chroma_cqt \\
45 & beat_synchronous_chroma_cqt \\
46 & chroma_cens \\
47 & beat_synchronous_chroma_cens \\
48 & chroma_cqt \\
49 & beat_synchronous_chroma_cqt \\
50 & chroma_cqt \\
&
\end{tabular}

statistical summarization basic_stats_2 basic_stats - 1 basic_stats ${ }^{2} 2$ diff_stats_ $\overline{1}$ diff stats $\overline{1}$ statistical_ ${ }^{-}$summarization basic stats 1 basic ${ }^{-}$stats $^{-} 1$ basic ${ }^{-}$stats ${ }^{-} 2$ diff stats 1 basic_stats_1 basic ${ }^{-}$stats $^{-}$ diff stats $\overline{1}$ diff $^{-}$stats ${ }^{-}$ diff $^{-}$stats diff - stats diff_stats_2 basic_stats_2 basic_stats _ 1 basic_stats diff_stats_1 diff_stats_ $\overline{1}^{1}$ diff basic $_{\text {stats }} 1$ basic ${ }^{-}$stats ${ }^{-} 1$ diff stats $\overline{1}$ diff stats 1 diff $^{-}$stats $^{-} 1$ diff stats 1 diff ${ }_{-}^{-}$stats ${ }_{-}^{-}$ diff_-stats -1 basic_stats 1 diff stats $\overline{2}$ vector_quantization_default statistical summarization chebyshev distance oti cosine distance oti euclidean_distance_oti euclidean ${ }^{-}$distance ${ }^{-}$oti cosine_distance_ot $\bar{i}$ cosine_distance_oti cosine_distance_oti euclidean_distance_oti cosine_distance_oti euclidean_distance oti manhattan_distance_ot euclidean_distance_oti manhattan distance ot manhattan distance manhattan ${ }^{-}$distance oti manhattan-distance-oti manhattan_distance cosine dist-distance manhattan_distance_oti manhattan_distan cosine_distance

euclidean_distance manhattan_distance
manhattan
distance ot manhattan-distanc

euclidean_distanc cosine_distance euclidean_distance cosine_distance manhattan distance oti euclidean distance manhattan distance euclidean $\overline{\text { distance }}$ euclidean_distance_oti manhattan distance manhattan_distance_oti cosine distance

euclidean distance oti euclidean_distance $-{ }^{-}$

\section{YouTubeCovers}

\begin{tabular}{|c|c|c|c|c|c|c|c|c|}
\hline line id & MR & MRR & MDR & MAP & Top 1 & Top 10 & Top 100 & Top 1000 \\
\hline 1 & 7.97143 & 0.6942 & 1.0 & 0.36114 & 219.0 & 296.0 & 345.0 & 350.0 \\
\hline 2 & 9.41143 & 0.63231 & 1.0 & 0.2977 & 189.0 & 279.0 & 345.0 & 350.0 \\
\hline 3 & 14.1 & 0.4344 & 3.5 & 0.19221 & 110.0 & 231.0 & 343.0 & 350.0 \\
\hline 4 & 14.13714 & 0.40846 & 5.0 & 0.1805 & 103.0 & 233.0 & 344.0 & 350.0 \\
\hline 5 & 14.71143 & 0.38021 & 5.0 & 0.15415 & 85.0 & 227.0 & 344.0 & 350.0 \\
\hline 6 & 15.89429 & 0.37811 & 5.0 & 0.15807 & 93.0 & 216.0 & 346.0 & 350.0 \\
\hline 7 & 15.89429 & 0.37811 & 5.0 & 0.15807 & 93.0 & 216.0 & 346.0 & 350.0 \\
\hline 8 & 16.46286 & 0.37605 & 5.5 & 0.15219 & 86.0 & 223.0 & 343.0 & 350.0 \\
\hline 9 & 16.85429 & 0.5271 & 2.0 & 0.21466 & 153.0 & 247.0 & 335.0 & 350.0 \\
\hline 10 & 17.06 & 0.35579 & 6.0 & 0.14139 & 78.0 & 225.0 & 342.0 & 350.0 \\
\hline 11 & 17.96 & 0.5303 & 2.0 & 0.21618 & 154.0 & 252.0 & 332.0 & 350.0 \\
\hline 12 & 18.41429 & 0.37785 & 6.0 & 0.15339 & 93.0 & 210.0 & 341.0 & 350.0 \\
\hline 13 & 18.8 & 0.38707 & 5.0 & 0.15379 & 95.0 & 213.0 & 338.0 & 350.0 \\
\hline 14 & 18.82857 & 0.36226 & 5.0 & 0.14261 & 81.0 & 219.0 & 337.0 & 350.0 \\
\hline 15 & 19.38571 & 0.38507 & 5.0 & 0.15785 & 93.0 & 218.0 & 339.0 & 350.0 \\
\hline 16 & 19.45143 & 0.37268 & 6.0 & 0.14618 & 90.0 & 218.0 & 336.0 & 350.0 \\
\hline 17 & 19.46857 & 0.36426 & 5.0 & 0.14654 & 84.0 & 215.0 & 336.0 & 350.0 \\
\hline 18 & 19.54571 & 0.39092 & 6.0 & 0.15383 & 100.0 & 205.0 & 340.0 & 350.0 \\
\hline 19 & 19.56 & 0.33607 & 8.0 & 0.12278 & 76.0 & 196.0 & 339.0 & 350.0 \\
\hline 20 & 19.61143 & 0.3506 & 7.0 & 0.13665 & 86.0 & 197.0 & 339.0 & 350.0 \\
\hline 21 & 19.63143 & 0.42627 & 4.0 & 0.1657 & 109.0 & 230.0 & 335.0 & 350.0 \\
\hline 22 & 19.76 & 0.40204 & 4.5 & 0.1571 & 101.0 & 221.0 & 333.0 & 350.0 \\
\hline 23 & 19.79429 & 0.35146 & 7.0 & 0.13543 & 80.0 & 214.0 & 338.0 & 350.0 \\
\hline 24 & 19.83143 & 0.37327 & 5.0 & 0.14771 & 87.0 & 213.0 & 338.0 & 350.0 \\
\hline 25 & 19.88857 & 0.37544 & 5.0 & 0.14857 & 88.0 & 214.0 & 338.0 & 350.0 \\
\hline 26 & 19.90857 & 0.38234 & 6.0 & 0.14595 & 93.0 & 218.0 & 336.0 & 350.0 \\
\hline 27 & 19.92286 & 0.35465 & 6.0 & 0.13542 & 84.0 & 210.0 & 335.0 & 350.0 \\
\hline 28 & 20.16571 & 0.35024 & 7.0 & 0.13404 & 80.0 & 209.0 & 337.0 & 350.0 \\
\hline 29 & 20.18 & 0.42428 & 5.0 & 0.16905 & 113.0 & 228.0 & 335.0 & 350.0 \\
\hline 30 & 20.20286 & 0.35045 & 7.0 & 0.13408 & 80.0 & 210.0 & 337.0 & 350.0 \\
\hline 31 & 20.21429 & 0.38504 & 7.0 & 0.14998 & 101.0 & 207.0 & 335.0 & 350.0 \\
\hline 32 & 20.32286 & 0.38765 & 6.5 & 0.15122 & 102.0 & 210.0 & 336.0 & 350.0 \\
\hline 33 & 20.36571 & 0.33463 & 7.0 & 0.12613 & 77.0 & 204.0 & 336.0 & 350.0 \\
\hline 34 & 20.48 & 0.3748 & 5.5 & 0.14459 & 92.0 & 211.0 & 337.0 & 350.0 \\
\hline 35 & 20.48571 & 0.33411 & 8.0 & 0.12859 & 78.0 & 194.0 & 340.0 & 350.0 \\
\hline 36 & 20.58571 & 0.3173 & 7.0 & 0.12465 & 71.0 & 200.0 & 339.0 & 350.0 \\
\hline 37 & 20.61429 & 0.36799 & 5.0 & 0.13742 & 85.0 & 224.0 & 331.0 & 350.0 \\
\hline 38 & 20.72286 & 0.33957 & 6.0 & 0.1267 & 74.0 & 204.0 & 336.0 & 350.0 \\
\hline 39 & 20.86571 & 0.35297 & 6.0 & 0.13521 & 82.0 & 208.0 & 340.0 & 350.0 \\
\hline 40 & 20.94857 & 0.39652 & 6.0 & 0.15371 & 101.0 & 215.0 & 333.0 & 350.0 \\
\hline 41 & 21.01143 & 0.34911 & 6.0 & 0.13439 & 79.0 & 207.0 & 339.0 & 350.0 \\
\hline 42 & 21.16571 & 0.38376 & 6.0 & 0.14662 & 97.0 & 207.0 & 331.0 & 350.0 \\
\hline 43 & 21.16571 & 0.36152 & 6.0 & 0.13785 & 87.0 & 210.0 & 335.0 & 350.0 \\
\hline 44 & 21.44286 & 0.33155 & 9.0 & 0.12398 & 78.0 & 193.0 & 340.0 & 350.0 \\
\hline 45 & 21.44857 & 0.3592 & 6.0 & 0.13646 & 87.0 & 209.0 & 331.0 & 350.0 \\
\hline 46 & 21.54286 & 0.33319 & 9.0 & 0.12406 & 79.0 & 194.0 & 340.0 & 350.0 \\
\hline 47 & 21.58571 & 0.36208 & 6.0 & 0.13483 & 88.0 & 210.0 & 336.0 & 350.0 \\
\hline 48 & 21.72286 & 0.38764 & 7.0 & 0.15387 & 101.0 & 204.0 & 328.0 & 350.0 \\
\hline 49 & 21.84571 & 0.36061 & 6.0 & 0.14888 & 92.0 & 200.0 & 334.0 & 350.0 \\
\hline 50 & 21.92 & 0.31323 & 7.5 & 0.11445 & 69.0 & 193.0 & 340.0 & 350.0 \\
\hline
\end{tabular}




\begin{tabular}{|c|c|c|c|}
\hline 1 & $\begin{array}{lll}\text { pitch } & \text { contour segmentation }\end{array}$ & octave abstraction & $\begin{array}{lll}\text { lcs } & \text { circular mean } \\
\end{array}$ \\
\hline 2 & pitch contour segmentation & octave $^{-}$abstraction & levenshtein circular max \\
\hline 3 & pitch contour segmentation & markov chain & circular mānhattan ${ }^{-}$ \\
\hline 4 & pitch contour-segmentation & markov_chain & circular cosine \\
\hline 5 & chroma stft - & statistical summarization & cosine $\overline{\text { distance }}$ oti \\
\hline 6 & pitch contour segmentation & markov chain & circular euclideān \\
\hline 7 & pitch ${ }^{-}$contour ${ }^{-}$segmentation & markov ${ }^{-}$chain & circular ${ }^{-}$chebyshev \\
\hline 8 & chroma stft & diff stats 1 & cosine $\overline{\text { distance oti }}$ \\
\hline 9 & pitch contour segmentation & octave abstraction & lcs circular max \\
\hline 10 & chroma stft - & basic stats 1 & cosine distance oti \\
\hline 11 & pitch_contour_segmentation & octave abstraction & levenshtein_circülar_mean \\
\hline 12 & beat_synchronous chroma_cens & basic_stats_2 & euclidean_distance_oti \\
\hline 13 & beat ${ }^{-}$synchronous ${ }^{-}$chroma ${ }^{-}$cens & basic ${ }^{-}$stats $^{-} 2$ & manhattan distance oti \\
\hline 14 & chroma cens & basic ${ }^{-}$stats ${ }^{-} 2$ & euclidean $\overline{\text { distance }} \overline{\text { oti }}$ \\
\hline 15 & beat synchronous chroma cens & basic ${ }^{-}$stats ${ }_{2}^{-}$ & cosine distance ot $\bar{i}$ \\
\hline 16 & chroma stft & diff $\overline{\text { stats }} \overline{1}$ & euclidean distance oti \\
\hline 17 & chroma ${ }^{-}$cens & basic stats 2 & cosine distance ot $\overline{\mathrm{i}}$ \\
\hline 18 & beat synchronous chroma cqt & diff $\overline{\text { stats }} \overline{1}$ & $\operatorname{cosine}^{-}$distance ${ }^{-}$oti \\
\hline 19 & beat ${ }^{-}$synchronous ${ }^{-}$chroma ${ }^{-}$stft & statistical ${ }^{-}$summarization & cosine $^{-}$distance ${ }^{-}$oti \\
\hline 20 & beat ${ }^{-}$synchronous ${ }^{-}$chroma ${ }^{-}$cqt & statistical ${ }^{-}$summarization & $\operatorname{cosine}^{-}$distance $^{-}$oti \\
\hline 21 & beat ${ }^{-}$synchronous ${ }^{-}$chroma ${ }^{-}$cens & diff stats 2 & manhattan distance oti \\
\hline 22 & beat ${ }^{-}$synchronous ${ }^{-}$chroma ${ }^{-}$cens & $\operatorname{diff}^{-}$stats ${ }^{-} 2$ & euclidean $\overline{\text { distance }} \overline{\text { oti }}$ \\
\hline 23 & chroma stft & basic stats 2 & cosine distance ot $\bar{i}$ \\
\hline 24 & chroma ${ }^{-}$stft & statistical summarization & manhattan distānce oti \\
\hline 25 & chroma ${ }^{-}$stft & diff stats 1 & manhattan ${ }^{-}$distance ${ }^{-}$oti \\
\hline 26 & chroma ${ }^{-}$cens & basic stats 2 & manhattan ${ }^{-}$distance ${ }^{-}$oti \\
\hline 27 & chroma ${ }^{-}$cens & statistical summarization & manhattan ${ }^{-}$distance ${ }^{-}$oti \\
\hline 28 & chroma ${ }^{-}$stft & basic stats 1 & euclidean $\overline{\text { distance }} \overline{\text { oti }}$ \\
\hline 29 & beat synchronous chroma cens & diff stats $\overline{2}$ & cosine distance ot $\overline{\mathrm{i}}$ \\
\hline 30 & chroma stft & basic stats 2 & euclidean distance oti \\
\hline 31 & beat synchronous chroma cens & diff $\overline{\text { stats }} \overline{1}$ & cosine distance ot $\overline{\mathrm{i}}$ \\
\hline 32 & beat ${ }^{-}$synchronous ${ }^{-}$chroma ${ }^{-}$cens & $\operatorname{diff}^{-}$stats $^{-1}$ & euclidean distance oti \\
\hline 33 & chroma cqt - & statistical ${ }^{-}$summarization & cosine distance ot $\overline{\mathrm{i}}$ \\
\hline 34 & beat synchronous chroma cens & statistical ${ }^{-}$summarization & manhattan distance oti \\
\hline 35 & beat ${ }^{-}$synchronous ${ }^{-}$chroma ${ }^{-}$cqt & basic stats 1 & cosine distance oti - \\
\hline 36 & beat ${ }^{-}$synchronous ${ }^{-}$chroma ${ }^{-}$stft & diff $\overline{\text { stats }} \overline{2}$ & manhattan distance oti \\
\hline 37 & beat ${ }^{-}$synchronous ${ }^{-}$chroma ${ }^{-}$cqt & statistical ${ }^{-}$summarization & manhattan ${ }^{-}$distance ${ }^{-}$oti \\
\hline 38 & chroma cqt - & statistical ${ }^{-}$summarization & manhattan ${ }^{-}$distance ${ }^{-}$oti \\
\hline 39 & chroma ${ }^{-}$stft & basic stats 1 & manhattan ${ }^{-}$distance ${ }^{-}$oti \\
\hline 40 & beat synchronous chroma cqt & diff $\overline{\text { stats }} \overline{1}$ & manhattan ${ }^{-}$distance ${ }^{-}$oti \\
\hline 41 & chroma stft & basic stats 2 & manhattan ${ }^{-}$distance ${ }^{-}$oti \\
\hline 42 & beat synchronous chroma cqt & diff $\overline{\text { stats }} \overline{1}$ & euclidean $\overline{\text { distance }} \overline{\text { oti }}$ \\
\hline 43 & chroma stft & statistical ${ }^{-}$summarization & euclidean ${ }^{-}$distance ${ }^{-}$oti \\
\hline 44 & beat synchronous chroma cens & basic stats 1 & euclidean ${ }^{-}$distance ${ }^{-}$oti \\
\hline 45 & beat ${ }^{-}$synchronous ${ }^{-}$chroma ${ }^{-}$stft & diff $\overline{\text { stats }} \overline{1}$ & cosine distance ot $\bar{i}$ \\
\hline 46 & beat ${ }^{-}$synchronous ${ }^{-}$chroma ${ }^{-}$cens & basic stats 1 & $\operatorname{cosine}^{-}$distance ${ }^{-}$oti \\
\hline 47 & beat ${ }^{-}$synchronous ${ }^{-}$chroma ${ }^{-}$stft & diff stats $\overline{1}$ & manhātan distance oti \\
\hline 48 & beat ${ }^{-}$synchronous ${ }^{-}$chroma ${ }^{-}$cens & $\operatorname{diff}^{-}$stats $^{-} 1$ & manhattan ${ }^{-}$distance ${ }^{-}$oti \\
\hline 49 & beat ${ }^{-}$synchronous ${ }^{-}$chroma ${ }^{-}$cqt & $\operatorname{diff}^{-}$stats $^{-} 2$ & manhattan ${ }^{-}$distance ${ }^{-}$oti \\
\hline 50 & chroma stft & $\operatorname{diff}^{-}$stats $^{-}$ & chebyshev distance oti \\
\hline
\end{tabular}

\section{Covers1000}

\begin{tabular}{|c|c|c|c|c|c|c|c|c|}
\hline line id & MR & MRR & MDR & MAP & Top 1 & Top 10 & Top 100 & Top 1000 \\
\hline 1 & 144.041 & 0.25731 & 35.0 & 0.19159 & 201.0 & 366.0 & 636.0 & 1000.0 \\
\hline 2 & 175.546 & 0.09845 & 74.5 & 0.06977 & 52.0 & 188.0 & 548.0 & 1000.0 \\
\hline 3 & 180.808 & 0.13566 & 75.0 & 0.09752 & 77.0 & 249.0 & 551.0 & 1000.0 \\
\hline 4 & 181.48 & 0.15934 & 72.0 & 0.11552 & 103.0 & 261.0 & 558.0 & 1000.0 \\
\hline 5 & 183.802 & 0.0861 & 95.0 & 0.06054 & 48.0 & 155.0 & 515.0 & 1000.0 \\
\hline 6 & 184.645 & 0.12143 & 81.0 & 0.08497 & 70.0 & 213.0 & 533.0 & 1000.0 \\
\hline 7 & 185.462 & 0.13359 & 79.0 & 0.09661 & 80.0 & 236.0 & 540.0 & 1000.0 \\
\hline 8 & 185.521 & 0.14401 & 84.5 & 0.10473 & 93.0 & 228.0 & 525.0 & 1000.0 \\
\hline 9 & 185.781 & 0.09549 & 97.0 & 0.06776 & 49.0 & 183.0 & 512.0 & 1000.0 \\
\hline 10 & 185.884 & 0.14356 & 86.0 & 0.10421 & 93.0 & 229.0 & 526.0 & 1000.0 \\
\hline 11 & 186.181 & 0.13101 & 79.5 & 0.09636 & 73.0 & 233.0 & 541.0 & 1000.0 \\
\hline 12 & 187.932 & 0.1348 & 72.0 & 0.09188 & 86.0 & 224.0 & 558.0 & 1000.0 \\
\hline 13 & 188.264 & 0.13732 & 86.0 & 0.10134 & 84.0 & 231.0 & 527.0 & 1000.0 \\
\hline 14 & 188.475 & 0.14861 & 87.0 & 0.10949 & 100.0 & 234.0 & 522.0 & 1000.0 \\
\hline 15 & 189.284 & 0.1337 & 84.5 & 0.09952 & 81.0 & 235.0 & 532.0 & 1000.0 \\
\hline 16 & 189.312 & 0.11524 & 84.0 & 0.08645 & 67.0 & 197.0 & 530.0 & 1000.0 \\
\hline 17 & 189.973 & 0.10335 & 84.5 & 0.07515 & 59.0 & 179.0 & 538.0 & 1000.0 \\
\hline 18 & 190.38 & 0.09767 & 96.0 & 0.06738 & 54.0 & 177.0 & 510.0 & 1000.0 \\
\hline 19 & 190.65 & 0.11334 & 94.0 & 0.08278 & 64.0 & 195.0 & 521.0 & 1000.0 \\
\hline 20 & 190.742 & 0.12131 & 87.0 & 0.09107 & 72.0 & 207.0 & 531.0 & 1000.0 \\
\hline 21 & 190.766 & 0.13448 & 87.0 & 0.09512 & 88.0 & 213.0 & 533.0 & 1000.0 \\
\hline 22 & 190.953 & 0.13206 & 86.0 & 0.09652 & 87.0 & 221.0 & 529.0 & 1000.0 \\
\hline 23 & 191.334 & 0.10765 & 90.0 & 0.07774 & 62.0 & 185.0 & 516.0 & 1000.0 \\
\hline 24 & 191.445 & 0.10792 & 90.0 & 0.07784 & 63.0 & 185.0 & 518.0 & 1000.0 \\
\hline 25 & 191.543 & 0.1073 & 91.5 & 0.07762 & 63.0 & 185.0 & 514.0 & 1000.0 \\
\hline 26 & 191.648 & 0.1072 & 91.5 & 0.07753 & 63.0 & 185.0 & 515.0 & 1000.0 \\
\hline 27 & 191.84 & 0.11782 & 96.0 & 0.08641 & 72.0 & 194.0 & 510.0 & 1000.0 \\
\hline 28 & 191.898 & 0.11821 & 95.0 & 0.08651 & 73.0 & 195.0 & 508.0 & 1000.0 \\
\hline 29 & 192.048 & 0.10912 & 82.0 & 0.07906 & 61.0 & 198.0 & 529.0 & 1000.0 \\
\hline 30 & 192.494 & 0.10853 & 96.5 & 0.07907 & 59.0 & 185.0 & 514.0 & 1000.0 \\
\hline 31 & 192.662 & 0.11786 & 90.0 & 0.08843 & 65.0 & 212.0 & 526.0 & 1000.0 \\
\hline 32 & 192.749 & 0.11648 & 98.5 & 0.08777 & 74.0 & 197.0 & 503.0 & 1000.0 \\
\hline 33 & 193.164 & 0.10728 & 97.5 & 0.07317 & 61.0 & 193.0 & 508.0 & 1000.0 \\
\hline 34 & 193.857 & 0.09469 & 91.0 & 0.06679 & 45.0 & 185.0 & 523.0 & 1000.0 \\
\hline 35 & 194.399 & 0.12253 & 80.0 & 0.09197 & 73.0 & 217.0 & 533.0 & 1000.0 \\
\hline 36 & 195.464 & 0.11381 & 93.5 & 0.08471 & 65.0 & 204.0 & 511.0 & 1000.0 \\
\hline 37 & 195.724 & 0.10344 & 90.0 & 0.0741 & 55.0 & 195.0 & 527.0 & 1000.0 \\
\hline 38 & 195.92 & 0.1074 & 93.0 & 0.0776 & 62.0 & 190.0 & 512.0 & 1000.0 \\
\hline 39 & 196.486 & 0.18707 & 81.0 & 0.13042 & 138.0 & 275.0 & 534.0 & 1000.0 \\
\hline 40 & 196.723 & 0.10651 & 91.5 & 0.07646 & 57.0 & 188.0 & 519.0 & 1000.0 \\
\hline 41 & 197.11 & 0.10332 & 95.5 & 0.07532 & 55.0 & 189.0 & 506.0 & 1000.0 \\
\hline 42 & 198.292 & 0.13356 & 91.0 & 0.10005 & 80.0 & 237.0 & 517.0 & 1000.0 \\
\hline
\end{tabular}




\begin{tabular}{l|llllllll}
43 & 198.426 & 0.10194 & 103.5 & 0.07181 & 53.0 & 193.0 & 497.0 & 1000.0 \\
44 & 199.148 & 0.11907 & 87.0 & 0.08307 & 73.0 & 207.0 & 521.0 & 1000.0 \\
45 & 199.706 & 0.07955 & 104.0 & 0.05329 & 43.0 & 144.0 & 495.0 & 1000.0 \\
46 & 199.788 & 0.103 & 99.0 & 0.07324 & 57.0 & 191.0 & 504.0 & 1000.0 \\
47 & 200.046 & 0.07752 & 108.0 & 0.05253 & 36.0 & 147.0 & 484.0 & 1000.0 \\
48 & 200.077 & 0.11595 & 88.5 & 0.0858 & 61.0 & 217.0 & 520.0 & 1000.0 \\
49 & 200.237 & 0.11163 & 103.0 & 0.07722 & 64.0 & 196.0 & 494.0 & 1000.0 \\
50 & 200.555 & 0.06977 & 119.0 & 0.04798 & 35.0 & 122.0 & 465.0 & 1000.0
\end{tabular}

\begin{tabular}{|c|c|c|c|}
\hline line id & extractor & aggregator & distance \\
\hline 1 & pitch_contour_segmentation & octave_abstraction & lcs_circular_mean \\
\hline 2 & beat synchronous chroma stft & diff stäts 1 & cosine distance oti \\
\hline 3 & beat_synchronous_chroma_cqt & diff $^{-}$stats $^{-} 1$ & manhattan_distance oti \\
\hline 4 & rhythm histogram & bypāss - & cosine distance - \\
\hline 5 & beat_synchronous_chroma_stft & basic_stats_ 1 & cosine $e^{-}$distance_oti \\
\hline 6 & chroma_cens $\quad--\quad-$ & basic_stats_-2 & manhattan_distānce_oti \\
\hline 7 & beat_synchronous_chroma_cqt & diff_stats_-̄i & euclidean_zistance_ōti \\
\hline 8 & beat_synchronous-chroma-cens & diff $^{-}$stats $^{-} 1$ & cosine_distance_ot $\overline{\mathrm{i}}$ \\
\hline 9 & beat_synchronous_chroma-stft & diff $^{-}$stats $^{-} 1$ & manhattan distance oti \\
\hline 10 & beat ${ }^{-}$synchronous ${ }^{-}$chroma_cens & $\operatorname{diff}^{-}$stats $^{-} 1$ & euclidean_ōistance_ōti \\
\hline 11 & beat ${ }^{-}$synchronous ${ }^{-}$chroma ${ }^{-}$cqt & $\operatorname{diff}^{-}$stats $^{-} 1$ & cosine distance ot $\overline{\mathrm{i}}$ \\
\hline 12 & chroma_cqt $\quad--\quad-1$ & statistical $^{-}$summarization & manhattan_distānce_oti \\
\hline 13 & beat synchronous chroma cens & basic stats 2 & manhattan ${ }^{-}$distance ${ }^{-}$oti \\
\hline 14 & beat_synchronous_chroma_cens & diff_stats_ $\overline{1}$ & manhattan_distance_oti \\
\hline 15 & beat_synchronous_chroma_cqt & statistical ${ }^{-}$summarization & manhattan ${ }^{-}$distance ${ }^{-}$oti \\
\hline 16 & beat ${ }^{-}$synchronous ${ }^{-}$chroma ${ }^{-}$cqt & basic stats 1 & cosine distance oti ${ }^{-}$ \\
\hline 17 & beat_synchronous_chroma-stft & statistical_summarization & cosine $_{-}^{-}$distance-oti \\
\hline 18 & beat ${ }^{-}$synchronous ${ }^{-}$chroma ${ }^{-}$stft & diff stats $^{-1}$ & euclidean distance oti \\
\hline 19 & chroma_cens $\quad--\quad-$ & diff $_{-}^{-}$stats $^{-} 1$ & manhattan $\bar{n}$ distance $\bar{y}_{\text {oti }}$ \\
\hline 20 & beat_synchronous_chroma_cens & basic_stats 2 & euclidean_ōistance_oti \\
\hline 21 & chroma_cens - & statistical summarization & manhattan distance $\bar{e}$ oti \\
\hline 22 & beat synchronous chroma cens & statistical $^{-}$summarization & manhattan ${ }^{-}$distance ${ }^{-}$oti \\
\hline 23 & chroma cens - & diff stats 1 & euclidean $\overline{\text { distance }} \overline{\text { oti }}$ \\
\hline 24 & chroma ${ }^{-}$cens & basic stats 1 & euclidean ${ }^{-}$distance ${ }^{-}$oti \\
\hline 25 & chroma-cens & diff_stats_ $\overline{1}$ & cosine_distance_ot $\overline{\mathrm{i}}$ \\
\hline 26 & chroma_cens & basic stats 1 & cosine $_{-}^{-}$distance $e_{\text {oti }}^{-}$ \\
\hline 27 & beat_synchronous_chroma_cens & basic ${ }^{-}$stats 1 & euclidean_distance_oti \\
\hline 28 & beat synchronous chroma cens & basic $^{-}$stats $^{-} 1$ & cosine distance ot $\bar{i}$ \\
\hline 29 & chroma cqt & diff $\overline{\text { stats }} \overline{1}$ & $\operatorname{cosine}^{-}$distance ${ }^{-}$oti \\
\hline 30 & chroma-cens & basic_stats 1 & manhattan_distance oti \\
\hline 31 & beat_synchronous_chroma_cqt & basic $^{-}$stats $^{-} 1$ & manhattan ${ }^{-}$distance ${ }^{-}$oti \\
\hline 32 & beat ${ }^{-}$synchronous ${ }^{-}$chroma_cens & basic $^{-}$stats $^{-} 1$ & manhattan ${ }^{-}$distance ${ }^{-}$oti \\
\hline 33 & chroma_cens $-{ }_{-}$ & basic ${ }^{-}$stats ${ }^{-} 2$ & euclidean_ōdistance_ōti \\
\hline 34 & chroma ${ }^{-}$stft & statistical summarization & cosine distance ot $\bar{i}$ \\
\hline 35 & beat synchronous chroma cens & basic stats 2 & $\operatorname{cosine}^{-}$distance $^{-}$oti \\
\hline 36 & beat_synchronous_chroma_cqt & basic_stats_- 1 & euclidēen_distance_oti \\
\hline 37 & chroma cqt & basic $^{-}$stats $^{-} 1$ & cosine distance ot $\overline{\mathrm{i}}$ \\
\hline 38 & chroma-cqt & diff_stats_ $\overline{1}$ & manhattan_distance oti \\
\hline 39 & pitch contour segmentation & octave abstraction & lcs circular max - \\
\hline 40 & chroma cqt - & basic stats 1 & manhhattan distance oti \\
\hline 41 & chroma_cqt & diff_stats_ $\overline{1}$ & euclidean_zistance_ōti \\
\hline 42 & beat_synchronous_chroma_cens & diff $_{-}^{-}$stats_ 2 & manhattan $\bar{n}$ distance $\bar{y}_{\text {oti }}$ \\
\hline 43 & beat_synchronous_chroma_stft & statistical_summarization & manhattan ${ }^{-}$distance $^{-}$oti \\
\hline 44 & chroma cqt $\quad--\quad-$ & statistical ${ }^{-}$summarization & cosine distance oti ${ }^{-}$ \\
\hline 45 & beat synchronous chroma stft & diff stats ${ }^{-} 2$ & manhattan distance oti \\
\hline 46 & chroma cqt & basic stats 1 & euclidean $\overline{\text { distance }} \overline{\text { oti }}$ \\
\hline 47 & beat_synchronous_chroma_stft & basic_stats_-2 & euclidean_distance_oti \\
\hline 48 & beat_synchronous_chroma_cens & diff_stats $\_\overline{2}$ & cosine_distance_ot $\overline{\mathrm{i}}$ \\
\hline 49 & chroma_cqt - & statistical ${ }^{-}$summarization & euclidean_distance_oti \\
\hline 50 & beat_synchronous_chroma_stft & diff_stats ${ }^{-} 1$ & chebyshev_distance oti \\
\hline
\end{tabular}

\section{Mazurkas}

\begin{tabular}{l|llllllll} 
line id & MR & MRR & MDR & MAP & Top 1 & Top 10 & Top 100 & Top 1000 \\
\hline 1 & 4.15724 & 0.95774 & 1.0 & 0.82286 & 2561.0 & 2714.0 & 2728.0 & 2739.0 \\
2 & 4.46479 & 0.87347 & 1.0 & 0.33623 & 2251.0 & 2638.0 & 2722.0 & 2740.0 \\
3 & 4.6297 & 0.83039 & 1.0 & 0.28564 & 2076.0 & 2620.0 & 2721.0 & 2740.0 \\
4 & 4.65268 & 0.85999 & 1.0 & 0.32391 & 2197.0 & 2622.0 & 2717.0 & 2741.0 \\
5 & 4.86173 & 0.8785 & 1.0 & 0.3994 & 2241.0 & 2671.0 & 2718.0 & 2741.0 \\
6 & 4.86647 & 0.83115 & 1.0 & 0.30325 & 2067.0 & 2615.0 & 2723.0 & 2739.0 \\
7 & 4.95659 & 0.81238 & 1.0 & 0.24961 & 2018.0 & 2583.0 & 2722.0 & 2740.0 \\
8 & 4.9865 & 0.80264 & 1.0 & 0.2673 & 1965.0 & 2593.0 & 2726.0 & 2741.0 \\
9 & 5.08063 & 0.95648 & 1.0 & 0.86734 & 2551.0 & 2713.0 & 2728.0 & 2737.0 \\
10 & 5.14447 & 0.79237 & 1.0 & 0.30303 & 1921.0 & 2602.0 & 2722.0 & 2741.0 \\
11 & 5.23896 & 0.75554 & 1.0 & 0.26523 & 1787.0 & 2572.0 & 2722.0 & 2741.0 \\
12 & 5.24298 & 0.75791 & 1.0 & 0.26433 & 1795.0 & 2574.0 & 2722.0 & 2740.0 \\
13 & 5.25939 & 0.80314 & 1.0 & 0.30854 & 1964.0 & 2605.0 & 2722.0 & 2740.0 \\
14 & 5.29734 & 0.83466 & 1.0 & 0.3252 & 2087.0 & 2614.0 & 2720.0 & 2739.0 \\
15 & 5.3185 & 0.89443 & 1.0 & 0.47089 & 2314.0 & 2676.0 & 2717.0 & 2740.0 \\
16 & 5.32908 & 0.83066 & 1.0 & 0.31932 & 2066.0 & 2630.0 & 2714.0 & 2741.0 \\
17 & 5.3371 & 0.78583 & 1.0 & 0.29183 & 1898.0 & 2603.0 & 2725.0 & 2740.0 \\
18 & 5.37468 & 0.76307 & 1.0 & 0.2816 & 1815.0 & 2577.0 & 2719.0 & 2741.0 \\
19 & 5.4097 & 0.81851 & 1.0 & 0.29689 & 2033.0 & 2590.0 & 2714.0 & 2741.0 \\
20 & 5.42868 & 0.76486 & 1.0 & 0.25983 & 1823.0 & 2564.0 & 2721.0 & 2740.0 \\
21 & 5.44655 & 0.85087 & 1.0 & 0.35608 & 2147.0 & 2635.0 & 2714.0 & 2741.0 \\
22 & 5.44874 & 0.76409 & 1.0 & 0.26173 & 1803.0 & 2593.0 & 2722.0 & 2739.0 \\
23 & 5.4513 & 0.75756 & 1.0 & 0.27149 & 1795.0 & 2579.0 & 2723.0 & 2740.0 \\
24 & 5.45275 & 0.78294 & 1.0 & 0.25122 & 1902.0 & 2563.0 & 2724.0 & 2741.0 \\
25 & 5.4564 & 0.84368 & 1.0 & 0.38897 & 2099.0 & 2643.0 & 2714.0 & 2741.0 \\
26 & 5.45677 & 0.81909 & 1.0 & 0.27946 & 2034.0 & 2592.0 & 2716.0 & 2740.0 \\
27 & 5.46333 & 0.75744 & 1.0 & 0.2692 & 1797.0 & 2578.0 & 2723.0 & 2740.0 \\
28 & 5.46771 & 0.75683 & 1.0 & 0.27046 & 1795.0 & 2579.0 & 2723.0 & 2740.0 \\
29 & 5.49945 & 0.8628 & 1.0 & 0.37915 & 2192.0 & 2651.0 & 2716.0 & 2741.0 \\
30 & 5.50967 & 0.80716 & 1.0 & 0.31016 & 1997.0 & 2595.0 & 2718.0 & 2739.0 \\
31 & 5.52171 & 0.81355 & 1.0 & 0.28902 & 2018.0 & 2592.0 & 2716.0 & 2740.0
\end{tabular}




\begin{tabular}{l|llllllll}
32 & 5.56841 & 0.79819 & 1.0 & 0.29974 & 1938.0 & 2611.0 & 2713.0 & 2741.0 \\
33 & 5.6421 & 0.77203 & 1.0 & 0.26002 & 1852.0 & 2563.0 & 2720.0 & 2739.0 \\
34 & 5.64757 & 0.76215 & 1.0 & 0.24718 & 1815.0 & 2566.0 & 2715.0 & 2739.0 \\
35 & 5.75593 & 0.80746 & 1.0 & 0.25716 & 2016.0 & 2558.0 & 2711.0 & 2741.0 \\
36 & 5.7822 & 0.88595 & 1.0 & 0.45548 & 2277.0 & 2676.0 & 2715.0 & 2739.0 \\
37 & 5.79022 & 0.95151 & 1.0 & 0.79202 & 2535.0 & 2703.0 & 2726.0 & 2735.0 \\
38 & 5.79168 & 0.68573 & 1.0 & 0.18034 & 1530.0 & 2526.0 & 2719.0 & 2741.0 \\
39 & 5.79387 & 0.84458 & 1.0 & 0.38471 & 2113.0 & 2641.0 & 2714.0 & 2741.0 \\
40 & 5.80482 & 0.88397 & 1.0 & 0.44858 & 2269.0 & 2669.0 & 2713.0 & 2741.0 \\
41 & 5.87888 & 0.84451 & 1.0 & 0.38817 & 2122.0 & 2643.0 & 2714.0 & 2741.0 \\
42 & 5.88034 & 0.79773 & 1.0 & 0.30175 & 1942.0 & 2598.0 & 2721.0 & 2739.0 \\
43 & 5.92886 & 0.85721 & 1.0 & 0.39831 & 2171.0 & 2648.0 & 2714.0 & 2740.0 \\
44 & 5.93506 & 0.92876 & 1.0 & 0.59167 & 2458.0 & 2685.0 & 2716.0 & 2739.0 \\
45 & 5.94163 & 0.84747 & 1.0 & 0.39376 & 2126.0 & 2639.0 & 2715.0 & 2741.0 \\
46 & 6.02007 & 0.80473 & 1.0 & 0.26676 & 1990.0 & 2563.0 & 2712.0 & 2740.0 \\
47 & 6.03101 & 0.75188 & 1.0 & 0.27081 & 1788.0 & 2581.0 & 2715.0 & 2741.0 \\
48 & 6.06421 & 0.80356 & 1.0 & 0.26642 & 1986.0 & 2563.0 & 2711.0 & 2740.0 \\
49 & 6.06567 & 0.84628 & 1.0 & 0.37895 & 2132.0 & 2636.0 & 2715.0 & 2741.0 \\
50 & 6.07224 & 0.86948 & 1.0 & 0.39357 & 2212.0 & 2658.0 & 2712.0 & 2740.0
\end{tabular}

\begin{tabular}{|c|c|c|c|}
\hline line id & extractor & aggregator & distance \\
\hline 1 & pitch contour segmentation & octave abstraction & levenshtein circular $\max$ \\
\hline 2 & beat synchronous chroma cqt & statistical summarization & manhattan distance oti \\
\hline 3 & chroma cqt & statistical ${ }^{-}$summarization & manhattan ${ }^{-}$distance ${ }^{-}$oti \\
\hline 4 & beat synchronous chroma cens & statistical ${ }^{-}$summarization & manhattan ${ }^{-}$distance $^{-}$oti \\
\hline 5 & beat ${ }^{-}$synchronous ${ }^{-}$chroma ${ }^{-}$stft & statistical summarization & manhattan ${ }^{-}$distance ${ }^{-}$oti \\
\hline 6 & beat ${ }^{-}$synchronous ${ }^{-}$chroma ${ }^{-}$cens & diff stats 2 & manhattan distance ${ }^{-}$oti \\
\hline 7 & chroma stft - & statistical ${ }^{-}$summarization & manhattan ${ }^{-}$distance $^{-}$oti \\
\hline 8 & chroma ${ }^{-}$stft & statistical ${ }^{-}$summarization & cosine distānce oti ${ }^{-}$ \\
\hline 9 & pitch contour segmentation & octave abstraction & lcs circular mean \\
\hline 10 & chroma_stft - & diff_stats_ 1 & cosine_distance oti \\
\hline 11 & chroma ${ }^{-}$stft & basic_stats 1 & manhattan_distānce_oti \\
\hline 12 & chroma- ${ }^{-}$stft & basic ${ }^{-}$stats ${ }^{-} 2$ & manhattan distance-oti \\
\hline 13 & chroma ${ }^{-}$stft & diff stats $\overline{1}$ & manhattan ${ }^{-}$distance $^{-}$oti \\
\hline 14 & beat synchronous chroma cqt & $\operatorname{diff}^{-}$stats $^{-} 1$ & manhattan ${ }^{-}$distance $^{-}$oti \\
\hline 15 & beat ${ }^{-}$synchronous ${ }^{-}$chroma ${ }^{-}$stft & $\operatorname{diff}^{-}$stats $^{-} 1$ & manhattan ${ }^{-}$distance ${ }^{-}$oti \\
\hline 16 & beat ${ }^{-}$synchronous ${ }^{-}$chroma ${ }^{-}$stft & $\operatorname{diff}^{-}$stats $^{-} 1$ & chebyshev distance oti \\
\hline 17 & chroma stft & $\operatorname{diff}^{-}$stats $^{-} 1$ & euclidean distance oti \\
\hline 18 & chroma ${ }^{-}$stft & basic stats 1 & cosine distance ot $\overline{\mathrm{i}}$ \\
\hline 19 & beat synchronous chroma cqt & diff stats $\overline{1}$ & $\operatorname{cosine}^{-}$distance ${ }^{-}$oti \\
\hline 20 & beat ${ }^{-}$synchronous ${ }^{-}$chroma ${ }^{-}$cens & $\operatorname{diff}^{-}$stats $^{-} 2$ & $\operatorname{cosine}^{-}$distance ${ }^{-}$oti \\
\hline 21 & beat ${ }^{-}$synchronous ${ }^{-}$chroma ${ }^{-}$stft & statistical summarization & euclidean distañce oti \\
\hline 22 & chroma stft & diff stats $^{-}$ & manhattan distance oti \\
\hline 23 & chroma ${ }^{-}$stft & basic stats 2 & cosine distānce oti ${ }^{-}$ \\
\hline 24 & chroma ${ }^{-}$stft & statistical summarization & euclidean distañe oti \\
\hline 25 & beat synchronous chroma stft & basic stats 1 & manhattan distance oti \\
\hline 26 & beat ${ }^{-}$synchronous ${ }^{-}$chroma ${ }^{-}$cens & diff stats $\overline{1}$ & manhattan ${ }^{-}$distance ${ }^{-}$oti \\
\hline 27 & chroma stft - & basic stats 2 & euclidean $\overline{\text { distance }} \overline{\text { oti }}$ \\
\hline 28 & chroma ${ }^{-}$stft & basic ${ }^{-}$stats 1 & euclidean ${ }^{-}$distance ${ }^{-}$oti \\
\hline 29 & beat synchronous chroma stft & statistical summarization & cosine distance ot $\overline{\mathrm{i}}$ \\
\hline 30 & beat ${ }^{-}$synchronous ${ }^{-}$chroma $^{-}$cens & basic stats 2 & manhattan distance oti \\
\hline 31 & beat - synchronous - chroma - cqt & diff $\overline{\text { stats }} \overline{1}$ & euclidean $\overline{\text { distance }}$ oti \\
\hline 32 & beat synchronous ${ }^{-}$chroma ${ }^{-}$stft & basic stats 1 & chebyshev $^{-}$distance ${ }^{-}$oti \\
\hline 33 & beat ${ }^{-}$synchronous ${ }^{-}$chroma ${ }^{-}$cens & diff $\overline{\text { stats }} \overline{2}$ & euclidean distance oti \\
\hline 34 & chroma_cqt -- & $\operatorname{diff}^{-}$stats $^{-} 1$ & manhattan distance oti \\
\hline 35 & beat synchronous chroma cqt & statistical ${ }^{-}$summarization & cosine distāance oti ${ }^{-}$ \\
\hline 36 & beat ${ }^{-}$sychronous ${ }^{-}$chroma $^{-}$stft & diff stats 1 & $\operatorname{cosine}^{-}$distance ${ }^{-}$oti \\
\hline 37 & pitch contour segmentation & octave abstraction & lcs circular $\max$ \\
\hline 38 & tonnetz & diff stats 2 & manhattan distance \\
\hline 39 & beat synchronous chroma stft & basic stats 1 & euclidean distance oti \\
\hline 40 & beat ${ }^{-}$synchronous ${ }^{-}$chroma $^{-}$stft & diff $\overline{\text { stats }} \overline{1}$ & euclidean ${ }^{-}$distance ${ }^{-}$oti \\
\hline 41 & beat ${ }^{-}$synchronous ${ }^{-}$chroma $^{-}$stft & basic stats 2 & euclidean ${ }^{-}$distance ${ }^{-}$oti \\
\hline 42 & beat ${ }^{-}$synchronous ${ }^{-}$chroma ${ }^{-}$cqt & diff $\overline{\text { stats }} \overline{2}$ & manhattan distance oti \\
\hline 43 & beat ${ }^{-}$synchronous ${ }^{-}$chroma ${ }^{-}$stft & basic stats 2 & manhattan ${ }^{-}$distance ${ }^{-}$oti \\
\hline 44 & beat ${ }^{-}$synchronous ${ }^{-}$chroma ${ }^{-}$cens & statistical summarization & manhattan ${ }^{-}$distance - \\
\hline 45 & beat ${ }^{-}$synchronous ${ }^{-}$chroma ${ }^{-}$stft & basic stats 1 & cosine distance oti \\
\hline 46 & beat ${ }^{-}$synchronous ${ }^{-}$chroma ${ }^{-}$cens & diff $\overline{\text { stats }} \overline{1}$ & $\operatorname{cosine}^{-}$distance $^{-}$oti \\
\hline 47 & beat ${ }^{-}$synchronous ${ }^{-}$chroma ${ }^{-}$stft & statistical ${ }^{-}$summarization & chebyshev distance oti \\
\hline 48 & beat ${ }^{-}$synchronous ${ }^{-}$chroma ${ }^{-}$cens & diff stats $^{-} 1$ & euclidean distance oti \\
\hline 49 & beat ${ }^{-}$synchronous ${ }^{-}$chroma ${ }^{-}$stft & basic stats 2 & cosine distance ot $\overline{\mathrm{i}}$ \\
\hline 50 & beat_synchronous_chroma_stft & diff_stats_ $\overline{1}$ & chebysh̄ev_distance \\
\hline
\end{tabular}

\section{SHS9K}

\begin{tabular}{l|llllllll} 
line id & MR & MRR & MDR & MAP & Top 1 & Top 10 & Top 100 & Top 1000 \\
\hline 1 & 47.57883 & 0.40387 & 6.0 & 0.05102 & 3008.0 & 5188.0 & 8067.0 & 9265.0 \\
2 & 56.67866 & 0.33549 & 11.0 & 0.03429 & 2385.0 & 4527.0 & 7741.0 & 9273.0 \\
3 & 59.06128 & 0.31115 & 13.0 & 0.02701 & 2182.0 & 4334.0 & 7658.0 & 9272.0 \\
4 & 62.9398 & 0.23255 & 15.0 & 0.02777 & 1275.0 & 3990.0 & 7836.0 & 9240.0 \\
5 & 63.73713 & 0.22736 & 16.0 & 0.02813 & 1234.0 & 3914.0 & 7809.0 & 9238.0 \\
6 & 64.75038 & 0.22813 & 16.0 & 0.02696 & 1265.0 & 3888.0 & 7787.0 & 9237.0 \\
7 & 65.22755 & 0.22587 & 16.0 & 0.02669 & 1222.0 & 3899.0 & 7766.0 & 9236.0 \\
8 & 65.88273 & 0.18724 & 20.0 & 0.02269 & 927.0 & 3407.0 & 7716.0 & 9243.0 \\
9 & 66.06203 & 0.29483 & 16.0 & 0.02617 & 2038.0 & 4100.0 & 7485.0 & 9270.0 \\
10 & 66.90114 & 0.18625 & 20.0 & 0.02065 & 903.0 & 3429.0 & 7646.0 & 9252.0 \\
11 & 67.6457 & 0.20722 & 18.0 & 0.02519 & 1073.0 & 3676.0 & 7713.0 & 9234.0 \\
12 & 68.04534 & 0.20876 & 18.0 & 0.02622 & 1107.0 & 3640.0 & 7739.0 & 9235.0 \\
13 & 68.12858 & 0.21972 & 18.0 & 0.0274 & 1199.0 & 3768.0 & 7697.0 & 9229.0 \\
14 & 68.27838 & 0.18492 & 21.0 & 0.02028 & 906.0 & 3393.0 & 7621.0 & 9251.0 \\
15 & 68.3067 & 0.18812 & 22.0 & 0.0214 & 953.0 & 3321.0 & 7576.0 & 9251.0 \\
16 & 68.89296 & 0.21759 & 18.0 & 0.02539 & 1200.0 & 3719.0 & 7666.0 & 9245.0 \\
17 & 69.16412 & 0.18338 & 20.0 & 0.02168 & 874.0 & 3397.0 & 7628.0 & 9249.0 \\
18 & 69.28484 & 0.2129 & 18.0 & 0.02628 & 1147.0 & 3730.0 & 7667.0 & 9238.0 \\
19 & 69.45402 & 0.15611 & 25.0 & 0.02011 & 698.0 & 2982.0 & 7538.0 & 9259.0 \\
20 & 69.52305 & 0.21339 & 19.0 & 0.02691 & 1162.0 & 3616.0 & 7615.0 & 9247.0
\end{tabular}




\begin{tabular}{l|llllllll}
21 & 69.53328 & 0.18706 & 21.0 & 0.01899 & 948.0 & 3317.0 & 7562.0 & 9256.0 \\
22 & 69.66078 & 0.19729 & 20.0 & 0.02591 & 1026.0 & 3507.0 & 7668.0 & 9231.0 \\
23 & 69.76125 & 0.17147 & 23.0 & 0.02174 & 837.0 & 3097.0 & 7556.0 & 9248.0 \\
24 & 69.93129 & 0.19109 & 20.0 & 0.02507 & 970.0 & 3431.0 & 7672.0 & 9230.0 \\
25 & 70.12966 & 0.17313 & 23.0 & 0.02266 & 851.0 & 3149.0 & 7559.0 & 9246.0 \\
26 & 70.39436 & 0.20682 & 18.0 & 0.02627 & 1072.0 & 3685.0 & 7658.0 & 9221.0 \\
27 & 70.79098 & 0.19835 & 20.0 & 0.02294 & 998.0 & 3558.0 & 7648.0 & 9234.0 \\
28 & 70.81542 & 0.15369 & 26.0 & 0.01974 & 699.0 & 2908.0 & 7439.0 & 9259.0 \\
29 & 70.87476 & 0.21268 & 19.0 & 0.02572 & 1144.0 & 3618.0 & 7610.0 & 9226.0 \\
30 & 70.98385 & 0.1995 & 19.0 & 0.02247 & 1008.0 & 3557.0 & 7629.0 & 9232.0 \\
31 & 71.17166 & 0.20504 & 18.0 & 0.02179 & 1039.0 & 3688.0 & 7635.0 & 9226.0 \\
32 & 71.31381 & 0.20525 & 19.0 & 0.02436 & 1065.0 & 3657.0 & 7615.0 & 9231.0 \\
33 & 71.43409 & 0.21136 & 19.0 & 0.02583 & 1145.0 & 3657.0 & 7607.0 & 9230.0 \\
34 & 71.47297 & 0.21097 & 19.0 & 0.02581 & 1144.0 & 3648.0 & 7597.0 & 9232.0 \\
35 & 71.59574 & 0.2026 & 21.0 & 0.02538 & 1076.0 & 3517.0 & 7569.0 & 9239.0 \\
36 & 71.59757 & 0.14174 & 27.0 & 0.01769 & 600.0 & 2731.0 & 7448.0 & 9263.0 \\
37 & 71.9048 & 0.1973 & 20.0 & 0.02269 & 1000.0 & 3488.0 & 7614.0 & 9236.0 \\
38 & 73.0967 & 0.16326 & 24.0 & 0.01826 & 757.0 & 3069.0 & 7454.0 & 9248.0 \\
39 & 73.18824 & 0.19294 & 21.0 & 0.02518 & 973.0 & 3459.0 & 7593.0 & 9238.0 \\
40 & 73.33954 & 0.18893 & 22.0 & 0.02217 & 986.0 & 3325.0 & 7515.0 & 9244.0 \\
41 & 73.81402 & 0.16483 & 24.0 & 0.01828 & 776.0 & 3060.0 & 7456.0 & 9249.0 \\
42 & 73.83524 & 0.23068 & 18.0 & 0.02266 & 1337.0 & 3793.0 & 7500.0 & 9239.0 \\
43 & 73.92936 & 0.18771 & 22.0 & 0.02398 & 950.0 & 3302.0 & 7477.0 & 9238.0 \\
44 & 74.06774 & 0.17857 & 24.0 & 0.0204 & 891.0 & 3234.0 & 7454.0 & 9247.0 \\
45 & 74.10984 & 0.1756 & 24.0 & 0.02056 & 856.0 & 3196.0 & 7342.0 & 9260.0 \\
46 & 74.27299 & 0.22651 & 18.0 & 0.02227 & 1272.0 & 3780.0 & 7460.0 & 9237.0 \\
47 & 74.5434 & 0.19064 & 21.0 & 0.02292 & 983.0 & 3399.0 & 7549.0 & 9241.0 \\
48 & 74.60338 & 0.19101 & 21.0 & 0.02346 & 968.0 & 3411.0 & 7579.0 & 9231.0 \\
49 & 74.8586 & 0.18576 & 23.0 & 0.02367 & 949.0 & 3281.0 & 7448.0 & 9238.0 \\
50 & 75.26696 & 0.18119 & 22.0 & 0.02033 & 903.0 & 3334.0 & 7446.0 & 9241.0
\end{tabular}

\begin{tabular}{|c|c|c|c|}
\hline line id & extractor & aggregator & distance \\
\hline 1 & pitch_contour_segmentation & octave_abstraction & lcs_circular_mean \\
\hline 2 & pitch_contour_segmentation & octave_abstraction & levenshtein_circular_max \\
\hline 3 & pitch ${ }^{-}$contour-segmentation & octave-abstraction & lcs_circular_max ${ }^{-}$ \\
\hline 4 & beat_synchronous_chroma_cqt & diff_stāts_1 & mañhattan_distance_oti \\
\hline 5 & beat_synchronous_chroma-cqt & $\operatorname{diff}^{-}$stats $^{-} 1$ & cosine_distance_oti ${ }^{-}$ \\
\hline 6 & beat_synchronous_chroma-cqt & statistical ${ }^{-}$summarization & manhātan_distance_oti \\
\hline 7 & beat_synchronous_chroma-cqt & diff_stats 1 & euclidean_distance_oti \\
\hline 8 & beat synchronous chroma cqt & $\operatorname{diff}^{-}$stats $^{-} 2$ & manhattan distance oti \\
\hline 9 & pitch_contour_segmentation & octāe abstraction & levenshtein ${ }^{-}$circular ${ }^{-}$mean \\
\hline 10 & beat_synchronous_chroma_stft & diff_stāts_1 & manhattan_distance_oti \\
\hline 11 & beat_synchronous_chroma-cqt & basic stats 1 & manhattan_distance ${ }^{-}$oti \\
\hline 12 & beat ${ }^{-}$synchronous ${ }^{-}$chroma ${ }^{-}$cens & basic ${ }^{-}$stats ${ }^{-} 2$ & manhattan ${ }^{-}$distance ${ }^{-}$oti \\
\hline 13 & beat_synchronous_chroma_cens & statistical_summarization & manhattan_distance_oti \\
\hline 14 & beat ${ }^{-}$synchronous ${ }^{-}$chroma ${ }^{-}$stft & diff stats 1 & euclidean distance oti \\
\hline 15 & beat_synchronous_chroma-stft & statistical_summarization & 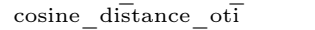 \\
\hline 16 & chroma_cqt - & statistical_summarization & manhattan_distānce_oti \\
\hline 17 & beat synchronous chroma stft & diff stats ${ }^{-} 1$ & cosine distance oti ${ }^{-}$ \\
\hline 18 & beat synchronous $^{-}$chroma ${ }^{-}$cqt & statistical ${ }^{-}$summarization & $\operatorname{cosine}^{-}$distance ${ }^{-}$oti \\
\hline 19 & beat synchronous ${ }^{-}$chroma ${ }^{-}$cqt & diff stats 2 & euclidean distance oti \\
\hline 20 & chroma cens & statistical ${ }^{-}$summarization & manhattan distance oti \\
\hline 21 & beat_sȳnchronous_chroma_stft & statistical_summarization & manhattan_distance_oti \\
\hline 22 & beat ${ }^{-}$synchronous ${ }^{-}$chroma ${ }^{-}$cens & basic stats 2 & cosine distance oti ${ }^{-}$ \\
\hline 23 & beat_synchronous ${ }^{-}$chroma ${ }^{-}$cens & diff_stats_ $\overline{2}$ & euclidean distance_oti \\
\hline 24 & beat ${ }^{-}$synchronous ${ }^{-}$chroma ${ }^{-}$cens & basic stats 2 & euclidean ${ }^{-}$distance ${ }^{-}$oti \\
\hline 25 & beat ${ }^{-}$synchronous ${ }^{-}$chroma ${ }^{-}$cens & diff $\overline{\text { stats }} \overline{2}$ & cosine distance ot $\overline{\mathrm{i}}$ \\
\hline 26 & beat_synchronous_chroma_cqt & basic_stats 1 & cosine $e^{-}$distance $e^{-}$oti \\
\hline 27 & beat_synchronous ${ }^{-}$chroma-cqt & basic_stats ${ }^{-} 2$ & euclidēean_distance_oti \\
\hline 28 & beat_synchronous ${ }^{-}$chroma_cqt & diff_stats_ $\overline{2}$ & cosine_distance_ot $\bar{i}$ \\
\hline 29 & beat_synchronous ${ }^{-}$chroma ${ }^{-}$cens & $\operatorname{diff}^{-}$stats $^{-} 1$ & manhātan_distānce_oti \\
\hline 30 & beat ${ }^{-}$synchronous ${ }^{-}$chroma ${ }^{-}$cqt & basic stats 2 & manhattan ${ }^{-}$distance ${ }^{-}$oti \\
\hline 31 & beat ${ }^{-}$ynchronous ${ }^{-}$chroma ${ }^{-}$cqt & statistical summarization 2 & monte carlo distance \\
\hline 32 & beat_synchronous_chroma-cqt & basic stats 1 & euclidean distance oti \\
\hline 33 & beat ${ }^{-}$synchronous ${ }^{-}$chroma ${ }^{-}$cens & diff $\overline{\text { stats }} \overline{1}$ & cosine distance ot $\bar{i}$ \\
\hline 34 & beat ${ }^{-}$synchronous ${ }^{-}$chroma ${ }^{-}$cens & $\operatorname{diff}^{-}$stats $^{-1}$ & euclidean distance oti \\
\hline 35 & chroma_cqt $-{ }^{-}-$ & statistical ${ }^{-}$summarization & cosine_distance_ot $\overline{\mathrm{i}}$ \\
\hline 36 & beat synchronous chroma stft & diff stats $^{-} 2$ & manhātan distance oti \\
\hline 37 & beat_synchronous_chroma-cqt & basic_stats_z 2 & cosine_distance_oti ${ }^{-}$ \\
\hline 38 & beat ${ }^{-}$synchronous ${ }^{-}$chroma $^{-}{ }^{-}$stft & basic_stats_1 & manhattan_distānce_oti \\
\hline 39 & chroma_cens $\quad--\quad-$ & basic $^{-}$stats $^{-} 2$ & manhattan ${ }^{-}$distance ${ }^{-}$oti \\
\hline 40 & chroma ${ }^{-}$cqt & $\operatorname{diff} \overline{\text { stats }} \overline{1}$ & manhattan ${ }^{-}$distance ${ }^{-}$oti \\
\hline 41 & beat synchronous chroma stft & basic stats 1 & euclidean $\overline{\text { distance }}$ oti \\
\hline 42 & beat ${ }^{-}$synchronous ${ }^{-}$chroma ${ }^{-}$cens & vector quantization & manhattan distance \\
\hline 43 & chroma cqt - & diff stats 1 & cosine distance oti \\
\hline 44 & chroma ${ }^{-}$cqt & basic stats 2 & manhātan distance oti \\
\hline 45 & chroma-stft & statistical summarization & cosine_distance_oti ${ }^{-}$ \\
\hline 46 & beat synchronous chroma cqt & vector quantization & manhātan distānce \\
\hline 47 & chroma cqt - - & basic stats 1 & manhattan distance oti \\
\hline 48 & beat synchronous chroma cens & basic $^{-}$stats $^{-} 1$ & manhattan ${ }^{-}$distance ${ }^{-}$oti \\
\hline 49 & chroma_cqt $-{ }^{-}-$ & basic_stats_-1 & cosine distance oti ${ }^{-}$ \\
\hline 50 & beat_synchronous_chroma_cqt & diff_stats_ $\overline{1}$ & chebysh̄ev_distance_oti \\
\hline
\end{tabular}




\section{Appendix C}

\section{Cover Song Identification: filtered results per dataset}

\section{Covers80}

\begin{tabular}{|c|c|c|c|c|c|c|c|c|c|c|}
\hline line id & \multicolumn{2}{|c|}{ original position } & \multicolumn{3}{|l|}{ extractor } & \multicolumn{3}{|l|}{ aggregator } & \multicolumn{2}{|c|}{ distance } \\
\hline 1 & \multicolumn{2}{|c|}{1} & \multirow{2}{*}{\multicolumn{3}{|c|}{ chroma_stft }} & & \multicolumn{2}{|c|}{ cosine distance oti } \\
\hline 2 & \multicolumn{2}{|l|}{2} & \multirow{2}{*}{\multicolumn{3}{|c|}{$\begin{array}{l}\text { chroma_stft } \\
\text { beat synchronous chroma cens }\end{array}$}} & basic stats & & & \multicolumn{2}{|c|}{ cosine $^{-}$distance ${ }^{-}$oti } \\
\hline 3 & \multicolumn{2}{|l|}{3} & & & & \multicolumn{3}{|l|}{$\begin{array}{l}\text { basic_stats_- } 1 \\
\text { basic }- \text { stats }-2\end{array}$} & \multicolumn{2}{|c|}{ manhattan_distance_ot } \\
\hline 4 & \multirow{2}{*}{\multicolumn{2}{|c|}{48}} & \multicolumn{3}{|c|}{ chroma_cqt - } & \multicolumn{3}{|c|}{ vector_quantization_default } & \multirow{2}{*}{\multicolumn{2}{|c|}{$\begin{array}{l}\text { cosine distance } \\
\text { manhattan distance }\end{array}$}} \\
\hline 5 & & & \multicolumn{3}{|l|}{ tonnetz ${ }^{-}-10$} & \multicolumn{3}{|c|}{ diff_stats_ 1} & & \\
\hline 6 & 125 & & \multicolumn{3}{|c|}{ beat_synchronous_chroma_cens } & \multirow{2}{*}{\multicolumn{3}{|c|}{ statistical_summarization ${ }^{2}$}} & \multicolumn{2}{|c|}{ monte carlo distance } \\
\hline 7 & \multicolumn{2}{|l|}{176} & \multirow{2}{*}{\multicolumn{3}{|c|}{$\begin{array}{l}\text { pitch_contour_segmentation } \\
\text { spectral_contrast }\end{array}$}} & \multirow{2}{*}{\multicolumn{3}{|c|}{$\begin{array}{l}\text { octave abstraction } \\
\text { statistical summarization } 2\end{array}$}} & les cir & lar mean \\
\hline 8 & 202 & & & & & & & & earth & overs distance \\
\hline 9 & 238 & & rhythm histog & & & bypass $\quad-$ & & & $\operatorname{cosin} \mathrm{e}^{-}$ & istance \\
\hline 10 & 312 & & $\operatorname{mfcc}-$ & & & statistical su & mariza & n 2 & monte & arlo distance \\
\hline 11 & 320 & & chroma stft & & & gaussian $\overline{\mathrm{mix}}$ & are mo & 1 default & manhat & an distance \\
\hline 12 & 387 & & zero crōssing & & & basic stāts & & & chebysl & $\mathrm{v}$ - distance \\
\hline 13 & 404 & & temporal_rhyt & 1_histo & $\mathrm{ram}$ & bypass $\overline{-}-$ & & & cosine & istance \\
\hline 14 & 413 & & pitch contour & ément & ion & markov chai & & & circular & manhattan \\
\hline 15 & 422 & & melspectrogran & & & basic_stats_ & & & cosine & istance \\
\hline 16 & 465 & & $\mathrm{rms}$ & & & diff_stats $\overline{1}$ & & & cosine & istance \\
\hline line id & MR & MRR & & MDR & MAP & & Top 1 & Top 10 & Top 100 & Top 1000 \\
\hline 1 & 41.575 & 0.193 & 9129898294591 & 31.0 & 0.1935 & 29898294591 & 21.0 & 46.0 & 142.0 & 160.0 \\
\hline 2 & 41.99375 & 0.192 & 5713147354658 & 32.0 & 0.1920 & 13147354658 & 20.0 & 44.0 & 142.0 & 160.0 \\
\hline 3 & 42.3625 & 0.205 & 799332525752 & 27.5 & 0.2059 & 9332525752 & 20.0 & 56.0 & 138.0 & 160.0 \\
\hline 4 & 46.7375 & 0.205 & 312990778413 & 26.5 & 0.2059 & 2990778413 & 22.0 & 51.0 & 129.0 & 160.0 \\
\hline 5 & 47.725 & 0.201 & 9125937259355 & 34.0 & 0.2014 & 25937259355 & 22.0 & 50.0 & 136.0 & 160.0 \\
\hline 6 & 49.675 & 0.235 & 5776927301804 & 30.5 & 0.2359 & 769273018 & 29.0 & 48.0 & 129.0 & 160.0 \\
\hline 7 & 52.2375 & 0.173 & 6189194579906 & 39.0 & 0.1734 & 89194579903 & 18.0 & 46.0 & 125.0 & 160.0 \\
\hline 8 & 53.55625 & 0.069 & 2528652541288 & 46.5 & 0.0693 & 28652541288 & 2.0 & 25.0 & 137.0 & 160.0 \\
\hline 9 & 55.29375 & 0.165 & 8027920050967 & 37.0 & 0.1654 & 27920050964 & 15.0 & 44.0 & 119.0 & 160.0 \\
\hline 10 & 59.58125 & 0.078 & 267105659147 & 53.5 & 0.0780 & 71056591469 & 3.0 & 23.0 & 128.0 & 160.0 \\
\hline 11 & 59.93125 & 0.084 & 995286521828 & 49.5 & 0.0842 & 52865218279 & 6.0 & 24.0 & 124.0 & 160.0 \\
\hline 12 & 64.25 & 0.041 & 5368472571946 & 58.5 & 0.0418 & 368472571945 & 0.0 & 18.0 & 121.0 & 160.0 \\
\hline 13 & 65.40625 & 0.081 & 231961881101 & 56.5 & 0.0812 & 31961881101 & 5.0 & 28.0 & 111.0 & 160.0 \\
\hline 14 & 66.425 & 0.088 & 0042156866501 & 61.5 & 0.0885 & 42156866501 & 7.0 & 26.0 & 114.0 & 160.0 \\
\hline 15 & 66.79375 & 0.080 & 2600860261674 & 58.0 & 0.0803 & 00860261674 & 3.0 & 30.0 & 110.0 & 160.0 \\
\hline 16 & 69.1 & 0.051 & 27264847166345 & 64.5 & 0.0519 & 264847166345 & 2.0 & 20.0 & 116.0 & 160.0 \\
\hline
\end{tabular}




\section{YouTubeCovers}

\begin{tabular}{|c|c|c|c|c|c|c|c|c|}
\hline line id & original position & \multicolumn{2}{|l|}{ extractor } & \multicolumn{2}{|l|}{ aggregator } & \multicolumn{3}{|c|}{ distance } \\
\hline 1 & 1 & \multirow{2}{*}{\multicolumn{2}{|c|}{$\begin{array}{l}\text { pitch_contour_segmentation } \\
\text { pitch }\end{array}$}} & \multicolumn{2}{|l|}{ octave_abstraction } & \multirow{2}{*}{\multicolumn{3}{|c|}{ lcs_circular_mean }} \\
\hline 2 & 3 & & & \multicolumn{2}{|l|}{ markov_chain } & & & circular_manhattan \\
\hline 3 & 4 & \multirow{2}{*}{\multicolumn{2}{|c|}{$\begin{array}{l}\text { pitch_contour_segmentation } \\
\text { chroma stft }\end{array}$}} & \multicolumn{2}{|l|}{ markov_chain } & \multirow{2}{*}{\multicolumn{3}{|c|}{ circular cosine }} \\
\hline 4 & 5 & & & statistical_summarizatio & & & & \\
\hline 5 & 8 & \multicolumn{2}{|l|}{ chroma_stft } & \multicolumn{2}{|l|}{ diff_stats ${ }^{-} 1$} & \multicolumn{3}{|c|}{ cosine $^{-}$distance ${ }^{-}$oti } \\
\hline 6 & 53 & \multicolumn{2}{|l|}{ rhythm_histogram } & \multicolumn{2}{|l|}{ bypāss - } & \multicolumn{3}{|c|}{$\operatorname{cosine}^{-}$distance $^{-}$} \\
\hline 7 & 55 & \multicolumn{2}{|c|}{ temporā_rhythm_histogram } & \multicolumn{2}{|l|}{ bypass } & \multicolumn{3}{|c|}{ cosine_distance } \\
\hline 8 & 119 & \multicolumn{2}{|c|}{ beat_synchronous_chroma_stft } & \multicolumn{2}{|l|}{ vector_quantization } & \multicolumn{3}{|c|}{ cosine $e^{-}$distance } \\
\hline 9 & 128 & \multicolumn{2}{|c|}{$\begin{array}{l}\text { chroma_cens } \\
\text { tonnetz }\end{array}$} & \multicolumn{2}{|c|}{ statistical_summarization_2 } & \multicolumn{3}{|c|}{ monte_carlo_distance } \\
\hline 10 & 160 & \multicolumn{2}{|l|}{ tonnetz ${ }^{-}$} & diff_stats_-1 & & manha & an_ēistan & \\
\hline 11 & 272 & $\mathrm{mfcc}$ & & diff_stats_2 $^{-}$s & & manha & an_distan & \\
\hline 12 & 338 & spectral_contrast & & vectōr_quāntization & & manha & an_distan & \\
\hline 13 & 362 & beat_synchronous_chrom & cqt & gaussian_mixture_mode & default & manha & an_distan & \\
\hline 14 & 376 & zero_crossing_ratē & & vector_quantization & & cosine & istānce & \\
\hline 15 & 416 & melspectrogram & & diff_stats_1 & & cosine & istance & \\
\hline 16 & 472 & $\mathrm{rms}$ & & diff $_{-}^{-}$stats $_{-}^{-} 1$ & & manha & an_distan & \\
\hline line id & MR & MRR & MDR & MAP & Top 1 & Top 10 & Top 100 & Top 1000 \\
\hline 1 & 7.9714285714285715 & 0.694195950993107 & 1.0 & 0.3611413162288402 & 219.0 & 296.0 & 345.0 & 350.0 \\
\hline 2 & 14.1 & 0.4343951990693004 & 3.5 & 0.19221026946238964 & 110.0 & 231.0 & 343.0 & 350.0 \\
\hline 3 & 14.137142857142857 & 0.4084621091100449 & 5.0 & 0.18049755463666442 & 103.0 & 233.0 & 344.0 & 350.0 \\
\hline 4 & 14.711428571428572 & 0.3802132780559847 & 5.0 & 0.15415423808967568 & 85.0 & 227.0 & 344.0 & 350.0 \\
\hline 5 & 16.462857142857143 & 0.3760507761162201 & 5.5 & 0.15219416288390122 & 86.0 & 223.0 & 343.0 & 350.0 \\
\hline 6 & 21.94 & 0.4074395921903713 & 5.0 & 0.1756347720915198 & 106.0 & 230.0 & 328.0 & 350.0 \\
\hline 7 & 22.042857142857144 & 0.3124044938703768 & 9.0 & 0.10795068713144258 & 72.0 & 177.0 & 338.0 & 350.0 \\
\hline 8 & 25.088571428571427 & 0.3539353998080258 & 7.0 & 0.14241939491512148 & 88.0 & 197.0 & 329.0 & 350.0 \\
\hline 9 & 25.47714285714286 & 0.42128384147654696 & 5.0 & 0.18007922766611834 & 116.0 & 210.0 & 333.0 & 350.0 \\
\hline 10 & 26.597142857142856 & 0.4081166554557799 & 7.0 & 0.1567165099024276 & 112.0 & 197.0 & 322.0 & 350.0 \\
\hline 11 & 29.605714285714285 & 0.20063578123307974 & 16.5 & 0.07573634001039234 & 37.0 & 136.0 & 331.0 & 350.0 \\
\hline 12 & 34.02857142857143 & 0.13802584574304955 & 20.0 & 0.0533298500932552 & 16.0 & 113.0 & 329.0 & 350.0 \\
\hline 13 & 36.505714285714284 & 0.1455764224149135 & 24.0 & 0.05635003857273054 & 21.0 & 108.0 & 318.0 & 350.0 \\
\hline 14 & 38.24285714285714 & 0.129752207125118 & 22.5 & 0.04984524115007655 & 19.0 & 99.0 & 324.0 & 350.0 \\
\hline 15 & 40.30285714285714 & 0.16630280449839283 & 26.5 & 0.05872635321775377 & 28.0 & 113.0 & 313.0 & 350.0 \\
\hline 16 & 42.22571428571428 & 0.0967731038801955 & 30.0 & 0.043335187664363124 & 8.0 & 81.0 & 319.0 & 350.0 \\
\hline
\end{tabular}

\section{Covers1000}

\begin{tabular}{|c|c|c|c|c|c|c|c|c|c|c|}
\hline line id & \multicolumn{2}{|c|}{ original position } & \multicolumn{3}{|l|}{ extractor } & \multicolumn{3}{|l|}{ aggregator } & \multicolumn{2}{|c|}{ distance } \\
\hline 1 & \multirow{2}{*}{\multicolumn{2}{|c|}{$\begin{array}{l}1 \\
2\end{array}$}} & \multirow{2}{*}{\multicolumn{3}{|c|}{$\begin{array}{l}\text { pitch_contour_segmentation } \\
\text { beat_synchronous chroma stft }\end{array}$}} & \multicolumn{3}{|c|}{ octave_abstraction } & \multicolumn{2}{|c|}{ lcs_circular_mean } \\
\hline 2 & & & & & & diff_stäts_1 & & & \multirow{2}{*}{\multicolumn{2}{|c|}{$\begin{array}{l}\text { cosine_distance_oti } \\
\text { manhattan distance oti }\end{array}$}} \\
\hline 3 & \multicolumn{2}{|l|}{3} & \multicolumn{3}{|c|}{ beat synchronous chroma-cqt } & \multicolumn{3}{|l|}{$\begin{array}{l}\text { diff } \\
\text { diff }^{-} \text {stats }\end{array}$} & & \\
\hline 4 & \multicolumn{2}{|l|}{4} & \multicolumn{3}{|c|}{ rhythm_histogram } & \multicolumn{3}{|c|}{ bypass } & \multicolumn{2}{|c|}{ cosine_distance } \\
\hline 5 & \multicolumn{2}{|l|}{5} & \multicolumn{3}{|c|}{ beat_synchronous_chroma_stft } & \multicolumn{3}{|c|}{ basic_stats_1 } & \multicolumn{2}{|c|}{ cosine_distance_oti } \\
\hline 6 & \multirow{2}{*}{\multicolumn{2}{|c|}{$\begin{array}{l}77 \\
79\end{array}$}} & \multirow{2}{*}{\multicolumn{3}{|c|}{ beat_synchronous_chroma_stft }} & \multicolumn{3}{|c|}{ vector_quantization } & \multicolumn{2}{|c|}{ manhattan_distance } \\
\hline 7 & & & \multirow{2}{*}{\multicolumn{3}{|c|}{$\begin{array}{l}\text { tonnètz } \\
\text { beat synchronous chroma cens }\end{array}$}} & \multirow{2}{*}{\multicolumn{3}{|c|}{ diff_stats 1 - }} & manhe & $\tan ^{-}$distance \\
\hline 8 & \multicolumn{2}{|l|}{$\begin{array}{l}79 \\
120\end{array}$} & & & & & & & monte & carlo_distance \\
\hline 9 & 153 & & pitch contou & segmen & tion & markov cha & & & circule & manhattan \\
\hline 10 & 192 & & $\mathrm{mfcc}^{-}$ & & & diff stats 2 & & & manhe & Ean distance \\
\hline 11 & 237 & & temporal rhy & $\mathrm{m}$ hist & rram & bypāss - & & & cosine & distance \\
\hline 12 & 300 & & spectral cont & & & diff stats 1 & & & manhi & tan distance \\
\hline 13 & 358 & & melspectrogra & & & $\operatorname{diff}^{-}$stats ${ }^{-}$ & & & cosine & distance \\
\hline 14 & 362 & & zero_crossing & ate & & vector quār & zation & & manhe & tan distance \\
\hline 15 & 397 & & beat ${ }^{-}$synchro & us chr & na cqt & gaussian m & ure $\mathrm{m}$ & el default & manh & $\tan ^{-}$distance \\
\hline 16 & 457 & & $\mathrm{rms}-\mathrm{ran}$ & & & diff stats 1 & & & cosine & distance \\
\hline line id & MR & MRR & & MDR & MAP & & Top 1 & Top 10 & Top 100 & Top 1000 \\
\hline 1 & 144.041 & 0.2573 & 452650966263 & 35.0 & 0.19159 & 9029548544 & 201.0 & 366.0 & 636.0 & 1000.0 \\
\hline 2 & 175.546 & 0.0984 & 970693153218 & 74.5 & 0.06976 & 4754591697 & 52.0 & 188.0 & 548.0 & 1000.0 \\
\hline 3 & 180.808 & 0.1356 & 338863815536 & 75.0 & 0.09752 & 9108235948 & 77.0 & 249.0 & 551.0 & 1000.0 \\
\hline 4 & 181.48 & 0.1593 & 515394861295 & 72.0 & 0.11552 & 0926621667 & 103.0 & 261.0 & 558.0 & 1000.0 \\
\hline 5 & 183.802 & 0.0861 & 445614781481 & 95.0 & 0.06054 & 8711264856 & 48.0 & 155.0 & 515.0 & 1000.0 \\
\hline 6 & 207.289 & 0.1508 & 76412071519 & 90.5 & 0.11276 & 4830950303 & 98.0 & 254.0 & 521.0 & 1000.0 \\
\hline 7 & 208.285 & 0.1363 & 161157172055 & 93.0 & 0.09963 & 9747778567 & 79.0 & 243.0 & 518.0 & 1000.0 \\
\hline 8 & 220.384 & 0.1709 & 22228089954 & 95.0 & 0.12686 & 6072808287 & 115.0 & 274.0 & 511.0 & 1000.0 \\
\hline 9 & 226.688 & 0.0907 & 560197206973 & 128.5 & 0.06050 & 1697078355 & 49.0 & 168.0 & 446.0 & 1000.0 \\
\hline 10 & 232.189 & 0.0425 & 757122885025 & 135.5 & 0.03086 & 77871570693 & 15.0 & 78.0 & 417.0 & 1000.0 \\
\hline 11 & 242.603 & 0.0923 & 102738320418 & 134.0 & 0.06269 & 859675943 & 53.0 & 162.0 & 445.0 & 1000.0 \\
\hline 12 & 263.229 & 0.0422 & 299953152908 & 185.5 & 0.02910 & 44291253885 & 21.0 & 72.0 & 325.0 & 1000.0 \\
\hline 13 & 284.52 & 0.0312 & 670487011743 & 199.5 & 0.02267 & 7253435587 & 7.0 & 72.0 & 324.0 & 1000.0 \\
\hline 14 & 285.036 & 0.0289 & 2807159794096 & 220.5 & 0.01960 & 0393456891 & 8.0 & 58.0 & 298.0 & 1000.0 \\
\hline 15 & 296.021 & 0.0335 & 064205683607 & 226.5 & 0.02246 & 20004721118 & 13.0 & 64.0 & 315.0 & 1000.0 \\
\hline 16 & 314.284 & 0.0201 & 7402933182406 & 227.0 & 0.01333 & 11728622089 & 5.0 & 24.0 & 274.0 & 1000.0 \\
\hline
\end{tabular}




\section{Mazurkas}

\begin{tabular}{|c|c|c|c|c|c|c|c|c|}
\hline line id & original position & \multicolumn{2}{|l|}{ extractor } & \multicolumn{2}{|l|}{ aggregator } & \multicolumn{3}{|c|}{ distance } \\
\hline 2 & 2 & \multicolumn{2}{|c|}{$\begin{array}{l}\text { pitch_contour_segmentation } \\
\text { beat_synchronous chroma cqt }\end{array}$} & \multicolumn{2}{|c|}{ statistical summarization } & \multicolumn{3}{|c|}{ manhattan distance oti } \\
\hline 3 & 6 & \multicolumn{2}{|c|}{ beat synchronous chroma-cens } & \multicolumn{2}{|c|}{ diff stats $^{-} 2$} & \multirow{2}{*}{\multicolumn{3}{|c|}{ manhattan - distance_oti }} \\
\hline 4 & 8 & \multicolumn{2}{|c|}{ chroma_stft -} & \multicolumn{2}{|c|}{ statistical ${ }^{-}$summarization } & & & \\
\hline 6 & 75 & $\begin{array}{l}\text { tonnetz } \\
\text { chroma cens }\end{array}$ & & \multicolumn{2}{|c|}{$\begin{array}{l}\text { diff_stats_2 } \\
\text { gaussian mixture model default }\end{array}$} & \multicolumn{3}{|c|}{ manhattan - distance } \\
\hline 7 & 140 & \multicolumn{2}{|c|}{ beat_synchronous_chroma_cqt } & \multicolumn{2}{|c|}{ vector_quantization } & \multicolumn{3}{|c|}{ chebyshev - distance } \\
\hline 8 & 186 & \multirow{2}{*}{\multicolumn{2}{|c|}{ pitch_contour_segmentation }} & \multicolumn{2}{|c|}{ markov_chain } & \multicolumn{3}{|c|}{ circular manhattan } \\
\hline 9 & 213 & & & statistical summarizat & $\begin{array}{l}\text { statistical_summarization_ }{ }^{2} \\
\text { vector quantization }\end{array}$ & \multicolumn{3}{|c|}{ monte_carlo_distance } \\
\hline 13 & 400 & zero crossing rate & & vector quāntization & & $\operatorname{cosin}$ & distance & \\
\hline 14 & 402 & temporal rhythm histog & & bypass & & manh & ttan dist & \\
\hline 15 & 464 & $\mathrm{rms}-\mathrm{C}^{-}-$ & & vector quantization & & manh & $\operatorname{ttan}^{-}$dist & \\
\hline 16 & 465 & statistical spectrum des & iptor & bypass & & manh & $\operatorname{ttan}^{-}$dist & \\
\hline line id & MR & MRR & MDR & MAP & Top 1 & Top 10 & Top 100 & Top 1000 \\
\hline 1 & 4.157241882524626 & 0.9577383028307782 & 1.0 & 0.8228559773164656 & 2561.0 & 2714.0 & 2728.0 & 2739.0 \\
\hline 2 & 4.4647938708500545 & 0.8734719826453456 & 1.0 & 0.3362310327306724 & 2251.0 & 2638.0 & 2722.0 & 2740.0 \\
\hline 8 & 7.771251368113827 & 0.8426504893474391 & 1.0 & 0.41661000115687447 & 2131.0 & 2614.0 & 2710.0 & 2737.0 \\
\hline 9 & 8.495074790222546 & 0.9360628322856959 & 1.0 & 0.6720956091182665 & 2474.0 & 2694.0 & 2714.0 & 2734.0 \\
\hline 10 & 8.673112002918643 & 0.6096829700733601 & 2.0 & 0.1548019689852552 & 1292.0 & 2390.0 & 2702.0 & 2740.0 \\
\hline 11 & 10.503101058008026 & 0.573401584809108 & 2.0 & 0.1334111314624724 & 1190.0 & 2314.0 & 2696.0 & 2741.0 \\
\hline 12 & 11.142283838015324 & 0.4944919319993829 & 3.0 & 0.12090178522543168 & 984.0 & 2161.0 & 2699.0 & 2741.0 \\
\hline 13 & 20.43962057643196 & 0.3767142478440718 & 5.0 & 0.07679918614424991 & 666.0 & 1832.0 & 2622.0 & 2741.0 \\
\hline 14 & 20.968624589565852 & 0.3679612676368265 & 6.0 & 0.06942143636338607 & 670.0 & 1716.0 & 2638.0 & 2740.0 \\
\hline 15 & 33.84312294782926 & 0.20316694543216524 & 14.0 & 0.03987678159635122 & 266.0 & 1176.0 & 2546.0 & 2738.0 \\
\hline 16 & 33.91499452754469 & 0.17980510863409532 & 16.0 & 0.03634632496485249 & 223.0 & 1092.0 & 2539.0 & 2740.0 \\
\hline
\end{tabular}

\section{SHS9K}

\begin{tabular}{|c|c|c|c|c|c|c|c|c|}
\hline line id & original position & \multicolumn{2}{|l|}{ extractor } & \multicolumn{2}{|l|}{ aggregator } & \multicolumn{3}{|c|}{ distance } \\
\hline 1 & 1 & \multicolumn{2}{|c|}{ pitch_contour_segmentation } & \multicolumn{2}{|l|}{ octave_abstraction } & \multicolumn{3}{|c|}{ lcs_circular_mean } \\
\hline 2 & 4 & \multirow{2}{*}{\multicolumn{2}{|c|}{$\begin{array}{l}\text { beat_synchronous_chroma_cqt } \\
\text { beat }- \text { synchronous } \\
\text { chroma }\end{array}$}} & \multicolumn{2}{|l|}{ diff_stats_1 } & \multirow{2}{*}{\multicolumn{3}{|c|}{ manhattan_distance_oti }} \\
\hline 3 & 5 & & & diff $^{-}$stats $^{-} 1$ & & cosine_distance_oti & & \\
\hline 4 & 6 & \multicolumn{2}{|c|}{ beat ${ }^{-}$synchronous ${ }^{-}$chroma ${ }^{-}$cqt } & $\begin{array}{l}\text { diff } \text { stats }^{1} \\
\text { statistical } \\
\text { summarization }\end{array}$ & & \multirow{2}{*}{\multicolumn{3}{|c|}{$\begin{array}{l}\text { manhattan distance_oti } \\
\text { monte carlo distance }\end{array}$}} \\
\hline 5 & 31 & \multicolumn{2}{|c|}{ beat_synchronous_chroma_cqt } & \multicolumn{2}{|c|}{ statistical ${ }^{-}$summarization 2} & & & \\
\hline 6 & 42 & \multicolumn{2}{|c|}{ beat_synchronous_chroma_cens } & \multicolumn{2}{|c|}{ vector_quantization } & \multicolumn{3}{|c|}{ manhattan_distance } \\
\hline 7 & 56 & \multirow{2}{*}{\multicolumn{2}{|c|}{$\begin{array}{l}\text { tonnētz } \\
\text { pitch contour segmentation }\end{array}$}} & \multicolumn{2}{|l|}{ vector-quantization } & \multicolumn{3}{|c|}{ manhattan distance } \\
\hline 8 & 128 & & & \multicolumn{2}{|l|}{ markov_chain } & \multicolumn{3}{|c|}{ circular_cosine } \\
\hline 9 & 229 & \multicolumn{2}{|l|}{$\begin{array}{l}\text { pitch_contour_segmentation } \\
\text { rhythm histogram }\end{array}$} & \multirow{2}{*}{\multicolumn{2}{|c|}{$\begin{array}{l}\text { bypass } \\
\text { diff stats } 2\end{array}$}} & \multicolumn{3}{|c|}{ cosine $\overline{\text { distance }}$} \\
\hline 10 & 257 & \multicolumn{2}{|l|}{ mfcc } & & & manhat & an distan & \\
\hline 11 & 272 & \multicolumn{2}{|l|}{ temporal rhythm histogram } & bypāss - & & manhat & an distan & \\
\hline 12 & 309 & spectral contrast & & vector quantization & & manhat & an distan & \\
\hline 13 & 335 & melspectrogram & & diff stats 1 & & manhat & n distan & \\
\hline 14 & 376 & chroma cqt & & gaussian mixture mode & default & manhat & $\mathrm{n}^{-}$distan & \\
\hline 15 & 409 & $\mathrm{rms}-\mathrm{H}^{\circ}$ & & diff stats 1 & & cosine & istance & \\
\hline 16 & 427 & zero_crossing_rate & & vectoror_quāntization & & manhat & an_distan & \\
\hline line id & MR & MRR & MDR & MAP & Top 1 & Top 10 & Top 100 & Top 1000 \\
\hline 1 & 47.57882834374327 & 0.40387101631290323 & 6.0 & 0.05102021990517946 & 3008.0 & 5188.0 & 8067.0 & 9265.0 \\
\hline 2 & 62.9398018522507 & 0.2325521006048516 & 15.0 & 0.02777233736708638 & 1275.0 & 3990.0 & 7836.0 & 9240.0 \\
\hline 3 & 63.73713116519492 & 0.22735912747253192 & 16.0 & 0.028126124449084335 & 1234.0 & 3914.0 & 7809.0 & 9238.0 \\
\hline 4 & 64.75037691147965 & 0.22813444987030118 & 16.0 & 0.026963736995958533 & 1265.0 & 3888.0 & 7787.0 & 9237.0 \\
\hline 5 & 71.17165625673056 & 0.20504453845846535 & 18.0 & 0.02178863340674668 & 1039.0 & 3688.0 & 7635.0 & 9226.0 \\
\hline 6 & 73.83523583889726 & 0.23068080412545208 & 18.0 & 0.022657830647124757 & 1337.0 & 3793.0 & 7500.0 & 9239.0 \\
\hline 7 & 75.95670902433771 & 0.24926315829542536 & 16.0 & 0.02451538944433362 & 1465.0 & 4018.0 & 7544.0 & 9222.0 \\
\hline 8 & 85.83469739392635 & 0.1384805174003356 & 33.0 & 0.015509588173725964 & 626.0 & 2576.0 & 6967.0 & 9248.0 \\
\hline 9 & 98.16121042429464 & 0.1535710358770886 & 31.0 & 0.01805656410673726 & 760.0 & 2726.0 & 6975.0 & 9202.0 \\
\hline 10 & 101.20019384018953 & 0.0831217277189994 & 51.0 & 0.01172026226874542 & 293.0 & 1624.0 & 6427.0 & 9265.0 \\
\hline 11 & 103.27019168640965 & 0.10511802347145303 & 46.0 & 0.01206894599791695 & 430.0 & 1936.0 & 6508.0 & 9240.0 \\
\hline 12 & 110.44841697178548 & 0.07411622238829096 & 58.0 & 0.011169863639562658 & 246.0 & 1452.0 & 6172.0 & 9253.0 \\
\hline 13 & 115.98847727762222 & 0.06701057859899788 & 68.0 & 0.009699406108035945 & 233.0 & 1251.0 & 5717.0 & 9270.0 \\
\hline 14 & 120.48212362696532 & 0.05083783264528273 & 74.0 & 0.009386275702521166 & 138.0 & 961.0 & 5571.0 & 9274.0 \\
\hline 15 & 126.26093043290976 & 0.055635538594680664 & 72.0 & 0.009932140745722282 & 175.0 & 993.0 & 5671.0 & 9238.0 \\
\hline 16 & 128.12416541029506 & 0.05927839890991961 & 71.0 & 0.010400864265275171 & 186.0 & 1101.0 & 5574.0 & 9236.0 \\
\hline
\end{tabular}


APPENDIX C 


\section{Appendix D}

\section{Cover Song Identification: unique top-50 results}

\begin{tabular}{|c|c|c|c|c|c|}
\hline position & MR (median) & $\begin{array}{l}\text { MR (median } \\
\text { absolute deviation) }\end{array}$ & extractor & aggregator & distance \\
\hline 1 & 41.58 & 36.19 & chroma_stft & diff_stats_1 & cosine_distance_oti \\
\hline 2 & 41.99 & 36.62 & chroma ${ }^{-}$stft & basic stats 1 & $\operatorname{cosine}^{-}$distance $^{-}$oti \\
\hline 3 & 42.36 & 25.68 & beat synchronous chroma cens & basic ${ }^{-}$stats ${ }^{-} 2$ & manhātan distance oti \\
\hline 4 & 42.44 & 27.49 & beat ${ }^{-}$synchronous ${ }^{-}$chroma ${ }^{-}$cens & basic ${ }^{-}$stats ${ }^{-} 2$ & euclidean $\overline{\text { distance }} \overline{\text { oti }}$ \\
\hline 5 & 42.46 & 37.2 & chroma stft & diff $\overline{\text { stats }} \overline{1}$ & manhattan distance oti \\
\hline 6 & 42.68 & 26.99 & beat synchronous chroma cens & basic stats 2 & cosine distance oti \\
\hline 7 & 42.72 & 33.87 & chroma_stft & statistical summarization & manhattan distance oti \\
\hline 8 & 43.02 & 37.14 & chroma ${ }^{-}$stft & diff stats 1 & euclidean $\overline{\text { distance }} \overline{\mathrm{oti}}$ \\
\hline 9 & 43.08 & 31.03 & chroma ${ }^{-}$stft & statistical ${ }^{-}$summarization & cosine distance ot $\overline{\mathrm{i}}$ \\
\hline 10 & 43.36 & 37.07 & chroma ${ }^{-}$stft & basic stats 1 & chebyshev distance oti \\
\hline 11 & 43.36 & 37.08 & chroma ${ }^{-}$stft & diff $\overline{\text { stats }} \overline{1}$ & chebyshev ${ }^{-}$distance ${ }^{-}$oti \\
\hline 12 & 43.36 & 37.09 & chroma ${ }^{-}$stft & basic stats 2 & chebyshev ${ }^{-}$distance ${ }^{-}$oti \\
\hline 13 & 43.45 & 36.2 & chroma ${ }^{-}$stft & statistical summarization & chebyshev ${ }^{-}$distance ${ }^{-}$oti \\
\hline 14 & 43.92 & 38.47 & chroma ${ }^{-}$stft & basic stats 2 & cosine distance oti ${ }^{-}$ \\
\hline 15 & 44.5 & 38.43 & chroma ${ }^{-}$stft & basic ${ }^{-}$stats 1 & euclidean distance oti \\
\hline 16 & 44.53 & 38.41 & chroma ${ }^{-}$stft & basic $^{-}$stats $^{-} 2$ & euclidean ${ }^{-}$distance ${ }^{-}$oti \\
\hline 17 & 44.78 & 25.24 & beat synchronous chroma cqt & diff $\overline{\text { stats }} \overline{1}$ & cosine distance ot $\overline{\mathrm{i}}$ \\
\hline 18 & 44.81 & 25.59 & beat ${ }^{-}$synchronous ${ }^{-}$chroma ${ }^{-}$cqt & basic stats 1 & cosine $^{-}$distance ${ }^{-}$oti \\
\hline 19 & 45.0 & 26.43 & beat ${ }^{-}$synchronous ${ }^{-}$chroma $^{-}$cens & diff $\overline{\text { stats }} \overline{1}$ & cosine $^{-}$distance ${ }^{-}$oti \\
\hline 20 & 45.01 & 36.2 & chroma stft & statistical ${ }^{-}$summarization & euclidean distance oti \\
\hline 21 & 45.06 & 30.92 & beat synchronous chroma cens & basic stats 1 & euclidean distance ${ }^{-}$oti \\
\hline 22 & 45.06 & 31.05 & beat ${ }^{-}$synchronous ${ }^{-}$chroma ${ }^{-}$cens & basic ${ }^{-}$stats -1 & cosine distance ot $\overline{\mathrm{i}}$ \\
\hline 23 & 45.15 & 26.32 & beat ${ }^{-}$synchronous chroma cens & diff $\overline{\text { stats }} \overline{1}$ & euclidean distance oti \\
\hline 24 & 45.15 & 37.43 & chroma stft - & basic stats $^{2}$ & manhattan distance oti \\
\hline 25 & 45.16 & 37.36 & chroma ${ }^{-}$stft & basic ${ }^{-}$stats 1 & manhattan ${ }^{-}$distance ${ }^{-}$oti \\
\hline 26 & 45.29 & 38.24 & beat synchronous chroma cens & basic ${ }^{-}$stats ${ }^{-} 2$ & manhattan ${ }^{-}$distance $^{-}$ \\
\hline 27 & 45.37 & 24.42 & beat ${ }^{-}$synchronous ${ }^{-}$chroma ${ }^{-}$cqt & diff $\overline{\text { stats }} \overline{1}$ & manhattan distance oti \\
\hline 28 & 45.38 & 23.54 & beat synchronous chroma cqt & $\operatorname{diff}^{-}$stats $^{-} 2$ & manhattan ${ }^{-}$distance ${ }^{-}$oti \\
\hline 29 & 45.46 & 33.1 & chroma stft - & $\operatorname{diff}^{-}$stats $^{-} 1$ & manhattan ${ }^{-}$distance ${ }^{-}$ \\
\hline 30 & 45.62 & 25.44 & beat synchronous chroma cens & $\operatorname{diff}^{-}$stats $^{-} 2$ & cosine distance oti \\
\hline 31 & 45.83 & 25.15 & beat ${ }^{-}$synchronous ${ }^{-}$chroma $^{-}$cqt & basic stats 2 & manhattan distance oti \\
\hline 32 & 46.04 & 39.29 & beat ${ }^{-}$synchronous chroma ${ }^{-}$cens & basic ${ }^{-}$stats $^{-} 1$ & cosine distance \\
\hline 33 & 46.07 & 39.33 & beat ${ }^{-}$synchronous ${ }^{-}$chroma ${ }^{-}$cens & basic - stats $^{-1}$ & euclidean distance \\
\hline 34 & 46.09 & 37.84 & chroma cqt & diff $\overline{\text { stats }} \overline{1}$ & manhattan distance \\
\hline 35 & 46.17 & 28.43 & beat synchronous chroma cens & basic $\quad$ stats 1 & manhattan - distance oti \\
\hline 36 & 46.19 & 39.2 & chroma cens & diff $\overline{\text { stats }} \overline{1}$ & cosine distance \\
\hline 37 & 46.2 & 39.21 & chroma ${ }^{-}$cens & $\operatorname{diff}^{-}$stats $^{-} 1$ & euclidean distance \\
\hline 38 & 46.22 & 39.23 & chroma ${ }^{-}$cens & basic stats 1 & cosine distance \\
\hline 39 & 46.24 & 39.24 & chroma ${ }^{-}$cens & basic $^{-}$stats $^{-} 1$ & euclidean distance \\
\hline 40 & 46.26 & 24.61 & beat synchronous chroma cens & diff $\overline{\text { stats }} \overline{1}$ & manhattan distance oti \\
\hline 41 & 46.26 & 38.26 & beat ${ }^{-}$synchronous ${ }^{-}$chroma ${ }^{-}$cens & $\operatorname{diff}^{-}$stats $^{-} 1$ & cosine distance \\
\hline 42 & 46.34 & 37.97 & beat ${ }^{-}$synchronous ${ }^{-}$chroma $^{-}$cens & diff $^{-}$stats $^{-} 1$ & euclidean distance \\
\hline 43 & 46.42 & 39.62 & chroma cens & diff $^{-}$stats $^{-} 1$ & manhattan distance \\
\hline 44 & 46.48 & 25.32 & beat synchronous chroma cqt & diff $^{-}$stats $^{-} 1$ & euclidean distance oti \\
\hline 45 & 46.48 & 38.95 & chroma cqt & $\operatorname{diff}^{-}$stats $^{-} 1$ & euclidean ${ }^{-}$distance ${ }^{-}$ \\
\hline 46 & 46.55 & 39.74 & chroma ${ }^{-}$cens & basic stats 1 & manhattan distance \\
\hline 47 & 46.61 & 41.75 & beat synchronous chroma cens & diff $\overline{\text { stats }} \overline{2}$ & manhattan - distance oti \\
\hline 48 & 46.74 & 37.09 & chroma cqt & vector quantization default & cosine distance \\
\hline 49 & 46.77 & 24.02 & beat synchronous chroma cqt & basic stats 2 & euclidean distance oti \\
\hline 50 & 46.8 & 39.94 & chroma cqt & statistical summarization & euclidean ${ }^{-}$distance \\
\hline
\end{tabular}


APPENDIX D 


\section{Appendix E}

\section{Cover Song Identification: unique filtered results}

\begin{tabular}{|c|c|c|c|c|c|}
\hline original position & MR (median) & $\begin{array}{l}\text { MR (median } \\
\text { absolute deviation) }\end{array}$ & extractor & aggregator & distance \\
\hline 1 & 41.58 & 36.19 & chroma_stft & diff_stats_1 & cosine_distance_oti \\
\hline 2 & 41.99 & 36.62 & chroma ${ }^{-}$stft & basic stats 1 & $\operatorname{cosine}^{-}$distance ${ }^{-}$oti \\
\hline 3 & 42.36 & 25.68 & beat synchronous chroma cens & basic $^{-}$stats $^{-} 2$ & manhattan distance oti \\
\hline 48 & 46.74 & 37.09 & chroma cqt - & vector quantization default & cosine distance \\
\hline 67 & 47.58 & 39.61 & pitch contour segmentation & octave abstraction - & lcs circular mean \\
\hline 69 & 47.72 & 38.41 & tonnetz & diff stats 1 & manhattan distance \\
\hline 126 & 49.68 & 28.96 & beat synchronous chroma cens & statistical ${ }^{-}$summarization 2 & monte carlo distance \\
\hline 202 & 53.56 & 14.32 & spectral_contrast - & statistical_summarization_ ${ }^{-} 2$ & earth_movers distance \\
\hline 238 & 55.29 & 33.35 & rhythm histogram & bypass -- & cosine distance \\
\hline 311 & 59.58 & 36.7 & $\mathrm{mfcc}$ & statistical summarization 2 & monte ${ }^{-}$carlo distance \\
\hline 319 & 59.93 & 44.35 & chroma_stft & gaussian_mixture_model_default & manhāttan_distance \\
\hline 386 & 64.25 & 22.06 & zero_crōssing_rate & basic_stāts_1 $\quad-\quad-$ & chebyshev_distance \\
\hline 403 & 65.41 & 40.87 & temporal_rhythm histogram & bypass $-\overline{-}$ & cosine_distance \\
\hline 413 & 66.42 & 52.32 & pitch contour segmentation & markov chain & circular manhattan \\
\hline 422 & 66.79 & 51.52 & melspectrogram & basic stats 1 & cosine $\overline{\text { distance }}$ \\
\hline 461 & 69.1 & 26.23 & $\mathrm{rms}$ & diff $\overline{\text { stats }} \overline{1}$ & $\operatorname{cosine}^{-}$distance \\
\hline
\end{tabular}


APPENDIX E 


\section{Bibliography}

$\left[\mathrm{AP}^{+} 02\right]$ Jean-Julien Aucouturier, Francois Pachet, et al. Music similarity measures: What's the use? In ISMIR, pages 13-17, 2002. 2, 3, 59, 60

[AP04] Jean-Julien Aucouturier and Francois Pachet. Improving timbre similarity: How high is the sky? Journal of Negative Results in Speech and Audio Sciences, 2004. 32

[Bel57] Richard Bellman. Dynamic Programming. Princeton University Press, 1957. 5

[Bel07] Juan Pablo Bello. Audio-based cover song retrieval using approximate chord sequences: Testing shifts, gaps, swaps and beats. In ISMIR, volume 7, pages 239-244, 2007. 37

[Bel10] Ron Weiss Juan Bello. Identifying repeated patterns in music using sparse convolutive non-negative matrix factorization. In 11th Int Society for Music Information Retrieval Conf, pages 123-128, 2010. 70

[BKAB10] RG Bachu, S Kopparthi, B Adapa, and Buket D Barkana. Voiced/unvoiced decision for speech signals based on zero-crossing rate and energy. In Advanced Techniques in Computing Sciences and Software Engineering, pages 279-282. Springer, 2010. 16

[BL81] Bladon and Bjorn Lindblom. Modeling the judgment of vowel quality differences. Journal of the Acoustical Society of America, 1981. 18

[BLEW04] Adam Berenzweig, Beth Logan, Daniel PW Ellis, and Brian Whitman. A large-scale evaluation of acoustic and subjective music-similarity measures. Computer Music Journal, 28(2):63-76, 2004. 2, 3, 59, 60

[BPVT08] Alessandro Bozzon, Giorgio Prandi, Giuseppe Valenzise, and Marco Tagliasacchi. A music recommendation system based on semantic audio segments similarity. Proceeding of Internet and Multimedia Systems and Applications-2008, 2008. 4, 50, 136

[BW05] Mark A Bartsch and Gregory H Wakefield. Audio thumbnailing of popular music using chroma-based representations. IEEE Transactions on multimedia, 7(1):96-104, 2005. 70

[BWFW04] Bernhard Burgeth, Martin Welk, Christian Feddern, and Joachim Weickert. Morphological operations on matrix-valued images. In European Conference on Computer Vision, pages 155-167. Springer, 2004. 50

[CF02] Matthew Cooper and Jonathan Foote. Automatic music summarization via similarity analysis. In ISMIR, 2002. 70

[Che00] Elaine Chew. Towards a mathematical model of tonality. PhD thesis, Massachusetts Institute of Technology, 2000. 13, 14

[CLX17] Ning Chen, Mingyu Li, and Haidong Xiao. Two-layer similarity fusion model for cover song identification. EURASIP Journal on Audio, Speech, and Music Processing, 2017(1):1-15, 2017. 3, 37, 56, 63, 96, 121, 127 
[CLX18] Ning Chen, Wei Li, and Haidong Xiao. Fusing similarity functions for cover song identification. Multimedia Tools and Applications, 77(2):2629-2652, 2018. xiv, 3, 42, 43

[DBEJ08] J Stephen Downie, Mert Bay, Andreas F Ehmann, and M Cameron Jones. Audio cover song identification: Mirex 2006-2007 results and analyses. In ISMIR, pages 468-474, 2008. 44

[DBVB17] Michael Defferrard, Kirell Benzi, Pierre Vandergheynst, and Xavier Bresson. Fma: A dataset for music analysis. In Proceedings of the 18th International Society for Music Information Retrieval Conference, 2017. 82

[DRD09] Jean-Louis Durrieu, Gaël Richard, and Bertrand David. An iterative approach to monaural musical mixture de-soloing. In 2009 IEEE International Conference on Acoustics, Speech and Signal Processing, pages 105-108. IEEE, 2009. 56

[DS14] Sander Dieleman and Benjamin Schrauwen. End-to-end learning for music audio. In 2014 IEEE International Conference on Acoustics, Speech and Signal Processing (ICASSP), pages 6964-6968. IEEE, 2014. 5

[Dub04] Shlomo Dubnov. Generalization of spectral flatness measure for non-gaussian linear processes. IEEE Signal Processing Letters, 11(8):698-701, 2004. 12

[EC07] Daniel PW Ellis and Courtenay Valentine Cotton. The 2007 labrosa cover song detection system. 2007. 37

[El107a] Dan Ellis. Chroma feature analysis and synthesis. Resources of Laboratory for the Recognition and Organization of Speech and Audio-LabROSA, 2007. 5

[Ell07b] Daniel PW Ellis. Classifying music audio with timbral and chroma features. 2007. 9

[EP07] Daniel PW Ellis and Graham E Poliner. Identifyingcover songs' with chroma features and dynamic programming beat tracking. In 2007 IEEE International Conference on Acoustics, Speech and Signal Processing-ICASSP'07, volume 4, pages IV-1429. IEEE, 2007. 38

[ET12] Daniel PW Ellis and Bertin-Mahieux Thierry. Large-scale cover song recognition using the $2 \mathrm{~d}$ fourier transform magnitude. 2012. 127

[Fal19] Kim Falk. Practical recommender systems. Manning Publications, 2019. 45

[Fas82] Hugo Fastl. Fluctuation strength and temporal masking patterns of amplitudemodulated broadband noise. Hearing Research, 1982. 18

[FDLR10] Rémi Foucard, Jean-Louis Durrieu, Mathieu Lagrange, and Gäel Richard. Multimodal similarity between musical streams for cover version detection. In 2010 IEEE International Conference on Acoustics, Speech and Signal Processing, pages 5514-5517. IEEE, 2010. xv, xvii, 4, 56, 57, 72

[FM05] Klaus Frieler and Daniel Müllensiefen. The simile algorithm for melodic similarity. Proceedings of the Annual Music Information Retrieval Evaluation exchange, 2005. 3, 59

[Foo97] Jonathan T Foote. Content-based retrieval of music and audio. In Multimedia Storage and Archiving Systems II, volume 3229, pages 138-147. International Society for Optics and Photonics, 1997. 2, 3, 59, 60 
[Foo00] Jonathan Foote. Automatic audio segmentation using a measure of audio novelty. In 2000 IEEE International Conference on Multimedia and Expo. ICME2000. Proceedings. Latest Advances in the Fast Changing World of Multimedia (Cat. No. 00TH8532), volume 1, pages 452-455. IEEE, 2000. 50

[FR21] Mohamadreza Sheikh Fathollahi and Farbod Razzazi. Music similarity measurement and recommendation system using convolutional neural networks. International Journal of Multimedia Information Retrieval, 10(1):43-53, 2021. 2

[GFK05] Todor Ganchev, Nikos Fakotakis, and George Kokkinakis. Comparative evaluation of various mfcc implementations on the speaker verification task. In Proceedings of the 10th International Conference on Speach and Computer, 2005. 9

[GHO06] Emilia Gómez, Perfecto Herrera, and Beesuan Ong. Automatic tonal analysis from music summaries for version identification. In Audio Engineering Society Convention 121. Audio Engineering Society, 2006. xiv, 3, 37, 38

[GLCS95] Asif Ghias, Jonathan Logan, David Chamberlin, and Brian C Smith. Query by humming: Musical information retrieval in an audio database. In Proceedings of the third ACM international conference on Multimedia, pages 231-236, 1995. 2, 3, 59, 60

[GLSC95] Asif Ghias, Jonathan Logan, Brian Smith, and David Chamberlin. Query by humming: Musical information retrieval in an audio database. In Proceedings of the 3rd ACM International Conference on Multimedia, 1995. 27

[Góm06] Emilia Gómez. Tonal description of polyphonic audio for music content processing. INFORMS Journal on Computing, 18(3):294-304, 2006. 38, 51

[Got03] Masataka Goto. A chorus-section detecting method for musical audio signals. In 2003 IEEE International Conference on Acoustics, Speech, and Signal Processing, 2003. Proceedings.(ICASSP'03)., volume 5, pages V-437. IEEE, 2003. 38

$\left[\mathrm{GPD}^{+} 00\right]$ Fabien Gouyon, François Pachet, Olivier Delerue, et al. On the use of zero-crossing rate for an application of classification of percussive sounds. In Proceedings of the COST G-6 conference on Digital Audio Effects (DAFX-00), Verona, Italy, volume 5, 2000. 16,59

[HCCP06] Wei Han, Cheong-Fat Chan, Chiu-Sing Choy, and Kong-Pang Pun. An efficient mfcc extraction method in speech recognition. In IEEE International Symposium on Circuits and Systems, 2006. 9

[HKKL17] Hoon Heo, Hyunwoo J Kim, Wan Soo Kim, and Kyogu Lee. Cover song identification with metric learning using distance as a feature. In ISMIR, pages 628-634, 2017. 2, 135

[HKVM20] Romain Hennequin, Anis Khlif, Felix Voituret, and Manuel Moussallam. Spleeter: a fast and efficient music source separation tool with pre-trained models. Journal of Open Source Software, 5(50):2154, 2020. Deezer Research. 136

[HRG01] Holger H Hoos, Kai Renz, and Marko Görg. Guido/mir-an experimental musical information retrieval system based on guido music notation. In ISMIR, pages 41-50, 2001. 3,60

[HSG06] Christopher Harte, Mark Sandler, and Martin Gasser. Detecting harmonic change in musical audio. In Proceedings of the 1st ACM workshop on Audio and music computing multimedia, pages 21-26, 2006. xiii, 14, 15 
[ISY12] Chadawan Ittichaichareon, Siwat Suksri, and Thaweesak Yingthawornsuk. Speech recognition using mfcc. In International Conference on Computer Graphics, Simulation and Modeling, 2012. 9

[JCEJ08] Jesper Hojvang Jensen, Mads G Christensen, Daniel PW Ellis, and Soren Holdt Jensen. A tempo-insensitive distance measure for cover song identification based on chroma features. In 2008 IEEE International Conference on Acoustics, Speech and Signal Processing, pages 2209-2212. IEEE, 2008. xiv, 2, 3, 38, 39, 59

[JLZ ${ }^{+}$02] Dan-Ning Jiang, Lie Lu, Hong-Jiang Zhang, Jian-Hua Tao, and Lian-Hong Cai. Music type classification by spectral contrast feature. In Proceedings. IEEE International Conference on Multimedia and Expo, volume 1, pages 113-116. IEEE, 2002. 12, 90

[Kel12] Matthew Kelly. Evaluation of melody similarity measures. PhD thesis, 2012. 3, 60

[KL51] S. Kullback and R. A. Leibler. On information and sufficiency. The Annals of Mathematical Statistics, 1951. 30

[KS13] Peter Knees and Markus Schedl. A survey of music similarity and recommendation from music context data. ACM Transactions on Multimedia Computing, Communications, and Applications (TOMM), 10(1):1-21, 2013. 2, 3, 60

[KS16] Peter Knees and Markus Schedl. Music Similarity and Retrieval: An Introduction to Audio- and Web-based Strategies. Springer, 2016. 2, 30, 33

[Ler12] Alexander Lerch. An introduction to audio content analysis: Applications in signal processing and music informatics. Wiley-IEEE Press, 2012. 10

[LH06] Cynthia CS Liem and Alan Hanjalic. Cover song retrieval: a comparative study of system component choices. system, 3(1), 2006. 37

[Lid07] Thomas Lidy. Music information retrieval: Audio feature extraction. http://ifs. tuwien.ac.at/mir/audiofeatureextraction.html, 2007. Accessed: 2020-06-12. 20, 21

[LO04] Tao Li and Mitsunori Ogihara. Content-based music similarity search and emotion detection. In 2004 IEEE International Conference on Acoustics, Speech, and Signal Processing, volume 5, pages V-705. IEEE, 2004. 2, 3, 59, 60

[LR05] Thomas Lidy and Andreas Rauber. Evaluation of feature extractors and psychoacoustic transformations for music genre classification. In Proceedings of the 6th International Conference on Music Information Retrieval, 2005. 19

[LS01] Beth Logan and Ariel Salomon. A music similarity function based on signal analysis. In ICME, pages 22-25, 2001. 2, 3, 59, 60

[LS06] Mark Levy and Mark Sandler. Application of segmentation and thumbnailing to music browsing and searching. In Audio Engineering Society Convention 120. Audio Engineering Society, 2006. 4, 49

[LS08] Mark Levy and Mark Sandler. Structural segmentation of musical audio by constrained clustering. IEEE transactions on audio, speech, and language processing, 16(2):318-326, 2008. 70

[LS10] Mathieu Lagrange and Joan Serrà. Unsupervised accuracy improvement for cover song detection using spectral connectivity network. 2010. 37 
[LSC06] Mark Levy, Mark Sandler, and Michael Casey. Extraction of high-level musical structure from audio data and its application to thumbnail generation. In 2006 IEEE International Conference on Acoustics Speech and Signal Processing Proceedings, volume 5, pages V-V. IEEE, 2006. 49

[LU00] Kjell Lemström and Esko Ukkonen. Including interval encoding into edit distance based music comparison and retrieval. In Proc. AISB, pages 53-60. Citeseer, 2000. 3, 60

[Mar05] Matija Marolt. Audio melody extraction based on timbral similarity of melodic fragments. In EUROCON 2005-The International Conference on" Computer as a Tool", volume 2, pages 1288-1291. IEEE, 2005. 40

[Mar06] Matija Marolt. A mid-level melody-based representation for calculating audio similarity. In ISMIR, pages 280-285, 2006. 3, 40, 59

[ME11] Meinard Müller and Sebastian Ewert. Chroma toolbox: Matlab implementations for extracting variants of chroma-based audio features. In Proceedings of the 12th International Conference on Music Information Retrieval (ISMIR), 2011. hal-00727791, version 2-22 Oct 2012. Citeseer, 2011. 8

[MEK09] Meinard Muller, Sebastian Ewert, and Sebastian Kreuzer. Making chroma features more robust to timbre changes. In 2009 IEEE International Conference on Acoustics, Speech and Signal Processing, pages 1877-1880. IEEE, 2009. 9

[MF05] Cory McKay and I Fujinaga. Automatic music classification and similarity analysis. In International Conference on Music Information Retrieval. Citeseer, 2005. 3, 59

[MND09] Matthias Mauch, Katy C Noland, and Simon Dixon. Using musical structure to enhance automatic chord transcription. In ISMIR, pages 231-236, 2009. 70

[MSC19] Manan Mehta, Anmol Sajnani, and Radhika Chapaneri. Cover song identification with pairwise cross-similarity matrix using deep learning. In 2019 IEEE Bombay Section Signature Conference (IBSSC), pages 1-5. IEEE, 2019. 2

[Mül15] Meinard Müller. Fundamentals of music processing: Audio, analysis, algorithms, applications. Springer, 2015. 5

$\left[\mathrm{ODZ}^{+} 16\right]$ Aaron van den Oord, Sander Dieleman, Heiga Zen, Karen Simonyan, Oriol Vinyals, Alex Graves, Nal Kalchbrenner, Andrew Senior, and Koray Kavukcuoglu. Wavenet: A generative model for raw audio. arXiv preprint arXiv:1609.03499, 2016. 5

[OH05] Bee Suan Ong and Perfecto Herrera. Semantic segmentation of music audio. In Proceedings of the... International Computer Music Conference, page 61. Computer Music Association, 2005. 50

[PA04] Francois Pachet and Jean-Julien Aucouturier. Improving timbre similarity: How high is the sky. Journal of negative results in speech and audio sciences, 1(1):1-13, 2004. $2,3,59,60$

[Pam06] Elias Pampalk. Computational Models of Music Similarity and their Application in Music Information Retrieval. PhD thesis, Vienna University of Technology, 2006. 9

$\left[\mathrm{PBD}^{+} 16\right]$ Maria Panteli, Emmanouil Benetos, Simon Dixon, et al. Learning a feature space for similarity in world music. ISMIR, 2016. 2, 135

[PD $\left.{ }^{+} 16\right]$ Maria Panteli, Simon Dixon, et al. On the evaluation of rhythmic and melodic descriptors for music similarity. 2016. 78,79 
[Pee04] Geoffroy Peeters. A large set of audio features for sound description (similarity and classification) in the cuidado project. CUIDADO IST Project Report, 54(0):1-25, 2004. $11,12,16$

$\left[\mathrm{PFW}^{+} 05\right]$ Elias Pampalk, Arthur Flexer, Gerhard Widmer, et al. Improvements of audio-based music similarity and genre classificaton. In ISMIR, volume 5, pages 634-637. London, UK, 2005. 3, 59

[PG79] Martin Piszczalski and Bernard A Galler. Predicting musical pitch from component frequency ratios. The Journal of the Acoustical Society of America, 66(3):710-720, 1979. 40

$\left[\mathrm{PNP}^{+} 17\right]$ Jordi Pons, Oriol Nieto, Matthew Prockup, Erik Schmidt, Andreas Ehmann, and Xavier Serra. End-to-end learning for music audio tagging at scale. arXiv preprint arXiv:1711.02520, 2017. 5

[PRM02a] Elias Pampalk, Andreas Rauber, and Dieter Merkl. Content-based organization and visualization of music archives. In Proceedings of the tenth ACM international conference on Multimedia, pages 570-579, 2002. xiii, 3, 18, 59

[PRM02b] Elias Pampalk, Andreas Rauber, and Dieter Merkl. Content-based organization and visualization of music archives. In Proceedings of the ACM International Conference on Multimedia, 2002. 18

[PT05] Costas Panagiotakis and Georgios Tziritas. A speech/music discriminator based on rms and zero-crossings. IEEE Transactions on multimedia, 7(1):155-166, 2005. 3, 59

[RE10] Suman Ravuri and Daniel PW Ellis. Cover song detection: from high scores to general classification. In 2010 IEEE International Conference on Acoustics, Speech and Signal Processing, pages 65-68. IEEE, 2010. xv, 2, 3, 37, 45, 46, 59

[RH06a] Seungmin Rho and Eenjun Hwang. Fmf: Query adaptive melody retrieval system. Journal of Systems and Software, 79(1):43-56, 2006. 3, 59, 60

[RH06b] Seungmin Rho and Eenjun Hwang. Fmf: Query adaptive melody retrieval system. The Journal of Systems and Software, 2006. 27

[SAH79] Manfred Schroeder, Bishnu Atal, and Hall. Optimizing digital speech coders by exploiting masking properties of the human ear. Journal of the Acoustical Society of America, 1979. 18

[SdSB15] Diego Furtado Silva, Vinícius MA de Souza, and Gustavo EAPA Batista. Music shapelets for fast cover song recognition. In ISMIR, pages 441-447, 2015. 4, 54

[Sey10a] Klaus Seyerlehner. Content-based music recommender systems: Beyond simple framelevel audio similarity. na, 2010. xv, 4, 55, 56, 71

[Sey10b] Klaus Seyerlehner. Content-Based Music Recommender Systems: Beyond simple Frame-Level Audio Similarity. PhD thesis, Johannes Kepler University Linz, 2010. 80

[SFW12] Dominik Schnitzer, Arthur Flexer, and Gerhard Widmer. A fast audio similarity retrieval method for millions of music tracks. Multimedia Tools and Applications, 58(1):23-40, 2012. 2, 3, 59, 60

[SG07] Joan Serrà and Emilia Gómez. A cover song identification system based on sequences of tonal descriptors. Music Information Retrieval Evaluation eXchange (MIREX), 2007. 46 
[SG12a] Justin Salamon and Emilia Gomez. Melody extraction from polyphonic music signals using pitch contour characteristics. IEEE Transactions on Audio, Speech and Language Processing, 2012. 17

[SG12b] Justin Salamon and Emilia Gómez. Melody extraction from polyphonic music signals using pitch contour characteristics. IEEE Transactions on Audio, Speech, and Language Processing, 20(6):1759-1770, 2012. 46

[SGH10] Joan Serrà, Emilia Gómez, and Perfecto Herrera. Audio cover song identification and similarity: background, approaches, evaluation, and beyond. In Advances in Music Information Retrieval, pages 307-332. Springer, 2010. 3, 35, 36, 37, 56, 96, 121

[SGHS08] Joan Serrà, Emilia Gómez, Perfecto Herrera, and Xavier Serra. Chroma binary similarity and local alignment applied to cover song identification. IEEE Transactions on Audio, Speech, and Language Processing, 16(6):1138-1151, 2008. 2, 38, 46, 59

[SHD19] Marc Sarfati, Anthony Hu, and Jonathan Donier. Ensemble-based cover song detection. arXiv preprint arXiv:1905.11700, 2019. 44

[SK10] Christian Schörkhuber and Anssi Klapuri. Constant-q transform toolbox for music processing. In 7th Sound and Music Computing Conference, Barcelona, Spain, pages 3-64, 2010. 7

[SMGA12] Joan Serra, Meinard Müller, Peter Grosche, and Josep Lluis Arcos. Unsupervised detection of music boundaries by time series structure features. In Proceedings of the AAAI Conference on Artificial Intelligence, volume 26, 2012. xv, 51, 52, 53

[SSA09] Joan Serra, Xavier Serra, and Ralph G Andrzejak. Cross recurrence quantification for cover song identification. New Journal of Physics, 11(9):093017, 2009. 41, 42, 46, 127

[SSG12] Justin Salamon, Joan Serrá, and Emilia Gómez. Melody, bass line, and harmony representations for music version identification. In Proceedings of the 21st International Conference on World Wide Web, pages 887-894, 2012. xv, 3, 37, 38, 46, 47, 56, 96, 121

[SSG13a] Justin Salamon, Joan Serra, and Emilia Gómez. Tonal representations for music retrieval: from version identification to query-by-humming. International Journal of Multimedia Information Retrieval, 2(1):45-58, 2013. 3, 59, 60

[SSG13b] Justin Salamon, Joan SerrÃ , and Emilia Gomez. Tonal representations for music retrieval: From version identification to query-by-humming. International Journal of Multimedia Information Retrieval, 2013. 26

[SSPK10] Klaus Seyerlehner, Markus Schedl, Tim Pohle, and Peter Knees. Using block-level features for genre classification, tag classification and music similarity estimation. Submission to Audio Music Similarity and Retrieval Task of MIREX, 2010, 2010. 2, $3,59,60$

[SULM19] Fabian-Robert Stöter, Stefan Uhlich, Antoine Liutkus, and Yuki Mitsufuji. Openunmix-a reference implementation for music source separation. 2019. 71, 72, 123

[SVN37] Stanley Smith Stevens, John Volkmann, and Edwin B Newman. A scale for the measurement of the psychological magnitude pitch. The Journal of the Acoustical Society of America, 8(3):185-190, 1937. 8 
[SWW08] Malcolm Slaney, Kilian Weinberger, and William White. Learning a metric for music similarity. In International Symposium on Music Information Retrieval (ISMIR), volume 148, 2008. 2, 135

[SYB $\left.{ }^{+} 16\right]$ Diego Furtado Silva, Chin-Chia Michael Yeh, Gustavo EAPA Batista, Eamonn J Keogh, et al. Simple: Assessing music similarity using subsequences joins. In ISMIR, pages 23-29, 2016. 127

[SZA09] Joan Serrà, Massimiliano Zanin, and Ralph G Andrzejak. Cover song retrieval by cross recurrence quantification and unsupervised set detection. 2009. 37

[TB15] Christopher J Tralie and Paul Bendich. Cover song identification with timbral shape sequences. arXiv preprint arXiv:150\%.05143, 2015. 41

[TC02a] George Tzanetakis and Perry Cook. Musical genre classification of audio signals. IEEE Transactions on speech and audio processing, 10(5):293-302, 2002. 71

[TC02b] George Tzanetakis and Perry Cook. Musical genre classification of audio signals. IEEE Transactions on Speech and Audio Processing, 2002. 77

[TEC01] George Tzanetakis, Georg Essl, and Perry Cook. Automatic musical genre classification of audio signals. In Proceedings of the 2nd international symposium on music information retrieval, Indiana, 2001. 10

[Tra17] Christopher J Tralie. Early mfcc and hpcp fusion for robust cover song identification. arXiv preprint arXiv:1707.04680, 2017. xiv, 2, 3, 41, 42, 44, 60, 127

[TWV05] Rainer Typke, Frans Wiering, and Remco C Veltkamp. Evaluating the earth mover's distance for measuring symbolic melodic similarity. In MIREX-ISMIR 2005: 6th International Conference on Music Information Retrieval. Citeseer, 2005. 2, 3, 60

[Tym09] Dmitri Tymoczko. Three conceptions of musical distance. In International Conference on Mathematics and Computation in Music, pages 258-272. Springer, 2009. 2, 59

$\left[\mathrm{TYW}^{+} 05\right]$ Wei-Ho Tsai, Hung-Ming Yu, Hsin-Min Wang, et al. Query-by-example technique for retrieving cover versions of popular songs with similar melodies. In ISMIR, volume 5, pages 183-190, 2005. 2, 3, 39, 40, 59

[UZ99] Alexandra Uitdenbogerd and Justin Zobel. Melodic matching techniques for large music databases. In Proceedings of the seventh ACM international conference on Multimedia (Part 1), pages 57-66, 1999. 3, 60

[VBBWV14] Jmh Van Balen, Dimitrios Bountouridis, Frans Wiering, and Remco C Veltkamp. Cognition-inspired descriptors for scalable cover song retrieval. In proceedings of the 15th international conference on Music Information Retrieval, 2014. 51, 52, 53

[WMD $\left.{ }^{+} 14\right]$ Bo Wang, Aziz M Mezlini, Feyyaz Demir, Marc Fiume, Zhuowen Tu, Michael Brudno, Benjamin Haibe-Kains, and Anna Goldenberg. Similarity network fusion for aggregating data types on a genomic scale. Nature methods, 11(3):333, 2014. 41

[YK09] Lexiang Ye and Eamonn Keogh. Time series shapelets: a new primitive for data mining. In Proceedings of the 15th ACM SIGKDD international conference on Knowledge discovery and data mining, pages 947-956, 2009. 54, 55

[YXCY20] Zhesong Yu, Xiaoshuo Xu, Xiaoou Chen, and Deshun Yang. Learning a representation for cover song identification using convolutional neural network. In ICASSP 2020-2020 IEEE International Conference on Acoustics, Speech and Signal Processing (ICASSP), pages 541-545. IEEE, 2020. 2, 135 
$\left[\mathrm{YZL}^{+}{ }^{+10]}\right.$ Xiaoqing Yu, Jing Zhang, Junwei Liu, Wanggen Wan, and Wei Yang. An audio retrieval method based on chromagram and distance metrics. In 2010 International Conference on Audio, Language and Image Processing, pages 425-428. IEEE, 2010. $2,3,59,60$

[ZF13] Eberhard Zwicker and Hugo Fastl. Psychoacoustics: Facts and models. Springer, 2013. 18 